NIST Special Publication 260-187

\title{
Certification of SRM 2493: Standard Reference Mortar for Rheological Measurements
}

\author{
Alex Olivas \\ Chiara F. Ferraris \\ Nicos S. Martys \\ William L. George \\ Edward J. Garboczi \\ Blaza Toman
}




\title{
Certification of SRM 2493: Standard Reference Mortar for Rheological Measurements
}

\author{
Alex Olivas \\ Chiara F. Ferraris \\ Nicos S. Martys \\ Materials and Structural Systems Division \\ Engineering Laboratory \\ William L. George \\ Applied and Computational Mathematics Division \\ Information Technology Laboratory \\ Edward J. Garboczi \\ Applied Chemicals and Materials Division \\ Material Measurement Laboratory \\ Blaza Toman \\ Statistical Engineering Division \\ Information Technology Laboratory
}

This publication is available free of charge from: https://doi.org/10.6028/NIST.SP.260-187

October 2017

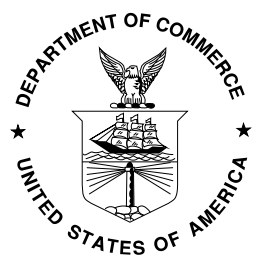

U.S. Department of Commerce Wilbur L. Ross, Jr., Secretary

National Institute of Standards and Technology Kent Rochford, Acting NIST Director and Under Secretary of Commerce for Standards and Technology 
Certain commercial entities, equipment, or materials may be identified in this document in order to describe an experimental procedure or concept adequately. Such identification is not intended to imply recommendation or endorsement by the National Institute of Standards and Technology, nor is it intended to imply that the entities, materials, or equipment are necessarily the best available for the purpose.

National Institute of Standards and Technology Special Publication 260-187

Natl. Inst. Stand. Technol. Spec. Publ. 260-187, 192 pages (October 2017) CODEN: NSPUE2

This publication is available free of charge from:

https://doi.org/10.6028/NIST.SP.260-187 


\section{$\underline{\text { Abstract }}$}

Rheological measurements are often performed using a rotational rheometer. In this type of rheometer, the tested fluid is sheared between two surfaces, one of which acts as the rotating surface [1]. Usually, the rotational velocity is imposed and the response of the material is monitored by the measurement of the torque. Typically, manufacturers of rheometers recommend using a standard oil of known viscosity to verify that the instrument is operating correctly. However, these oils are expensive, and they cannot be used for the large volumes employed in concrete rheometers. Furthermore, the oils do not contain aggregates and may not offer a valid representation of concrete since concrete is a suspension with solid particulates and display non-Newtonian behavior. Therefore, a relatively inexpensive, accurate standard reference material (SRM) for concrete rheometers is needed. The development of this new SRM is based on a multiphase approach: (1) the first phase established the SRM 2492 for cementitious paste mixtures [2], (2) the second phase developed and certified the mortar phase SRM 2493, and (3) the completion of an SRM 2497 for concrete mixtures will mark the third stage. This third stage is currently in progress.

The purpose of this report is to describe the process used to certify SRM 2493, a "Standard Reference Mortar for Rheological Measurements". The results of the rheological testing used to determine the rheological characteristics of SRM 2493 are discussed, along with the corresponding statistical analyses that was conducted to certify that the proposed models and reference values are valid. 


\section{Acknowledgements}

The authors would also like to thank some key persons at NIST without whom this certification could not have been completed: John "Rusty" Hettenhouser and Michelle Helsel. We would also like to thank Max Peltz for all of his assistance on properly running the equipment at the laboratory.

We would also like to acknowledge that this research used resources of the Argonne Leadership Computing Facility at Argonne National Laboratory, which is supported by the Office of Science of the U.S. Department of Energy under contract DE-AC02-06CH11357.

The authors would also like to thanks the reviewers Mr. Dale Bentz, Drs. Raissa Ferron and Kenneth Snyder for their detailed comments and discussions. 


\section{Table of Contents}

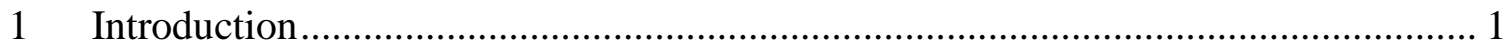

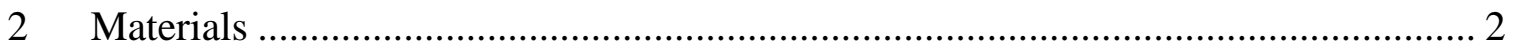

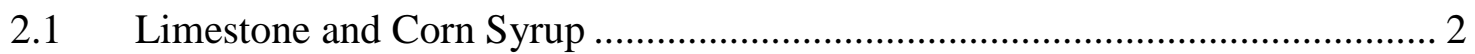

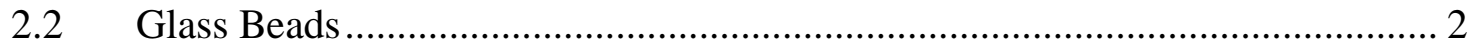

2.2.1 Sample Selection and Analysis Schedule ………………………………..... 2

2.2.2 Specimen Set-up for Shape Analysis ............................................................. 3

2.2.3 Light Microscope ................................................................................ 4

2.2.4 X-ray Computed Tomography and Spherical Harmonic Analysis ................... 6

2.2.5 Bead Analysis Results................................................................................. 9

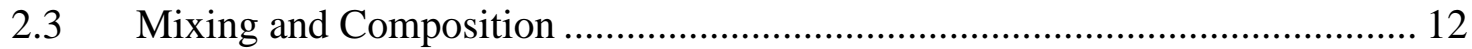

2.3.1 Instruction for the Certificate to Prepare the Mixture..................................... 13

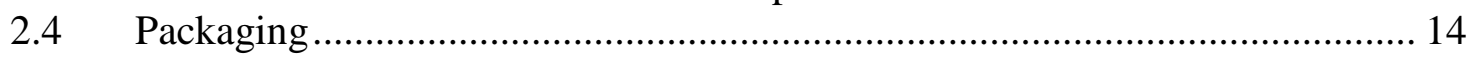

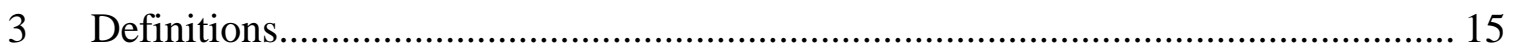

4 Modeling Approach ............................................................................................ 16

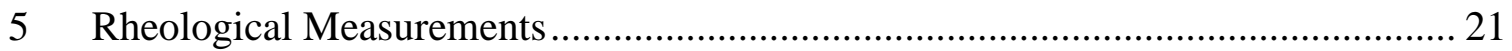

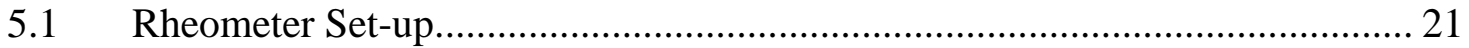

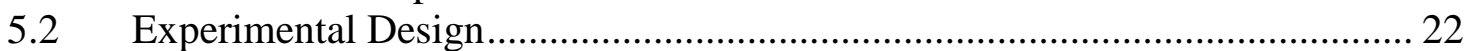

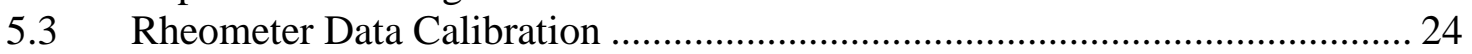

5.3.1 Calibration Based on the Bingham Parameters ........................................... 24

5.3.2 Calibration Using the Viscosity vs. Shear Rate Curve .................................. 27

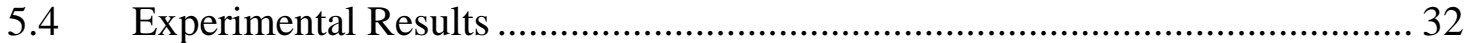

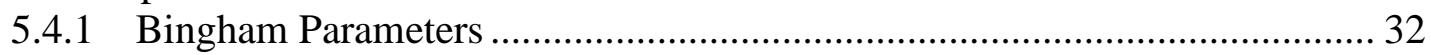

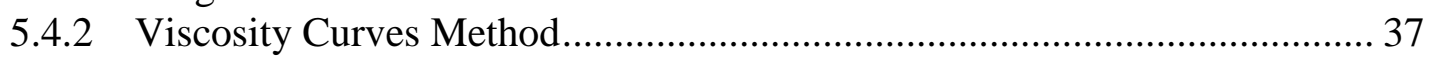

5.4.3 Comparison of Two Calibration Methods: Bingham or Viscosity Curves Fit 40

6 Comparison Between Model and Experimental ....................................................... 46

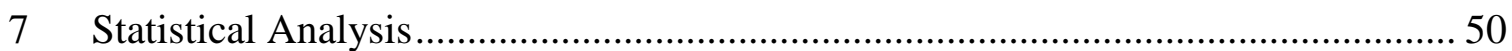

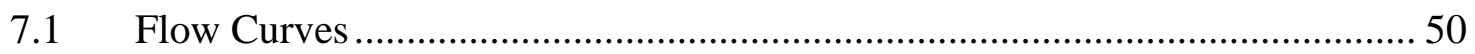

7.1.1 Uncertainty Calculation .......................................................................... 50

7.1.2 OpenBUGS Code.................................................................................... 57

7.1.3 Uncertainty of the Estimates of the Parameters of Equation 1 ….................. 57

7.2 Bingham Parameters ..................................................................................... 59

7.3 Flow Curve Fundamental Data in Fundamental Units .................................... 62

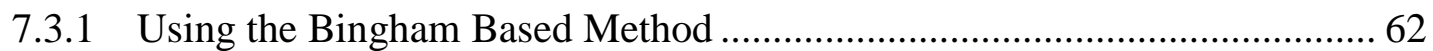

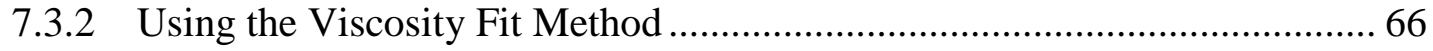

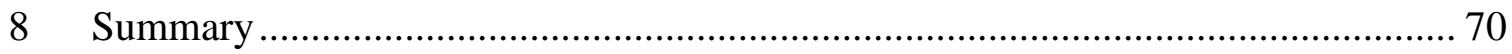

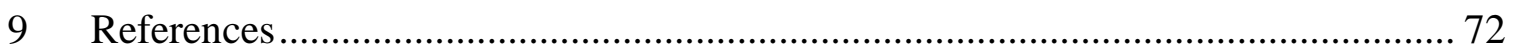

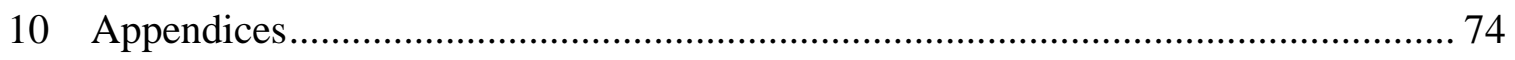

Appendix A: Original Data Measured from Rheometer..............................A-1

Appendix B: Data for Calibration................................................... 


\section{List of Figures}

Figure 1: Light microscope with adjustable lighting of the specimen. The black area under the specimen is the grid system shown in Figure 2.

Figure 2: Grid system placed under specimen allows easy selection of analysis areas. The grid system creates (row, col) coordinates for random selection analysis. .......5

Figure 3: Snap shot image taken with the light microscope. ....................................5

Figure 4: X-ray CT instrument. ......................................................................

Figure 5: A typical reconstructed slice from the X-ray CT results for bead sample D7. The image is $1000 \times 1000$ pixels, and the length per pixel is approximately $20.9 \mu \mathrm{m}$. Note the several non-spherical particles, one double particle, and one particle with internal porosity (marked with white circles).

Figure 6: Deformed beads from the two analysis methods. The LM method provided an uncertainty due to estimation, shown by error bars. The uncertainty for the CT data was estimated at $1 \%$.

Figure 7: An example of plunger mixer.

Figure 8: Numerical model simulation model data and predicted data from scaling parameters are compared to experimental data results (see Table 6 for values). The uncertainty is about $10 \%$ (see text)....

Figure 9: Three spindle types were used for testing, each representing a different family

Figure 10: Schematic for the calibration of rheometer data from raw torque and rotational speed to fundamental rheological variables, viscosity and shear rate...26

Figure 11: Screenshot of the running of the above software code and of the results for the SRM 2493 six-blade vane data using the method for fitting equation (1).

Figure 12: Schematic for the calibration of rheometer data from raw torque and rotational speed to fundamental rheological variables, viscosity and shear rate, applicable only to the linear portion of the curve.

Figure 13: Flow curve comparison of the calibrated experimental data for SRM 2492 paste ( $0 \%$ beads) for all three spindles. The flow curves are compared to their reference curve shown in black, SRM2492 certified values. Uncertainities are analyzed in Section 6.

Figure 14: Calibrated, by Bingham parameters, experimental flow curves for different geometries of spindles at bead volume concentrations of $0 \%, 20 \%$ and $40 \%$. The $0 \%$ curve (black) represents the certified SRM2492 flow curve values. Uncertainities are analyzed in Section 7

Figure 15: Viscosity curve at concentrations of $20 \%$ and $40 \%$ by volume of glass beads. The uncertainty is estimated to be $10 \%$ of the viscosity. The $0 \%$ curves are omitted for clarity. Full discussion of the uncertainties can be found in Section 6.

Figure 16: An enlargement of Figure 15 for the higher shear rates to better show the difference in viscosity depending on the spindle used. The $0 \%$ curves are omitted for clarity. Full discussion of the uncertainties can be found in Section 6. 
Figure 17: Flow curve comparison of the calibrated experimental data for SRM 2492 paste ( $0 \%$ beads) for all three spindles. The flow curves are compared to their reference curve shown in black, SRM2492 certified values, using the L factors shown in Table 11. Uncertainities are analyzed in Section 6.

Figure 18: Calibrated, by viscosity curve method, experimental flow curves for each spindle at bead volume concentrations of $0 \%, 20 \%$ and $40 \%$. The $0 \%$ curve (black) represents the certified SRM2492 flow curve values. Uncertainities are analyzed in Section 6.

Figure 19: Viscosity curve at concentrations of $20 \%$ and $40 \%$ by volume of glass beads. The uncertainty is estimated to be $10 \%$ of the viscosity. Full discussion on the uncertainties can be found in Section 7.

Figure 20: Graphic comparison between the curves obtained by Bingham (K factors) or by the viscosity curves ( $\mathrm{L}$ factors). Uncertainities are analyzed in Section 6 ......

Figure 21: Graphical representation of the data in Table 15 representing the relative viscosity as a function of the beads volume concentration and the spindle used. The uncertainity is as listed in the Tables 15.

Figure 22: Viscosity vs. shear rate (using the Viscosity fit method) for all data collected including the scaled prediction values determined from the numerical simulation model. The $0 \%$ data for the measurements were omitted for clarity. The uncertainties are discussed in Section 6.

Figure 23: Comparison of model vs experimental viscosity curves (obtained using the viscosity fit method) for A) $20 \%$ and B) $40 \%$ glass beads volume fraction mortar mixture. Relative viscosity, $\mu_{\mathrm{R}}$, was modeled with respect to the SRM 2492 (1 day) baseline curve at $20 \mathrm{~s}^{-1}$. The uncertainties are discussed in Section 6

Figure 24: Graphic representation of the relative viscosity from Table 15 and Table 16 , with the values for the model added. The uncertainities are also from these two tables with the model assigned an uncertainity of $10 \%$

Figure 25: Example of torque vs. rotational speed for $20 \%$ bead volume fraction using six-blade vane. The red line is the least squares line fitted to all of the points.

Figure 26 Example of fitted line and $95 \%$ uncertainty bounds for $20 \%$ beads using six-blade vane with data for mixture 1 , whose median value is shown by red line 


\section{List of Tables}

Table 1: Randomly selected bottles for bead analysis ..................................................... 3

Table 2: Schedule for bead analysis........................................................................... 3

Table 3: Grid boxes randomly selected for analysis. The coordinates (row, col) indicate to the operator which grid box to scan. The most-right column "testing order" was also randomized................................................................................... 6

Table 4: Total and deformed bead count for the LM and CT analysis methods. The LM method counts are estimates, while the CT counts are calculated via computer. Samples are listed in alphabetical order by bucket bottle \#.

Table 5: Deformed ratio separated into percentage of either distorted or double sphere category. All percentages are respective to the total amount of beads in each sample.

Table 6: Rheological values estimated from numerical simulation model for SRM2492 paste containg $0 \%, 20 \%$ and $40 \%$ spherical inclusions. Simulation data portrayed as X's and O's in Figure 8. The uncertainty in values of viscosity, based on the standard deviation, is $10 \%$ or lower.

Table 7: Scaling parameters to factor the SRM2492 curve to a mortar curve with known concentration. The uncertainty in scaling parameters, based on a least square fit of simulation data to equation (1), is $10 \%$ or less.

Table 8: Values for model with uncertainties provided in fundamental units (data were rounded to nearest hundreth). The shear rates for the $0 \%$ were arbitrary selected while the shear stress and viscosity were calculated using equation 1. The $20 \%$ and $40 \%$ were determined using the scaling factors of Table 7 ............. 19

Table 9: Bingham parameters extracted from the model data displayed in Table 8........ 20

Table 10: Testing schedule complemented by the key on the right side. The waiting periods were used to create the mixes listed along the gray rows. ……………….... 23

Table 11: Calibration factors for experimental data of each spindle tested in this study.

Table 12: Plastic viscosity and yield stress at $0 \%, 20 \%$ and $40 \%$ bead volume concentrations for all three spindles. See Section 7.1 for standard uncertainties. Note that the values for $0 \%$ are all identical as all curves were calibrated against the $0 \%$ data of SRM 2492 .

Table 13: Calibration factors for experimental data of each spindle tested in this study.

Table 14: The differences between viscosity calculated using Bingham equation and viscosity fits are shown for three shear rates. The differences was calculated by (Values by Bingham - Value by viscosity fit). The uncertainity is estimated to be less than $10 \%$ of the measured viscosity.

Table 15: Relative viscosity $\left(\mu_{\mathrm{R}}\right)$ values based on the calibrated experimental data at concentrations of $20 \%$ and $40 \%$ by volume, labeled $\mu_{\mathrm{R} 20}$ and $\mu_{\mathrm{R} 40}$, respectively. These were calculated using the Bingham method of conversion.

Table 16: Relative viscosity $\left(\mu_{\mathrm{R}}\right)$ values based on the calibrated experimental data at concentrations of $20 \%$ and $40 \%$ by volume, labeled $\mu_{\mathrm{R} 20}$ and $\mu_{\mathrm{R} 40}$, respectively.

These are calculated using the viscosity curve approach.....

Table 17: Relative viscosity $\left(\mu_{\mathrm{R}}\right)$ values for the scaled prediction numerical model. Standard uncertainties are estimated at $10 \%$. 
Table 18: Torque and rotational speed with the calculated uncertainity for the double Spiral spindle with $0 \%$ beads

Table 19: Torque and rotational speed with the calculated uncertainity for the double Spiral spindle with $20 \%$ beads

Table 20: Torque and rotational speed with the calculated uncertainity for the double Spiral spindle with $40 \%$ beads 52

Table 21: Torque and rotational speed with the calculated uncertainity for the sixblade vane spindle with $0 \%$ beads

Table 22: Torque and rotational speed with the calculated uncertainity for the sixblad vane spindle with $20 \%$ beads

Table 23: Torque and rotational speed with the calculated uncertainity for the sixblade vane spindle with $40 \%$ beads

Table 24: Torque and rotational speed with the calculated uncertainity for the Serrated Coaxial Cylinder with $0 \%$ beads

Table 25: Torque and rotational speed with the calculated uncertainity for the Serrated Coaxial Cylinder with $20 \%$ beads

Table 26: Torque and rotational speed with the calculated uncertainity for the Serrated Coaxial Cylinder with $40 \%$ beads

Table 27: Double spiral spindle: Posterior means, standard deviations and $95 \%$ uncertainty bounds for $\alpha$ (yield stress)...

Table 28: Six-blade Vane: Posterior means, standard deviations and $95 \%$ uncertainty bounds for $\alpha$ (yield stress)...

Table 29: Serrated Coaxial Cylinder: Posterior means, standard deviations and $95 \%$ uncertainty bounds for $\alpha$ (yield stress).

Table 30: Double spiral spindle: posterior means, standard deviations and $95 \%$ uncertainty bounds for $\beta$ (plastic viscosity).

Table 31: Six-Blade Vane: posterior means, standard deviations and $95 \%$ uncertainty bounds for $\beta$ (plastic viscosity)

Table 32: Serrated Coaxial Cylinder: posterior means, standard deviations and 95\% uncertainty bounds for $\beta$ (plastic viscosity).

Table 33: Values for Double spiral spindle with uncertainties provided in fundamental units. - these are based on Bingham parameters

Table 34: Values for Six Blade Vane with uncertainties provided in fundamental units. - these are based on Bingham parameters

Table 35: Values for serrated coaxial cylinder with uncertainties provided in fundamental units. - these are based on Bingham parameters

Table 36: Values for double spiral spindle with uncertainties provided in fundamental units, based on viscosity fit method.

Table 37: Values for six blade vane with uncertainties provided in fundamental units, based on viscosity fit method....

Table 38: Values for cylinder coaxial with uncertainties provided in fundamental units, based on viscosity fit method

Table 39: Certified values based on the Bingham parameters. A) $0 \%$ paste, B) $20 \%$ mortar and C) $40 \%$ mortar . 


\section{Notations:}

SD: Standard Deviation

SRM: Standard Reference Material

$\dot{\gamma}$ : Shear rate $\left[s^{-1}\right]$

$\tau$ : Shear stress $[\mathrm{Pa}]$

$\tau_{0}$ : Yield stress $[\mathrm{Pa}]$

$\mathrm{N}$ : Rotational speed [ $\mathrm{rad} / \mathrm{s}]$ or [rpm]

$\Gamma$ : Measured torque $[N \cdot m]$

$\mu$ : Viscosity [Pa s]

$\mu_{\mathrm{B}}$ : plastic viscosity (Bingham model) $[\mathrm{Pa} \cdot \mathrm{s}]$

$\mu_{\mathrm{R}}$ : Relative viscosity $[-]$

$\mu_{\mathrm{R} 20}$ : Relative viscosity for $20 \%$ mortar $[-]$

$\mu_{\mathrm{R} 40}$ : Relative viscosity for $40 \%$ mortar $\quad[-]$

$\mu_{\mathrm{sc}}:$ Viscosity scaling factor $[-]$

$\dot{\gamma}_{\text {sc }}$ : Shear rate scaling factor $[-]$

$\mathrm{K}_{\tau}$ : Shear stress calibration factor based on Bingham parameters $[\mathrm{Pa} /(\mathrm{N} \cdot \mathrm{m})]$

$\mathrm{K}_{\mu}$ : Viscosity calibration factor based on Bingham parameters $[\mathrm{Pa} \cdot \mathrm{s} /(\mathrm{N} \cdot \mathrm{m} \cdot \mathrm{s})]$

$\mathrm{K}_{\gamma}$ : Shear rate calibration factor based on Bingham parameters [-]

$\mathrm{L}_{\tau}$ : Shear stress calibration factor based on viscosity curves $[\mathrm{Pa} /(\mathrm{N} \cdot \mathrm{m})]$

$\mathrm{L}_{\mu}$ : Viscosity calibration factor based on viscosity curves $[\mathrm{Pa} \cdot \mathrm{s} /(\mathrm{N} \cdot \mathrm{m} \cdot \mathrm{s})]$

$\mathrm{L}_{\gamma}$ : Shear rate calibration factor based on viscosity curves [-] 


\section{Introduction}

A National Institute of Standards and Technology (NIST) Standard Reference Material ${ }^{\circledR}$ (SRM) meets specific certification criteria [https://www.nist.gov/srm/srm-definitions] and is issued with a certificate of analysis that reports the results of its characterization and provides information regarding the appropriate use(s) of the material. An SRM is prepared and used for three main purposes: 1 ) to help develop accurate methods of analysis; 2) to calibrate measurement systems used to facilitate exchange of goods, institute quality control, determine performance characteristics, or measure a property at the state-of-the-art limit; and 3) to ensure the long-term adequacy and integrity of measurement quality assurance programs. NIST provides over 1300 different SRMs to industry and academia. Every NIST SRM is provided with a certificate of analysis that gives the official characterization of the material's properties and and provides information regarding the appropriate use(s) of the SRM. In addition, supplementary documentation, such as this report, describing the development, analysis, and use of SRMs, is also often provided to ensure effective use of the SRM.

Rheological measurements are commonly performed using a rotational rheometer. In this type of rheometer, the test fluid is sheared between two surfaces: one surface is rotating while the other is stationary [3]. The rate of the rotating surface is precisely controlled by a computer, while measuring the torque resulting from the material response. Laboratory rheometers are mainly designed for homogeneous liquids containing no particles, such as oils. After completing two interlaboratory studies related to rheological properties of concrete, it was determined that the use of expensive oils was not suitable for calibrating concrete rheometers [4] [5]. Thus, as requested by the industry, a need exists for a granular reference material specifically designed for concrete rheometers. The first step to fulfilling that need was the development of SRM 2492, a cement paste reference material. The second step is a mortar reference material, SRM 2493. To created SRM 2493, the operator needs to mix the SRM 2492 as certified [1] [2] with the addition of $1 \mathrm{~mm}$ glass spheres as the fine aggregate component. Once mixed, the shelf life of the mixture is $7 \mathrm{~d}$.

In this report, the simulation results of flow in an ideal Couette system are provided to determine the certified data for the rheological properties of the SRM 2493. Reported values obtained through rheological testing performed at NIST using a double spiral, a coaxial, and a vane spindles are also provided for comparision purposes. In addition, NIST is working on developing a concrete SRM, which would involve adding $10 \mathrm{~mm}$ beads to SRM 2493.

The objective of this report is to describe the mortar reference material, SRM 2493, and its rheological properties. A description of the methodology and all measurements used are provided, along with the statistical analysis. 


\section{Materials}

\subsection{Limestone and Corn Syrup}

The SRM 2492 paste, which serves as a matrix for the SRM mortar, consists of a limestone powder, corn syrup and distilled water mixture. The limestone is referred to as a micro-limestone flour with an average particle size of $45 \mu \mathrm{m}$ and a density of $2724 \mathrm{~kg} / \mathrm{m}^{3} \pm 15 \mathrm{~kg} / \mathrm{m}^{3}$. The corn syrup used was specified, by the manufacturer, as a $100 \%$ glucose corn syrup with no additives and verified via NIST testing. Analyses were conducted at NIST on samples of the limestone powder to determine its mineralogical, chemical, and physical properties, as well as its particle size distribution and density. This limestone powder and corn syrup were the same as those used for the certification of SRM 2492; refer to [1] [2] for more details about these materials.

\subsection{Glass Beads}

To produce SRM 2493, $1 \mathrm{~mm}$ glass beads were added to SRM 2492 to transform it into a suspension representative of a mortar. The glass beads were received in 5-gal buckets that were labelled from A to $\mathrm{G}$. The beads from each bucket were subsequently distributed in $1.5 \mathrm{~L}$ plastic bottles (See Section 2.4).

The density of the glass beads was found to be $2465 \mathrm{~kg} / \mathrm{m}^{3} \pm 3 \mathrm{~kg} / \mathrm{m}^{3}$ at NIST by using ASTM C188-09 [6]. The sphericity and size distribution of the $1 \mathrm{~mm}$ beads were verified as described in Sections 2.2.1 to 2.2.6 using light microscope analysis, and computed tomography scan analysis.

\subsubsection{Sample Selection and Analysis Schedule}

As stated in Section 2.2, the beads were received in several 5-gal buckets and then portioned into plastic bottles. To ensure a statistical representation of the bottles of beads, a pseudo-random number generator was used to randomly select two bottles from each bucket (see section 2.4). Table 1 lists the bottles selected for analysis and their corresponding buckets. These bottles contained enough beads to be used during both the bead analysis and the subsequent rheological testing.

The order in which these bottles were analyzed was also randomized, resulting in the schedule displayed in Table 2. The schedule took into consideration the operator's availability and the required equipment time needed for test completion. For the bead shape analysis, only a small portion of the total beads (less than $5 \%$ ) in each bottle was used for testing. Therefore, the remaining beads were used for the rheological testing. 
Table 1: Randomly selected bottles for bead analysis

\begin{tabular}{|c|c|c|}
\cline { 2 - 3 } \multicolumn{1}{c|}{} & \multicolumn{2}{c|}{ Random Bottle Selection } \\
\hline Bucket A & 3 & 10 \\
\hline Bucket B & 2 & 11 \\
\hline Bucket C & 9 & 14 \\
\hline Bucket D & 7 & 5 \\
\hline Bucket E & 7 & 10 \\
\hline Bucket F & 5 & 14 \\
\hline Bucket G & 4 & 8 \\
\hline
\end{tabular}

Table 2: Schedule for bead analysis.

\begin{tabular}{|c|c|c|c|c|c|}
\hline \multicolumn{6}{|c|}{ Bead Analysis Sche dule } \\
\hline \multicolumn{2}{|r|}{ M } & $\mathrm{T}$ & $\mathrm{W}$ & $\mathrm{T}$ & \multirow{2}{*}{$\frac{\mathrm{F}}{\mathrm{G} 4}$} \\
\hline Week 1 & & & & & \\
\hline 3/31/2014 & & & & & \\
\hline Week 2 & & D7 & & F5 & \\
\hline 4/14/2014 & & & & & \\
\hline Week 3 & B2 & & C9 & & B11 \\
\hline $4 / 21 / 2014$ & & & & & \\
\hline Week 4 & & C14 & & A10 & \\
\hline $4 / 28 / 2014$ & & & & & \\
\hline Week 5 & G8 & & F14 & & A3 \\
\hline $5 / 5 / 2014$ & & & & & \\
\hline Week 6 & E10 & & E7 & & D5 \\
\hline $5 / 12 / 2014$ & & & & & \\
\hline
\end{tabular}

\subsubsection{Specimen Set-up for Shape Analysis}

The method for holding the beads needed to be usable for both analysis tests. This required that the specimen holder have transparency for the light microscope and flexibility for the X-ray computed tomography (CT) scan. The size of the specimen was specified to be a $76 \mathrm{~mm}$ x 178 $\mathrm{mm}$ (3 in $\mathrm{x} 7$ in) piece of self-adhesive laminated sheet. This size was governed by the required sample size for the X-ray CT test.

The first step in setting up the specimen was removing the protective cover from the adhesive sheet, and placing it on a flat surface such that the adhesive side was facing up. Then, the beads that were to be analyzed were hand stirred in a cup to ensure a random particle distribution. Finally, the beads were evenly sprinkled across the adhesive sheet with the help of a scoop. Typically, the goal was to obtain a uniform distribution in which the beads were not touching each other, i.e., not very dense in terms of number of beads per unit area. This goal was necessary to avoid the appearance of beads touching in the X-ray CT reconstruction process. A total sample of around 2000 to 3000 beads was considered adequate for statistical shape analysis [7]. 


\subsubsection{Light Microscope}

This step of the bead analysis process required a microscope with adjustable lighting (Figure 1). The microscope in conjunction with an image analysis software, ImagePro, enabled capturing images of the specimen during analysis. The specimen set-up, as described in Section 2.2.2, was placed onto an analysis template and the lighting was adjusted to minimize shadows or any other factors that affected image visibility.

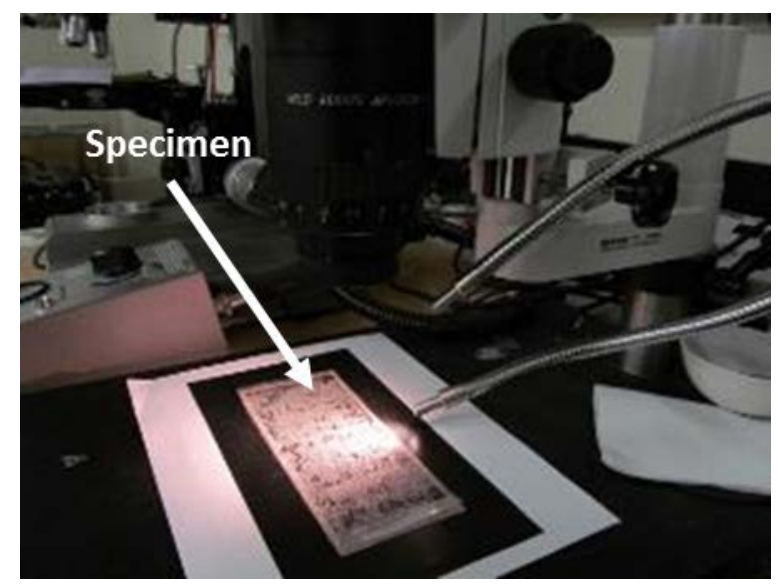

Figure 1: Light microscope with adjustable lighting of the specimen. The black area under the specimen is the grid system shown in Figure 2.

A grid template was developed to place under the specimen in order to construct a grid system that would guide the operator when using the microscope, see Figure 2. ImagePro's “video mode" enabled seeing the specimen in real-time, and the grid system enabled easy identification of the grid boxed for analysis. The system was designed to have grids (6 x 11= 66 total) of dimensions equal to the best window size that provided clear microscope images. Measurements of this window size using a metric ruler concluded that the individual grids should measure 11.5 $\mathrm{mm} \times 15 \mathrm{~mm}$, since this ratio developed an image of the beads at a clear size for human eye analysis, as seen in Figure 3. A black background was selected for the template to increase the contrast of the glass beads under light. The grey areas that made up the perimeter of this base template allow the operator to handle the sheet without altering the number of beads under analysis.

A total of 10 grid boxes were chosen as the standard number to analyze, and were randomly selected. This generator was modified to retrieve random selections with a fairer weight balance, as described in Section 2.2.6. The areas selected can be found in Table 3. Once the images were stored, the total number of beads and deformities in each grid box image were counted and recorded by the operator. Deformities were defined as either double beads (i.e., two or more beads that were physically attached to each other) or distorted beads (i.e., single beads that were visually non-spherical). This methodology gathered an average count per grid box image, and estimates the total number of beads and deformities in the entire specimen. This number was then compared to the bead count calculated using the X-ray CT spherical harmonic analysis, in Section 2.2.5. 
Figure 2: Grid system placed under specimen allows easy selection of analysis areas. The

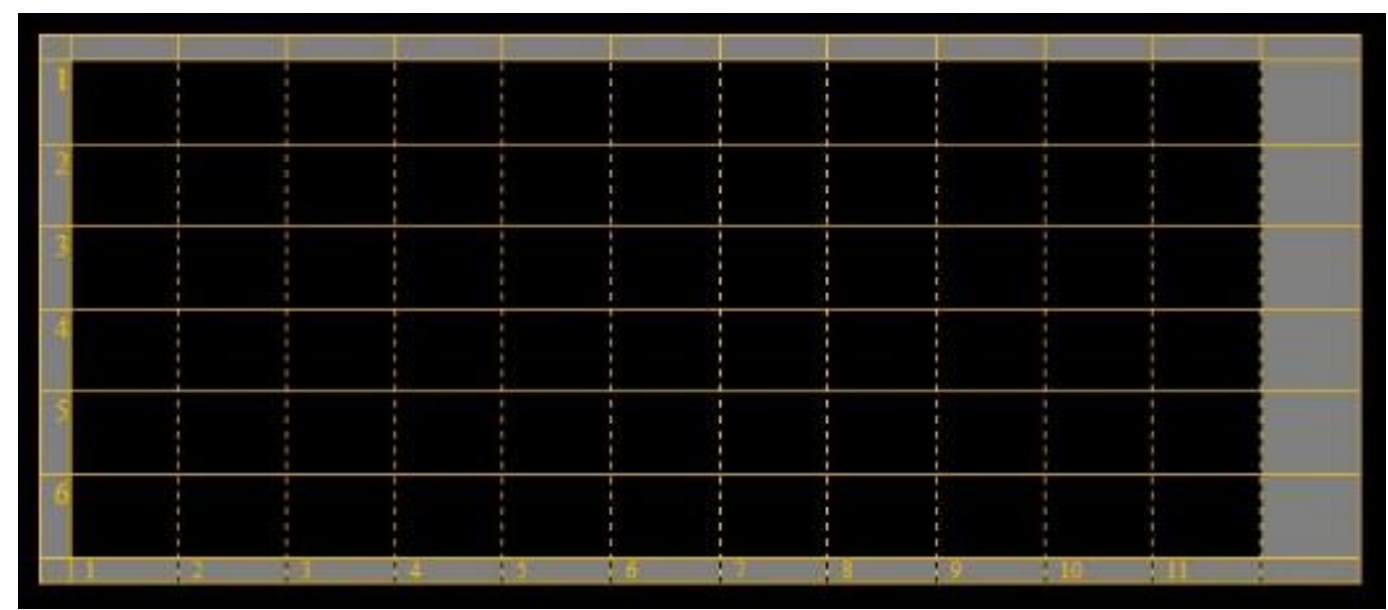

grid system creates (row, col) coordinates for random selection analysis.

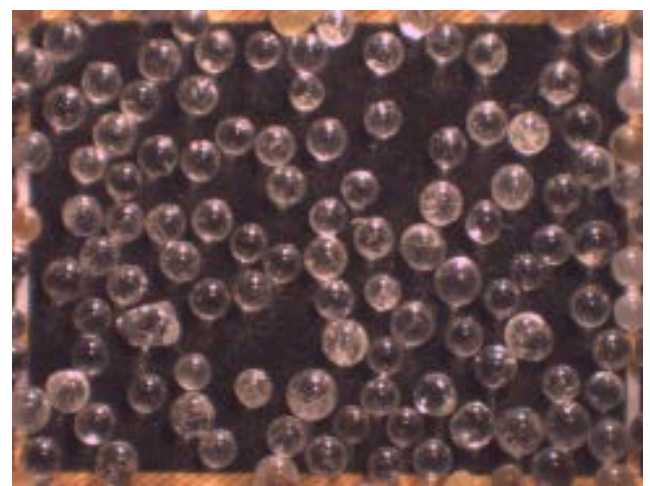

Figure 3: Snap shot image taken with the light microscope. 
Table 3: Grid boxes randomly selected for analysis. The coordinates (row, col) indicate to the operator which grid box to scan. The most-right column "testing order" was also randomized.

\begin{tabular}{|c|c|c|c|c|c|c|c|c|c|c|c|c|c|}
\hline \multicolumn{13}{|c|}{ Random Selection of Grids To Scan } & \multirow{3}{*}{$\begin{array}{c}\text { Testing } \\
\text { Order }\end{array}$} \\
\hline \multirow{2}{*}{ Bucket } & \multirow{2}{*}{ Bottle \# } & & \multicolumn{10}{|c|}{ Grid Box Selected } & \\
\hline & & & $1 \mathrm{st}$ & 2nd & 3rd & 4th & 5th & 6th & 7 th & 8th & 9th & 10th & \\
\hline \multirow{4}{*}{ A } & \multirow{2}{*}{3} & Row & 3 & 4 & 4 & 6 & 4 & 1 & 6 & 2 & 5 & 4 & \multirow{2}{*}{11} \\
\hline & & $\mathrm{Col}$ & 5 & 11 & 1 & 2 & 5 & 4 & 5 & 8 & 3 & 6 & \\
\hline & \multirow{2}{*}{10} & Row & 2 & 5 & 3 & 6 & 2 & 1 & 2 & 3 & 1 & 3 & \multirow{2}{*}{8} \\
\hline & & $\mathrm{Col}$ & 3 & 8 & 8 & 1 & 9 & 2 & 7 & 1 & 7 & 1 & \\
\hline \multirow{4}{*}{ B } & \multirow{2}{*}{2} & Row & 1 & 1 & 2 & 3 & 5 & 5 & 3 & 3 & 2 & 6 & \multirow{2}{*}{4} \\
\hline & & $\mathrm{Col}$ & 7 & 2 & 7 & 2 & 9 & 10 & 8 & 6 & 6 & 3 & \\
\hline & \multirow{2}{*}{11} & Row & 3 & 6 & 3 & 2 & 1 & 6 & 3 & 5 & 5 & 2 & \multirow{2}{*}{6} \\
\hline & & Col & 7 & 4 & 6 & 10 & 7 & 8 & 8 & 6 & 1 & 2 & \\
\hline \multirow{4}{*}{ C } & \multirow{2}{*}{9} & Row & 1 & 3 & 6 & 3 & 2 & 3 & 2 & 4 & 1 & 4 & \multirow{2}{*}{5} \\
\hline & & Col & 2 & 6 & 2 & 7 & 6 & 8 & 6 & 9 & 7 & 4 & \\
\hline & \multirow{2}{*}{14} & Row & 2 & 3 & 1 & 2 & 2 & 1 & 4 & 1 & 6 & 4 & \multirow{2}{*}{7} \\
\hline & & $\mathrm{Col}$ & 6 & 11 & 4 & 9 & 5 & 3 & 9 & 9 & 5 & 2 & \\
\hline \multirow{4}{*}{$\mathrm{D}$} & \multirow{2}{*}{7} & Row & 1 & 2 & 1 & 3 & 6 & 3 & 2 & 2 & 6 & 6 & \multirow{2}{*}{2} \\
\hline & & $\mathrm{Col}$ & 5 & 5 & 1 & 3 & 7 & 5 & 1 & 1 & 5 & 3 & \\
\hline & \multirow{2}{*}{5} & Row & 1 & 4 & 3 & 2 & 5 & 5 & 5 & 2 & 6 & 3 & \multirow{2}{*}{14} \\
\hline & & $\mathrm{Col}$ & 6 & 4 & 6 & 11 & 6 & 9 & 5 & 10 & 6 & 10 & \\
\hline \multirow{4}{*}{$\mathrm{E}$} & \multirow{2}{*}{7} & Row & 5 & 6 & 3 & 6 & 3 & 6 & 6 & 4 & 5 & 1 & 12 \\
\hline & & $\mathrm{Col}$ & 11 & 2 & 4 & 9 & 6 & 11 & 5 & 2 & 1 & 2 & 13 \\
\hline & 10 & Row & 6 & 1 & 6 & 5 & 1 & 3 & 5 & 3 & 2 & 5 & 12 \\
\hline & 10 & $\mathrm{Col}$ & 5 & 4 & 3 & 7 & 7 & 3 & 8 & 9 & 2 & 6 & 12 \\
\hline & 5 & Row & 5 & 3 & 4 & 1 & 3 & 3 & 3 & 6 & 5 & 1 & 3 \\
\hline $\mathrm{F}$ & $J$ & $\mathrm{Col}$ & 7 & 9 & 10 & 7 & 11 & 1 & 8 & 3 & 4 & 6 & 3 \\
\hline $\mathbf{r}$ & 14 & Row & 2 & 3 & 1 & 5 & 6 & 5 & 6 & 4 & 6 & 5 & 10 \\
\hline & 14 & $\mathrm{Col}$ & 4 & 11 & 4 & 6 & 5 & 5 & 4 & 1 & 8 & 7 & 10 \\
\hline & 4 & Row & 2 & 2 & 6 & 2 & 3 & 1 & 5 & 4 & 6 & 3 & 1 \\
\hline $\mathrm{G}$ & 4 & $\mathrm{Col}$ & 5 & 3 & 10 & 6 & 11 & 10 & 11 & 8 & 11 & 3 & \\
\hline U & & Row & 4 & 5 & 5 & 2 & 4 & 1 & 1 & 1 & 5 & 2 & 0 \\
\hline & 8 & $\mathrm{Col}$ & 5 & 1 & 5 & 2 & 5 & 2 & 11 & 9 & 8 & 4 & $y$ \\
\hline
\end{tabular}

\subsubsection{X-ray Computed Tomography and Spherical Harmonic Analysis}

The final step in the bead shape analysis process consisted of an X-ray computed tomography (CT) analysis, which uses X-ray technology and a reconstruction algorithm [7] to acquire the 3-D particle shapes, which are then analyzed by a spherical harmonic process. The specimen used for the light microscope was also used for this final step. The CT scans were performed using a Skyscan 1172 instrument (see Figure 4). To begin this analysis process, the flat specimen, from the previous step (Section 2.2.3), was first rolled into a cylindrical shape, and fit into a sample support that was placed upright into the Skyscan. 


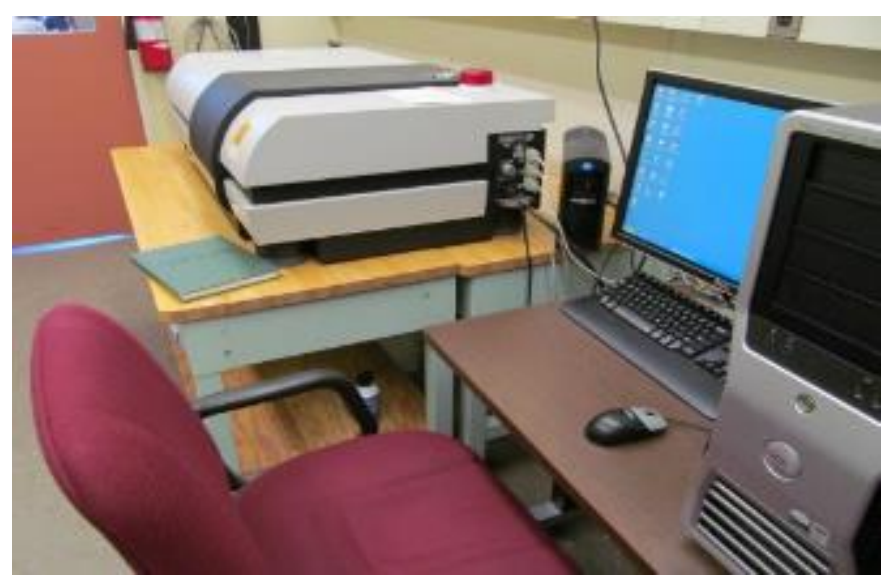

Figure 4: X-ray CT instrument.

The X-ray CT instrument takes X-ray absorption images as the sample is rotated. The reconstruction algorithm takes this image data and constructs accurate $2 \mathrm{D}$ slices orthogonal to the cylindrical axis of the specimen [7]. A typical slice, for the D7 sample, is shown in Figure 5. In this image, a double particle, several quite non-spherical particles, and some internal porosity can be clearly seen (marked with white circles). These slices were thresholded so that the particles were white and the background is black. By stacking the slices on top of each other computationally the result was a 3D model of the specimen. A FORTRAN program scanned each 3D structure, extracted the particles and fit them with a 3D spherical harmonic series. Any internal porosity was removed before this mathematical fit, since the only interest was in the shape of the outer envelope, which would affect suspension rheology physics. Then, it automatically computed various geometrical parameters of each bead, such as volume, surface area, and dimensions of a box that minimally surrounds the beads (length $(L)$, width $(W)$, and thickness $(T)$ ). The volume equivalent spherical diameter (VESD), the mean curvature integrated over the surface, and other factors were also included in these automatic calculations [8]. 


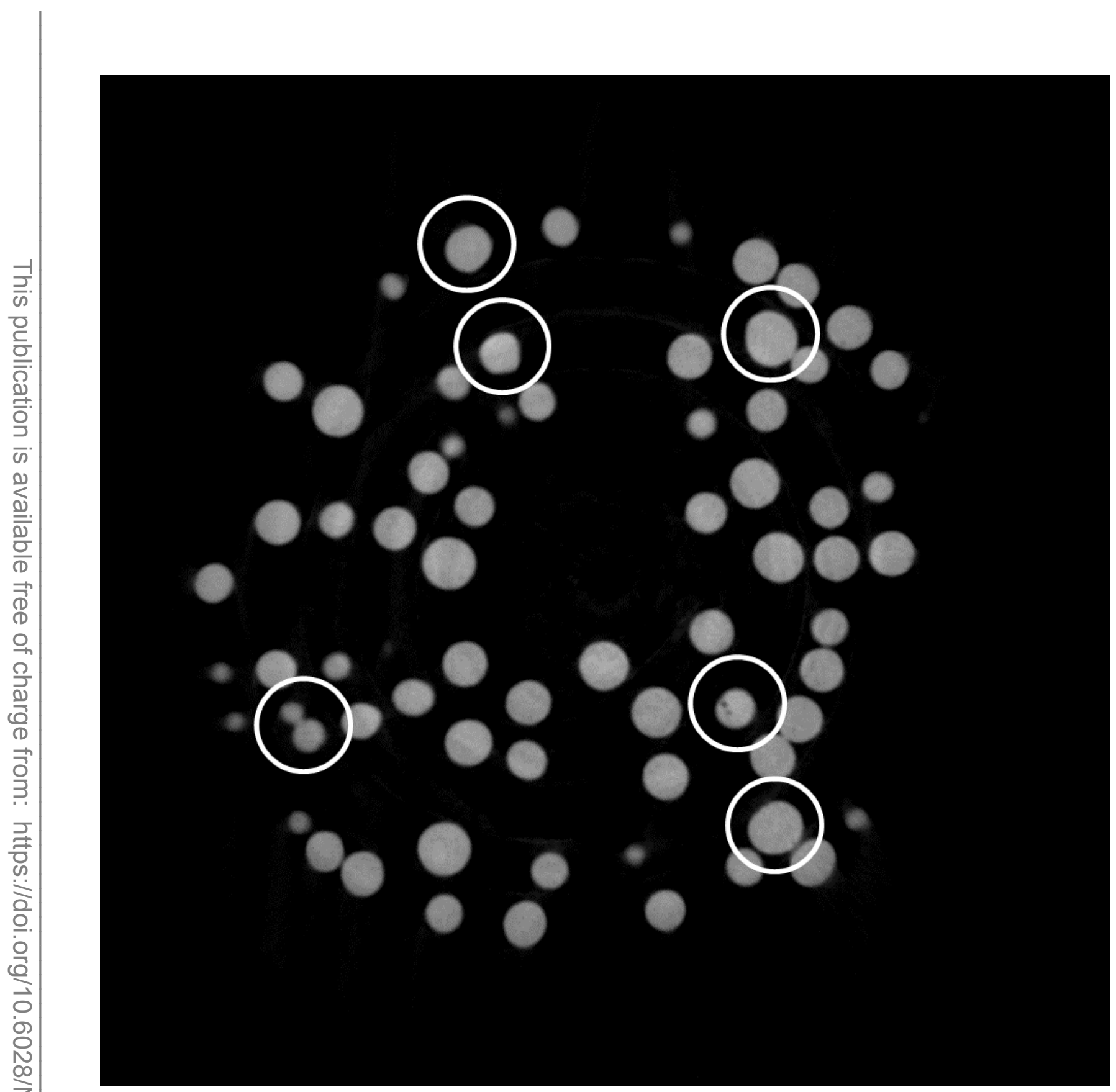

Figure 5: A typical reconstructed slice from the X-ray CT results for bead sample D7. The image is $1000 \times 1000$ pixels, and the length per pixel is approximately $20.9 \mu \mathrm{m}$. Note the several non-spherical particles, one double particle, and one particle with internal porosity (marked with white circles).

The dimensional data of the beads were analyzed to verify that most were indeed very close to being $1 \mathrm{~mm}$ spheres $(\mathrm{L}=\mathrm{W}=\mathrm{T}=1 \mathrm{~mm})$. To analyze non-sphericity, consider ratios of these dimensions. A sphere has $\mathrm{L} / \mathrm{T}=\mathrm{W} / \mathrm{T}=1$. A double sphere (or two identical spheres glued lightly together) has $\mathrm{L} / \mathrm{T}=2$, and $\mathrm{W} / \mathrm{T}=1$, since by definition, $\mathrm{L}>\mathrm{W}>\mathrm{T}$. Any particle that had values of $\mathrm{L} / \mathrm{T}$ and $\mathrm{W} / \mathrm{T}$ outside what was considered to be the spherical range (see section 2.2.5 for details) 
were considered to be distorted, and were compared to the number developed from the light microscope analysis for confirmation.

\subsubsection{Bead Analysis Results}

The analysis of the two tests showed similar characteristics but differed slightly due to distinct parameters between the two methods. The analyses did confirm the existence of deformed beads, which were categorized as either distorted or double-spheres. These deformities taint $1 \%$ to $4 \%$ of the sample population, by number, according to the light microscope (LM) and X-ray CT scan tests. Table 4 represents the total amount of deformed beads for each sample analyzed from both methods, but doesn't highlight which are distorted or double-spheres. The individual percent of distorted or double-spheres for both analysis methods can be found in Table 5. The "total beads estimate" and "deformed beads estimate" from the LM method were calculated by taking the average count of the ten grid boxes analyzed, and proportionally estimating the count for the total specimen area. The CT scan method numbers come from the automatic calculations described in Section 2.2.4.

The deformed beads were categorized as either double-spheres or distorted as shown in Table 5. These values were obtained from the CT scan data, which gives the dimensions of each reconstructed sphere. A perfect sphere will have an $\mathrm{L} / \mathrm{T}$ ratio of 1 , thus doubling that value would signify a double sphere. Therefore, the number of double spheres was calculated by counting the beads with length/thickness ratios greater than 1.8, to allow for some size variation in the spheres. Two unequal spheres, with diameters $D_{1}$ and $D_{2}, D_{2}>D_{1}$, will have $L=D_{2}+D_{1}$ but $T=D_{2}$, so that $L / T=1+D_{1} / D_{2}<2$. The remaining deformed beads were classified as distorted, if the $L / T$ ratio was between 1.3 and 1.8, which would indicate a non-spherical single particle. A value of L/T below about 1.3 was considered close to being spherical, hence was not counted as distorted or as a double particle. These approximate cutoff values of $\mathrm{L} / \mathrm{T}$ were confirmed by a visual study of randomly-selected particles. The uncertainty was estimated at $10 \%$ based on previous data using the same instrument [7].

The deformed bead count presented for the LM method is an estimate calculated via operator visual analysis of 10 randomly selected grid boxes. This attaches an uncertainty to the LM estimated deformed count, as shown in Figure 6, since the 10 selected grid boxes could have missed better representations of deformed beads. Furthermore, the LM method is a basic 2D analysis while the X-ray CT is a true 3D method. The CT method computes its numbers from reconstructed models, which can over count the deformed percentage by considering two neighboring, non-connected spheres that are too close together as double-spheres. The reconstruction process can blend two particles together that were geometrically very close in the original sample. The CT scan can be safely assumed to be the more accurate representation since it is actually analyzing every single bead in the specimen, and at worst it will over count as previously mentioned. The main uncertainty in the CT data comes from the segmentation of the CT images into particles (white) and background (black), and this mostly affects the volume determination [7]. The shape analysis is quite insensitive to the exact segmentation, so we estimate the uncertainty in the CT shape analysis, determining which spheres are irregular or double, to be about $1 \%$, which is almost certainly an upper bound. 
Table 4: Total and deformed bead count for the LM and CT analysis methods. The LM method counts are estimates, while the CT counts are calculated via computer. Samples are listed in alphabetical order by bucket bottle \#.

\begin{tabular}{|c|c|c|c|c|}
\hline \multicolumn{2}{|c|}{ Light Micros cope } & \multirow{2}{*}{$\begin{array}{c}\text { Total Beads } \\
\text { Es timate }\end{array}$} & \multirow{2}{*}{$\begin{array}{c}\text { Deformed Beads } \\
\text { Es timate } \\
\end{array}$} & \multirow{2}{*}{$\begin{array}{c}\text { Deformed Ratio } \\
{[\%]}\end{array}$} \\
\hline Sample \# & Bucket Bottle \# & & & \\
\hline 11 & A 3 & 3729 & 34 & 0.91 \\
\hline 8 & A 10 & 3518 & 27 & 0.77 \\
\hline 4 & B 2 & 3802 & 6 & 0.16 \\
\hline 6 & B 11 & 3788 & 34 & 0.90 \\
\hline 5 & C 9 & 3610 & 47 & 1.30 \\
\hline 7 & C 14 & 3656 & 42 & 1.15 \\
\hline 2 & D 7 & 3201 & 41 & 1.28 \\
\hline 14 & D 5 & 4376 & 19 & 0.43 \\
\hline 13 & E 7 & 3755 & 48 & 1.28 \\
\hline 12 & E 10 & 3445 & 35 & 1.02 \\
\hline 3 & F 5 & 3821 & 36 & 0.94 \\
\hline 10 & F 14 & 3927 & 36 & 0.92 \\
\hline 1 & G 4 & 4666 & 51 & 1.09 \\
\hline \multirow[t]{3}{*}{9} & G 8 & 3775 & 30 & 0.79 \\
\hline & Average & 3791 & 35 & 0.92 \\
\hline & $S D$ & 351 & 11 & 0.31 \\
\hline \multicolumn{2}{|c|}{ CT Scan } & \multirow{2}{*}{ Total Beads } & \multirow{2}{*}{ Deformed Beads } & \multirow{2}{*}{$\begin{array}{c}\text { Deformed Ratio } \\
{[\%]} \\
\end{array}$} \\
\hline Sample \# & Bucket Bottle \# & & & \\
\hline 11 & A3 & 2969 & 152 & 5.12 \\
\hline 8 & A10 & 3082 & 150 & 4.87 \\
\hline 4 & B2 & 3053 & 177 & 5.80 \\
\hline 6 & B11 & 2986 & 160 & 5.36 \\
\hline 5 & C9 & 2380 & 120 & 5.04 \\
\hline 7 & C14 & 2925 & 174 & 5.95 \\
\hline 2 & D7 & 2688 & 188 & 6.99 \\
\hline 14 & D5 & 3564 & 149 & 4.18 \\
\hline 13 & E7 & 3418 & 204 & 5.97 \\
\hline 12 & E10 & 2903 & 193 & 6.65 \\
\hline 3 & F5 & 2945 & 175 & 5.94 \\
\hline 10 & F14 & 3077 & 163 & 5.30 \\
\hline 1 & G4 & 4105 & 249 & 6.07 \\
\hline \multirow[t]{3}{*}{9} & G8 & 3026 & 134 & 4.43 \\
\hline & Average & 3080 & 171 & 5.55 \\
\hline & $S D$ & 391 & 31 & 0.77 \\
\hline
\end{tabular}


Table 5: Deformed ratio separated into percentage of either distorted or double sphere category. All percentages are respective to the total amount of beads in each sample.

\begin{tabular}{rcccc}
\multicolumn{2}{c}{ Light Microscope } & Deformed Beads & $\begin{array}{c}\text { Double Sphere } \\
{[\%]}\end{array}$ & $\begin{array}{c}\text { Distorted } \\
{[\%]}\end{array}$ \\
\hline Sample \# & Bucket Bottle \# & {$[\%]$} & 0.20 & 0.71 \\
\hline 11 & A 3 & 0.91 & 0.17 & 0.60 \\
8 & A 10 & 0.77 & 0.03 & 0.12 \\
4 & B 2 & 0.16 & 0.20 & 0.70 \\
6 & B 11 & 0.90 & 0.29 & 1.02 \\
5 & C 9 & 1.30 & 0.25 & 0.90 \\
7 & C 14 & 1.15 & 0.28 & 1.00 \\
2 & D 7 & 1.28 & 0.10 & 0.34 \\
14 & D 5 & 0.43 & 0.28 & 1.00 \\
13 & E 7 & 1.28 & 0.22 & 0.79 \\
12 & E 10 & 1.02 & 0.21 & 0.73 \\
3 & F 5 & 0.94 & 0.20 & 0.72 \\
10 & F 14 & 0.92 & 0.24 & 0.85 \\
1 & G 4 & 1.09 & 0.17 & 0.62 \\
\hline 9 & G 8 & 0.79 & $\mathbf{0 . 2 0}$ & $\mathbf{0 . 7 2}$ \\
\hline & Average & $\mathbf{0 . 9 2}$ & $\mathbf{0 . 0 7}$ & $\mathbf{0 . 2 4}$ \\
\hline & SD & $\mathbf{0 . 3 1}$ &
\end{tabular}

\begin{tabular}{|c|c|c|c|c|}
\hline \multicolumn{2}{|c|}{ CT Scan } & \multirow{2}{*}{$\begin{array}{c}\text { Deformed Beads } \\
{[\%]}\end{array}$} & \multirow{2}{*}{$\begin{array}{c}\text { Double Sphere } \\
{[\%]}\end{array}$} & \multirow{2}{*}{$\begin{array}{c}\text { Distorted } \\
{[\%]}\end{array}$} \\
\hline Sample \# & Bucket Bottle \# & & & \\
\hline 11 & A 3 & 5.12 & 2.63 & 2.49 \\
\hline 8 & A 10 & 4.87 & 2.76 & 2.11 \\
\hline 4 & B 2 & 5.80 & 2.69 & 3.11 \\
\hline 6 & B 11 & 5.36 & 2.95 & 2.41 \\
\hline 5 & С 9 & 5.04 & 1.93 & 3.11 \\
\hline 7 & C 14 & 5.95 & 3.04 & 2.91 \\
\hline 2 & D 7 & 6.99 & 3.98 & 3.01 \\
\hline 14 & D 5 & 4.18 & 1.74 & 2.44 \\
\hline 13 & E 7 & 5.97 & 3.10 & 2.87 \\
\hline 12 & E 10 & 6.65 & 3.69 & 2.96 \\
\hline 3 & F 5 & 5.94 & 3.43 & 2.51 \\
\hline 10 & F 14 & 5.30 & 2.86 & 2.44 \\
\hline 1 & G 4 & 6.07 & 3.58 & 2.48 \\
\hline \multirow[t]{3}{*}{9} & G 8 & 4.43 & 2.18 & 2.25 \\
\hline & Average & 5.55 & 2.90 & 2.65 \\
\hline & $S D$ & 0.77 & 0.63 & 0.32 \\
\hline
\end{tabular}




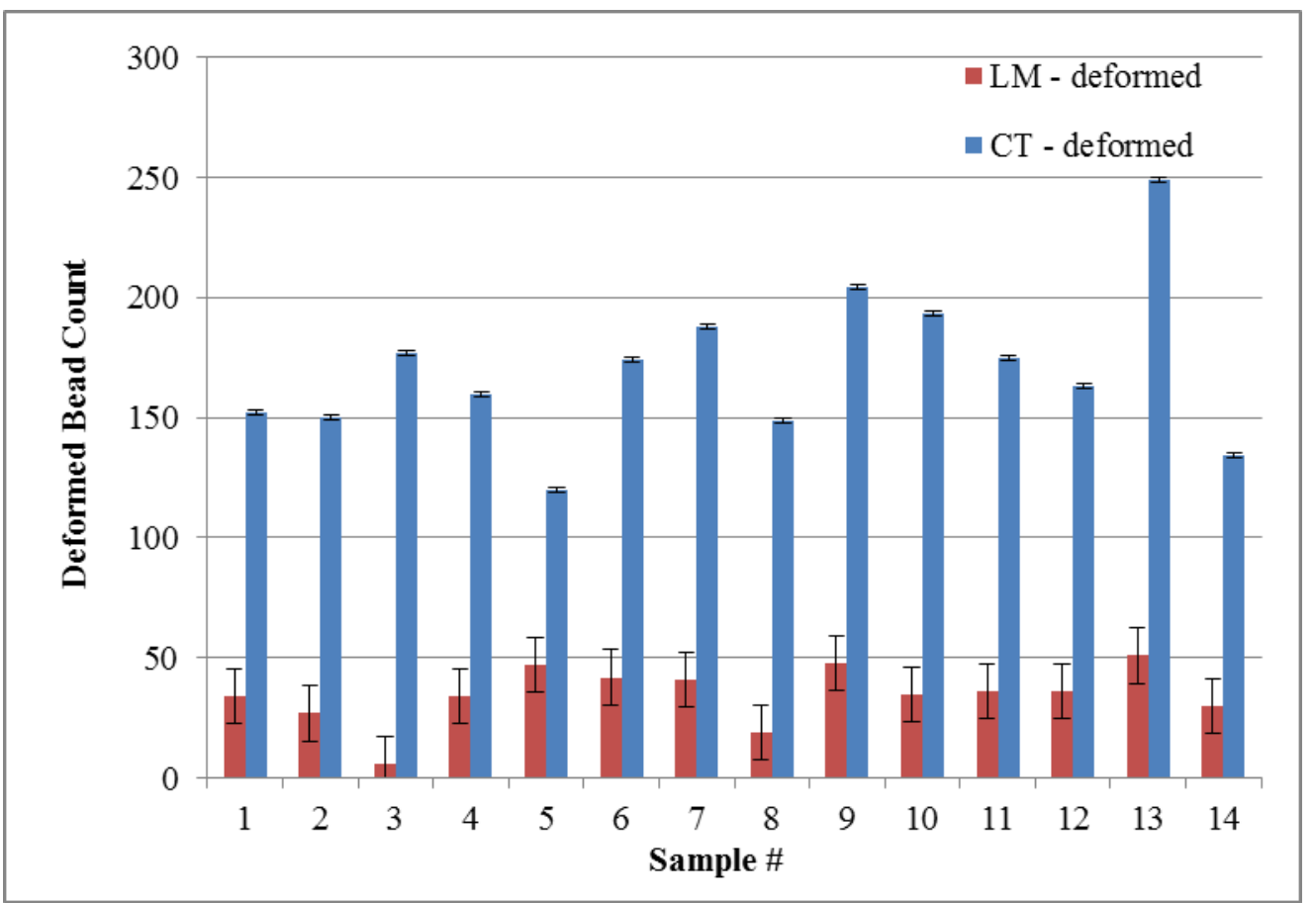

Figure 6: Deformed beads from the two analysis methods. The LM method provided an uncertainty due to estimation, shown by error bars. The uncertainty for the CT data was estimated at $1 \%$.

\subsection{Mixing and Composition}

The SRM paste portion for SRM 2493 was mixed according to ASTM C1738, as described in the previous reports [1] [2]. Once the SRM paste was mixed, it was stored in a polypropylene sealed container for one night. The following day, the beads were mixed into the SRM paste using a high-speed plunger blender at $31.42 \mathrm{rad} / \mathrm{s}$ (300 RPM) for 5 min to reach homogeneity. The lag for the addition of beads was due to the fact that SRM 2492 paste was measured in the rheometer the day before the beads were added.

The initial step of the mixing procedure is dependent on the composition of the mortar. For mortar mixes with a high percentage of bead composition, the initial mixing time may take longer than mortars with low bead percentages ( $<25 \%$ by vol.) due to the slow addition of the beads (longer if more beads). This suggestion allows the blender to mix a portion of the added beads without clogging during blending. It is also recommended to mix for at least 2 additional min (i.e., 7 min total) after all the beads have been introduced in order to give the SRM mortar enough time to homogenize. The shelf life of the mortar is $6 \mathrm{~d}$ (limited by the shelf life of the paste [9]). If the SRM mortar will be stored and reused some other day after mixing (but less than $7 \mathrm{~d}$ ), it is recommended to verify that no changes have occurred to the mortar's composition. The user should measure the bead concentration by volume. To avoid issues with assuring that the SRM 
was stored and its composition was not altered during future reuse, the certification values were only recorded at an age of one day and the material was not reused.

During this study, two compositions of the mortar were measured, with the glass beads having a volume fraction of $20 \%$ and $40 \%$. Pre-certification testing showed that the mortar becomes too stiff as the mortar composition approaches the maximum packing fraction for spherical monosize particles, $64 \%$ by volume. In fact, compositions higher than $50 \%$ caused our rheometer to exceed its maximum torque limit. Considering these observations, a $40 \%$ by volume mortar was concluded to be a good representation of a concentrated bead percentage. Furthermore, for future interpolations and model comparisons, a $20 \%$ composition was also tested to portray semi-concentrated mortars. The experimental results of this study (in Section 4) were drawn from samples of compositions as discussed in this section.

\subsubsection{Instruction for the Certificate to Prepare the Mixture}

Follow the instructions below. The mixture's composition is:

- Corn syrup:

- Distilled water: $200.00 \mathrm{~g}$

- Limestone:

- Glass beads (Asuming a total paste volume of $320 \mathrm{~cm}^{3}$ or see calculation below): $20 \%$ volume fraction $196.8 \mathrm{~g}$ $40 \%$ volume fraction $\quad 524.8 \mathrm{~g}$

Mixing the material is a two phase process by first preparing the paste as for SRM 2492 and then adding the beads. The whole process is described here. The water baths of the high shear blender and the rheometer should be maintained at $23^{\circ} \mathrm{C} \pm 1{ }^{\circ} \mathrm{C}$.

Phase 1: Preparation of the paste fraction (limestone, corn syrup and water). The equipment and the method are described in ASTM C1738 [3], with the following sequence to introduce the ingredients. Pour the correct amount of distilled water in a jar that already contains the correct mass of corn syrup, and mix with a spatula until homogeneous. On average, it will take 5 min of mixing to dilute the visible glucose chains in the corn syrup. Note that assuring the syrup is diluted prior to adding it into the high shear blender results in a more effective transfer of the corn syrup into the mixture. Then, add all of the mixture into the high shear blender that conforms to the specifications detailed in ASTM C1738 and proceed as described in ASTM C1738 to introduce the limestone and mix. (note, the limestone is taking the place of the cement in ASTM C1738).

Phase 2: Addition of the beads

- Tare a plastic container preferably made of polypropylene [9] and pour the mixed paste into it. Make sure that the container has a lid. Measure the mass of the paste, Pm.

- Calculate the mass of beads needed for the mixture using the formula below:

$$
\begin{array}{ll}
20 \% \text { beads } & \text { Mass beads }=0.329 * \mathrm{Pm} \\
40 \% \text { beads } & \text { Mass beads }=0.877 * \mathrm{Pm}
\end{array}
$$

is $2.46 \mathrm{~g} / \mathrm{cm}^{3}$

Note: the density of the paste is $(1.87 \pm 0.01) \mathrm{g} / \mathrm{cm}^{3}$ and the glass beads densities 
- After the mass of beads is determined, the best method to mix the beads into the paste is to use a plunger mixer (rotation speed of $31.4 \mathrm{rad} / \mathrm{s}(300 \mathrm{rpm})$ ). Add the calculated amount of beads and mix for 5 min using the plunger mixer (See Figure 7). It is recommended to use a plunger mixer (rotation speed of $31.4 \mathrm{rad} / \mathrm{s}(300 \mathrm{rpm})$ ) for $30 \mathrm{~s}$ to remix the prepared SRM before each use, especially after a few days of rest.

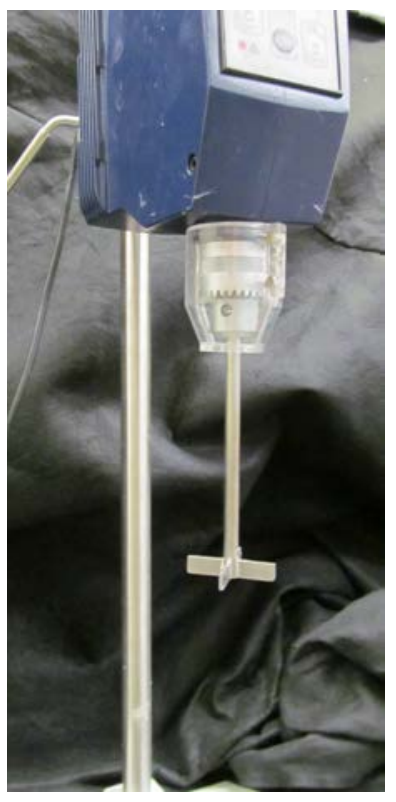

Figure 7: An example of plunger mixer

\subsection{Packaging}

The corn syrup and the limestone used were taken from SRM 2492 supplies [1]. The beads were purchased in one lot and were delivered in 5-gal buckets. The seven buckets were labelled from A to $\mathrm{G}$. The beads were then packaged in $500 \mathrm{~mL}$ plastic bottles (100 bottles in total) each containing about $1500 \mathrm{~g}$. About 14 bottles were obtained from each bucket.

The final packaging was in single boxes, each containing:

- 2 containers of limestone (600 g each)

- 1 container of corn syrup (500 g)

- 1 container of 1 -mm beads (1500 g)

Each box contains enough material to make two batches of SRM 2493 at $40 \%$ beads by volume. 


\section{Definitions}

Here some definitions of terminology used throughout this report are provided for more detailed discussion on rheological properties of fluid review ref [3].

Viscosity of a fluid, $\mu$, is the shear stress, $\tau$, divided by the shear rate, $\dot{\gamma}$.

Bingham equation is a linear relationship between shear stress and shear rate

$$
\tau=\mu_{B} \dot{\gamma}+\tau_{0}
$$

where $\tau$ is the shear stress, $\mu_{B}$, is the plastic viscosity and, $\tau_{0}$ is the yield stress.

The Bingham equation is a two parameter model used for describing viscoplastic fluid exhibiting a yield response. The ideal Bingham material is an elastic solid at low shear stress values and a Newtonian fluid above a critical value called the yield stress. The plastic viscosity region exhibit a differential viscosity called the plastic viscosity.

Pseudo-viscosity, $\Gamma / \mathrm{N}$, is defined as the ratio of the measured torque and the applied rotational speed. This value is related to the viscosity. 


\section{Modeling Approach}

A two-step approach was used to predict the viscosity of the mortar SRM. First, a direct numerical simulation was used that incorporates the paste SRM rheological properties in a hard sphere suspension computational model. Once the viscosity was calculated for a finite set of shear rates, two scaling parameters were determined that map the viscosity vs. shear rate data of the suspension to the viscosity vs. shear rate data of the matrix fluid (SRM paste). By performing the inverse of this transformation to the matrix viscosity vs. shear rate curve, the full viscosity vs shear rate curve is obtained for the suspension. The full description and validation of this scaling ansatz is given in [10].

The computational approach used in this work for modeling suspension is based on Smooth Particle Hydrodynamics (SPH) [11]. SPH is a Lagrangian formulation of the Navier-Stokes equations that has been adapted to model non-Newtonian fluids containing solid inclusions. While a full description of this approach is beyond the scope of this work, it is worth mentioning a few features of this simulation. A Lees-Edwards boundary condition is used to model Couette flow in the simulation cell [12]. This approach allows for the establishment of a Couette-like velocity profile in an infinite quasi-periodic system. As a result, wall effects, which might produce an inhomogeneous density variation or slip effects, are avoided. For an applied rate of strain, the volume average stress is calculated. The viscosity is then determined by dividing the volume averaged stress by the shear rate. An additional feature in this simulation is that lubrication forces are included to properly model the interactions between solid inclusions when they are in close proximity, as the numerical resolution needed to model such effects is too demanding computationally to accomplish using SPH alone. The approach utilized for this work has been validated for a variety of flow scenarios where excellent agreement with analytic solutions of flow fields for non-Newtonian continuum fluids in channel tube geometries and in experimental measurements of suspensions composed of micrometer sized spheres with different power-law matrix fluids in a Couette geometry $[10,13]$.

SRM 2493 (mortar) utilized SRM 2492 (paste) to serve as the matrix fluid of a suspension composed of mono-sized glass bead inclusions. Due to the different power law behavior of the viscosity vs. shear rate in low and high shear rate, the data were divided into two regions. The division between low and high shear rate was selected to be at a shear rate of $1 \mathrm{~s}^{-1}$. The high shear portion could be approximated with the Bingham equation or linear behavior of shear stress vs. shear rate. The data in each region was fitted to functions $f_{1}$ and $f_{2}$ (see equation 2 and 3 respectively). Function $f_{1}$ fit the viscosity vs. shear rate curve with $\dot{\gamma}<1$ and function $f_{2}$ fit for $\dot{\gamma}>1$. To represent the full dataset with a single function, equation 1 was developed, which seamlessly combines the two functions without any significant deviations from $f_{1}$ and $f_{2}$, 


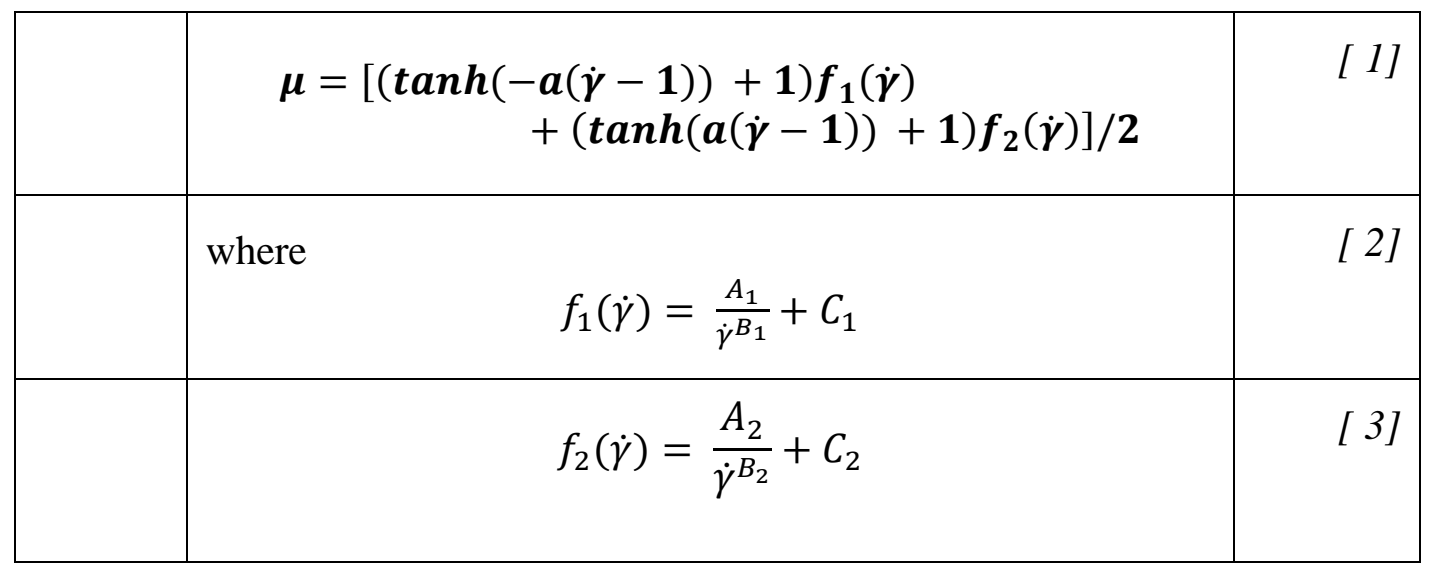

where

$\dot{\gamma}=$ Shear rate at the outer rim of the plate

$\mu=$ Viscosity

$A_{i}, B_{i}$, and $C_{i}(i=1,2)=$ Coefficients to fit the curve

$a=$ Fitting parameter to smoothly combine curves $\mathrm{f}_{1}$ and $f_{2}$.

The coefficients were determined by a least square fit to the paste SRM 2492 data obtained with the parallel plate geometry. The difference was estimated at less than $3 \%$ of the experimental values. The viscosity $(\mu)$ can be calculated at any given shear rate with equation (1) (uncertainity discussion on Section 7):

$$
\begin{aligned}
& \mathrm{A}_{1}=16.411 ; \mathrm{B}_{1}=0.988 ; \mathrm{C}_{1}=9.641 \\
& \mathrm{~A}_{2}=19.178 ; \mathrm{B}_{2}=0.727 ; \mathrm{C}_{2}=7.116 \\
& a=4
\end{aligned}
$$

The viscosity from equation (1) was input into the simulation code to serve as the matrix fluid. The glass beads were modeled as spherical inclusions with 472 spheres and 944 spheres used to model the $20 \%$ and $40 \%$ suspensions, respectively. The uncertainty of the simulated suspension viscosity was derived from calculating the standard deviation of the stress values, which was proportional to viscosity, over several strains (usually strains values of approximately 5 to 10). Four different shear rates were used in the simulation and the viscosity at each shear rate was determined (Table 6). When suitably rescaled [10], the simulation data falls on top of the viscosity vs. shear rate curve of the matrix fluid. The scaling parameters, shown in Table 7, are then used to generate predictive curves of the suspension's viscosity vs shear-rate, as shown in Figure 8 for the $20 \%$ and $40 \%$ volume fraction suspensions. In other words, to produce the scaled predictive curves, the $\mu_{\mathrm{sc}}$ scaling parameter was factored into the viscosity variable, $\mu$, in equation (1). Similarly, the $\gamma_{\mathrm{sc}}$ scaling parameter was factored into the shear rate variable, $\dot{\gamma}$, in equation (1).

In Figure 8, the SRM 2492 (paste) certified data baseline is shown as a solid black line, along with the simulation data for $20 \%$ volume fraction (O's) and $40 \%$ volume fraction (X's). From the data obtained using equation 1 and the scaling parameters of Table 7, it is possible to generate Table 8 using arbitrary shear rates for the paste and then calculate the plastic viscosity and yield stress, i.e., the Bingham parameters. 
Table 6: Rheological values estimated from numerical simulation model for SRM2492 paste containg $0 \%, 20 \%$ and $40 \%$ spherical inclusions. Simulation data portrayed as $X$ 's and $O$ 's in Figure 8. The uncertainty in values of viscosity, based on the standard deviation, is $10 \%$ or lower.

\begin{tabular}{|c|c|c|c|}
\hline $\begin{array}{c}\text { Shear Rate } \\
{[\mathbf{1} / \mathbf{s}]}\end{array}$ & $\begin{array}{c}\text { 0 \% Simulated } \\
\text { Viscosity } \\
\text { [Pa-s] }\end{array}$ & $\begin{array}{c}\text { 20 \% Simulated } \\
\text { Viscosity } \\
\text { [Pa-s] }\end{array}$ & $\begin{array}{c}\mathbf{4 0} \text { \% Simulated } \\
\text { Viscosity } \\
\text { [Pa-s] }\end{array}$ \\
\hline 0.1 & 175.0 & 214.0 & 297 \\
\hline 1 & 27.4 & 39.4 & 85 \\
\hline 10 & 10.6 & 18.1 & 66 \\
\hline 100 & 7.76 & 14.1 & 59 \\
\hline
\end{tabular}

Table 7: Scaling parameters to factor the SRM2492 curve to a mortar curve with known concentration. The uncertainty in scaling parameters, based on a least square fit of simulation data to equation (1), is $10 \%$ or less

\begin{tabular}{|c|c|c|}
\hline Concentration [\%] & $\boldsymbol{\mu}_{\text {sc }}$ factor & $\dot{\boldsymbol{\gamma}}_{\mathbf{s c}}$ factor \\
\hline 20 & 1.85 & 0.66 \\
\hline 40 & 7.20 & 0.23 \\
\hline
\end{tabular}

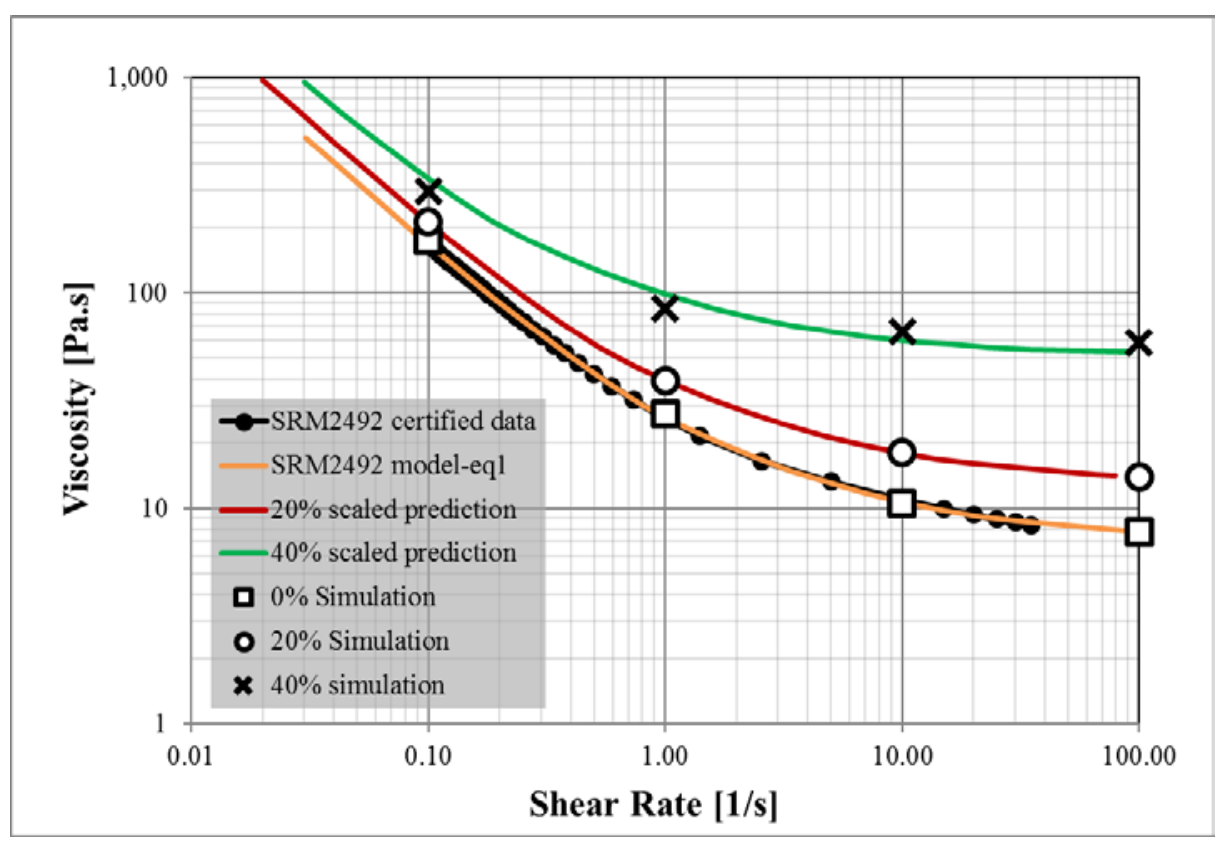

Figure 8: Numerical model simulation model data and predicted data from scaling parameters are compared to experimental data results (see Table 6 for values). The uncertainty is about $10 \%$ (see text) 
Table 8: Values for model with uncertainties provided in fundamental units (data were rounded to nearest hundreth). The shear rates for the $0 \%$ were arbitrary selected while the shear stress and viscosity were calculated using equation 1. The $20 \%$ and $40 \%$ were determined using the scaling factors of Table 7.

\begin{tabular}{|c|c|c|c|c|c|c|c|c|c|c|c|c|c|c|}
\hline \multicolumn{15}{|c|}{ Model } \\
\hline \multicolumn{5}{|c|}{$0 \%$ Model } & \multicolumn{5}{|c|}{ 20\% Model } & \multicolumn{5}{|c|}{$40 \%$ Model } \\
\hline $\begin{array}{c}\text { Shear } \\
\text { Rate } \\
{[1 / \mathrm{s}]}\end{array}$ & $\begin{array}{c}\text { Shear } \\
\text { Stress } \\
\text { [Pa] }\end{array}$ & $\begin{array}{c}\text { Shear } \\
\text { Stress } \\
\text { Expanded } \\
\text { uncertainity } \\
\text { [Pa] }\end{array}$ & $\begin{array}{c}\text { Viscosity } \\
{[\mathrm{Pa} \cdot \mathrm{s}]}\end{array}$ & $\begin{array}{c}\text { Viscosity } \\
\text { Expanded } \\
\text { uncertainity } \\
{[\text { Pa] }}\end{array}$ & $\begin{array}{c}\text { Shear } \\
\text { Rate } \\
{[1 / \mathrm{s}]}\end{array}$ & $\begin{array}{c}\text { Shear } \\
\text { Stress } \\
{[\mathrm{Pa}]}\end{array}$ & $\begin{array}{c}\text { Shear } \\
\text { Stress } \\
\text { Expanded } \\
\text { uncertainity } \\
\text { [Pa] }\end{array}$ & $\begin{array}{c}\text { Viscosity } \\
{[\mathrm{Pa} \cdot \mathrm{s}]}\end{array}$ & $\begin{array}{c}\text { Viscosity } \\
\text { Expanded } \\
\text { uncertainity } \\
{[\text { Pa] }}\end{array}$ & $\begin{array}{c}\text { Shear } \\
\text { Rate } \\
{[1 / \mathrm{s}]}\end{array}$ & $\begin{array}{c}\text { Shear } \\
\text { Stress } \\
\text { [Pa] }\end{array}$ & $\begin{array}{c}\text { Shear } \\
\text { Stress } \\
\text { Expanded } \\
\text { uncertainity } \\
{[\text { Pa] }}\end{array}$ & $\begin{array}{c}\text { Viscosity } \\
{[\mathrm{Pa} \cdot \mathrm{s}]}\end{array}$ & $\begin{array}{c}\text { Viscosity } \\
\text { Expanded } \\
\text { uncertainity } \\
{[\mathrm{Pa}]}\end{array}$ \\
\hline 30 & 262 & 26 & 8.7 & 0.9 & 20.0 & 323 & 32 & 16 & 2 & 6.90 & 454 & 45 & 63 & 6 \\
\hline 21 & 193 & 19 & 9.2 & 0.9 & 13.9 & 223 & 22 & 17 & 2 & 4.83 & 332 & 33 & 66 & 7 \\
\hline 14 & 139 & 14 & 9.9 & 1.0 & 9.7 & 167 & 17 & 18 & 2 & 3.22 & 247 & 25 & 72 & 7 \\
\hline 10 & 107 & 11 & 10.7 & 1.1 & 6.7 & 128 & 13 & 20 & 2 & 2.30 & 186 & 19 & 77 & 8 \\
\hline 7 & 82 & 8 & 11.8 & 1.2 & 4.7 & 99 & 10 & 22 & 2 & 1.61 & 142 & 14 & 85 & 8 \\
\hline 5 & 65 & 7 & 13.1 & 1.3 & 3.2 & 78 & 8 & 24 & 2 & 1.15 & 110 & 11 & 94 & 9 \\
\hline 3 & 47 & 5 & 15.7 & 1.6 & 2.3 & 63 & 6 & 29 & 3 & 0.69 & 87 & 9 & 113 & 11 \\
\hline 2.3 & 40 & 4 & 17.6 & 1.8 & 1.6 & 51 & 5 & 33 & 3 & 0.53 & 70 & 7 & 127 & 13 \\
\hline 1.6 & 33 & 3 & 20.7 & 2.1 & 1.1 & 42 & 4 & 38 & 4 & 0.37 & 57 & 6 & 149 & 15 \\
\hline 1.1 & 28 & 3 & 25.0 & 2.5 & 0.8 & 36 & 4 & 46 & 5 & 0.25 & 47 & 5 & 180 & 18 \\
\hline 0.8 & 24.1 & 2.4 & 30 & 3 & 0.53 & 32 & 3 & 56 & 6 & 0.18 & 41 & 4 & 217 & 22 \\
\hline 0.5 & 21.1 & 2.1 & 42 & 4 & 0.36 & 29 & 3 & 78 & 8 & 0.12 & 36 & 4 & 304 & 30 \\
\hline 0.4 & 20.1 & 2.0 & 50 & 5 & 0.26 & 27 & 3 & 93 & 9 & 0.09 & 33 & 3 & 362 & 36 \\
\hline 0.3 & 19.1 & 1.9 & 64 & 6 & 0.18 & 26 & 3 & 118 & 12 & 0.07 & 31 & 3 & 458 & 46 \\
\hline 0.2 & 18.0 & 1.8 & 90 & 9 & 0.13 & 25 & 3 & 167 & 17 & 0.05 & 30 & 3 & 649 & 65 \\
\hline 0.1 & 16.9 & 1.7 & 169 & 17 & 0.09 & 24 & 2 & 313 & 31 & 0.023 & 29 & 3 & 1219 & 122 \\
\hline 0.09 & 16.8 & 1.7 & 187 & 19 & 0.06 & 24 & 2 & 346 & 35 & 0.021 & 28 & 3 & 1345 & 134 \\
\hline 0.06 & 16.4 & 1.6 & 274 & 27 & 0.04 & 23 & 2 & 507 & 51 & 0.014 & 27 & 3 & 1973 & 197 \\
\hline 0.04 & 16.2 & 1.6 & 404 & 40 & 0.03 & 23 & 2 & 748 & 75 & 0.009 & 26.9 & 3 & 2911 & 291 \\
\hline 0.03 & 16.0 & 1.6 & 534 & 53 & 0.02 & 23 & 2 & 988 & 99 & 0.007 & 26.6 & 3 & 3846 & 385 \\
\hline
\end{tabular}


Table 9: Bingham parameters extracted from the model data displayed in Table 8.

\begin{tabular}{|c|c|c|c|}
\hline & \multicolumn{2}{|c|}{$\begin{array}{c}\text { Bingham } \\
\text { Parameters }\end{array}$} \\
\hline & & $\begin{array}{c}\text { Yield } \\
\text { Stress, } \\
\text { To }[\mathrm{Pa}]\end{array}$ & $\begin{array}{c}\text { Plastic } \\
\text { Viscosity, } \\
\boldsymbol{\mu}_{\text {B }}[\mathrm{Pa} \cdot \mathrm{s}]\end{array}$ \\
\hline \multirow{6}{*}{ Model } & $0 \%$ & 23.1 & 8.07 \\
\hline & $\begin{array}{l}\text { Std. } \\
\text { Unc. }\end{array}$ & 1.8 & 0.8 \\
\hline & $20 \%$ & 31.0 & 14.9 \\
\hline & $\begin{array}{l}\text { Std. } \\
\text { Unc. }\end{array}$ & 3.6 & 2.3 \\
\hline & $40 \%$ & 38.8 & 57.9 \\
\hline & $\begin{array}{l}\text { Std. } \\
\text { Unc. }\end{array}$ & 5.2 & 8.7 \\
\hline
\end{tabular}




\section{Rheological Measurements}

\subsection{Rheometer Set-up}

Many industrial rheometers available today allow the users to test a variety of configurations of containers and spindles. In this study, the container is a cylindrical steel cup with a height of $80 \mathrm{~mm}$ and a diameter of $43 \mathrm{~mm}$, with serrated ribs. Various spindle designs were used. A computer controls the rotational speed of the spindle and measures the torque, while the container is stationary.

Three types of spindles were used for this certification study, selected to represent spindle families: six-blade vane (vane-type), a serrated coaxial cylinder (cylindrical-type) and a double helical spiral (sometimes referred to as helical ribbon mixer or mixer-type). See Figure 9 for spindles' dimensions.

A coaxial rheometer with a smooth or serrated surface of the spindle is the most widely used design. The serration is needed to reduce slippage of the suspension. The modified spindle with a serrated surface is shown in Fig. 9a.

The vane spindle (Fig. $9 b$ ) provides a shearing plane made from the material in shear itself. As the vane spins, it is generally assumed that a cylindrical wall of the material is formed on the perimeter of the blades, thus the material shears on a wall made of itself. This shearing scenario, presumably, increases the frictional force, therefore reducing slippage.

The third type of spindle tested in this study is the mixer-type or impeller spindle, known as the double spiral (Fig. 9c). This spindle type is useful due to its capability to mix the material during testing. By mixing during the testing, any sedimentation or migration issues are minimized.

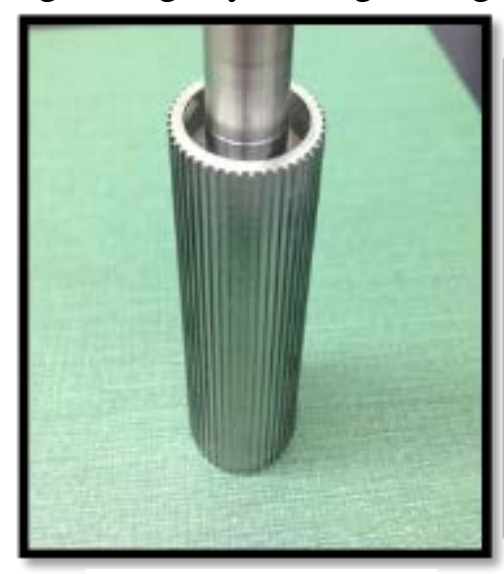

Fig. 9a: Serrated coaxial cylinder.

$D=18 \mathrm{~mm}$

$H=55 \mathrm{~mm}$

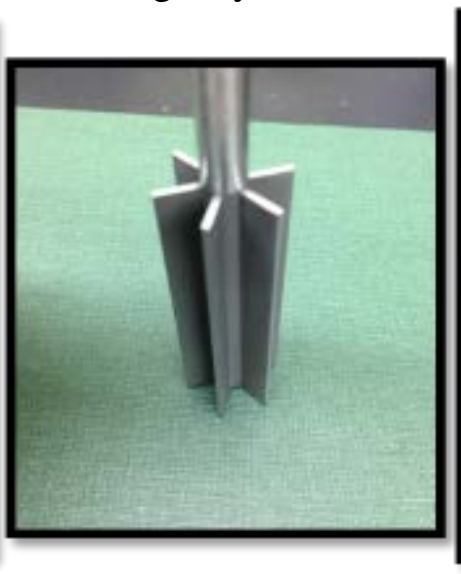

Fig. 9b: Six-blade vane.

$D=18 \mathrm{~mm}$

$H=55 \mathrm{~mm}$

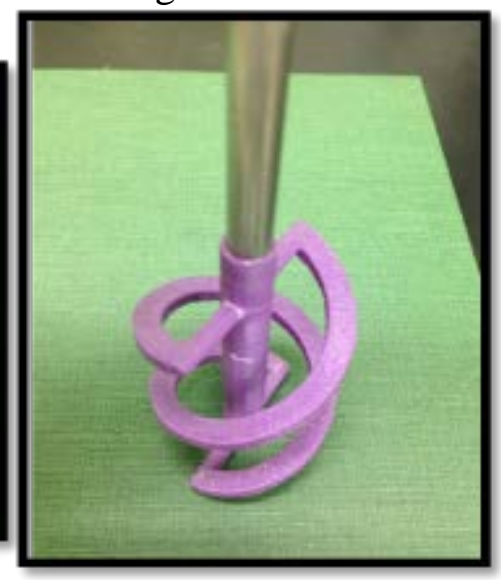

Fig. 9c: Double helical spiral.

$D=35 \mathrm{~mm}$

$H=50 \mathrm{~mm}$

Figure 9: Three spindle types were used for testing, each representing a different family 


\subsection{Experimental Design}

Rheological testing was performed on a MARS III rheometer in conjunction with the spindle geometries described in the previous section. The certification of SRM 2492 [2] used one batch from 11 units (or boxes). The second batch from these same units were used for the certification of SRM 2493 along with 11 randomly selected container of beads ${ }^{1}$. The first batch of 11 was incorrectly tested and was consequentl not considered for the certification. See Appendices $\mathrm{A}$ and $\mathrm{B}$ for the data obtained from the 10 mixtures used for certification.

All 10 mixtures used for the certification were tested at an age of one day after mixing the paste portion of the SRM. Each reported value was the average of three consecutive tests run back-toback with a 5 min rest in between each test. The test protocol consisted of ramping 15 steps up from $0.1 \mathrm{rpm}(0.01047 \mathrm{rad} / \mathrm{s})$ to $100 \mathrm{rpm}(10.47 \mathrm{rad} / \mathrm{s})$ and 20 steps back down to $0.1 \mathrm{rpm}$ $(0.01047 \mathrm{rad} / \mathrm{s})$ to capture any hysteresis. Schedule codes for testing were assigned and represented in the schedule shown in Table 10 and complemented by the legend on the side. The pseudo-random generator was used to establish the order in which the spindles were tested each day. The gray row in the schedule refers to the time period it takes the rheometer to complete its readings. This waiting period was used to create the mixture samples as scheduled.

The testing model was developed to observe three concentrations of beads in the amounts of $0 \%, 20 \%$ and $40 \%$ by volume. The amount of material developed by one batch of SRM was sufficient enough to yield two containers of SRM that overfilled the rheometer's holding container. Therefore, half of the 10 usable batches were used to test SRM compositions of $0 \%$ and $20 \%$ beads by volume, while the other half tested compositions of $0 \%$ and $40 \%$. Ultimately, the results will report five values for $20 \%$, five for $40 \%$ and 10 values for $0 \%$ concentrations.

\footnotetext{
${ }^{1}$ Eventually a portion of the SRM 2492 units (or boxes) were repackaged to include the beads container and re-labelled
} SRM 2493 
Table 10: Testing schedule complemented by the key on the right side. The waiting periods were used to create the mixes listed along the gray rows.

\begin{tabular}{|c|c|c|c|c|c|c|}
\hline \multirow[b]{2}{*}{ 8:00 AM } & & \multicolumn{5}{|c|}{ Full Testing Schedule } \\
\hline & & $\mathrm{M}$ & $\mathrm{T}$ & $\mathrm{W}$ & $\mathrm{T}$ & $\mathrm{F}$ \\
\hline 9:30 AM & Week 1 & \multirow{7}{*}{$\begin{array}{c}\text { Columbus } \\
\text { Day }\end{array}$} & & $1 \mathrm{~B}$ & $2 \mathrm{~A}$ & \\
\hline $11: 00 \mathrm{AM}$ & \multirow{5}{*}{$10 / 13 / 2015$} & & & $1 \mathrm{C}$ & $2 \mathrm{~B}$ & \\
\hline $12: 30 \mathrm{PM}$ & & & & $1 \mathrm{~A}$ & $2 \mathrm{C}$ & \\
\hline 3:00 PM & & & & 1B & $2 \mathrm{~A}$ & \\
\hline 4:30 PM & & & & $1 \mathrm{C}$ & $2 \mathrm{~B}$ & \\
\hline 6:00 PM & & & & $1 \mathrm{~A}$ & $2 \mathrm{C}$ & \\
\hline \multirow[t]{7}{*}{ Waiting Period } & & & Mix 1 & Mix 2 & & \\
\hline & Week 3 & & $3 \mathrm{~B}$ & $4 \mathrm{~A}$ & & \\
\hline & & & $3 \mathrm{~A}$ & $4 \mathrm{C}$ & & \\
\hline & & & $3 \mathrm{C}$ & 4B & & \\
\hline & $10 / 20 / 2015$ & & $3 B$ & $4 \mathrm{~A}$ & & \\
\hline & & & $3 \mathrm{~A}$ & $4 \mathrm{C}$ & & \\
\hline & & & $3 \mathrm{C}$ & 4B & & \\
\hline \multirow[t]{8}{*}{ Waiting Period } & & Mix 3 & Mix 4 & & & \\
\hline & $\begin{array}{l}\text { Week } 4 \\
10 / 27 / 2015 \\
\end{array}$ & \multicolumn{3}{|c|}{ ACI Convention } & & \\
\hline & Week 5 & & & & $5 B$ & $6 \mathrm{~B}$ \\
\hline & \multirow{5}{*}{$11 / 3 / 2015$} & & & & $5 \mathrm{~A}$ & $6 \mathrm{C}$ \\
\hline & & & & & $5 \mathrm{C}$ & $6 \mathrm{~A}$ \\
\hline & & & & & $5 B$ & $6 \mathrm{~B}$ \\
\hline & & & & & $5 \mathrm{~A}$ & $6 \mathrm{C}$ \\
\hline & & & & & $5 \mathrm{C}$ & $6 \mathrm{~A}$ \\
\hline \multirow[t]{7}{*}{ Waiting Period } & & & & Mix 5 & Mix 6 & \\
\hline & Week 6 & & \multirow{7}{*}{$\begin{array}{c}\text { Veterans } \\
\text { Day }\end{array}$} & & $7 \mathrm{~B}$ & $8 \mathrm{C}$ \\
\hline & \multirow{5}{*}{$11 / 10 / 2015$} & & & & $7 \mathrm{~A}$ & $8 B$ \\
\hline & & & & & $7 \mathrm{C}$ & $8 \mathrm{~A}$ \\
\hline & & & & & $7 \mathrm{~B}$ & $8 \mathrm{C}$ \\
\hline & & & & & $7 \mathrm{~A}$ & $8 \mathrm{~B}$ \\
\hline & & & & & $7 \mathrm{C}$ & $8 \mathrm{~A}$ \\
\hline \multirow[t]{7}{*}{ Waiting Pe riod } & & & & Mix 7 & Mix 8 & \\
\hline & Week 7 & & 9B & 10B & $11 \mathrm{~B}$ & \\
\hline & & & $9 \mathrm{~A}$ & $10 \mathrm{C}$ & $11 \mathrm{~A}$ & \\
\hline & & & $9 \mathrm{C}$ & $10 \mathrm{~A}$ & $11 \mathrm{C}$ & \\
\hline & $11 / 17 / 2015$ & & 9B & 10B & $11 \mathrm{~B}$ & \\
\hline & & & $9 \mathrm{~A}$ & $10 \mathrm{C}$ & $11 \mathrm{~A}$ & \\
\hline & & & $9 \mathrm{C}$ & $10 \mathrm{~A}$ & $11 \mathrm{C}$ & \\
\hline Waiting Period & & Mix 9 & Mix 10 & Mix 11 & & \\
\hline
\end{tabular}

\begin{tabular}{|c|c|}
\hline \multicolumn{2}{|c|}{ Schedule Key } \\
\hline \multirow{2}{*}{$\frac{\text { Testing }}{\text { Parameters }}$} & \\
\hline & Mixing Day \\
\hline \multirow{3}{*}{$\begin{array}{c}\text { All Mixes } \\
\text { tested at } 1 \\
\text { day. }\end{array}$} & Test $0 \%(11)$ \\
\hline & Test $20 \%(5)$ \\
\hline & Test $40 \%(6)$ \\
\hline Schedule Code & Spindle \\
\hline $\mathbf{A}$ & $\begin{array}{c}\text { RHN-83A } \\
\text { Double Spiral }\end{array}$ \\
\hline B & $\begin{array}{c}\text { RHN-83C } \\
\text { Six Blade Vane }\end{array}$ \\
\hline C & $\begin{array}{c}\text { SS18 } \\
\text { Serr. Coaxial Cylinder }\end{array}$ \\
\hline
\end{tabular}




\subsection{Rheometer Data Calibration}

To calibrate a rheometer using the data developed in this report, two methods are suggested: 1) using the approximation of the Bingham equation and 2) using a method based on the viscosity vs. shear rate curve and the model described in Section 4. Here, step by step instruction will be provided on how to proceed using these two suggested methods.

The first step with either method is to prepare the SRM 2493 mortar mixture and to load it in the rheometer to be calibrated. Run at least three tests and use the average to extract the raw data, which consists of the measured torque $(I)[\mathrm{N} \cdot \mathrm{m}]$ and rotational speed $(N)[\mathrm{rad} / \mathrm{s}]$ for at least 10 points in the decreasing rotations speed curve (down-curve). The next goal is to convert this values in fundamental units of $\mathrm{Pa}$ and $\mathrm{s}^{-1}$, respectively, by using one of the two suggested methods. Either methods are acceptable, the method based on Bingham parameters (Section 4.3.1) is faster and easy to implement, but the method based on viscosity vs. shear rate (Section 4.3.2) could be more accurate as it takes into account the whole curve measured not just the linear portion.

The certified values for SRM 2492 tested in a parallel plate rheometer are used here as reference points. It should be noted that the SRM 2492 paste materials are the same as the SRM 2493 matrix materials. Ideally, the results of any other spindle that is used to test the SRM 2492 or paste part of SRM 2493 should reproduce the certified values. Using such criteria, the raw data simply needs to be calibrated to the SRM 2492 certified values. Thus, the next step would be to convert the data obtained with the different tools (Section 5.2) into fundamental units by using SRM 2492 or paste SRM 2493. The next two sections, 5.3.1 and 4.3.2, will describe how this is done.

The same process described in two Sections 5.3.1 and 4.3.2 could be used to calibrate any rheometer by the user of SRM 2493, by selecting either method as described in the certificate for SRM $2493^{2}$. A web based software and a spreadsheet are posted on the NIST website that should help the user to calculate the necessary calibration factors.

\subsubsection{Calibration Based on the Bingham Parameters}

To transform the data generated in this report using the Bingham approach, the SRM 2492 certified values were used as the base line. The Bingham parameters of the SRM 2492 paste were extracted from the certificate and compared to the parameters calculated from the experimental or model data, using only the linear part of the curve (torque vs. rotational speed). The schematic shown in Figure 10 explains the calibration process in a sequential form. A spreadsheet was developed for this procedure, and is posted on the SRM 2493 website ${ }^{2}$. This file can be used by following a "how to" fact sheet, which will be available on the same spreadsheet.

The calibration process begins by running at least three tests with a geometry of choice on a sample of SRM 2493, and finding the average values from the produced torque and respective rotational speed. Since the raw data should match the SRM 2493 certified values either for the paste or the model data, then the torque and rotational speed can be scaled to match the known

\footnotetext{
${ }^{2}$ www.nist.gov under “Standard Reference Materials”
} 
certified viscosity and shear rate. This implies that the shear stress $(\tau)$, shear rate $(\dot{\gamma})$, and apparent viscosity $(\mu)$ are proportional to the torque $(\Gamma)$, rotational speed $(N)$, and angular momentum $(\Gamma / N)$, respectively. In other words, the ( $\Gamma$ vs. $N$ ) results from the new geometry are scaled to match the ( $\tau$ vs. $\dot{\gamma}$ ) certified SRM 2493 paste values by calculating scaling factors $K_{\tau}$ and $K_{\mu}$. Figure 9 provides a schematic of the procedure. The calibration factors convert the two raw variables into fundamental rheology units by using proportionality relationships. Also, if preferable, the two calibration factors listed are able to produce a direct shear rate scaling factor, $K_{\gamma}$, by means of equation (5) which is derived from the known relationship shown in equation (4):

$$
\mu=\tau / \dot{\gamma} \quad[\mathrm{Pa} \cdot \mathrm{s}] .
$$

Thus, the shear rate calibration factor would be:

$$
K_{\gamma}=K_{\tau} / K_{\mu} .
$$

The best results using this method are obtained when the shear rate is larger than $1 \mathrm{~s}^{-1}$ or when using the linear portion of the curve. The flow curve of shear stress vs. shear rate is clearly not linear for shear rates lower than $1 \mathrm{~s}^{-1}$, while the Bingham model implies that the relationship remains linear. The factors $K$ represent the influence of the rheometer geometry on the results. 
Obtain the average of the raw data at any day between $1 \mathrm{~d}$ and $7 \mathrm{~d}$.

(Torque, $\boldsymbol{\Gamma}[\mu \mathrm{Nm}]$ and rotational speed, $\mathbf{N}$ [rpm]) for at least three runs.

\section{1}

Use the average data to find the slope (S) and y-intercept (I) of the torque vs. rotational speed relationship. Note: use only the linear part of the curve, with at least 10 data points.

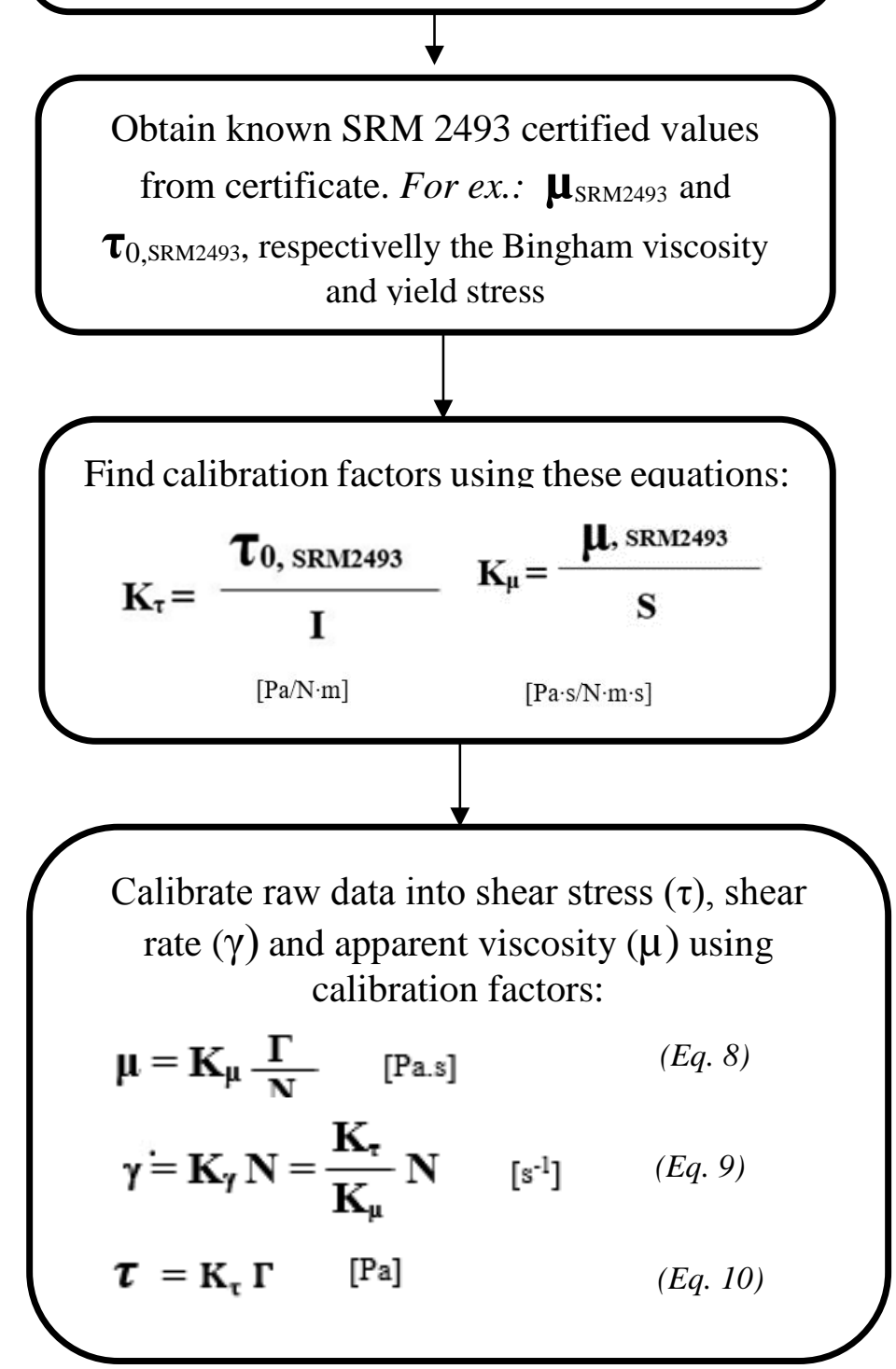

Figure 10: Schematic for the calibration of rheometer data from raw torque and rotational speed to fundamental rheological variables, viscosity and shear rate.

Note: a) This process is applied for calibration of any spindle used in coaxial rheometer.

b) The conversion from rpm to rad/s requires multiplication by $2 \pi / 60$. 


\subsubsection{Calibration Using the Viscosity vs. Shear Rate Curve}

The second method proposed is to use the viscosity vs. shear rate curve, as represented by equation (1) and compared with the data measured using the same material (i.e., same beads concentration). This comparison can be achieved by calculating two scaling factors, $L_{\mu}$ and $L \gamma$, that will be used for all future calculation to convert torque to shear rate when measuring unknown materials. See Figure 12 for a scheme of the calibration process.

$$
\begin{array}{cr}
\boldsymbol{\mu}=\boldsymbol{L}_{\boldsymbol{\mu}} \frac{\boldsymbol{\Gamma}}{\boldsymbol{N}} & {[\mathrm{Pa} \cdot \mathrm{s}]} \\
\dot{\gamma}=L_{\gamma} N & {\left[\mathrm{~s}^{-1}\right]} \\
\tau=L_{\mu} L_{\gamma} \Gamma & {[\mathrm{Pa}] .}
\end{array}
$$

To calculate the factors $L$, the measured curve needs to be rescaled both along the $\mathrm{Y}$ and $\mathrm{X}$ axes, until it matches the reference curve (SRM 2492 paste or the model curve). An algorithm could be developed to fit by least square regression, to equation (1). This equation can be simplified by simple algebra , using the same parameters as equation (1):

$$
\mu=\frac{\left(C_{2}+\frac{A_{2}}{\dot{j}^{\beta_{2}}}\right)+\left(C_{1}+\frac{A_{1}}{\dot{j}^{\beta_{1}}}\right) e^{2 a(j-1)}}{1+e^{2 a(j-1)}}
$$

The computation of the $L$ factors can be accomplished as follows:

1. Define a statistical model for the $n$ measurement pairs $\left(N_{i}, \Gamma_{i}\right)$ based on the SRM 2493 paste as:

$\dot{\gamma}_{i}=\boldsymbol{L}_{\boldsymbol{\gamma}} \cdot N_{i}, \quad i=1, \ldots, n$

$\tau_{i} \sim \operatorname{Gaussian}\left(\mu_{i}, \sigma^{2}\right), i=1, \ldots, n$

where

$\mu_{i}=\boldsymbol{L}_{\mu} \cdot \frac{\left(C_{2}+\frac{A_{2}}{\dot{\gamma}_{i}^{B}}\right)+\left(C_{1}+\frac{A_{1}}{\dot{\gamma}_{i}^{B}}\right) e^{2 a\left(\dot{\gamma}_{i}-1\right)}}{1+e^{2 a\left(\dot{\gamma}_{i}-1\right)}}, i=1, \ldots, n$,

$\mathrm{A}_{1}=16.411 ; \mathrm{B}_{1}=0.988 ; \mathrm{C}_{1}=9.641$

$\mathrm{A}_{2}=19.178 ; \mathrm{B}_{2}=0.727 ; \mathrm{C}_{2}=7.116$

$a=4$

Prior distributions:

$\sigma \sim \operatorname{Gamma}(1.0 E-5,1.0 E-5)$

$\boldsymbol{L}_{\boldsymbol{\gamma}} \sim$ Uniform $(1,8)$

$\boldsymbol{L}_{\mu} \sim$ Uniform $(10,10000)$ 
2. Compute the values of $\boldsymbol{L}_{\gamma}$ and $\boldsymbol{L}_{\mu}$ using a Markov Chain Monte Carlo method [14] implemented in OpenBUGS [15], executed using the following R code [16].

The code requires that both $\mathrm{R}$ and OpenBUGS be installed, both are free to download. $\mathrm{R}$ can be obtained at http://www.R-project.org/ , OpenBUGS at http://www.openbugs.net/w/Downloads . The only input to the program that is needed are the values $N$ and $\Gamma$, and the sample size $n$. Pasting the entire program below into an R window will produce the estimates of the $L$ factors as well as a plot which shows the fit to the SRM 2492 function.

Here is an example using the measurement data for SRM 2493 with six-blade vane configuration (Figure 11). This software will also be available from the SRM 2493 website $^{2}$.

In the program below $\mathrm{X}=\mathrm{N}$ in $\mathrm{rad} / \mathrm{s}$, and $\mathrm{Y}=\Gamma$ in $\mu \mathrm{Nm}$

\#\#\#\#\#\#\#\#\#\#\#\#\#\#\#\#\#\#\#\#\#\#\#\#\#\#\#\#\#\#\#\#\#\#\#\#\#\#\#\#\#\#\#\#\#\#\#\#\#\#\#\#\#\#\#\#\#\#\#\#\#\#\#\#\#\#\# library(R2OpenBUGS)

\#\#\#\#\#\#\#\#\#\#\#\# type data $X, Y, n$ here:

linedata $<-$ list $(y=c(7246.99,5435.07,4136.84,3203.2,2528.2,2035.31,1672.22,1399.73$, 1190,1031.01,907.89,812.84,740.87), $x=c(10.472,7.278,5.058,3.519,2.45,1.696,1.183,0.822,0.571,0.397,0.276,0.192,0.133), n=13)$

\#\#\#\#\#\#\#\#\#\#\#\#\#

\#\#\#\#SR 2492 curve for comparison to see the fit visc92<-c(8.72,9.21,9.84,10.66,11.73,13.15,14.94,17.33,20.44,24.44,30.1, $39.27,51.25,69.47,94.31,132.83,186.79,274.08,404.37,534.13)$

sr92<-c(30.32,21.07,14.64,10.19,7.09,4.91,3.43,2.38,1.65,1.15,0.8, $0.55,0.39,0.27,0.19,0.13,0.09,0.06,0.04,0.03)$

\#\#\#\#

\#\#\#\#\# OpenBUGS code and inits

lineinits $<$-function()\{list(sig=1)\}

linemodel $<-$ function() \{sig dgamma(1.0E-5,1.0E-5)

$\operatorname{Lg} \sim \operatorname{dunif}(1,8)$

$\operatorname{Lm} \sim$ dunif $(10,10000)$

$\mathrm{Lmu}<-1 / \mathrm{Lm}$

$\mathrm{Ltau}<-\mathrm{Lg} / \mathrm{Lm}$

$a 1 n<-16.411$

a1sig $<-1 /(0.64 * 0.64)$

b1 $<-0.988$

b1sig $<-1 /(0.018 * 0.018)$

$c 1 n<-9.641$ 


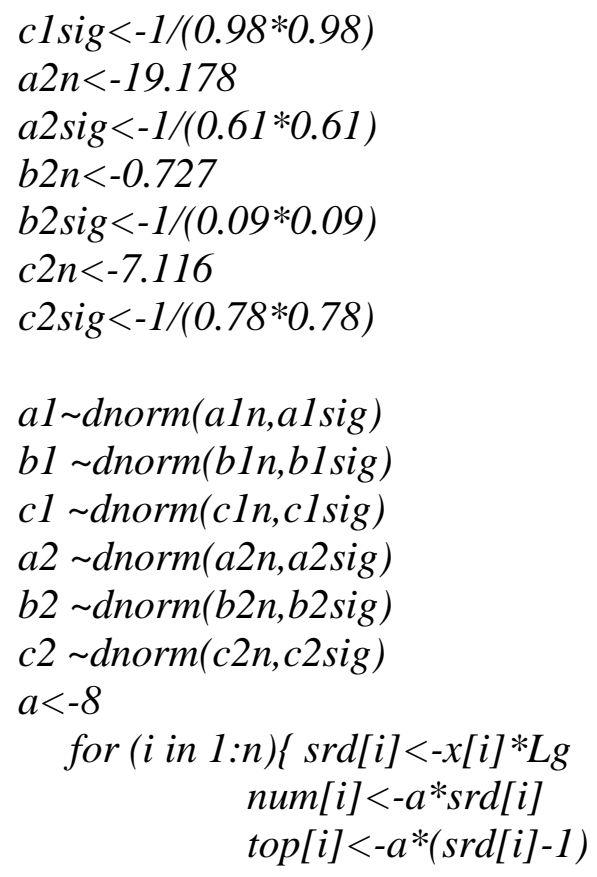




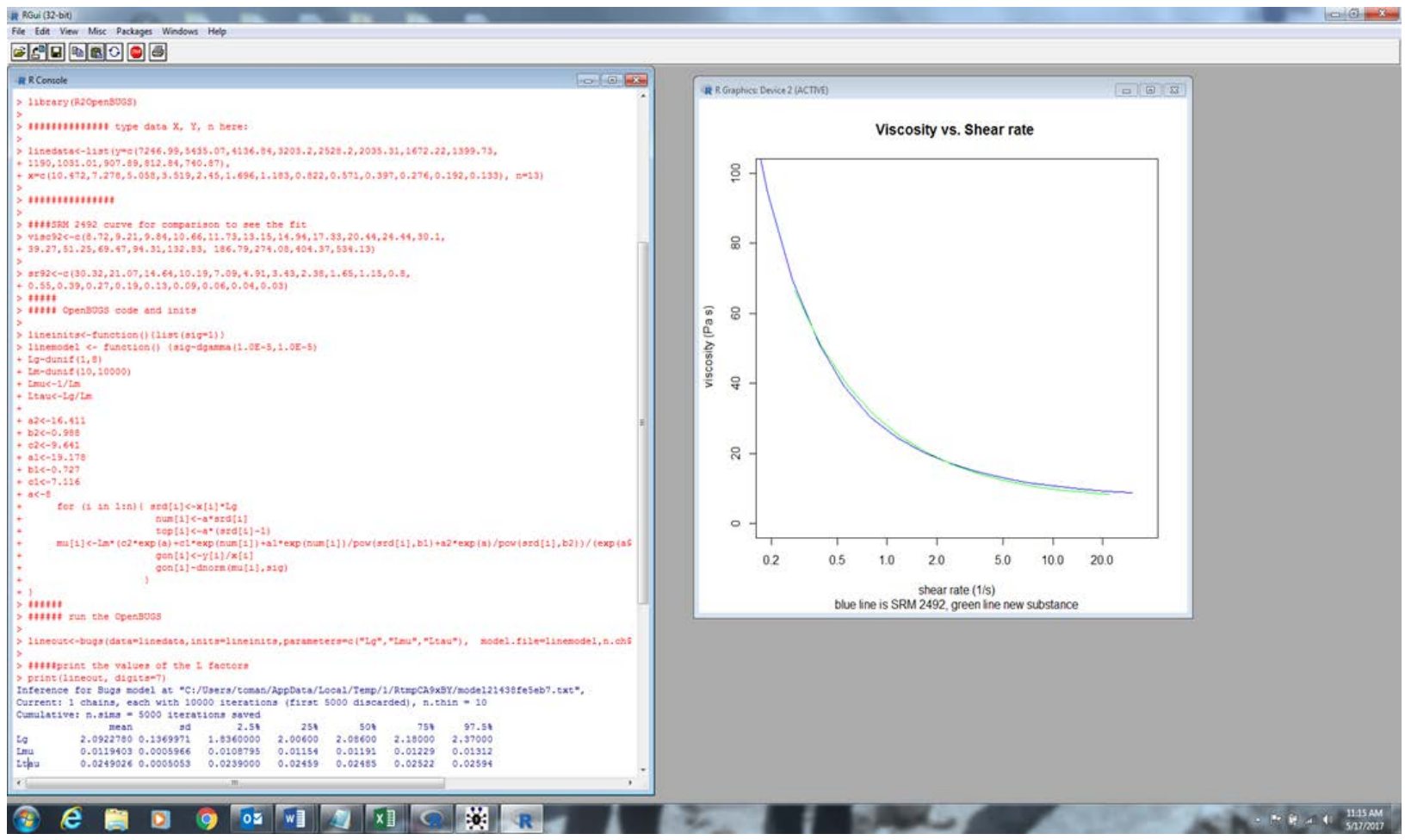

Figure 11: Screenshot of the running of the above software code and of the results for the SRM 2493 six-blade vane data using the method for fitting equation (1). 
Obtain the average of the raw data (Torque, $\boldsymbol{\Gamma}$

$[\mu \mathrm{Nm}]$ and rotational speed, $\mathbf{N}$ [rpm]) for at least three runs using an SRM 2493 mixture

Convert $\mathbf{N}$ units to [rad/s] and $\boldsymbol{\Gamma}$ to $[\mathrm{Nm}]$.

Use the average data to calculate the ratio $(\boldsymbol{\Gamma} /$ $\mathbf{N}[\mathrm{Nm} /(\mathrm{rad} / \mathrm{s})])$ for each $\mathbf{N}[\mathrm{rad} / \mathrm{s}]$.

Fit the data to model curve equation (1), scaled with the coefficient of Table 4 with the same concentration used for the tests and obtain two factors $\mathbf{L}_{\boldsymbol{\mu}}$ and $\mathbf{L} \boldsymbol{\gamma}$ (See methodology above)

Calibrate raw data into shear stress $(\tau)$, shear rate $(\dot{\gamma})$ and viscosity $(\mu)$ using scaling factors:

$$
\begin{array}{ccc}
\boldsymbol{\mu}=\boldsymbol{L}_{\boldsymbol{\mu}} \frac{\boldsymbol{\Gamma}}{\boldsymbol{N}} & {[\mathrm{Pa} \cdot \mathrm{s}]} & (\text { Eq.14 }) \\
\dot{\gamma}=\boldsymbol{L}_{\gamma} \boldsymbol{N} & {\left[\mathrm{s}^{-1}\right]} & (\text { Eq. 15) } \\
\boldsymbol{\tau}=\boldsymbol{L}_{\boldsymbol{\mu}} \boldsymbol{L}_{\boldsymbol{\gamma}} \boldsymbol{\Gamma} & {[\mathrm{Pa}]} & (\text { Eq.16) }
\end{array}
$$

Figure 12: Schematic for the calibration of rheometer data from raw torque and rotational speed to fundamental rheological variables, viscosity and shear rate, applicable only to the linear portion of the curve.

Note: a) This process is applied for calibration of any spindle used in coaxial rheometer.

b) The conversion from rpm to rad/s requires multiplication by $2 \pi / 60$. 


\subsection{Experimental Results}

Rheological data represented in the form of flow curves were obtained for three different spindles, as mentioned in Section 4.1. The flow curves portray the flow behavior of the material as a simple continuum fluid without needing to regard the existence of the complex inter-particle flow [10]. The flow curves are useful representations because they help compare the flow behavior of two different materials, or two different spindles, so long as the testing shear rates are similar. In addition, flow curve data is typically represented by the data acquired during the downward ramp (decreasing shear rate) which is why the protocol contains more steps for the down ramp compared to the up ramp, see protocol in Section 4.2. Therefore, flow curve comparisons will use only down curve data in the rest of this report. These results were expected to match the behavior from the simulation models described in Section 3.

As explained in the rheometer calibration process in Section 4.3 for the reference curves developed in this report, testing requires that the SRM paste (0\% beads) be tested when introducing a new spindle geometry. Therefore, every batch used for SRM mortar testing was divided into two containers prior to testing on the rheometer. In doing so, half the batch is used to complete the required calibration step where the operator performs the test on the SRM paste (0\% beads). Since this step is required for data calibration, the calibrated $0 \%$ values for any new geometry is expected to match the SRM 2492 certified values using a parallel plate.

As stated in Section 4.2, half of the mixtures analyzed during this study represent a concentrated mortar composition of $40 \%$ monosized beads by volume. Half of the batches were used to analyze a concentrated mortar composition of $20 \%$ beads by volume. After the calibration step was taken, the same testing procedure was conducted on both the $20 \%$ and $40 \%$ mortars.

Then two processes were applied to the raw data of torque and rotational velocity to convert the data into fundamental units: either by using the Bingham approximation (Section 4.4.1) or by rescaling the viscosity curves (Section 4.4.2) as described in the calibration Section 5.3.

\subsubsection{Bingham Parameters}

As mentioned in Section 4.3, the calibration factors are calculated in order to convert the rheometer's raw data into fundamental rheological units. A different calibration factor is calculated for each test since each set of tests data is independent of the other tests. The three calibration factors $\left(K_{\gamma}, K_{\tau}, K_{\mu}\right)$ were calculated for each mixture, and were found not to change with the beads concentration. Thus, they were averaged to represent the geometry being used (Table 9), along with the standard uncertainty, calculated from one standard deviation of all the values obtained. The calculation of such factors are produced when testing the SRM paste without beads. The table shows the calibration factors operators could use to calibrate their experimental data if using the same exact spindles as were used in this study.

The resulting flow curves of the calibrated data for both mortar types and the paste without beads $(0 \%)$ can be found in Figure 13 to Figure 16. The figures compare the results of the $0 \%$ paste (Figure 13) to the certified SRM 2492 values, as well as the changes in the flow curve behavior when $20 \%$ or $40 \%$ beads by volume are added to the paste. As shown, the $20 \%$ and 40 
\% mortars increase the viscosity of the mixtures, as expected when increasing the concentration of particles in a suspended fluid. The flow curves shown in Figure 13 track each other closely, within the uncertainity of the measurements, providing confidence in the calibration process of the SRM 2493 mortars.

The torque and rotational speed, obtained from the set of raw data in this study showed that the SRM 2493 materials acted like Bingham materials. Bingham materials are known for having a linear shear stress vs. shear rate relationship, which makes the Bingham parameters, yield stress and plastic viscosity, simple to calculate, where the plastic viscosity is the slope of the linear relationship and the yield stress is its y-intercept. The similarity in behavior found in the raw data encouraged the calculation of a "yield stress" and "plastic viscosity" based on the torque vs. rotational speed relationship. These values were calculated for all three spindles discussed in this study at $0 \%, 20 \%$ and $40 \%$ bead concentrations and are summarized in Table 12 .

The accuracy of this calibration process is shown in Figure 13, which compares the calibrated data after testing SRM paste (no beads) with the three spindles observed in this study. As shown, using any of the three spindles allows the experimental curves to be shifted on top of the certified SRM 2492 values (shown in black) especially in the range of equal shear rates. Equal shear rates refer to those that were applied during the SRM 2492 certification testing. A larger range of shear rates was used in this study to observe the behavior at lower shear rates.

Table 11: Calibration factors for experimental data of each spindle tested in this study.

\begin{tabular}{|c|c|c|c|}
\hline & \multicolumn{3}{|c|}{ Data Calibration Factors } \\
\hline & $\begin{array}{c}\text { Serrated } \\
\text { Coaxial } \\
\text { Cylinder }\end{array}$ & $\begin{array}{c}\text { Six Blade } \\
\text { Vane }\end{array}$ & $\begin{array}{c}\text { Double } \\
\text { Spiral }\end{array}$ \\
\hline $\begin{array}{c}\mathbf{K} \boldsymbol{\tau} \\
{[\mathrm{Pa} / \mathrm{N} \cdot \mathrm{m}]}\end{array}$ & 29951.7 & 28470.5 & $11,540.5$ \\
\hline Std. Unc. & 927.8 & 657.7 & 282.4 \\
\hline $\begin{array}{l}\mathbf{K} \gamma \\
{[-]}\end{array}$ & 2.66 & 2.29 & 3.63 \\
\hline Std. Unc. & 0.16 & 0.11 & 0.19 \\
\hline $\begin{array}{c}\mathbf{K} \boldsymbol{\mu} \\
{[\mathrm{Pa} \cdot \mathrm{s} / \mathrm{N} \cdot \mathrm{m} \cdot \mathrm{s}]}\end{array}$ & $11,307.6$ & $12,485.0$ & $3,185.2$ \\
\hline Std. Unc. & 574.4 & 555.3 & 146.9 \\
\hline
\end{tabular}


Table 12: Plastic viscosity and yield stress at $0 \%, 20 \%$ and $40 \%$ bead volume concentrations for all three spindles. See Section 7.1 for standard uncertainties. Note that the values for $0 \%$ are all identical as all curves were calibrated against the $0 \%$ data of SRM 2492.

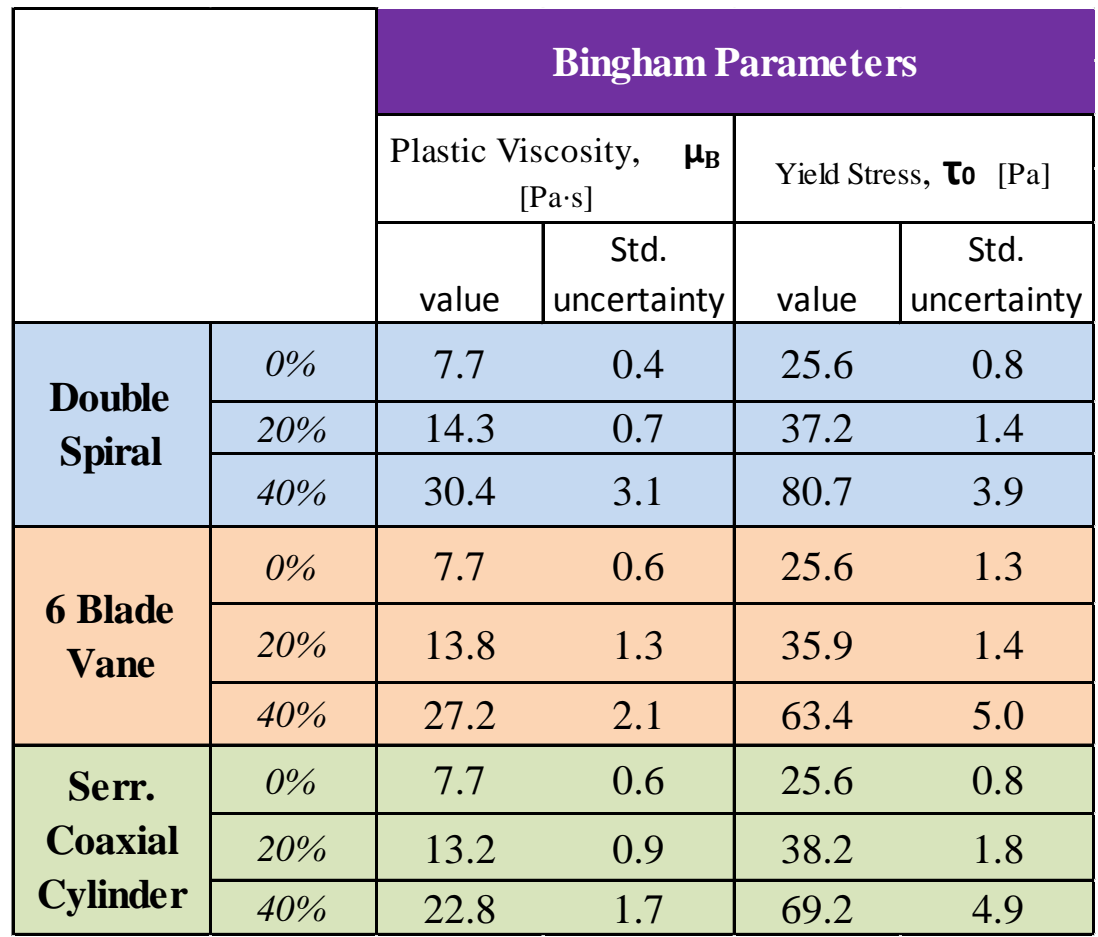

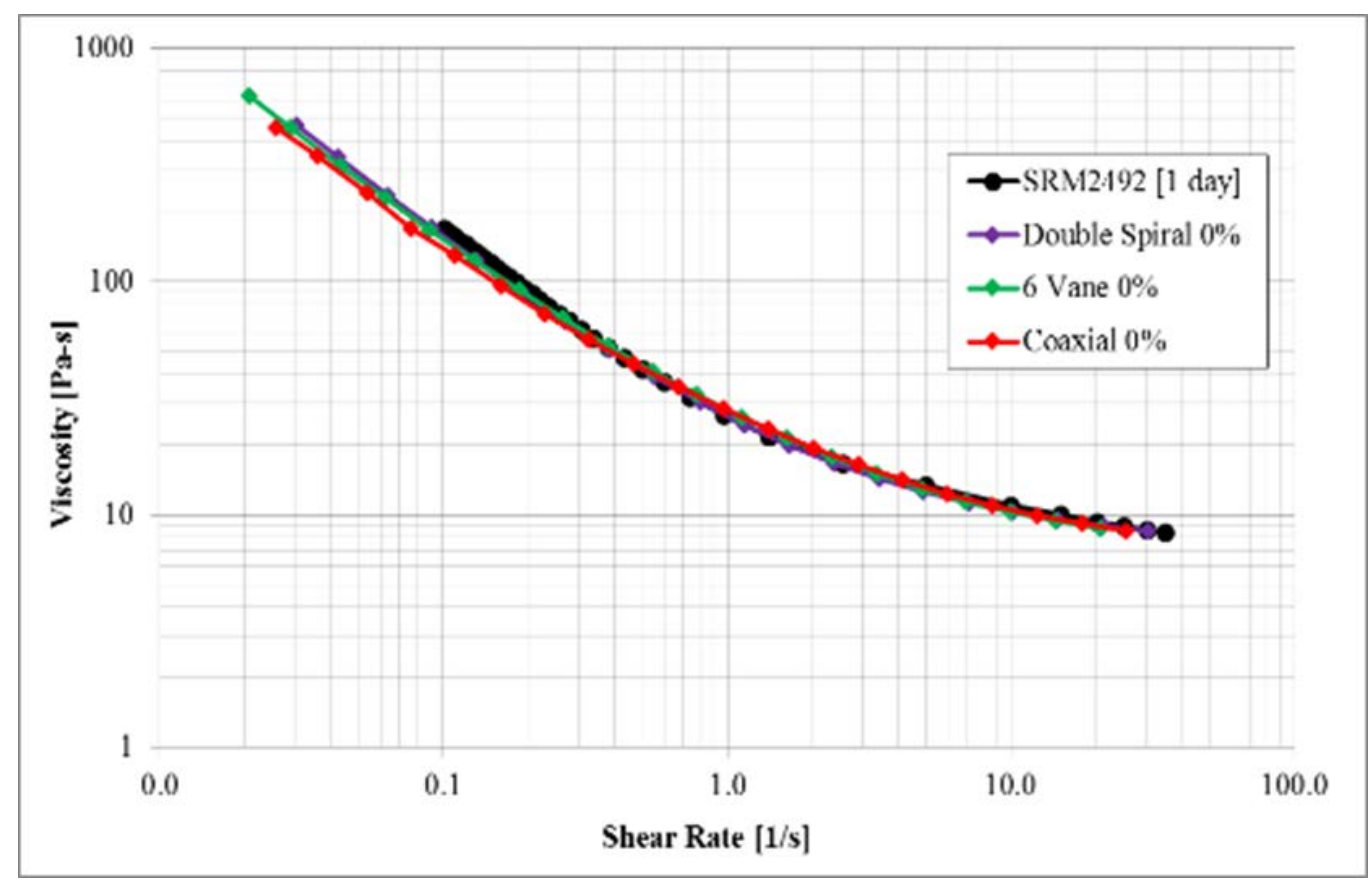

Figure 13: Flow curve comparison of the calibrated experimental data for SRM 2492 paste ( $0 \%$ beads) for all three spindles. The flow curves are compared to their reference curve shown in black, SRM2492 certified values. Uncertainities are analyzed in Section 6. 


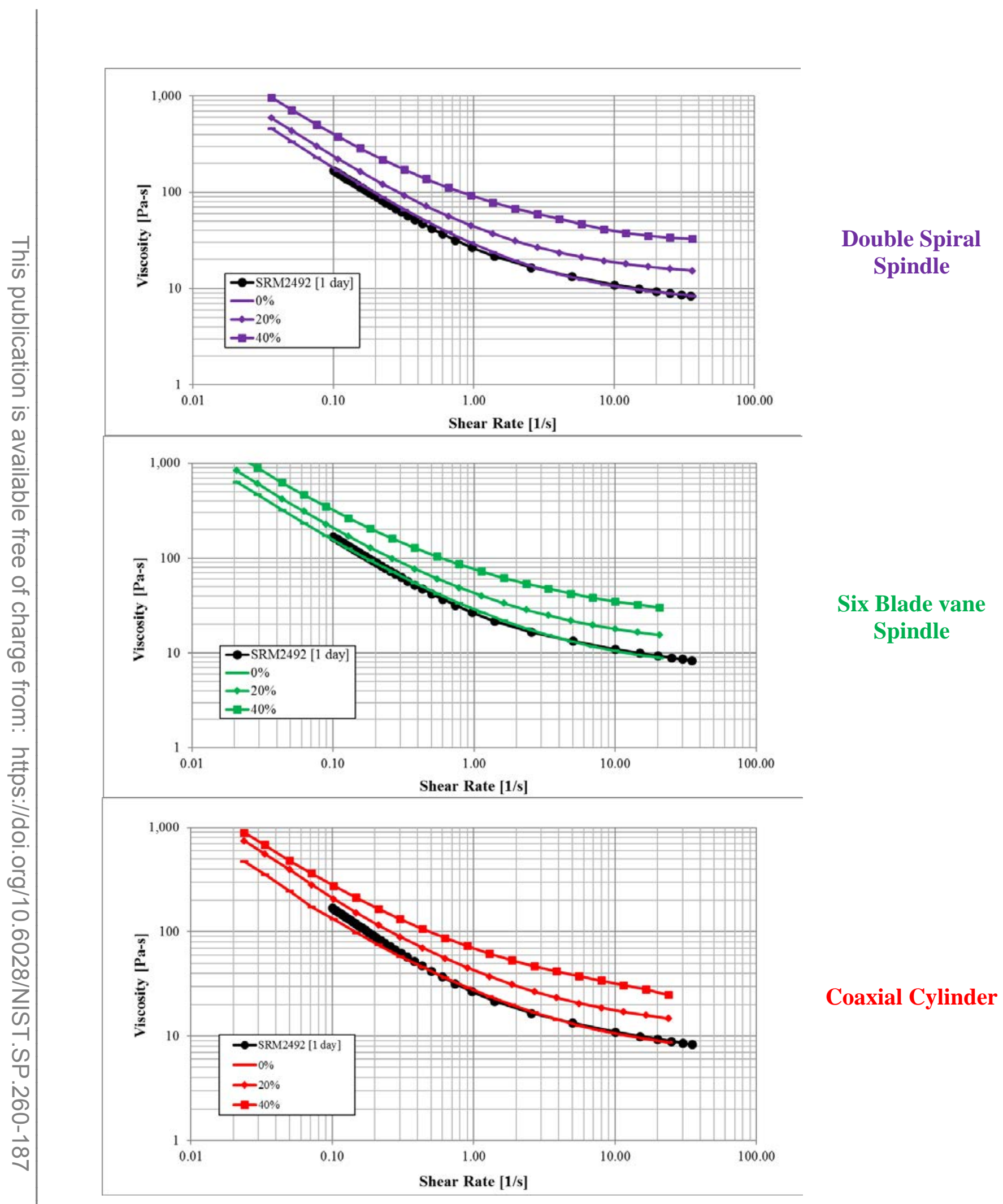

Figure 14: Calibrated, by Bingham parameters, experimental flow curves for different geometries of spindles at bead volume concentrations of $0 \%, 20 \%$ and $40 \%$. The $0 \%$ curve (black) represents the certified SRM2492 flow curve values. Uncertainities are analyzed in Section 7. 


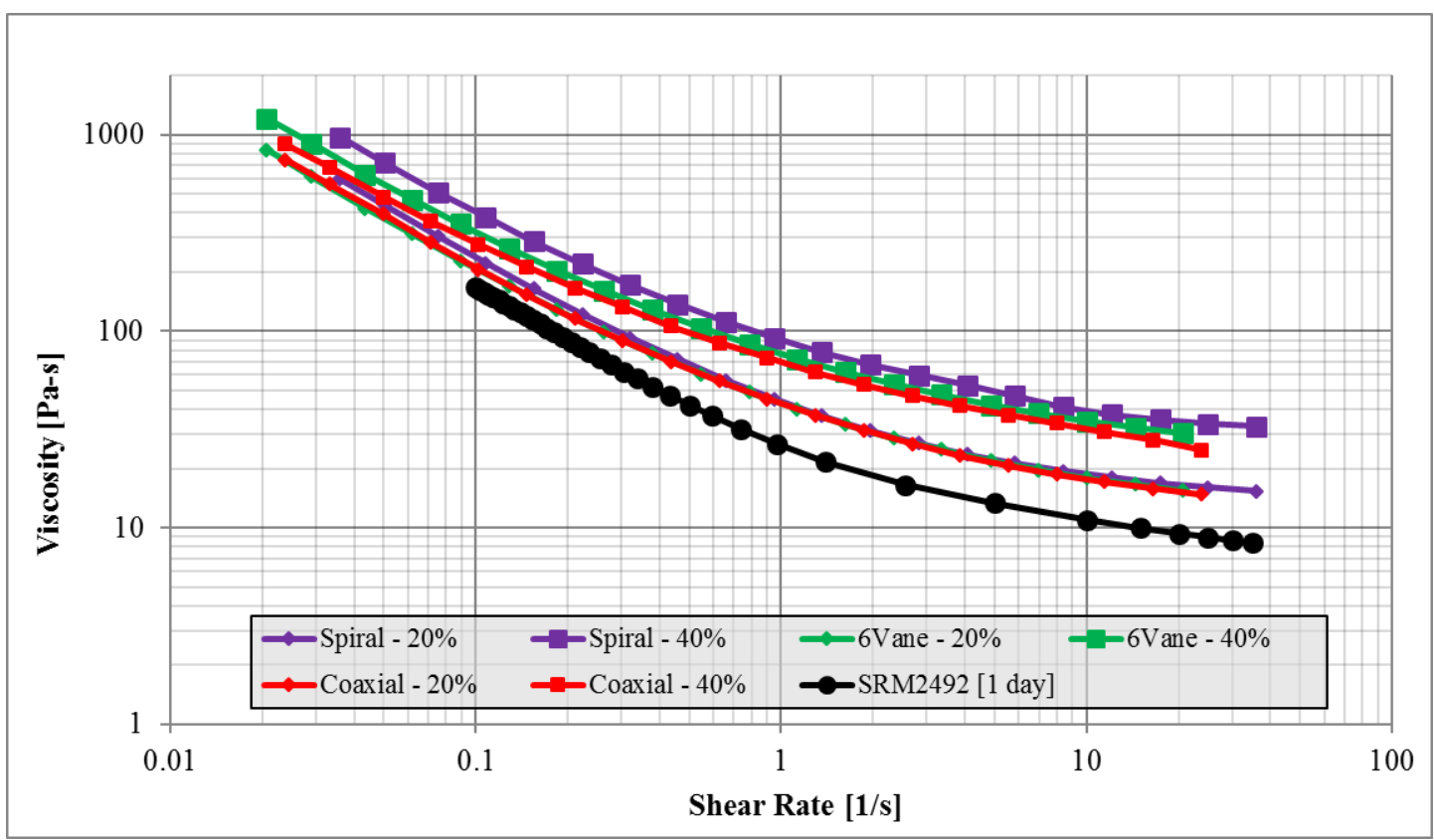

Figure 15: Viscosity curve at concentrations of $20 \%$ and $40 \%$ by volume of glass beads. The uncertainty is estimated to be $10 \%$ of the viscosity. The $0 \%$ curves are omitted for clarity. Full discussion of the uncertainties can be found in Section 6.

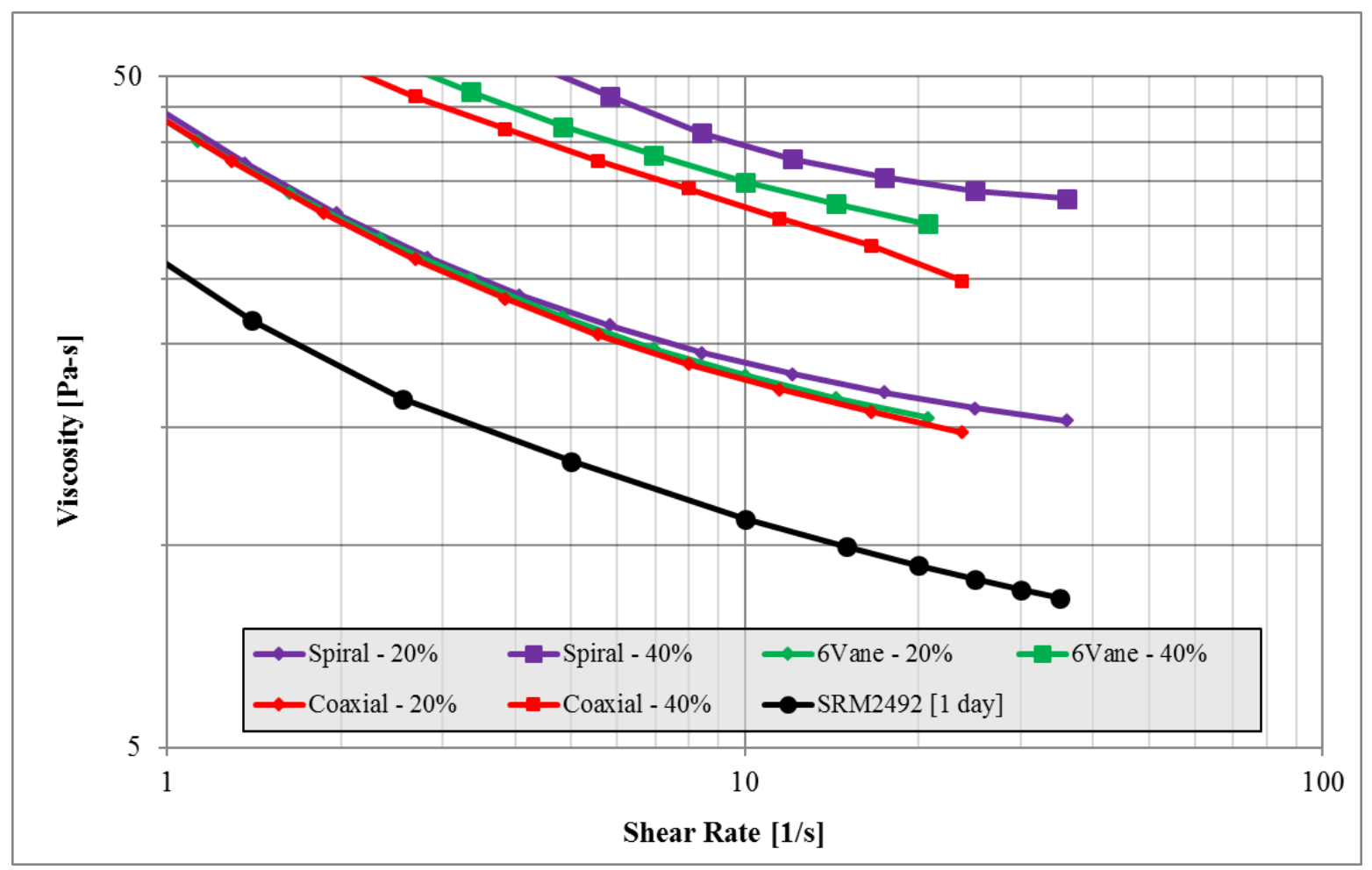

Figure 16: An enlargement of Figure 15 for the higher shear rates to better show the difference in viscosity depending on the spindle used. The $0 \%$ curves are omitted for clarity. Full discussion of the uncertainties can be found in Section 6. 


\subsubsection{Viscosity Curves Method}

As mentioned in Section 4.3 the calibration factors are calculated in order to convert the rheometer's raw data into fundamental rheological units. The three calibration factors $\left(L_{\gamma}, L_{\tau}, L_{\mu}\right)$ were calculated by rescaling the measured curves of the paste ( $0 \%$ beads) to the reference curve found for SRM 2492. The values obtained are shown in Table 13, along with the standard uncertainty. These $\mathrm{L}$ factors could be used to calibrate new experimental data if using the exact spindles as in this study. Uncertainty in the L factors was estimated using the method as described in Section 6.1.3 for the coefficients of Equation 1. This means that the $\mathrm{L}$ factor values were produced by a ordinary least squares procedure (OLS) and also by a Bayesian hierarchical model (BHM). The uncertainties are square roots of sums of squared uncertainties of the BHM and the squared bias.

The resulting flow curves of the calibrated data for both mortar types and the paste without beads ( $0 \%$ ) can be found in Figure 17. Figure 18 compares the results of the $0 \%$ paste to the certified SRM2492 values, as well as the changes in the flow curve behavior when $20 \%$ or $40 \%$ beads by volume are added to the paste. As expected again, the $20 \%$ and $40 \%$ mortars increase the viscosity of the mixtures. The calibrated data that compose the viscosity curves can be found Table 36 to Table 38. The agreement of the flow curves shown in Figure 19 with each other provided reliability in the calibration process which was enacted on the study subjects, the SRM mortars, when compared with the SRM 2492 as measured using a parallel plate rheometer. Figure 18 shows all the curves in one graph. It highlights the higher viscosity measured using the spiral spindle compared with the vane or coaxial spindles.

Table 13: Calibration factors for experimental data of each spindle tested in this study.

\begin{tabular}{|c|c|c|c|}
\hline & \multicolumn{3}{|c|}{ Data Calibration Factors based on } \\
\hline & $\begin{array}{l}\text { Serrated } \\
\text { Coaxial } \\
\text { Cylinder }\end{array}$ & $\begin{array}{l}\text { Six } \\
\text { Blade } \\
\text { Vane }\end{array}$ & Double Spiral \\
\hline $\begin{array}{l}\mathbf{L} \boldsymbol{\tau} \\
{[\mathrm{Pa} / \mathrm{N} \cdot \mathrm{m}]}\end{array}$ & 32,000 & 26,000 & 9,700 \\
\hline Std. Unc. & 6,280 & 4,970 & 1,050 \\
\hline $\begin{array}{l}\mathbf{L} \gamma \\
{[-]}\end{array}$ & 3.24 & 2.28 & 3.09 \\
\hline Std. Unc. & 0.56 & 0.42 & 0.26 \\
\hline $\begin{array}{l}\mathbf{L} \boldsymbol{\mu} \\
{[\mathrm{Pa} \cdot \mathrm{s} / \mathrm{N} \cdot \mathrm{m} \cdot \mathrm{s}]}\end{array}$ & 9,718 & 11,419 & 3,145 \\
\hline Std. Unc. & 954 & 558 & 214 \\
\hline
\end{tabular}




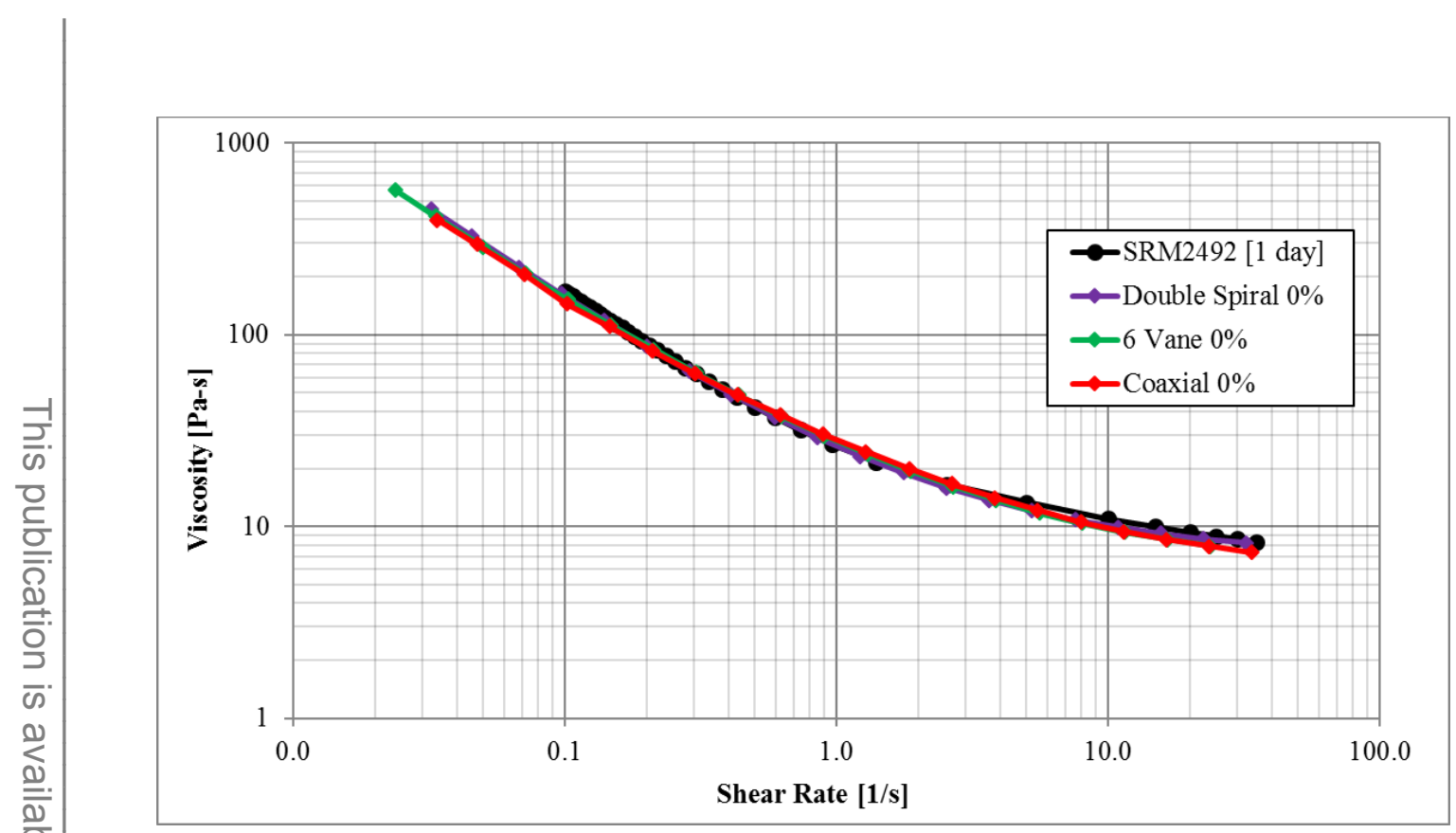

Figure 17: Flow curve comparison of the calibrated experimental data for SRM 2492 paste ( $0 \%$ beads) for all three spindles. The flow curves are compared to their reference curve shown in black, SRM2492 certified values, using the $L$ factors shown in Table 11. Uncertainities are analyzed in Section 6. 


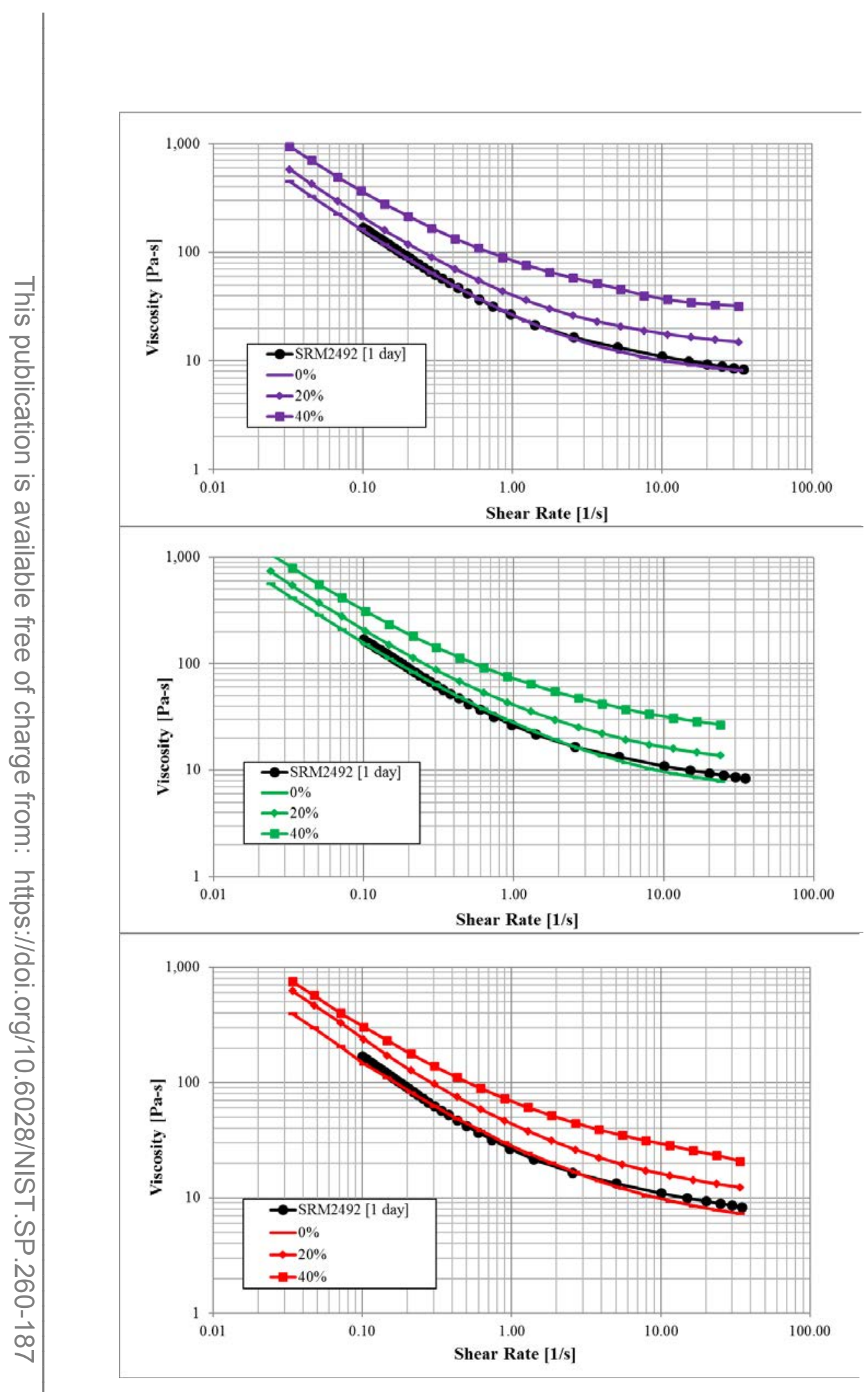

Double Spiral Spindle

Six Blade Vane Spindle

Coaxial Cylinder

Figure 18: Calibrated, by viscosity curve method, experimental flow curves for each spindle at bead volume concentrations of $0 \%, 20 \%$ and $40 \%$. The $0 \%$ curve (black) represents the certified SRM2492 flow curve values. Uncertainities are analyzed in Section 6. 


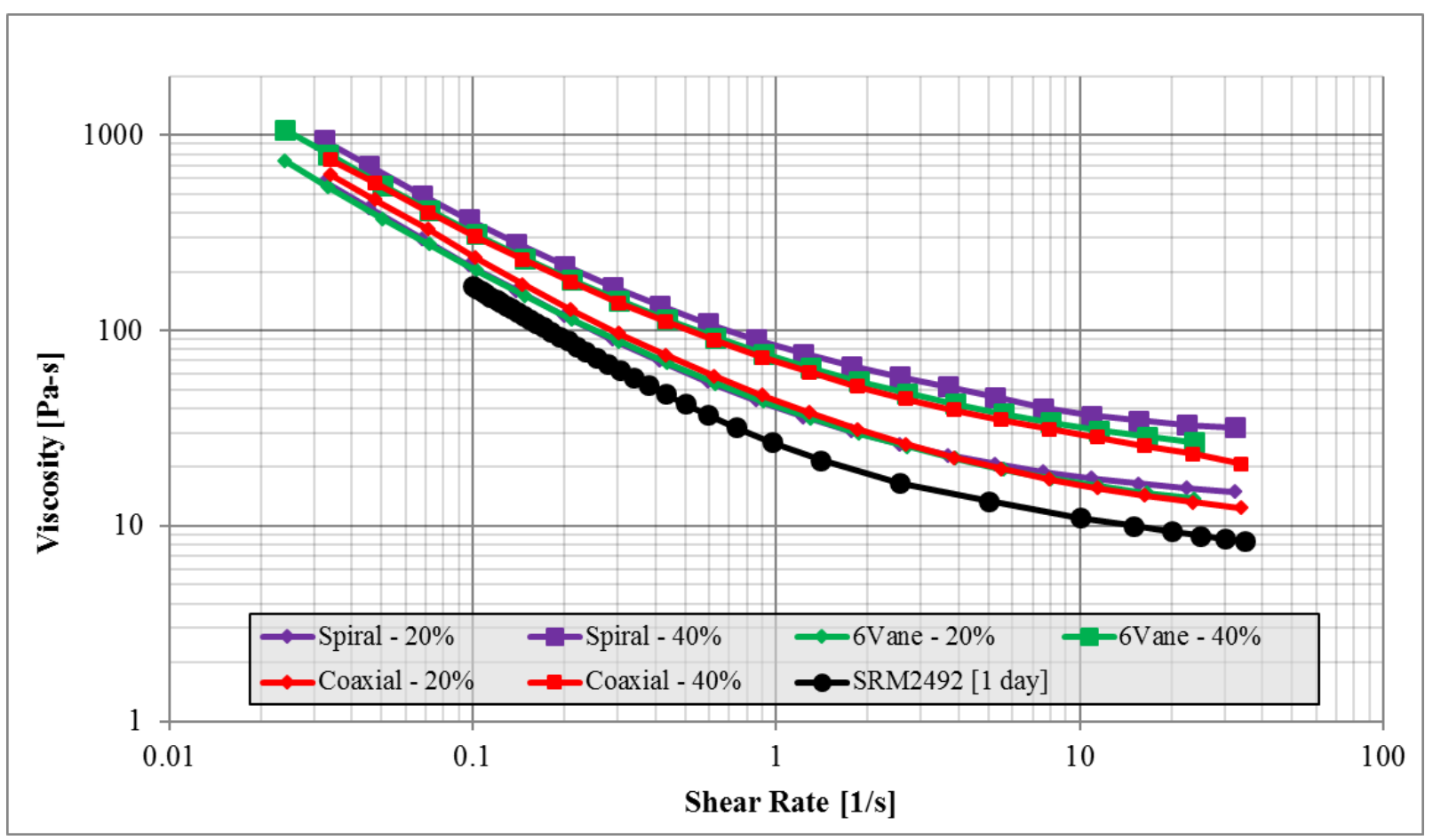

Figure 19: Viscosity curve at concentrations of $20 \%$ and $40 \%$ by volume of glass beads. The uncertainty is estimated to be $\mathbf{1 0} \%$ of the viscosity. Full discussion on the uncertainties can be found in Section 7.

\subsubsection{Comparison of Two Calibration Methods: Bingham or Viscosity Curves Fit}

It is important to check how much the choice of either method of calibration influences the results obtained. As all the data were first calibrated on the SRM 2492 (paste or 0\% beads), the fit there should be optimal as shown in Figure 13 and Figure 17. The differences should be more evident on the mixtures of $20 \%$ and $40 \%$ beads concentration. Figure 20 show the curves for each of the spindles used and calculated using either the K (Bingham method) or the L factors (viscosity fit). The visual differences from the figures are clearly small. To quantify the differences Table 14 was prepared. It is clear that the differences between the two methods are within the uncertainity of the measurements, or less than $10 \%$ of the viscosity values. Thus the two methods are considered equivalent. The advantage of the Bingham method is that it also produces the yield stress, while the viscosity fit does not. 

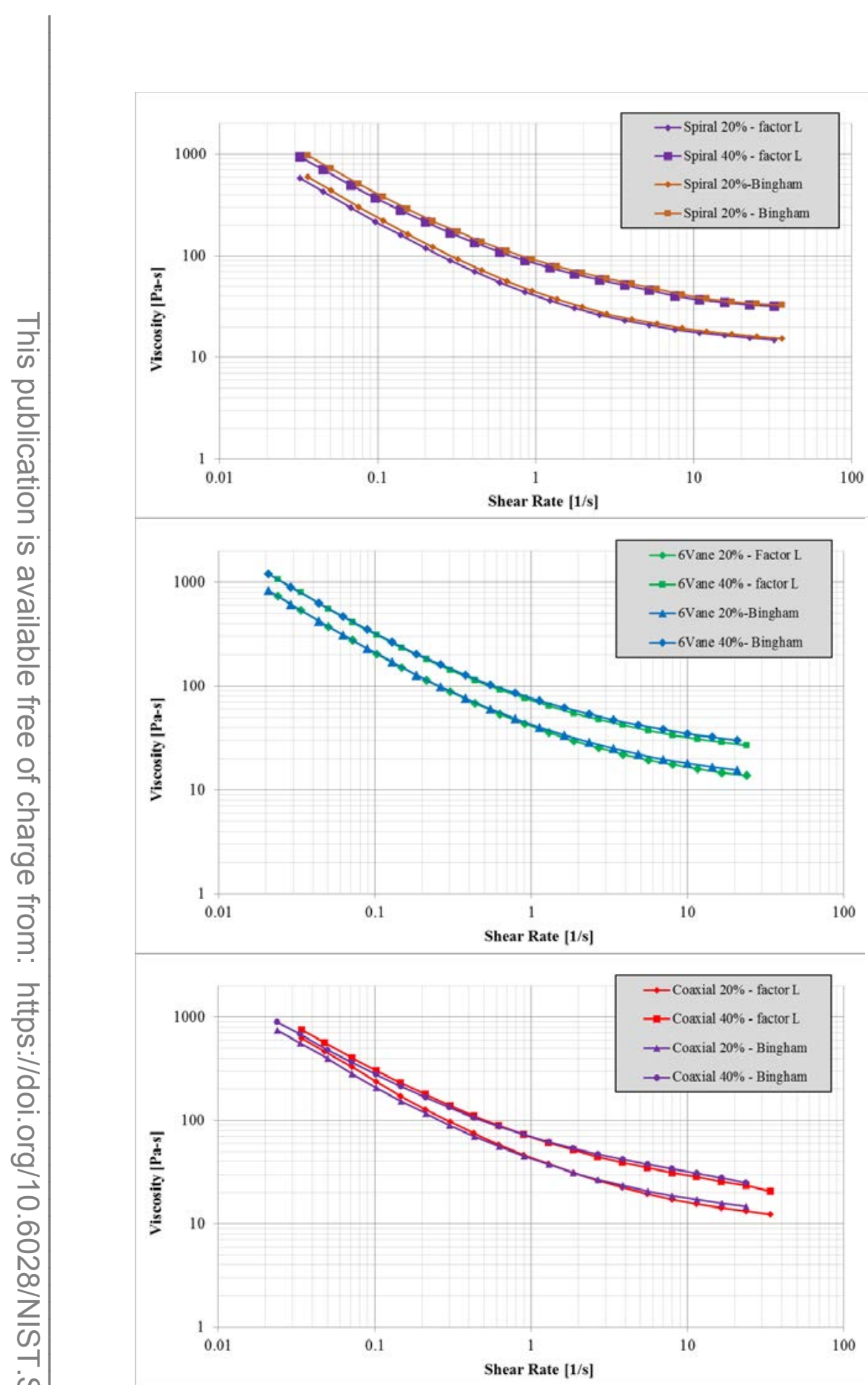

Figure 20: Graphic comparison between the curves obtained by Bingham (K factors) or by the viscosity curves (L factors). Uncertainities are analyzed in Section 6. 
Table 14: The differences between viscosity calculated using Bingham equation and viscosity fits are shown for three shear rates. The differences was calculated by (Values by Bingham - Value by viscosity fit). The uncertainity is estimated to be less than $10 \%$ of the measured viscosity.

\begin{tabular}{|c|c|c|c|c|c|}
\hline & \multirow{2}{*}{$\begin{array}{l}\text { Volume } \\
\text { fraction of } \\
\text { glass beads }\end{array}$} & \multicolumn{3}{|c|}{ Viscosity Difference $[\mathrm{Pa} \cdot \mathrm{s}]$} & \\
\hline & & $0.1 \mathrm{~s}^{-1}$ & $1 \mathrm{~s}^{-1}$ & $20 \mathrm{~s}^{-1}$ & \\
\hline \multirow{3}{*}{$\begin{array}{l}\text { Double } \\
\text { Spiral }\end{array}$} & $0 \%$ & 20.1 & 2.3 & 0.4 & Pa.s \\
\hline & $20 \%$ & 25.8 & 3.3 & 0.7 & Pa.s \\
\hline & $40 \%$ & 41.1 & 6.1 & 1.4 & Pa.s \\
\hline \multirow{3}{*}{$\begin{array}{c}6 \text { Blade } \\
\text { Vane }\end{array}$} & $0 \%$ & 6.9 & 1.1 & 0.7 & Pa.s \\
\hline & $20 \%$ & 9.9 & 1.9 & 1.3 & Pa.s \\
\hline & $40 \%$ & 16.0 & 4.1 & 2.4 & Pa.s \\
\hline \multirow{3}{*}{$\begin{array}{l}\text { Coaxial } \\
\text { Cylinder }\end{array}$} & $0 \%$ & -12.2 & -0.7 & 0.9 & Pa.s \\
\hline & $20 \%$ & -30.6 & -1.0 & 1.6 & Pa.s \\
\hline & $40 \%$ & -26.2 & 0.5 & 1.9 & Pa.s \\
\hline
\end{tabular}

Another concept that should be introduced now to compare the various methods is the relative viscosity $\left(\mu_{\mathrm{R}}\right)$. The definition of the relative viscosity is the ratio of the viscosity of a mortar (with beads) to the viscosity of the paste at the same shear rate. Table 15 and Table 16 show the calculated relative viscosities at selected shear rates. Table 15 used the data calibrated using the Bingham method, while Table 16 used the data from the viscosity fit. Figure 21 shows the data from Table 15. Only the data from Table 15 is represented for clarity of the figure, as it can be seen from the two tables that the difference between the two methods is very small: the highest difference is 0.2 within the uncertainty. It can be seen that for $20 \%$ mortar, the relative viscosity is within the uncertainty for all the spindles used. On the other hand for the $40 \%$ mortar, a wide spread occurs with the spiral having always the highest relative viscosity. In Section 6, an attempted explanation for this difference will be offered. 
Table 15: Relative viscosity $\left(\mu_{\mathrm{R}}\right)$ values based on the calibrated experimental data at concentrations of $20 \%$ and $40 \%$ by volume, labeled $\mu_{\mathrm{R} 20}$ and $\mu_{\mathrm{R} 40}$, respectively. These were calculated using the Bingham method of conversion.

\begin{tabular}{|c|c|c|c|c|}
\hline & \multicolumn{3}{|c|}{ Shear Rate [1/s] } \\
\hline & & 0.1 & 1 & 20 \\
\hline \multirow{4}{*}{$\begin{array}{l}\text { Double } \\
\text { Spiral }\end{array}$} & $\mu_{\mathrm{R} 20}$ & 1.3 & 1.6 & 1.8 \\
\hline & $\begin{array}{l}\text { Std. } \\
\text { Unc. }\end{array}$ & 0.04 & 0.06 & 0.09 \\
\hline & $\mu_{\mathrm{R} 40}$ & 2.3 & 3.3 & 3.8 \\
\hline & $\begin{array}{l}\text { Std. } \\
\text { Unc. }\end{array}$ & 0.12 & 0.21 & 0.34 \\
\hline \multirow{4}{*}{$\begin{array}{l}6 \text { Blade } \\
\text { Vane }\end{array}$} & $\mu_{\mathrm{R} 20}$ & 1.3 & 1.5 & 1.7 \\
\hline & $\begin{array}{l}\text { Std. } \\
\text { Unc. }\end{array}$ & 0.03 & 0.03 & 0.05 \\
\hline & $\mu_{\mathrm{R} 40}$ & 2.0 & 2.7 & 3.4 \\
\hline & $\begin{array}{l}\text { Std. } \\
\text { Unc. }\end{array}$ & 0.11 & 0.17 & 0.24 \\
\hline \multirow{4}{*}{$\begin{array}{l}\text { Coaxial } \\
\text { Cylinder }\end{array}$} & $\mu_{\mathrm{R} 20}$ & 1.6 & 1.5 & 1.7 \\
\hline & $\begin{array}{l}\text { Std. } \\
\text { Unc. }\end{array}$ & 0.08 & 0.06 & 0.08 \\
\hline & $\mu_{\mathrm{R} 40}$ & 2.1 & 2.5 & 2.8 \\
\hline & $\begin{array}{l}\text { Std. } \\
\text { Unc. }\end{array}$ & 0.10 & 0.10 & 0.19 \\
\hline \multirow{2}{*}{ Model } & $\mu_{\mathrm{R} 20}$ & 1.3 & 1.5 & 1.7 \\
\hline & $\mu_{\mathrm{R} 40}$ & 2.0 & 3.8 & 6.1 \\
\hline (mul & Unc. & $\begin{array}{l}\text { andar } \\
\text { obta }\end{array}$ & ertat & \\
\hline
\end{tabular}


Table 16: Relative viscosity $\left(\mu_{\mathrm{R}}\right)$ values based on the calibrated experimental data at concentrations of $20 \%$ and $40 \%$ by volume, labeled $\mu_{\mathrm{R} 20}$ and $\mu_{\mathrm{R} 40}$, respectively. These are calculated using the viscosity curve approach.

\begin{tabular}{|c|c|c|c|c|}
\hline & & \multicolumn{3}{|c|}{ Shear Rate [1/s] } \\
\hline & & 0.1 & 1 & 20 \\
\hline \multirow{4}{*}{$\begin{array}{l}\text { Double } \\
\text { Spiral }\end{array}$} & $\mu_{\mathrm{R} 20}$ & 1.3 & 1.5 & 1.8 \\
\hline & $\begin{array}{l}\text { Std. } \\
\text { Unc. }\end{array}$ & 0.04 & 0.06 & 0.09 \\
\hline & $\mu_{\mathrm{R} 40}$ & 2.3 & 3.2 & 3.8 \\
\hline & $\begin{array}{l}\text { Std. } \\
\text { Unc. }\end{array}$ & 0.12 & 0.21 & 0.34 \\
\hline \multirow{4}{*}{$\begin{array}{c}6 \text { Blade } \\
\text { Vane }\end{array}$} & $\mu_{\mathrm{R} 20}$ & 1.3 & 1.5 & 1.7 \\
\hline & $\begin{array}{l}\text { Std. } \\
\text { Unc. }\end{array}$ & 0.03 & 0.03 & 0.05 \\
\hline & $\mu_{\mathrm{R} 40}$ & 2.0 & 2.7 & 3.4 \\
\hline & $\begin{array}{l}\text { Std. } \\
\text { Unc. }\end{array}$ & 0.11 & 0.17 & 0.24 \\
\hline \multirow{4}{*}{$\begin{array}{l}\text { Coaxial } \\
\text { Cylinder }\end{array}$} & $\mu_{\mathrm{R} 20}$ & 1.6 & 1.5 & 1.7 \\
\hline & $\begin{array}{l}\text { Std. } \\
\text { Unc. }\end{array}$ & 0.08 & 0.06 & 0.08 \\
\hline & $\mu_{\mathrm{R} 40}$ & 2.1 & 2.4 & 3.0 \\
\hline & $\begin{array}{l}\text { Std. } \\
\text { Unc. }\end{array}$ & 0.10 & 0.10 & 0.19 \\
\hline (multif & $\begin{array}{l}\text { 1. Unc. } \\
\text { by } 1.9\end{array}$ & $\begin{array}{l}\text { andarc } \\
\text { btain }\end{array}$ & $\begin{array}{l}\text { ertain } \\
\text { xpande }\end{array}$ & \\
\hline
\end{tabular}




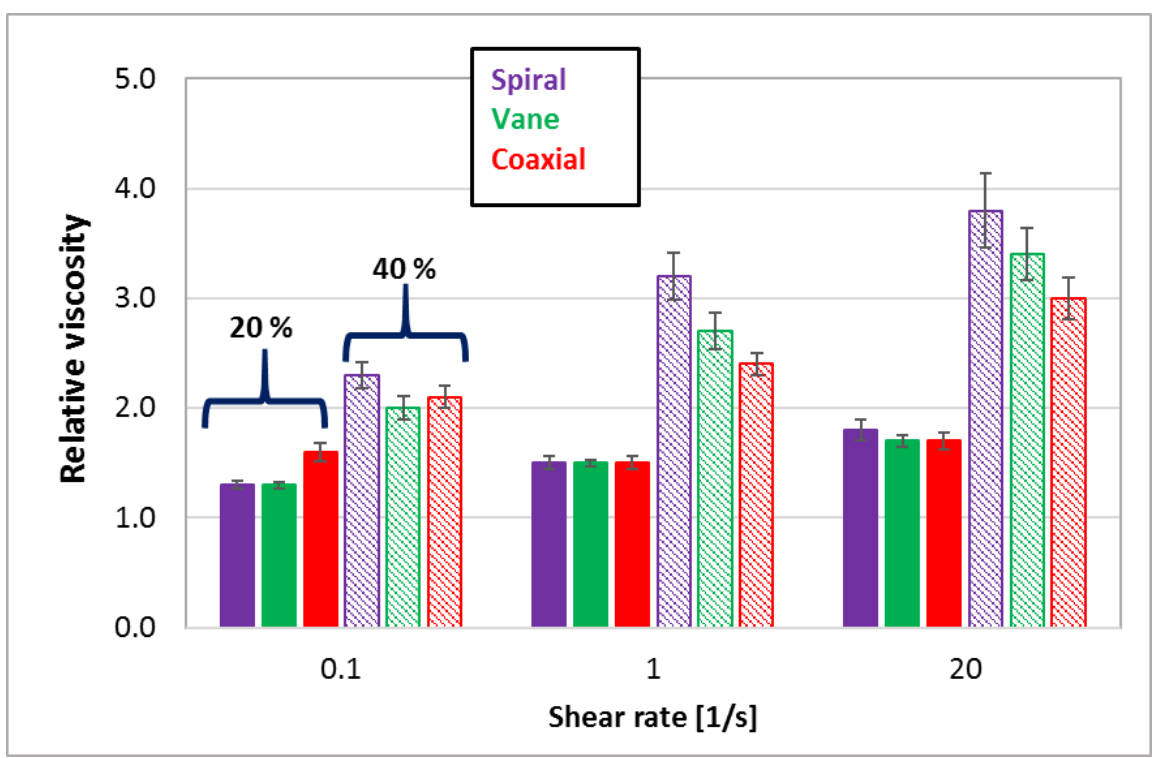

Figure 21: Graphical representation of the data in Table 15 representing the relative viscosity as a function of the beads volume concentration and the spindle used. The uncertainity is as listed in the Tables 15. 


\section{Comparison Between Model and Experimental}

Figure 22 shows the average curves for all type of spindles and concentrations used in this study. The three spindel geometries (see Section 5.1) used on a mortar reference material (SRM2493) of $20 \%$ and $40 \%$ volume concentrations are shown. In Figure 23, the model results (see Section 0) were added to the data from Figure 22. The graphs compare all the experimental viscosity curves to the certified SRM2492 curve (black), which is the reference baseline for this study. The difference in viscosities between the baseline and any other viscosity curve of interest is considered the relative viscosity $(\mu R)$, as stated above. The relative viscosity is a factor that represents the effects on viscous behavior of a material caused by using different rheometers geometries and the addition of beads to the matrix (i.e. bead concentration).

As shown previously (Section 5.3), the viscosity depends on the spindle used for performing the rheological measurement. For $40 \%$ beads concentration the model values are higher than the measured one. The different rheometer spindles vary in effectiveness of shearing. This effectiveness can be correlated to how well the shearing walls are actually able to grasp the material and shear it without slippage. Any slippage (non-effective shearing) results in lower readings of viscosity. This issue is more evident at higher bead concentrations, which is shown when comparing the $40 \%$ curves with the SRM 2492 paste (shown in black) in Figure 22 or Figure 23.

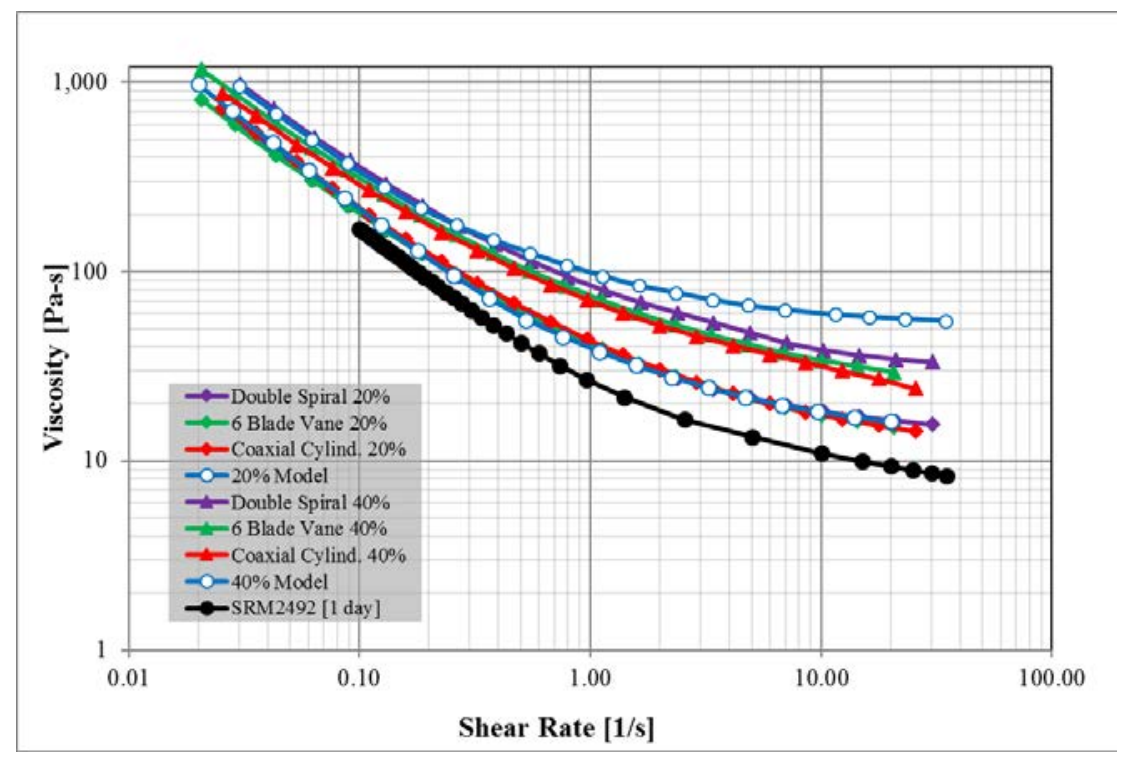

Figure 22: Viscosity vs. shear rate (using the Viscosity fit method) for all data collected including the scaled prediction values determined from the numerical simulation model. The $0 \%$ data for the measurements were omitted for clarity. The uncertainties are discussed in Section 6. 


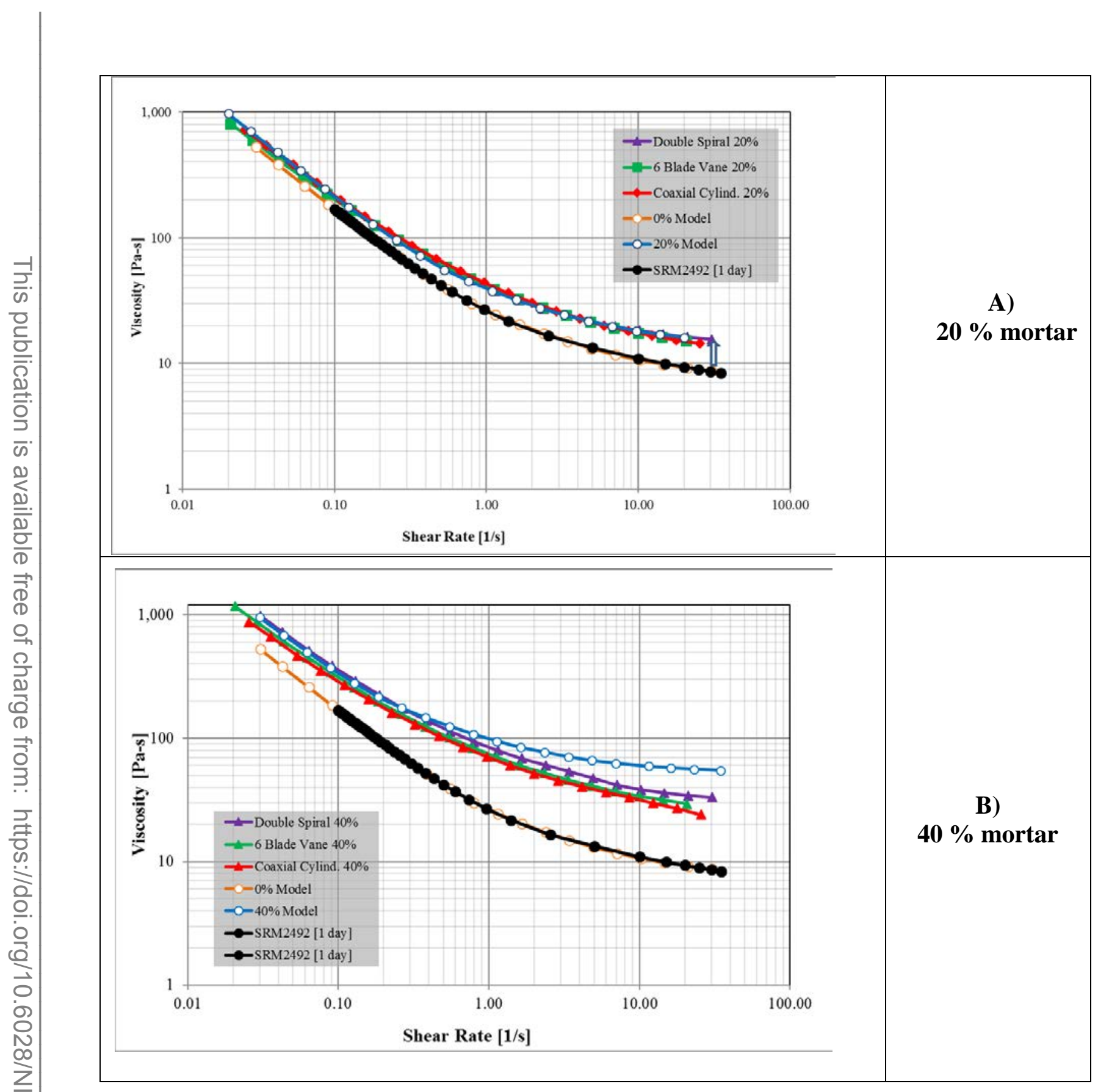

Figure 23: Comparison of model vs experimental viscosity curves (obtained using the viscosity fit method) for A) $20 \%$ and B) $\mathbf{4 0 \%}$ glass beads volume fraction mortar mixture. Relative viscosity, $\mu_{R}$, was modeled with respect to the SRM 2492 (1 day) baseline curve at $20 \mathrm{~s}^{-1}$. The uncertainties are discussed in Section 6. 
Table 17: Relative viscosity $\left(\mu_{\mathrm{R}}\right)$ values for the scaled prediction numerical model. Standard uncertainties are estimated at $10 \%$.

\begin{tabular}{|c|c|c|c|c|}
\hline & \multicolumn{3}{|c|}{ Shear Rate [1/s] } \\
\hline & & 0.1 & 1 & 20 \\
\hline \multirow{2}{*}{ Model } & $\mu_{\mathrm{R} 20}$ & 1.3 & 1.5 & 1.7 \\
\hline & $\mu_{\mathrm{R} 40}$ & 2.0 & 3.8 & 6.1 \\
\hline
\end{tabular}

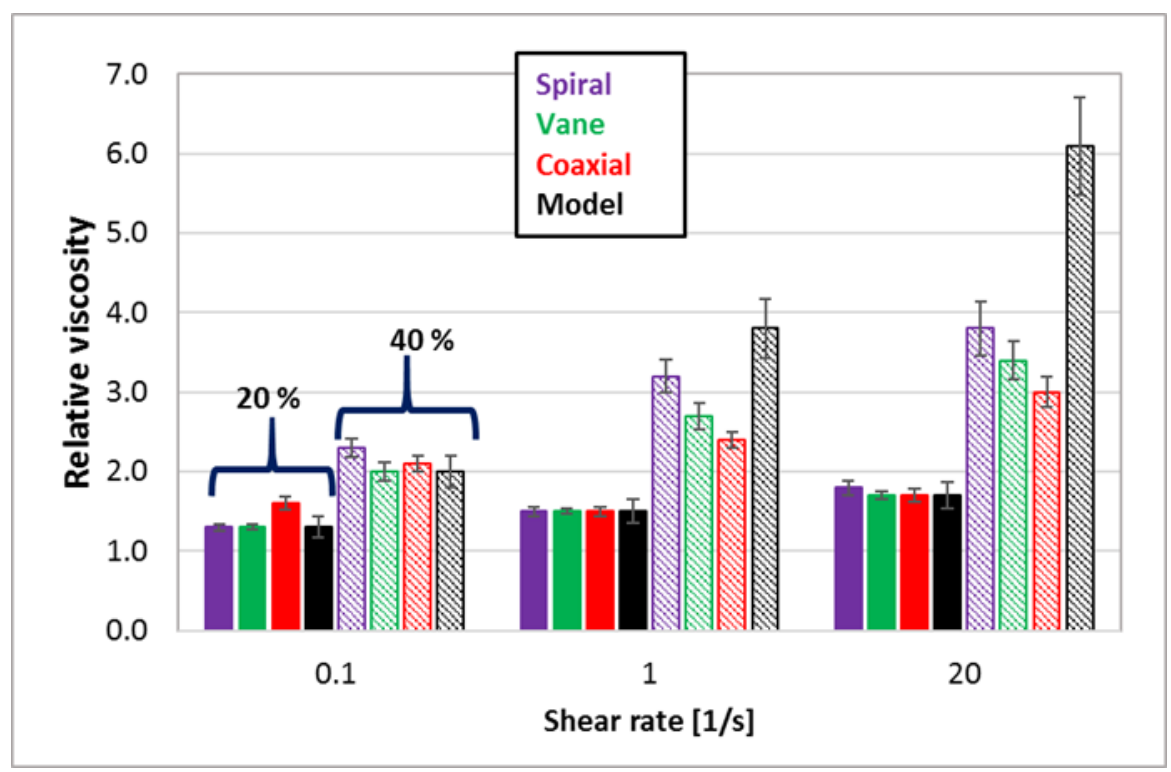

Figure 24: Graphic representation of the relative viscosity from Table 15 and Table 16, with the values for the model added. The uncertainities are also from these two tables with the model assigned an uncertainity of $10 \%$

The relative viscosity for all spindle/material combinations are displayed in Table 15, Table 16 and Table 17 at three shear rates and represented graphically in The three shear rates were selected to portray the relative viscosity at low, medium and high measured shear rates. It is evident from these tables and Figure 24 that major differences in $\mu_{\mathrm{R}}$ values are caused by two factors: shear rate and bead concentration. First, when the mortar tested has a high volume fraction of glass beads ( $40 \%$ ) the relative viscosity $\left(\mu_{\mathrm{R} 40}\right)$ differs greatly depending on the spindle used and the model; however, when testing the $20 \%$ mortar the relative viscosities $\left(\mu_{\mathrm{R} 20}\right)$ don't differ nearly as much (see discussion below). The second noticeable trend is caused by the shear rate, at high shear rates major differences also exist based on the spindle used, yet at low shear rates the relative viscosities are identical regardless of spindle.

The model is, ideally, a prediction for the experimental results, but experimental results and theory don't always match perfectly. Figure 23a portrays the $20 \%$ predicted (computer modeled) 
viscosity curve and its relative viscosity $\left(\mu_{R}\right)$ in order to compare with the experimental relative viscosities discussed formerly. Similarly, the 40 \% curves are shown in Figure $23 b$. The relative viscosities at the high shear rates are good comparison points for a quantitative analysis between experimental vs. model curves. However, Table 16 and Table 17 display the $\mu_{\mathrm{R}}$ at low, medium and high shear rates for the experimental and compares them to the predicted $\mu_{\mathrm{R}}$ values from the model. As shown, the model predicted the viscosity to be higher than the resulting experimental data for all three spindles types, with the difference more evident for $\mu_{R 40}$ than $\mu_{R 20}$. It should be noted that once again, a trend exists between the three types of spindle which highlights the double spiral being the most accurate when compared to the prediction model at high shear rates and 40 $\%$ beads. High shear, $40 \%$ conditions are the least accurate for the serrated coaxial cylinder. The reason for the model and experimental relative viscosity discrepancy is possibly due to several possible explanations, which can be present in experiments but are not present in the model:

- Slippage of the material on the spindle or the cup. The spiral tool provides a higher connection between the material and the spindle thus has a higher torque or shear stress than measured with the other spindles at the same shear rate.

- The model is an ideal couette flow with no walls and no changes in the material composition, while the experimental measurement could have also particle migration away from the spindle and also vertically on the open surface.

- The open surface at the top allows material to dilate and thus create an artificial reduction in particle concentration that would tend to reduce the viscosity.

More detailed analysis of the discrepancies between the model and the experimental data are provided in Ref. [17]. 


\section{Statistical Analysis}

\subsection{Flow Curves}

\subsubsection{Uncertainty Calculation}

As stated above, for each of the three spindles: double spiral, 6 blade vane and serrated coaxial cylinder, and different bead volume fractions, $0 \%$, $20 \%$ and $40 \%$, five different batches (See Section 5.2) were measured for torque $\Gamma[\mu \mathrm{Nm}]$ at 35 different rotational speed $N[1 / \mathrm{min}]$. The shear rate increased from 0.1 to 100 [1/min] and then decreased back down to 0.1 .

The five mixtures were treated as independent replicates of the flow curves for each geometry and bead volume fraction. Only the "down curve" data was used, that is, the torques for the 20 shear rates starting at $100 \mathrm{~s}^{-1}$ and decreasing to $0.1 \mathrm{~s}^{-1}$. For $0 \%$ beads the curves were computed individually for $0 \%$ pre $20 \%$ and $0 \%$ pre $40 \%$ and also combined. Pre $20 \%$ or pre $40 \%$ represent the paste measured before the beads were added in the volume concentration indicated. They represent repeats of paste measurements (see Section 4.2).

For each of the shear rates, a consensus torque was computed together with an uncertainty value using the model

$$
\Gamma_{i j}=\theta_{i}+\varepsilon_{i j}, i=1, \ldots, 20, j=1, \ldots, 5
$$

where $\theta_{i}$ were the consensus torque values for the 5 replicates.

Estimation was performed via Markov Chain Monte Carlo methods [14] (which require prior distributions on the parameters of the model) with

$$
\theta_{i} \sim N(0,1.0 E 5), \varepsilon_{i j} \sim N\left(0, \sigma_{i}^{2}\right), \sigma_{i}^{2} \sim \operatorname{Gamma}(1.0 E 5,1.0 E 5), i=1, \ldots, 20 .
$$

The computations were done using the software OpenBUGS [15] using the code given in Section 6.4. The posterior mean, standard deviation, and $95 \%$ highest posterior density interval at each shear rate were used for the estimate $(\Gamma)$, standard $(u(\Gamma))$ and expanded uncertainty. These estimates were very stable with respect to the Gaussian and Gamma distributions of the parameters. The following tables contain the results. 
Table 18: Torque and rotational speed with the calculated uncertainity for the double Spiral spindle with $0 \%$ beads

\begin{tabular}{|c|c|c|c|c|c|c|c|c|c|}
\hline & \multicolumn{3}{|c|}{$0 \%$ pre $40 \%$} & \multicolumn{3}{|c|}{$0 \%$ pre $20 \%$} & \multicolumn{3}{|c|}{$0 \%$ combined } \\
\hline $\begin{array}{l}\mathrm{N} \\
1 / \text { min }\end{array}$ & $\begin{array}{l}\Gamma \\
\mu \mathrm{Nm}\end{array}$ & $\begin{array}{l}\mathrm{U}(\Gamma) \\
\mu \mathrm{Nm}\end{array}$ & $\begin{array}{l}\text { expand } \\
\text { ed } \\
\mathrm{U}(\Gamma)\end{array}$ & $\Gamma \mu \mathrm{Nm}$ & $\begin{array}{l}\mathrm{U}(\Gamma) \\
\mu \mathrm{Nm}\end{array}$ & $\begin{array}{l}\text { expande } \\
\mathrm{d} U(\Gamma)\end{array}$ & $\Gamma \mu \mathrm{Nm}$ & $\begin{array}{l}\mathrm{U}(\Gamma) \\
\mu \mathrm{Nm}\end{array}$ & $\begin{array}{c}\text { expanded } \\
\mathrm{U}(\Gamma)\end{array}$ \\
\hline 100 & 26920.36 & 781.44 & $\begin{array}{l}1531.6 \\
3\end{array}$ & 27766.15 & 1194.69 & 2341.59 & 27347.39 & 525.87 & 1030.71 \\
\hline 69.5 & 19691.11 & 516.52 & $\begin{array}{l}1012.3 \\
9 \\
\end{array}$ & 20292.41 & 787.99 & 1544.47 & 19997.13 & 383.05 & 750.78 \\
\hline 48.3 & 14570.1 & 389.66 & 763.73 & 15024.64 & 607.37 & 1190.45 & 14789.76 & 279.16 & 547.15 \\
\hline 33.6 & 10903.95 & 282.69 & 554.07 & 11252.44 & 438.87 & 860.19 & 11068.28 & 213.07 & 417.62 \\
\hline 23.4 & 8296.96 & 217.09 & 425.49 & 8546.34 & 337.43 & 661.35 & 8420.6 & 156.62 & 306.9 \\
\hline 16.2 & 6428.55 & 159.78 & 313.17 & 6612.42 & 243.16 & 476.60 & 6522.95 & 118.41 & 232.06 \\
\hline 11.3 & 5085.59 & 123.58 & 242.22 & 5228.63 & 185.78 & 364.12 & 5159.47 & 90.9 & 178.16 \\
\hline 7.85 & 4121.02 & 101.99 & 199.89 & 4229.29 & 148.83 & 291.70 & 4174.73 & 72.55 & 142.20 \\
\hline 5.45 & 3417.81 & 86.5 & 169.53 & 3502.89 & 120.90 & 236.96 & 3461.13 & 56.69 & 111.11 \\
\hline 3.79 & 2908.17 & 71.35 & 139.83 & 2978.17 & 95.00 & 186.21 & 2943.22 & 46.99 & 92.10 \\
\hline 2.64 & 2534.37 & 61.8 & 121.11 & 2590.92 & 81.59 & 159.91 & 2561.62 & 39.82 & 78.05 \\
\hline 1.83 & 2257.35 & 55.33 & 108.43 & 2304.34 & 69.47 & 136.16 & 2280.26 & 34.1 & 66.84 \\
\hline 1.27 & 2048.01 & 50.13 & 98.25 & 2090.88 & 60.53 & 118.65 & 2070.09 & 30.53 & 59.84 \\
\hline 0.89 & 1891.55 & 46.73 & 91.59 & 1931.35 & 56.21 & 110.16 & 1911.94 & 28.62 & 56.09 \\
\hline 0.62 & 1772.84 & 41.09 & 80.53 & 1808.58 & 48.53 & 95.11 & 1789.91 & 25.03 & 49.06 \\
\hline 0.43 & 1687.08 & 41.19 & 80.71 & 1718.48 & 44.04 & 86.33 & 1702.75 & 23.15 & 45.38 \\
\hline 0.3 & 1614.25 & 40.21 & 78.80 & 1647.44 & 43.07 & 84.43 & 1631.25 & 22.83 & 44.75 \\
\hline 0.21 & 1558.58 & 40.73 & 79.83 & 1591.92 & 41.27 & 80.88 & 1575.38 & 21.78 & 42.7 \\
\hline 0.14 & 1515.53 & 37.03 & 72.59 & 1546.64 & 40.82 & 80.02 & 1531.65 & 21.86 & 42.9 \\
\hline 0.1 & 1481.95 & 39.03 & 76.49 & 1511.95 & 43.28 & 84.83 & 1496.99 & 20.97 & 41.11 \\
\hline
\end{tabular}


Table 19: Torque and rotational speed with the calculated uncertainity for the double Spiral spindle with $20 \%$ beads

\begin{tabular}{|l|l|l|l|}
\hline $\begin{array}{l}\mathrm{N} \\
1 / \mathrm{min}\end{array}$ & $\begin{array}{l}\Gamma \\
\mu \mathrm{Nm}\end{array}$ & $\begin{array}{l}\mathrm{U}(\Gamma) \\
\mu \mathrm{Nm}\end{array}$ & $\begin{array}{l}\text { expanded } \\
\mathrm{U}(\Gamma)\end{array}$ \\
\hline 100 & 49833.47 & 2318.27 & 4543.82 \\
\hline 69.5 & 36132.02 & 1606.74 & 3149.21 \\
\hline 48.3 & 26493.03 & 1174.34 & 2301.70 \\
\hline 33.6 & 19615.71 & 861.06 & 1687.67 \\
\hline 23.4 & 14721.38 & 642.11 & 1258.54 \\
\hline 16.2 & 11191.44 & 453.54 & 888.94 \\
\hline 11.3 & 8661.45 & 341.25 & 668.86 \\
\hline 7.85 & 6840.30 & 265.94 & 521.24 \\
\hline 5.45 & 5526.94 & 204.34 & 400.51 \\
\hline 3.79 & 4570.18 & 158.89 & 311.42 \\
\hline 2.64 & 3865.92 & 129.02 & 252.89 \\
\hline 1.83 & 3351.13 & 106.17 & 208.10 \\
\hline 1.27 & 2963.43 & 89.93 & 176.26 \\
\hline 0.89 & 2674.21 & 79.32 & 155.47 \\
\hline 0.62 & 2456.19 & 68.63 & 134.51 \\
\hline 0.43 & 2286.19 & 61.16 & 119.87 \\
\hline 0.3 & 2161 & 57.11 & 111.94 \\
\hline 0.21 & 2064.17 & 53.30 & 104.46 \\
\hline 0.14 & 1987.19 & 50.42 & 98.82 \\
\hline 0.1 & 1931.65 & 53.27 & 104.41 \\
\hline & & & \\
\hline
\end{tabular}

Table 20: Torque and rotational speed with the calculated uncertainity for the double Spiral spindle with $40 \%$ beads

\begin{tabular}{|l|l|l|l|}
\hline $\begin{array}{l}\mathrm{N} \\
1 / \mathrm{min}\end{array}$ & $\begin{array}{l}\Gamma \\
\mu \mathrm{Nm}\end{array}$ & $\begin{array}{l}\mathrm{U}(\Gamma) \\
\mu \mathrm{Nm}\end{array}$ & $\begin{array}{l}\text { expanded } \\
\mathrm{U}(\Gamma)\end{array}$ \\
\hline 100 & 106683 & 9561.52 & 18740.58 \\
\hline 69.5 & 76155.25 & 6656.63 & 13046.99 \\
\hline 48.3 & 55491.24 & 4734.68 & 9279.97 \\
\hline 33.6 & 41062.72 & 3314.46 & 6496.33 \\
\hline 23.4 & 31257.54 & 2036.05 & 3990.67 \\
\hline 16.2 & 24606.85 & 1205.36 & 2362.50 \\
\hline 11.3 & 19427.01 & 1094.86 & 2145.93 \\
\hline 7.85 & 15158.49 & 996.95 & 1954.02 \\
\hline 5.45 & 11973.31 & 788.09 & 1544.65 \\
\hline 3.79 & 9621.73 & 614.26 & 1203.95 \\
\hline 2.64 & 7915.19 & 502.65 & 985.19 \\
\hline 1.83 & 6631.62 & 414.27 & 811.97 \\
\hline 1.27 & 5665.15 & 330.41 & 647.59 \\
\hline 0.89 & 4954.07 & 277.95 & 544.77 \\
\hline 0.62 & 4416.82 & 245.72 & 481.62 \\
\hline 0.43 & 3998.80 & 214.90 & 421.20 \\
\hline 0.3 & 3694.45 & 195.37 & 382.93 \\
\hline 0.21 & 3445.4 & 176.09 & 345.13 \\
\hline 0.14 & 3264.46 & 166.73 & 326.80 \\
\hline 0.1 & 3142.72 & 156.89 & 307.51 \\
\hline & & & \\
\hline
\end{tabular}


Table 21: Torque and rotational speed with the calculated uncertainity for the sixblade vane spindle with $0 \%$ beads

\begin{tabular}{|c|c|c|c|c|c|c|c|c|c|}
\hline & \multicolumn{3}{|c|}{$0 \%$ pre $40 \%$} & \multicolumn{3}{|c|}{$0 \%$ pre $20 \%$} & \multicolumn{3}{|c|}{$0 \%$ combined } \\
\hline $\begin{array}{l}\mathrm{N} \\
1 / \min \end{array}$ & $\begin{array}{l}\Gamma \\
\mu \mathrm{Nm}\end{array}$ & $\begin{array}{l}\mathrm{U}(\Gamma) \\
\mu \mathrm{Nm}\end{array}$ & $\begin{array}{r}\text { expanded } \\
U(\Gamma)\end{array}$ & $\begin{array}{l}\Gamma \\
\mu \mathrm{Nm}\end{array}$ & $\begin{array}{l}\mathrm{U}(\Gamma) \\
\mu \mathrm{Nm}\end{array}$ & $\begin{array}{r}\text { expanded } \\
U(\Gamma)\end{array}$ & $\begin{array}{l}\Gamma \\
\mu \mathrm{Nm}\end{array}$ & $\begin{array}{l}\mathrm{U}(\Gamma) \\
\mu \mathrm{Nm}\end{array}$ & $\begin{array}{l}\text { expanded } \\
\mathrm{U}(\Gamma)\end{array}$ \\
\hline 100 & 7309.29 & 182.61 & 357.91 & 7185.95 & 195.45 & 383.09 & 7246.99 & 103.12 & 202.12 \\
\hline 69.5 & 5483.12 & 133.88 & 262.40 & 5387.10 & 146.27 & 286.70 & 5435.07 & 75.67 & 148.32 \\
\hline 48.3 & 4176.09 & 95.39 & 186.97 & 4102.92 & 106.86 & 209.44 & 4136.84 & 55.33 & 108.44 \\
\hline 33.6 & 3228.94 & 73.28 & 143.62 & 3175.48 & 82.43 & 161.57 & 3203.20 & 41.76 & 81.85 \\
\hline 23.4 & 2549.10 & 55.00 & 107.80 & 2507.84 & 64.35 & 126.12 & 2528.2 & 31.77 & 62.28 \\
\hline 16.2 & 2049.33 & 40.63 & 79.63 & 2020.66 & 49.08 & 96.20 & 2035.31 & 24.49 & 48.01 \\
\hline 11.3 & 1683.84 & 30.00 & 58.78 & 1659.8 & 36.54 & 71.62 & 1672.22 & 18.84 & 36.93 \\
\hline 7.85 & 1409.06 & 25.84 & 50.66 & 1389.63 & 34.45 & 67.51 & 1399.73 & 16.28 & 31.90 \\
\hline 5.45 & 1197.40 & 20.72 & 40.62 & 1182.76 & 26.86 & 52.64 & 1190.00 & 13.20 & 25.87 \\
\hline 3.79 & 1034.64 & 17.44 & 34.19 & 1027.23 & 23.27 & 45.61 & 1031.01 & 11.00 & 21.55 \\
\hline 2.64 & 910.59 & 14.93 & 29.26 & 904.97 & 20.13 & 39.45 & 907.89 & 9.61 & 18.83 \\
\hline 1.83 & 814.18 & 13.15 & 25.77 & 811.41 & 17.49 & 34.27 & 812.84 & 8.33 & 16.32 \\
\hline 1.27 & 738.89 & 11.74 & 23.01 & 742.40 & 17.97 & 35.23 & 740.87 & 8.20 & 16.08 \\
\hline 0.89 & 681.35 & 10.71 & 20.99 & 685.53 & 15.36 & 30.10 & 683.41 & 7.26 & 14.23 \\
\hline 0.62 & 633.74 & 10.08 & 19.76 & 639.61 & 12.59 & 24.68 & 636.65 & 6.23 & 12.21 \\
\hline 0.43 & 597.61 & 10.59 & 20.76 & 605.64 & 13.95 & 27.34 & 601.52 & 6.71 & 13.15 \\
\hline 0.3 & 566.48 & 9.61 & 18.83 & 583.08 & 10.78 & 21.12 & 574.89 & 6.18 & 12.12 \\
\hline 0.21 & 543.32 & 10.26 & 20.11 & 558.76 & 12.17 & 23.85 & 551.14 & 6.85 & 13.43 \\
\hline 0.14 & 525.05 & 11.90 & 23.33 & 542.44 & 8.30 & 16.27 & 533.65 & 6.60 & 12.93 \\
\hline 0.1 & 508.48 & 9.61 & 18.84 & 530.70 & 11.34 & 22.22 & 519.51 & 7.08 & 13.88 \\
\hline
\end{tabular}


Table 22: Torque and rotational speed with the calculated uncertainity for the six-blad vane spindle with $20 \%$ beads

\begin{tabular}{|l|l|l|l|}
\hline $\begin{array}{l}\mathrm{N} \\
1 / \mathrm{min}\end{array}$ & $\begin{array}{l}\Gamma \mathrm{Nm} \\
\mu \mathrm{C})\end{array}$ & $\begin{array}{l}\text { expanded } \\
\mathrm{U}(\Gamma)\end{array}$ \\
\hline 100 & 12606.57 & 327.67 & 642.23 \\
\hline 69.5 & 9373.63 & 230.94 & 452.65 \\
\hline 48.3 & 7049.17 & 168.46 & 330.18 \\
\hline 33.6 & 5372.77 & 131.21 & 257.17 \\
\hline 23.4 & 4167.39 & 92.38 & 181.07 \\
\hline 16.2 & 3285.99 & 65.02 & 127.43 \\
\hline 11.3 & 2629.74 & 47.13 & 92.38 \\
\hline 7.85 & 2140.88 & 41.93 & 82.18 \\
\hline 5.45 & 1777.44 & 30.19 & 59.17 \\
\hline 3.79 & 1506.62 & 26.62 & 52.17 \\
\hline 2.64 & 1298.55 & 23.60 & 46.26 \\
\hline 1.83 & 1141.87 & 18.93 & 37.10 \\
\hline 1.27 & 1021.81 & 17.50 & 34.30 \\
\hline 0.89 & 926.39 & 16.95 & 33.22 \\
\hline 0.62 & 859.88 & 14.40 & 28.23 \\
\hline 0.43 & 800.90 & 13.28 & 26.04 \\
\hline 0.3 & 761.74 & 11.39 & 22.32 \\
\hline 0.21 & 720.38 & 10.48 & 20.54 \\
\hline 0.14 & 696.30 & 8.03 & 15.74 \\
\hline 0.1 & 678.22 & 10.35 & 20.28 \\
\hline & & & \\
\hline
\end{tabular}

Table 23: Torque and rotational speed with the calculated uncertainity for the six-blade vane spindle with $\mathbf{4 0} \%$ beads

\begin{tabular}{|c|c|c|c|}
\hline $\begin{array}{l}N \\
1 / \text { min }\end{array}$ & $\begin{array}{l}\Gamma \\
\mu N m\end{array}$ & $\begin{array}{l}\mathrm{U}(\Gamma) \\
\mu N m\end{array}$ & $\begin{array}{l}\text { expanded } \\
\mathrm{U}(\Gamma)\end{array}$ \\
\hline 100 & 24512.57 & 1328.32 & 2603.51 \\
\hline 69.5 & 18264.38 & 1252.74 & 2455.37 \\
\hline 48.3 & 13673.2 & 894.37 & 1752.96 \\
\hline 33.6 & 10432.25 & 719.23 & 1409.69 \\
\hline 23.4 & 8018.50 & 576.80 & 1130.52 \\
\hline 16.2 & 6251.92 & 435.50 & 853.57 \\
\hline 11.3 & 4938.09 & 347.48 & 681.06 \\
\hline 7.85 & 3947.76 & 270.61 & 530.40 \\
\hline 5.45 & 3204.74 & 218.71 & 428.67 \\
\hline 3.79 & 2649.68 & 175.61 & 344.20 \\
\hline 2.64 & 2221.24 & 154.61 & 303.04 \\
\hline 1.83 & 1903.85 & 118.53 & 232.32 \\
\hline 1.27 & 1662.50 & 101.78 & 199.48 \\
\hline 0.89 & 1475.63 & 87.58 & 171.65 \\
\hline 0.62 & 1334.05 & 76.63 & 150.20 \\
\hline 0.43 & 1226.54 & 71.98 & 141.07 \\
\hline 0.3 & 1139.02 & 62.75 & 122.99 \\
\hline 0.21 & 1067.44 & 53.08 & 104.03 \\
\hline 0.14 & 1021.97 & 54.27 & 106.37 \\
\hline 0.1 & 980.35 & 47.81 & 93.715 \\
\hline
\end{tabular}


Table 24: Torque and rotational speed with the calculated uncertainity for the Serrated Coaxial Cylinder with 0 \% beads

\begin{tabular}{|c|c|c|c|c|c|c|c|c|c|}
\hline & \multicolumn{3}{|c|}{$0 \%$ pre $40 \%$} & \multicolumn{3}{|c|}{$0 \%$ pre $20 \%$} & \multicolumn{3}{|c|}{$0 \%$ combined } \\
\hline $\begin{array}{l}\mathrm{N} \\
1 / \text { min }\end{array}$ & $\begin{array}{l}\Gamma \\
\mu N m\end{array}$ & $\begin{array}{l}\mathrm{U}(\Gamma) \\
\mu \mathrm{Nm}\end{array}$ & $\begin{array}{l}\text { expand } \\
\text { ed } \\
\mathrm{U}(\Gamma) \\
\end{array}$ & $\begin{array}{l}\Gamma \\
\mu N m\end{array}$ & $\begin{array}{l}\mathrm{U}(\Gamma) \\
\mu \mathrm{Nm}\end{array}$ & $\begin{array}{l}\text { expande } \\
\mathrm{d} U(\Gamma)\end{array}$ & $\begin{array}{l}\Gamma \\
\mu \mathrm{Nm}\end{array}$ & $\begin{array}{l}\mathrm{U}(\Gamma) \\
\mu \mathrm{Nm}\end{array}$ & $\begin{array}{c}\text { expanded } \\
\mathrm{U}(\Gamma)\end{array}$ \\
\hline 100 & 7686.6 & 245.91 & 481.98 & 8072.5 & 422.36 & 827.83 & 7879.8 & 198.52 & 389.10 \\
\hline 69.5 & 5765 & 189.55 & 371.52 & 6040.8 & 321.69 & 630.51 & 5903.4 & 147.36 & 288.83 \\
\hline 48.3 & 4356.1 & 138.46 & 271.38 & 4562.1 & 233.25 & 457.17 & 4455.2 & 108.8 & 213.25 \\
\hline 33.6 & 3335.1 & 106.5 & 208.74 & 3484.7 & 180.31 & 353.41 & 3411.8 & 81.95 & 160.62 \\
\hline 23.4 & 2604.6 & 80.21 & 157.21 & 2719.1 & 136.97 & 268.46 & 2661.6 & 61.67 & 120.87 \\
\hline 16.2 & 2068.7 & 61.76 & 121.06 & 2162 & 100.61 & 197.20 & 2115.6 & 48.44 & 94.95 \\
\hline 11.3 & 1676.4 & 46.34 & 90.82 & 1751.6 & 76.51 & 149.96 & 1714.4 & 37.47 & 73.45 \\
\hline 7.85 & 1378.2 & 40.22 & 78.82 & 1442.6 & 67.23 & 131.78 & 1411.1 & 31.15 & 61.054 \\
\hline 5.45 & 1148.1 & 33.16 & 65.00 & 1208.9 & 54.39 & 106.61 & 1178.3 & 26.91 & 52.75 \\
\hline 3.79 & 972.15 & 28.38 & 55.63 & 1028.6 & 47.76 & 93.61 & 1000.4 & 23.38 & 45.82 \\
\hline 2.64 & 834.34 & 25.589 & 50.15 & 886.06 & 41.88 & 82.083 & 860.36 & 21.14 & 41.43 \\
\hline 1.83 & 725.18 & 22.68 & 44.45 & 782.41 & 37.35 & 73.20 & 753.94 & 19.76 & 38.72 \\
\hline 1.27 & 638.3 & 20.45 & 40.08 & 691.91 & 33.07 & 64.82 & 665.63 & 17.97 & 35.22 \\
\hline 0.89 & 574.36 & 25.75 & 50.46 & 625.49 & 25.39 & 49.76 & 599.94 & 16.91 & 33.14 \\
\hline 0.62 & 515.41 & 21.60 & 42.33 & 584.88 & 26.20 & 51.36 & 550.06 & 18.65 & 36.56 \\
\hline 0.43 & 469.95 & 19.82 & 38.85 & 557.07 & 27.10 & 53.11 & 513.17 & 20.75 & 40.67 \\
\hline 0.3 & 433.3 & 19.77 & 38.76 & 502.27 & 24.99 & 48.98 & 467.9 & 17.56 & 34.43 \\
\hline 0.21 & 407.72 & 24.60 & 48.21 & 521.34 & 36.60 & 71.72 & 465.01 & 27.33 & 53.57 \\
\hline 0.14 & 380.95 & 19.99 & 39.18 & 515.81 & 33.80 & 66.24 & 448.35 & 30.14 & 59.06 \\
\hline 0.1 & 365.28 & 24.45 & 47.91 & 487.25 & 36.59 & 71.72 & 426.01 & 28.47 & 55.79 \\
\hline
\end{tabular}


Table 25: Torque and rotational speed with the calculated uncertainity for the Serrated Coaxial Cylinder with $20 \%$ beads

\begin{tabular}{|l|r|r|r|}
\hline $\begin{array}{l}\mathrm{N} \\
1 / \mathrm{min}\end{array}$ & \multicolumn{1}{l|}{$\begin{array}{l}\Gamma \\
\mu \mathrm{Nm}\end{array}$} & \multicolumn{1}{l|}{$\mathrm{U}(\Gamma)$} & \multicolumn{1}{l|}{$\begin{array}{l}\text { expanded } \\
\mathrm{UN}(\Gamma)\end{array}$} \\
\hline 100 & 13300 & 503.36 & 986.59 \\
\hline 69.5 & 9908 & 381.61 & 747.96 \\
\hline 48.3 & 7441.6 & 284.2 & 557.03 \\
\hline 33.6 & 5642.8 & 221.28 & 433.71 \\
\hline 23.4 & 4349.3 & 169.49 & 332.20 \\
\hline 16.2 & 3408 & 125.47 & 245.92 \\
\hline 11.3 & 2722.8 & 95.51 & 187.19 \\
\hline 7.85 & 2214.5 & 79.91 & 156.62 \\
\hline 5.45 & 1834.2 & 62.09 & 121.70 \\
\hline 3.79 & 1548.6 & 52.99 & 103.85 \\
\hline 2.64 & 1327.1 & 43.62 & 85.49 \\
\hline 1.83 & 1155.1 & 39.07 & 76.57 \\
\hline 1.27 & 1023.3 & 35.49 & 69.55 \\
\hline 0.89 & 931.6 & 32.40 & 63.50 \\
\hline 0.62 & 852.62 & 29.35 & 57.53 \\
\hline 0.43 & 797.47 & 27.16 & 53.23 \\
\hline 0.3 & 764.83 & 21.20 & 41.56 \\
\hline 0.21 & 747.97 & 53.35 & 104.56 \\
\hline 0.14 & 704.58 & 26.99 & 52.90 \\
\hline 0.1 & 673.3 & 27.04 & 52.99 \\
\hline
\end{tabular}

Table 26: Torque and rotational speed with the calculated uncertainity for the Serrated Coaxial Cylinder with $40 \%$ beads

\begin{tabular}{|l|r|r|r|}
\hline $\begin{array}{l}\mathrm{N} \\
1 / \mathrm{min}\end{array}$ & \multicolumn{1}{l|}{$\begin{array}{l}\Gamma \\
\mu \mathrm{Nm}\end{array}$} & \multicolumn{1}{l|}{$\begin{array}{l}\mathrm{U}(\Gamma) \\
\mu \mathrm{Nm}\end{array}$} & \multicolumn{1}{l|}{$\begin{array}{l}\text { expanded } \\
\mathrm{U}(\Gamma)\end{array}$} \\
\hline 100 & 22339 & 1084.4 & 2125.42 \\
\hline 69.5 & 17512 & 1003.6 & 1967.06 \\
\hline 48.3 & 13364 & 708.69 & 1389.03 \\
\hline 33.6 & 10316 & 467.7 & 916.69 \\
\hline 23.4 & 7897.2 & 326.89 & 640.70 \\
\hline 16.2 & 6096 & 236.56 & 463.66 \\
\hline 11.3 & 4756.4 & 177.22 & 347.35 \\
\hline 7.85 & 3771.1 & 142.64 & 279.57 \\
\hline 5.45 & 3036.4 & 105.82 & 207.41 \\
\hline 3.79 & 2490.5 & 85.29 & 167.16 \\
\hline 2.64 & 2071.7 & 66.05 & 129.47 \\
\hline 1.83 & 1755.6 & 53.08 & 104.03 \\
\hline 1.27 & 1520 & 43.06 & 84.40 \\
\hline 0.89 & 1329.9 & 34.61 & 67.84 \\
\hline 0.62 & 1189.4 & 31.72 & 62.16 \\
\hline 0.43 & 1071.2 & 27.77 & 54.46 \\
\hline 0.3 & 979.14 & 24.57 & 48.16 \\
\hline 0.21 & 907.41 & 22.34 & 43.79 \\
\hline 0.14 & 860.1 & 37.25 & 73.01 \\
\hline 0.1 & 807.42 & 32.96 & 64.60 \\
\hline
\end{tabular}




\subsubsection{OpenBUGS Code}

This program computes the yield stress (alpha) in $1000 \cdot \mathrm{Nm}$, and viscosity (betad) in $1000 \cdot \mathrm{Nm} \cdot \mathrm{s}$, for the cases where there are 5 replicates of 20 points each. It also computes the average torque (theta) in $10^{-2} \cdot \mu \mathrm{Nm}$. For alpha, beta, and theta, it also computes the standard uncertainties and 95\% uncertainty intervals. The data is first reduced so that only the downcurve is included, that is, the first 25 points are eliminated. It also obtains the average curves in microNm/1000 (theta).

\{\#\# prior distributions for Bingham parameters

\#\# dnorm represents the Gaussian distribution

\#\# dgamma represents the Gamma distribution.

alpha dnorm(0,1.0E-5)

beta dnorm $(0,1.0 \mathrm{E}-5)$

siga dgamma(1.0E-5,1.0E-5)

sigb dgamma(1.0E-5,1.0E-5)

sigd dgamma(1.0E-5,1.0E-5)

for(i in 1:5)\{ a[i] dnorm(alpha,siga)

$b[i] \sim \operatorname{dnorm}($ beta,sigb)

\}

\#\#\#

\#\# regression model for Bingham parameters

for(i in 1:100)\{ mean[i] $<-a[\operatorname{mix}[i]]+b[\operatorname{mix}[i]] * \operatorname{sig}[i]$

$M[i] \sim \operatorname{dnorm}($ mean[i],sigd)\}

betad $<-$ beta/0.105

\#\#\#

\#\# prior distributions for flow curve model

for $(j$ in $1: 20)\{$ theta $[j] \sim$ dnorm $(0,1.0 E-5)$

sigthe[j] dgamma(1.0E-5,1.0E-5)

\}

\#\#\#

\#\# flow curves

for(i in 1:100)\{M2[i] dnorm(theta[posit[i]],sigthe[posit[i]])\}

\#\#

\}

\#\# initial values

$\operatorname{list}(\operatorname{sig} a=1, \operatorname{sig} b=1, \operatorname{sigd}=1$, sigthe $=c(1,1,1,1,1,1,1,1,1,1,1,1,1,1,1,1,1,1,1,1))$

\subsubsection{Uncertainty of the Estimates of the Parameters of Equation 1}

Section 3 provides an equation (Equation 1) for viscosity as a function of shear rate to be used for calibration of the user's rheometer data. This equation (1) can be written as:

$$
\mu=\frac{\left(C_{2}+\frac{A_{2}}{\dot{\gamma}^{B}}\right)+\left(C_{1}+\frac{A_{1}}{\dot{\gamma}^{B} 1}\right) e^{a \times(\dot{\gamma}-1)}}{1+e^{a \times(\dot{\gamma}-1)}}
$$

where $\mu$ is viscosity, $\dot{\gamma}$ is shear rate, $A_{i}, B_{i}, C_{i}$ are parameters estimated based on the SRM 2492 paste data, and $a$ is a fitting parameter. Note that 


$$
\begin{aligned}
\lim _{a \rightarrow-\infty} \mu= & \left(C_{1}+\frac{A_{1}}{\dot{\gamma}^{B_{1}}}\right), \text { for } \dot{\gamma}<1 s^{-1}, \\
& \left(C_{2}+\frac{A_{2}}{\dot{\gamma}^{B_{2}}}\right), \text { for } \dot{\gamma}>1 s^{-1}, \\
& \frac{\left(C_{1}+\frac{A_{1}}{\dot{\gamma}_{1}}\right)+\left(C_{2}+\frac{A_{2}}{\dot{\gamma}^{B_{2}}}\right)}{2}, \text { for } \dot{\gamma}=1 s^{-1} .
\end{aligned}
$$

The parameter estimates given in Section 3 were obtained using ordinary least squares (OLS) fitting separately for viscosity values where $\dot{\gamma}<1 s^{-1}$ and for values where $\dot{\gamma}>1 s^{-1}$. To obtain an estimate of total uncertainty for these coefficients, equation 1 was also fitted using a Bayesian hierarchical model (BHM) using the code given below. This model accounts for the uncertainty of the fit as well as the uncertainty in the SRM 2492 measurements. As the two sets of estimates were not identical, the uncertainty estimates based on the BHM could not be used for the OLS coefficients without accounting for the bias. This component was computed by subtracting the OLS estimate from the BHM estimate. Squaring the bias and adding it to the squared BHM standard uncertainty forms an estimate of the total uncertainty of the OLS values. Thus the expanded total uncertainty is $e t u=2 \sqrt{\left(u^{2}\right)+{b i a s^{2}}}$ given below.

\begin{tabular}{|c|l|l|l|l|l|}
\hline Coefficients & $\begin{array}{l}\text { Estimate via } \\
\text { OLS }\end{array}$ & $\begin{array}{l}\text { Estimate } \\
\text { via BHM }\end{array}$ & $\begin{array}{l}\text { standard } \\
\text { uncertainty } \\
\text { via BHM }(u)\end{array}$ & $\begin{array}{l}\text { Bias : } \\
\text { BHM - OLS } \\
\text { (bias })\end{array}$ & $\begin{array}{l}\text { Expanded total } \\
\text { uncertainty of the } \\
\text { OLS estimates } \\
(\text { etu })\end{array}$ \\
\hline$A_{1}$ & 16.411 & 16.99 & 0.30 & 0.58 & 1.30 \\
\hline$A_{2}$ & 19.178 & 18.74 & 0.53 & -0.44 & 1.38 \\
\hline$B_{1}$ & 0.988 & 0.97 & 0.01 & -0.018 & 0.04 \\
\hline$B_{2}$ & 0.727 & 0.63 & 0.07 & -0.097 & 0.24 \\
\hline$C_{1}$ & 9.641 & 8.79 & 0.54 & -0.851 & 2.09 \\
\hline$C_{2}$ & 7.116 & 6.39 & 0.70 & -0.726 & 2.02 \\
\hline
\end{tabular}

OpenBUGS code:

\{sig dgamma(1.0E-5,1.0E-5)

a1 dunif $(5,25)$

b1 dunif $(0,1.5)$

c1 dunif $(1,15)$

a2 $\operatorname{dunif}(5,25)$

b2 dunif $(0,1.5)$

c2 dunif(1,15)

for(i in 1:29) \{ $m u[i]<-a 1 / \operatorname{pow}(\operatorname{sr}[i], b 1)+c 1$ $\operatorname{visc}[i] \sim \operatorname{dnorm}(\mathrm{mu}[i], \operatorname{sig})\}$

for(i in 30:38) $\{m u[i]<-a 2 / p o w(s r[i], b 2)+c 2$ visc[i] dnorm(mu[i],sig)\}

for(i in 1:38)\{dif[i]<-(visc[i]-mu[i])/visc[i]\}

\}

list(sig=1)

$\begin{array}{lll}s r[ & \text { visc[] } & \text { viscu[] } \\ 0.1 & 168.527 & 11.924 \\ 0.103 & 163.838 & 11.734 \\ 0.107 & 159.082 & 11.595 \\ 0.11 & 154.122 & 11.1 \\ 0.114 & 149.277 & 10.891 \\ 0.119 & 144.355 & 10.569\end{array}$




$\begin{array}{lll}0.123 & 139.263 & 10.168 \\ 0.129 & 134.355 & 9.843 \\ 0.134 & 129.328 & 9.458 \\ 0.14 & 124.294 & 8.993 \\ 0.147 & 119.303 & 8.653 \\ 0.154 & 114.252 & 8.293 \\ 0.162 & 109.06 & 7.965 \\ 0.171 & 103.957 & 7.549 \\ 0.181 & 98.739 & 7.162 \\ 0.192 & 93.497 & 6.794 \\ 0.205 & 88.39 & 6.428 \\ 0.219 & 83.193 & 6.054 \\ 0.235 & 78.004 & 5.66 \\ 0.255 & 72.871 & 5.345 \\ 0.277 & 67.72 & 4.904 \\ 0.305 & 62.549 & 4.536 \\ 0.338 & 57.373 & 4.157 \\ 0.379 & 52.333 & 3.731 \\ 0.431 & 47.235 & 3.326 \\ 0.5 & 42.127 & 2.946 \\ 0.596 & 37.011 & 2.536 \\ 0.737 & 31.881 & 2.139 \\ 0.966 & 26.782 & 1.73 \\ 1.402 & 21.659 & 1.357 \\ 2.55 & 16.554 & 1.104 \\ 5 & 13.353 & 0.944 \\ 10 & 10.966 & 0.777 \\ 15 & 9.972 & 0.722 \\ 20 & 9.349 & 0.676 \\ 25 & 8.925 & 0.655 \\ 30 & 8.601 & 0.62 \\ 35 & 8.354 & 0.617 \\ E N D & & \end{array}$

\subsection{Bingham Parameters}

Yield stress and plastic viscosity are respectively the intercept and slope of a straight line fitted to the graph of torque plotted against rotational speed (Figure 25). Only the "down curve" data was used to fit the regression, that is, the torques for the 10 shear rates starting at $100 \mathrm{~s}^{-1}$ and decreasing to $1 \mathrm{~s}^{-1}$. This is considered to use only the linear portion of the curve. A straight line model fits the flow curve data reasonably well and provides an approximation to the flow curves given in the previous section. Figure 25 shows an example.

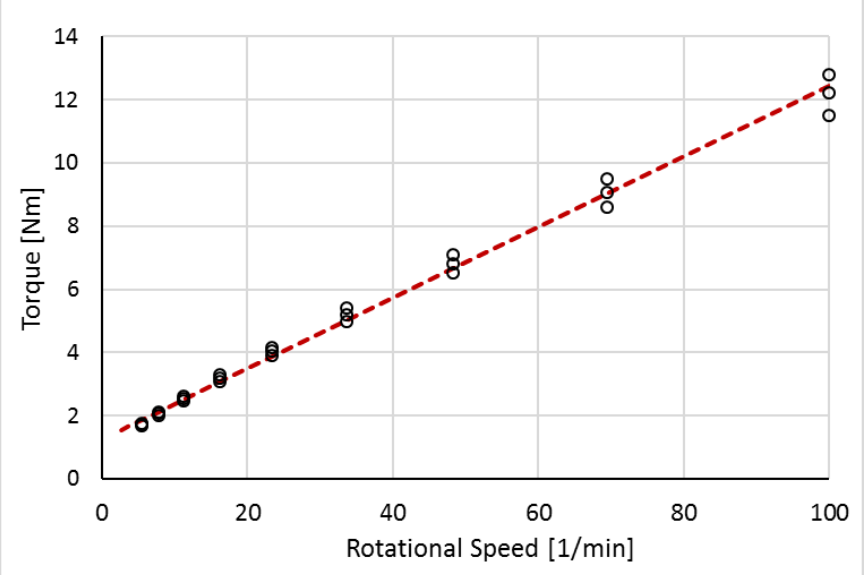

Figure 25: Example of torque vs. rotational speed for $20 \%$ bead volume fraction using six-blade vane. The red line is the least squares line fitted to all of the points. 
Yield stress and viscosity were estimated using a random effects regression model:

$$
\Gamma_{i j}=a_{i}+b_{i} \Omega_{j}+\varepsilon_{i j}
$$

where $a_{i} \sim N\left(\alpha, \sigma_{\alpha}^{2}\right), b_{i} \sim N\left(\beta, \sigma_{\beta}^{2}\right), \varepsilon_{i j} \sim N\left(0, \sigma_{i}^{2}\right), i=1, \ldots, 5, j=1, \ldots, 20$.

In this model, $a_{i}$ are the yield stress values for the 5 individual mixtures, and $\alpha$ is the consensus yield stress value for the particular geometry and \% beads. Similarly, $\boldsymbol{b}_{\boldsymbol{i}}$ are the viscosity values for the 5 individual mixtures and $\beta$ is the consensus viscosity for the geometry and \% beads. Unlike the simple least squares fit shown in the above figure, this model accounts for measurement uncertainty and lack of fit of each replicate using $\sigma_{i}$, and also for between replicate variability using $\sigma_{\alpha}$ and $\sigma_{\beta}$.

Fitting was done using Markov Chain Monte Carlo methods which require prior distributions on the parameters of the model. These were

$$
\begin{aligned}
& \alpha \sim N(0,1.0 E 5), \beta \sim N(0,1.0 E 5), \sigma_{\alpha}^{2} \sim \operatorname{Gamma}(1.0 E 5,1.0 E 5) \\
& \sigma_{\beta}^{2} \sim \operatorname{Gamma(1.0E5,1.0E5),\sigma _{i}^{2}\sim \operatorname {Gamma}(1.0E5,1.0E5),i=1,\ldots ,5} .
\end{aligned}
$$

Figure 26 shows the fitted line with 95\% uncertainty bounds based on the estimates of slope ( $\beta$ - plastic viscosity) and intercept ( $\alpha$ - yield stress) with data points in green for mixture $1,20 \%$ bead volume fraction, using the six-blade vane.

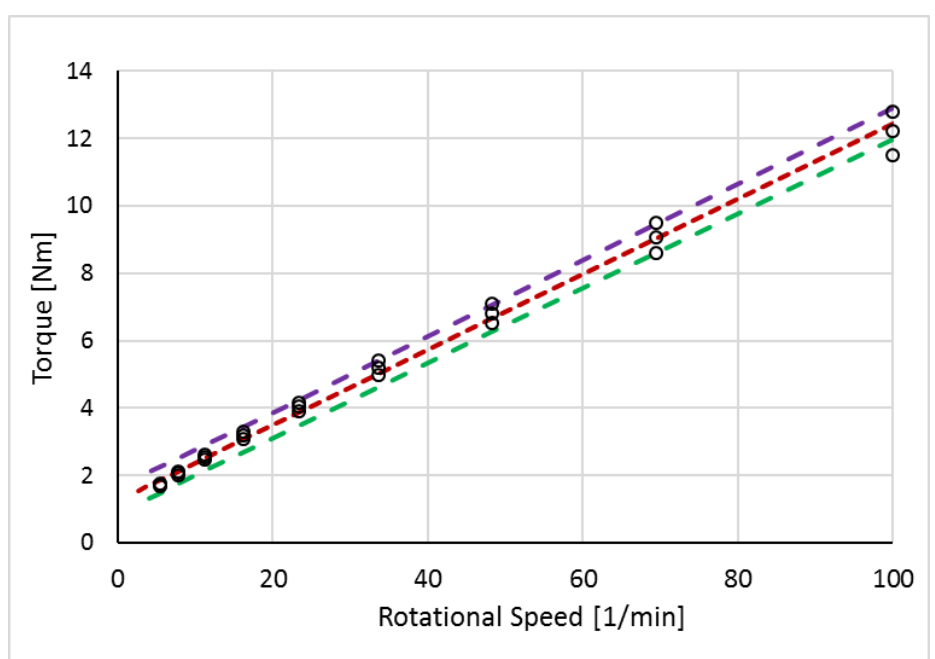

Figure 26 Example of fitted line and $95 \%$ uncertainty bounds for $20 \%$ beads using six-blade vane with data for mixture 1 , whose median value is shown by red line.

Tables 25 to Table 27 contain the posterior means, standard deviations and 95\% uncertainty bounds for $\alpha$ (yield stress) and Tables 28 to Table 30 contain the posterior means, standard deviations and $95 \%$ uncertainty bounds for $\beta$ (plastic viscosity). 
Table 27: Double spiral spindle: Posterior means, standard deviations and $95 \%$ uncertainty bounds for $\alpha$ (yield stress)

\begin{tabular}{|c|c|c|c|}
\hline Mixture & $\begin{array}{c}\text { Yield Stress } \\
{[\mathrm{Nm}]}\end{array}$ & $\begin{array}{c}\text { standard } \\
\text { uncertainty } \\
{[\mathrm{Nm}]}\end{array}$ & $\begin{array}{c}\text { Expanded } \\
\text { uncertainty } \\
{[\mathrm{Nm}]}\end{array}$ \\
\hline $0 \%($ pre $40 \%)$ & 0.002198 & 0.000054 & 0.000108 \\
\hline $0 \%($ pre $20 \%)$ & 0.002247 & 0.000061 & 0.000122 \\
\hline $0 \%$ & 0.002222 & 0.000037 & 0.000074 \\
\hline $20 \%$ & 0.003223 & 0.000095 & 0.000190 \\
\hline $40 \%$ & 0.007004 & 0.000290 & 0.000580 \\
\hline
\end{tabular}

Table 28: Six-blade Vane: Posterior means, standard deviations and $95 \%$ uncertainty bounds for $\alpha$ (yield stress)

\begin{tabular}{|c|c|c|c|}
\hline Mixture & $\begin{array}{c}\text { Yield Stress } \\
{[\mathrm{Nm}]}\end{array}$ & $\begin{array}{c}\text { standard } \\
\text { uncertainty } \\
{[\mathrm{Nm}]}\end{array}$ & $\begin{array}{c}\text { Expanded } \\
\text { uncertainty } \\
{[\mathrm{Nm}]}\end{array}$ \\
\hline $0 \%($ pre $40 \%)$ & 0.00090 & 0.00002 & 0.00005 \\
\hline $0 \%($ pre $20 \%)$ & 0.00089 & 0.00002 & 0.00004 \\
\hline $0 \%$ & 0.00090 & 0.00002 & 0.00004 \\
\hline $20 \%$ & 0.00126 & 0.00004 & 0.00008 \\
\hline $40 \%$ & 0.00223 & 0.00007 & 0.00014 \\
\hline
\end{tabular}

Table 29: Serrated Coaxial Cylinder: Posterior means, standard deviations and $95 \%$ uncertainty bounds for $\alpha$ (yield stress)

\begin{tabular}{|c|c|c|c|}
\hline Mixture & $\begin{array}{c}\text { Yield Stress } \\
{[\mathrm{Nm}]}\end{array}$ & $\begin{array}{c}\text { standard } \\
\text { uncertainty } \\
{[\mathrm{Nm}]}\end{array}$ & $\begin{array}{c}\text { Expanded } \\
\text { uncertainty } \\
{[\mathrm{Nm}]}\end{array}$ \\
\hline $0 \%($ pre $40 \%)$ & 0.000837 & 0.000029 & 0.000058 \\
\hline $0 \%($ pre $20 \%)$ & 0.000876 & 0.000032 & 0.000064 \\
\hline $0 \%$ & 0.000856 & 0.000020 & 0.000040 \\
\hline $20 \%$ & 0.001279 & 0.000046 & 0.000092 \\
\hline $40 \%$ & 0.002316 & 0.000146 & 0.000292 \\
\hline
\end{tabular}

Table 30: Double spiral spindle: posterior means, standard deviations and $95 \%$ uncertainty bounds for $\beta$ (plastic viscosity)

\begin{tabular}{|c|c|c|c|}
\hline Mixture & $\begin{array}{c}\text { Viscosity } \\
{[\mathrm{Nm} \cdot \mathrm{s}]}\end{array}$ & $\begin{array}{c}\text { Standard } \\
\text { uncertainty } \\
{[\mathrm{Nm} \cdot \mathrm{s}]}\end{array}$ & $\begin{array}{c}\text { Expanded } \\
\text { uncertainty } \\
{[\mathrm{Nm} \cdot \mathrm{s}]}\end{array}$ \\
\hline $0 \%($ pre $40 \%)$ & 0.00239 & 0.00008 & 0.00015 \\
\hline $0 \%($ pre $20 \%)$ & 0.00247 & 0.00011 & 0.00022 \\
\hline $0 \%$ & 0.00243 & 0.00005 & 0.00010 \\
\hline $20 \%$ & 0.00450 & 0.00023 & 0.00045 \\
\hline $40 \%$ & 0.00954 & 0.00088 & 0.00176 \\
\hline
\end{tabular}


Table 31: Six-Blade Vane: posterior means, standard deviations and $95 \%$ uncertainty bounds for $\beta$ (plastic viscosity)

\begin{tabular}{|c|c|c|c|}
\hline Mixture & $\begin{array}{c}\text { Viscosity } \\
{[\mathrm{Nm} \cdot \mathrm{s}]}\end{array}$ & $\begin{array}{c}\text { Standard } \\
\text { uncertainty } \\
{[\mathrm{Nm} \cdot \mathrm{s}]}\end{array}$ & $\begin{array}{c}\text { Expanded } \\
\text { uncertainty } \\
{[\mathrm{Nm} \cdot \mathrm{s}]}\end{array}$ \\
\hline $0 \%($ pre $40 \%)$ & 0.00062 & 0.00002 & 0.00004 \\
\hline $0 \%($ pre $20 \%)$ & 0.00061 & 0.00002 & 0.00004 \\
\hline $0 \%$ & 0.00062 & 0.00001 & 0.00002 \\
\hline $20 \%$ & 0.00111 & 0.00003 & 0.00006 \\
\hline $40 \%$ & 0.00218 & 0.00014 & 0.00028 \\
\hline
\end{tabular}

Table 32: Serrated Coaxial Cylinder: posterior means, standard deviations and 95\% uncertainty bounds for $\beta$ (plastic viscosity)

\begin{tabular}{|c|c|c|c|}
\hline Mixture & $\begin{array}{c}\text { Viscosity } \\
{[\mathrm{Nm} \cdot \mathrm{s}]}\end{array}$ & $\begin{array}{c}\text { Standard } \\
\text { uncertainty } \\
{[\mathrm{Nm} \cdot \mathrm{s}]}\end{array}$ & $\begin{array}{c}\text { Expanded } \\
\text { uncertainty } \\
{[\mathrm{Nm} \cdot \mathrm{s}]}\end{array}$ \\
\hline $0 \%($ pre $40 \%)$ & 0.000669 & 0.000029 & 0.000057 \\
\hline $0 \%($ pre $20 \%)$ & 0.000702 & 0.000042 & 0.000084 \\
\hline $0 \%$ & 0.000685 & 0.000020 & 0.000040 \\
\hline $20 \%$ & 0.001172 & 0.000052 & 0.000104 \\
\hline $40 \%$ & 0.002018 & 0.000114 & 0.000228 \\
\hline
\end{tabular}

\subsection{Flow Curve Fundamental Data in Fundamental Units}

\subsubsection{Using the Bingham Based Method}

Flow curves should be displayed in the fundamental units of viscosity [Pa·s] and shear rate $[1 / \mathrm{s}]$; however, the original data (raw data) obtained from the rheometer is in units of torque $[\mathrm{Nm}]$ and rotational speed $[1 / \mathrm{min}]$. This section provides the reader with the fundamental data that is directly used to compose the flow curves in this study, which were derived from calibrating the raw data into fundamental data. Table 33 to Table 35 provide the uncertainties for the fundamental data of all three spindles. These tables provide the values in fundamental units based on the Bingham method. 
Table 33: Values for Double spiral spindle with uncertainties provided in fundamental units. - these are based on Bingham parameters

\begin{tabular}{|c|c|c|c|c|c|c|c|c|c|c|c|c|c|}
\hline \multicolumn{14}{|c|}{ SPIRAL } \\
\hline \multicolumn{2}{|c|}{ Shear Rate } & \multicolumn{4}{|c|}{ 0\% Exp. Data - Calibrated } & \multicolumn{4}{|c|}{ 20\% Exp. Data - Calibrated } & \multicolumn{4}{|c|}{ 40\% Exp. Data - Calibrated } \\
\hline $\begin{array}{l}\text { Shear } \\
\text { Rate } \\
{[1 / s]}\end{array}$ & $\begin{array}{c}\text { uncertainity } \\
{[1 / \mathrm{s}]}\end{array}$ & $\begin{array}{c}\text { Shear } \\
\text { Stress } \\
{[\text { Pa] }}\end{array}$ & $\begin{array}{c}\text { Shear } \\
\text { Stress } \\
\text { uncertainity } \\
\text { [Pa] }\end{array}$ & $\begin{array}{c}\text { Viscosity } \\
{[\mathrm{Pa} \cdot \mathrm{s}]}\end{array}$ & $\begin{array}{c}\text { Viscosity } \\
\text { uncertainity } \\
\text { [Pa.s] }\end{array}$ & $\begin{array}{c}\text { Shear } \\
\text { Stress } \\
\text { [Pa] }\end{array}$ & $\begin{array}{c}\text { Shear } \\
\text { Stress } \\
\text { uncertainity } \\
\text { [Pa] }\end{array}$ & $\begin{array}{c}\text { Viscosity } \\
{[\mathrm{Pa} \cdot \mathrm{s}]}\end{array}$ & $\begin{array}{c}\text { Viscosity } \\
\text { uncertainity } \\
\text { [Pa.s] }\end{array}$ & $\begin{array}{c}\text { Shear } \\
\text { Stress } \\
{[\mathbf{P a}]}\end{array}$ & $\begin{array}{c}\text { Shear } \\
\text { Stress } \\
\text { uncertainity } \\
{[\text { Pa] }}\end{array}$ & $\begin{array}{c}\text { Viscosity } \\
{[\mathrm{Pa} \cdot \mathrm{s}]}\end{array}$ & $\begin{array}{c}\text { Viscosity } \\
\text { uncertainity } \\
\text { [Pa.s] }\end{array}$ \\
\hline 38.95 & 2.014 & 315.7 & 9.8 & 8.1 & 0.4 & 575.2 & 30.0 & 14.8 & 1.0 & 1230.0 & 113.0 & 31.7 & 3.2 \\
\hline 27.07 & 1.400 & 230.9 & 7.1 & 8.6 & 0.4 & 416.8 & 21.3 & 15.4 & 1.0 & 878.5 & 79.9 & 32.5 & 3.2 \\
\hline 18.82 & 0.973 & 170.8 & 5.2 & 9.1 & 0.5 & 305.8 & 15.5 & 16.3 & 1.0 & 639.9 & 56.8 & 34.1 & 3.3 \\
\hline 13.09 & 0.677 & 127.8 & 4.0 & 9.8 & 0.5 & 226.3 & 11.4 & 17.3 & 1.1 & 473.2 & 40.3 & 36.2 & 3.4 \\
\hline 9.11 & 0.471 & 97.2 & 3.0 & 10.7 & 0.5 & 169.8 & 8.5 & 18.7 & 1.2 & 359.9 & 24.9 & 39.6 & 3.1 \\
\hline 6.31 & 0.326 & 75.3 & 2.3 & 12.0 & 0.6 & 129.1 & 6.1 & 20.5 & 1.3 & 284.1 & 15.5 & 45.1 & 3.0 \\
\hline 4.4 & 0.228 & 59.6 & 1.8 & 13.6 & 0.7 & 99.9 & 4.6 & 22.8 & 1.4 & 224.2 & 13.8 & 51.1 & 3.7 \\
\hline 3.06 & 0.158 & 48.2 & 1.4 & 15.8 & 0.8 & 78.9 & 3.7 & 25.9 & 1.6 & 174.8 & 12.4 & 57.3 & 4.6 \\
\hline 2.12 & 0.110 & 40.0 & 1.2 & 18.9 & 0.9 & 63.8 & 2.9 & 30.1 & 1.8 & 138.1 & 9.7 & 65.2 & 5.3 \\
\hline 1.48 & 0.076 & 34.0 & 1.0 & 23.1 & 1.1 & 52.7 & 2.3 & 35.8 & 2.1 & 111.0 & 7.6 & 75.3 & 5.9 \\
\hline 1.03 & 0.053 & 29.6 & 0.9 & 28.9 & 1.4 & 44.6 & 1.8 & 43.5 & 2.5 & 91.4 & 6.2 & 89.2 & 6.9 \\
\hline 0.71 & 0.037 & 26.3 & 0.8 & 36.9 & 1.8 & 38.7 & 1.6 & 54.3 & 3.0 & 76.6 & 5.1 & 107.5 & 8.3 \\
\hline 0.49 & 0.026 & 23.9 & 0.7 & 48.4 & 2.4 & 34.2 & 1.3 & 69.3 & 3.8 & 65.4 & 4.1 & 132.4 & 9.8 \\
\hline 0.35 & 0.018 & 22.1 & 0.6 & 63.9 & 3.1 & 30.9 & 1.2 & 89.4 & 4.8 & 57.2 & 3.5 & 165.5 & 12.0 \\
\hline 0.24 & 0.013 & 20.7 & 0.6 & 85.6 & 4.2 & 28.4 & 1.0 & 117.5 & 6.3 & 50.9 & 3.1 & 211.1 & 15.2 \\
\hline 0.17 & 0.009 & 19.7 & 0.6 & 117.7 & 5.7 & 26.4 & 1.0 & 158.0 & 8.4 & 46.1 & 2.7 & 275.7 & 19.4 \\
\hline 0.12 & 0.006 & 18.8 & 0.5 & 163.6 & 7.9 & 24.9 & 0.9 & 216.7 & 11.5 & 42.6 & 2.5 & 370.4 & 26.0 \\
\hline 0.08 & 0.004 & 18.2 & 0.5 & 222.7 & 10.8 & 23.8 & 0.8 & 291.6 & 15.5 & 39.7 & 2.2 & 486.6 & 33.1 \\
\hline 0.06 & 0.003 & 17.7 & 0.5 & 317.6 & 15.4 & 22.9 & 0.8 & 411.9 & 21.7 & 37.7 & 2.1 & 676.5 & 46.2 \\
\hline 0.04 & 0.002 & 17.3 & 0.5 & 465.4 & 22.6 & 22.3 & 0.8 & 600.4 & 32.4 & 36.3 & 2.0 & 977.4 & 65.9 \\
\hline
\end{tabular}


Table 34: Values for Six Blade Vane with uncertainties provided in fundamental units. - these are based on Bingham parameters

\begin{tabular}{|c|c|c|c|c|c|c|c|c|c|c|c|c|c|}
\hline \multicolumn{14}{|c|}{ Vane } \\
\hline \multicolumn{2}{|c|}{ Shear Rate } & \multicolumn{4}{|c|}{ 0\% Exp. Data - Calibrated } & \multicolumn{4}{|c|}{$20 \%$ Exp. Data - Calibrated } & \multicolumn{4}{|c|}{ 40\% Exp. Data - Calibrated } \\
\hline $\begin{array}{l}\text { Shear } \\
\text { Rate } \\
{[1 / \mathrm{s}]}\end{array}$ & $\begin{array}{c}\text { uncertainit } \\
\text { y }[1 / \mathrm{s}]\end{array}$ & $\begin{array}{c}\text { Shear } \\
\text { Stress } \\
{[\text { Pa] }}\end{array}$ & $\begin{array}{c}\text { Shear } \\
\text { Stress } \\
\text { uncertainit } \\
\text { y [Pa] }\end{array}$ & $\begin{array}{c}\text { Viscosity } \\
{[\mathrm{Pa} \cdot \mathrm{s}]}\end{array}$ & $\begin{array}{c}\text { Viscosity } \\
\text { uncertainit } \\
\text { y [Pa.s] }\end{array}$ & $\begin{array}{c}\text { Shear } \\
\text { Stress } \\
{[\mathrm{Pa}]}\end{array}$ & $\begin{array}{c}\text { Shear } \\
\text { Stress } \\
\text { uncertainit } \\
\text { y [Pa] }\end{array}$ & $\begin{array}{c}\text { Viscosity } \\
{[\mathrm{Pa} \cdot \mathrm{s}]}\end{array}$ & $\begin{array}{c}\text { Viscosity } \\
\text { uncertainit } \\
\text { y [Pa.s] }\end{array}$ & $\begin{array}{c}\text { Shear } \\
\text { Stress } \\
{[\mathrm{Pa}]}\end{array}$ & $\begin{array}{c}\text { Shear } \\
\text { Stress } \\
\text { uncertainit } \\
\text { y [Pa] }\end{array}$ & $\begin{array}{c}\text { Viscosity } \\
{[\mathrm{Pa} \cdot \mathrm{s}]}\end{array}$ & $\begin{array}{c}\text { Viscosity } \\
\text { uncertainit } \\
\text { y [Pa.s] }\end{array}$ \\
\hline 23.92 & 1.200 & 206.4 & 5.6 & 8.6 & 0.4 & 358.9 & 12.5 & 15.0 & 0.8 & 697.5 & 40.6 & 29.2 & 2.0 \\
\hline 16.63 & 0.834 & 154.8 & 4.2 & 9.3 & 0.4 & 266.8 & 9.1 & 16.1 & 0.8 & 519.7 & 37.7 & 31.3 & 2.6 \\
\hline 11.56 & 0.579 & 117.8 & 3.1 & 10.2 & 0.5 & 200.7 & 6.7 & 17.4 & 0.9 & 389.0 & 27.0 & 33.7 & 2.6 \\
\hline 8.04 & 0.403 & 91.2 & 2.4 & 11.4 & 0.5 & 152.9 & 5.2 & 19.1 & 1.0 & 296.6 & 21.8 & 37.0 & 3.0 \\
\hline 5.60 & 0.281 & 72.0 & 1.9 & 12.9 & 0.6 & 118.6 & 3.8 & 21.2 & 1.1 & 227.7 & 17.1 & 40.8 & 3.4 \\
\hline 3.88 & 0.194 & 58.0 & 1.5 & 15.0 & 0.7 & 93.5 & 2.9 & 24.2 & 1.2 & 178.1 & 13.0 & 46.0 & 3.7 \\
\hline 2.70 & 0.136 & 47.6 & 1.2 & 17.7 & 0.8 & 74.8 & 2.2 & 27.8 & 1.3 & 140.6 & 10.4 & 52.1 & 4.3 \\
\hline 1.88 & 0.094 & 39.9 & 1.0 & 21.3 & 1.0 & 60.9 & 1.9 & 32.5 & 1.6 & 112.3 & 8.2 & 59.9 & 4.9 \\
\hline 1.31 & 0.065 & 33.9 & 0.9 & 26.0 & 1.2 & 50.6 & 1.5 & 38.9 & 1.9 & 91.2 & 6.6 & 70.0 & 5.8 \\
\hline 0.91 & 0.045 & 29.4 & 0.7 & 32.4 & 1.5 & 42.9 & 1.3 & 47.4 & 2.3 & 75.4 & 5.3 & 83.3 & 6.6 \\
\hline 0.63 & 0.032 & 25.9 & 0.7 & 41.1 & 1.9 & 37.0 & 1.1 & 58.7 & 2.9 & 63.3 & 4.6 & 100.6 & 8.2 \\
\hline 0.44 & 0.022 & 23.2 & 0.6 & 52.9 & 2.4 & 32.5 & 0.9 & 74.3 & 3.5 & 54.3 & 3.6 & 123.9 & 9.4 \\
\hline 0.30 & 0.015 & 21.1 & 0.5 & 69.6 & 3.2 & 29.1 & 0.8 & 95.9 & 4.6 & 47.3 & 3.1 & 156.0 & 11.7 \\
\hline 0.21 & 0.011 & 19.5 & 0.5 & 91.8 & 4.2 & 26.4 & 0.8 & 124.4 & 5.9 & 42.0 & 2.7 & 198.0 & 14.6 \\
\hline 0.15 & 0.007 & 18.1 & 0.5 & 122.3 & 5.6 & 24.5 & 0.7 & 165.2 & 7.8 & 37.9 & 2.3 & 256.0 & 18.5 \\
\hline 0.10 & 0.005 & 17.1 & 0.4 & 166.9 & 7.6 & 22.8 & 0.7 & 222.2 & 10.5 & 34.9 & 2.2 & 339.6 & 24.8 \\
\hline 0.07 & 0.004 & 16.4 & 0.4 & 231.6 & 10.6 & 21.7 & 0.6 & 306.8 & 14.4 & 32.4 & 1.9 & 458.6 & 32.5 \\
\hline 0.05 & 0.003 & 15.7 & 0.4 & 312.9 & 14.5 & 20.5 & 0.6 & 408.7 & 19.2 & 30.4 & 1.7 & 605.3 & 39.9 \\
\hline 0.03 & 0.002 & 15.2 & 0.4 & 444.5 & 20.7 & 19.8 & 0.5 & 579.6 & 26.7 & 29.1 & 1.7 & 850.4 & 58.6 \\
\hline 0.02 & 0.001 & 14.8 & 0.4 & 648.8 & 30.3 & 19.3 & 0.5 & 846.7 & 39.9 & 27.9 & 1.5 & 1224.0 & 80.2 \\
\hline
\end{tabular}


Table 35: Values for serrated coaxial cylinder with uncertainties provided in fundamental units. - these are based on Bingham parameters

\begin{tabular}{|c|c|c|c|c|c|c|c|c|c|c|c|c|c|}
\hline \multicolumn{14}{|c|}{ Coaxial } \\
\hline \multicolumn{2}{|c|}{ Shear Rate } & \multicolumn{4}{|c|}{ 0\% Exp. Data - Calibrated } & \multicolumn{4}{|c|}{$20 \%$ Exp. Data - Calibrated } & \multicolumn{4}{|c|}{ 40\% Exp. Data - Calibrated } \\
\hline $\begin{array}{l}\text { Shear } \\
\text { Rate } \\
{[1 / \mathrm{s}]}\end{array}$ & $\begin{array}{c}\text { uncertainit } \\
\text { y }[1 / s]\end{array}$ & $\begin{array}{c}\text { Shear } \\
\text { Stress } \\
{[\text { Pa] }}\end{array}$ & $\begin{array}{c}\text { Shear } \\
\text { Stress } \\
\text { uncertainit } \\
\text { y [Pa] }\end{array}$ & $\begin{array}{c}\text { Viscosity } \\
{[\mathrm{Pa} \cdot \mathrm{s}]}\end{array}$ & $\begin{array}{c}\text { Viscosity } \\
\text { uncertainit } \\
\text { y [Pa.s] }\end{array}$ & $\begin{array}{c}\text { Shear } \\
\text { Stress } \\
{[\text { Pa] }}\end{array}$ & $\begin{array}{c}\text { Shear } \\
\text { Stress } \\
\text { uncertainit } \\
\text { y [Pa] }\end{array}$ & $\begin{array}{c}\text { Viscosity } \\
{[\mathrm{Pa} \cdot \mathrm{s}]}\end{array}$ & $\begin{array}{c}\text { Viscosity } \\
\text { uncertainit } \\
\text { y [Pa.s] }\end{array}$ & $\begin{array}{c}\text { Shear } \\
\text { Stress } \\
{[\mathrm{Pa}]}\end{array}$ & $\begin{array}{c}\text { Shear } \\
\text { Stress } \\
\text { uncertainit } \\
\text { y [Pa] }\end{array}$ & $\begin{array}{c}\text { Viscosity } \\
{[\mathrm{Pa} \cdot \mathrm{s}]}\end{array}$ & $\begin{array}{c}\text { Viscosity } \\
\text { uncertainit } \\
\text { y [Pa.s] }\end{array}$ \\
\hline 27.81 & 1.650 & 236.0 & 9.5 & 8.5 & 0.5 & 398.2 & 19.4 & 14.4 & 0.9 & 668.6 & 38.0 & 24.1 & 1.7 \\
\hline 19.32 & 1.150 & 176.9 & 7.0 & 9.2 & 0.5 & 296.5 & 14.8 & 15.4 & 1.0 & 524.2 & 34.2 & 27.2 & 2.1 \\
\hline 13.43 & 0.800 & 133.5 & 5.2 & 10.0 & 0.6 & 222.8 & 11.0 & 16.6 & 1.1 & 399.9 & 24.6 & 29.9 & 2.2 \\
\hline 9.34 & 0.560 & 102.2 & 4.0 & 11.0 & 0.6 & 168.9 & 8.4 & 18.1 & 1.2 & 308.6 & 17.1 & 33.1 & 2.3 \\
\hline 6.51 & 0.390 & 79.7 & 3.1 & 12.3 & 0.7 & 130.1 & 6.5 & 20.1 & 1.3 & 236.1 & 12.2 & 36.4 & 2.4 \\
\hline 4.50 & 0.270 & 63.4 & 2.4 & 14.1 & 0.8 & 102.0 & 4.9 & 22.7 & 1.4 & 182.6 & 9.1 & 40.7 & 2.6 \\
\hline 3.14 & 0.190 & 51.3 & 2.0 & 16.4 & 0.9 & 81.5 & 3.8 & 26.0 & 1.6 & 142.4 & 6.9 & 45.5 & 2.9 \\
\hline 2.18 & 0.130 & 42.3 & 1.6 & 19.4 & 1.1 & 66.3 & 3.2 & 30.5 & 1.9 & 112.8 & 5.6 & 51.8 & 3.3 \\
\hline 1.52 & 0.090 & 35.3 & 1.4 & 23.3 & 1.3 & 54.9 & 2.5 & 36.3 & 2.2 & 90.9 & 4.2 & 60.1 & 3.7 \\
\hline 1.05 & 0.060 & 30.0 & 1.2 & 28.5 & 1.6 & 46.3 & 2.2 & 44.1 & 2.7 & 74.5 & 3.5 & 70.9 & 4.3 \\
\hline 0.73 & 0.040 & 25.8 & 1.0 & 35.3 & 2.0 & 39.7 & 1.8 & 54.3 & 3.3 & 62.1 & 2.8 & 84.9 & 5.1 \\
\hline 0.51 & 0.030 & 22.6 & 0.9 & 44.4 & 2.5 & 34.6 & 1.6 & 68.0 & 4.2 & 52.6 & 2.3 & 103.4 & 6.1 \\
\hline 0.35 & 0.020 & 19.9 & 0.8 & 56.6 & 3.3 & 30.6 & 1.4 & 87.0 & 5.4 & 45.5 & 1.9 & 129.2 & 7.4 \\
\hline 0.25 & 0.010 & 18.0 & 0.8 & 73.0 & 4.2 & 27.9 & 1.3 & 113.2 & 6.9 & 39.8 & 1.6 & 161.7 & 9.2 \\
\hline 0.17 & 0.010 & 16.5 & 0.8 & 95.7 & 5.8 & 25.5 & 1.2 & 148.3 & 9.1 & 35.6 & 1.5 & 206.8 & 11.8 \\
\hline 0.12 & 0.010 & 15.4 & 0.8 & 129.0 & 8.4 & 23.9 & 1.1 & 200.4 & 12.3 & 32.0 & 1.3 & 268.9 & 15.2 \\
\hline 0.08 & 0.000 & 14.0 & 0.7 & 170.8 & 10.8 & 22.9 & 1.0 & 278.9 & 16.2 & 29.3 & 1.2 & 357.1 & 20.1 \\
\hline 0.06 & 0.000 & 13.9 & 0.9 & 239.2 & 18.6 & 22.4 & 1.7 & 384.0 & 33.8 & 27.2 & 1.1 & 466.2 & 26.0 \\
\hline 0.04 & 0.000 & 13.5 & 1.0 & 338.6 & 28.9 & 21.1 & 1.0 & 531.0 & 33.8 & 25.8 & 1.4 & 648.3 & 42.9 \\
\hline 0.03 & 0.000 & 12.8 & 1.0 & 481.9 & 40.8 & 20.1 & 1.0 & 760.9 & 49.6 & 24.2 & 1.2 & 913.2 & 59.1 \\
\hline
\end{tabular}




\subsubsection{Using the Viscosity Fit Method}

This section provides the reader with the fundamental data that is directly used to compose the flow curves in this study, which were derived from calibrating the raw data into fundamental data. Table 36 to Table 38 provide the uncertainties for the fundamental data of all three spindles and the model. These tables provide the values in fundamental units based on the viscosity fit method. 
Table 36: Values for double spiral spindle with uncertainties provided in fundamental units, based on viscosity fit method

\begin{tabular}{|c|c|c|c|c|c|c|c|c|c|c|c|c|c|}
\hline \multicolumn{14}{|c|}{ SPIRAL } \\
\hline \multicolumn{2}{|c|}{ Shear Rate } & \multicolumn{4}{|c|}{ 0\% Exp. Data - Calibrated } & \multicolumn{4}{|c|}{$20 \%$ Exp. Data - Calibrated } & \multicolumn{4}{|c|}{ 40\% Exp. Data - Calibrated } \\
\hline $\begin{array}{l}\text { Shear } \\
\text { Rate } \\
{[1 / s]}\end{array}$ & $\begin{array}{c}\text { uncertainity } \\
{[1 / \mathrm{s}]}\end{array}$ & $\begin{array}{c}\text { Shear } \\
\text { Stress } \\
{[\text { Pa] }}\end{array}$ & $\begin{array}{c}\text { Shear } \\
\text { Stress } \\
\text { uncertainity } \\
{[\mathrm{Pa}]} \\
\end{array}$ & $\begin{array}{c}\text { Viscosity } \\
\text { [Pa's] }\end{array}$ & $\begin{array}{c}\text { Viscosity } \\
\text { uncertainity } \\
\text { [Pa.s] }\end{array}$ & $\begin{array}{c}\text { Shear } \\
\text { Stress } \\
{[\text { Pa] }}\end{array}$ & $\begin{array}{c}\text { Shear } \\
\text { Stress } \\
\text { uncertainity } \\
{[\text { Pa] }} \\
\end{array}$ & $\begin{array}{c}\text { Viscosity } \\
\text { [Pa·s] }\end{array}$ & $\begin{array}{c}\text { Viscosity } \\
\text { uncertainity } \\
\text { [Pa.s] }\end{array}$ & $\begin{array}{c}\text { Shear } \\
\text { Stress } \\
{[\text { Pa] }}\end{array}$ & $\begin{array}{c}\text { Shear } \\
\text { Stress } \\
\text { uncertainity } \\
{[\mathrm{Pa}]}\end{array}$ & $\begin{array}{c}\text { Viscosity } \\
\text { [Pa-s] }\end{array}$ & $\begin{array}{c}\text { Viscosity } \\
\text { uncertainity } \\
\text { [Pa.s] }\end{array}$ \\
\hline 32.92 & 2.34 & 265.4 & 29.6 & 8.1 & 0.7 & 484.6 & 58.6 & 14.7 & 1.4 & 1041.0 & 147.4 & 31.6 & 3.9 \\
\hline 22.88 & 1.63 & 194.0 & 21.6 & 8.5 & 0.7 & 351.7 & 42.6 & 15.4 & 1.5 & 742.4 & 104.6 & 32.4 & 3.9 \\
\hline 15.90 & 1.13 & 143.6 & 16.0 & 9.0 & 0.8 & 257.6 & 30.9 & 16.2 & 1.5 & 540.1 & 74.8 & 33.9 & 4.0 \\
\hline 11.06 & 0.79 & 107.4 & 12.0 & 9.7 & 0.8 & 190.7 & 22.8 & 17.2 & 1.6 & 400.7 & 54.7 & 36.2 & 4.2 \\
\hline 7.70 & 0.55 & 81.7 & 9.1 & 10.6 & 0.9 & 143.1 & 17.2 & 18.6 & 1.8 & 304.6 & 39.1 & 39.5 & 4.2 \\
\hline 5.33 & 0.38 & 63.3 & 7.1 & 11.9 & 1.0 & 108.8 & 12.9 & 20.4 & 1.9 & 240.1 & 29.1 & 45.0 & 4.4 \\
\hline 3.72 & 0.26 & 50.1 & 5.6 & 13.5 & 1.2 & 84.3 & 10.0 & 22.7 & 2.1 & 189.4 & 23.5 & 50.9 & 5.2 \\
\hline 2.58 & 0.18 & 40.5 & 4.5 & 15.7 & 1.4 & 66.5 & 7.9 & 25.7 & 2.4 & 147.7 & 19.1 & 57.1 & 6.1 \\
\hline 1.80 & 0.13 & 33.6 & 3.7 & 18.7 & 1.6 & 53.7 & 6.4 & 29.9 & 2.8 & 116.8 & 15.0 & 65.0 & 6.9 \\
\hline 1.25 & 0.09 & 28.6 & 3.2 & 22.9 & 2.0 & 44.5 & 5.2 & 35.6 & 3.3 & 93.8 & 12.1 & 75.1 & 8.0 \\
\hline 0.87 & 0.06 & 24.9 & 2.8 & 28.7 & 2.5 & 37.6 & 4.4 & 43.3 & 4.0 & 77.1 & 9.8 & 88.8 & 9.3 \\
\hline 0.60 & 0.04 & 22.1 & 2.5 & 36.7 & 3.1 & 32.6 & 3.8 & 54.0 & 4.9 & 64.7 & 8.3 & 107.0 & 11.3 \\
\hline 0.42 & 0.03 & 20.1 & 2.2 & 48.0 & 4.1 & 28.8 & 3.3 & 68.9 & 6.2 & 55.3 & 7.0 & 132.0 & 13.6 \\
\hline 0.29 & 0.02 & 18.6 & 2.1 & 63.5 & 5.5 & 26.0 & 3.0 & 88.9 & 8.0 & 48.3 & 6.0 & 164.9 & 16.5 \\
\hline 0.20 & 0.02 & 17.4 & 1.9 & 85.0 & 7.3 & 23.9 & 2.7 & 116.8 & 10.4 & 43.1 & 5.3 & 210.6 & 21.2 \\
\hline 0.14 & 0.01 & 16.5 & 1.8 & 116.8 & 10.0 & 22.2 & 2.5 & 157.1 & 13.9 & 39.0 & 4.8 & 275.5 & 27.4 \\
\hline 0.10 & 0.01 & 15.8 & 1.8 & 162.5 & 13.9 & 21.0 & 2.4 & 215.5 & 19.2 & 36.0 & 4.4 & 369.1 & 36.8 \\
\hline 0.07 & 0.01 & 15.3 & 1.7 & 221.1 & 18.9 & 20.1 & 2.3 & 290.0 & 25.6 & 33.6 & 4.1 & 485.1 & 47.7 \\
\hline 0.05 & 0.00 & 14.9 & 1.7 & 315.3 & 27.0 & 19.3 & 2.2 & 409.5 & 36.2 & 31.8 & 3.9 & 673.8 & 66.5 \\
\hline 0.03 & 0.00 & 14.5 & 1.6 & 462.1 & 39.6 & 18.8 & 2.2 & 597.3 & 53.4 & 30.6 & 3.7 & 973.1 & 95.6 \\
\hline
\end{tabular}


Table 37: Values for six blade vane with uncertainties provided in fundamental units, based on viscosity fit method

\begin{tabular}{|c|c|c|c|c|c|c|c|c|c|c|c|c|c|}
\hline \multicolumn{14}{|c|}{ Vane } \\
\hline \multicolumn{2}{|c|}{ Shear Rate } & \multicolumn{4}{|c|}{ 0\% Exp. Data - Calibrated } & \multicolumn{4}{|c|}{$20 \%$ Exp. Data - Calibrated } & \multicolumn{4}{|c|}{ 40\% Exp. Data - Calibrated } \\
\hline $\begin{array}{l}\text { Shear } \\
\text { Rate } \\
{[1 / s]}\end{array}$ & $\begin{array}{c}\text { uncertainity } \\
{[1 / \mathrm{s}]}\end{array}$ & $\begin{array}{c}\text { Shear } \\
\text { Stress } \\
\text { [Pa] }\end{array}$ & $\begin{array}{c}\text { Shear } \\
\text { Stress } \\
\text { uncertainity } \\
\text { [Pa] }\end{array}$ & $\begin{array}{c}\text { Viscosity } \\
\text { [Pa-s] }\end{array}$ & $\begin{array}{c}\text { Viscosity } \\
\text { uncertainity } \\
\text { [Pa.s] }\end{array}$ & $\begin{array}{c}\text { Shear } \\
\text { Stress } \\
\text { [Pa] }\end{array}$ & $\begin{array}{c}\text { Shear } \\
\text { Stress } \\
\text { uncertainity } \\
{[\text { [Pa] }} \\
\end{array}$ & $\begin{array}{c}\text { Viscosity } \\
\text { [Pa·s] }\end{array}$ & $\begin{array}{c}\text { Viscosity } \\
\text { uncertainity } \\
\text { [Pa.s] }\end{array}$ & $\begin{array}{c}\text { Shear } \\
\text { Stress } \\
\text { [Pa] }\end{array}$ & $\begin{array}{c}\text { Shear Stress } \\
\text { uncertainity } \\
\text { [Pa] }\end{array}$ & $\begin{array}{c}\text { Viscosity } \\
\text { [Pa·s] }\end{array}$ & $\begin{array}{c}\text { Viscosity } \\
\text { uncertainity } \\
\text { [Pa.s] }\end{array}$ \\
\hline 23.84 & 4.40 & 188.3 & 36.0 & 7.9 & 0.4 & 328.6 & 63.3 & 13.8 & 0.8 & 640.5 & 127.4 & 26.8 & 1.9 \\
\hline 16.57 & 3.06 & 141.2 & 27.0 & 8.5 & 0.4 & 244.4 & 47.2 & 14.7 & 0.8 & 477.1 & 97.5 & 28.7 & 2.4 \\
\hline 11.52 & 2.12 & 107.5 & 20.6 & 9.3 & 0.5 & 183.7 & 35.3 & 15.9 & 0.9 & 356.7 & 72.0 & 30.9 & 2.5 \\
\hline 8.01 & 1.48 & 83.2 & 15.9 & 10.4 & 0.5 & 140.0 & 26.9 & 17.4 & 1.0 & 272.6 & 55.3 & 33.9 & 2.9 \\
\hline 5.58 & 1.03 & 65.7 & 12.6 & 11.8 & 0.6 & 108.6 & 20.9 & 19.4 & 1.1 & 209.3 & 42.8 & 37.4 & 3.3 \\
\hline 3.86 & 0.71 & 52.9 & 10.1 & 13.7 & 0.7 & 85.7 & 16.4 & 22.1 & 1.2 & 163.5 & 33.4 & 42.2 & 3.6 \\
\hline 2.69 & 0.50 & 43.5 & 8.3 & 16.1 & 0.8 & 68.6 & 13.1 & 25.4 & 1.3 & 129.0 & 26.3 & 47.7 & 4.1 \\
\hline 1.87 & 0.35 & 36.4 & 7.0 & 19.4 & 1.0 & 55.8 & 10.7 & 29.8 & 1.6 & 103.0 & 21.0 & 54.9 & 4.6 \\
\hline 1.30 & 0.24 & 30.9 & 5.9 & 23.8 & 1.2 & 46.3 & 8.9 & 35.6 & 1.9 & 83.8 & 17.0 & 64.2 & 5.4 \\
\hline 0.90 & 0.17 & 26.8 & 5.1 & 29.6 & 1.5 & 39.3 & 7.5 & 43.4 & 2.3 & 69.2 & 14.1 & 76.3 & 6.3 \\
\hline 0.63 & 0.12 & 23.6 & 4.5 & 37.6 & 1.9 & 33.9 & 6.5 & 53.7 & 2.8 & 58.0 & 11.8 & 92.0 & 7.8 \\
\hline 0.44 & 0.08 & 21.1 & 4.0 & 48.3 & 2.4 & 29.8 & 5.7 & 68.0 & 3.5 & 49.7 & 10.1 & 113.4 & 9.1 \\
\hline 0.30 & 0.06 & 19.3 & 3.7 & 63.6 & 3.2 & 26.6 & 5.1 & 87.8 & 4.6 & 43.4 & 8.8 & 143.0 & 11.4 \\
\hline 0.21 & 0.04 & 17.8 & 3.4 & 83.9 & 4.2 & 24.2 & 4.6 & 113.8 & 6.0 & 38.5 & 7.7 & 181.4 & 13.8 \\
\hline 0.15 & 0.03 & 16.5 & 3.2 & 111.8 & 5.6 & 22.4 & 4.3 & 151.1 & 7.8 & 34.9 & 7.0 & 234.8 & 17.7 \\
\hline 0.10 & 0.02 & 15.6 & 3.0 & 152.6 & 7.7 & 20.9 & 4.0 & 203.3 & 10.5 & 32.1 & 6.4 & 312.0 & 23.8 \\
\hline 0.07 & 0.01 & 14.9 & 2.9 & 211.7 & 10.6 & 19.9 & 3.8 & 280.7 & 14.5 & 29.7 & 5.9 & 420.1 & 31.0 \\
\hline 0.05 & 0.01 & 14.3 & 2.7 & 285.9 & 14.5 & 18.8 & 3.6 & 374.0 & 19.1 & 27.9 & 5.5 & 554.8 & 38.7 \\
\hline 0.03 & 0.01 & 13.9 & 2.7 & 406.1 & 20.6 & 18.2 & 3.5 & 530.2 & 26.8 & 26.7 & 5.3 & 778.8 & 56.6 \\
\hline 0.02 & 0.00 & 13.5 & 2.6 & 592.9 & 30.2 & 17.7 & 3.4 & 774.8 & 40.1 & 25.6 & 5.1 & 1121.0 & 78.0 \\
\hline
\end{tabular}


Table 38: Values for cylinder coaxial with uncertainties provided in fundamental units, based on viscosity fit method

\begin{tabular}{|c|c|c|c|c|c|c|c|c|c|c|c|c|c|}
\hline \multicolumn{14}{|c|}{ Coaxial } \\
\hline \multicolumn{2}{|c|}{ Shear Rate } & \multicolumn{4}{|c|}{ 0\% Exp. Data - Calibrated } & \multicolumn{4}{|c|}{$20 \%$ Exp. Data - Calibrated } & \multicolumn{4}{|c|}{ 40\% Exp. Data - Calibrated } \\
\hline $\begin{array}{l}\text { Shear } \\
\text { Rate } \\
{[1 / s]}\end{array}$ & $\begin{array}{c}\text { uncertainity } \\
{[1 / \mathrm{s}]}\end{array}$ & $\begin{array}{c}\text { Shear } \\
\text { Stress } \\
\text { [Pa] }\end{array}$ & $\begin{array}{c}\text { Shear } \\
\text { Stress } \\
\text { uncertainity } \\
{[\text { [Pa] }}\end{array}$ & $\begin{array}{c}\text { Viscosity } \\
\text { [Pa·s] }\end{array}$ & $\begin{array}{c}\text { Viscosity } \\
\text { uncertainity } \\
\text { [Pa.s] }\end{array}$ & $\begin{array}{c}\text { Shear } \\
\text { Stress } \\
{[\text { Pa] }}\end{array}$ & $\begin{array}{c}\text { Shear } \\
\text { Stress } \\
\text { uncertainity } \\
{[\text { [Pa] }} \\
\end{array}$ & $\begin{array}{c}\text { Viscosity } \\
{[\text { Pa's] }}\end{array}$ & $\begin{array}{c}\text { Viscosity } \\
\text { uncertainity } \\
\text { [Pa.s] }\end{array}$ & $\begin{array}{c}\text { Shear } \\
\text { Stress } \\
\text { [Pa] }\end{array}$ & $\begin{array}{c}\text { Shear Stress } \\
\text { uncertainity } \\
\text { [Pa] }\end{array}$ & $\begin{array}{c}\text { Viscosity } \\
\text { [Pa-s] }\end{array}$ & $\begin{array}{c}\text { Viscosity } \\
\text { uncertainity } \\
\text { [Pa.s] }\end{array}$ \\
\hline 33.89 & 5.86 & 248.4 & 50.1 & 7.3 & 0.8 & 420.8 & 86.0 & 12.4 & 1.3 & 703.9 & 144.4 & 20.7 & 2.3 \\
\hline 23.55 & 4.07 & 186.2 & 37.6 & 7.9 & 0.8 & 313.4 & 64.0 & 13.3 & 1.4 & 552.1 & 115.0 & 23.4 & 2.7 \\
\hline 16.37 & 2.83 & 140.5 & 28.3 & 8.6 & 0.9 & 235.6 & 48.0 & 14.4 & 1.5 & 421.1 & 87.3 & 25.7 & 2.9 \\
\hline 11.39 & 1.97 & 107.6 & 21.7 & 9.4 & 1.0 & 178.5 & 36.4 & 15.6 & 1.6 & 325.3 & 66.5 & 28.5 & 3.1 \\
\hline 7.93 & 1.37 & 83.9 & 17.0 & 10.6 & 1.1 & 137.6 & 28.0 & 17.3 & 1.8 & 249.0 & 51.0 & 31.4 & 3.4 \\
\hline 5.49 & 0.95 & 66.7 & 13.5 & 12.1 & 1.2 & 107.8 & 21.9 & 19.6 & 2.1 & 192.1 & 39.2 & 34.9 & 3.7 \\
\hline 3.83 & 0.66 & 54.1 & 10.9 & 14.1 & 1.4 & 86.1 & 17.5 & 22.4 & 2.3 & 150.0 & 30.5 & 39.1 & 4.1 \\
\hline 2.66 & 0.46 & 44.5 & 9.0 & 16.7 & 1.7 & 70.1 & 14.2 & 26.3 & 2.7 & 118.9 & 24.3 & 44.6 & 4.7 \\
\hline 1.85 & 0.32 & 37.2 & 7.5 & 20.1 & 2.0 & 58.0 & 11.8 & 31.3 & 3.2 & 95.8 & 19.5 & 51.7 & 5.4 \\
\hline 1.29 & 0.22 & 31.6 & 6.4 & 24.5 & 2.5 & 49.0 & 10.0 & 38.0 & 4.0 & 78.6 & 16.0 & 61.1 & 6.4 \\
\hline 0.89 & 0.15 & 27.1 & 5.5 & 30.3 & 3.1 & 42.0 & 8.5 & 46.9 & 4.8 & 65.3 & 13.2 & 73.0 & 7.5 \\
\hline 0.62 & 0.11 & 23.8 & 4.8 & 38.2 & 3.9 & 36.5 & 7.4 & 58.6 & 6.1 & 55.3 & 11.2 & 88.9 & 9.2 \\
\hline 0.43 & 0.07 & 21.0 & 4.3 & 48.7 & 5.0 & 32.4 & 6.6 & 75.0 & 7.8 & 47.9 & 9.7 & 111.2 & 11.4 \\
\hline 0.30 & 0.05 & 18.9 & 3.8 & 62.7 & 6.4 & 29.5 & 6.0 & 97.6 & 10.2 & 41.9 & 8.5 & 139.1 & 14.1 \\
\hline 0.21 & 0.04 & 17.3 & 3.5 & 82.3 & 8.6 & 27.0 & 5.5 & 127.9 & 13.3 & 37.5 & 7.6 & 178.0 & 18.1 \\
\hline 0.15 & 0.03 & 16.2 & 3.3 & 110.9 & 11.8 & 25.2 & 5.1 & 172.7 & 17.9 & 33.8 & 6.8 & 231.6 & 23.6 \\
\hline 0.10 & 0.02 & 14.8 & 3.0 & 146.8 & 15.5 & 24.2 & 4.9 & 240.5 & 24.5 & 30.9 & 6.2 & 307.4 & 31.2 \\
\hline 0.07 & 0.01 & 14.7 & 3.1 & 205.6 & 23.7 & 23.6 & 5.0 & 331.1 & 39.8 & 28.6 & 5.8 & 401.3 & 40.7 \\
\hline 0.05 & 0.01 & 14.1 & 3.0 & 290.4 & 34.9 & 22.3 & 4.5 & 457.8 & 48.2 & 27.1 & 5.6 & 557.6 & 59.8 \\
\hline 0.03 & 0.01 & 13.4 & 2.9 & 413.8 & 49.7 & 21.3 & 4.4 & 655.8 & 69.1 & 25.5 & 5.2 & 785.0 & 83.7 \\
\hline
\end{tabular}




\section{Summary}

A reference material that simulates mortar was developed that consists of a noncolloidal suspension comprised of corn syrup and fine limestone, and $1 \mathrm{~mm}$ diameter nominally spherical beads. The user needs to prepare the mixture according to the proportions defined in the certificate by following ASTM C1738 and the mixing procedure outlined in Section 4.2 of this report.

The uniqueness of this reference material is that it, like mortar, manifests Bingham rheological behavior. This reference material is the second of a series of three reference materials: 1) SRM 2492 paste with no beads; 2) SRM 2493, which is SRM 2492 paste with the addition of $1 \mathrm{~mm}$ beads, and 3) SRM 2497, which is SRM 2493 plus $10 \mathrm{~mm}$ beads (under preparation). Two different bead volume fractions, $20 \%$ and $40 \%$, were investigated in work. A shape analysis was carried out to verify the sphercity of the glass beads. The addition of glass beads enables SRM 2493 to be used as a mortar reference material and the addition of $10 \mathrm{~mm}$ glass beads enables SRM 2497 to be used a concrete reference material and it is envisioned that the addition of $10 \mathrm{~mm}$ glass beads will enable SRM 2497 to be used a concrete reference material.

The certified values for the mortar reference material, SRM2493, were determined using an extensive experimental design, based on the paste rheological properties measured using a parallel plate calibrated rheometer, parallel computational modeling, and statistical analysis. The uniqueness of this SRM is that it based on the paste rheological properties measured using a parallel plate calibrated rheometer and a computational model. The model was necessary to obtain the fundamental values for the $20 \%$ and $40 \%$ bead volume fractions.

The user is provided with two methods to use SRM 2493 for calibration of rheometers for mortar:

- one method is based on experimental parameters determined from fitting the Bingham equation to the rheological data; (Table 39)

- second method is based on the computational model, by fitting the rheological data to an equation provided in this report (Equation (1) in Section 4).

In both cases, the operator will obtain calibration factors that subsequently can be used for transforming rheometer dependent untis (N.m and rad/s or rpm) into fundamental units (Pa and $1 / \mathrm{s}$ ) for the shear stress and the shear rate.

The last step for this series of SRMs is the concrete rheology reference materials, which will be based on the same procedure as the mortar, but with the addition of $10 \mathrm{~mm}$ beads. This work is in progress. 
Table 39: Certified values based on the Bingham parameters. A) $0 \%$ paste, B) $20 \%$ mortar and C) $\mathbf{4 0} \%$ mortar

\begin{tabular}{|c|l|c|c|c|c|}
\hline & Parameter & $\begin{array}{c}\text { Type of } \\
\text { geometry }\end{array}$ & $\begin{array}{c}\text { Certified } \\
\text { Value } \\
(\mathrm{Pa})\end{array}$ & $\begin{array}{c}\text { Standard } \\
\text { Uncertainty } \\
(\mathrm{Pa})\end{array}$ & $\begin{array}{c}\text { Expanded } \\
\text { Uncertainty } \\
(\mathrm{Pa})\end{array}$ \\
\cline { 2 - 6 } $\mathrm{A})$ & $\begin{array}{l}\text { Yield } \\
\text { Stress }\end{array}$ & $\begin{array}{c}\text { Parallel } \\
\text { plate }\end{array}$ & 24.7 & 0.5 & 1.0 \\
\cline { 2 - 6 } $\begin{array}{c}\text { beads } \\
\text { (paste) }\end{array}$ & Model & 23.1 & 1.8 & 3.6 \\
\cline { 2 - 6 } & Plastic & $\begin{array}{c}\text { Type of } \\
\text { geometry }\end{array}$ & $\begin{array}{c}\text { Certified } \\
\text { Value } \\
(\mathrm{Pa} \cdot \mathrm{s})\end{array}$ & $\begin{array}{c}\text { Standard } \\
\text { Uncertainty } \\
(\mathrm{Pa} \cdot \mathrm{s})\end{array}$ & $\begin{array}{c}\text { Expanded } \\
\text { Uncertainty } \\
(\mathrm{Pa} \cdot \mathrm{s})\end{array}$ \\
\cline { 2 - 6 } & Viscosity & $\begin{array}{c}\text { Parallel } \\
\text { plate }\end{array}$ & 7.9 & 0.2 & 0.5 \\
\cline { 2 - 6 } & Model & 8.1 & 0.8 & 1.6 \\
\hline
\end{tabular}

\begin{tabular}{|c|c|c|c|c|}
\hline \multirow{4}{*}{$\begin{array}{l}20 \% \text { Beads } \\
\text { by volume } \\
\text { concentration }\end{array}$} & Parameter & $\begin{array}{l}\text { Certified } \\
\text { Value } \\
(\mathrm{Pa})\end{array}$ & $\begin{array}{c}\text { Standard } \\
\text { Uncertainty } \\
(\mathrm{Pa})\end{array}$ & $\begin{array}{c}\text { Expanded } \\
\text { Uncertainty } \\
(\mathrm{Pa})\end{array}$ \\
\hline & $\begin{array}{l}\text { Yield } \\
\text { Stress }\end{array}$ & 31.0 & 3.6 & 5.2 \\
\hline & $\begin{array}{l}\text { Plastic } \\
\text { Viscosity }\end{array}$ & $\begin{array}{c}\text { Certified } \\
\text { Value } \\
(\mathrm{Pa} \cdot \mathrm{s})\end{array}$ & $\begin{array}{c}\text { Standard } \\
\text { Uncertainty } \\
(\mathrm{Pa} \cdot \mathrm{s})\end{array}$ & $\begin{array}{c}\text { Expanded } \\
\text { Uncertainty } \\
(\mathrm{Pa} \cdot \mathrm{s})\end{array}$ \\
\hline & & 14.9 & 2.3 & 3.0 \\
\hline
\end{tabular}

\begin{tabular}{|c|c|c|c|c|}
\hline \multirow{4}{*}{$\begin{array}{l}\text { 40\% Beads } \\
\text { by volume } \\
\text { concentration }\end{array}$} & Parameter & $\begin{array}{l}\text { Certified } \\
\text { Value } \\
(\mathrm{Pa})\end{array}$ & $\begin{array}{c}\text { Standard } \\
\text { Uncertainty } \\
(\mathrm{Pa})\end{array}$ & $\begin{array}{c}\text { Expanded } \\
\text { Uncertainty } \\
(\mathrm{Pa})\end{array}$ \\
\hline & $\begin{array}{l}\text { Yield } \\
\text { Stress }\end{array}$ & 38.8 & 5.2 & 6.2 \\
\hline & $\begin{array}{l}\text { Plastic } \\
\text { Viscosity }\end{array}$ & $\begin{array}{c}\text { Certified } \\
\text { Value } \\
(\mathrm{Pa} \cdot \mathrm{s})\end{array}$ & $\begin{array}{c}\text { Standard } \\
\text { Uncertainty } \\
(\mathrm{Pa} \cdot \mathrm{s})\end{array}$ & $\begin{array}{c}\text { Expanded } \\
\text { Uncertainty } \\
(\mathrm{Pa} \cdot \mathrm{s})\end{array}$ \\
\hline & & 57.9 & 8.7 & 11.8 \\
\hline
\end{tabular}




\section{References}

[1] C. F. Ferraris , P. Stutzman, J. Winpigler and W. Guthrie, "Certification of SRM 2492: Bingham Paste Mixture for Rheological Measurements," SP-260-174 Rev. 2012, June 2012.

[2] A. Olivas, C. F. Ferraris, W. F. Guthrie and B. Toman , "Re-certification of SRM 2492: Bingham Paste Mixture for Rheological Measurements," National Institute of Standards and Technology. (NIST Special Publication 260-182). U.S. Department of Commerce, Gaithersburg, MD, August 2015.

[3] V. A. Hackley and C. F. Ferraris, "The Use of Nomenclature in Dispersion Science and Technology," NIST Recommended Practice Guide, SP 960-3, 2001.

[4] C. F. Ferraris and L. E. Brower, "Comparison of concrete rheometers: International tests at LCPC (Nantes, France) in October 2000 (NISTIR 6819)," National Institute of Standards and Technology, Gaithersburg, MD.

[5] C. F. Ferraris and L. E. Brower, "Comparison of concrete rheometers: International tests at MB (Cleveland OH, USA) in May 2003 (NISTIR 7154)," National Institute of Standards and Technology , Gaithersburg, MD.

[6] A. C188-09, "Standard Test Method for Density of Hydraulic Cement," ASTM vol 04.01, 2009.

[7] E. J. Garboczi, "Three-dimensional mathematical analysis of particle shape using xray tomography and spehical harmonics: applications to aggregates used in concrete.," Cement Concrete Research 32, 2002.

[8] M. A. Taylor, E. J. Garboczi, S. T. Erdogan and D. W. Fowler, "Some properties of irregular particles in 3-D," Powder Technology 162, pp. 1-15, 2006.

[9] A. Olivas, C. F. Ferraris, B. Lang, J. Richter, R.P. Ferron. "Cement Paste Reference Material (SRM 2492) Shelf-Life Extension"," NIST - TN 1934, 2016.

[10] L. Maxime, N.S. Martys, W. L. George, D. Lootens and P. Hebraud, "Scaling laws for the flow of generalized Newtonian suspensions," Journal of Rheology , vol. 58, 2014.

[11] H. Zhu, N.S. Martys, C.F. Ferraris, D. De Kee, "A numerical study for the flow of Bingham-like Fluids in two Dimensional Vane and Coaxial cylinders using Smoothed Particle Hydrodynamic (SPH) based Method"," J. of Non-Newtonian Fluid Mechanics, vol. 165, pp. 363-375, 2010.

[12] M. Allen and D. Tildesley, Computer simulations of liquids, Clarendon, Oxford, 1987.

[13] N. S. Martys, W. L. George, B.-W. Chun and D. Lootens, "A smoothed particle hydrodynamics-based fluid model with a spatially dependent viscosity: application to flow of a suspension with a non-Newtonian fluid matrix.," Rheologica acta, vol. 49, no. 10, pp. 1059-1069, 2010.

[14] A. Gelman, J. Carlin , H. Stern , D. Dunson, A. Vehatri and D. Rubin, Bayesian Data Analysis, Boca Raton: Chapman \& Hall 3rd Edition, 2013.

[15] D. J. Lunn, D. Spiegelhalter, A. Thomas and N. Best, "The BUGS project: evolution, critique and future directions (with discussion)," Statistics in Medicine , pp. 30493082, 2009.

[16] R. C. Team, "A language and Environment for Statistical Computing," R Foundation for statistica Computing,, Vienna, Austria, http://www.R-project.org/, 2015. 
[17] A. Olivas, M.A. Helsel, N.S. Martys, C.F. Ferraris, R.P. Ferron, "Rheological Measurements of Suspensions Without Slippage: experimental and Model," NIST-TN 1946, 2016. 


\section{Appendices}

\section{Appendix A: Original Data Measured from Rheometer}

This appendix provides all the original data obtained from each test that was recorded from the rheometer. Also included are graphs needed for interpretation of the results, which portray the repeatability of the measured data.

\section{Appendix B: Data for Calibration}

This appendix provides the averaged data for each mixture which was calculated from the original data shown in Appendix A. The average data was calculated for each geometry analyzed and mortar composition (bead \%). This set of data was then used in the calibration process described in Section 4.3. 


\section{Appendix A: Original Data Measured from Rheometer}

This appendix provides all the original data obtained from each test that was recorded directly from the rheometer. Also included are graphs needed for interpretation of the results, which portray the repeatability of the measured data.

\section{Notes:}

Mix\#1 was discarded due to technical issues encountered during testing, thus the data tables in this appendix begin with Mix\#2. The data extends from Mix\#2 and continues up to Mix\#11, thus a total of 10 mixes were used (See Section 4.2). Each mix was tested with three spindles used at two compositions (either $20 \%$ or $40 \%$ beads by volume). Therefore, six tables and corresponding figures are attached in this appendix per Mix\#.

Each table will contain NIST codes showing the spindle and cup used, which are intended for NIST reference only.

\section{Unit Notations:}

$N$ : Rotational Speed $[1 / \mathrm{min}]$

$\Gamma$ : Measured Torque $[\mu N \cdot m]$

The columns showing the average values contain the following unit conversions:

$N:$ Rotational Speed $\rightarrow[1 / \mathrm{min}] * 2 \pi / 60=[\mathrm{rad} / \mathrm{s}]$

$\Gamma:$ Measured Torque $\rightarrow[\mu N \cdot m] * 10^{-6}=[N \cdot m]$ 
Table A - 1: Measured data for Mix\#2 with 0 \% beads using Double Spiral spindle.

\begin{tabular}{|c|c|c|c|c|c|c|c|c|}
\hline \multicolumn{6}{|c|}{ Double Spiral } & \multicolumn{3}{|c|}{ Bead \% } \\
\hline Geom.: & $R H N-83 A$ & & & $\mathrm{Cu}$ & Z43S & & $0 \%$ & \\
\hline \multicolumn{2}{|c|}{ Run \#1 } & \multicolumn{2}{|c|}{ Run \#2 } & \multicolumn{2}{|c|}{ Run \#3 } & \multirow{2}{*}{\multicolumn{3}{|c|}{$\begin{array}{c}\text { Average } \\
\text { values }\end{array}$}} \\
\hline \multicolumn{2}{|c|}{ NIST Code: SMC-113D } & \multicolumn{2}{|c|}{ NIST Code: SMC-113E } & \multicolumn{2}{|c|}{ NIST Code: SMC-113F } & & & \\
\hline $\mathrm{N}$ & $\Gamma$ & $\mathrm{N}$ & $\Gamma$ & $\mathrm{N}$ & $\Gamma$ & $\mathbf{N}$ & $\Gamma$ & $\Gamma / N$ (Angular \\
\hline $1 / \mathrm{min}$ & $\mu \mathrm{Nm}$ & $1 / \mathrm{min}$ & $\mu \mathrm{Nm}$ & $1 / \mathrm{min}$ & $\mu \mathrm{Nm}$ & $\mathrm{rad} / \mathrm{s}$ & $\mathbf{N m}$ & momentum) \\
\hline 0.0943 & 1020 & 0.0945 & 959 & 0.0944 & 998 & 0.010 & $9.92 E-04$ & $1.00 E-01$ \\
\hline 0.161 & 1500 & 0.161 & 1430 & 0.161 & 1480 & 0.017 & $1.47 E-03$ & $8.72 E-02$ \\
\hline 0.268 & 1700 & 0.268 & 1640 & 0.268 & 1690 & 0.028 & $1.68 E-03$ & $5.97 E-02$ \\
\hline 0.44 & 1800 & 0.44 & 1760 & 0.44 & 1810 & 0.046 & $1.79 E-03$ & $3.88 E-02$ \\
\hline 0.72 & 1910 & 0.72 & 1880 & 0.72 & 1930 & 0.075 & $1.91 E-03$ & $2.53 E-02$ \\
\hline 1.18 & 2080 & 1.18 & 2050 & 1.18 & 2110 & 0.124 & $2.08 E-03$ & $1.68 E-02$ \\
\hline 1.93 & 2320 & 1.93 & 2300 & 1.93 & 2370 & 0.202 & $2.33 E-03$ & $1.15 E-02$ \\
\hline 3.16 & 2710 & 3.16 & 2710 & 3.16 & 2780 & 0.331 & $2.73 E-03$ & $8.26 E-03$ \\
\hline 5.18 & 3320 & 5.18 & 3330 & 5.18 & 3420 & 0.542 & $3.36 E-03$ & $6.19 E-03$ \\
\hline 8.48 & 4260 & 8.48 & 4290 & 8.48 & 4410 & 0.888 & $4.32 E-03$ & $4.86 E-03$ \\
\hline 13.9 & 5780 & 13.9 & 5860 & 13.9 & 6000 & 1.456 & $5.88 E-03$ & $4.04 E-03$ \\
\hline 22.8 & 8160 & 22.8 & 8220 & 22.8 & 8470 & 2.388 & $8.28 E-03$ & $3.47 E-03$ \\
\hline 37.3 & 11900 & 37.3 & 12000 & 37.3 & 12400 & 3.906 & $1.21 E-02$ & $3.10 E-03$ \\
\hline 61.1 & 17700 & 61.1 & 18000 & 61.1 & 18500 & 6.398 & $1.81 E-02$ & $2.82 E-03$ \\
\hline 100 & 26800 & 100 & 27200 & 100 & 28100 & 10.472 & $2.74 E-02$ & $2.61 E-03$ \\
\hline 100 & 26600 & 100 & 27100 & 100 & 27900 & 10.472 & $2.72 E-02$ & $2.60 E-03$ \\
\hline 69.5 & 19400 & 69.5 & 19800 & 69.5 & 20400 & 7.278 & $1.99 E-02$ & $2.73 E-03$ \\
\hline 48.3 & 14400 & 48.3 & 14700 & 48.3 & 15100 & 5.058 & $1.47 E-02$ & $2.91 E-03$ \\
\hline 33.6 & 10800 & 33.6 & 11000 & 33.6 & 11300 & 3.519 & $1.10 E-02$ & $3.14 E-03$ \\
\hline 23.4 & 8190 & 23.4 & 8350 & 23.4 & 8590 & 2.450 & $8.38 E-03$ & $3.42 E-03$ \\
\hline 16.2 & 6350 & 16.2 & 6470 & 16.2 & 6650 & 1.696 & $6.49 E-03$ & $3.83 E-03$ \\
\hline 11.3 & 5020 & 11.3 & 5110 & 11.3 & 5250 & 1.183 & $5.13 E-03$ & 4.33E-03 \\
\hline 7.85 & 4070 & 7.85 & 4140 & 7.85 & 4240 & 0.822 & $4.15 E-03$ & $5.05 E-03$ \\
\hline 5.46 & 3380 & 5.46 & 3430 & 5.46 & 3510 & 0.572 & $3.44 E-03$ & $6.02 E-03$ \\
\hline 3.79 & 2870 & 3.79 & 2920 & 3.79 & 2980 & 0.397 & $2.92 E-03$ & 7.37E-03 \\
\hline 2.64 & 2500 & 2.64 & 2540 & 2.64 & 2590 & 0.276 & $2.54 E-03$ & $9.20 E-03$ \\
\hline 1.83 & 2230 & 1.83 & 2260 & 1.83 & 2300 & 0.192 & $2.26 E-03$ & $1.18 E-02$ \\
\hline 1.27 & 2020 & 1.27 & 2050 & 1.27 & 2090 & 0.133 & $2.05 E-03$ & $1.54 E-02$ \\
\hline 0.886 & 1870 & 0.886 & 1890 & 0.886 & 1930 & 0.093 & $1.90 E-03$ & $2.04 E-02$ \\
\hline 0.615 & 1750 & 0.616 & 1770 & 0.616 & 1800 & 0.064 & $1.77 E-03$ & $2.75 E-02$ \\
\hline 0.428 & 1670 & 0.428 & 1680 & 0.427 & 1720 & 0.045 & $1.69 E-03$ & $3.77 E-02$ \\
\hline 0.3 & 1600 & 0.297 & 1620 & 0.298 & 1650 & 0.031 & $1.62 E-03$ & $5.20 E-02$ \\
\hline 0.206 & 1550 & 0.207 & 1580 & 0.207 & 1580 & 0.022 & $1.57 E-03$ & $7.25 E-02$ \\
\hline 0.144 & 1500 & 0.144 & 1510 & 0.145 & 1540 & 0.015 & $1.52 E-03$ & $1.00 E-01$ \\
\hline 0.0994 & 1470 & 0.0997 & 1480 & 0.101 & 1510 & 0.010 & $1.49 E-03$ & $1.42 E-01$ \\
\hline
\end{tabular}


Table A - 2: Measured data for Mix\#2 with 40 \% beads using Double Spiral spindle.

\begin{tabular}{|c|c|c|c|c|c|c|c|c|}
\hline \multicolumn{6}{|c|}{ Double Spiral } & \multirow{2}{*}{\multicolumn{3}{|c|}{$\begin{array}{c}\text { Bead } \% \\
40 \%\end{array}$}} \\
\hline Geom.: & RHN-83A & & & \multicolumn{2}{|c|}{ Cup: } & & & \\
\hline \multicolumn{2}{|c|}{ Run \#1 } & \multicolumn{2}{|c|}{ Run \#2 } & \multicolumn{2}{|c|}{ Run \#3 } & \multirow{2}{*}{\multicolumn{3}{|c|}{$\begin{array}{c}\text { Average } \\
\text { values }\end{array}$}} \\
\hline \multicolumn{2}{|c|}{ NIST Code: SMC-79J } & \multicolumn{2}{|c|}{ NIST Code: SMC-79K } & \multicolumn{2}{|c|}{ NIST Code: SMC-79L } & & & \\
\hline $\mathrm{N}$ & $\Gamma$ & $\mathrm{N}$ & $\Gamma$ & $\mathrm{N}$ & $\Gamma$ & $\mathbf{N}$ & $\Gamma$ & $\Gamma / \boldsymbol{N}$ (Angular \\
\hline $1 / \mathrm{min}$ & $\mu \mathrm{Nm}$ & $1 / \mathrm{min}$ & $\mu \mathrm{Nm}$ & $1 / \mathrm{min}$ & $\mu \mathrm{Nm}$ & $\mathrm{rad} / \mathrm{s}$ & $\mathrm{Nm}$ & momentum) \\
\hline 0.09 & 2200 & 0.0894 & 2260 & 0.0891 & 2150 & 0.009 & $2.20 E-03$ & $2.35 E-01$ \\
\hline 0.158 & 3110 & 0.162 & 3170 & 0.16 & 3060 & 0.017 & $3.11 E-03$ & $1.86 E-01$ \\
\hline 0.263 & 3700 & 0.267 & 3610 & 0.269 & 3580 & 0.028 & 3.63E-03 & $1.30 E-01$ \\
\hline 0.435 & 4310 & 0.439 & 4050 & 0.438 & 4020 & 0.046 & 4.13E-03 & $9.01 E-02$ \\
\hline 0.719 & 5050 & 0.725 & 4620 & 0.716 & 4620 & 0.075 & $4.76 E-03$ & $6.32 E-02$ \\
\hline 1.18 & 5950 & 1.19 & 5480 & 1.17 & 5410 & 0.124 & $5.61 E-03$ & $4.54 E-02$ \\
\hline 1.93 & 7320 & 1.93 & 6660 & 1.93 & 6640 & 0.202 & $6.87 E-03$ & $3.40 E-02$ \\
\hline 3.17 & 9470 & 3.17 & 8700 & 3.15 & 8560 & 0.331 & 8.91E-03 & 2.69E-02 \\
\hline 5.18 & 12700 & 5.17 & 11800 & 5.18 & 11500 & 0.542 & $1.20 E-02$ & $2.21 E-02$ \\
\hline 8.48 & 17900 & 8.48 & 16700 & 8.48 & 16600 & 0.888 & $1.71 E-02$ & $1.92 E-02$ \\
\hline 13.9 & 25200 & 13.9 & 23600 & 13.9 & 23800 & 1.456 & $2.42 E-02$ & $1.66 E-02$ \\
\hline 22.8 & 35600 & 22.8 & 33700 & 22.7 & 33900 & 2.384 & $3.44 E-02$ & $1.44 E-02$ \\
\hline 37.3 & 51500 & 37.3 & 49700 & 37.3 & 50800 & 3.906 & $5.07 E-02$ & $1.30 E-02$ \\
\hline 61.1 & 74500 & 61 & 73900 & 61 & 76300 & 6.391 & $7.49 E-02$ & $1.17 E-02$ \\
\hline 100 & 106000 & 100 & 101000 & 100 & 108000 & 10.472 & $1.05 E-01$ & $1.00 E-02$ \\
\hline 100 & 101000 & 100 & 95800 & 100 & 104000 & 10.472 & $1.00 E-01$ & $9.57 E-03$ \\
\hline 69.6 & 71900 & 69.6 & 68200 & 69.5 & 75000 & 7.285 & 7.17E-02 & $9.84 E-03$ \\
\hline 48.3 & 52700 & 48.3 & 49300 & 48.3 & 55200 & 5.058 & $5.24 E-02$ & $1.04 E-02$ \\
\hline 33.6 & 39100 & 33.6 & 36300 & 33.6 & 41300 & 3.519 & $3.89 E-02$ & $1.11 E-02$ \\
\hline 23.3 & 29900 & 23.4 & 27600 & 23.4 & 31700 & 2.447 & 2.97E-02 & $1.22 E-02$ \\
\hline 16.2 & 23100 & 16.2 & 22700 & 16.2 & 24800 & 1.696 & $2.35 E-02$ & $1.39 E-02$ \\
\hline 11.3 & 17600 & 11.3 & 17700 & 11.3 & 19000 & 1.183 & $1.81 E-02$ & $1.53 E-02$ \\
\hline 7.83 & 13600 & 7.85 & 13800 & 7.86 & 14700 & 0.822 & $1.40 E-02$ & $1.71 E-02$ \\
\hline 5.44 & 10800 & 5.45 & 10900 & 5.46 & 11600 & 0.571 & $1.11 E-02$ & $1.94 E-02$ \\
\hline 3.79 & 8700 & 3.78 & 8950 & 3.78 & 9310 & 0.396 & 8.99E-03 & $2.27 E-02$ \\
\hline 2.65 & 7120 & 2.64 & 7260 & 2.63 & 7750 & 0.276 & $7.38 E-03$ & 2.67E-02 \\
\hline 1.82 & 5940 & 1.83 & 6100 & 1.82 & 6510 & 0.191 & $6.18 E-03$ & $3.24 E-02$ \\
\hline 1.27 & 5150 & 1.27 & 5200 & 1.28 & 5540 & 0.133 & $5.30 E-03$ & 3.97E-02 \\
\hline 0.889 & 4470 & 0.884 & 4540 & 0.88 & 4830 & 0.093 & $4.61 E-03$ & $4.98 E-02$ \\
\hline 0.608 & 3980 & 0.617 & 4060 & 0.611 & 4320 & 0.064 & $4.12 E-03$ & $6.43 E-02$ \\
\hline 0.426 & 3630 & 0.425 & 3680 & 0.428 & 3890 & 0.045 & $3.73 E-03$ & 8.36E-02 \\
\hline 0.295 & 3320 & 0.298 & 3390 & 0.297 & 3590 & 0.031 & $3.43 E-03$ & $1.11 E-01$ \\
\hline 0.203 & 3100 & 0.208 & 3180 & 0.205 & 3380 & 0.022 & $3.22 E-03$ & $1.50 E-01$ \\
\hline 0.144 & 2980 & 0.147 & 3020 & 0.145 & 3140 & 0.015 & $3.05 E-03$ & $2.00 E-01$ \\
\hline 0.0999 & 2870 & 0.0987 & 2890 & 0.101 & 3040 & 0.010 & $2.93 E-03$ & $2.80 E-01$ \\
\hline
\end{tabular}




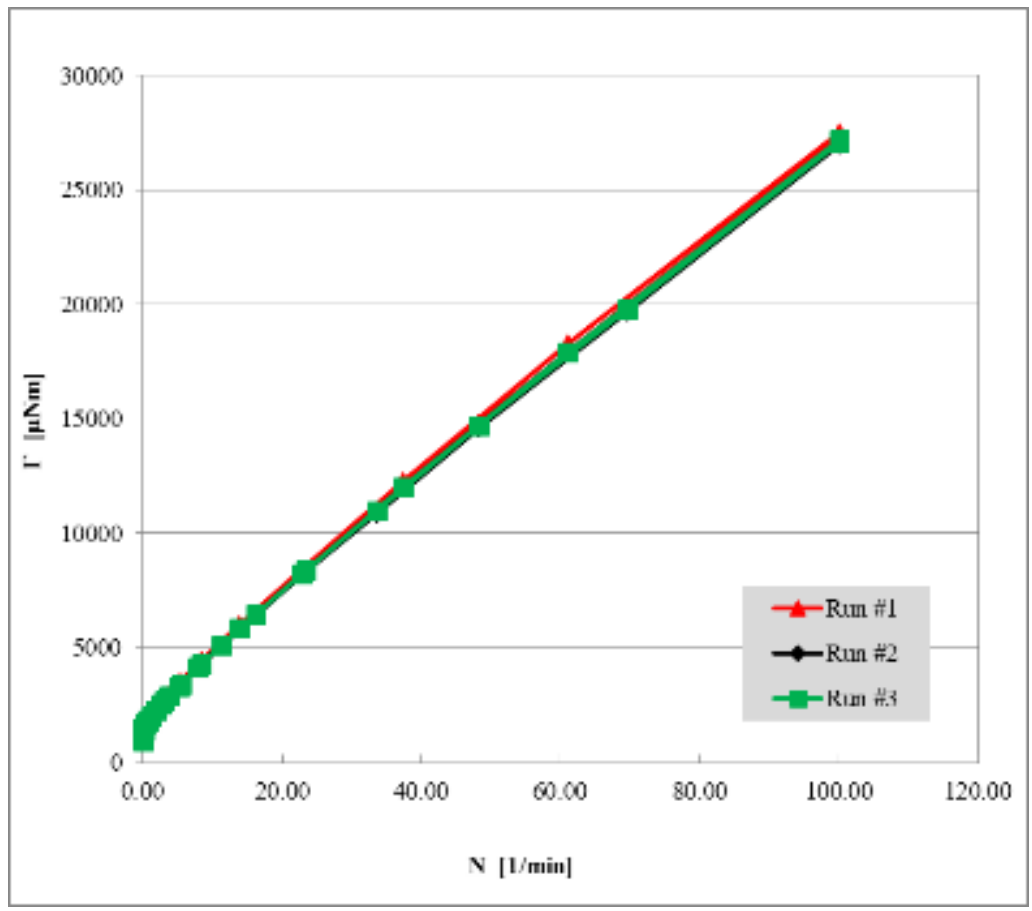

Figure A - 1: Torque vs. Angular Speed using Double Spiral spindle on Mix\#2, with $0 \%$ beads by volume. Portrays data from Table A-1.

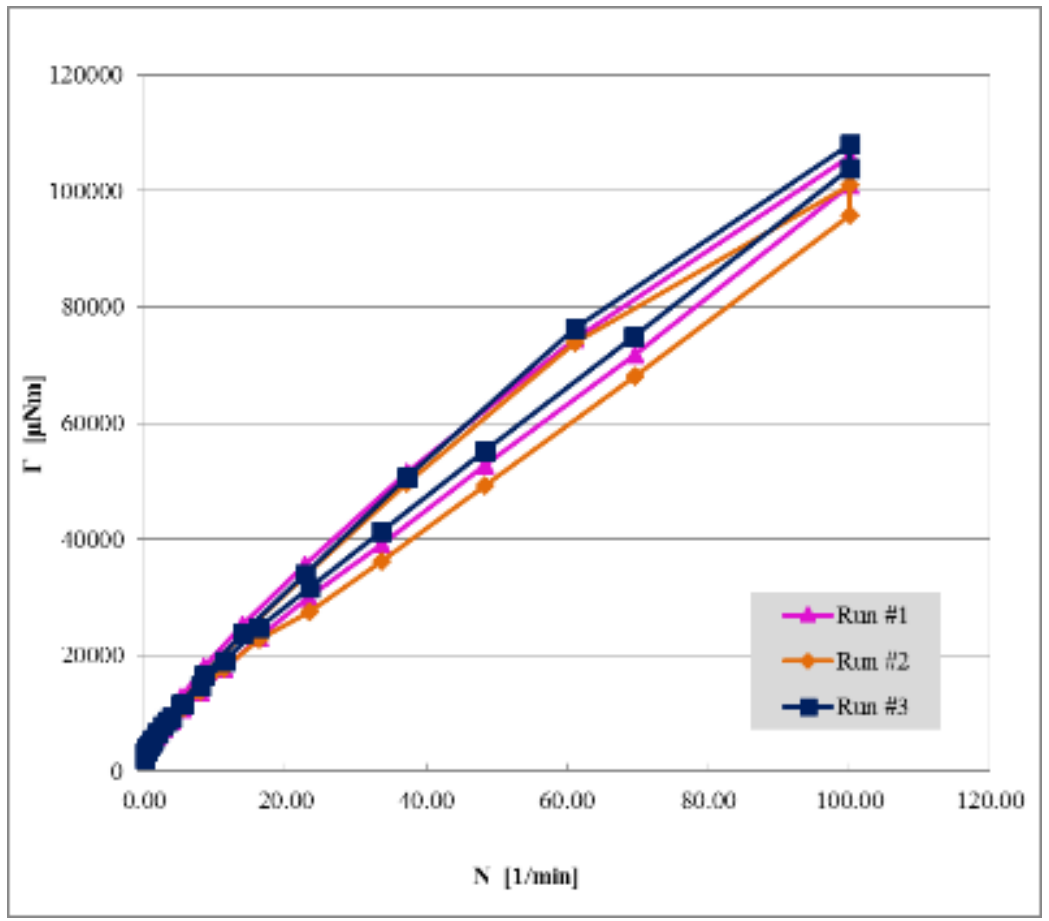

Figure A - 2: Torque vs. Angular Speed using Double spiral spindle on Mix\#2, with $40 \%$ beads by volume. Portrays data from Table A-2. 
Table A - 3: Measured data for Mix\#2 with 0 \% beads using Six-blade Vane.

\begin{tabular}{|c|c|c|c|c|c|c|c|c|}
\hline \multicolumn{6}{|c|}{6 Blade Vane } & \multicolumn{3}{|c|}{$\begin{array}{c}\text { Bead } \% \\
0 \%\end{array}$} \\
\hline \multicolumn{2}{|c|}{ Run \#1 } & \multicolumn{2}{|c|}{ Run \#2 } & \multicolumn{2}{|c|}{ Run \#3 } & \multirow{2}{*}{\multicolumn{3}{|c|}{$\begin{array}{c}\text { Average } \\
\text { values }\end{array}$}} \\
\hline NIST Cod & SMC-79D & NIST Code & SMC-79E & NIST Coc & SMC-79F & & & \\
\hline $\mathrm{N}$ & $\Gamma$ & $\mathrm{N}$ & $\Gamma$ & $\mathrm{N}$ & $\Gamma$ & $\mathbf{N}$ & $\Gamma$ & $\Gamma / \boldsymbol{N}$ (Angular \\
\hline $1 / \mathrm{min}$ & $\mu \mathrm{Nm}$ & $1 / \mathrm{min}$ & $\mu \mathrm{Nm}$ & $1 / \mathrm{min}$ & $\mu \mathrm{Nm}$ & $\mathrm{rad} / \mathrm{s}$ & $\mathrm{Nm}$ & momentum) \\
\hline 0.0977 & 434 & 0.1 & 520 & 0.0979 & 400 & 0.010 & $4.51 E-04$ & 4.37E-02 \\
\hline 0.163 & 573 & 0.164 & 541 & 0.163 & 537 & 0.017 & $5.50 E-04$ & $3.22 E-02$ \\
\hline 0.268 & 614 & 0.268 & 571 & 0.268 & 581 & 0.028 & $5.89 E-04$ & $2.10 E-02$ \\
\hline 0.439 & 649 & 0.439 & 613 & 0.439 & 618 & 0.046 & $6.27 E-04$ & $1.36 E-02$ \\
\hline 0.72 & 702 & 0.72 & 669 & 0.72 & 669 & 0.075 & $6.80 E-04$ & $9.02 E-03$ \\
\hline 1.18 & 776 & 1.18 & 745 & 1.18 & 744 & 0.124 & $7.55 E-04$ & $6.11 E-03$ \\
\hline 1.93 & 879 & 1.93 & 847 & 1.93 & 849 & 0.202 & $8.58 E-04$ & $4.25 E-03$ \\
\hline 3.16 & 1030 & 3.16 & 993 & 3.16 & 1000 & 0.331 & $1.01 E-03$ & $3.05 E-03$ \\
\hline 5.18 & 1240 & 5.18 & 1200 & 5.18 & 1210 & 0.542 & $1.22 E-03$ & $2.24 E-03$ \\
\hline 8.48 & 1540 & 8.48 & 1490 & 8.48 & 1520 & 0.888 & $1.52 E-03$ & $1.71 E-03$ \\
\hline 13.9 & 1980 & 13.9 & 1930 & 13.9 & 1970 & 1.456 & $1.96 E-03$ & $1.35 E-03$ \\
\hline 22.8 & 2620 & 22.8 & 2570 & 22.8 & 2630 & 2.388 & $2.61 E-03$ & $1.09 E-03$ \\
\hline 37.3 & 3620 & 37.3 & 3560 & 37.3 & 3650 & 3.906 & $3.61 E-03$ & $9.24 E-04$ \\
\hline 61 & 5170 & 61 & 5080 & 61 & 5210 & 6.388 & $5.15 E-03$ & 8.07E-04 \\
\hline 100 & 7590 & 100 & 7490 & 100 & 7620 & 10.472 & $7.57 E-03$ & $7.23 E-04$ \\
\hline 100 & 7580 & 100 & 7470 & 100 & 7600 & 10.472 & $7.55 E-03$ & $7.21 E-04$ \\
\hline 69.5 & 5670 & 69.5 & 5600 & 69.5 & 5680 & 7.278 & $5.65 E-03$ & $7.76 E-04$ \\
\hline 48.3 & 4310 & 48.3 & 4260 & 48.3 & 4310 & 5.058 & $4.29 E-03$ & $8.49 E-04$ \\
\hline 33.6 & 3330 & 33.6 & 3290 & 33.6 & 3330 & 3.519 & $3.32 E-03$ & $9.43 E-04$ \\
\hline 23.4 & 2620 & 23.4 & 2590 & 23.4 & 2620 & 2.450 & $2.61 E-03$ & $1.07 E-03$ \\
\hline 16.2 & 2100 & 16.2 & 2080 & 16.2 & 2100 & 1.696 & $2.09 E-03$ & $1.23 E-03$ \\
\hline 11.3 & 1720 & 11.3 & 1700 & 11.3 & 1720 & 1.183 & $1.71 E-03$ & $1.45 E-03$ \\
\hline 7.85 & 1440 & 7.85 & 1430 & 7.85 & 1440 & 0.822 & $1.44 E-03$ & $1.75 E-03$ \\
\hline 5.45 & 1220 & 5.45 & 1210 & 5.45 & 1220 & 0.571 & $1.22 E-03$ & $2.13 E-03$ \\
\hline 3.79 & 1060 & 3.79 & 1040 & 3.79 & 1050 & 0.397 & $1.05 E-03$ & $2.65 E-03$ \\
\hline 2.64 & 929 & 2.64 & 917 & 2.64 & 921 & 0.276 & $9.22 E-04$ & $3.34 E-03$ \\
\hline 1.83 & 830 & 1.83 & 820 & 1.83 & 822 & 0.192 & $8.24 E-04$ & $4.30 E-03$ \\
\hline 1.27 & 752 & 1.27 & 741 & 1.27 & 744 & 0.133 & $7.46 E-04$ & $5.61 E-03$ \\
\hline 0.886 & 691 & 0.886 & 681 & 0.885 & 690 & 0.093 & $6.87 E-04$ & $7.41 E-03$ \\
\hline 0.616 & 643 & 0.617 & 648 & 0.616 & 635 & 0.065 & $6.42 E-04$ & $9.95 E-03$ \\
\hline 0.428 & 604 & 0.428 & 608 & 0.428 & 596 & 0.045 & $6.03 E-04$ & $1.34 E-02$ \\
\hline 0.298 & 574 & 0.297 & 569 & 0.297 & 567 & 0.031 & $5.70 E-04$ & $1.83 E-02$ \\
\hline 0.207 & 548 & 0.21 & 545 & 0.207 & 541 & 0.022 & $5.45 E-04$ & $2.50 E-02$ \\
\hline 0.144 & 529 & 0.144 & 520 & 0.144 & 521 & 0.015 & $5.23 E-04$ & $3.47 E-02$ \\
\hline 0.0999 & 515 & 0.0998 & 505 & 0.101 & 507 & 0.010 & $5.09 E-04$ & $4.85 E-02$ \\
\hline
\end{tabular}


Table A - 4: Measured data for Mix\#2 with 40 \% beads using Six-blade Vane.

\begin{tabular}{|c|c|c|c|c|c|c|c|c|}
\hline \multicolumn{6}{|c|}{6 Blade Vane } & \multicolumn{3}{|c|}{$\begin{array}{c}\text { Bead } \% \\
40 \%\end{array}$} \\
\hline \multicolumn{2}{|c|}{ Run \#1 } & \multirow{2}{*}{\multicolumn{2}{|c|}{ Run \#2 }} & \multicolumn{2}{|c|}{ Run \#3 } & \multirow{2}{*}{\multicolumn{3}{|c|}{$\begin{array}{c}\text { Average } \\
\text { values }\end{array}$}} \\
\hline NIST Code: & SMC-79M & NIST Code & & NIST Co & AC-79O & & & \\
\hline $\mathrm{N}$ & $\Gamma$ & $\mathrm{N}$ & $\Gamma$ & $\mathrm{N}$ & $\Gamma$ & $\mathbf{N}$ & $\Gamma$ & $\boldsymbol{\Gamma} / \mathbf{N}$ (Angular \\
\hline $1 / \mathrm{min}$ & $\mu \mathrm{Nm}$ & $1 / \mathrm{min}$ & $\mu \mathrm{Nm}$ & $1 / \mathrm{min}$ & $\mu \mathrm{Nm}$ & $\mathrm{rad} / \mathrm{s}$ & $\mathrm{Nm}$ & momentum) \\
\hline 0.096 & 1070 & 0.096 & 1030 & 0.0947 & 1130 & 0.010 & $1.08 E-03$ & $1.08 E-01$ \\
\hline 0.162 & 1310 & 0.161 & 1300 & 0.165 & 1430 & 0.017 & $1.35 E-03$ & $7.91 E-02$ \\
\hline 0.267 & 1440 & 0.267 & 1390 & 0.271 & 1580 & 0.028 & $1.47 E-03$ & $5.23 E-02$ \\
\hline 0.435 & 1550 & 0.44 & 1520 & 0.44 & 1630 & 0.046 & $1.57 E-03$ & $3.41 E-02$ \\
\hline 0.717 & 1710 & 0.719 & 1670 & 0.717 & 1860 & 0.075 & $1.75 E-03$ & $2.32 E-02$ \\
\hline 1.18 & 1960 & 1.18 & 1950 & 1.17 & 2180 & 0.123 & $2.03 E-03$ & $1.65 E-02$ \\
\hline 1.93 & 2340 & 1.93 & 2330 & 1.92 & 2540 & 0.202 & $2.40 E-03$ & $1.19 E-02$ \\
\hline 3.16 & 2860 & 3.16 & 2880 & 3.18 & 3130 & 0.332 & $2.96 E-03$ & $8.92 E-03$ \\
\hline 5.17 & 3620 & 5.19 & 3640 & 5.17 & 4050 & 0.542 & $3.77 E-03$ & $6.95 E-03$ \\
\hline 8.48 & 4690 & 8.49 & 4740 & 8.47 & 5260 & 0.888 & $4.90 E-03$ & $5.51 E-03$ \\
\hline 13.9 & 6190 & 13.9 & 6430 & 13.9 & 7100 & 1.456 & $6.57 E-03$ & $4.52 E-03$ \\
\hline 22.8 & 8410 & 22.8 & 8820 & 22.7 & 9640 & 2.384 & $8.96 E-03$ & $3.76 E-03$ \\
\hline 37.3 & 11900 & 37.3 & 12500 & 37.3 & 13400 & 3.906 & $1.26 E-02$ & $3.23 E-03$ \\
\hline 61.1 & 16700 & 61 & 17400 & 61 & 18400 & 6.391 & $1.75 E-02$ & $2.74 E-03$ \\
\hline 100 & 23700 & 100 & 25600 & 100 & 26600 & 10.472 & $2.53 E-02$ & $2.42 E-03$ \\
\hline 100 & 23300 & 100 & 24400 & 100 & 25600 & 10.472 & $2.44 E-02$ & $2.33 E-03$ \\
\hline 69.5 & 17400 & 69.5 & 18300 & 69.5 & 19400 & 7.278 & $1.84 E-02$ & $2.52 E-03$ \\
\hline 48.3 & 13300 & 48.3 & 13900 & 48.3 & 14500 & 5.058 & 1.39E-02 & $2.75 E-03$ \\
\hline 33.6 & 10200 & 33.6 & 10700 & 33.6 & 11100 & 3.519 & $1.07 E-02$ & 3.03E-03 \\
\hline 23.4 & 7880 & 23.3 & 8270 & 23.4 & 8530 & 2.447 & $8.23 E-03$ & $3.36 E-03$ \\
\hline 16.2 & 6110 & 16.2 & 6390 & 16.2 & 6640 & 1.696 & $6.38 E-03$ & $3.76 E-03$ \\
\hline 11.3 & 4860 & 11.3 & 5070 & 11.3 & 5240 & 1.183 & $5.06 E-03$ & 4.27E-03 \\
\hline 7.84 & 3890 & 7.85 & 4040 & 7.85 & 4200 & 0.822 & $4.04 E-03$ & $4.92 E-03$ \\
\hline 5.46 & 3170 & 5.45 & 3250 & 5.45 & 3390 & 0.571 & $3.27 E-03$ & $5.73 E-03$ \\
\hline 3.8 & 2590 & 3.79 & 2730 & 3.8 & 2790 & 0.398 & $2.70 E-03$ & $6.80 E-03$ \\
\hline 2.64 & 2190 & 2.64 & 2290 & 2.64 & 2300 & 0.276 & $2.26 E-03$ & 8.17E-03 \\
\hline 1.84 & 1880 & 1.83 & 1940 & 1.83 & 1970 & 0.192 & $1.93 E-03$ & $1.01 E-02$ \\
\hline 1.27 & 1640 & 1.28 & 1710 & 1.27 & 1720 & 0.133 & $1.69 E-03$ & $1.27 E-02$ \\
\hline 0.889 & 1460 & 0.884 & 1500 & 0.886 & 1530 & 0.093 & $1.50 E-03$ & $1.61 E-02$ \\
\hline 0.614 & 1320 & 0.62 & 1350 & 0.617 & 1370 & 0.065 & $1.35 E-03$ & $2.08 E-02$ \\
\hline 0.428 & 1210 & 0.427 & 1240 & 0.428 & 1250 & 0.045 & $1.23 E-03$ & $2.75 E-02$ \\
\hline 0.3 & 1120 & 0.294 & 1150 & 0.295 & 1170 & 0.031 & $1.15 E-03$ & $3.70 E-02$ \\
\hline 0.206 & 1080 & 0.209 & 1070 & 0.208 & 1100 & 0.022 & $1.08 E-03$ & $4.98 E-02$ \\
\hline 0.144 & 1010 & 0.145 & 1000 & 0.143 & 1030 & 0.015 & $1.01 E-03$ & $6.72 E-02$ \\
\hline 0.0992 & 951 & 0.0998 & 989 & 0.1 & 983 & 0.010 & $9.74 E-04$ & $9.34 E-02$ \\
\hline
\end{tabular}




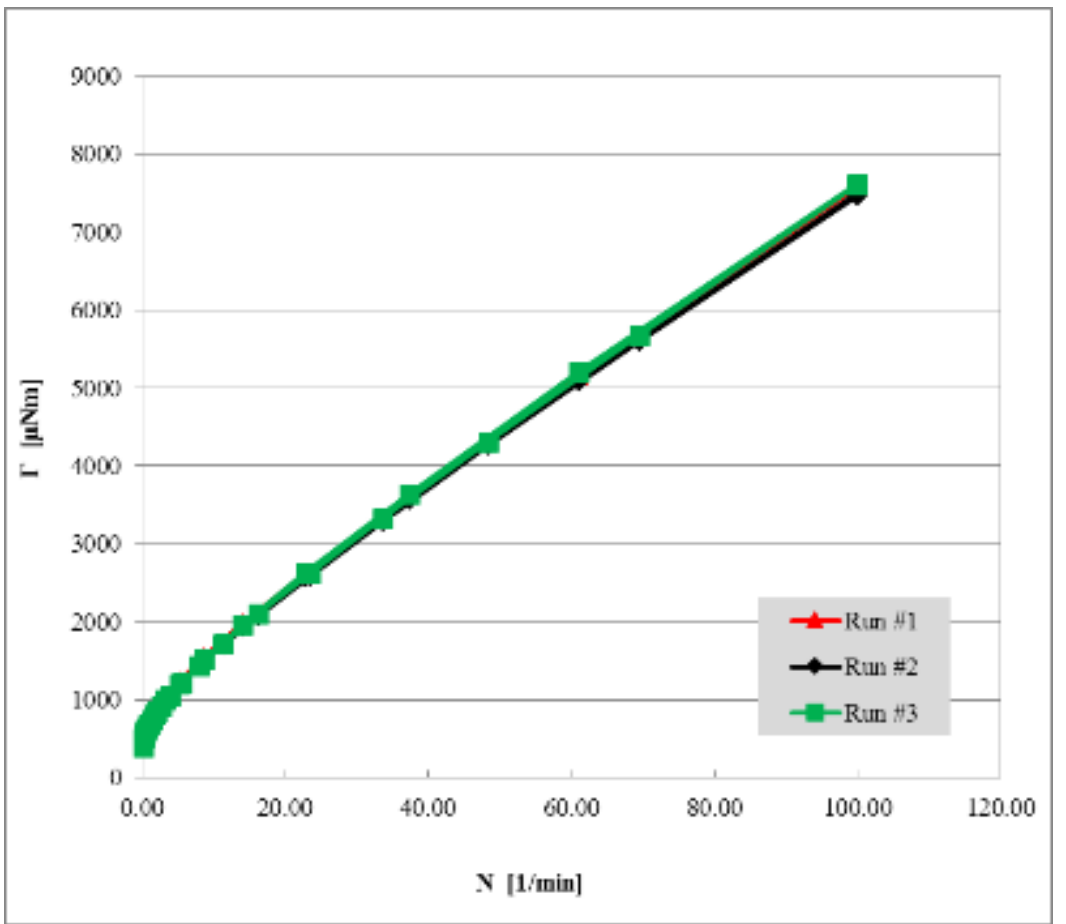

Figure A - 1: Torque vs. Angular Speed using six-blade vane on Mix\#2, with $0 \%$ beads by volume. Portrays data from Table A-3.

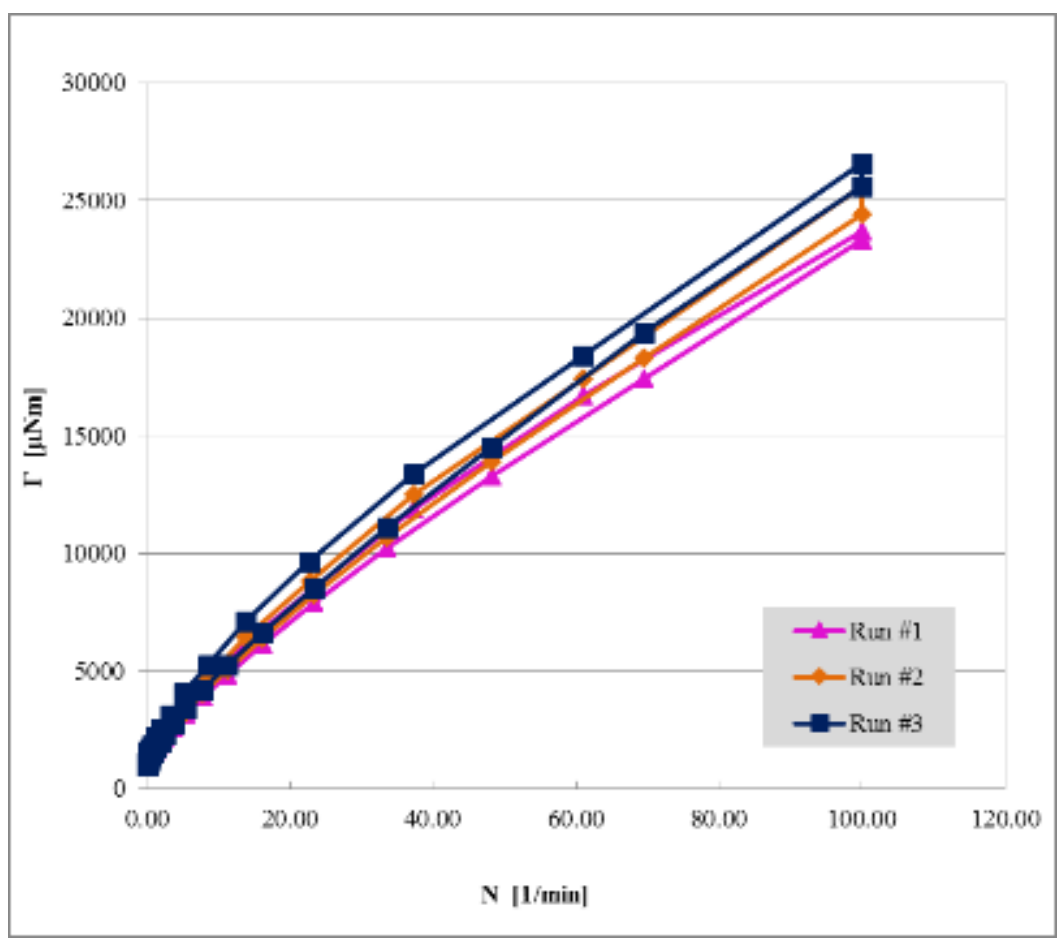

Figure A - 2: Torque vs. Angular Speed using six-blade vane on Mix\#2, with 40 \% beads by volume. Portrays data from Table A-4. 
Table A - 5: Measured data for Mix\#2 with 0 \% beads using Serrated Coaxial Cylinder.

\begin{tabular}{|c|c|c|c|c|c|c|c|c|}
\hline \multicolumn{6}{|c|}{ Serrated Coaxial Cylinder } & \multirow{2}{*}{\multicolumn{3}{|c|}{$\begin{array}{c}\text { Bead } \% \\
0 \%\end{array}$}} \\
\hline Geom.: & & & & \multicolumn{2}{|c|}{ Z43S } & & & \\
\hline \multicolumn{2}{|c|}{ Run \#1 } & \multicolumn{2}{|c|}{ Run \#2 } & \multicolumn{2}{|c|}{ Run \#3 } & \multirow{2}{*}{\multicolumn{3}{|c|}{$\begin{array}{c}\text { Average } \\
\text { values }\end{array}$}} \\
\hline NIST Code: & SMC-79G & NIST Code: & $S M C-79 H$ & NIST Co & MC-79I & & & \\
\hline $\mathrm{N}$ & $\Gamma$ & $\mathrm{N}$ & $\Gamma$ & $\mathrm{N}$ & $\Gamma$ & $\mathbf{N}$ & $\Gamma$ & $\Gamma / N$ (Angular \\
\hline $1 / \mathrm{min}$ & $\mu \mathrm{Nm}$ & $1 / \mathrm{min}$ & $\mu \mathrm{Nm}$ & $1 / \mathrm{min}$ & $\mu \mathrm{Nm}$ & $\mathrm{rad} / \mathrm{s}$ & $\mathrm{Nm}$ & momentum) \\
\hline 0.0978 & 480 & 0.1 & 375 & 0.099 & 331 & 0.010 & $3.95 E-04$ & $3.82 E-02$ \\
\hline 0.164 & 624 & 0.164 & 405 & 0.164 & 405 & 0.017 & $4.78 E-04$ & $2.78 E-02$ \\
\hline 0.268 & 661 & 0.268 & 445 & 0.268 & 449 & 0.028 & $5.18 E-04$ & $1.85 E-02$ \\
\hline 0.44 & 708 & 0.439 & 501 & 0.439 & 502 & 0.046 & $5.70 E-04$ & $1.24 E-02$ \\
\hline 0.72 & 774 & 0.719 & 578 & 0.72 & 570 & 0.075 & $6.41 E-04$ & 8.50E-03 \\
\hline 1.18 & 865 & 1.18 & 675 & 1.18 & 660 & 0.124 & 7.33E-04 & $5.93 E-03$ \\
\hline 1.93 & 989 & 1.93 & 808 & 1.93 & 783 & 0.202 & 8.60E-04 & $4.26 E-03$ \\
\hline 3.16 & 1160 & 3.16 & 986 & 3.16 & 955 & 0.331 & $1.03 E-03$ & $3.12 E-03$ \\
\hline 5.18 & 1410 & 5.18 & 1230 & 5.18 & 1200 & 0.542 & $1.28 E-03$ & $2.36 E-03$ \\
\hline 8.48 & 1770 & 8.48 & 1580 & 8.48 & 1540 & 0.888 & $1.63 E-03$ & $1.84 E-03$ \\
\hline 13.9 & 2280 & 13.9 & 2070 & 13.9 & 2040 & 1.456 & $2.13 E-03$ & $1.46 E-03$ \\
\hline 22.8 & 3030 & 22.8 & 2780 & 22.8 & 2760 & 2.388 & $2.86 E-03$ & $1.20 E-03$ \\
\hline 37.3 & 4190 & 37.3 & 3880 & 37.3 & 3850 & 3.906 & $3.97 E-03$ & $1.02 E-03$ \\
\hline 61.1 & 5930 & 61.1 & 5570 & 61.1 & 5540 & 6.398 & $5.68 E-03$ & 8.88E-04 \\
\hline 100 & 8570 & 100 & 8150 & 100 & 8100 & 10.472 & $8.27 E-03$ & $7.90 E-04$ \\
\hline 100 & 8450 & 100 & 8110 & 100 & 8060 & 10.472 & $8.21 E-03$ & $7.84 E-04$ \\
\hline 69.5 & 6340 & 69.5 & 6090 & 69.5 & 6070 & 7.278 & $6.17 E-03$ & $8.47 E-04$ \\
\hline 48.3 & 4780 & 48.3 & 4600 & 48.3 & 4570 & 5.058 & 4.65E-03 & $9.19 E-04$ \\
\hline 33.6 & 3650 & 33.6 & 3520 & 33.6 & 3490 & 3.519 & $3.55 E-03$ & $1.01 E-03$ \\
\hline 23.4 & 2840 & 23.4 & 2730 & 23.4 & 2710 & 2.450 & $2.76 E-03$ & $1.13 E-03$ \\
\hline 16.2 & 2250 & 16.2 & 2170 & 16.2 & 2150 & 1.696 & $2.19 E-03$ & $1.29 E-03$ \\
\hline 11.3 & 1820 & 11.3 & 1740 & 11.3 & 1730 & 1.183 & $1.76 E-03$ & $1.49 E-03$ \\
\hline 7.85 & 1490 & 7.85 & 1430 & 7.85 & 1410 & 0.822 & $1.44 E-03$ & $1.76 E-03$ \\
\hline 5.46 & 1240 & 5.46 & 1190 & 5.46 & 1170 & 0.572 & $1.20 E-03$ & $2.10 E-03$ \\
\hline 3.79 & 1050 & 3.79 & 999 & 3.79 & 985 & 0.397 & $1.01 E-03$ & $2.55 E-03$ \\
\hline 2.64 & 900 & 2.64 & 852 & 2.64 & 840 & 0.276 & 8.64E-04 & $3.13 E-03$ \\
\hline 1.83 & 780 & 1.83 & 737 & 1.83 & 728 & 0.192 & $7.48 E-04$ & $3.90 E-03$ \\
\hline 1.27 & 685 & 1.27 & 646 & 1.27 & 637 & 0.133 & $6.56 E-04$ & $4.93 E-03$ \\
\hline 0.886 & 608 & 0.886 & 573 & 0.886 & 566 & 0.093 & $5.82 E-04$ & $6.28 E-03$ \\
\hline 0.616 & 545 & 0.616 & 515 & 0.616 & 508 & 0.065 & $5.23 E-04$ & 8.10E-03 \\
\hline 0.428 & 495 & 0.428 & 468 & 0.428 & 462 & 0.045 & $4.75 E-04$ & $1.06 E-02$ \\
\hline 0.298 & 454 & 0.298 & 430 & 0.298 & 425 & 0.031 & $4.36 E-04$ & $1.40 E-02$ \\
\hline 0.207 & 421 & 0.207 & 399 & 0.207 & 396 & 0.022 & $4.05 E-04$ & $1.87 E-02$ \\
\hline 0.144 & 394 & 0.144 & 374 & 0.144 & 371 & 0.015 & $3.80 E-04$ & $2.52 E-02$ \\
\hline 0.1 & 373 & 0.1 & 355 & 0.1 & 352 & 0.010 & $3.60 E-04$ & $3.44 E-02$ \\
\hline
\end{tabular}


Table A - 6: Measured data for Mix\#2 with 40 \% beads using Serrated Coaxial Cylinder.

\begin{tabular}{|c|c|c|c|c|c|c|c|c|}
\hline \multicolumn{6}{|c|}{ Serrated Coaxial Cylinder } & \multirow{2}{*}{\multicolumn{3}{|c|}{$\begin{array}{c}\text { Bead } \% \\
40 \%\end{array}$}} \\
\hline \multicolumn{4}{|c|}{ SS18 } & \multicolumn{2}{|c|}{ Z43S } & & & \\
\hline Run & \#1 & Run & \#2 & Run & & \multirow{2}{*}{\multicolumn{3}{|c|}{$\begin{array}{c}\text { Average } \\
\text { values }\end{array}$}} \\
\hline NIST Code: & SMC-79P & NIST Code: & SMC-79Q & NIST Code: & $S M C-79 R$ & & & \\
\hline $\mathrm{N}$ & $\Gamma$ & $\mathrm{N}$ & $\Gamma$ & $\mathrm{N}$ & $\Gamma$ & $\mathbf{N}$ & $\Gamma$ & $\boldsymbol{\Gamma} / \mathbf{N}$ (Angular \\
\hline $1 / \mathrm{min}$ & $\mu \mathrm{Nm}$ & $1 / \mathrm{min}$ & $\mu \mathrm{Nm}$ & $1 / \mathrm{min}$ & $\mu \mathrm{Nm}$ & $\mathrm{rad} / \mathrm{s}$ & $\mathrm{Nm}$ & momentum) \\
\hline 0.0987 & 1050 & 0.0984 & 1040 & 0.0994 & 766 & 0.010 & $9.52 E-04$ & 9.20E-02 \\
\hline 0.163 & 1270 & 0.164 & 1180 & 0.164 & 819 & 0.017 & $1.09 E-03$ & $6.36 E-02$ \\
\hline 0.27 & 1260 & 0.268 & 1200 & 0.268 & 896 & 0.028 & $1.12 E-03$ & 3.98E-02 \\
\hline 0.439 & 1270 & 0.441 & 1280 & 0.439 & 1010 & 0.046 & $1.19 E-03$ & $2.58 E-02$ \\
\hline 0.719 & 1400 & 0.72 & 1440 & 0.719 & 1160 & 0.075 & $1.33 E-03$ & $1.77 E-02$ \\
\hline 1.18 & 1620 & 1.18 & 1680 & 1.18 & 1400 & 0.124 & $1.57 E-03$ & $1.27 E-02$ \\
\hline 1.93 & 1920 & 1.93 & 2030 & 1.93 & 1720 & 0.202 & $1.89 E-03$ & $9.35 E-03$ \\
\hline 3.17 & 2400 & 3.17 & 2550 & 3.16 & 2200 & 0.332 & $2.38 E-03$ & 7.19E-03 \\
\hline 5.18 & 3070 & 5.18 & 3300 & 5.18 & 2890 & 0.542 & $3.09 E-03$ & $5.69 E-03$ \\
\hline 8.48 & 4080 & 8.48 & 4420 & 8.48 & 3930 & 0.888 & $4.14 E-03$ & 4.67E-03 \\
\hline 13.9 & 5560 & 13.9 & 6020 & 13.9 & 5460 & 1.456 & $5.68 E-03$ & $3.90 E-03$ \\
\hline 22.8 & 7710 & 22.8 & 8370 & 22.8 & 7780 & 2.388 & $7.95 E-03$ & $3.33 E-03$ \\
\hline 37.3 & 10800 & 37.3 & 11900 & 37.3 & 11300 & 3.906 & $1.13 E-02$ & $2.90 E-03$ \\
\hline 61.1 & 14800 & 61 & 16800 & 61.1 & 16600 & 6.395 & $1.61 E-02$ & $2.51 E-03$ \\
\hline 100 & 19900 & 100 & 21900 & 100 & 24400 & 10.472 & $2.21 E-02$ & $2.11 E-03$ \\
\hline 100 & 19900 & 100 & 21000 & 100 & 24100 & 10.472 & $2.17 E-02$ & $2.07 E-03$ \\
\hline 69.5 & 15800 & 69.5 & 17100 & 69.5 & 17900 & 7.278 & $1.69 E-02$ & 2.33E-03 \\
\hline 48.3 & 12400 & 48.3 & 12900 & 48.3 & 13400 & 5.058 & $1.29 E-02$ & $2.55 E-03$ \\
\hline 33.6 & 9700 & 33.6 & 10200 & 33.6 & 10200 & 3.519 & $1.00 E-02$ & $2.85 E-03$ \\
\hline 23.4 & 7450 & 23.4 & 7890 & 23.4 & 7730 & 2.450 & $7.69 E-03$ & $3.14 E-03$ \\
\hline 16.2 & 5700 & 16.2 & 6070 & 16.2 & 5930 & 1.696 & $5.90 E-03$ & $3.48 E-03$ \\
\hline 11.3 & 4430 & 11.3 & 4720 & 11.3 & 4640 & 1.183 & $4.60 E-03$ & $3.88 E-03$ \\
\hline 7.85 & 3540 & 7.84 & 3760 & 7.85 & 3680 & 0.822 & $3.66 E-03$ & $4.45 E-03$ \\
\hline 5.46 & 2860 & 5.45 & 2980 & 5.46 & 2950 & 0.571 & $2.93 E-03$ & $5.13 E-03$ \\
\hline 3.79 & 2330 & 3.79 & 2460 & 3.79 & 2420 & 0.397 & $2.40 E-03$ & $6.06 E-03$ \\
\hline 2.64 & 1940 & 2.64 & 2050 & 2.64 & 2000 & 0.276 & $2.00 E-03$ & $7.22 E-03$ \\
\hline 1.84 & 1650 & 1.83 & 1730 & 1.83 & 1710 & 0.192 & $1.70 E-03$ & $8.84 E-03$ \\
\hline 1.27 & 1430 & 1.27 & 1490 & 1.27 & 1480 & 0.133 & $1.47 E-03$ & $1.10 E-02$ \\
\hline 0.885 & 1250 & 0.885 & 1310 & 0.886 & 1290 & 0.093 & $1.28 E-03$ & $1.38 E-02$ \\
\hline 0.615 & 1110 & 0.617 & 1170 & 0.616 & 1140 & 0.065 & $1.14 E-03$ & $1.77 E-02$ \\
\hline 0.427 & 1000 & 0.427 & 1060 & 0.428 & 1030 & 0.045 & $1.03 E-03$ & $2.30 E-02$ \\
\hline 0.298 & 917 & 0.297 & 962 & 0.298 & 945 & 0.031 & $9.41 E-04$ & $3.02 E-02$ \\
\hline 0.206 & 853 & 0.207 & 884 & 0.207 & 880 & 0.022 & $8.72 E-04$ & $4.03 E-02$ \\
\hline 0.144 & 789 & 0.143 & 815 & 0.144 & 813 & 0.015 & $8.06 E-04$ & 5.36E-02 \\
\hline 0.1 & 746 & 0.0994 & 776 & 0.1 & 771 & 0.010 & 7.64E-04 & 7.31E-02 \\
\hline
\end{tabular}




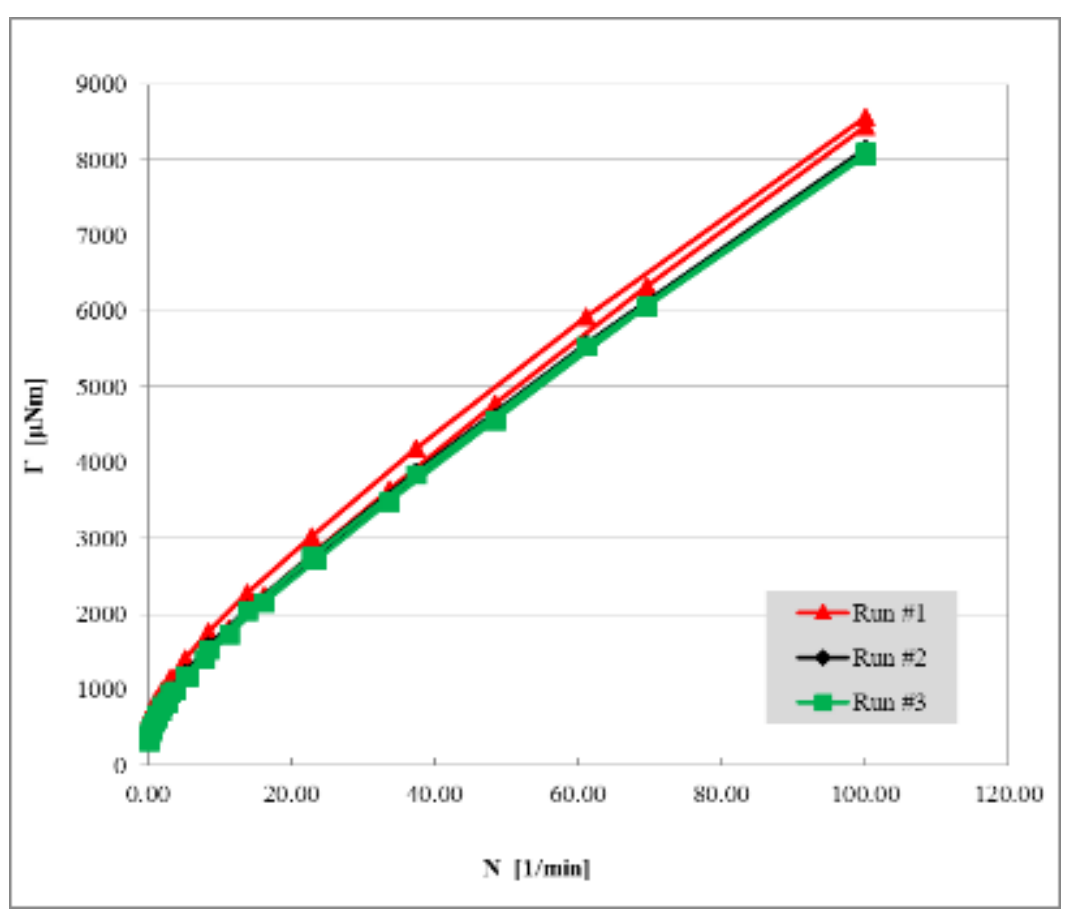

Figure A - 3: Torque vs. Angular Speed using Serrated Coaxial Cylinder on Mix\#2, with 0 \% beads by volume. Portrays data from Table A-5.

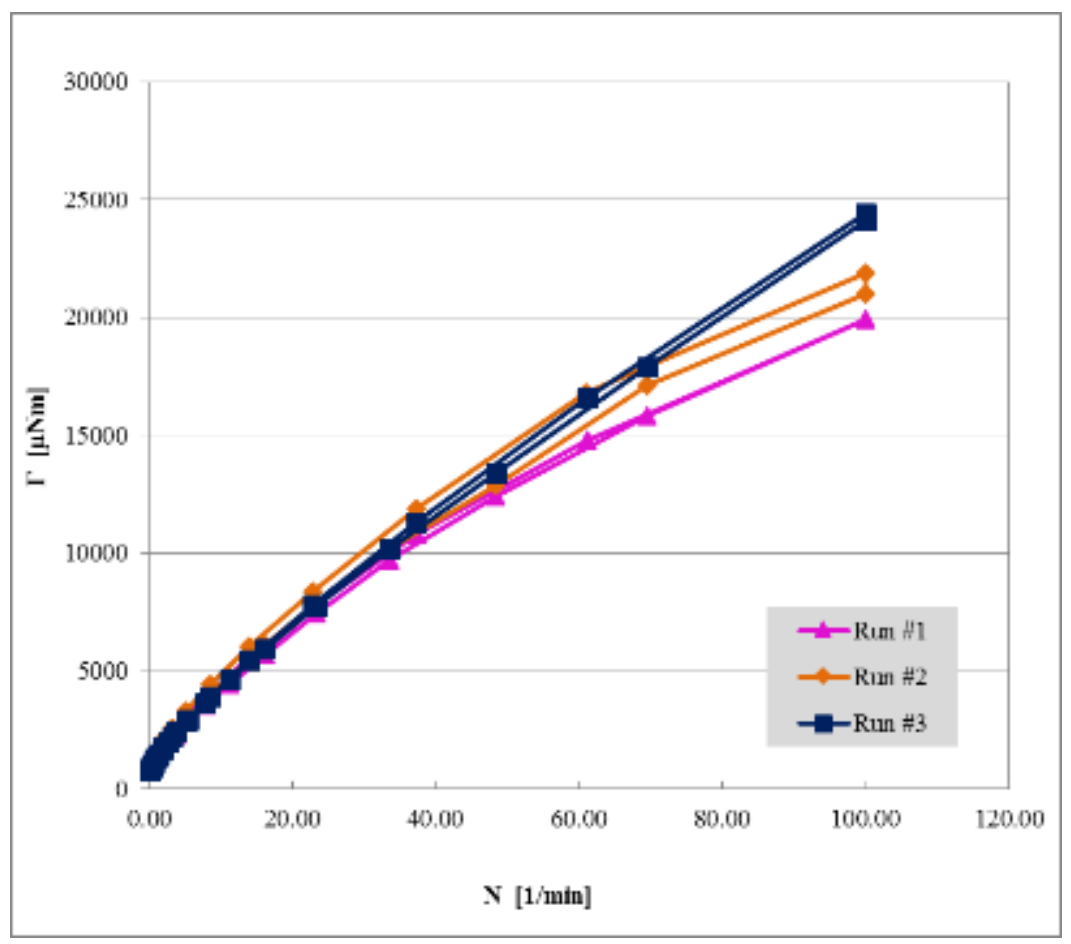

Figure A - 4: Torque vs. Angular Speed using Serrated Coaxial Cylinder on Mix\#2, with $40 \%$ beads by volume. Portrays data from Table A-6. 
Table A - 7: Measured data for Mix\#3 with 0 \% beads using Double spiral spindle.

\begin{tabular}{|c|c|c|c|c|c|c|c|c|}
\hline \multicolumn{6}{|c|}{ Double Spiral } & \multirow{2}{*}{\multicolumn{3}{|c|}{$\begin{array}{c}\text { Bead } \% \\
0 \%\end{array}$}} \\
\hline Geom.: & RHN-83A & & & Cup: & Z43S & & & \\
\hline \multicolumn{2}{|c|}{ Run \#1 } & \multicolumn{2}{|c|}{ Run \#2 } & \multicolumn{2}{|c|}{ Run \#3 } & \multirow{2}{*}{\multicolumn{3}{|c|}{$\begin{array}{c}\text { Average } \\
\text { values }\end{array}$}} \\
\hline NIST Code: & SMC-81D & NIST Code: & SMC-81E & NIST Code: & $M C-81 F$ & & & \\
\hline $\mathrm{N}$ & $\Gamma$ & $\mathrm{N}$ & $\Gamma$ & $\mathrm{N}$ & $\Gamma$ & $\mathbf{N}$ & $\Gamma$ & $\boldsymbol{\Gamma} / \boldsymbol{N}$ (Angular \\
\hline $1 / \mathrm{min}$ & $\mu \mathrm{Nm}$ & $1 / \mathrm{min}$ & $\mu \mathrm{Nm}$ & $1 / \mathrm{min}$ & $\mu \mathrm{Nm}$ & $\mathrm{rad} / \mathrm{s}$ & $\mathrm{Nm}$ & momentum) \\
\hline 0.0941 & 1070 & 0.1 & 1520 & 0.1 & 1520 & 0.010 & $1.37 E-03$ & $1.33 E-01$ \\
\hline 0.161 & 1570 & 0.164 & 1570 & 0.164 & 1560 & 0.017 & $1.57 E-03$ & $9.18 E-02$ \\
\hline 0.268 & 1760 & 0.269 & 1630 & 0.268 & 1620 & 0.028 & $1.67 E-03$ & $5.94 E-02$ \\
\hline 0.44 & 1870 & 0.441 & 1720 & 0.44 & 1710 & 0.046 & $1.77 E-03$ & $3.83 E-02$ \\
\hline 0.72 & 1990 & 0.72 & 1850 & 0.72 & 1840 & 0.075 & $1.89 E-03$ & $2.51 E-02$ \\
\hline 1.18 & 2160 & 1.18 & 2040 & 1.18 & 2020 & 0.124 & $2.07 E-03$ & $1.68 E-02$ \\
\hline 1.93 & 2420 & 1.93 & 2310 & 1.93 & 2290 & 0.202 & $2.34 E-03$ & $1.16 E-02$ \\
\hline 3.16 & 2820 & 3.16 & 2740 & 3.16 & 2720 & 0.331 & $2.76 E-03$ & 8.34E-03 \\
\hline 5.18 & 3460 & 5.18 & 3390 & 5.18 & 3370 & 0.542 & $3.41 E-03$ & $6.28 E-03$ \\
\hline 8.48 & 4450 & 8.48 & 4380 & 8.48 & 4350 & 0.888 & 4.39E-03 & $4.95 E-03$ \\
\hline 13.9 & 6010 & 13.9 & 5890 & 13.9 & 5840 & 1.456 & $5.91 E-03$ & $4.06 E-03$ \\
\hline 22.8 & 8460 & 22.8 & 8360 & 22.8 & 8310 & 2.388 & 8.38E-03 & $3.51 E-03$ \\
\hline 37.3 & 12400 & 37.3 & 12200 & 37.3 & 12100 & 3.906 & $1.22 E-02$ & $3.13 E-03$ \\
\hline 61.1 & 18500 & 61.1 & 18200 & 61.1 & 18100 & 6.398 & $1.83 E-02$ & $2.85 E-03$ \\
\hline 100 & 28000 & 100 & 27700 & 100 & 27500 & 10.472 & $2.77 E-02$ & $2.65 E-03$ \\
\hline 100 & 27800 & 100 & 27600 & 100 & 27300 & 10.472 & $2.76 E-02$ & 2.63E-03 \\
\hline 69.5 & 20300 & 69.5 & 20200 & 69.5 & 20000 & 7.278 & $2.02 E-02$ & $2.77 E-03$ \\
\hline 48.3 & 15000 & 48.3 & 14900 & 48.3 & 14800 & 5.058 & $1.49 E-02$ & $2.95 E-03$ \\
\hline 33.6 & 11200 & 33.6 & 11100 & 33.6 & 11000 & 3.519 & $1.11 E-02$ & $3.15 E-03$ \\
\hline 23.4 & 8500 & 23.4 & 8440 & 23.4 & 8380 & 2.450 & 8.44E-03 & $3.44 E-03$ \\
\hline 16.2 & 6570 & 16.2 & 6530 & 16.2 & 6480 & 1.696 & $6.53 E-03$ & $3.85 E-03$ \\
\hline 11.3 & 5180 & 11.3 & 5150 & 11.3 & 5120 & 1.183 & $5.15 E-03$ & $4.35 E-03$ \\
\hline 7.85 & 4190 & 7.85 & 4170 & 7.85 & 4140 & 0.822 & $4.17 E-03$ & $5.07 E-03$ \\
\hline 5.46 & 3470 & 5.46 & 3450 & 5.46 & 3430 & 0.572 & $3.45 E-03$ & $6.03 E-03$ \\
\hline 3.79 & 2950 & 3.79 & 2930 & 3.79 & 2910 & 0.397 & 2.93E-03 & $7.38 E-03$ \\
\hline 2.64 & 2560 & 2.64 & 2550 & 2.64 & 2530 & 0.276 & $2.55 E-03$ & $9.21 E-03$ \\
\hline 1.83 & 2280 & 1.83 & 2270 & 1.83 & 2260 & 0.192 & $2.27 E-03$ & $1.18 E-02$ \\
\hline 1.27 & 2070 & 1.27 & 2060 & 1.27 & 2050 & 0.133 & $2.06 E-03$ & $1.55 E-02$ \\
\hline 0.886 & 1910 & 0.886 & 1900 & 0.886 & 1890 & 0.093 & $1.90 E-03$ & $2.05 E-02$ \\
\hline 0.616 & 1780 & 0.616 & 1780 & 0.616 & 1770 & 0.065 & $1.78 E-03$ & $2.75 E-02$ \\
\hline 0.428 & 1700 & 0.427 & 1690 & 0.428 & 1680 & 0.045 & $1.69 E-03$ & $3.77 E-02$ \\
\hline 0.297 & 1620 & 0.298 & 1620 & 0.298 & 1610 & 0.031 & $1.62 E-03$ & $5.19 E-02$ \\
\hline 0.207 & 1570 & 0.207 & 1560 & 0.207 & 1550 & 0.022 & $1.56 E-03$ & $7.20 E-02$ \\
\hline 0.144 & 1520 & 0.144 & 1520 & 0.144 & 1510 & 0.015 & $1.52 E-03$ & $1.01 E-01$ \\
\hline 0.0993 & 1490 & 0.0998 & 1480 & 0.0998 & 1470 & 0.010 & $1.48 E-03$ & $1.42 E-01$ \\
\hline
\end{tabular}


Table A - 8: Measured data for Mix\#3 with $40 \%$ beads using Double spiral spindle.

\begin{tabular}{|c|c|c|c|c|c|c|c|c|}
\hline \multicolumn{6}{|c|}{ Double Spiral } & \multirow{2}{*}{\multicolumn{3}{|c|}{$\begin{array}{c}\text { Bead } \% \\
40 \%\end{array}$}} \\
\hline Geom.: & \multicolumn{3}{|l|}{ RHN-83A } & \multicolumn{2}{|l|}{ Cup: } & & & \\
\hline \multicolumn{2}{|c|}{ Run \#1 } & \multicolumn{2}{|c|}{ Run \#2 } & \multicolumn{2}{|c|}{ Run \#3 } & \multirow{2}{*}{\multicolumn{3}{|c|}{$\begin{array}{c}\text { Average } \\
\text { values }\end{array}$}} \\
\hline NIST Code: & $S M C-81 M$ & NIST Code: & SMC-81N & NIST Code: & SMC-81O & & & \\
\hline $\mathrm{N}$ & $\Gamma$ & $\mathrm{N}$ & $\Gamma$ & $\mathrm{N}$ & $\Gamma$ & $\mathbf{N}$ & $\Gamma$ & $\Gamma / N$ (Angular \\
\hline $1 / \mathrm{min}$ & $\mu \mathrm{Nm}$ & $1 / \mathrm{min}$ & $\mu \mathrm{Nm}$ & $1 / \mathrm{min}$ & $\mu \mathrm{Nm}$ & $\mathrm{rad} / \mathrm{s}$ & $\mathrm{Nm}$ & momentum) \\
\hline 0.0883 & 2530 & 0.0873 & 2390 & 0.093 & 3160 & 0.009 & $2.69 E-03$ & $2.87 E-01$ \\
\hline 0.158 & 3600 & 0.16 & 3390 & 0.162 & 3680 & 0.017 & $3.56 E-03$ & $2.12 E-01$ \\
\hline 0.267 & 4240 & 0.264 & 4020 & 0.268 & 4160 & 0.028 & $4.14 E-03$ & $1.48 E-01$ \\
\hline 0.442 & 4850 & 0.444 & 4580 & 0.445 & 4620 & 0.046 & 4.68E-03 & $1.01 E-01$ \\
\hline 0.711 & 5560 & 0.72 & 5310 & 0.717 & 5440 & 0.075 & $5.44 E-03$ & $7.25 E-02$ \\
\hline 1.17 & 6520 & 1.19 & 6250 & 1.18 & 6580 & 0.124 & $6.45 E-03$ & $5.22 E-02$ \\
\hline 1.93 & 8090 & 1.92 & 7710 & 1.94 & 8290 & 0.202 & 8.03E-03 & 3.97E-02 \\
\hline 3.16 & 10600 & 3.14 & 10100 & 3.16 & 10900 & 0.330 & $1.05 E-02$ & 3.19E-02 \\
\hline 5.17 & 14500 & 5.17 & 13900 & 5.18 & 14600 & 0.542 & $1.43 E-02$ & $2.65 E-02$ \\
\hline 8.47 & 20000 & 8.48 & 19700 & 8.5 & 20000 & 0.888 & 1.99E-02 & $2.24 E-02$ \\
\hline 13.9 & 27500 & 13.9 & 27700 & 13.9 & 28400 & 1.456 & $2.79 E-02$ & $1.91 E-02$ \\
\hline 22.8 & 38300 & 22.8 & 38500 & 22.7 & 41200 & 2.384 & $3.93 E-02$ & $1.65 E-02$ \\
\hline 37.3 & 56400 & 37.3 & 57200 & 37.3 & 60600 & 3.906 & $5.81 E-02$ & $1.49 E-02$ \\
\hline 61.1 & 80100 & 61.1 & 83000 & 61.1 & 88900 & 6.398 & $8.40 E-02$ & $1.31 E-02$ \\
\hline 100 & 110000 & 100 & 116000 & 100 & 123000 & 10.472 & $1.16 E-01$ & $1.11 E-02$ \\
\hline 100 & 105000 & 100 & 111000 & 100 & 118000 & 10.472 & $1.11 E-01$ & $1.06 E-02$ \\
\hline 69.6 & 74500 & 69.5 & 78900 & 69.6 & 84000 & 7.285 & $7.91 E-02$ & $1.09 E-02$ \\
\hline 48.3 & 54000 & 48.3 & 57100 & 48.4 & 61100 & 5.061 & $5.74 E-02$ & $1.13 E-02$ \\
\hline 33.6 & 39700 & 33.6 & 42100 & 33.6 & 44900 & 3.519 & $4.22 E-02$ & $1.20 E-02$ \\
\hline 23.4 & 29700 & 23.4 & 31600 & 23.4 & 33600 & 2.450 & $3.16 E-02$ & $1.29 E-02$ \\
\hline 16.2 & 24300 & 16.3 & 25000 & 16.2 & 26300 & 1.700 & $2.52 E-02$ & $1.48 E-02$ \\
\hline 11.3 & 19100 & 11.3 & 19900 & 11.3 & 20600 & 1.183 & $1.99 E-02$ & $1.68 E-02$ \\
\hline 7.86 & 15100 & 7.85 & 15500 & 7.88 & 16200 & 0.823 & $1.56 E-02$ & 1.89E-02 \\
\hline 5.46 & 11900 & 5.45 & 12200 & 5.46 & 12700 & 0.571 & $1.23 E-02$ & $2.15 E-02$ \\
\hline 3.8 & 9520 & 3.79 & 9990 & 3.79 & 10200 & 0.397 & $9.90 E-03$ & $2.49 E-02$ \\
\hline 2.64 & 7760 & 2.64 & 8190 & 2.63 & 8360 & 0.276 & $8.10 E-03$ & $2.93 E-02$ \\
\hline 1.83 & 6510 & 1.83 & 6790 & 1.83 & 6950 & 0.192 & $6.75 E-03$ & $3.52 E-02$ \\
\hline 1.27 & 5560 & 1.28 & 5780 & 1.28 & 5930 & 0.134 & $5.76 E-03$ & $4.31 E-02$ \\
\hline 0.89 & 4840 & 0.882 & 5030 & 0.884 & 5190 & 0.093 & $5.02 E-03$ & $5.41 E-02$ \\
\hline 0.618 & 4350 & 0.62 & 4530 & 0.62 & 4650 & 0.065 & $4.51 E-03$ & $6.95 E-02$ \\
\hline 0.422 & 3890 & 0.422 & 4080 & 0.427 & 4220 & 0.044 & $4.06 E-03$ & $9.16 E-02$ \\
\hline 0.3 & 3620 & 0.299 & 3770 & 0.297 & 3920 & 0.031 & $3.77 E-03$ & $1.21 E-01$ \\
\hline 0.204 & 3350 & 0.207 & 3470 & 0.208 & 3610 & 0.022 & $3.48 E-03$ & $1.61 E-01$ \\
\hline 0.143 & 3150 & 0.143 & 3290 & 0.144 & 3390 & 0.015 & $3.28 E-03$ & $2.18 E-01$ \\
\hline 0.0992 & 3050 & 0.101 & 3170 & 0.0989 & 3250 & 0.010 & $3.16 E-03$ & $3.02 E-01$ \\
\hline
\end{tabular}




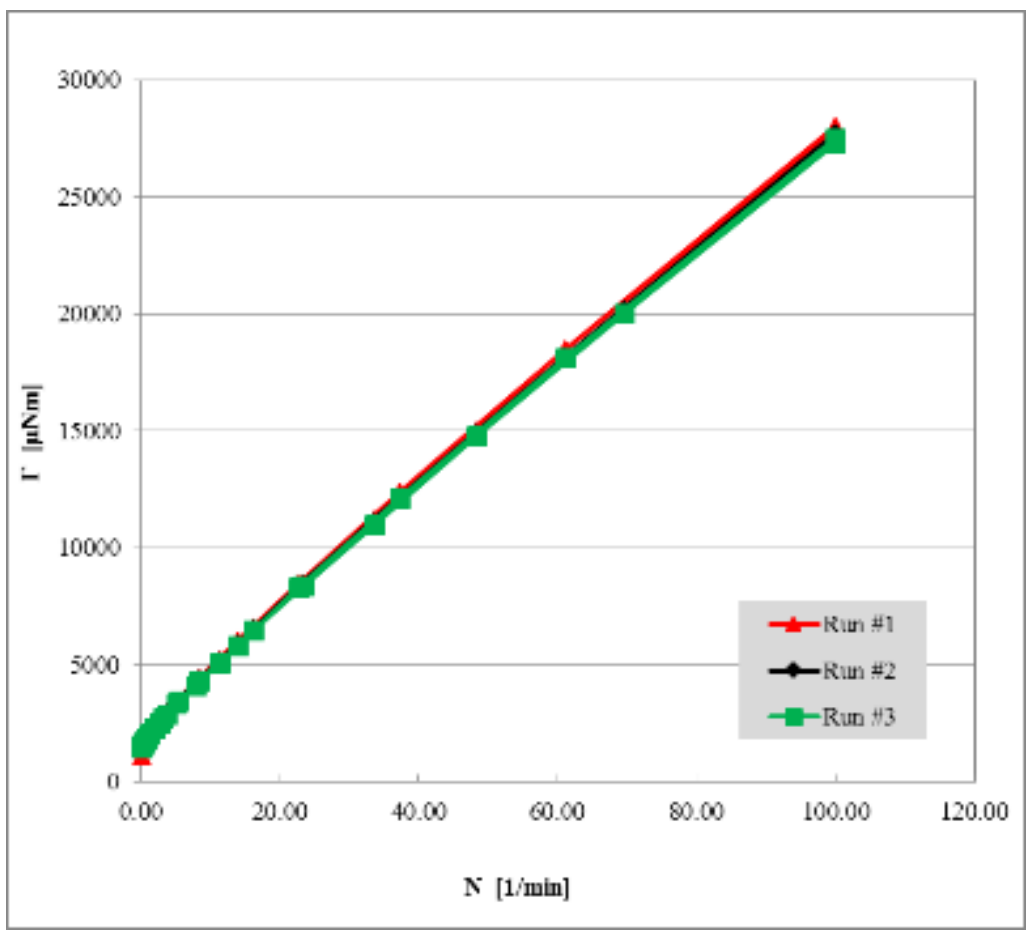

Figure A - 7: Torque vs. Angular Speed using Double spiral spindle on Mix\#3, with $0 \%$ beads by volume. Portrays data from Table A-7.

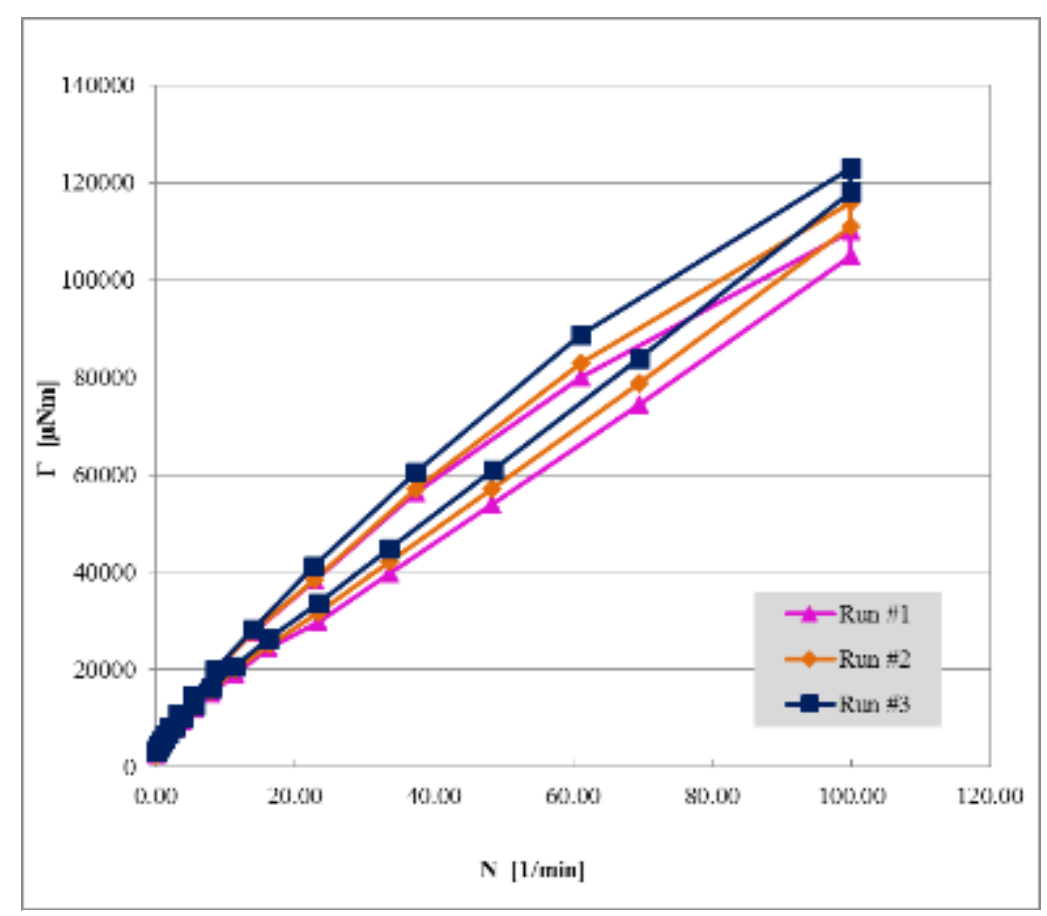

Figure A - 8: Torque vs. Angular Speed using Double spiral spindle on Mix\#3, with $40 \%$ beads by volume. Portrays data from Table A-8. 
Table A - 9: Measured data for Mix\#3 with 0 \% beads using Six-blade Vane.

\begin{tabular}{|c|c|c|c|c|c|c|c|c|}
\hline \multicolumn{6}{|c|}{6 Blade Vane } & \multirow{2}{*}{\multicolumn{3}{|c|}{$\begin{array}{c}\text { Bead } \% \\
0 \%\end{array}$}} \\
\hline Geom.: & $R H N-\varepsilon$ & & & \multicolumn{2}{|c|}{ Z43S } & & & \\
\hline \multicolumn{2}{|c|}{ Run \#1 } & \multicolumn{2}{|c|}{ Run \#2 } & \multicolumn{2}{|c|}{ Run \#3 } & \multirow{2}{*}{\multicolumn{3}{|c|}{$\begin{array}{l}\text { Average } \\
\text { values }\end{array}$}} \\
\hline \multicolumn{2}{|c|}{ NIST Code: SMC-81A } & \multicolumn{2}{|c|}{ NIST Code: SMC-81B } & \multicolumn{2}{|c|}{ NIST Code: SMC-81C } & & & \\
\hline $\mathrm{N}$ & $\Gamma$ & $\mathrm{N}$ & $\Gamma$ & $\mathrm{N}$ & $\Gamma$ & $\mathbf{N}$ & $\Gamma$ & $\boldsymbol{\Gamma} / \mathbf{N}$ (Angular \\
\hline $1 / \mathrm{min}$ & $\mu \mathrm{Nm}$ & $1 / \mathrm{min}$ & $\mu \mathrm{Nm}$ & $1 / \mathrm{min}$ & $\mu \mathrm{Nm}$ & $\mathrm{rad} / \mathrm{s}$ & $\mathrm{Nm}$ & momentum) \\
\hline 0.0978 & 433 & 0.0984 & 525 & 0.0977 & 430 & 0.010 & 4.63E-04 & $4.51 E-02$ \\
\hline 0.163 & 574 & 0.164 & 538 & 0.163 & 575 & 0.017 & $5.62 E-04$ & $3.29 E-02$ \\
\hline 0.268 & 618 & 0.268 & 567 & 0.268 & 615 & 0.028 & $6.00 E-04$ & $2.14 E-02$ \\
\hline 0.439 & 653 & 0.44 & 612 & 0.439 & 649 & 0.046 & $6.38 E-04$ & $1.39 E-02$ \\
\hline 0.72 & 704 & 0.72 & 662 & 0.72 & 700 & 0.075 & $6.89 E-04$ & $9.13 E-03$ \\
\hline 1.18 & 777 & 1.18 & 736 & 1.18 & 774 & 0.124 & $7.62 E-04$ & 6.17E-03 \\
\hline 1.93 & 877 & 1.93 & 838 & 1.93 & 876 & 0.202 & $8.64 E-04$ & 4.27E-03 \\
\hline 3.16 & 1020 & 3.16 & 983 & 3.16 & 1020 & 0.331 & $1.01 E-03$ & $3.05 E-03$ \\
\hline 5.18 & 1230 & 5.18 & 1190 & 5.18 & 1230 & 0.542 & $1.22 E-03$ & $2.24 E-03$ \\
\hline 8.48 & 1520 & 8.48 & 1470 & 8.48 & 1530 & 0.888 & $1.51 E-03$ & $1.70 E-03$ \\
\hline 13.9 & 1960 & 13.9 & 1900 & 13.9 & 1970 & 1.456 & $1.94 E-03$ & $1.34 E-03$ \\
\hline 22.8 & 2590 & 22.8 & 2530 & 22.8 & 2610 & 2.388 & $2.58 E-03$ & $1.08 E-03$ \\
\hline 37.3 & 3570 & 37.3 & 3500 & 37.3 & 3600 & 3.906 & $3.56 E-03$ & $9.11 E-04$ \\
\hline 61 & 5060 & 61 & 4990 & 61 & 5110 & 6.388 & $5.05 E-03$ & 7.91E-04 \\
\hline 100 & 7410 & 100 & 7330 & 100 & 7480 & 10.472 & $7.41 E-03$ & $7.07 E-04$ \\
\hline 100 & 7390 & 100 & 7320 & 100 & 7460 & 10.472 & $7.39 E-03$ & $7.06 E-04$ \\
\hline 69.5 & 5560 & 69.5 & 5500 & 69.5 & 5590 & 7.278 & $5.55 E-03$ & 7.63E-04 \\
\hline 48.3 & 4230 & 48.3 & 4190 & 48.3 & 4250 & 5.058 & $4.22 E-03$ & $8.35 E-04$ \\
\hline 33.6 & 3270 & 33.6 & 3240 & 33.6 & 3280 & 3.519 & $3.26 E-03$ & $9.27 E-04$ \\
\hline 23.4 & 2570 & 23.4 & 2550 & 23.4 & 2590 & 2.450 & 2.57E-03 & $1.05 E-03$ \\
\hline 16.2 & 2070 & 16.2 & 2050 & 16.2 & 2080 & 1.696 & 2.07E-03 & $1.22 E-03$ \\
\hline 11.3 & 1700 & 11.3 & 1690 & 11.3 & 1700 & 1.183 & $1.70 E-03$ & $1.43 E-03$ \\
\hline 7.85 & 1420 & 7.85 & 1410 & 7.85 & 1420 & 0.822 & $1.42 E-03$ & $1.72 E-03$ \\
\hline 5.45 & 1210 & 5.45 & 1190 & 5.45 & 1210 & 0.571 & $1.20 E-03$ & $2.11 E-03$ \\
\hline 3.79 & 1040 & 3.79 & 1030 & 3.79 & 1040 & 0.397 & $1.04 E-03$ & $2.61 E-03$ \\
\hline 2.64 & 917 & 2.64 & 907 & 2.64 & 916 & 0.276 & $9.13 E-04$ & $3.30 E-03$ \\
\hline 1.83 & 820 & 1.83 & 810 & 1.83 & 818 & 0.192 & 8.16E-04 & $4.26 E-03$ \\
\hline 1.27 & 744 & 1.27 & 735 & 1.27 & 742 & 0.133 & $7.40 E-04$ & $5.57 E-03$ \\
\hline 0.886 & 684 & 0.886 & 675 & 0.886 & 682 & 0.093 & $6.80 E-04$ & 7.33E-03 \\
\hline 0.616 & 637 & 0.616 & 628 & 0.616 & 634 & 0.065 & $6.33 E-04$ & $9.81 E-03$ \\
\hline 0.428 & 599 & 0.426 & 596 & 0.428 & 596 & 0.045 & $5.97 E-04$ & $1.33 E-02$ \\
\hline 0.298 & 569 & 0.298 & 561 & 0.298 & 565 & 0.031 & $5.65 E-04$ & $1.81 E-02$ \\
\hline 0.207 & 544 & 0.207 & 537 & 0.207 & 541 & 0.022 & $5.41 E-04$ & $2.49 E-02$ \\
\hline 0.144 & 525 & 0.144 & 518 & 0.144 & 521 & 0.015 & $5.21 E-04$ & $3.46 E-02$ \\
\hline 0.0999 & 510 & 0.0999 & 502 & 0.0996 & 506 & 0.010 & $5.06 E-04$ & $4.84 E-02$ \\
\hline
\end{tabular}


Table A - 10: Measured data for Mix\#3 with 40 \% beads using Six-blade Vane.

\begin{tabular}{|c|c|c|c|c|c|c|c|c|}
\hline \multicolumn{6}{|c|}{6 Blade Vane } & \multicolumn{3}{|c|}{$\begin{array}{c}\text { Bead \% } \\
40 \%\end{array}$} \\
\hline Run & & Run & & Run & $\# 3$ & \multirow{2}{*}{\multicolumn{3}{|c|}{$\begin{array}{c}\text { Average } \\
\text { values }\end{array}$}} \\
\hline NIST Code: & $S M C-81 \mathrm{~J}$ & NIST Code: & $S M C-81 K$ & NIST Code: & $S M C-81 L$ & & & \\
\hline $\mathrm{N}$ & $\Gamma$ & $\mathrm{N}$ & $\Gamma$ & $\mathrm{N}$ & $\Gamma$ & $\mathbf{N}$ & $\Gamma$ & $\Gamma / N$ (Angular \\
\hline $1 / \mathrm{min}$ & $\mu \mathrm{Nm}$ & $1 / \mathrm{min}$ & $\mu \mathrm{Nm}$ & $1 / \mathrm{min}$ & $\mu \mathrm{Nm}$ & $\mathrm{rad} / \mathrm{s}$ & $\mathrm{Nm}$ & momentum) \\
\hline 0.0957 & 1070 & 0.0963 & 1080 & 0.0976 & 1050 & 0.010 & $1.07 E-03$ & $1.06 E-01$ \\
\hline 0.163 & 1360 & 0.164 & 1340 & 0.161 & 1320 & 0.017 & $1.34 E-03$ & $7.87 E-02$ \\
\hline 0.266 & 1480 & 0.269 & 1460 & 0.266 & 1430 & 0.028 & $1.46 E-03$ & $5.21 E-02$ \\
\hline 0.441 & 1640 & 0.443 & 1610 & 0.438 & 1580 & 0.046 & $1.61 E-03$ & $3.49 E-02$ \\
\hline 0.721 & 1800 & 0.717 & 1750 & 0.717 & 1750 & 0.075 & $1.77 E-03$ & $2.35 E-02$ \\
\hline 1.18 & 2070 & 1.18 & 2020 & 1.19 & 2040 & 0.124 & $2.04 E-03$ & $1.65 E-02$ \\
\hline 1.94 & 2430 & 1.94 & 2390 & 1.93 & 2440 & 0.203 & $2.42 E-03$ & $1.19 E-02$ \\
\hline 3.17 & 2970 & 3.17 & 2960 & 3.16 & 3000 & 0.332 & $2.98 E-03$ & $8.98 E-03$ \\
\hline 5.17 & 3730 & 5.18 & 3770 & 5.18 & 3820 & 0.542 & $3.77 E-03$ & $6.96 E-03$ \\
\hline 8.48 & 4890 & 8.49 & 4890 & 8.48 & 5050 & 0.888 & $4.94 E-03$ & $5.56 E-03$ \\
\hline 13.9 & 6430 & 13.9 & 6430 & 13.9 & 6770 & 1.456 & $6.54 E-03$ & $4.50 E-03$ \\
\hline 22.8 & 8770 & 22.8 & 8730 & 22.8 & 9210 & 2.388 & $8.90 E-03$ & $3.73 E-03$ \\
\hline 37.3 & 11900 & 37.3 & 12300 & 37.3 & 12700 & 3.906 & $1.23 E-02$ & $3.15 E-03$ \\
\hline 61.1 & 16200 & 61.1 & 17200 & 61.1 & 17900 & 6.398 & $1.71 E-02$ & $2.67 E-03$ \\
\hline 100 & 23800 & 100 & 24600 & 100 & 25700 & 10.472 & $2.47 E-02$ & $2.36 E-03$ \\
\hline 100 & 22600 & 99.9 & 23800 & 99.9 & 25200 & 10.465 & $2.39 E-02$ & $2.28 E-03$ \\
\hline 69.5 & 17100 & 69.5 & 17400 & 69.5 & 18000 & 7.278 & $1.75 E-02$ & $2.40 E-03$ \\
\hline 48.3 & 12600 & 48.3 & 13000 & 48.3 & 13500 & 5.058 & $1.30 E-02$ & $2.58 E-03$ \\
\hline 33.6 & 9590 & 33.6 & 10000 & 33.6 & 10400 & 3.519 & $1.00 E-02$ & $2.84 E-03$ \\
\hline 23.4 & 7380 & 23.4 & 7650 & 23.4 & 7990 & 2.450 & 7.67E-03 & $3.13 E-03$ \\
\hline 16.2 & 5800 & 16.2 & 5960 & 16.2 & 6240 & 1.696 & $6.00 E-03$ & $3.54 E-03$ \\
\hline 11.3 & 4590 & 11.3 & 4730 & 11.3 & 4910 & 1.183 & $4.74 E-03$ & $4.01 E-03$ \\
\hline 7.85 & 3680 & 7.85 & 3790 & 7.85 & 3950 & 0.822 & $3.81 E-03$ & $4.63 E-03$ \\
\hline 5.46 & 3000 & 5.45 & 3070 & 5.46 & 3170 & 0.571 & $3.08 E-03$ & $5.39 E-03$ \\
\hline 3.79 & 2490 & 3.79 & 2550 & 3.79 & 2600 & 0.397 & $2.55 E-03$ & $6.42 E-03$ \\
\hline 2.64 & 2080 & 2.64 & 2130 & 2.64 & 2170 & 0.276 & $2.13 E-03$ & 7.69E-03 \\
\hline 1.83 & 1800 & 1.83 & 1800 & 1.83 & 1870 & 0.192 & $1.82 E-03$ & $9.51 E-03$ \\
\hline 1.27 & 1570 & 1.28 & 1580 & 1.27 & 1630 & 0.133 & $1.59 E-03$ & 1.19E-02 \\
\hline 0.888 & 1380 & 0.884 & 1400 & 0.884 & 1460 & 0.093 & $1.41 E-03$ & $1.52 E-02$ \\
\hline 0.613 & 1260 & 0.611 & 1260 & 0.62 & 1310 & 0.064 & $1.28 E-03$ & $1.98 E-02$ \\
\hline 0.429 & 1150 & 0.429 & 1160 & 0.429 & 1200 & 0.045 & $1.17 E-03$ & $2.60 E-02$ \\
\hline 0.297 & 1090 & 0.298 & 1090 & 0.298 & 1110 & 0.031 & $1.10 E-03$ & $3.52 E-02$ \\
\hline 0.207 & 1020 & 0.206 & 1040 & 0.207 & 1050 & 0.022 & $1.04 E-03$ & $4.79 E-02$ \\
\hline 0.145 & 974 & 0.143 & 970 & 0.143 & 995 & 0.015 & $9.80 E-04$ & $6.51 E-02$ \\
\hline 0.101 & 930 & 0.0992 & 945 & 0.1 & 958 & 0.010 & $9.44 E-04$ & $9.01 E-02$ \\
\hline
\end{tabular}




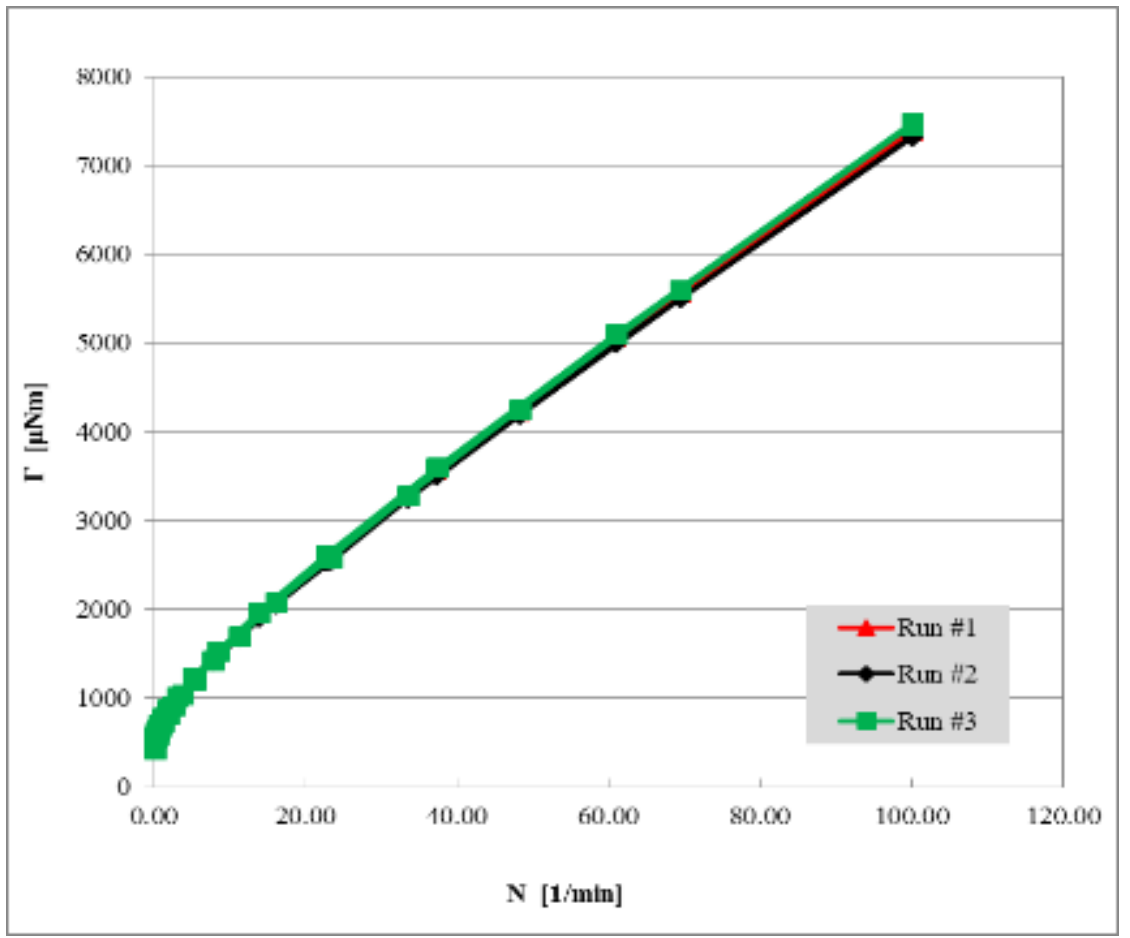

Figure A - 5: Torque vs. Angular Speed using six-blade vane on Mix\#3, with 0 \% beads by volume. Portrays data from Table A-9.

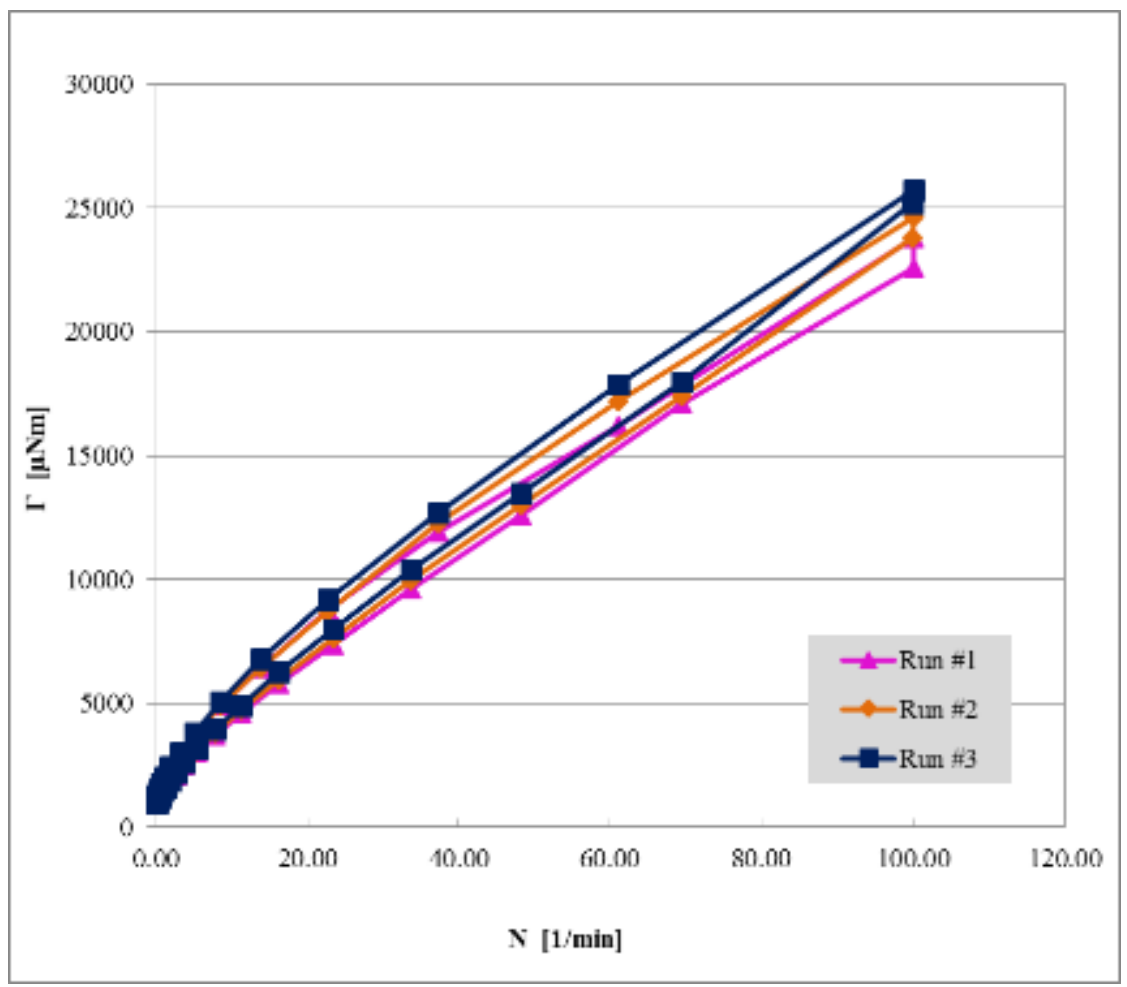

Figure A - 6: Torque vs. Angular Speed using six-blade vane on Mix\#3, with $40 \%$ beads by volume. Portrays data from Table A-10. 
Table A - 11: Measured data for Mix\#3 with 0 \% beads using Serrated Coaxial Cylinder.

\begin{tabular}{|c|c|c|c|c|c|c|c|c|}
\hline \multicolumn{6}{|c|}{ Serrated Coaxial Cylinder } & \multirow{2}{*}{\multicolumn{3}{|c|}{$\begin{array}{c}\text { Bead } \% \\
0 \%\end{array}$}} \\
\hline \multicolumn{4}{|c|}{ SS18 } & \multicolumn{2}{|c|}{ Z43S } & & & \\
\hline \multicolumn{2}{|c|}{ Run \#1 } & \multicolumn{2}{|c|}{ Run \#2 } & \multicolumn{2}{|c|}{ Run \#3 } & \multirow{2}{*}{\multicolumn{3}{|c|}{$\begin{array}{c}\text { Average } \\
\text { values }\end{array}$}} \\
\hline NIST Code: & $S M C-81 G$ & NIST Code: & $S M C-81 H$ & NIST Co & $S M C-81 I$ & & & \\
\hline $\mathrm{N}$ & \begin{tabular}{|c|}
$\Gamma$ \\
\end{tabular} & $\mathrm{N}$ & $\Gamma$ & $\mathrm{N}$ & $\Gamma$ & $\mathbf{N}$ & $\Gamma$ & $\Gamma / N$ (Angular \\
\hline $1 / \mathrm{min}$ & $\mu \mathrm{Nm}$ & $1 / \mathrm{min}$ & $\mu \mathrm{Nm}$ & $1 / \mathrm{min}$ & $\mu \mathrm{Nm}$ & $\mathrm{rad} / \mathrm{s}$ & $\mathrm{Nm}$ & momentum) \\
\hline 0.0977 & 442 & 0.1 & 351 & 0.099 & 322 & 0.010 & $3.72 E-04$ & $3.59 E-02$ \\
\hline 0.164 & 591 & 0.164 & 379 & 0.164 & 393 & 0.017 & $4.54 E-04$ & $2.65 E-02$ \\
\hline 0.268 & 623 & 0.268 & 420 & 0.268 & 438 & 0.028 & 4.94E-04 & $1.76 E-02$ \\
\hline 0.44 & 665 & 0.439 & 475 & 0.439 & 496 & 0.046 & $5.45 E-04$ & $1.19 E-02$ \\
\hline 0.72 & 725 & 0.72 & 551 & 0.719 & 573 & 0.075 & $6.16 E-04$ & $8.18 E-03$ \\
\hline 1.18 & 808 & 1.18 & 650 & 1.18 & 666 & 0.124 & $7.08 E-04$ & $5.73 E-03$ \\
\hline 1.93 & 925 & 1.93 & 765 & 1.93 & 783 & 0.202 & $8.24 E-04$ & $4.08 E-03$ \\
\hline 3.16 & 1090 & 3.16 & 932 & 3.16 & 937 & 0.331 & $9.86 E-04$ & $2.98 E-03$ \\
\hline 5.18 & 1320 & 5.18 & 1160 & 5.18 & 1160 & 0.542 & $1.21 E-03$ & $2.24 E-03$ \\
\hline 8.48 & 1640 & 8.48 & 1480 & 8.48 & 1480 & 0.888 & $1.53 E-03$ & $1.73 E-03$ \\
\hline 13.9 & 2110 & 13.9 & 1930 & 13.9 & 1940 & 1.456 & $1.99 E-03$ & $1.37 E-03$ \\
\hline 22.8 & 2800 & 22.8 & 2590 & 22.8 & 2610 & 2.388 & $2.67 E-03$ & $1.12 E-03$ \\
\hline 37.3 & 3860 & 37.3 & 3610 & 37.3 & 3650 & 3.906 & $3.71 E-03$ & $9.49 E-04$ \\
\hline 61 & 5520 & 61 & 5190 & 61 & 5240 & 6.388 & $5.32 E-03$ & $8.32 E-04$ \\
\hline 100 & 8030 & 100 & 7590 & 100 & 7640 & 10.472 & $7.75 E-03$ & $7.40 E-04$ \\
\hline 100 & 7900 & 100 & 7520 & 100 & 7590 & 10.472 & 7.67E-03 & $7.32 E-04$ \\
\hline 69.5 & 5910 & 69.5 & 5640 & 69.5 & 5680 & 7.278 & $5.74 E-03$ & 7.89E-04 \\
\hline 48.3 & 4460 & 48.3 & 4260 & 48.3 & 4290 & 5.058 & 4.34E-03 & 8.57E-04 \\
\hline 33.6 & 3410 & 33.6 & 3270 & 33.6 & 3280 & 3.519 & 3.32E-03 & $9.44 E-04$ \\
\hline 23.4 & 2660 & 23.4 & 2550 & 23.4 & 2550 & 2.450 & $2.59 E-03$ & $1.06 E-03$ \\
\hline 16.2 & 2110 & 16.2 & 2020 & 16.2 & 2030 & 1.696 & $2.05 E-03$ & $1.21 E-03$ \\
\hline 11.3 & 1710 & 11.3 & 1640 & 11.3 & 1640 & 1.183 & $1.66 E-03$ & $1.41 E-03$ \\
\hline 7.85 & 1410 & 7.85 & 1340 & 7.85 & 1340 & 0.822 & $1.36 E-03$ & $1.66 E-03$ \\
\hline 5.45 & 1170 & 5.45 & 1110 & 5.45 & 1110 & 0.571 & $1.13 E-03$ & $1.98 E-03$ \\
\hline 3.79 & 991 & 3.79 & 940 & 3.79 & 941 & 0.397 & $9.57 E-04$ & $2.41 E-03$ \\
\hline 2.64 & 850 & 2.64 & 804 & 2.64 & 806 & 0.276 & $8.20 E-04$ & $2.97 E-03$ \\
\hline 1.83 & 737 & 1.83 & 697 & 1.83 & 700 & 0.192 & $7.11 E-04$ & $3.71 E-03$ \\
\hline 1.27 & 647 & 1.27 & 612 & 1.27 & 616 & 0.133 & $6.25 E-04$ & $4.70 E-03$ \\
\hline 0.886 & 574 & 0.886 & 543 & 0.886 & 548 & 0.093 & $5.55 E-04$ & $5.98 E-03$ \\
\hline 0.616 & 514 & 0.616 & 485 & 0.616 & 494 & 0.065 & $4.98 E-04$ & 7.71E-03 \\
\hline 0.428 & 466 & 0.427 & 441 & 0.428 & 449 & 0.045 & $4.52 E-04$ & $1.01 E-02$ \\
\hline 0.298 & 427 & 0.298 & 407 & 0.298 & 413 & 0.031 & $4.16 E-04$ & $1.33 E-02$ \\
\hline 0.207 & 395 & 0.207 & 377 & 0.207 & 383 & 0.022 & $3.85 E-04$ & $1.78 E-02$ \\
\hline 0.144 & 370 & 0.144 & 355 & 0.144 & 360 & 0.015 & $3.62 E-04$ & $2.40 E-02$ \\
\hline 0.1 & 348 & 0.1 & 336 & 0.1 & 343 & 0.010 & $3.42 E-04$ & $3.27 E-02$ \\
\hline
\end{tabular}


Table A - 12: Measured data for Mix\#3 with 40 \% beads using Serrated Coaxial Cylinder.

\begin{tabular}{|c|c|c|c|c|c|c|c|c|}
\hline \multicolumn{6}{|c|}{ Serrated Coaxial Cylinder } & \multirow{2}{*}{\multicolumn{3}{|c|}{$\begin{array}{c}\text { Bead } \% \\
40 \%\end{array}$}} \\
\hline Geom.: & SS18 & & & \multicolumn{2}{|c|}{ Z43S } & & & \\
\hline \multicolumn{2}{|c|}{ Run \#1 } & \multicolumn{2}{|c|}{ Run \#2 } & \multicolumn{2}{|c|}{ Run \#3 } & \multirow{2}{*}{\multicolumn{3}{|c|}{$\begin{array}{c}\text { Average } \\
\text { values }\end{array}$}} \\
\hline NIST Code: & SMC-81P & NIST Code: & SMC-81Q & NIST Cod & SMC-81R & & & \\
\hline $\mathrm{N}$ & $\Gamma$ & $\mathrm{N}$ & $\Gamma$ & $\mathrm{N}$ & $\Gamma$ & $\mathbf{N}$ & $\Gamma$ & $\Gamma / N$ (Angular \\
\hline $1 / \mathrm{min}$ & $\mu \mathrm{Nm}$ & $1 / \mathrm{min}$ & $\mu \mathrm{Nm}$ & $1 / \mathrm{min}$ & $\mu \mathrm{Nm}$ & $\mathrm{rad} / \mathrm{s}$ & $\mathrm{Nm}$ & momentum) \\
\hline 0.0949 & 1530 & 0.0973 & 1140 & 0.0963 & 1250 & 0.010 & $1.31 E-03$ & $1.30 E-01$ \\
\hline 0.162 & 1920 & 0.164 & 1340 & 0.164 & 1480 & 0.017 & $1.58 E-03$ & $9.24 E-02$ \\
\hline 0.271 & 2020 & 0.268 & 1360 & 0.271 & 1510 & 0.028 & $1.63 E-03$ & $5.76 E-02$ \\
\hline 0.442 & 2040 & 0.44 & 1460 & 0.441 & 1590 & 0.046 & $1.70 E-03$ & 3.67E-02 \\
\hline 0.717 & 2200 & 0.722 & 1590 & 0.719 & 1770 & 0.075 & $1.85 E-03$ & $2.46 E-02$ \\
\hline 1.18 & 2320 & 1.18 & 1860 & 1.18 & 2080 & 0.124 & $2.09 E-03$ & $1.69 E-02$ \\
\hline 1.93 & 2660 & 1.93 & 2290 & 1.93 & 2560 & 0.202 & $2.50 E-03$ & $1.24 E-02$ \\
\hline 3.16 & 3140 & 3.16 & 2850 & 3.16 & 3200 & 0.331 & $3.06 E-03$ & $9.26 E-03$ \\
\hline 5.18 & 3900 & 5.18 & 3720 & 5.18 & 4140 & 0.542 & $3.92 E-03$ & $7.23 E-03$ \\
\hline 8.48 & 5050 & 8.48 & 4990 & 8.48 & 5520 & 0.888 & $5.19 E-03$ & $5.84 E-03$ \\
\hline 13.9 & 6690 & 13.9 & 6890 & 13.9 & 7480 & 1.456 & $7.02 E-03$ & $4.82 E-03$ \\
\hline 22.8 & 9090 & 22.8 & 9700 & 22.8 & 10400 & 2.388 & $9.73 E-03$ & $4.08 E-03$ \\
\hline 37.3 & 12700 & 37.3 & 13900 & 37.3 & 14600 & 3.906 & $1.37 E-02$ & $3.52 E-03$ \\
\hline 61 & 16500 & 61 & 18900 & 61.1 & 20400 & 6.391 & $1.86 E-02$ & $2.91 E-03$ \\
\hline 99.9 & 23000 & 100 & 24500 & 100 & 26300 & 10.468 & $2.46 E-02$ & $2.35 E-03$ \\
\hline 100 & 22300 & 100 & 26600 & 100 & 25500 & 10.472 & $2.48 E-02$ & $2.37 E-03$ \\
\hline 69.5 & 18400 & 69.5 & 20200 & 69.5 & 20600 & 7.278 & $1.97 E-02$ & $2.71 E-03$ \\
\hline 48.3 & 14100 & 48.3 & 15200 & 48.3 & 15600 & 5.058 & $1.50 E-02$ & $2.96 E-03$ \\
\hline 33.6 & 10800 & 33.6 & 11600 & 33.6 & 11900 & 3.519 & $1.14 E-02$ & $3.25 E-03$ \\
\hline 23.4 & 8270 & 23.4 & 8780 & 23.4 & 9020 & 2.450 & 8.69E-03 & $3.55 E-03$ \\
\hline 16.2 & 6400 & 16.2 & 6750 & 16.2 & 6950 & 1.696 & $6.70 E-03$ & $3.95 E-03$ \\
\hline 11.3 & 4980 & 11.3 & 5250 & 11.3 & 5400 & 1.183 & $5.21 E-03$ & $4.40 E-03$ \\
\hline 7.85 & 3950 & 7.85 & 4130 & 7.84 & 4290 & 0.822 & $4.12 E-03$ & $5.02 E-03$ \\
\hline 5.45 & 3170 & 5.46 & 3290 & 5.45 & 3420 & 0.571 & $3.29 E-03$ & $5.77 E-03$ \\
\hline 3.79 & 2600 & 3.79 & 2690 & 3.79 & 2810 & 0.397 & $2.70 E-03$ & $6.80 E-03$ \\
\hline 2.64 & 2140 & 2.64 & 2230 & 2.64 & 2320 & 0.276 & $2.23 E-03$ & 8.07E-03 \\
\hline 1.83 & 1800 & 1.83 & 1890 & 1.83 & 1950 & 0.192 & $1.88 E-03$ & $9.81 E-03$ \\
\hline 1.27 & 1560 & 1.27 & 1610 & 1.27 & 1700 & 0.133 & $1.62 E-03$ & $1.22 E-02$ \\
\hline 0.886 & 1350 & 0.886 & 1410 & 0.884 & 1470 & 0.093 & $1.41 E-03$ & $1.52 E-02$ \\
\hline 0.615 & 1200 & 0.616 & 1260 & 0.614 & 1310 & 0.064 & $1.26 E-03$ & $1.95 E-02$ \\
\hline 0.427 & 1080 & 0.427 & 1120 & 0.429 & 1170 & 0.045 & $1.12 E-03$ & $2.51 E-02$ \\
\hline 0.299 & 994 & 0.299 & 1020 & 0.298 & 1080 & 0.031 & $1.03 E-03$ & $3.30 E-02$ \\
\hline 0.207 & 909 & 0.207 & 949 & 0.207 & 989 & 0.022 & $9.49 E-04$ & 4.38E-02 \\
\hline 0.143 & 839 & 0.144 & 876 & 0.143 & 917 & 0.015 & 8.77E-04 & $5.85 E-02$ \\
\hline 0.1 & 794 & 0.101 & 828 & 0.0995 & 863 & 0.010 & $8.28 E-04$ & $7.90 E-02$ \\
\hline
\end{tabular}




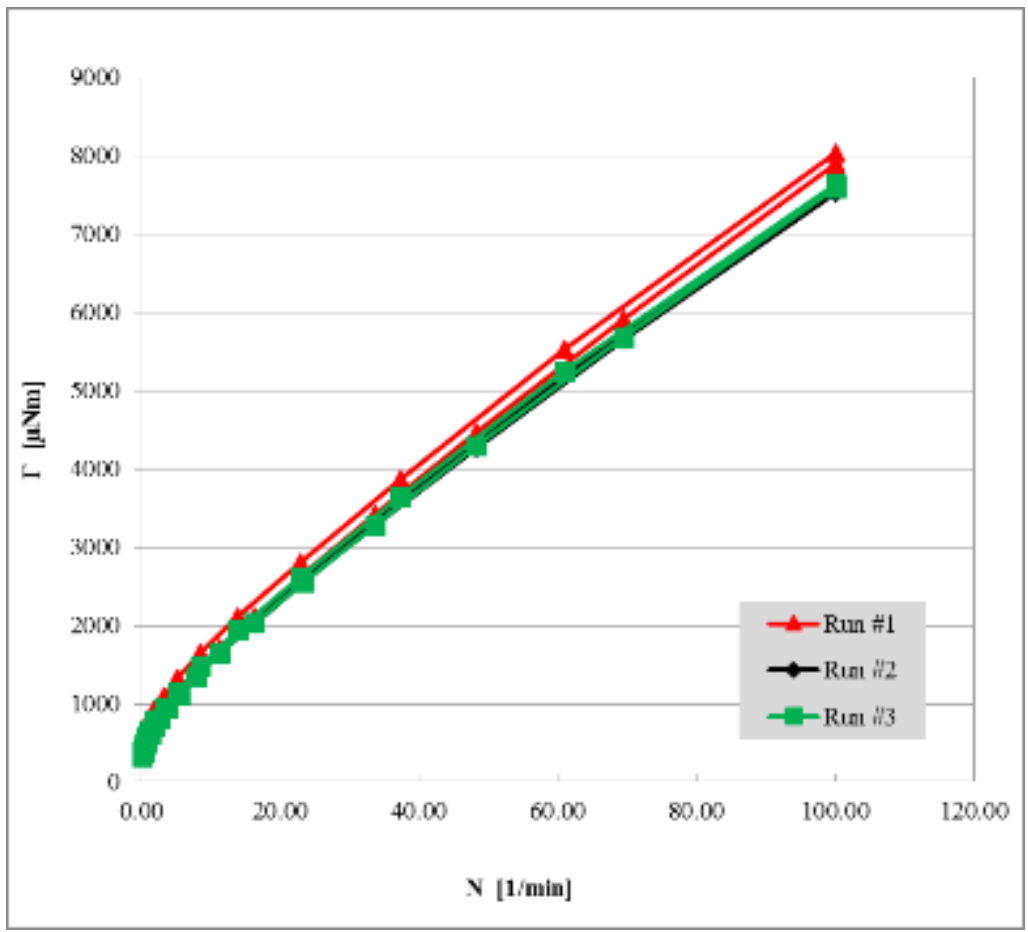

Figure A - 7: Torque vs. Angular Speed using Serrated Coaxial Cylinder on Mix\#3, with 0 \% beads by volume. Portrays data from Table A-11.

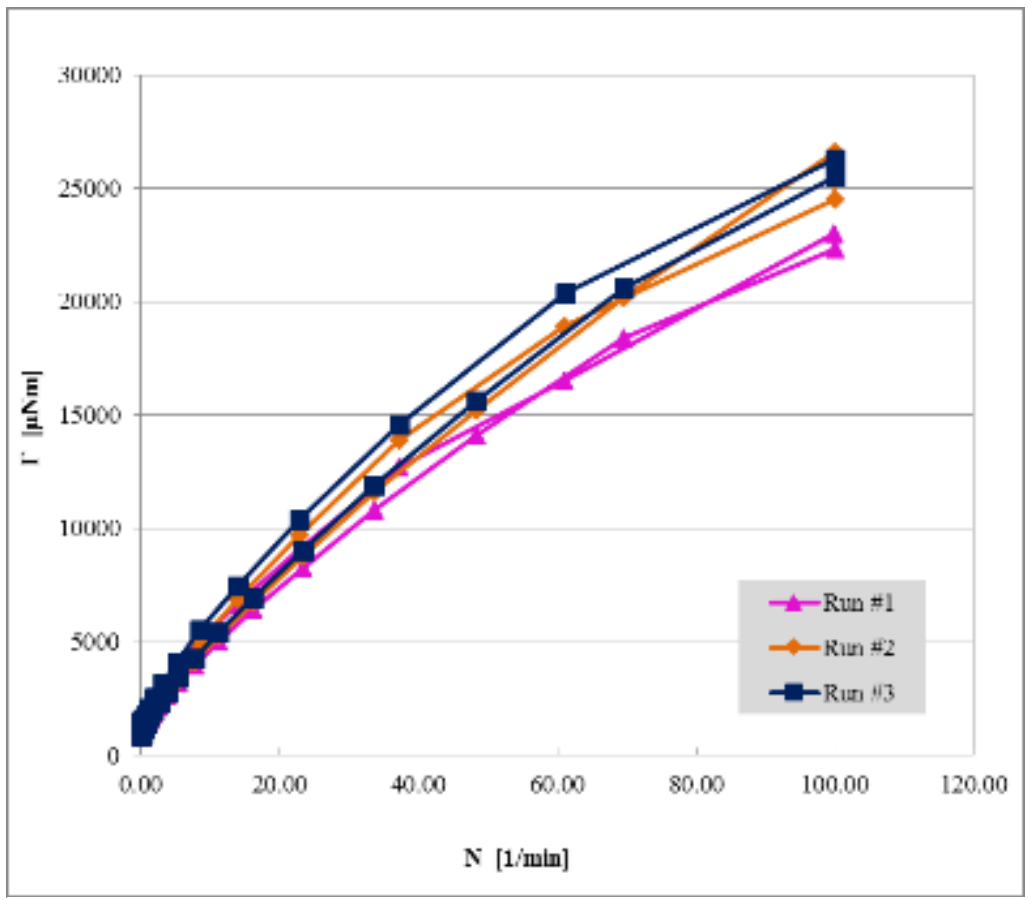

Figure A - 8: Torque vs. Angular Speed using Serrated Coaxial Cylinder on Mix\#3, with 40 \% beads by volume. Portrays data from Table A-12. 
Table A - 13: Measured data for Mix\#4 with $0 \%$ beads using Double spiral spindle.

\begin{tabular}{|c|c|c|c|c|c|c|c|c|}
\hline Geom.: & \multicolumn{3}{|l|}{$R H N-83 A$} & \multicolumn{2}{|c|}{ Cup: $\quad$ Z43S } & \multicolumn{3}{|c|}{$\begin{array}{c}\text { Bead } \% \\
0 \% \\
\end{array}$} \\
\hline \multicolumn{2}{|c|}{ Run \#1 } & \multicolumn{2}{|c|}{ Run \#2 } & \multicolumn{2}{|c|}{ Run \#3 } & \multirow{2}{*}{\multicolumn{3}{|c|}{$\begin{array}{c}\text { Average } \\
\text { values }\end{array}$}} \\
\hline \multicolumn{2}{|c|}{ NIST Code: SMC-85J } & \multicolumn{2}{|c|}{ NIST Code: SMC-85K } & \multicolumn{2}{|c|}{ NIST Code: SMC-85L } & & & \\
\hline $\mathrm{N}$ & $\Gamma$ & $\mathrm{N}$ & $\Gamma$ & $\mathrm{N}$ & $\Gamma$ & $\mathbf{N}$ & $\Gamma$ & $\boldsymbol{\Gamma} / \mathbf{N}$ (Angular \\
\hline $1 / \mathrm{min}$ & $\mu \mathrm{Nm}$ & $1 / \mathrm{min}$ & $\mu \mathrm{Nm}$ & $1 / \mathrm{min}$ & $\mu \mathrm{Nm}$ & $\mathrm{rad} / \mathrm{s}$ & $\mathrm{Nm}$ & momentum) \\
\hline 0.0942 & 1080 & 0.0948 & 964 & 0.0949 & 952 & 0.010 & $9.99 E-04$ & $1.01 E-01$ \\
\hline 0.161 & 1570 & 0.162 & 1420 & 0.162 & 1390 & 0.017 & $1.46 E-03$ & 8.62E-02 \\
\hline 0.268 & 1750 & 0.268 & 1610 & 0.268 & 1590 & 0.028 & $1.65 E-03$ & $5.88 E-02$ \\
\hline 0.44 & 1860 & 0.439 & 1720 & 0.439 & 1700 & 0.046 & $1.76 E-03$ & 3.83E-02 \\
\hline 0.72 & 1970 & 0.72 & 1850 & 0.72 & 1830 & 0.075 & $1.88 E-03$ & $2.50 E-02$ \\
\hline 1.18 & 2140 & 1.18 & 2030 & 1.18 & 2010 & 0.124 & $2.06 E-03$ & $1.67 E-02$ \\
\hline 1.93 & 2380 & 1.93 & 2280 & 1.93 & 2270 & 0.202 & $2.31 E-03$ & $1.14 E-02$ \\
\hline 3.16 & 2770 & 3.16 & 2680 & 3.16 & 2670 & 0.331 & $2.71 E-03$ & 8.18E-03 \\
\hline 5.18 & 3370 & 5.18 & 3290 & 5.18 & 3290 & 0.542 & $3.32 E-03$ & $6.11 E-03$ \\
\hline 8.48 & 4310 & 8.48 & 4220 & 8.48 & 4220 & 0.888 & $4.25 E-03$ & 4.79E-03 \\
\hline 13.9 & 5780 & 13.9 & 5710 & 13.9 & 5700 & 1.456 & 5.73E-03 & $3.94 E-03$ \\
\hline 22.8 & 8100 & 22.8 & 8020 & 22.8 & 8020 & 2.388 & $8.05 E-03$ & $3.37 E-03$ \\
\hline 37.3 & 11800 & 37.3 & 11700 & 37.3 & 11700 & 3.906 & $1.17 E-02$ & $3.00 E-03$ \\
\hline 61.1 & 17500 & 61.1 & 17500 & 61.1 & 17400 & 6.398 & $1.75 E-02$ & $2.73 E-03$ \\
\hline 100 & 26400 & 100 & 26500 & 100 & 26400 & 10.472 & $2.64 E-02$ & $2.52 E-03$ \\
\hline 100 & 26200 & 100 & 26300 & 100 & 26300 & 10.472 & $2.63 E-02$ & $2.51 E-03$ \\
\hline 69.5 & 19100 & 69.5 & 19200 & 69.5 & 19200 & 7.278 & $1.92 E-02$ & $2.63 E-03$ \\
\hline 48.3 & 14100 & 48.3 & 14200 & 48.3 & 14200 & 5.058 & $1.42 E-02$ & $2.80 E-03$ \\
\hline 33.6 & 10600 & 33.6 & 10600 & 33.6 & 10600 & 3.519 & $1.06 E-02$ & $3.01 E-03$ \\
\hline 23.4 & 8060 & 23.4 & 8110 & 23.4 & 8110 & 2.450 & 8.09E-03 & $3.30 E-03$ \\
\hline 16.2 & 6260 & 16.2 & 6290 & 16.2 & 6290 & 1.696 & $6.28 E-03$ & $3.70 E-03$ \\
\hline 11.3 & 4960 & 11.3 & 4990 & 11.3 & 4990 & 1.183 & $4.98 E-03$ & $4.21 E-03$ \\
\hline 7.85 & 4030 & 7.85 & 4050 & 7.85 & 4050 & 0.822 & $4.04 E-03$ & $4.92 E-03$ \\
\hline 5.46 & 3350 & 5.46 & 3370 & 5.46 & 3360 & 0.572 & $3.36 E-03$ & $5.88 E-03$ \\
\hline 3.79 & 2860 & 3.79 & 2870 & 3.79 & 2870 & 0.397 & $2.87 E-03$ & $7.22 E-03$ \\
\hline 2.64 & 2500 & 2.64 & 2510 & 2.64 & 2500 & 0.276 & $2.50 E-03$ & $9.05 E-03$ \\
\hline 1.83 & 2240 & 1.83 & 2240 & 1.83 & 2230 & 0.192 & $2.24 E-03$ & $1.17 E-02$ \\
\hline 1.27 & 2030 & 1.27 & 2030 & 1.27 & 2030 & 0.133 & $2.03 E-03$ & $1.53 E-02$ \\
\hline 0.886 & 1880 & 0.886 & 1880 & 0.886 & 1880 & 0.093 & $1.88 E-03$ & $2.03 E-02$ \\
\hline 0.616 & 1770 & 0.616 & 1770 & 0.616 & 1760 & 0.065 & $1.77 E-03$ & $2.74 E-02$ \\
\hline 0.428 & 1690 & 0.428 & 1680 & 0.428 & 1680 & 0.045 & $1.68 E-03$ & $3.76 E-02$ \\
\hline 0.297 & 1620 & 0.297 & 1610 & 0.297 & 1610 & 0.031 & $1.61 E-03$ & $5.19 E-02$ \\
\hline 0.207 & 1560 & 0.207 & 1560 & 0.207 & 1550 & 0.022 & $1.56 E-03$ & 7.18E-02 \\
\hline 0.144 & 1520 & 0.144 & 1520 & 0.144 & 1510 & 0.015 & $1.52 E-03$ & $1.01 E-01$ \\
\hline 0.0998 & 1490 & 0.0998 & 1490 & 0.0998 & 1480 & 0.010 & $1.49 E-03$ & $1.42 E-01$ \\
\hline
\end{tabular}


Table A - 14: Measured data for Mix\#4 with $40 \%$ beads using Double spiral spindle.

\begin{tabular}{|c|c|c|c|c|c|c|c|c|}
\hline \multicolumn{6}{|c|}{ Double Spiral } & \multirow{2}{*}{\multicolumn{3}{|c|}{$\begin{array}{c}\text { Bead \% } \\
40 \%\end{array}$}} \\
\hline Geom.: & \multicolumn{3}{|l|}{$R H N-83 A$} & \multicolumn{2}{|c|}{ Cup: $\quad$ Z43S } & & & \\
\hline \multicolumn{2}{|c|}{ Run \#1 } & \multicolumn{2}{|c|}{ Run \#2 } & \multicolumn{2}{|c|}{ Run \#3 } & \multirow{2}{*}{\multicolumn{3}{|c|}{$\begin{array}{c}\text { Average } \\
\text { values }\end{array}$}} \\
\hline \multicolumn{2}{|c|}{ NIST Code: SMC-85A } & \multicolumn{2}{|c|}{ NIST Code: SMC-85B } & \multicolumn{2}{|c|}{ NIST Code: SMC-85C } & & & \\
\hline $\mathrm{N}$ & $\Gamma$ & $\mathrm{N}$ & $\Gamma$ & $\mathrm{N}$ & $\Gamma$ & $\mathbf{N}$ & $\Gamma$ & \multirow{2}{*}{$\begin{array}{c}\Gamma / \boldsymbol{N} \text { (Angular } \\
\text { momentum) }\end{array}$} \\
\hline $1 / \mathrm{min}$ & $\mu \mathrm{Nm}$ & $1 / \mathrm{min}$ & $\mu \mathrm{Nm}$ & $1 / \mathrm{min}$ & $\mu \mathrm{Nm}$ & $\mathrm{rad} / \mathrm{s}$ & $\mathrm{Nm}$ & \\
\hline 0.44 & 4720 & 0.0873 & 2370 & 0.0887 & 2490 & 0.022 & 3.19E-03 & $1.49 E-01$ \\
\hline 0.44 & 4720 & 0.161 & 3330 & 0.155 & 3500 & 0.026 & $3.85 E-03$ & $1.46 E-01$ \\
\hline 0.44 & 4720 & 0.263 & 3810 & 0.267 & 4060 & 0.034 & $4.20 E-03$ & $1.24 E-01$ \\
\hline 0.44 & 4720 & 0.436 & 4360 & 0.441 & 4620 & 0.046 & 4.57E-03 & $9.93 E-02$ \\
\hline 0.719 & 5410 & 0.721 & 4930 & 0.724 & 5190 & 0.076 & $5.18 E-03$ & $6.85 E-02$ \\
\hline 1.17 & 6440 & 1.19 & 5780 & 1.19 & 6120 & 0.124 & $6.11 E-03$ & $4.93 E-02$ \\
\hline 1.93 & 7750 & 1.93 & 7130 & 1.93 & 7410 & 0.202 & 7.43E-03 & $3.68 E-02$ \\
\hline 3.16 & 9950 & 3.16 & 9060 & 3.17 & 9460 & 0.331 & $9.49 E-03$ & $2.86 E-02$ \\
\hline 5.19 & 13300 & 5.17 & 12300 & 5.18 & 12800 & 0.542 & $1.28 E-02$ & $2.36 E-02$ \\
\hline 8.48 & 18700 & 8.49 & 17400 & 8.48 & 18100 & 0.888 & $1.81 E-02$ & $2.03 E-02$ \\
\hline 13.9 & 26300 & 13.9 & 24700 & 13.9 & 25400 & 1.456 & $2.55 E-02$ & $1.75 E-02$ \\
\hline 22.8 & 37400 & 22.8 & 35000 & 22.8 & 35500 & 2.388 & 3.60E-02 & $1.51 E-02$ \\
\hline 37.3 & 54100 & 37.3 & 51700 & 37.3 & 52100 & 3.906 & $5.26 E-02$ & $1.35 E-02$ \\
\hline 61 & 78400 & 61.1 & 76300 & 61.1 & 76300 & 6.395 & $7.70 E-02$ & $1.20 E-02$ \\
\hline 100 & 90500 & 100 & 89600 & 100 & 95100 & 10.472 & $9.17 E-02$ & $8.76 E-03$ \\
\hline 100 & 82600 & 100 & 85000 & 100 & 90400 & 10.472 & 8.60E-02 & $8.21 E-03$ \\
\hline 69.6 & 59200 & 69.5 & 60900 & 69.5 & 64700 & 7.282 & $6.16 E-02$ & $8.46 E-03$ \\
\hline 48.3 & 43400 & 48.4 & 44700 & 48.4 & 47200 & 5.065 & $4.51 E-02$ & $8.90 E-03$ \\
\hline 33.6 & 33100 & 33.6 & 33900 & 33.6 & 35500 & 3.519 & $3.42 E-02$ & $9.71 E-03$ \\
\hline 23.4 & 26900 & 23.3 & 27200 & 23.4 & 27700 & 2.447 & $2.73 E-02$ & $1.11 E-02$ \\
\hline 16.2 & 22000 & 16.2 & 22700 & 16.2 & 23400 & 1.696 & $2.27 E-02$ & $1.34 E-02$ \\
\hline 11.3 & 17800 & 11.3 & 18600 & 11.3 & 19100 & 1.183 & $1.85 E-02$ & $1.56 E-02$ \\
\hline 7.86 & 13800 & 7.84 & 14400 & 7.84 & 14800 & 0.822 & $1.43 E-02$ & $1.74 E-02$ \\
\hline 5.45 & 10800 & 5.45 & 11300 & 5.45 & 11700 & 0.571 & $1.13 E-02$ & $1.97 E-02$ \\
\hline 3.8 & 8790 & 3.8 & 9080 & 3.8 & 9360 & 0.398 & $9.08 E-03$ & $2.28 E-02$ \\
\hline 2.63 & 7280 & 2.64 & 7470 & 2.63 & 7660 & 0.276 & 7.47E-03 & $2.71 E-02$ \\
\hline 1.84 & 6090 & 1.83 & 6300 & 1.82 & 6470 & 0.192 & $6.29 E-03$ & $3.28 E-02$ \\
\hline 1.27 & 5230 & 1.27 & 5450 & 1.28 & 5550 & 0.133 & $5.41 E-03$ & $4.06 E-02$ \\
\hline 0.886 & 4640 & 0.887 & 4780 & 0.879 & 4910 & 0.093 & $4.78 E-03$ & $5.16 E-02$ \\
\hline 0.615 & 4120 & 0.617 & 4260 & 0.615 & 4380 & 0.064 & 4.25E-03 & $6.60 E-02$ \\
\hline 0.428 & 3730 & 0.428 & 3870 & 0.43 & 3990 & 0.045 & $3.86 E-03$ & 8.61E-02 \\
\hline 0.3 & 3510 & 0.296 & 3570 & 0.298 & 3670 & 0.031 & $3.58 E-03$ & $1.15 E-01$ \\
\hline 0.207 & 3280 & 0.207 & 3390 & 0.206 & 3440 & 0.022 & 3.37E-03 & $1.56 E-01$ \\
\hline 0.145 & 3080 & 0.146 & 3190 & 0.142 & 3290 & 0.015 & $3.19 E-03$ & $2.11 E-01$ \\
\hline 0.101 & 2970 & 0.1 & 3080 & 0.0991 & 3150 & 0.010 & 3.07E-03 & $2.93 E-01$ \\
\hline
\end{tabular}




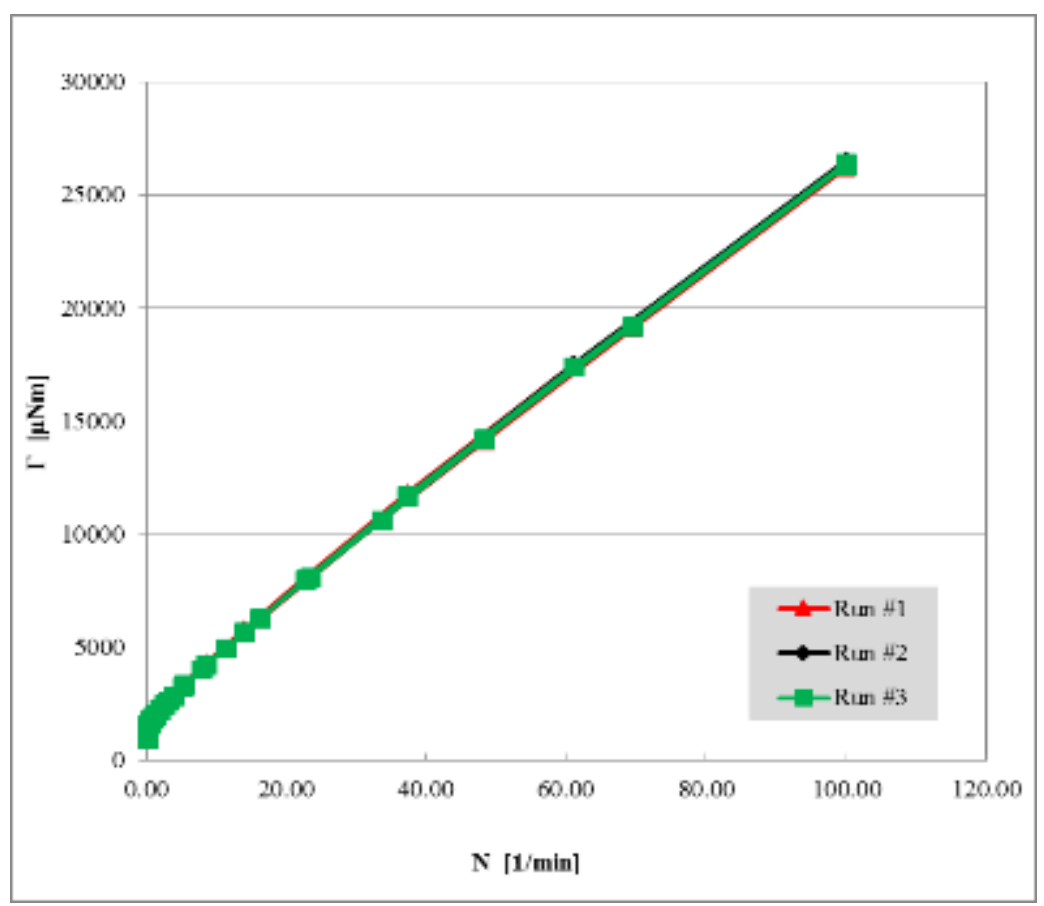

Figure A - 9: Torque vs. Angular Speed using Double spiral spindle on Mix\#4, with 0 \% beads by volume. Portrays data from Table A-13.

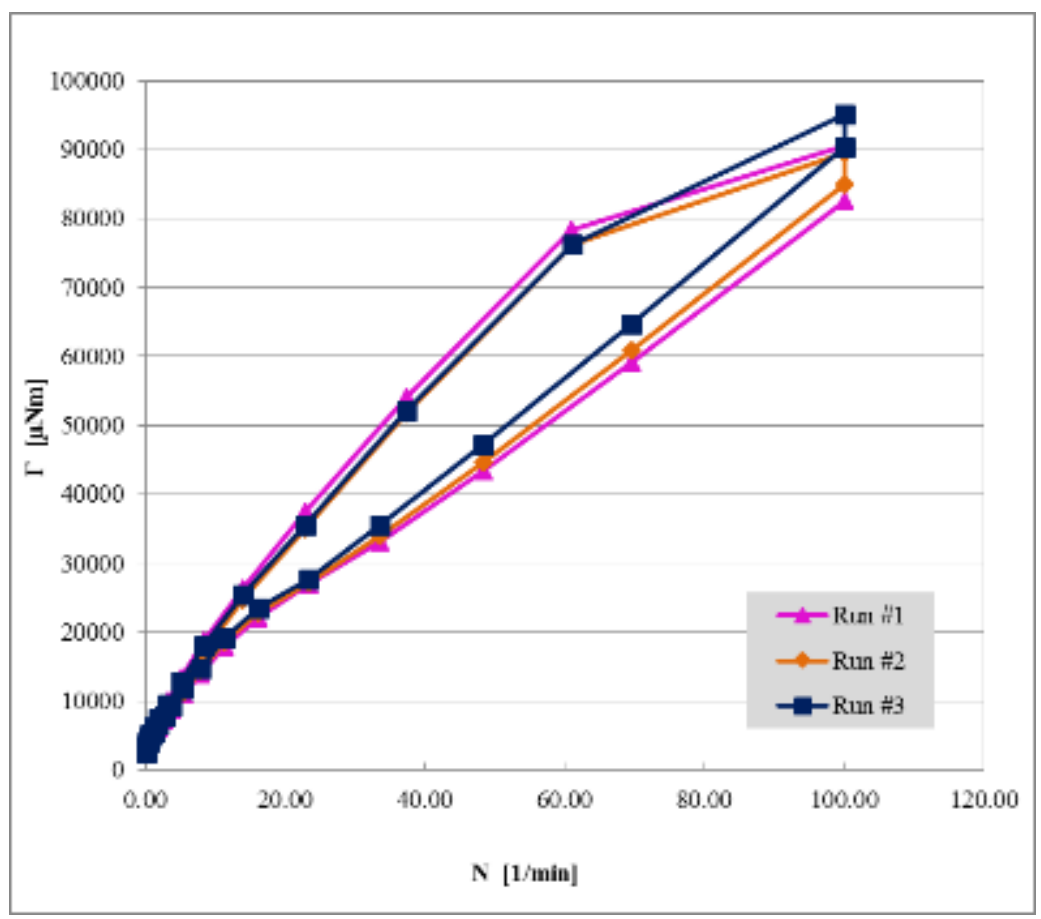

Figure A - 10: Torque vs. Angular Speed using Double spiral spindle on Mix\#4, with $40 \%$ beads by volume. Portrays data from Table A-14. 
Table A - 15: Measured data for Mix\#4 with 0 \% beads using Six-blade Vane

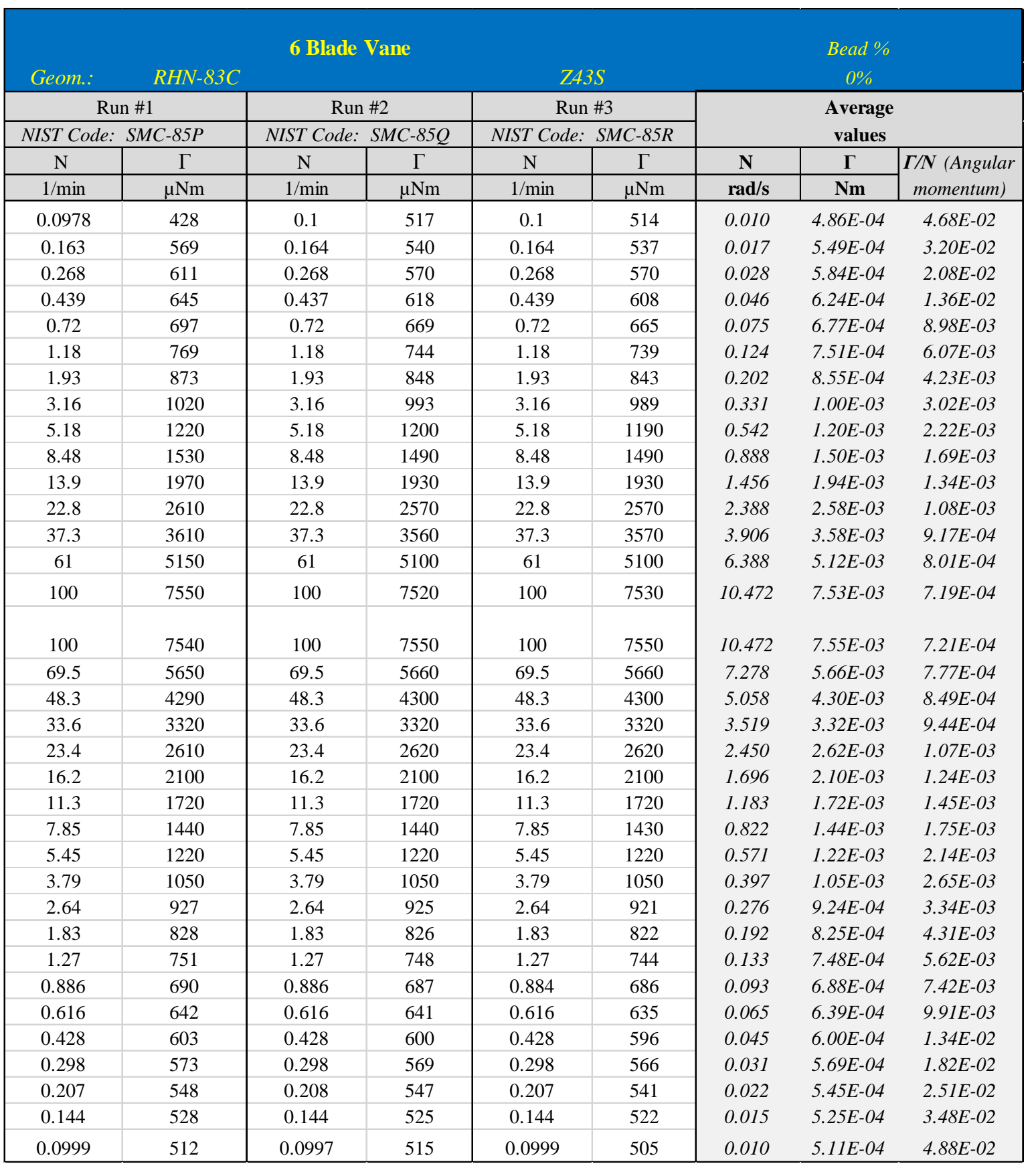


Table A - 16: Measured data for Mix\#4 with 40 \% beads using Six-blade Vane.

\begin{tabular}{|c|c|c|c|c|c|c|c|c|}
\hline \multicolumn{4}{|c|}{6 Blade Vane } & \multicolumn{2}{|c|}{$Z 43 S$} & \multicolumn{3}{|c|}{$\begin{array}{c}\text { Bead } \% \\
40 \% \\
\end{array}$} \\
\hline \multicolumn{2}{|c|}{ Run \#1 } & \multirow{2}{*}{\multicolumn{2}{|c|}{$\frac{\text { Run } \# 2}{\text { NIST Code: } S M C-85 H}$}} & \multicolumn{2}{|c|}{ Run \#3 } & \multirow{2}{*}{\multicolumn{3}{|c|}{$\begin{array}{l}\text { Average } \\
\text { values }\end{array}$}} \\
\hline NIST Code: & $S M C-85 G$ & & $M C-85 H$ & NIST Cod & $M C-85 I$ & & & \\
\hline $\mathrm{N}$ & $\Gamma$ & $\mathrm{N}$ & $\Gamma$ & $\mathrm{N}$ & $\Gamma$ & $\mathbf{N}$ & $\Gamma$ & $\boldsymbol{\Gamma} / \boldsymbol{N}$ (Angular \\
\hline $1 / \mathrm{min}$ & $\mu \mathrm{Nm}$ & $1 / \mathrm{min}$ & $\mu \mathrm{Nm}$ & $1 / \mathrm{min}$ & $\mu \mathrm{Nm}$ & $\mathrm{rad} / \mathrm{s}$ & $\mathrm{Nm}$ & momentum) \\
\hline 0.0941 & 1350 & 0.095 & 1420 & 0.0927 & 1500 & 0.010 & $1.42 E-03$ & $1.45 E-01$ \\
\hline 0.161 & 1710 & 0.166 & 1830 & 0.163 & 1900 & 0.017 & $1.81 E-03$ & $1.06 E-01$ \\
\hline 0.274 & 1840 & 0.264 & 1950 & 0.272 & 2090 & 0.028 & $1.96 E-03$ & $6.93 E-02$ \\
\hline 0.44 & 2020 & 0.439 & 2130 & 0.44 & 2210 & 0.046 & $2.12 E-03$ & 4.60E-02 \\
\hline 0.72 & 2240 & 0.72 & 2330 & 0.719 & 2470 & 0.075 & $2.35 E-03$ & $3.11 E-02$ \\
\hline 1.18 & 2530 & 1.18 & 2680 & 1.18 & 2830 & 0.124 & $2.68 E-03$ & $2.17 E-02$ \\
\hline 1.93 & 3010 & 1.94 & 3110 & 1.93 & 3390 & 0.202 & $3.17 E-03$ & $1.57 E-02$ \\
\hline 3.16 & 3760 & 3.16 & 3840 & 3.15 & 4170 & 0.331 & $3.92 E-03$ & 1.19E-02 \\
\hline 5.17 & 4710 & 5.17 & 4860 & 5.18 & 5230 & 0.542 & $4.93 E-03$ & $9.11 E-03$ \\
\hline 8.48 & 6130 & 8.48 & 6310 & 8.49 & 6790 & 0.888 & $6.41 E-03$ & $7.22 E-03$ \\
\hline 13.9 & 8070 & 13.9 & 8290 & 13.9 & 8870 & 1.456 & $8.41 E-03$ & $5.78 E-03$ \\
\hline 22.7 & 10800 & 22.8 & 11100 & 22.8 & 11900 & 2.384 & $1.13 E-02$ & $4.73 E-03$ \\
\hline 37.3 & 14500 & 37.3 & 15300 & 37.3 & 16300 & 3.906 & $1.54 E-02$ & $3.93 E-03$ \\
\hline 61 & 20700 & 61.1 & 21300 & 61.1 & 22100 & 6.395 & $2.14 E-02$ & $3.34 E-03$ \\
\hline 100 & 28100 & 100 & 30400 & 100 & 30500 & 10.472 & $2.97 E-02$ & $2.83 E-03$ \\
\hline 100 & 27000 & 100 & 28300 & 100 & 28800 & 10.472 & $2.80 E-02$ & $2.68 E-03$ \\
\hline 69.5 & 20800 & 69.5 & 21700 & 69.5 & 22200 & 7.278 & $2.16 E-02$ & $2.96 E-03$ \\
\hline 48.3 & 15500 & 48.3 & 16000 & 48.3 & 16500 & 5.058 & $1.60 E-02$ & $3.16 E-03$ \\
\hline 33.6 & 11800 & 33.6 & 12300 & 33.6 & 12600 & 3.519 & $1.22 E-02$ & $3.48 E-03$ \\
\hline 23.4 & 9180 & 23.4 & 9420 & 23.4 & 9680 & 2.450 & $9.43 E-03$ & $3.85 E-03$ \\
\hline 16.2 & 7190 & 16.2 & 7360 & 16.2 & 7570 & 1.696 & $7.37 E-03$ & $4.35 E-03$ \\
\hline 11.3 & 5640 & 11.3 & 5900 & 11.3 & 5980 & 1.183 & $5.84 E-03$ & $4.94 E-03$ \\
\hline 7.84 & 4510 & 7.83 & 4610 & 7.84 & 4730 & 0.821 & $4.62 E-03$ & $5.63 E-03$ \\
\hline 5.45 & 3650 & 5.46 & 3760 & 5.45 & 3870 & 0.571 & $3.76 E-03$ & $6.58 E-03$ \\
\hline 3.79 & 3000 & 3.8 & 3080 & 3.79 & 3200 & 0.397 & $3.09 E-03$ & $7.79 E-03$ \\
\hline 2.64 & 2560 & 2.63 & 2630 & 2.63 & 2660 & 0.276 & $2.62 E-03$ & $9.49 E-03$ \\
\hline 1.84 & 2180 & 1.84 & 2190 & 1.83 & 2250 & 0.192 & $2.21 E-03$ & $1.15 E-02$ \\
\hline 1.28 & 1890 & 1.27 & 1920 & 1.28 & 1970 & 0.134 & $1.93 E-03$ & $1.44 E-02$ \\
\hline 0.882 & 1670 & 0.882 & 1720 & 0.885 & 1720 & 0.092 & $1.70 E-03$ & $1.84 E-02$ \\
\hline 0.617 & 1500 & 0.615 & 1540 & 0.613 & 1570 & 0.064 & $1.54 E-03$ & $2.39 E-02$ \\
\hline 0.425 & 1400 & 0.43 & 1390 & 0.431 & 1440 & 0.045 & $1.41 E-03$ & $3.14 E-02$ \\
\hline 0.3 & 1270 & 0.3 & 1310 & 0.3 & 1320 & 0.031 & 1.30E-03 & 4.14E-02 \\
\hline 0.205 & 1180 & 0.208 & 1210 & 0.205 & 1230 & 0.022 & $1.21 E-03$ & $5.59 E-02$ \\
\hline 0.144 & 1140 & 0.143 & 1160 & 0.144 & 1200 & 0.015 & $1.17 E-03$ & $7.75 E-02$ \\
\hline 0.1 & 1090 & 0.101 & 1110 & 0.0993 & 1110 & 0.010 & $1.10 E-03$ & $1.05 E-01$ \\
\hline
\end{tabular}




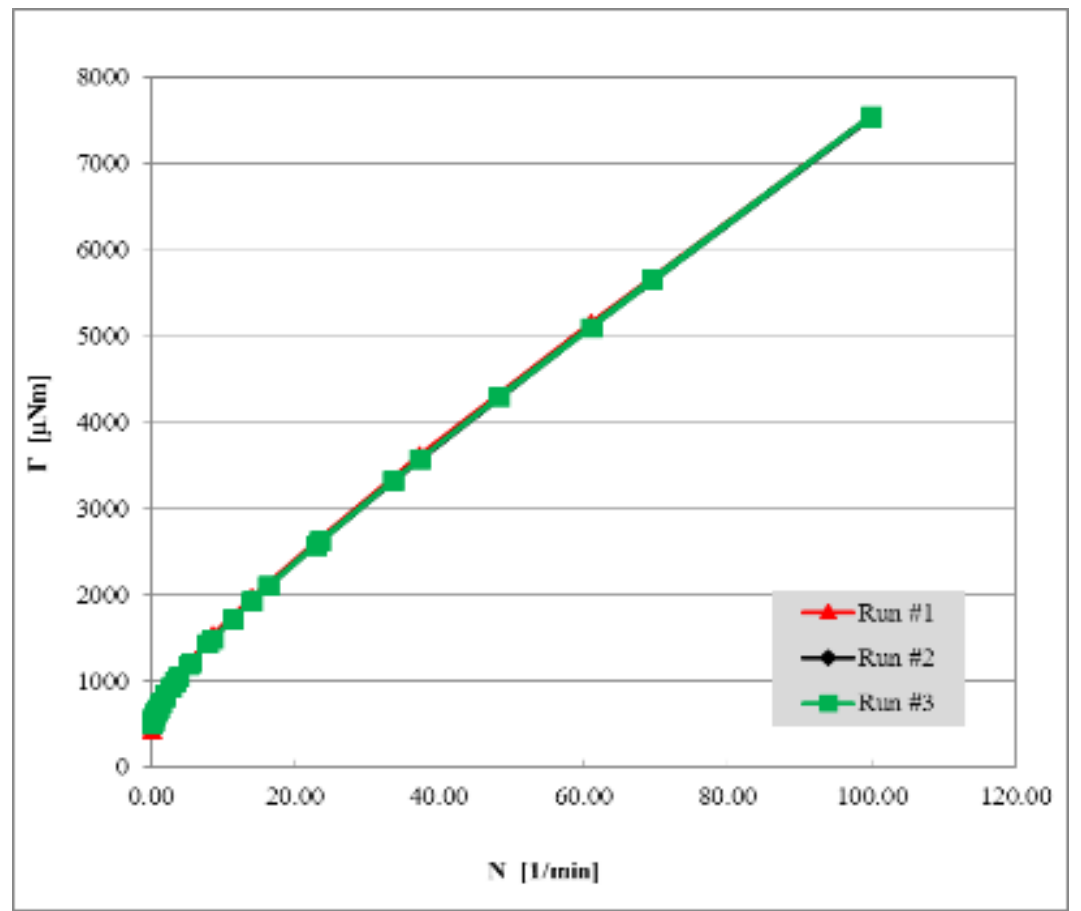

Figure A - 12: Torque vs. Angular Speed using six-blade vane on Mix\#4, with $0 \%$ beads by volume. Portrays data from Table A-15.

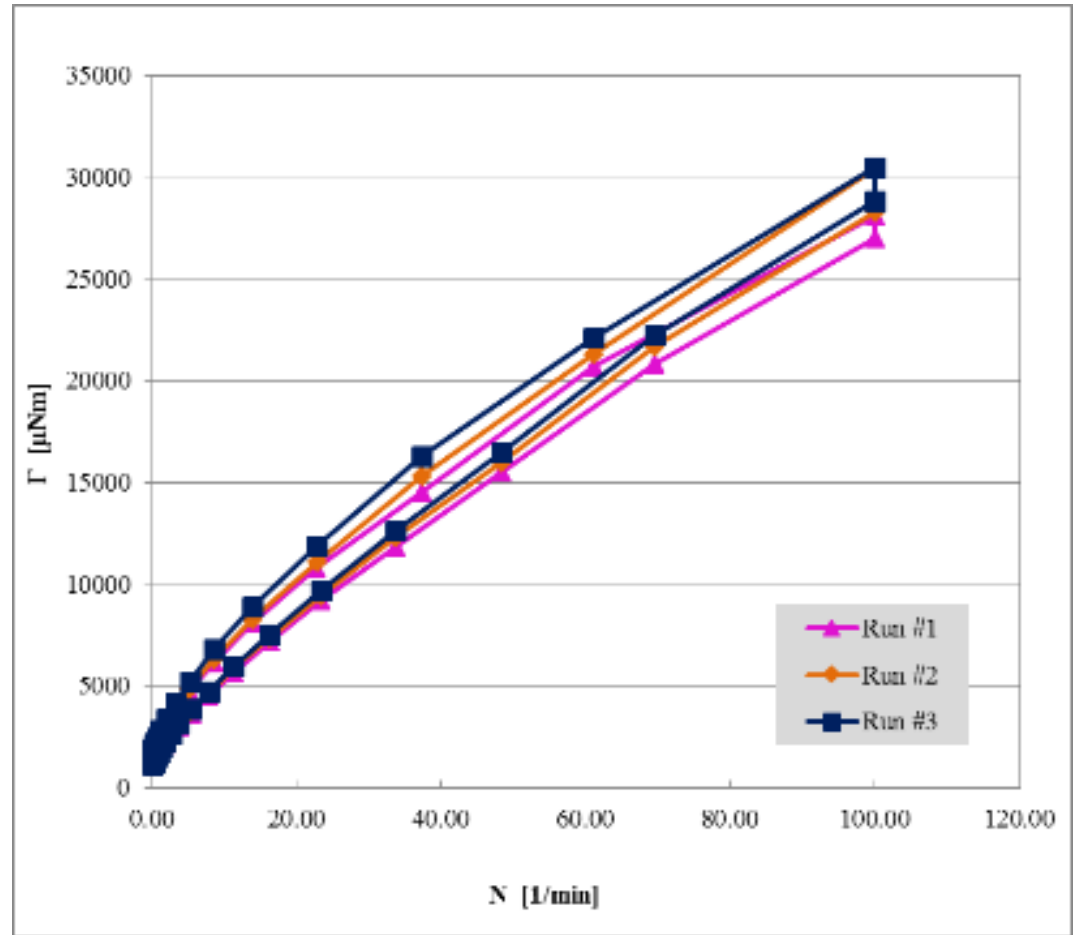

Figure A - 11: Torque vs. Angular Speed using six-blade vane on Mix\#4, with $40 \%$ beads by volume. Portrays data from Table A-16. 
Table A - 17: Measured data for Mix\#4 with 0 \% beads using Serrated Coaxial Cylinder.

\begin{tabular}{|c|c|c|c|c|c|c|c|c|}
\hline \multicolumn{6}{|c|}{ Serrated Coaxial Cylinder } & \multicolumn{3}{|c|}{$\begin{array}{c}\text { Bead } \% \\
0 \%\end{array}$} \\
\hline \multirow{2}{*}{\multicolumn{2}{|c|}{ Run \#1 }} & \multirow{2}{*}{\multicolumn{2}{|c|}{ Run \#2 }} & \multicolumn{2}{|c|}{ Z43S } & \multirow{3}{*}{\multicolumn{3}{|c|}{$\begin{array}{c}\text { Average } \\
\text { values }\end{array}$}} \\
\hline & & & & \multicolumn{2}{|c|}{ Run \#3 } & & & \\
\hline \multicolumn{2}{|c|}{ NIST Code: SMC-85M } & \multicolumn{2}{|c|}{ NIST Code: SMC-85N } & \multicolumn{2}{|c|}{ NIST Code: SMC-85O } & & & \\
\hline $\mathrm{N}$ & $\Gamma$ & $\mathrm{N}$ & $\Gamma$ & $\mathrm{N}$ & $\Gamma$ & $\mathbf{N}$ & $\Gamma$ & $\Gamma / \mathbf{N}$ (Angular \\
\hline $1 / \mathrm{min}$ & $\mu \mathrm{Nm}$ & $1 / \mathrm{min}$ & $\mu \mathrm{Nm}$ & $1 / \mathrm{min}$ & $\mu \mathrm{Nm}$ & $\mathrm{rad} / \mathrm{s}$ & $\mathrm{Nm}$ & momentum) \\
\hline 0.0983 & 449 & 0.099 & 340 & 0.1 & 348 & 0.010 & $3.79 E-04$ & $3.65 E-02$ \\
\hline 0.164 & 564 & 0.164 & 413 & 0.164 & 373 & 0.017 & 4.50E-04 & $2.62 E-02$ \\
\hline 0.268 & 598 & 0.268 & 454 & 0.268 & 409 & 0.028 & $4.87 E-04$ & $1.74 E-02$ \\
\hline 0.44 & 641 & 0.439 & 503 & 0.439 & 454 & 0.046 & $5.33 E-04$ & $1.16 E-02$ \\
\hline 0.72 & 700 & 0.72 & 568 & 0.72 & 516 & 0.075 & $5.95 E-04$ & $7.89 E-03$ \\
\hline 1.18 & 781 & 1.18 & 656 & 1.18 & 604 & 0.124 & $6.80 E-04$ & $5.51 E-03$ \\
\hline 1.93 & 891 & 1.93 & 773 & 1.93 & 718 & 0.202 & $7.94 E-04$ & 3.93E-03 \\
\hline 3.16 & 1040 & 3.16 & 932 & 3.16 & 881 & 0.331 & $9.51 E-04$ & 2.87E-03 \\
\hline 5.18 & 1260 & 5.18 & 1150 & 5.18 & 1100 & 0.542 & 1.17E-03 & $2.16 E-03$ \\
\hline 8.48 & 1580 & 8.48 & 1460 & 8.48 & 1400 & 0.888 & $1.48 E-03$ & $1.67 E-03$ \\
\hline 13.9 & 2030 & 13.9 & 1910 & 13.9 & 1830 & 1.456 & $1.92 E-03$ & $1.32 E-03$ \\
\hline 22.8 & 2680 & 22.8 & 2560 & 22.8 & 2460 & 2.388 & $2.57 E-03$ & $1.07 E-03$ \\
\hline 37.3 & 3690 & 37.3 & 3540 & 37.3 & 3430 & 3.906 & $3.55 E-03$ & $9.10 E-04$ \\
\hline 61 & 5240 & 61 & 5060 & 61 & 4920 & 6.388 & $5.07 E-03$ & 7.94E-04 \\
\hline 100 & 7710 & 100 & 7420 & 100 & 7240 & 10.472 & $7.46 E-03$ & 7.12E-04 \\
\hline 100 & 7600 & 100 & 7380 & 100 & 7200 & 10.472 & 7.39E-03 & $7.06 E-04$ \\
\hline 69.5 & 5690 & 69.5 & 5540 & 69.5 & 5400 & 7.278 & $5.54 E-03$ & $7.62 E-04$ \\
\hline 48.3 & 4300 & 48.3 & 4180 & 48.3 & 4090 & 5.058 & $4.19 E-03$ & $8.28 E-04$ \\
\hline 33.6 & 3300 & 33.6 & 3210 & 33.6 & 3140 & 3.519 & $3.22 E-03$ & $9.14 E-04$ \\
\hline 23.4 & 2580 & 23.4 & 2510 & 23.4 & 2460 & 2.450 & $2.52 E-03$ & $1.03 E-03$ \\
\hline 16.2 & 2050 & 16.2 & 1990 & 16.2 & 1950 & 1.696 & $2.00 E-03$ & $1.18 E-03$ \\
\hline 11.3 & 1670 & 11.3 & 1620 & 11.3 & 1580 & 1.183 & $1.62 E-03$ & $1.37 E-03$ \\
\hline 7.85 & 1380 & 7.85 & 1330 & 7.85 & 1300 & 0.822 & $1.34 E-03$ & $1.63 E-03$ \\
\hline 5.46 & 1150 & 5.45 & 1110 & 5.45 & 1080 & 0.571 & $1.11 E-03$ & $1.95 E-03$ \\
\hline 3.79 & 980 & 3.79 & 936 & 3.79 & 912 & 0.397 & $9.43 E-04$ & $2.38 E-03$ \\
\hline 2.64 & 843 & 2.64 & 802 & 2.64 & 781 & 0.276 & $8.09 E-04$ & $2.93 E-03$ \\
\hline 1.83 & 734 & 1.83 & 696 & 1.83 & 679 & 0.192 & 7.03E-04 & 3.67E-03 \\
\hline 1.27 & 646 & 1.27 & 612 & 1.27 & 597 & 0.133 & $6.18 E-04$ & 4.65E-03 \\
\hline 0.886 & 576 & 0.886 & 545 & 0.886 & 532 & 0.093 & $5.51 E-04$ & $5.94 E-03$ \\
\hline 0.616 & 519 & 0.616 & 491 & 0.616 & 480 & 0.065 & $4.97 E-04$ & 7.70E-03 \\
\hline 0.428 & 473 & 0.428 & 449 & 0.429 & 441 & 0.045 & $4.54 E-04$ & $1.01 E-02$ \\
\hline 0.298 & 435 & 0.298 & 415 & 0.298 & 405 & 0.031 & $4.18 E-04$ & $1.34 E-02$ \\
\hline 0.207 & 405 & 0.207 & 386 & 0.207 & 377 & 0.022 & $3.89 E-04$ & $1.80 E-02$ \\
\hline 0.144 & 381 & 0.144 & 363 & 0.144 & 355 & 0.015 & $3.66 E-04$ & $2.43 E-02$ \\
\hline 0.1 & 361 & 0.1 & 345 & 0.1 & 337 & 0.010 & $3.48 E-04$ & $3.32 E-02$ \\
\hline
\end{tabular}


Table A - 18: Measured data for Mix\#4 with 40 \% beads using Serrated Coaxial Cylinder.

\begin{tabular}{|c|c|c|c|c|c|c|c|c|}
\hline \multicolumn{6}{|c|}{ Serrated Coaxial Cylinder } & \multicolumn{3}{|c|}{$\begin{array}{c}\text { Bead } \% \\
40 \%\end{array}$} \\
\hline \multirow{2}{*}{\multicolumn{2}{|c|}{ Run \#1 }} & \multirow{2}{*}{\multicolumn{2}{|c|}{ Run \#2 }} & \multicolumn{2}{|c|}{ Z43S } & \multirow{3}{*}{\multicolumn{3}{|c|}{$\begin{array}{c}\text { Average } \\
\text { values }\end{array}$}} \\
\hline & & & & \multicolumn{2}{|c|}{ Run \#3 } & & & \\
\hline NIST Code: & SMC-85D & NIST Code: & SMC-85E & NIST Cod & $M C-85 F$ & & & \\
\hline $\mathrm{N}$ & $\Gamma$ & $\mathrm{N}$ & $\Gamma$ & $\mathrm{N}$ & $\Gamma$ & $\mathbf{N}$ & $\Gamma$ & $\boldsymbol{\Gamma} / \mathbf{N}$ (Angular \\
\hline $1 / \mathrm{min}$ & $\mu \mathrm{Nm}$ & $1 / \mathrm{min}$ & $\mu \mathrm{Nm}$ & $1 / \mathrm{min}$ & $\mu \mathrm{Nm}$ & $\mathrm{rad} / \mathrm{s}$ & $\mathbf{N m}$ & momentum) \\
\hline 0.0975 & 1400 & 0.0964 & 1340 & 0.0972 & 1290 & 0.010 & $1.34 E-03$ & $1.32 E-01$ \\
\hline 0.163 & 1660 & 0.165 & 1510 & 0.165 & 1450 & 0.017 & $1.54 E-03$ & $8.95 E-02$ \\
\hline 0.272 & 1740 & 0.268 & 1480 & 0.268 & 1450 & 0.028 & $1.56 E-03$ & $5.52 E-02$ \\
\hline 0.444 & 1810 & 0.441 & 1580 & 0.44 & 1550 & 0.046 & $1.65 E-03$ & $3.56 E-02$ \\
\hline 0.718 & 1890 & 0.722 & 1770 & 0.722 & 1720 & 0.075 & $1.79 E-03$ & $2.38 E-02$ \\
\hline 1.18 & 2100 & 1.18 & 2050 & 1.18 & 2040 & 0.124 & $2.06 E-03$ & $1.67 E-02$ \\
\hline 1.93 & 2370 & 1.93 & 2460 & 1.93 & 2470 & 0.202 & $2.43 E-03$ & $1.20 E-02$ \\
\hline 3.16 & 2820 & 3.16 & 3050 & 3.17 & 3100 & 0.331 & $2.99 E-03$ & $9.03 E-03$ \\
\hline 5.18 & 3500 & 5.18 & 3870 & 5.17 & 3990 & 0.542 & 3.79E-03 & $6.99 E-03$ \\
\hline 8.48 & 4530 & 8.49 & 5040 & 8.48 & 5210 & 0.888 & 4.93E-03 & $5.55 E-03$ \\
\hline 13.9 & 5990 & 13.9 & 6730 & 13.9 & 7030 & 1.456 & $6.58 E-03$ & $4.52 E-03$ \\
\hline 22.8 & 8060 & 22.8 & 9150 & 22.8 & 9630 & 2.388 & 8.95E-03 & $3.75 E-03$ \\
\hline 37.3 & 11200 & 37.3 & 12500 & 37.3 & 13400 & 3.906 & $1.24 E-02$ & $3.17 E-03$ \\
\hline 61 & 15500 & 61.1 & 16500 & 61.1 & 17400 & 6.395 & $1.65 E-02$ & $2.57 E-03$ \\
\hline 100 & 20100 & 100 & 21900 & 100 & 25000 & 10.472 & $2.23 E-02$ & $2.13 E-03$ \\
\hline 100 & 20400 & 100 & 22100 & 99.9 & 24900 & 10.468 & $2.25 E-02$ & $2.15 E-03$ \\
\hline 69.5 & 16400 & 69.5 & 17800 & 69.5 & 19300 & 7.278 & $1.78 E-02$ & $2.45 E-03$ \\
\hline 48.3 & 12600 & 48.3 & 13600 & 48.3 & 14400 & 5.058 & $1.35 E-02$ & $2.68 E-03$ \\
\hline 33.6 & 9640 & 33.6 & 10400 & 33.6 & 11000 & 3.519 & $1.03 E-02$ & $2.94 E-03$ \\
\hline 23.3 & 7400 & 23.3 & 7910 & 23.4 & 8360 & 2.443 & $7.89 E-03$ & $3.23 E-03$ \\
\hline 16.2 & 5740 & 16.2 & 6140 & 16.2 & 6470 & 1.696 & $6.12 E-03$ & 3.61E-03 \\
\hline 11.3 & 4490 & 11.3 & 4810 & 11.3 & 5090 & 1.183 & 4.80E-03 & $4.05 E-03$ \\
\hline 7.85 & 3570 & 7.84 & 3800 & 7.85 & 4030 & 0.822 & 3.80E-03 & 4.62E-03 \\
\hline 5.46 & 2900 & 5.46 & 3090 & 5.46 & 3240 & 0.572 & $3.08 E-03$ & $5.38 E-03$ \\
\hline 3.79 & 2380 & 3.79 & 2530 & 3.79 & 2660 & 0.397 & $2.52 E-03$ & $6.36 E-03$ \\
\hline 2.64 & 1980 & 2.64 & 2100 & 2.64 & 2210 & 0.276 & $2.10 E-03$ & $7.58 E-03$ \\
\hline 1.83 & 1690 & 1.83 & 1790 & 1.83 & 1880 & 0.192 & $1.79 E-03$ & $9.32 E-03$ \\
\hline 1.27 & 1460 & 1.28 & 1550 & 1.28 & 1620 & 0.134 & $1.54 E-03$ & $1.15 E-02$ \\
\hline 0.886 & 1280 & 0.885 & 1350 & 0.886 & 1420 & 0.093 & $1.35 E-03$ & $1.46 E-02$ \\
\hline 0.617 & 1150 & 0.614 & 1210 & 0.616 & 1260 & 0.064 & $1.21 E-03$ & $1.87 E-02$ \\
\hline 0.429 & 1030 & 0.429 & 1080 & 0.427 & 1140 & 0.045 & $1.08 E-03$ & $2.42 E-02$ \\
\hline 0.297 & 945 & 0.298 & 992 & 0.298 & 1040 & 0.031 & $9.92 E-04$ & $3.18 E-02$ \\
\hline 0.209 & 882 & 0.206 & 914 & 0.208 & 967 & 0.022 & $9.21 E-04$ & $4.24 E-02$ \\
\hline 0.144 & 814 & 0.145 & 867 & 0.144 & 888 & 0.015 & 8.56E-04 & 5.67E-02 \\
\hline 0.0994 & 763 & 0.0989 & 799 & 0.0998 & 831 & 0.010 & $7.98 E-04$ & 7.67E-02 \\
\hline
\end{tabular}




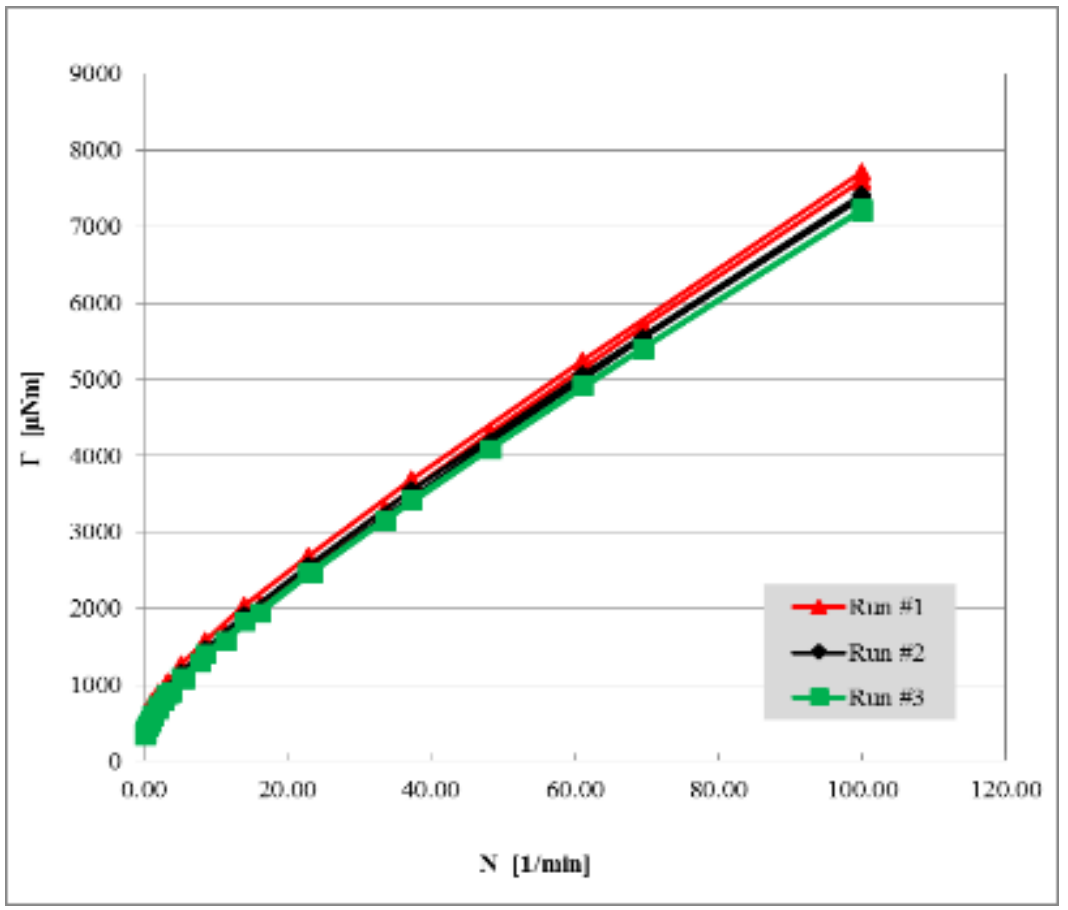

Figure A - 13: Torque vs. Angular Speed using Serrated Coaxial Cylinder on Mix\#4, with 0 \% beads by volume. Portrays data from Table A-17.

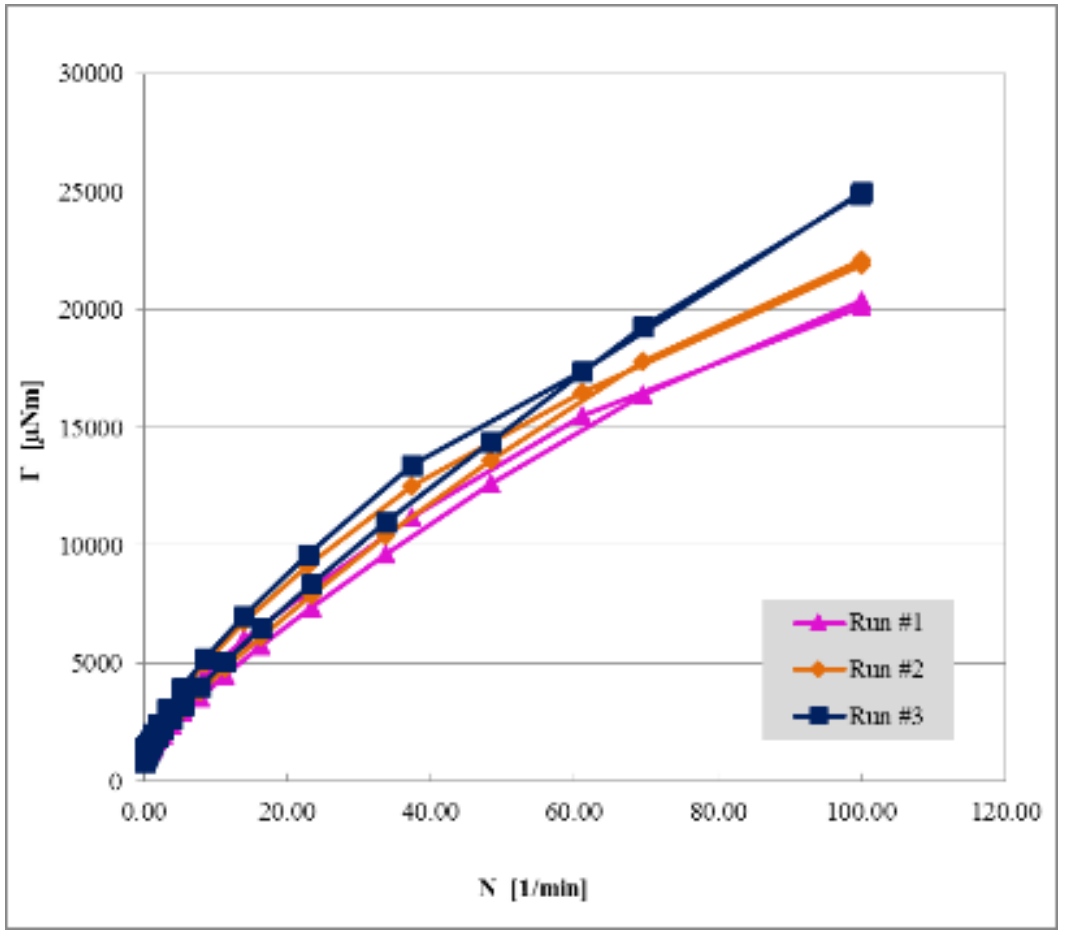

Figure A - 14: Torque vs. Angular Speed using Serrated Coaxial Cylinder on Mix\#4, with $40 \%$ beads by volume. Portrays data from Table A-18. 
Table A - 19: Measured data for Mix\#5 with $0 \%$ beads using Double spiral spindle.

\begin{tabular}{|c|c|c|c|c|c|c|c|c|}
\hline \multicolumn{6}{|c|}{ Double Spiral } & \multirow{2}{*}{\multicolumn{3}{|c|}{$\begin{array}{c}\text { Bead } \% \\
0 \%\end{array}$}} \\
\hline Geom.: & \multicolumn{3}{|l|}{ RHN-83A } & \multicolumn{2}{|c|}{ Cup: } & & & \\
\hline \multicolumn{2}{|c|}{ Run \#1 } & \multicolumn{2}{|c|}{ Run \#2 } & \multicolumn{2}{|c|}{ Run \#3 } & \multirow{2}{*}{\multicolumn{3}{|c|}{$\begin{array}{c}\text { Average } \\
\text { values }\end{array}$}} \\
\hline \multicolumn{2}{|c|}{ NIST Code: SMC-95D } & \multicolumn{2}{|c|}{ NIST Code: SMC-95E } & \multicolumn{2}{|c|}{ NIST Code: SMC-95F } & & & \\
\hline $\mathrm{N}$ & $\Gamma$ & $\mathrm{N}$ & $\Gamma$ & $\mathrm{N}$ & $\Gamma$ & $\mathbf{N}$ & $\Gamma$ & \multirow{2}{*}{$\begin{array}{c}\Gamma / \boldsymbol{N} \text { (Angular } \\
\text { momentum) }\end{array}$} \\
\hline $1 / \mathrm{min}$ & $\mu \mathrm{Nm}$ & $1 / \mathrm{min}$ & $\mu \mathrm{Nm}$ & $1 / \mathrm{min}$ & $\mu \mathrm{Nm}$ & $\mathrm{rad} / \mathrm{s}$ & $\mathrm{Nm}$ & \\
\hline 0.0947 & 973 & 0.1 & 1420 & 0.095 & 902 & 0.010 & $1.10 E-03$ & 1.09E-01 \\
\hline 0.162 & 1420 & 0.164 & 1470 & 0.162 & 1330 & 0.017 & $1.41 E-03$ & $8.26 E-02$ \\
\hline 0.268 & 1590 & 0.268 & 1540 & 0.268 & 1510 & 0.028 & $1.55 E-03$ & $5.51 E-02$ \\
\hline 0.44 & 1690 & 0.44 & 1620 & 0.44 & 1620 & 0.046 & $1.64 E-03$ & $3.57 E-02$ \\
\hline 0.72 & 1800 & 0.72 & 1740 & 0.72 & 1740 & 0.075 & $1.76 E-03$ & $2.33 E-02$ \\
\hline 1.18 & 1980 & 1.18 & 1920 & 1.18 & 1920 & 0.124 & $1.94 E-03$ & $1.57 E-02$ \\
\hline 1.93 & 2220 & 1.93 & 2180 & 1.93 & 2180 & 0.202 & $2.19 E-03$ & $1.09 E-02$ \\
\hline 3.16 & 2620 & 3.16 & 2570 & 3.16 & 2570 & 0.331 & $2.59 E-03$ & $7.82 E-03$ \\
\hline 5.18 & 3230 & 5.18 & 3170 & 5.18 & 3160 & 0.542 & $3.19 E-03$ & $5.87 E-03$ \\
\hline 8.48 & 4150 & 8.48 & 4090 & 8.48 & 4070 & 0.888 & 4.10E-03 & 4.62E-03 \\
\hline 13.9 & 5580 & 13.9 & 5490 & 13.9 & 5510 & 1.456 & $5.53 E-03$ & $3.80 E-03$ \\
\hline 22.8 & 7940 & 22.8 & 7760 & 22.8 & 7740 & 2.388 & $7.81 E-03$ & $3.27 E-03$ \\
\hline 37.3 & 11500 & 37.3 & 11300 & 37.3 & 11300 & 3.906 & $1.14 E-02$ & $2.91 E-03$ \\
\hline 61.1 & 17100 & 61.1 & 16700 & 61.1 & 16800 & 6.398 & $1.69 E-02$ & $2.64 E-03$ \\
\hline 100 & 25800 & 100 & 25300 & 100 & 25300 & 10.472 & $2.55 E-02$ & $2.43 E-03$ \\
\hline 100 & 25600 & 100 & 25200 & 100 & 25200 & 10.472 & $2.53 E-02$ & $2.42 E-03$ \\
\hline 69.5 & 18700 & 69.5 & 18500 & 69.5 & 18500 & 7.278 & $1.86 E-02$ & $2.55 E-03$ \\
\hline 48.3 & 13900 & 48.3 & 13700 & 48.3 & 13700 & 5.058 & $1.38 E-02$ & $2.72 E-03$ \\
\hline 33.6 & 10400 & 33.6 & 10300 & 33.6 & 10300 & 3.519 & $1.03 E-02$ & $2.94 E-03$ \\
\hline 23.4 & 7900 & 23.4 & 7830 & 23.4 & 7840 & 2.450 & $7.86 E-03$ & $3.21 E-03$ \\
\hline 16.2 & 6120 & 16.2 & 6070 & 16.2 & 6080 & 1.696 & $6.09 E-03$ & $3.59 E-03$ \\
\hline 11.3 & 4840 & 11.3 & 4810 & 11.3 & 4820 & 1.183 & $4.82 E-03$ & $4.08 E-03$ \\
\hline 7.85 & 3920 & 7.85 & 3900 & 7.85 & 3900 & 0.822 & $3.91 E-03$ & $4.75 E-03$ \\
\hline 5.46 & 3250 & 5.46 & 3230 & 5.46 & 3240 & 0.572 & $3.24 E-03$ & $5.67 E-03$ \\
\hline 3.79 & 2760 & 3.79 & 2750 & 3.79 & 2760 & 0.397 & $2.76 E-03$ & $6.95 E-03$ \\
\hline 2.64 & 2410 & 2.64 & 2400 & 2.64 & 2400 & 0.276 & $2.40 E-03$ & 8.69E-03 \\
\hline 1.83 & 2140 & 1.83 & 2130 & 1.83 & 2140 & 0.192 & $2.14 E-03$ & $1.11 E-02$ \\
\hline 1.27 & 1940 & 1.27 & 1940 & 1.27 & 1940 & 0.133 & $1.94 E-03$ & $1.46 E-02$ \\
\hline 0.886 & 1790 & 0.886 & 1790 & 0.886 & 1790 & 0.093 & $1.79 E-03$ & $1.93 E-02$ \\
\hline 0.616 & 1680 & 0.616 & 1680 & 0.616 & 1680 & 0.065 & $1.68 E-03$ & $2.60 E-02$ \\
\hline 0.428 & 1590 & 0.428 & 1590 & 0.428 & 1600 & 0.045 & $1.59 E-03$ & $3.55 E-02$ \\
\hline 0.297 & 1530 & 0.298 & 1520 & 0.297 & 1530 & 0.031 & $1.53 E-03$ & $4.90 E-02$ \\
\hline 0.207 & 1470 & 0.207 & 1470 & 0.207 & 1470 & 0.022 & $1.47 E-03$ & $6.78 E-02$ \\
\hline 0.144 & 1430 & 0.144 & 1430 & 0.144 & 1430 & 0.015 & $1.43 E-03$ & $9.48 E-02$ \\
\hline 0.0998 & 1400 & 0.0999 & 1400 & 0.0998 & 1400 & 0.010 & $1.40 E-03$ & $1.34 E-01$ \\
\hline
\end{tabular}


Table A - 20: Measured data for Mix\#5 with $40 \%$ beads using Double spiral spindle.

\begin{tabular}{|c|c|c|c|c|c|c|c|c|}
\hline \multicolumn{6}{|c|}{ Double Spiral } & \multirow{2}{*}{\multicolumn{3}{|c|}{$\begin{array}{c}\text { Bead } \% \\
40 \%\end{array}$}} \\
\hline Geom.: & \multicolumn{3}{|l|}{$R H N-83 A$} & \multicolumn{2}{|c|}{ Cup: $\quad$ Z43S } & & & \\
\hline \multicolumn{2}{|c|}{ Run \#1 } & \multicolumn{2}{|c|}{ Run \#2 } & \multicolumn{2}{|c|}{ Run \#3 } & \multirow{2}{*}{\multicolumn{3}{|c|}{$\begin{array}{c}\text { Average } \\
\text { values }\end{array}$}} \\
\hline \multicolumn{2}{|c|}{ NIST Code: SMC-95M } & \multicolumn{2}{|c|}{ NIST Code: SMC-95N } & \multicolumn{2}{|c|}{ NIST Code: SMC-95O } & & & \\
\hline $\mathrm{N}$ & $\Gamma$ & $\mathrm{N}$ & $\Gamma$ & $\mathrm{N}$ & $\Gamma$ & $\mathbf{N}$ & $\Gamma$ & $\boldsymbol{\Gamma} / \mathbf{N}$ (Angular \\
\hline $1 / \mathrm{min}$ & $\mu \mathrm{Nm}$ & $1 / \mathrm{min}$ & $\mu \mathrm{Nm}$ & $1 / \mathrm{min}$ & $\mu \mathrm{Nm}$ & $\mathrm{rad} / \mathrm{s}$ & $\mathrm{Nm}$ & momentum) \\
\hline 0.0882 & 2330 & 0.0916 & 2020 & 0.0879 & 2380 & 0.009 & $2.24 E-03$ & $2.40 E-01$ \\
\hline 0.162 & 3320 & 0.16 & 2810 & 0.157 & 3400 & 0.017 & $3.18 E-03$ & $1.90 E-01$ \\
\hline 0.266 & 3850 & 0.267 & 3240 & 0.272 & 3890 & 0.028 & $3.66 E-03$ & $1.30 E-01$ \\
\hline 0.435 & 4490 & 0.44 & 3730 & 0.438 & 4480 & 0.046 & $4.23 E-03$ & $9.24 E-02$ \\
\hline 0.72 & 5170 & 0.721 & 4350 & 0.72 & 5130 & 0.075 & $4.88 E-03$ & $6.47 E-02$ \\
\hline 1.17 & 6030 & 1.18 & 5150 & 1.18 & 6050 & 0.123 & $5.74 E-03$ & $4.66 E-02$ \\
\hline 1.93 & 7470 & 1.94 & 6340 & 1.92 & 7380 & 0.202 & $7.06 E-03$ & $3.49 E-02$ \\
\hline 3.18 & 9760 & 3.17 & 8140 & 3.17 & 9520 & 0.332 & $9.14 E-03$ & $2.75 E-02$ \\
\hline 5.18 & 13500 & 5.18 & 11000 & 5.17 & 12800 & 0.542 & $1.24 E-02$ & $2.29 E-02$ \\
\hline 8.5 & 18600 & 8.49 & 15500 & 8.51 & 17800 & 0.890 & $1.73 E-02$ & $1.94 E-02$ \\
\hline 13.9 & 26000 & 13.9 & 22100 & 13.9 & 25200 & 1.456 & $2.44 E-02$ & $1.68 E-02$ \\
\hline 22.8 & 37000 & 22.8 & 32500 & 22.8 & 35700 & 2.388 & $3.51 E-02$ & $1.47 E-02$ \\
\hline 37.3 & 54000 & 37.2 & 48600 & 37.3 & 52100 & 3.903 & $5.16 E-02$ & $1.32 E-02$ \\
\hline 61 & 79000 & 61.1 & 73300 & 61.1 & 76000 & 6.395 & 7.61E-02 & $1.19 E-02$ \\
\hline 100 & 116000 & 100 & 111000 & 100 & 108000 & 10.472 & $1.12 E-01$ & $1.07 E-02$ \\
\hline 100 & 114000 & 100 & 110000 & 100 & 103000 & 10.472 & $1.09 E-01$ & $1.04 E-02$ \\
\hline 69.5 & 81200 & 69.5 & 80000 & 69.6 & 73900 & 7.282 & 7.84E-02 & $1.08 E-02$ \\
\hline 48.4 & 58800 & 48.4 & 58100 & 48.3 & 54000 & 5.065 & $5.70 E-02$ & $1.12 E-02$ \\
\hline 33.6 & 43300 & 33.6 & 42800 & 33.6 & 40400 & 3.519 & $4.22 E-02$ & $1.20 E-02$ \\
\hline 23.4 & 32000 & 23.4 & 31800 & 23.4 & 31400 & 2.450 & $3.17 E-02$ & $1.30 E-02$ \\
\hline 16.2 & 24000 & 16.3 & 23800 & 16.2 & 24100 & 1.700 & $2.40 E-02$ & $1.41 E-02$ \\
\hline 11.3 & 18200 & 11.3 & 18200 & 11.3 & 18500 & 1.183 & $1.83 E-02$ & $1.55 E-02$ \\
\hline 7.85 & 14100 & 7.84 & 14100 & 7.86 & 14300 & 0.822 & $1.42 E-02$ & $1.72 E-02$ \\
\hline 5.46 & 11100 & 5.47 & 11100 & 5.46 & 11400 & 0.572 & $1.12 E-02$ & $1.96 E-02$ \\
\hline 3.79 & 8850 & 3.78 & 8850 & 3.8 & 9140 & 0.397 & 8.95E-03 & $2.25 E-02$ \\
\hline 2.65 & 7280 & 2.64 & 7290 & 2.64 & 7660 & 0.277 & $7.41 E-03$ & $2.68 E-02$ \\
\hline 1.83 & 6080 & 1.83 & 6090 & 1.84 & 6480 & 0.192 & $6.22 E-03$ & $3.24 E-02$ \\
\hline 1.28 & 5220 & 1.27 & 5220 & 1.27 & 5520 & 0.133 & $5.32 E-03$ & 3.99E-02 \\
\hline 0.888 & 4580 & 0.887 & 4590 & 0.896 & 4820 & 0.093 & $4.66 E-03$ & $5.00 E-02$ \\
\hline 0.615 & 4040 & 0.618 & 4100 & 0.616 & 4270 & 0.065 & $4.14 E-03$ & $6.41 E-02$ \\
\hline 0.425 & 3680 & 0.431 & 3750 & 0.428 & 3880 & 0.045 & $3.77 E-03$ & $8.41 E-02$ \\
\hline 0.296 & 3420 & 0.3 & 3450 & 0.297 & 3590 & 0.031 & $3.49 E-03$ & $1.12 E-01$ \\
\hline 0.207 & 3190 & 0.21 & 3240 & 0.208 & 3330 & 0.022 & $3.25 E-03$ & $1.49 E-01$ \\
\hline 0.142 & 3030 & 0.145 & 3040 & 0.145 & 3210 & 0.015 & $3.09 E-03$ & $2.05 E-01$ \\
\hline 0.101 & 2940 & 0.101 & 2940 & 0.101 & 3070 & 0.011 & $2.98 E-03$ & $2.82 E-01$ \\
\hline
\end{tabular}




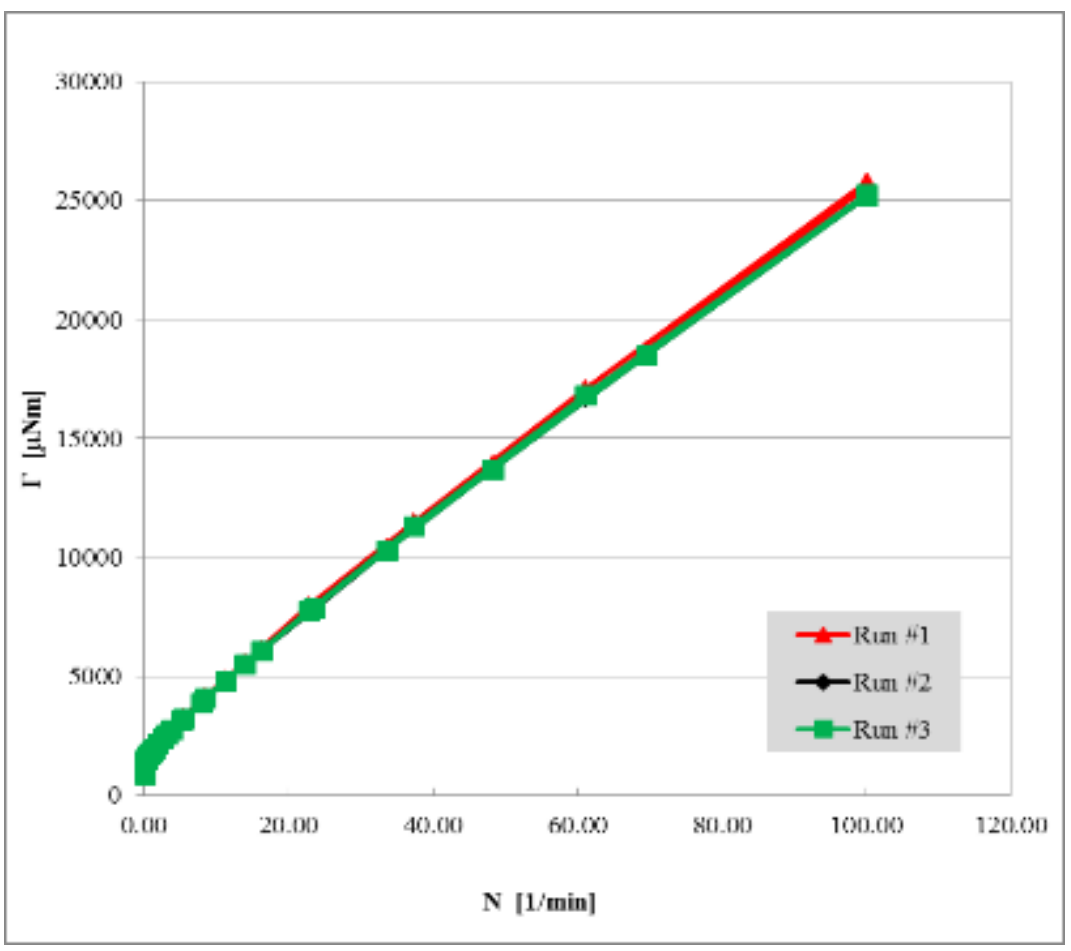

Figure A - 19: Torque vs. Angular Speed using Double spiral spindle on Mix\#5, with $0 \%$ beads by volume. Portrays data from Table A-19.

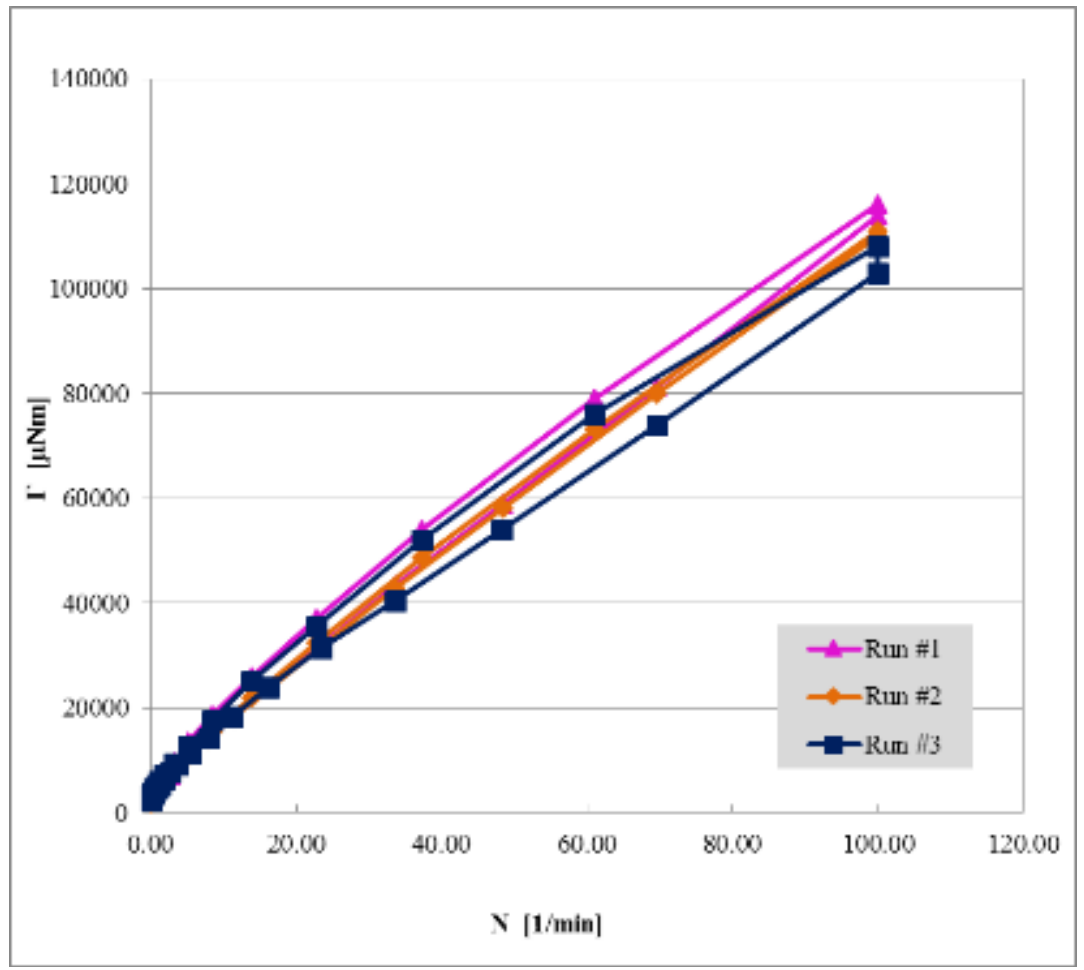

Figure A - 20: Torque vs. Angular Speed using Double spiral spindle on Mix\#5, with $\mathbf{4 0} \%$ beads by volume. Portrays data from Table A-20. 
Table A - 21: Measured data for Mix\#5 with 0 \% beads using Six-blade Vane.

\begin{tabular}{|c|c|c|c|c|c|c|c|c|}
\hline \multicolumn{6}{|c|}{6 Blade Vane } & \multirow{2}{*}{\multicolumn{3}{|c|}{$\begin{array}{c}\text { Bead } \% \\
0 \% \\
\end{array}$}} \\
\hline Geom.: & RHN-8 & & & \multicolumn{2}{|c|}{ Z43S } & & & \\
\hline \multicolumn{2}{|c|}{ Run \#1 } & \multicolumn{2}{|c|}{ Run \#2 } & \multicolumn{2}{|c|}{ Run \#3 } & \multirow{2}{*}{\multicolumn{3}{|c|}{$\begin{array}{c}\text { Average } \\
\text { values }\end{array}$}} \\
\hline \multicolumn{2}{|c|}{ NIST Code: SMC-95A } & \multicolumn{2}{|c|}{ NIST Code: SMC-95B } & \multicolumn{2}{|c|}{ NIST Code: SMC-95C } & & & \\
\hline $\mathrm{N}$ & $\Gamma$ & $\mathrm{N}$ & $\Gamma$ & $\mathrm{N}$ & $\Gamma$ & $\mathbf{N}$ & $\Gamma$ & $\Gamma / \boldsymbol{N}$ (Angular \\
\hline $1 / \mathrm{min}$ & $\mu \mathrm{Nm}$ & $1 / \mathrm{min}$ & $\mu \mathrm{Nm}$ & $1 / \mathrm{min}$ & $\mu \mathrm{Nm}$ & $\mathrm{rad} / \mathrm{s}$ & $\mathrm{Nm}$ & momentum) \\
\hline 0.0979 & 413 & 0.0999 & 502 & 0.098 & 385 & 0.010 & 4.33E-04 & 4.20E-02 \\
\hline 0.163 & 547 & 0.164 & 518 & 0.163 & 513 & 0.017 & $5.26 E-04$ & $3.08 E-02$ \\
\hline 0.268 & 588 & 0.268 & 553 & 0.268 & 558 & 0.028 & $5.66 E-04$ & $2.02 E-02$ \\
\hline 0.439 & 624 & 0.439 & 586 & 0.439 & 591 & 0.046 & $6.00 E-04$ & $1.31 E-02$ \\
\hline 0.72 & 675 & 0.724 & 647 & 0.72 & 640 & 0.076 & $6.54 E-04$ & $8.66 E-03$ \\
\hline 1.18 & 745 & 1.18 & 707 & 1.18 & 710 & 0.124 & $7.21 E-04$ & $5.83 E-03$ \\
\hline 1.93 & 840 & 1.93 & 821 & 1.93 & 808 & 0.202 & $8.23 E-04$ & 4.07E-03 \\
\hline 3.16 & 976 & 3.16 & 945 & 3.16 & 946 & 0.331 & $9.56 E-04$ & $2.89 E-03$ \\
\hline 5.18 & 1170 & 5.18 & 1140 & 5.18 & 1140 & 0.542 & $1.15 E-03$ & $2.12 E-03$ \\
\hline 8.48 & 1450 & 8.48 & 1400 & 8.48 & 1430 & 0.888 & $1.43 E-03$ & $1.61 E-03$ \\
\hline 13.9 & 1860 & 13.9 & 1800 & 13.9 & 1840 & 1.456 & $1.83 E-03$ & $1.26 E-03$ \\
\hline 22.8 & 2450 & 22.8 & 2380 & 22.8 & 2450 & 2.388 & $2.43 E-03$ & $1.02 E-03$ \\
\hline 37.3 & 3360 & 37.3 & 3280 & 37.3 & 3370 & 3.906 & $3.34 E-03$ & $8.54 E-04$ \\
\hline 61 & 4760 & 61 & 4660 & 61 & 4760 & 6.388 & $4.73 E-03$ & $7.40 E-04$ \\
\hline 100 & 6940 & 100 & 6820 & 100 & 6910 & 10.472 & $6.89 E-03$ & $6.58 E-04$ \\
\hline 100 & 6900 & 100 & 6810 & 100 & 6890 & 10.472 & $6.87 E-03$ & $6.56 E-04$ \\
\hline 69.5 & 5180 & 69.5 & 5120 & 69.5 & 5170 & 7.278 & $5.16 E-03$ & 7.09E-04 \\
\hline 48.3 & 3950 & 48.3 & 3910 & 48.3 & 3940 & 5.058 & $3.93 E-03$ & $7.78 E-04$ \\
\hline 33.6 & 3060 & 33.6 & 3030 & 33.6 & 3050 & 3.519 & $3.05 E-03$ & 8.66E-04 \\
\hline 23.4 & 2420 & 23.4 & 2400 & 23.4 & 2410 & 2.450 & $2.41 E-03$ & $9.83 E-04$ \\
\hline 16.2 & 1950 & 16.2 & 1930 & 16.2 & 1940 & 1.696 & $1.94 E-03$ & $1.14 E-03$ \\
\hline 11.3 & 1610 & 11.3 & 1590 & 11.3 & 1600 & 1.183 & $1.60 E-03$ & $1.35 E-03$ \\
\hline 7.85 & 1350 & 7.85 & 1330 & 7.85 & 1340 & 0.822 & $1.34 E-03$ & $1.63 E-03$ \\
\hline 5.45 & 1150 & 5.45 & 1130 & 5.45 & 1140 & 0.571 & $1.14 E-03$ & $2.00 E-03$ \\
\hline 3.79 & 993 & 3.79 & 982 & 3.79 & 985 & 0.397 & $9.87 E-04$ & $2.49 E-03$ \\
\hline 2.64 & 876 & 2.64 & 865 & 2.64 & 868 & 0.276 & 8.70E-04 & $3.15 E-03$ \\
\hline 1.83 & 784 & 1.83 & 774 & 1.83 & 777 & 0.192 & $7.78 E-04$ & $4.06 E-03$ \\
\hline 1.27 & 713 & 1.27 & 703 & 1.27 & 705 & 0.133 & $7.07 E-04$ & $5.32 E-03$ \\
\hline 0.886 & 657 & 0.886 & 648 & 0.887 & 655 & 0.093 & $6.53 E-04$ & 7.04E-03 \\
\hline 0.616 & 612 & 0.616 & 604 & 0.616 & 604 & 0.065 & $6.07 E-04$ & $9.40 E-03$ \\
\hline 0.428 & 577 & 0.428 & 569 & 0.428 & 568 & 0.045 & $5.71 E-04$ & $1.27 E-02$ \\
\hline 0.298 & 550 & 0.298 & 540 & 0.298 & 540 & 0.031 & $5.43 E-04$ & $1.74 E-02$ \\
\hline 0.207 & 525 & 0.207 & 517 & 0.208 & 518 & 0.022 & $5.20 E-04$ & $2.40 E-02$ \\
\hline 0.144 & 507 & 0.144 & 498 & 0.144 & 498 & 0.015 & $5.01 E-04$ & $3.32 E-02$ \\
\hline 0.1 & 493 & 0.0999 & 484 & 0.0999 & 483 & 0.010 & 4.87E-04 & 4.65E-02 \\
\hline
\end{tabular}


Table A - 22: Measured data for Mix\#5 with 40 \% beads using Six-blade Vane.

\begin{tabular}{|c|c|c|c|c|c|c|c|c|}
\hline \multicolumn{6}{|c|}{6 Blade Vane } & \multirow{2}{*}{\multicolumn{3}{|c|}{$\begin{array}{c}\text { Bead } \% \\
40 \% \\
\end{array}$}} \\
\hline Geom.: & $R H N-83 C$ & & & \multicolumn{2}{|c|}{ Z43S } & & & \\
\hline \multicolumn{2}{|c|}{ Run \#1 } & \multicolumn{2}{|c|}{ Run \#2 } & \multicolumn{2}{|c|}{ Run \#3 } & \multirow{2}{*}{\multicolumn{3}{|c|}{$\begin{array}{c}\text { Average } \\
\text { values }\end{array}$}} \\
\hline NIST Code: & SMC-95J & NIST Code: & SMC-95K & NIST Coc & SMC-95L & & & \\
\hline $\mathrm{N}$ & $\Gamma$ & $\mathrm{N}$ & $\Gamma$ & $\mathrm{N}$ & $\Gamma$ & $\mathbf{N}$ & $\Gamma$ & $\Gamma / N$ (Angular \\
\hline $1 / \mathrm{min}$ & $\mu \mathrm{Nm}$ & $1 / \mathrm{min}$ & $\mu \mathrm{Nm}$ & $1 / \mathrm{min}$ & $\mu \mathrm{Nm}$ & $\mathrm{rad} / \mathrm{s}$ & $\mathrm{Nm}$ & momentum) \\
\hline 0.0958 & 1050 & 0.0973 & 967 & 0.0976 & 1010 & 0.010 & $1.01 E-03$ & $9.94 E-02$ \\
\hline 0.163 & 1330 & 0.163 & 1230 & 0.163 & 1260 & 0.017 & $1.27 E-03$ & $7.46 E-02$ \\
\hline 0.27 & 1420 & 0.271 & 1340 & 0.272 & 1370 & 0.028 & $1.38 E-03$ & $4.85 E-02$ \\
\hline 0.438 & 1550 & 0.441 & 1420 & 0.444 & 1510 & 0.046 & $1.49 E-03$ & $3.23 E-02$ \\
\hline 0.72 & 1700 & 0.717 & 1630 & 0.719 & 1640 & 0.075 & $1.66 E-03$ & $2.20 E-02$ \\
\hline 1.18 & 1960 & 1.18 & 1850 & 1.18 & 1900 & 0.124 & $1.90 E-03$ & $1.54 E-02$ \\
\hline 1.93 & 2270 & 1.93 & 2200 & 1.93 & 2200 & 0.202 & $2.22 E-03$ & $1.10 E-02$ \\
\hline 3.16 & 2740 & 3.17 & 2680 & 3.17 & 2720 & 0.332 & $2.71 E-03$ & $8.18 E-03$ \\
\hline 5.18 & 3480 & 5.18 & 3440 & 5.18 & 3510 & 0.542 & $3.48 E-03$ & $6.41 E-03$ \\
\hline 8.5 & 4450 & 8.48 & 4470 & 8.47 & 4610 & 0.888 & $4.51 E-03$ & $5.08 E-03$ \\
\hline 13.9 & 5970 & 13.9 & 5960 & 13.9 & 6170 & 1.456 & $6.03 E-03$ & 4.14E-03 \\
\hline 22.8 & 8080 & 22.7 & 8330 & 22.8 & 8510 & 2.384 & 8.31E-03 & $3.48 E-03$ \\
\hline 37.3 & 11100 & 37.3 & 11700 & 37.3 & 12000 & 3.906 & $1.16 E-02$ & 2.97E-03 \\
\hline 61.1 & 15400 & 61.1 & 16700 & 61 & 17100 & 6.395 & $1.64 E-02$ & $2.56 E-03$ \\
\hline 100 & 21500 & 100 & 23800 & 100 & 24500 & 10.472 & 2.33E-02 & $2.22 E-03$ \\
\hline 100 & 20900 & 100 & 22900 & 100 & 23700 & 10.472 & $2.25 E-02$ & $2.15 E-03$ \\
\hline 69.5 & 15600 & 69.5 & 16600 & 69.5 & 17300 & 7.278 & $1.65 E-02$ & $2.27 E-03$ \\
\hline 48.3 & 11900 & 48.3 & 12300 & 48.3 & 12800 & 5.058 & $1.23 E-02$ & $2.44 E-03$ \\
\hline 33.6 & 9090 & 33.6 & 9260 & 33.6 & 9600 & 3.519 & $9.32 E-03$ & $2.65 E-03$ \\
\hline 23.4 & 6960 & 23.3 & 7070 & 23.3 & 7340 & 2.443 & 7.12E-03 & $2.92 E-03$ \\
\hline 16.2 & 5420 & 16.2 & 5510 & 16.2 & 5680 & 1.696 & $5.54 E-03$ & $3.26 E-03$ \\
\hline 11.3 & 4270 & 11.3 & 4300 & 11.3 & 4470 & 1.183 & $4.35 E-03$ & $3.67 E-03$ \\
\hline 7.84 & 3450 & 7.85 & 3480 & 7.85 & 3560 & 0.822 & $3.50 E-03$ & $4.26 E-03$ \\
\hline 5.46 & 2800 & 5.46 & 2830 & 5.45 & 2880 & 0.571 & $2.84 E-03$ & $4.96 E-03$ \\
\hline 3.79 & 2340 & 3.79 & 2320 & 3.79 & 2400 & 0.397 & $2.35 E-03$ & $5.93 E-03$ \\
\hline 2.64 & 1960 & 2.64 & 1960 & 2.64 & 2000 & 0.276 & $1.97 E-03$ & $7.14 E-03$ \\
\hline 1.83 & 1700 & 1.83 & 1690 & 1.83 & 1740 & 0.192 & $1.71 E-03$ & $8.92 E-03$ \\
\hline 1.27 & 1490 & 1.27 & 1460 & 1.27 & 1530 & 0.133 & $1.49 E-03$ & $1.12 E-02$ \\
\hline 0.884 & 1320 & 0.886 & 1320 & 0.887 & 1360 & 0.093 & $1.33 E-03$ & $1.44 E-02$ \\
\hline 0.615 & 1210 & 0.616 & 1200 & 0.619 & 1230 & 0.065 & $1.21 E-03$ & $1.88 E-02$ \\
\hline 0.43 & 1090 & 0.431 & 1090 & 0.427 & 1140 & 0.045 & $1.11 E-03$ & $2.46 E-02$ \\
\hline 0.298 & 1020 & 0.295 & 1040 & 0.296 & 1040 & 0.031 & $1.03 E-03$ & 3.33E-02 \\
\hline 0.206 & 962 & 0.208 & 971 & 0.207 & 990 & 0.022 & $9.74 E-04$ & $4.49 E-02$ \\
\hline 0.143 & 921 & 0.144 & 932 & 0.144 & 949 & 0.015 & $9.34 E-04$ & $6.21 E-02$ \\
\hline 0.101 & 889 & 0.0985 & 895 & 0.1 & 903 & 0.010 & 8.96E-04 & 8.57E-02 \\
\hline
\end{tabular}




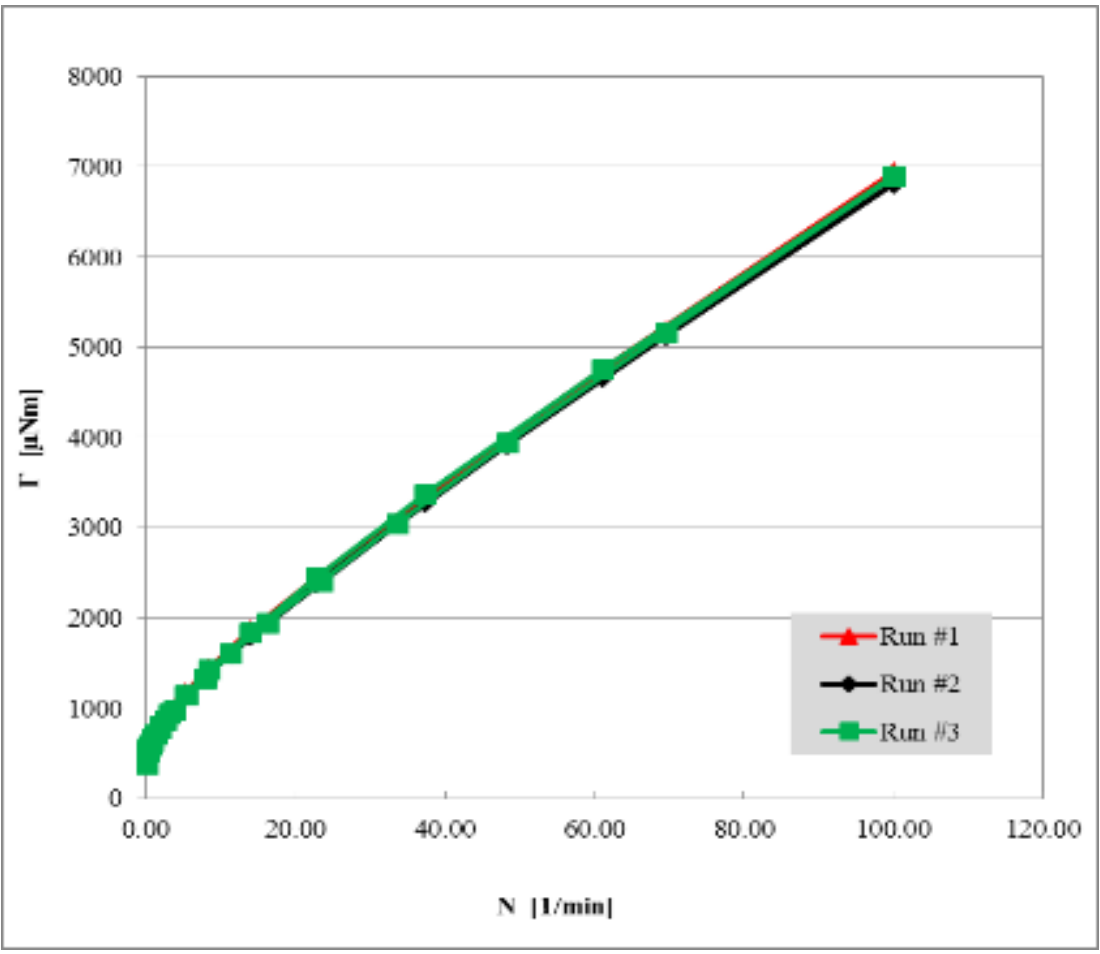

Figure A - 15: Torque vs. Angular Speed using six-blade vane on Mix\#5, with $0 \%$ beads by volume. Portrays data from Table A-21.

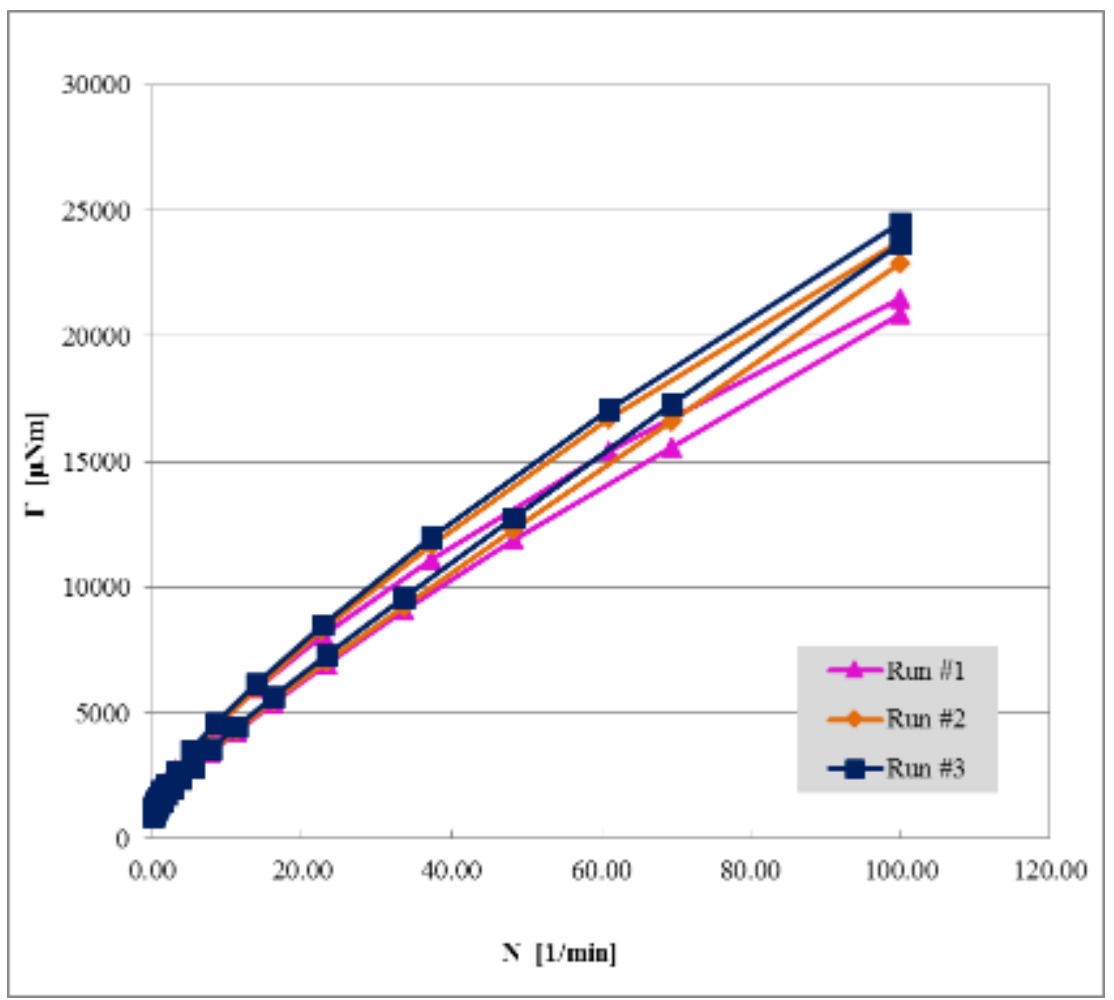

Figure A - 16: Torque vs. Angular Speed using six-blade vane on Mix\#5, with $40 \%$ beads by volume. Portrays data from Table A-22. 
Table A - 23: Measured data for Mix\#5 with 0 \% beads using Serrated Coaxial Cylinder.

\begin{tabular}{|c|c|c|c|c|c|c|c|c|}
\hline \multicolumn{6}{|c|}{ Serrated Coaxial Cylinder } & \multirow{2}{*}{\multicolumn{3}{|c|}{$\begin{array}{c}\text { Bead } \% \\
0 \% \\
\end{array}$}} \\
\hline Geom.: & & & & \multicolumn{2}{|c|}{ Z43S } & & & \\
\hline \multicolumn{2}{|c|}{ Run \#1 } & \multicolumn{2}{|c|}{ Run \#2 } & \multicolumn{2}{|c|}{ Run \#3 } & \multirow{2}{*}{\multicolumn{3}{|c|}{$\begin{array}{c}\text { Average } \\
\text { values }\end{array}$}} \\
\hline \multicolumn{2}{|c|}{ NIST Code: SMC-95G } & \multicolumn{2}{|c|}{ NIST Code: SMC-95H } & \multicolumn{2}{|c|}{ NIST Code: SMC-95I } & & & \\
\hline $\mathrm{N}$ & $\Gamma$ & $\mathrm{N}$ & $\Gamma$ & $\mathrm{N}$ & $\Gamma$ & $\mathbf{N}$ & $\Gamma$ & $\Gamma / N$ (Angular \\
\hline $1 / \mathrm{min}$ & $\mu \mathrm{Nm}$ & $1 / \mathrm{min}$ & $\mu \mathrm{Nm}$ & $1 / \mathrm{min}$ & $\mu \mathrm{Nm}$ & $\mathrm{rad} / \mathrm{s}$ & $\mathrm{Nm}$ & momentum) \\
\hline 0.0985 & 441 & 0.0999 & 353 & 0.0986 & 367 & 0.010 & 3.87E-04 & 3.73E-02 \\
\hline 0.164 & 545 & 0.164 & 376 & 0.164 & 473 & 0.017 & $4.65 E-04$ & $2.71 E-02$ \\
\hline 0.268 & 574 & 0.268 & 412 & 0.268 & 510 & 0.028 & $4.99 E-04$ & $1.78 E-02$ \\
\hline 0.439 & 612 & 0.439 & 462 & 0.439 & 550 & 0.046 & $5.41 E-04$ & $1.18 E-02$ \\
\hline 0.72 & 669 & 0.72 & 526 & 0.72 & 606 & 0.075 & $6.00 E-04$ & $7.96 E-03$ \\
\hline 1.18 & 746 & 1.18 & 613 & 1.18 & 683 & 0.124 & $6.81 E-04$ & $5.51 E-03$ \\
\hline 1.93 & 853 & 1.93 & 730 & 1.93 & 793 & 0.202 & 7.92E-04 & $3.92 E-03$ \\
\hline 3.16 & 1000 & 3.16 & 888 & 3.16 & 951 & 0.331 & $9.46 E-04$ & $2.86 E-03$ \\
\hline 5.18 & 1210 & 5.18 & 1100 & 5.18 & 1170 & 0.542 & $1.16 E-03$ & $2.14 E-03$ \\
\hline 8.48 & 1510 & 8.48 & 1400 & 8.48 & 1490 & 0.888 & $1.47 E-03$ & $1.65 E-03$ \\
\hline 13.9 & 1950 & 13.9 & 1830 & 13.9 & 1950 & 1.456 & $1.91 E-03$ & $1.31 E-03$ \\
\hline 22.8 & 2580 & 22.8 & 2440 & 22.8 & 2600 & 2.388 & $2.54 E-03$ & $1.06 E-03$ \\
\hline 37.3 & 3560 & 37.3 & 3380 & 37.3 & 3590 & 3.906 & $3.51 E-03$ & 8.99E-04 \\
\hline 61 & 5050 & 61 & 4840 & 61 & 5100 & 6.388 & $5.00 E-03$ & $7.82 E-04$ \\
\hline 100 & 7410 & 100 & 7120 & 100 & 7460 & 10.472 & 7.33E-03 & $7.00 E-04$ \\
\hline 100 & 7300 & 100 & 7100 & 100 & 7350 & 10.472 & $7.25 E-03$ & $6.92 E-04$ \\
\hline 69.5 & 5480 & 69.5 & 5320 & 69.5 & 5500 & 7.278 & $5.43 E-03$ & $7.47 E-04$ \\
\hline 48.3 & 4140 & 48.3 & 4030 & 48.3 & 4150 & 5.058 & $4.11 E-03$ & $8.12 E-04$ \\
\hline 33.6 & 3180 & 33.6 & 3090 & 33.6 & 3180 & 3.519 & $3.15 E-03$ & $8.95 E-04$ \\
\hline 23.4 & 2490 & 23.4 & 2420 & 23.4 & 2480 & 2.450 & $2.46 E-03$ & $1.01 E-03$ \\
\hline 16.2 & 1980 & 16.2 & 1920 & 16.2 & 1970 & 1.696 & $1.96 E-03$ & $1.15 E-03$ \\
\hline 11.3 & 1610 & 11.3 & 1560 & 11.3 & 1600 & 1.183 & $1.59 E-03$ & $1.34 E-03$ \\
\hline 7.85 & 1330 & 7.85 & 1280 & 7.85 & 1310 & 0.822 & $1.31 E-03$ & $1.59 E-03$ \\
\hline 5.45 & 1110 & 5.45 & 1070 & 5.45 & 1090 & 0.571 & $1.09 E-03$ & $1.91 E-03$ \\
\hline 3.79 & 943 & 3.79 & 902 & 3.79 & 924 & 0.397 & $9.23 E-04$ & $2.33 E-03$ \\
\hline 2.64 & 810 & 2.64 & 772 & 2.64 & 793 & 0.276 & 7.92E-04 & $2.86 E-03$ \\
\hline 1.83 & 705 & 1.83 & 670 & 1.83 & 691 & 0.192 & $6.89 E-04$ & $3.59 E-03$ \\
\hline 1.27 & 622 & 1.27 & 589 & 1.27 & 609 & 0.133 & $6.07 E-04$ & $4.56 E-03$ \\
\hline 0.886 & 554 & 0.886 & 525 & 0.886 & 545 & 0.093 & $5.41 E-04$ & $5.83 E-03$ \\
\hline 0.616 & 499 & 0.616 & 473 & 0.615 & 490 & 0.064 & $4.87 E-04$ & $7.56 E-03$ \\
\hline 0.428 & 455 & 0.428 & 431 & 0.428 & 451 & 0.045 & $4.46 E-04$ & $9.94 E-03$ \\
\hline 0.298 & 418 & 0.298 & 397 & 0.298 & 416 & 0.031 & $4.10 E-04$ & $1.31 E-02$ \\
\hline 0.207 & 389 & 0.207 & 371 & 0.207 & 388 & 0.022 & $3.83 E-04$ & $1.77 E-02$ \\
\hline 0.144 & 365 & 0.144 & 349 & 0.144 & 366 & 0.015 & $3.60 E-04$ & $2.39 E-02$ \\
\hline 0.1 & 346 & 0.1 & 331 & 0.1 & 347 & 0.010 & $3.41 E-04$ & $3.26 E-02$ \\
\hline
\end{tabular}


Table A - 24: Measured data for Mix\#5 with 40 \% beads using Serrated Coaxial Cylinder.

\begin{tabular}{|c|c|c|c|c|c|c|c|c|}
\hline \multicolumn{6}{|c|}{ Serrated Coaxial Cylinder } & \multirow{2}{*}{\multicolumn{3}{|c|}{$\begin{array}{c}\text { Bead \% } \\
40 \%\end{array}$}} \\
\hline Geom.: & SS18 & & & \multicolumn{2}{|c|}{ Z43S } & & & \\
\hline \multicolumn{2}{|c|}{ Run \#1 } & \multicolumn{2}{|c|}{ Run \#2 } & \multicolumn{2}{|c|}{ Run \#3 } & \multirow{2}{*}{\multicolumn{3}{|c|}{$\begin{array}{c}\text { Average } \\
\text { values }\end{array}$}} \\
\hline NIST Code: & SMC-95P & NIST Code: & SMC-95Q & NIST Code: & SMC-95R & & & \\
\hline $\mathrm{N}$ & $\Gamma$ & $\mathrm{N}$ & $\Gamma$ & $\mathrm{N}$ & $\Gamma$ & $\mathbf{N}$ & $\Gamma$ & $\boldsymbol{\Gamma} / \mathbf{N}$ (Angular \\
\hline $1 / \mathrm{min}$ & $\mu \mathrm{Nm}$ & $1 / \mathrm{min}$ & $\mu \mathrm{Nm}$ & $1 / \mathrm{min}$ & $\mu \mathrm{Nm}$ & $\mathrm{rad} / \mathrm{s}$ & $\mathrm{Nm}$ & momentum) \\
\hline 0.0989 & 1270 & 0.0967 & 1080 & 0.0971 & 1090 & 0.010 & $1.15 E-03$ & $1.12 E-01$ \\
\hline 0.161 & 1520 & 0.163 & 1210 & 0.165 & 1220 & 0.017 & $1.32 E-03$ & $7.71 E-02$ \\
\hline 0.269 & 1580 & 0.27 & 1220 & 0.27 & 1250 & 0.028 & $1.35 E-03$ & $4.78 E-02$ \\
\hline 0.445 & 1640 & 0.44 & 1300 & 0.438 & 1320 & 0.046 & $1.42 E-03$ & $3.07 E-02$ \\
\hline 0.724 & 1710 & 0.719 & 1450 & 0.722 & 1510 & 0.076 & $1.56 E-03$ & $2.06 E-02$ \\
\hline 1.18 & 1820 & 1.18 & 1700 & 1.18 & 1740 & 0.124 & $1.75 E-03$ & $1.42 E-02$ \\
\hline 1.93 & 2100 & 1.93 & 2080 & 1.93 & 2130 & 0.202 & $2.10 E-03$ & $1.04 E-02$ \\
\hline 3.16 & 2500 & 3.16 & 2580 & 3.16 & 2700 & 0.331 & $2.59 E-03$ & $7.84 E-03$ \\
\hline 5.18 & 3140 & 5.18 & 3340 & 5.18 & 3470 & 0.542 & $3.32 E-03$ & $6.11 E-03$ \\
\hline 8.49 & 4090 & 8.48 & 4410 & 8.49 & 4580 & 0.889 & $4.36 E-03$ & $4.91 E-03$ \\
\hline 13.9 & 5470 & 13.9 & 5930 & 13.9 & 6220 & 1.456 & $5.87 E-03$ & $4.03 E-03$ \\
\hline 22.8 & 7460 & 22.7 & 8170 & 22.8 & 8520 & 2.384 & 8.05E-03 & $3.38 E-03$ \\
\hline 37.3 & 10100 & 37.3 & 11400 & 37.3 & 11900 & 3.906 & $1.11 E-02$ & $2.85 E-03$ \\
\hline 61.1 & 14000 & 61.1 & 16000 & 61.1 & 16600 & 6.398 & $1.55 E-02$ & $2.43 E-03$ \\
\hline 100 & 19300 & 100 & 22400 & 100 & 22700 & 10.472 & $2.15 E-02$ & $2.05 E-03$ \\
\hline 100 & 19400 & 100 & 20100 & 99.9 & 20800 & 10.468 & $2.01 E-02$ & $1.92 E-03$ \\
\hline 69.5 & 14900 & 69.5 & 15500 & 69.5 & 15800 & 7.278 & $1.54 E-02$ & $2.12 E-03$ \\
\hline 48.3 & 11700 & 48.3 & 11800 & 48.3 & 12100 & 5.058 & $1.19 E-02$ & $2.35 E-03$ \\
\hline 33.6 & 9150 & 33.6 & 9540 & 33.6 & 9660 & 3.519 & $9.45 E-03$ & $2.69 E-03$ \\
\hline 23.4 & 7010 & 23.4 & 7400 & 23.3 & 7570 & 2.447 & 7.33E-03 & $2.99 E-03$ \\
\hline 16.2 & 5430 & 16.2 & 5730 & 16.2 & 5910 & 1.696 & $5.69 E-03$ & $3.35 E-03$ \\
\hline 11.3 & 4250 & 11.3 & 4470 & 11.3 & 4620 & 1.183 & $4.45 E-03$ & $3.76 E-03$ \\
\hline 7.84 & 3390 & 7.85 & 3560 & 7.85 & 3640 & 0.822 & $3.53 E-03$ & 4.30E-03 \\
\hline 5.46 & 2720 & 5.45 & 2880 & 5.45 & 2960 & 0.571 & $2.85 E-03$ & $5.00 E-03$ \\
\hline 3.79 & 2260 & 3.79 & 2360 & 3.79 & 2430 & 0.397 & $2.35 E-03$ & $5.92 E-03$ \\
\hline 2.63 & 1890 & 2.64 & 1970 & 2.63 & 2030 & 0.276 & $1.96 E-03$ & $7.12 E-03$ \\
\hline 1.83 & 1620 & 1.83 & 1670 & 1.83 & 1710 & 0.192 & $1.67 E-03$ & 8.70E-03 \\
\hline 1.27 & 1400 & 1.27 & 1460 & 1.27 & 1490 & 0.133 & $1.45 E-03$ & $1.09 E-02$ \\
\hline 0.886 & 1230 & 0.887 & 1280 & 0.886 & 1310 & 0.093 & $1.27 E-03$ & $1.37 E-02$ \\
\hline 0.614 & 1100 & 0.615 & 1140 & 0.616 & 1170 & 0.064 & $1.14 E-03$ & $1.76 E-02$ \\
\hline 0.429 & 995 & 0.428 & 1030 & 0.428 & 1040 & 0.045 & $1.02 E-03$ & $2.28 E-02$ \\
\hline 0.296 & 905 & 0.298 & 953 & 0.298 & 962 & 0.031 & $9.40 E-04$ & $3.02 E-02$ \\
\hline 0.207 & 830 & 0.208 & 872 & 0.207 & 898 & 0.022 & 8.67E-04 & $3.99 E-02$ \\
\hline 0.145 & 776 & 0.144 & 814 & 0.143 & 835 & 0.015 & $8.08 E-04$ & $5.36 E-02$ \\
\hline 0.0995 & 725 & 0.101 & 768 & 0.0984 & 781 & 0.010 & $7.58 E-04$ & 7.27E-02 \\
\hline
\end{tabular}




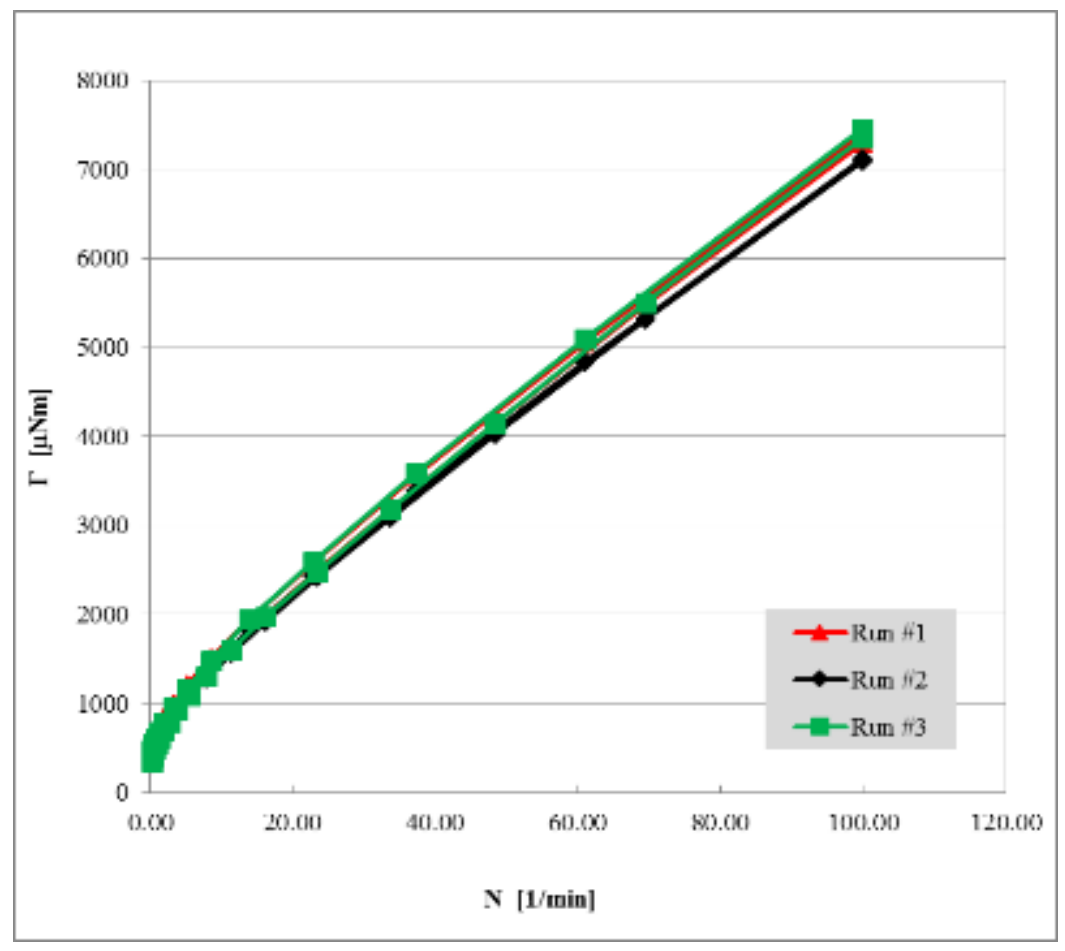

Figure A - 18: : Torque vs. Angular Speed using Serrated Coaxial Cylinder on Mix\#5, with $0 \%$ beads by volume. Portrays data from Table A-23.

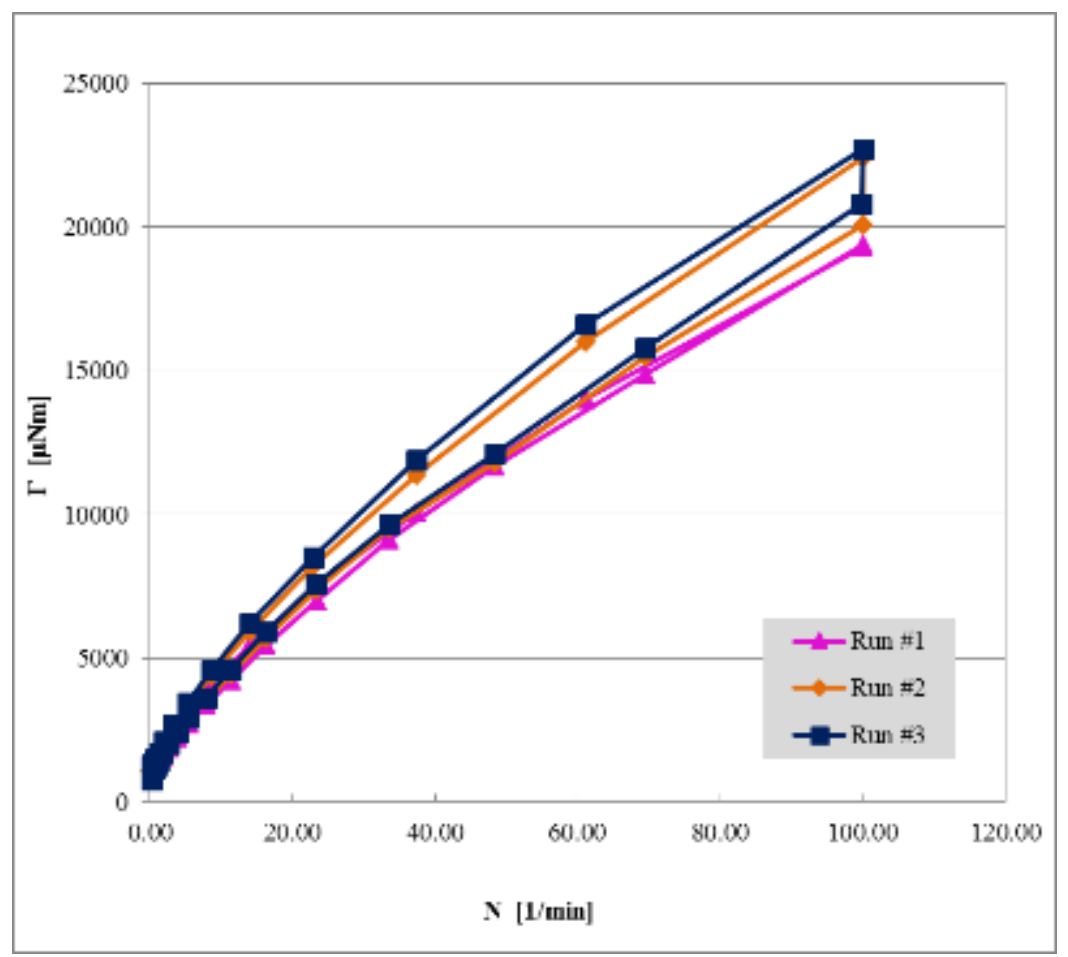

Figure A - 17: : Torque vs. Angular Speed using Serrated Coaxial Cylinder on Mix\#5, with $40 \%$ beads by volume. Portrays data from Table A-24. 
Table A - 25: Measured data for Mix\#6 with 0 \% beads using Double spiral spindle.

\begin{tabular}{|c|c|c|c|c|c|c|c|c|}
\hline \multicolumn{6}{|c|}{ Double Spiral } & \multicolumn{3}{|c|}{$\begin{array}{c}\text { Bead } \% \\
0 \% \\
\end{array}$} \\
\hline \multicolumn{2}{|c|}{ Run \#1 } & \multicolumn{2}{|c|}{ Run \#2 } & \multicolumn{2}{|c|}{ Run \#3 } & \multirow{2}{*}{\multicolumn{3}{|c|}{$\begin{array}{c}\text { Average } \\
\text { values }\end{array}$}} \\
\hline NIST Code: & SMC-97G & NIST Code: & SMC-97H & NIST Code: & SMC-97I & & & \\
\hline \begin{tabular}{l|l}
$\mathrm{N}$ \\
\end{tabular} & $\Gamma$ & $\mathrm{N}$ & $\Gamma$ & $\mathrm{N}$ & $\Gamma$ & $\mathbf{N}$ & $\Gamma$ & $\Gamma / \boldsymbol{N}$ (Angular \\
\hline $1 / \mathrm{min}$ & $\mu \mathrm{Nm}$ & $1 / \mathrm{min}$ & $\mu \mathrm{Nm}$ & $1 / \mathrm{min}$ & $\mu \mathrm{Nm}$ & $\mathrm{rad} / \mathrm{s}$ & $\mathrm{Nm}$ & momentum) \\
\hline 0.0941 & 1080 & 0.0945 & 988 & 0.0945 & 1000 & 0.010 & $1.02 E-03$ & $1.03 E-01$ \\
\hline 0.161 & 1580 & 0.161 & 1470 & 0.161 & 1480 & 0.017 & $1.51 E-03$ & 8.96E-02 \\
\hline 0.268 & 1790 & 0.268 & 1670 & 0.268 & 1690 & 0.028 & $1.72 E-03$ & $6.12 E-02$ \\
\hline 0.44 & 1900 & 0.439 & 1800 & 0.439 & 1820 & 0.046 & $1.84 E-03$ & $4.00 E-02$ \\
\hline 0.72 & 2030 & 0.72 & 1940 & 0.72 & 1960 & 0.075 & $1.98 E-03$ & $2.62 E-02$ \\
\hline 1.18 & 2200 & 1.18 & 2130 & 1.18 & 2150 & 0.124 & $2.16 E-03$ & $1.75 E-02$ \\
\hline 1.93 & 2480 & 1.93 & 2420 & 1.93 & 2430 & 0.202 & $2.44 E-03$ & $1.21 E-02$ \\
\hline 3.16 & 2900 & 3.16 & 2850 & 3.16 & 2870 & 0.331 & $2.87 E-03$ & $8.68 E-03$ \\
\hline 5.18 & 3550 & 5.18 & 3510 & 5.18 & 3530 & 0.542 & $3.53 E-03$ & $6.51 E-03$ \\
\hline 8.48 & 4560 & 8.48 & 4530 & 8.48 & 4540 & 0.888 & $4.54 E-03$ & $5.12 E-03$ \\
\hline 13.9 & 6180 & 13.9 & 6120 & 13.9 & 6190 & 1.456 & $6.16 E-03$ & $4.23 E-03$ \\
\hline 22.8 & 8710 & 22.8 & 8610 & 22.8 & 8690 & 2.388 & 8.67E-03 & $3.63 E-03$ \\
\hline 37.3 & 12700 & 37.3 & 12500 & 37.3 & 12700 & 3.906 & $1.26 E-02$ & $3.23 E-03$ \\
\hline 61.1 & 18900 & 61.1 & 18700 & 61.1 & 18900 & 6.398 & $1.88 E-02$ & $2.94 E-03$ \\
\hline 100 & 28500 & 100 & 28200 & 100 & 28600 & 10.472 & $2.84 E-02$ & $2.72 E-03$ \\
\hline 100 & 28300 & 100 & 28100 & 100 & 28500 & 10.472 & $2.83 E-02$ & $2.70 E-03$ \\
\hline 69.5 & 20700 & 69.5 & 20600 & 69.5 & 20900 & 7.278 & 2.07E-02 & $2.85 E-03$ \\
\hline 48.3 & 15300 & 48.3 & 15200 & 48.3 & 15400 & 5.058 & $1.53 E-02$ & $3.02 E-03$ \\
\hline 33.6 & 11500 & 33.6 & 11400 & 33.6 & 11600 & 3.519 & $1.15 E-02$ & $3.27 E-03$ \\
\hline 23.4 & 8720 & 23.4 & 8690 & 23.4 & 8810 & 2.450 & 8.74E-03 & $3.57 E-03$ \\
\hline 16.2 & 6760 & 16.2 & 6740 & 16.2 & 6830 & 1.696 & $6.78 E-03$ & $3.99 E-03$ \\
\hline 11.3 & 5350 & 11.3 & 5330 & 11.3 & 5410 & 1.183 & 5.36E-03 & $4.53 E-03$ \\
\hline 7.85 & 4330 & 7.85 & 4320 & 7.85 & 4380 & 0.822 & $4.34 E-03$ & $5.28 E-03$ \\
\hline 5.46 & 3590 & 5.46 & 3590 & 5.46 & 3630 & 0.572 & $3.60 E-03$ & $6.30 E-03$ \\
\hline 3.79 & 3060 & 3.79 & 3060 & 3.79 & 3090 & 0.397 & 3.07E-03 & $7.74 E-03$ \\
\hline 2.64 & 2670 & 2.64 & 2660 & 2.64 & 2690 & 0.276 & 2.67E-03 & $9.67 E-03$ \\
\hline 1.83 & 2370 & 1.83 & 2370 & 1.83 & 2390 & 0.192 & $2.38 E-03$ & $1.24 E-02$ \\
\hline 1.27 & 2160 & 1.27 & 2150 & 1.27 & 2170 & 0.133 & $2.16 E-03$ & $1.62 E-02$ \\
\hline 0.886 & 1990 & 0.886 & 1990 & 0.886 & 2010 & 0.093 & $2.00 E-03$ & $2.15 E-02$ \\
\hline 0.616 & 1860 & 0.616 & 1860 & 0.616 & 1870 & 0.065 & $1.86 E-03$ & $2.89 E-02$ \\
\hline 0.428 & 1770 & 0.428 & 1770 & 0.428 & 1780 & 0.045 & $1.77 E-03$ & $3.96 E-02$ \\
\hline 0.297 & 1700 & 0.298 & 1700 & 0.298 & 1710 & 0.031 & $1.70 E-03$ & $5.46 E-02$ \\
\hline 0.207 & 1640 & 0.207 & 1640 & 0.208 & 1650 & 0.022 & $1.64 E-03$ & 7.57E-02 \\
\hline 0.144 & 1600 & 0.144 & 1590 & 0.143 & 1600 & 0.015 & $1.60 E-03$ & $1.06 E-01$ \\
\hline 0.0995 & 1560 & 0.0991 & 1560 & 0.0998 & 1560 & 0.010 & $1.56 E-03$ & $1.50 E-01$ \\
\hline
\end{tabular}


Table A - 26: Measured data for Mix\#6 with 40 \% beads using Double spiral spindle.

\begin{tabular}{|c|c|c|c|c|c|c|c|c|}
\hline \multicolumn{6}{|c|}{ Double Spiral } & \multirow{2}{*}{\multicolumn{3}{|c|}{$\begin{array}{c}\text { Bead } \% \\
40 \% \\
\end{array}$}} \\
\hline Geom.: & $R H N-83 A$ & & & Cup: & Z43S & & & \\
\hline \multicolumn{2}{|c|}{ Run \#1 } & \multicolumn{2}{|c|}{ Run \#2 } & \multicolumn{2}{|c|}{ Run \#3 } & \multirow{2}{*}{\multicolumn{3}{|c|}{$\begin{array}{c}\text { Average } \\
\text { values }\end{array}$}} \\
\hline NIST Code: & SMC-97P & NIST Code: & SMC-97Q & NIST Code: & $S M C-97 R$ & & & \\
\hline \begin{tabular}{|l|l}
$\mathrm{N}$ & \\
\end{tabular} & $\Gamma$ & \begin{tabular}{l|l}
$\mathrm{N}$ \\
\end{tabular} & \begin{tabular}{|c|}
$\Gamma$ \\
\end{tabular} & \begin{tabular}{|c|}
$\mathrm{N}$ \\
\end{tabular} & $\Gamma$ & $\mathbf{N}$ & $\Gamma$ & $\boldsymbol{\Gamma} / \mathbf{N}$ (Angular \\
\hline $1 / \mathrm{min}$ & $\mu \mathrm{Nm}$ & $1 / \mathrm{min}$ & $\mu \mathrm{Nm}$ & $1 / \mathrm{min}$ & $\mu \mathrm{Nm}$ & $\mathrm{rad} / \mathrm{s}$ & $\mathrm{Nm}$ & momentum) \\
\hline 0.0815 & 2760 & 0.0861 & 2810 & 0.0862 & 2810 & 0.009 & $2.79 E-03$ & $3.15 E-01$ \\
\hline 0.16 & 4030 & 0.159 & 4170 & 0.157 & 4010 & 0.017 & $4.07 E-03$ & $2.45 E-01$ \\
\hline 0.261 & 4890 & 0.26 & 5010 & 0.267 & 4670 & 0.028 & 4.86E-03 & $1.77 E-01$ \\
\hline 0.445 & 5810 & 0.438 & 5670 & 0.445 & 5310 & 0.046 & $5.60 E-03$ & $1.21 E-01$ \\
\hline 0.721 & 6680 & 0.728 & 6490 & 0.728 & 6110 & 0.076 & $6.43 E-03$ & $8.46 E-02$ \\
\hline 1.16 & 8000 & 1.18 & 7650 & 1.17 & 7020 & 0.123 & $7.56 E-03$ & 6.17E-02 \\
\hline 1.94 & 9820 & 1.93 & 9420 & 1.94 & 8760 & 0.203 & $9.33 E-03$ & 4.60E-02 \\
\hline 3.17 & 12800 & 3.16 & 12200 & 3.17 & 11500 & 0.332 & $1.22 E-02$ & 3.67E-02 \\
\hline 5.19 & 17300 & 5.18 & 16600 & 5.19 & 15600 & 0.543 & $1.65 E-02$ & $3.04 E-02$ \\
\hline 8.49 & 24200 & 8.49 & 23500 & 8.45 & 22100 & 0.888 & 2.33E-02 & $2.62 E-02$ \\
\hline 13.9 & 33700 & 13.9 & 33300 & 13.9 & 31600 & 1.456 & $3.29 E-02$ & $2.26 E-02$ \\
\hline 22.8 & 47000 & 22.8 & 47400 & 22.8 & 44800 & 2.388 & $4.64 E-02$ & $1.94 E-02$ \\
\hline 37.3 & 68000 & 37.3 & 68900 & 37.3 & 65400 & 3.906 & $6.74 E-02$ & $1.73 E-02$ \\
\hline 61.1 & 96700 & 61.1 & 100000 & 61.1 & 90800 & 6.398 & $9.58 E-02$ & $1.50 E-02$ \\
\hline 100 & 133000 & 100 & 143000 & 100 & 127000 & 10.472 & $1.34 E-01$ & $1.28 E-02$ \\
\hline 100 & 125000 & 100 & 135000 & 99.9 & 122000 & 10.468 & $1.27 E-01$ & $1.22 E-02$ \\
\hline 69.6 & 88200 & 69.6 & 95800 & 69.5 & 86300 & 7.285 & $9.01 E-02$ & $1.24 E-02$ \\
\hline 48.3 & 63700 & 48.4 & 70200 & 48.4 & 62600 & 5.065 & $6.55 E-02$ & $1.29 E-02$ \\
\hline 33.6 & 46600 & 33.6 & 51900 & 33.6 & 45800 & 3.519 & $4.81 E-02$ & $1.37 E-02$ \\
\hline 23.4 & 34500 & 23.4 & 38900 & 23.4 & 34300 & 2.450 & $3.59 E-02$ & $1.47 E-02$ \\
\hline 16.2 & 26500 & 16.2 & 29500 & 16.2 & 26900 & 1.696 & $2.76 E-02$ & $1.63 E-02$ \\
\hline 11.3 & 21600 & 11.3 & 23000 & 11.3 & 22500 & 1.183 & $2.24 E-02$ & $1.89 E-02$ \\
\hline 7.84 & 17100 & 7.84 & 18000 & 7.85 & 17900 & 0.821 & $1.77 E-02$ & $2.15 E-02$ \\
\hline 5.46 & 13500 & 5.45 & 14200 & 5.46 & 14400 & 0.571 & $1.40 E-02$ & $2.46 E-02$ \\
\hline 3.79 & 10700 & 3.8 & 11300 & 3.8 & 11600 & 0.398 & $1.12 E-02$ & $2.82 E-02$ \\
\hline 2.65 & 8860 & 2.64 & 9290 & 2.63 & 9480 & 0.276 & $9.21 E-03$ & 3.33E-02 \\
\hline 1.84 & 7490 & 1.84 & 7760 & 1.83 & 7940 & 0.192 & 7.73E-03 & $4.02 E-02$ \\
\hline 1.28 & 6400 & 1.27 & 6580 & 1.28 & 6700 & 0.134 & $6.56 E-03$ & $4.91 E-02$ \\
\hline 0.886 & 5540 & 0.889 & 5730 & 0.885 & 5830 & 0.093 & 5.70E-03 & $6.14 E-02$ \\
\hline 0.609 & 4920 & 0.612 & 5130 & 0.62 & 5150 & 0.064 & $5.07 E-03$ & $7.88 E-02$ \\
\hline 0.428 & 4380 & 0.427 & 4640 & 0.422 & 4660 & 0.045 & $4.56 E-03$ & $1.02 E-01$ \\
\hline 0.301 & 4060 & 0.299 & 4270 & 0.298 & 4270 & 0.031 & $4.20 E-03$ & $1.34 E-01$ \\
\hline 0.204 & 3770 & 0.205 & 4010 & 0.207 & 3980 & 0.022 & $3.92 E-03$ & $1.82 E-01$ \\
\hline 0.141 & 3580 & 0.14 & 3770 & 0.145 & 3800 & 0.015 & $3.72 E-03$ & $2.50 E-01$ \\
\hline 0.101 & 3470 & 0.1 & 3610 & 0.101 & 3630 & 0.011 & 3.57E-03 & 3.39E-01 \\
\hline
\end{tabular}




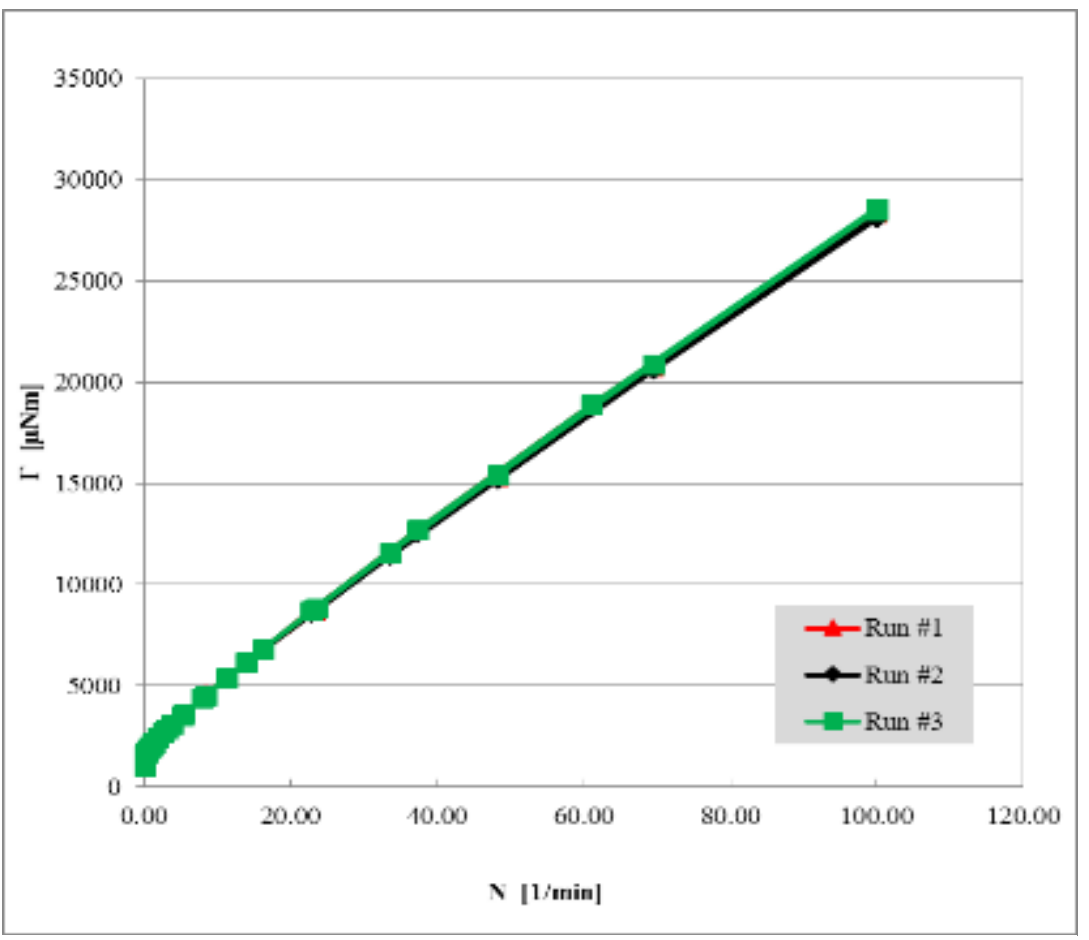

Figure A - 19: Torque vs. Angular Speed using Double spiral spindle on Mix\#6, with $0 \%$ beads by volume. Portrays data from Table A-25.

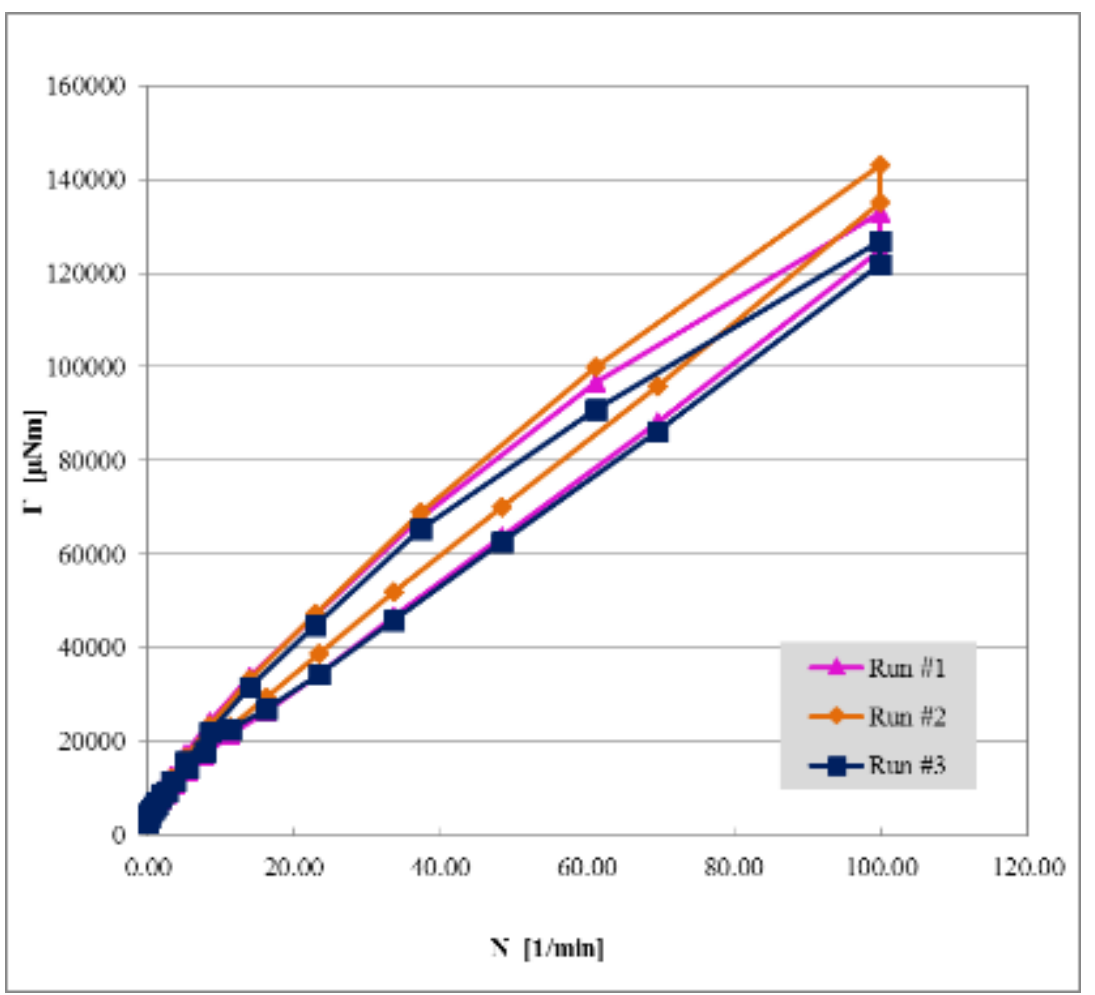

Figure A - 20: Torque vs. Angular Speed using Double spiral spindle on Mix\#6, with 40 \% beads by volume. Portrays data from Table A-26. 
Table A - 27: Measured data for Mix\#6 with 0 \% beads using Six-blade Vane.

\begin{tabular}{|c|c|c|c|c|c|c|c|c|}
\hline \multicolumn{6}{|c|}{6 Blade Vane } & \multirow{2}{*}{\multicolumn{3}{|c|}{$\begin{array}{c}\text { Bead \% } \\
0 \%\end{array}$}} \\
\hline Geom.: & RHN-83C & & & \multicolumn{2}{|c|}{ Z43S } & & & \\
\hline \multicolumn{2}{|c|}{ Run \#1 } & \multicolumn{2}{|c|}{ Run \#2 } & \multicolumn{2}{|c|}{ Run \#3 } & \multirow{2}{*}{\multicolumn{3}{|c|}{$\begin{array}{c}\text { Average } \\
\text { values }\end{array}$}} \\
\hline \multicolumn{2}{|c|}{ NIST Code: SMC-97A } & \multicolumn{2}{|c|}{ NIST Code: SMC-97B } & \multicolumn{2}{|c|}{ NIST Code: SMC-97C } & & & \\
\hline $\mathrm{N}$ & $\Gamma$ & $\mathrm{N}$ & $\Gamma$ & $\mathrm{N}$ & $\Gamma$ & $\mathbf{N}$ & $\Gamma$ & $\Gamma / N$ (Angular \\
\hline $1 / \mathrm{min}$ & $\mu \mathrm{Nm}$ & $1 / \mathrm{min}$ & $\mu \mathrm{Nm}$ & $1 / \mathrm{min}$ & $\mu \mathrm{Nm}$ & $\mathrm{rad} / \mathrm{s}$ & $\mathrm{Nm}$ & momentum) \\
\hline 0.0978 & 452 & 0.0978 & 419 & 0.0981 & 416 & 0.010 & $4.29 E-04$ & $4.18 E-02$ \\
\hline 0.163 & 591 & 0.163 & 554 & 0.164 & 553 & 0.017 & $5.66 E-04$ & $3.31 E-02$ \\
\hline 0.268 & 634 & 0.268 & 593 & 0.267 & 592 & 0.028 & $6.06 E-04$ & $2.16 E-02$ \\
\hline 0.44 & 670 & 0.44 & 627 & 0.439 & 625 & 0.046 & $6.41 E-04$ & $1.39 E-02$ \\
\hline 0.72 & 722 & 0.72 & 679 & 0.72 & 677 & 0.075 & $6.93 E-04$ & $9.19 E-03$ \\
\hline 1.18 & 793 & 1.18 & 750 & 1.18 & 756 & 0.124 & 7.66E-04 & $6.20 E-03$ \\
\hline 1.93 & 892 & 1.93 & 855 & 1.93 & 852 & 0.202 & $8.66 E-04$ & $4.29 E-03$ \\
\hline 3.16 & 1030 & 3.16 & 991 & 3.16 & 998 & 0.331 & $1.01 E-03$ & $3.04 E-03$ \\
\hline 5.18 & 1230 & 5.18 & 1200 & 5.18 & 1200 & 0.542 & $1.21 E-03$ & $2.23 E-03$ \\
\hline 8.48 & 1520 & 8.48 & 1490 & 8.48 & 1500 & 0.888 & $1.50 E-03$ & $1.69 E-03$ \\
\hline 13.9 & 1950 & 13.9 & 1920 & 13.9 & 1930 & 1.456 & $1.93 E-03$ & $1.33 E-03$ \\
\hline 22.8 & 2570 & 22.8 & 2540 & 22.8 & 2560 & 2.388 & $2.56 E-03$ & $1.07 E-03$ \\
\hline 37.3 & 3520 & 37.3 & 3490 & 37.3 & 3520 & 3.906 & $3.51 E-03$ & 8.99E-04 \\
\hline 61 & 4950 & 61 & 4940 & 61 & 4970 & 6.388 & $4.95 E-03$ & $7.75 E-04$ \\
\hline 100 & 7190 & 100 & 7220 & 100 & 7230 & 10.472 & $7.21 E-03$ & $6.89 E-04$ \\
\hline 100 & 7150 & 100 & 7210 & 100 & 7220 & 10.472 & 7.19E-03 & $6.87 E-04$ \\
\hline 69.5 & 5370 & 69.5 & 5420 & 69.5 & 5420 & 7.278 & $5.40 E-03$ & $7.42 E-04$ \\
\hline 48.3 & 4100 & 48.3 & 4140 & 48.3 & 4140 & 5.058 & $4.13 E-03$ & $8.16 E-04$ \\
\hline 33.6 & 3190 & 33.6 & 3210 & 33.6 & 3210 & 3.519 & $3.20 E-03$ & $9.10 E-04$ \\
\hline 23.4 & 2530 & 23.4 & 2540 & 23.4 & 2540 & 2.450 & $2.54 E-03$ & $1.04 E-03$ \\
\hline 16.2 & 2040 & 16.2 & 2050 & 16.2 & 2050 & 1.696 & $2.05 E-03$ & $1.21 E-03$ \\
\hline 11.3 & 1690 & 11.3 & 1690 & 11.3 & 1690 & 1.183 & $1.69 E-03$ & $1.43 E-03$ \\
\hline 7.85 & 1410 & 7.85 & 1420 & 7.85 & 1420 & 0.822 & $1.42 E-03$ & $1.72 E-03$ \\
\hline 5.45 & 1200 & 5.45 & 1210 & 5.45 & 1210 & 0.571 & $1.21 E-03$ & $2.11 E-03$ \\
\hline 3.79 & 1050 & 3.79 & 1050 & 3.79 & 1050 & 0.397 & $1.05 E-03$ & $2.65 E-03$ \\
\hline 2.64 & 923 & 2.64 & 926 & 2.64 & 922 & 0.276 & $9.24 E-04$ & $3.34 E-03$ \\
\hline 1.83 & 828 & 1.83 & 829 & 1.83 & 826 & 0.192 & $8.28 E-04$ & $4.32 E-03$ \\
\hline 1.27 & 757 & 1.27 & 755 & 1.27 & 752 & 0.133 & $7.55 E-04$ & $5.67 E-03$ \\
\hline 0.886 & 694 & 0.886 & 709 & 0.886 & 692 & 0.093 & $6.98 E-04$ & $7.53 E-03$ \\
\hline 0.615 & 650 & 0.616 & 649 & 0.616 & 644 & 0.064 & $6.48 E-04$ & $1.00 E-02$ \\
\hline 0.429 & 621 & 0.429 & 617 & 0.427 & 613 & 0.045 & $6.17 E-04$ & $1.38 E-02$ \\
\hline 0.296 & 590 & 0.298 & 579 & 0.299 & 586 & 0.031 & $5.85 E-04$ & $1.88 E-02$ \\
\hline 0.207 & 564 & 0.205 & 567 & 0.208 & 569 & 0.022 & $5.67 E-04$ & $2.62 E-02$ \\
\hline 0.143 & 567 & 0.145 & 544 & 0.145 & 552 & 0.015 & $5.54 E-04$ & $3.67 E-02$ \\
\hline 0.0999 & 523 & 0.0999 & 548 & 0.101 & 518 & 0.010 & $5.30 E-04$ & $5.04 E-02$ \\
\hline
\end{tabular}


Table A - 28: Measured data for Mix\#6 with 40 \% beads using Six-blade Vane.

\begin{tabular}{|c|c|c|c|c|c|c|c|c|}
\hline \multicolumn{6}{|c|}{6 Blade Vane } & \multirow{2}{*}{\multicolumn{3}{|c|}{$\begin{array}{c}\text { Bead \% } \\
40 \%\end{array}$}} \\
\hline Geom.: & $R H N-83 C$ & & & \multicolumn{2}{|c|}{ Z43S } & & & \\
\hline \multicolumn{2}{|c|}{ Run \#1 } & \multicolumn{2}{|c|}{ Run \#2 } & \multicolumn{2}{|c|}{ Run \#3 } & \multirow{2}{*}{\multicolumn{3}{|c|}{$\begin{array}{c}\text { Average } \\
\text { values }\end{array}$}} \\
\hline NIST Code: & $S M C-97 J$ & NIST Code: & $M C-97 K$ & NIST Cod & MC-97L & & & \\
\hline $\mathrm{N}$ & $\Gamma$ & $\mathrm{N}$ & $\Gamma$ & $\mathrm{N}$ & $\Gamma$ & $\mathbf{N}$ & $\Gamma$ & $\Gamma / \mathbf{N}$ (Angular \\
\hline $1 / \mathrm{min}$ & $\mu \mathrm{Nm}$ & $1 / \mathrm{min}$ & $\mu \mathrm{Nm}$ & $1 / \mathrm{min}$ & $\mu \mathrm{Nm}$ & $\mathrm{rad} / \mathrm{s}$ & $\mathrm{Nm}$ & momentum) \\
\hline 0.0958 & 1160 & 0.0958 & 1210 & 0.0951 & 1300 & 0.010 & $1.22 E-03$ & $1.22 E-01$ \\
\hline 0.163 & 1420 & 0.167 & 1500 & 0.162 & 1590 & 0.017 & $1.50 E-03$ & $8.75 E-02$ \\
\hline 0.267 & 1560 & 0.27 & 1660 & 0.265 & 1700 & 0.028 & $1.64 E-03$ & $5.86 E-02$ \\
\hline 0.437 & 1730 & 0.438 & 1780 & 0.445 & 1800 & 0.046 & $1.77 E-03$ & $3.84 E-02$ \\
\hline 0.723 & 1880 & 0.729 & 1920 & 0.726 & 2050 & 0.076 & $1.95 E-03$ & $2.56 E-02$ \\
\hline 1.18 & 2140 & 1.19 & 2170 & 1.18 & 2270 & 0.124 & $2.19 E-03$ & $1.77 E-02$ \\
\hline 1.93 & 2460 & 1.94 & 2580 & 1.94 & 2720 & 0.203 & $2.59 E-03$ & $1.28 E-02$ \\
\hline 3.16 & 2980 & 3.16 & 3150 & 3.16 & 3300 & 0.331 & $3.14 E-03$ & $9.50 E-03$ \\
\hline 5.19 & 3710 & 5.18 & 3940 & 5.19 & 4160 & 0.543 & $3.94 E-03$ & $7.25 E-03$ \\
\hline 8.49 & 4790 & 8.48 & 5100 & 8.48 & 5380 & 0.888 & $5.09 E-03$ & $5.73 E-03$ \\
\hline 13.9 & 6270 & 13.9 & 6790 & 13.9 & 7180 & 1.456 & $6.75 E-03$ & $4.63 E-03$ \\
\hline 22.7 & 8390 & 22.8 & 9250 & 22.8 & 9790 & 2.384 & $9.14 E-03$ & $3.84 E-03$ \\
\hline 37.3 & 11500 & 37.3 & 12900 & 37.3 & 13700 & 3.906 & $1.27 E-02$ & $3.25 E-03$ \\
\hline 61.1 & 15900 & 61 & 18000 & 61.1 & 19200 & 6.395 & $1.77 E-02$ & $2.77 E-03$ \\
\hline 100 & 23200 & 100 & 25100 & 100 & 26800 & 10.472 & $2.50 E-02$ & $2.39 E-03$ \\
\hline 100 & 22000 & 100 & 23700 & 100 & 25500 & 10.472 & $2.37 E-02$ & $2.27 E-03$ \\
\hline 69.5 & 16400 & 69.5 & 17400 & 69.5 & 18400 & 7.278 & $1.74 E-02$ & $2.39 E-03$ \\
\hline 48.3 & 12200 & 48.3 & 13200 & 48.3 & 13700 & 5.058 & $1.30 E-02$ & $2.58 E-03$ \\
\hline 33.6 & 9400 & 33.6 & 10100 & 33.6 & 10500 & 3.519 & $1.00 E-02$ & $2.84 E-03$ \\
\hline 23.4 & 7150 & 23.4 & 7660 & 23.3 & 8050 & 2.447 & 7.62E-03 & $3.11 E-03$ \\
\hline 16.2 & 5660 & 16.2 & 5970 & 16.2 & 6280 & 1.696 & $5.97 E-03$ & $3.52 E-03$ \\
\hline 11.3 & 4470 & 11.3 & 4740 & 11.3 & 4930 & 1.183 & $4.71 E-03$ & $3.98 E-03$ \\
\hline 7.85 & 3590 & 7.85 & 3790 & 7.85 & 3990 & 0.822 & $3.79 E-03$ & 4.61E-03 \\
\hline 5.46 & 2920 & 5.45 & 3080 & 5.46 & 3220 & 0.571 & $3.07 E-03$ & $5.38 E-03$ \\
\hline 3.79 & 2460 & 3.8 & 2540 & 3.79 & 2660 & 0.397 & $2.55 E-03$ & $6.43 E-03$ \\
\hline 2.63 & 2070 & 2.64 & 2140 & 2.63 & 2190 & 0.276 & $2.13 E-03$ & 7.74E-03 \\
\hline 1.83 & 1800 & 1.83 & 1850 & 1.83 & 1910 & 0.192 & $1.85 E-03$ & $9.67 E-03$ \\
\hline 1.27 & 1560 & 1.27 & 1620 & 1.28 & 1670 & 0.133 & $1.62 E-03$ & $1.21 E-02$ \\
\hline 0.886 & 1400 & 0.884 & 1440 & 0.887 & 1460 & 0.093 & $1.43 E-03$ & $1.55 E-02$ \\
\hline 0.616 & 1260 & 0.614 & 1310 & 0.616 & 1320 & 0.064 & $1.30 E-03$ & $2.01 E-02$ \\
\hline 0.425 & 1170 & 0.433 & 1220 & 0.427 & 1240 & 0.045 & $1.21 E-03$ & $2.70 E-02$ \\
\hline 0.298 & 1100 & 0.293 & 1110 & 0.296 & 1150 & 0.031 & $1.12 E-03$ & $3.62 E-02$ \\
\hline 0.207 & 1000 & 0.204 & 1060 & 0.207 & 1060 & 0.022 & $1.04 E-03$ & $4.82 E-02$ \\
\hline 0.144 & 985 & 0.143 & 1020 & 0.143 & 1040 & 0.015 & $1.02 E-03$ & $6.76 E-02$ \\
\hline 0.0996 & 938 & 0.098 & 978 & 0.0996 & 1030 & 0.010 & $9.82 E-04$ & $9.47 E-02$ \\
\hline
\end{tabular}




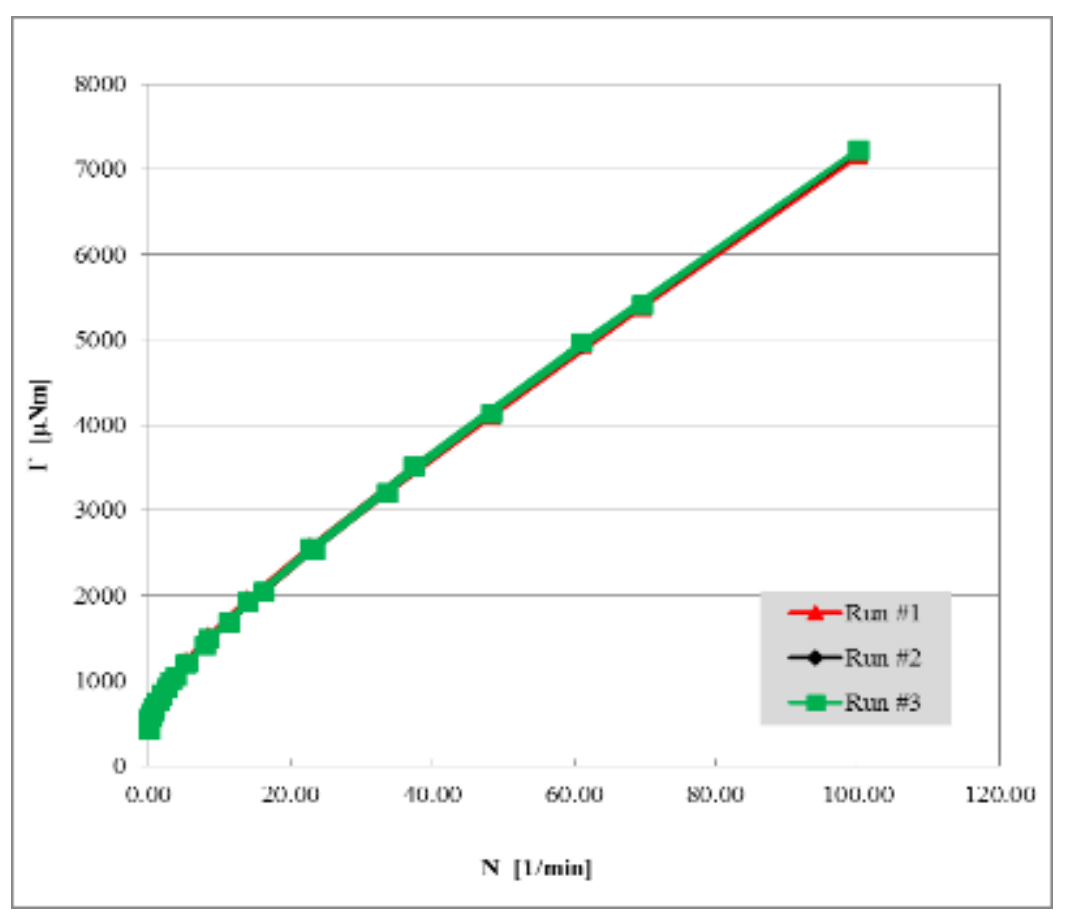

Figure A - 21: Torque vs. Angular Speed using six-blade vane on Mix\#6, with $0 \%$ beads by volume. Portrays data from Table A-27.

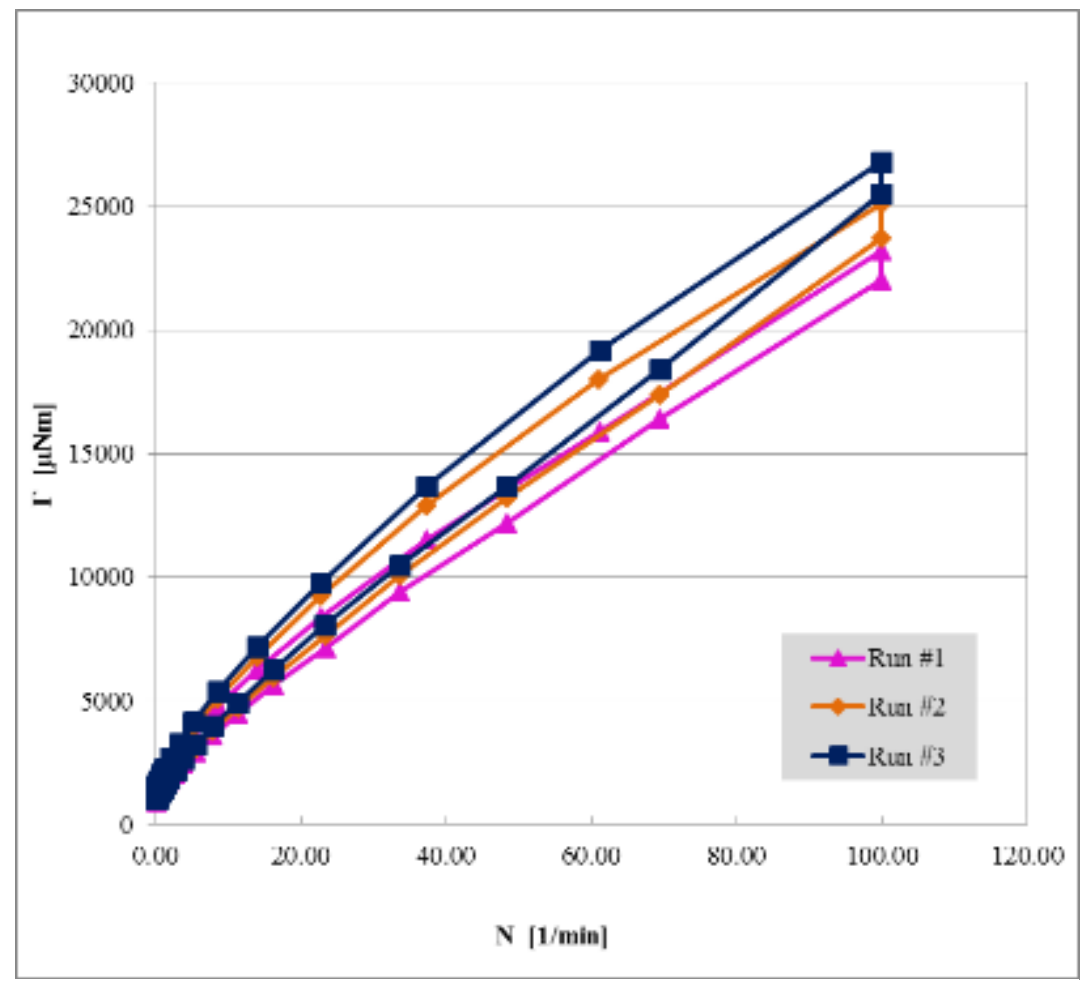

Figure A - 22: Torque vs. Angular Speed using six-blade vane on Mix\#6, with $40 \%$ beads by volume. Portrays data from Table A-28. 
Table A - 29: Measured data for Mix\#6 with 0 \% beads using Serrated Coaxial Cylinder.

\begin{tabular}{|c|c|c|c|c|c|c|c|c|}
\hline \multicolumn{6}{|c|}{ Serrated Coaxial Cylinder } & \multirow{2}{*}{\multicolumn{3}{|c|}{$\begin{array}{c}\text { Bead } \% \\
0 \%\end{array}$}} \\
\hline \multicolumn{4}{|l|}{ Geom.: } & \multicolumn{2}{|c|}{ Z43S } & & & \\
\hline \multicolumn{2}{|c|}{ Run \#1 } & \multicolumn{2}{|c|}{ Run \#2 } & \multicolumn{2}{|c|}{ Run \#3 } & \multirow{2}{*}{\multicolumn{3}{|c|}{$\begin{array}{c}\text { Average } \\
\text { values }\end{array}$}} \\
\hline NIST Code: & $S M C-97 D$ & NIST Code & $S M C-97 E$ & NIST Co & MC-97F & & & \\
\hline $\mathrm{N}$ & $\Gamma$ & $\mathrm{N}$ & $\Gamma$ & $\mathrm{N}$ & $\Gamma$ & $\mathbf{N}$ & $\Gamma$ & $\Gamma / \mathbf{N}$ (Angular \\
\hline $1 / \mathrm{min}$ & $\mu \mathrm{Nm}$ & $1 / \mathrm{min}$ & $\mu \mathrm{Nm}$ & $1 / \mathrm{min}$ & $\mu \mathrm{Nm}$ & $\mathrm{rad} / \mathrm{s}$ & Nm & momentum) \\
\hline 0.0981 & 465 & 0.0983 & 458 & 0.0982 & 438 & 0.010 & $4.54 E-04$ & $4.41 E-02$ \\
\hline 0.164 & 592 & 0.164 & 576 & 0.164 & 564 & 0.017 & $5.77 E-04$ & $3.36 E-02$ \\
\hline 0.268 & 626 & 0.268 & 613 & 0.268 & 606 & 0.028 & $6.15 E-04$ & $2.19 E-02$ \\
\hline 0.439 & 670 & 0.439 & 660 & 0.439 & 657 & 0.046 & $6.62 E-04$ & $1.44 E-02$ \\
\hline 0.72 & 732 & 0.72 & 726 & 0.72 & 724 & 0.075 & 7.27E-04 & $9.65 E-03$ \\
\hline 1.18 & 816 & 1.18 & 815 & 1.18 & 816 & 0.124 & $8.16 E-04$ & $6.60 E-03$ \\
\hline 1.93 & 931 & 1.93 & 937 & 1.93 & 939 & 0.202 & $9.36 E-04$ & $4.63 E-03$ \\
\hline 3.16 & 1090 & 3.16 & 1110 & 3.16 & 1110 & 0.331 & $1.10 E-03$ & $3.33 E-03$ \\
\hline 5.18 & 1320 & 5.18 & 1340 & 5.18 & 1350 & 0.542 & $1.34 E-03$ & $2.46 E-03$ \\
\hline 8.48 & 1640 & 8.48 & 1680 & 8.48 & 1680 & 0.888 & 1.67E-03 & $1.88 E-03$ \\
\hline 13.9 & 2100 & 13.9 & 2160 & 13.9 & 2170 & 1.456 & $2.14 E-03$ & $1.47 E-03$ \\
\hline 22.8 & 2770 & 22.8 & 2860 & 22.8 & 2870 & 2.388 & $2.83 E-03$ & $1.19 E-03$ \\
\hline 37.3 & 3800 & 37.3 & 3930 & 37.3 & 3960 & 3.906 & $3.90 E-03$ & $9.98 E-04$ \\
\hline 61 & 5370 & 61 & 5560 & 61 & 5620 & 6.388 & $5.52 E-03$ & $8.64 E-04$ \\
\hline 100 & 7840 & 100 & 8000 & 100 & 8130 & 10.472 & $7.99 E-03$ & 7.63E-04 \\
\hline 100 & 7770 & 100 & 7930 & 100 & 8040 & 10.472 & $7.91 E-03$ & $7.56 E-04$ \\
\hline 69.5 & 5830 & 69.5 & 5960 & 69.5 & 6030 & 7.278 & $5.94 E-03$ & $8.16 E-04$ \\
\hline 48.3 & 4410 & 48.3 & 4500 & 48.3 & 4550 & 5.058 & $4.49 E-03$ & 8.87E-04 \\
\hline 33.6 & 3390 & 33.6 & 3450 & 33.6 & 3490 & 3.519 & $3.44 E-03$ & $9.79 E-04$ \\
\hline 23.4 & 2650 & 23.4 & 2700 & 23.4 & 2730 & 2.450 & $2.69 E-03$ & $1.10 E-03$ \\
\hline 16.2 & 2120 & 16.2 & 2150 & 16.2 & 2170 & 1.696 & $2.15 E-03$ & $1.27 E-03$ \\
\hline 11.3 & 1720 & 11.3 & 1750 & 11.3 & 1760 & 1.183 & $1.74 E-03$ & $1.47 E-03$ \\
\hline 7.85 & 1420 & 7.85 & 1450 & 7.85 & 1460 & 0.822 & $1.44 E-03$ & $1.76 E-03$ \\
\hline 5.46 & 1190 & 5.45 & 1210 & 5.45 & 1220 & 0.571 & $1.21 E-03$ & $2.11 E-03$ \\
\hline 3.79 & 1010 & 3.79 & 1030 & 3.79 & 1040 & 0.397 & $1.03 E-03$ & $2.59 E-03$ \\
\hline 2.64 & 872 & 2.64 & 891 & 2.64 & 901 & 0.276 & $8.88 E-04$ & $3.21 E-03$ \\
\hline 1.83 & 760 & 1.83 & 780 & 1.83 & 786 & 0.192 & $7.75 E-04$ & $4.05 E-03$ \\
\hline 1.27 & 670 & 1.27 & 689 & 1.28 & 702 & 0.133 & $6.87 E-04$ & $5.15 E-03$ \\
\hline 0.886 & 599 & 0.886 & 616 & 0.892 & 713 & 0.093 & $6.43 E-04$ & $6.91 E-03$ \\
\hline 0.615 & 545 & 0.622 & 588 & 0.616 & 585 & 0.065 & $5.73 E-04$ & $8.85 E-03$ \\
\hline 0.428 & 500 & 0.419 & 539 & 0.428 & 527 & 0.045 & $5.22 E-04$ & $1.17 E-02$ \\
\hline 0.298 & 452 & 0.308 & 508 & 0.295 & 499 & 0.031 & $4.86 E-04$ & $1.55 E-02$ \\
\hline 0.207 & 423 & 0.207 & 504 & 0.194 & 507 & 0.021 & $4.78 E-04$ & $2.25 E-02$ \\
\hline 0.144 & 400 & 0.142 & 428 & 0.137 & 482 & 0.015 & 4.37E-04 & $2.96 E-02$ \\
\hline 0.102 & 390 & 0.101 & 445 & 0.0998 & 467 & 0.011 & 4.34E-04 & $4.11 E-02$ \\
\hline
\end{tabular}


Table A - 30: Measured data for Mix\#6 with $40 \%$ beads using Serrated Coaxial Cylinder.

\begin{tabular}{|c|c|c|c|c|c|c|c|c|}
\hline \multicolumn{6}{|c|}{ Serrated Coaxial Cylinder } & \multirow{2}{*}{\multicolumn{3}{|c|}{$\begin{array}{c}\text { Bead } \% \\
40 \%\end{array}$}} \\
\hline \multicolumn{4}{|c|}{ SS18 } & \multicolumn{2}{|c|}{ Z43S } & & & \\
\hline Run & $\# 1$ & \multicolumn{2}{|c|}{ Run \#2 } & \multicolumn{2}{|c|}{ Run \#3 } & \multirow{2}{*}{\multicolumn{3}{|c|}{$\begin{array}{c}\text { Average } \\
\text { values }\end{array}$}} \\
\hline \multicolumn{2}{|c|}{ NIST Code: SMC-97M } & NIST Coc & SMC-97N & \multicolumn{2}{|c|}{ NIST Code: SMC-97O } & & & \\
\hline $\mathrm{N}$ & $\Gamma$ & $\mathrm{N}$ & $\Gamma$ & $\mathrm{N}$ & $\Gamma$ & $\mathbf{N}$ & $\Gamma$ & $\Gamma / N$ (Angular \\
\hline $1 / \mathrm{min}$ & $\mu \mathrm{Nm}$ & $1 / \mathrm{min}$ & $\mu \mathrm{Nm}$ & $1 / \mathrm{min}$ & $\mu \mathrm{Nm}$ & $\mathrm{rad} / \mathrm{s}$ & $\mathrm{Nm}$ & momentum) \\
\hline 0.0973 & 1230 & 0.0967 & 1190 & 0.0979 & 1170 & 0.010 & $1.20 E-03$ & 1.17E-01 \\
\hline 0.164 & 1480 & 0.163 & 1350 & 0.165 & 1330 & 0.017 & $1.39 E-03$ & 8.07E-02 \\
\hline 0.27 & 1550 & 0.27 & 1380 & 0.268 & 1390 & 0.028 & $1.44 E-03$ & $5.11 E-02$ \\
\hline 0.441 & 1570 & 0.441 & 1470 & 0.442 & 1490 & 0.046 & $1.51 E-03$ & $3.27 E-02$ \\
\hline 0.721 & 1670 & 0.718 & 1660 & 0.722 & 1670 & 0.075 & $1.67 E-03$ & $2.21 E-02$ \\
\hline 1.18 & 1840 & 1.18 & 1960 & 1.18 & 1980 & 0.124 & $1.93 E-03$ & $1.56 E-02$ \\
\hline 1.93 & 2150 & 1.93 & 2350 & 1.93 & 2410 & 0.202 & $2.30 E-03$ & $1.14 E-02$ \\
\hline 3.17 & 2640 & 3.16 & 2950 & 3.16 & 2990 & 0.331 & $2.86 E-03$ & 8.63E-03 \\
\hline 5.18 & 3380 & 5.18 & 3800 & 5.18 & 3870 & 0.542 & $3.68 E-03$ & 6.79E-03 \\
\hline 8.49 & 4420 & 8.49 & 4990 & 8.49 & 5090 & 0.889 & 4.83E-03 & $5.44 E-03$ \\
\hline 13.9 & 5870 & 13.9 & 6700 & 13.9 & 6890 & 1.456 & $6.49 E-03$ & 4.46E-03 \\
\hline 22.8 & 8090 & 22.8 & 9210 & 22.8 & 9490 & 2.388 & 8.93E-03 & $3.74 E-03$ \\
\hline 37.3 & 11300 & 37.3 & 12800 & 37.3 & 13300 & 3.906 & $1.25 E-02$ & 3.19E-03 \\
\hline 61 & 16000 & 61 & 16400 & 61.1 & 17300 & 6.391 & $1.66 E-02$ & $2.59 E-03$ \\
\hline 100 & 21300 & 100 & 23700 & 100 & 24700 & 10.472 & $2.32 E-02$ & $2.22 E-03$ \\
\hline 100 & 21000 & 99.9 & 23200 & 100 & 23800 & 10.468 & $2.27 E-02$ & $2.17 E-03$ \\
\hline 69.5 & 16200 & 69.5 & 18100 & 69.5 & 18700 & 7.278 & $1.77 E-02$ & $2.43 E-03$ \\
\hline 48.3 & 12600 & 48.3 & 13800 & 48.3 & 14100 & 5.058 & $1.35 E-02$ & 2.67E-03 \\
\hline 33.6 & 9850 & 33.6 & 10400 & 33.6 & 10800 & 3.519 & $1.04 E-02$ & $2.94 E-03$ \\
\hline 23.4 & 7570 & 23.3 & 7900 & 23.3 & 8160 & 2.443 & $7.88 E-03$ & $3.22 E-03$ \\
\hline 16.2 & 5800 & 16.2 & 6100 & 16.2 & 6320 & 1.696 & $6.07 E-03$ & $3.58 E-03$ \\
\hline 11.3 & 4530 & 11.3 & 4760 & 11.3 & 4920 & 1.183 & $4.74 E-03$ & $4.00 E-03$ \\
\hline 7.85 & 3580 & 7.85 & 3770 & 7.85 & 3900 & 0.822 & $3.75 E-03$ & $4.56 E-03$ \\
\hline 5.46 & 2890 & 5.45 & 3040 & 5.45 & 3150 & 0.571 & $3.03 E-03$ & $5.30 E-03$ \\
\hline 3.79 & 2370 & 3.79 & 2490 & 3.79 & 2570 & 0.397 & $2.48 E-03$ & $6.24 E-03$ \\
\hline 2.64 & 1980 & 2.64 & 2080 & 2.64 & 2160 & 0.276 & $2.07 E-03$ & $7.50 E-03$ \\
\hline 1.83 & 1670 & 1.83 & 1760 & 1.83 & 1820 & 0.192 & $1.75 E-03$ & $9.13 E-03$ \\
\hline 1.27 & 1450 & 1.28 & 1520 & 1.28 & 1590 & 0.134 & $1.52 E-03$ & $1.14 E-02$ \\
\hline 0.885 & 1280 & 0.887 & 1330 & 0.885 & 1390 & 0.093 & $1.33 E-03$ & $1.44 E-02$ \\
\hline 0.615 & 1140 & 0.614 & 1230 & 0.617 & 1250 & 0.064 & $1.21 E-03$ & $1.87 E-02$ \\
\hline 0.428 & 1030 & 0.423 & 1090 & 0.429 & 1170 & 0.045 & $1.10 E-03$ & $2.45 E-02$ \\
\hline 0.294 & 954 & 0.295 & 1000 & 0.294 & 1020 & 0.031 & $9.91 E-04$ & $3.22 E-02$ \\
\hline 0.204 & 873 & 0.204 & 967 & 0.208 & 949 & 0.022 & $9.30 E-04$ & $4.32 E-02$ \\
\hline 0.142 & 871 & 0.141 & 915 & 0.151 & 1070 & 0.015 & $9.52 E-04$ & $6.28 E-02$ \\
\hline 0.0959 & 864 & 0.105 & 921 & 0.101 & 877 & 0.011 & 8.87E-04 & $8.42 E-02$ \\
\hline
\end{tabular}




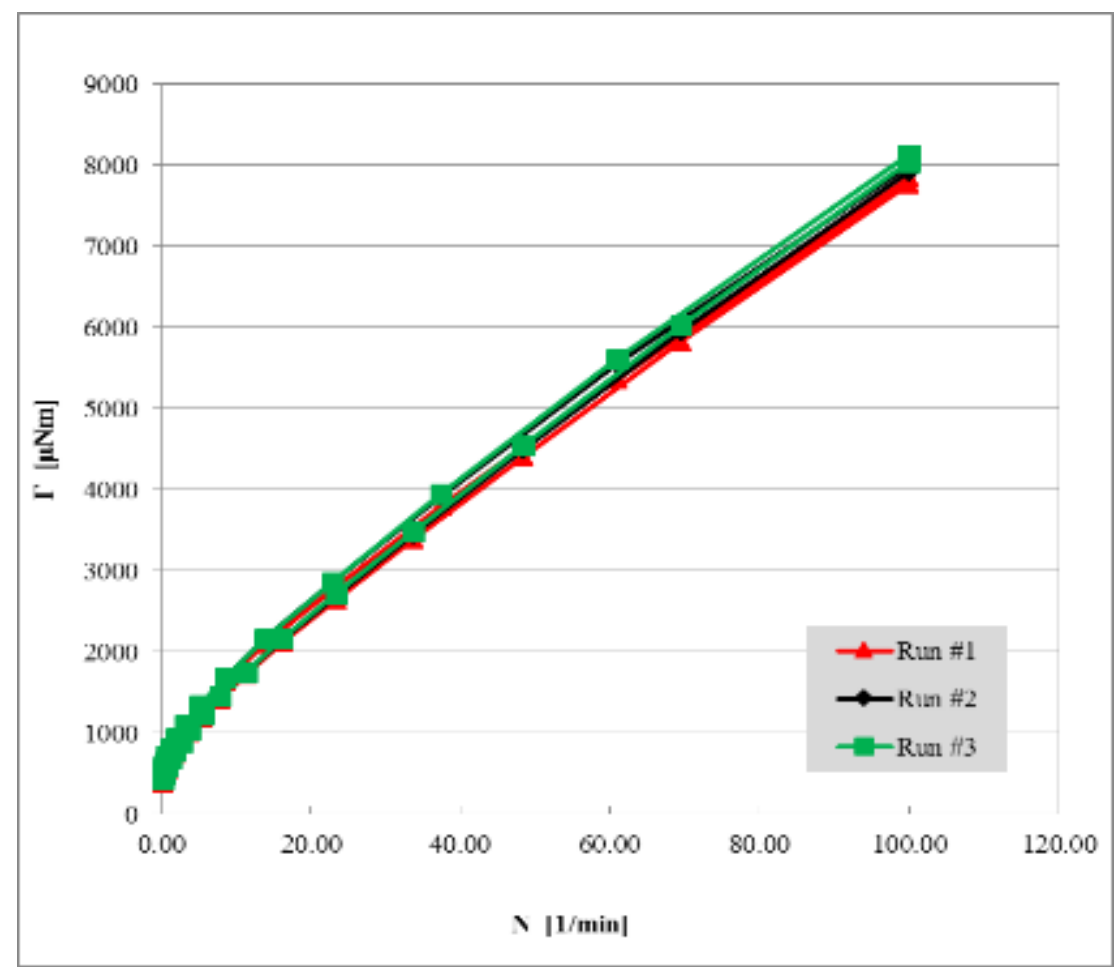

Figure A - 29: Torque vs. Angular Speed using Serrated Coaxial Cylinder on Mix\#6, with 0 \% beads by volume. Portrays data from Table A-29.

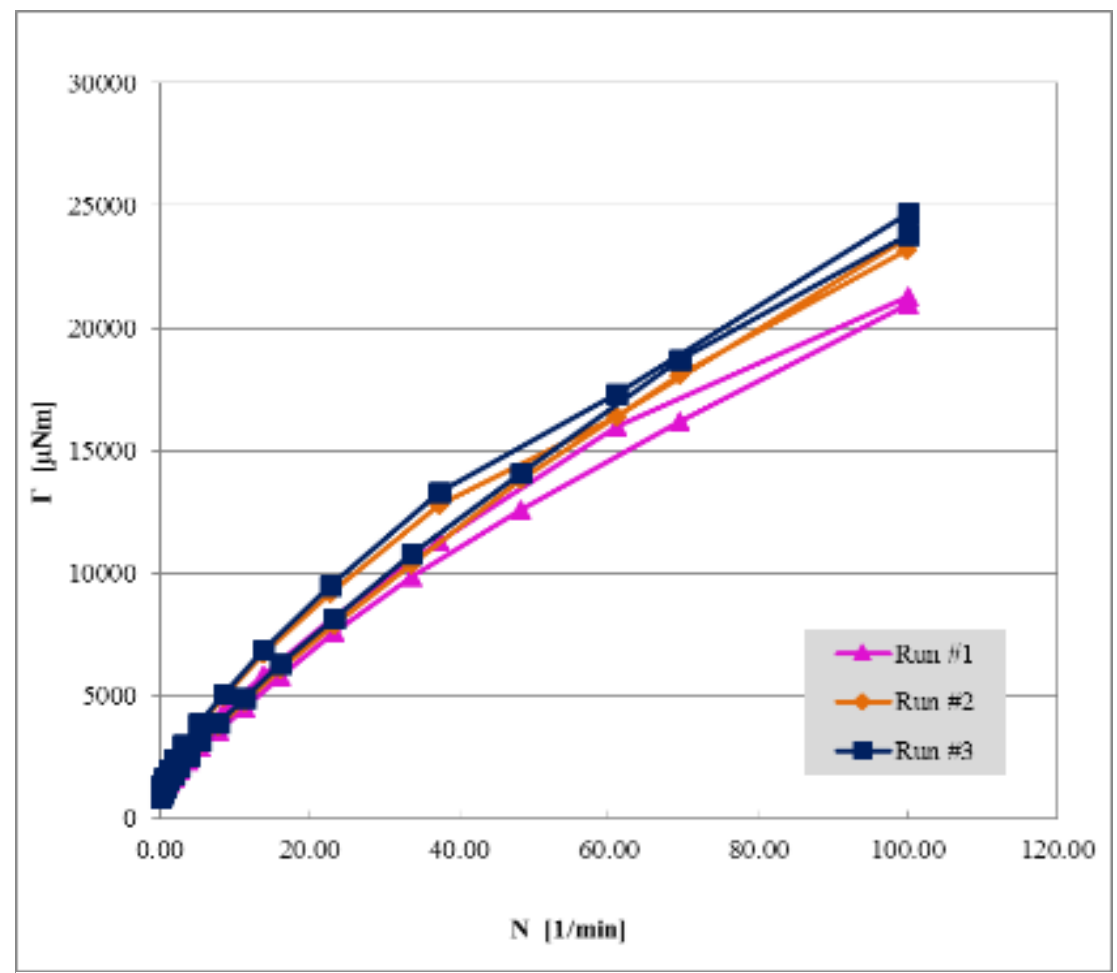

Figure A - 30: Torque vs. Angular Speed using Serrated Coaxial Cylinder on Mix\#6, with $40 \%$ beads by volume. Portrays data from Table A-30. 
Table A - 31: Measured data for Mix\#7 with 0 \% beads using Double spiral spindle.

\begin{tabular}{|c|c|c|c|c|c|c|c|c|}
\hline \multicolumn{6}{|c|}{ Double Spiral } & \multirow{2}{*}{\multicolumn{3}{|c|}{$\begin{array}{c}\text { Bead } \% \\
0 \%\end{array}$}} \\
\hline Geom.: & \multicolumn{3}{|l|}{$R H N-83 A$} & \multicolumn{2}{|c|}{ Cup: } & & & \\
\hline \multicolumn{2}{|c|}{ Run \#1 } & \multicolumn{2}{|c|}{ Run \#2 } & \multicolumn{2}{|c|}{ Run \#3 } & \multirow{2}{*}{\multicolumn{3}{|c|}{$\begin{array}{c}\text { Average } \\
\text { values }\end{array}$}} \\
\hline \multicolumn{2}{|c|}{ NIST Code: SMC-101D } & \multicolumn{2}{|c|}{ NIST Code: SMC-101E } & \multicolumn{2}{|c|}{ NIST Code: SMC-101F } & & & \\
\hline $\mathrm{N}$ & $\Gamma$ & $\mathrm{N}$ & $\Gamma$ & $\mathrm{N}$ & $\Gamma$ & $\mathbf{N}$ & $\Gamma$ & $\boldsymbol{\Gamma} / \mathbf{N}$ (Angular \\
\hline $1 / \mathrm{min}$ & $\mu \mathrm{Nm}$ & $1 / \mathrm{min}$ & $\mu \mathrm{Nm}$ & $1 / \mathrm{min}$ & $\mu \mathrm{Nm}$ & $\mathrm{rad} / \mathrm{s}$ & $\mathrm{Nm}$ & momentum) \\
\hline 0.0943 & 1020 & 0.0949 & 921 & 0.0949 & 918 & 0.010 & $9.53 E-04$ & $9.61 E-02$ \\
\hline 0.161 & 1500 & 0.162 & 1370 & 0.162 & 1360 & 0.017 & $1.41 E-03$ & 8.33E-02 \\
\hline 0.268 & 1690 & 0.268 & 1550 & 0.268 & 1540 & 0.028 & $1.59 E-03$ & $5.68 E-02$ \\
\hline 0.439 & 1790 & 0.439 & 1660 & 0.439 & 1660 & 0.046 & $1.70 E-03$ & $3.71 E-02$ \\
\hline 0.72 & 1900 & 0.72 & 1780 & 0.72 & 1780 & 0.075 & $1.82 E-03$ & $2.41 E-02$ \\
\hline 1.18 & 2060 & 1.18 & 1960 & 1.18 & 1960 & 0.124 & $1.99 E-03$ & $1.61 E-02$ \\
\hline 1.93 & 2290 & 1.93 & 2210 & 1.93 & 2210 & 0.202 & $2.24 E-03$ & $1.11 E-02$ \\
\hline 3.16 & 2660 & 3.16 & 2590 & 3.16 & 2600 & 0.331 & $2.62 E-03$ & $7.91 E-03$ \\
\hline 5.18 & 3230 & 5.18 & 3180 & 5.18 & 3190 & 0.542 & $3.20 E-03$ & $5.90 E-03$ \\
\hline 8.48 & 4130 & 8.48 & 4090 & 8.48 & 4100 & 0.888 & $4.11 E-03$ & $4.62 E-03$ \\
\hline 13.9 & 5550 & 13.9 & 5510 & 13.9 & 5520 & 1.456 & $5.53 E-03$ & $3.80 E-03$ \\
\hline 22.8 & 7800 & 22.8 & 7730 & 22.8 & 7750 & 2.388 & $7.76 E-03$ & $3.25 E-03$ \\
\hline 37.3 & 11300 & 37.3 & 11200 & 37.3 & 11300 & 3.906 & $1.13 E-02$ & $2.88 E-03$ \\
\hline 61.1 & 16900 & 61.1 & 16700 & 61.1 & 16800 & 6.398 & $1.68 E-02$ & 2.63E-03 \\
\hline 100 & 25500 & 100 & 25400 & 100 & 25500 & 10.472 & $2.55 E-02$ & $2.43 E-03$ \\
\hline 100 & 25300 & 100 & 25300 & 100 & 25400 & 10.472 & $2.53 E-02$ & $2.42 E-03$ \\
\hline 69.5 & 18500 & 69.5 & 18500 & 69.5 & 18600 & 7.278 & $1.85 E-02$ & $2.55 E-03$ \\
\hline 48.3 & 13700 & 48.3 & 13700 & 48.3 & 13800 & 5.058 & $1.37 E-02$ & $2.72 E-03$ \\
\hline 33.6 & 10300 & 33.6 & 10300 & 33.6 & 10300 & 3.519 & $1.03 E-02$ & 2.93E-03 \\
\hline 23.4 & 7830 & 23.4 & 7830 & 23.4 & 7870 & 2.450 & $7.84 E-03$ & $3.20 E-03$ \\
\hline 16.2 & 6080 & 16.2 & 6090 & 16.2 & 6110 & 1.696 & $6.09 E-03$ & $3.59 E-03$ \\
\hline 11.3 & 4830 & 11.3 & 4830 & 11.3 & 4850 & 1.183 & $4.84 E-03$ & 4.09E-03 \\
\hline 7.85 & 3920 & 7.85 & 3920 & 7.85 & 3940 & 0.822 & $3.93 E-03$ & 4.78E-03 \\
\hline 5.46 & 3260 & 5.46 & 3270 & 5.46 & 3280 & 0.572 & $3.27 E-03$ & $5.72 E-03$ \\
\hline 3.79 & 2790 & 3.79 & 2790 & 3.79 & 2790 & 0.397 & $2.79 E-03$ & $7.03 E-03$ \\
\hline 2.64 & 2430 & 2.64 & 2430 & 2.64 & 2440 & 0.276 & $2.43 E-03$ & 8.80E-03 \\
\hline 1.83 & 2170 & 1.83 & 2170 & 1.83 & 2180 & 0.192 & $2.17 E-03$ & 1.13E-02 \\
\hline 1.27 & 1980 & 1.27 & 1980 & 1.27 & 1980 & 0.133 & $1.98 E-03$ & $1.49 E-02$ \\
\hline 0.886 & 1830 & 0.886 & 1830 & 0.886 & 1830 & 0.093 & $1.83 E-03$ & $1.97 E-02$ \\
\hline 0.616 & 1720 & 0.616 & 1720 & 0.616 & 1720 & 0.065 & $1.72 E-03$ & 2.67E-02 \\
\hline 0.428 & 1640 & 0.428 & 1630 & 0.428 & 1630 & 0.045 & $1.63 E-03$ & $3.64 E-02$ \\
\hline 0.298 & 1570 & 0.297 & 1560 & 0.297 & 1570 & 0.031 & $1.57 E-03$ & $5.03 E-02$ \\
\hline 0.207 & 1510 & 0.207 & 1510 & 0.209 & 1530 & 0.022 & $1.52 E-03$ & 6.97E-02 \\
\hline 0.144 & 1470 & 0.143 & 1470 & 0.144 & 1480 & 0.015 & $1.47 E-03$ & $9.79 E-02$ \\
\hline 0.0999 & 1440 & 0.1 & 1440 & 0.0996 & 1440 & 0.010 & $1.44 E-03$ & $1.38 E-01$ \\
\hline
\end{tabular}


Table A - 32: Measured data for Mix\#7 with 20 \% beads using Double spiral spindle.

\begin{tabular}{|c|c|c|c|c|c|c|c|c|}
\hline \multicolumn{6}{|c|}{ Double Spiral } & \multicolumn{3}{|c|}{$\begin{array}{c}\text { Bead } \% \\
20 \%\end{array}$} \\
\hline \multicolumn{2}{|c|}{ Run \#1 } & \multicolumn{2}{|c|}{ Run \#2 } & \multicolumn{2}{|c|}{ Run \#3 } & \multirow{2}{*}{\multicolumn{3}{|c|}{$\begin{array}{c}\text { Average } \\
\text { values }\end{array}$}} \\
\hline NIST Code: & SMC-101M & NIST Code & MC-101N & NIST Code & $I C-1010$ & & & \\
\hline $\mathrm{N}$ & $\Gamma$ & $\mathrm{N}$ & $\Gamma$ & $\mathrm{N}$ & $\Gamma$ & $\mathbf{N}$ & $\Gamma$ & $\Gamma / \mathbf{N}$ (Angular \\
\hline $1 / \mathrm{min}$ & $\mu \mathrm{Nm}$ & $1 / \mathrm{min}$ & $\mu \mathrm{Nm}$ & $1 / \mathrm{min}$ & $\mu \mathrm{Nm}$ & $\mathrm{rad} / \mathrm{s}$ & $\mathrm{Nm}$ & momentum) \\
\hline 0.0935 & 1330 & 0.0972 & 1670 & 0.0933 & 1220 & 0.010 & $1.41 E-03$ & $1.42 E-01$ \\
\hline 0.162 & 1890 & 0.162 & 1900 & 0.161 & 1760 & 0.017 & $1.85 E-03$ & $1.09 E-01$ \\
\hline 0.269 & 2140 & 0.268 & 2040 & 0.268 & 1990 & 0.028 & $2.06 E-03$ & 7.32E-02 \\
\hline 0.439 & 2290 & 0.44 & 2200 & 0.439 & 2170 & 0.046 & $2.22 E-03$ & 4.83E-02 \\
\hline 0.719 & 2510 & 0.72 & 2400 & 0.72 & 2390 & 0.075 & $2.43 E-03$ & $3.23 E-02$ \\
\hline 1.18 & 2830 & 1.18 & 2730 & 1.18 & 2710 & 0.124 & $2.76 E-03$ & $2.23 E-02$ \\
\hline 1.93 & 3320 & 1.93 & 3230 & 1.93 & 3210 & 0.202 & $3.25 E-03$ & $1.61 E-02$ \\
\hline 3.16 & 4100 & 3.16 & 3990 & 3.16 & 3940 & 0.331 & $4.01 E-03$ & $1.21 E-02$ \\
\hline 5.18 & 5310 & 5.18 & 5130 & 5.18 & 5060 & 0.542 & $5.17 E-03$ & $9.52 E-03$ \\
\hline 8.49 & 7190 & 8.48 & 6830 & 8.48 & 6750 & 0.888 & $6.92 E-03$ & 7.79E-03 \\
\hline 13.9 & 9840 & 13.9 & 9330 & 13.9 & 9310 & 1.456 & $9.49 E-03$ & $6.52 E-03$ \\
\hline 22.7 & 13800 & 22.8 & 13400 & 22.8 & 13400 & 2.384 & $1.35 E-02$ & $5.68 E-03$ \\
\hline 37.3 & 20400 & 37.3 & 19900 & 37.3 & 19800 & 3.906 & $2.00 E-02$ & $5.13 E-03$ \\
\hline 61 & 30900 & 61.1 & 30100 & 61 & 30000 & 6.391 & $3.03 E-02$ & 4.75E-03 \\
\hline 100 & 46800 & 100 & 45800 & 100 & 45800 & 10.472 & 4.61E-02 & $4.41 E-03$ \\
\hline 100 & 46200 & 100 & 45500 & 100 & 45500 & 10.472 & $4.57 E-02$ & 4.37E-03 \\
\hline 69.5 & 33500 & 69.5 & 33000 & 69.5 & 33100 & 7.278 & 3.32E-02 & $4.56 E-03$ \\
\hline 48.3 & 24500 & 48.3 & 24200 & 48.3 & 24300 & 5.058 & $2.43 E-02$ & $4.81 E-03$ \\
\hline 33.6 & 18100 & 33.6 & 18000 & 33.6 & 18000 & 3.519 & $1.80 E-02$ & $5.13 E-03$ \\
\hline 23.3 & 13600 & 23.4 & 13500 & 23.4 & 13600 & 2.447 & $1.36 E-02$ & $5.54 E-03$ \\
\hline 16.2 & 10400 & 16.2 & 10300 & 16.2 & 10300 & 1.696 & $1.03 E-02$ & $6.09 E-03$ \\
\hline 11.3 & 8050 & 11.3 & 7980 & 11.3 & 8020 & 1.183 & 8.02E-03 & 6.77E-03 \\
\hline 7.84 & 6380 & 7.85 & 6330 & 7.85 & 6380 & 0.822 & $6.36 E-03$ & $7.74 E-03$ \\
\hline 5.46 & 5140 & 5.46 & 5140 & 5.45 & 5170 & 0.571 & $5.15 E-03$ & $9.01 E-03$ \\
\hline 3.79 & 4280 & 3.79 & 4270 & 3.8 & 4280 & 0.397 & $4.28 E-03$ & $1.08 E-02$ \\
\hline 2.63 & 3630 & 2.64 & 3620 & 2.64 & 3630 & 0.276 & 3.63E-03 & $1.31 E-02$ \\
\hline 1.83 & 3150 & 1.83 & 3150 & 1.83 & 3160 & 0.192 & $3.15 E-03$ & $1.65 E-02$ \\
\hline 1.27 & 2780 & 1.27 & 2790 & 1.28 & 2810 & 0.133 & $2.79 E-03$ & $2.09 E-02$ \\
\hline 0.886 & 2510 & 0.886 & 2520 & 0.886 & 2540 & 0.093 & $2.52 E-03$ & $2.72 E-02$ \\
\hline 0.616 & 2320 & 0.617 & 2310 & 0.616 & 2340 & 0.065 & $2.32 E-03$ & 3.60E-02 \\
\hline 0.429 & 2160 & 0.431 & 2180 & 0.428 & 2180 & 0.045 & $2.17 E-03$ & 4.83E-02 \\
\hline 0.297 & 2050 & 0.294 & 2070 & 0.297 & 2060 & 0.031 & $2.06 E-03$ & $6.65 E-02$ \\
\hline 0.205 & 1950 & 0.205 & 1980 & 0.208 & 1980 & 0.022 & $1.97 E-03$ & $9.13 E-02$ \\
\hline 0.143 & 1880 & 0.142 & 1900 & 0.142 & 1900 & 0.015 & $1.89 E-03$ & $1.27 E-01$ \\
\hline 0.101 & 1820 & 0.0997 & 1820 & 0.101 & 1830 & 0.011 & $1.82 E-03$ & $1.73 E-01$ \\
\hline
\end{tabular}




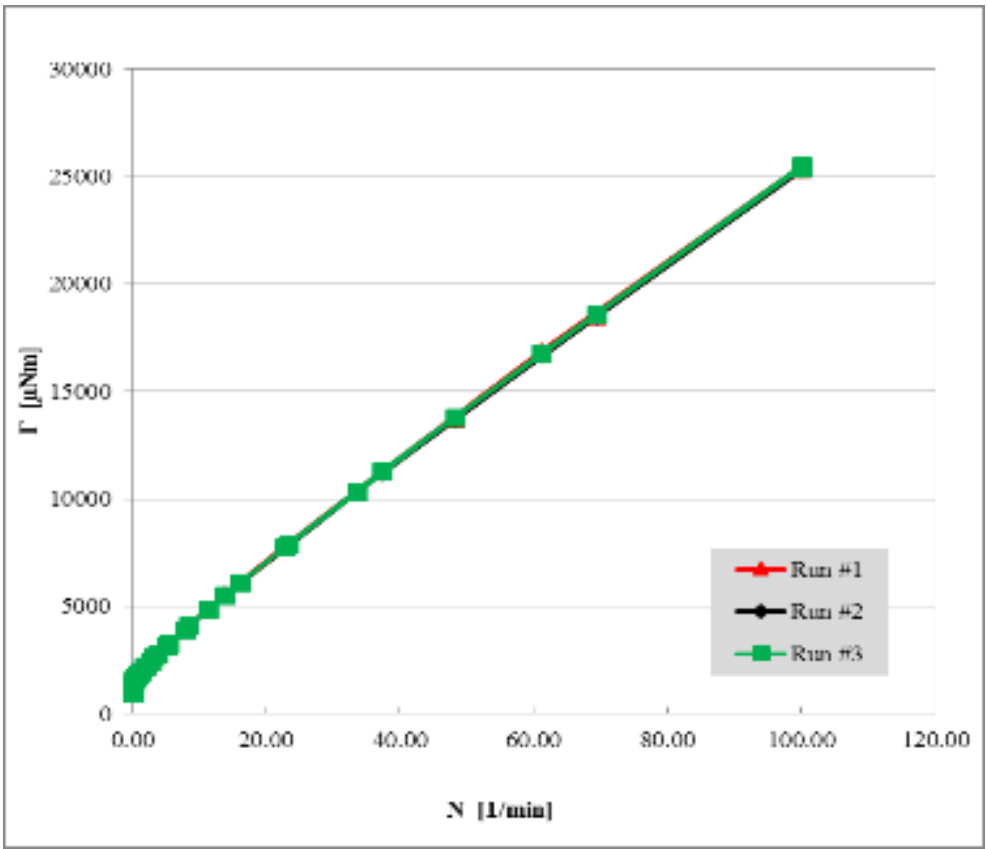

Figure A - 23: Torque vs. Angular Speed using Double spiral spindle on Mix\#7, with 0 \% beads by volume. Portrays data from Table A-31.

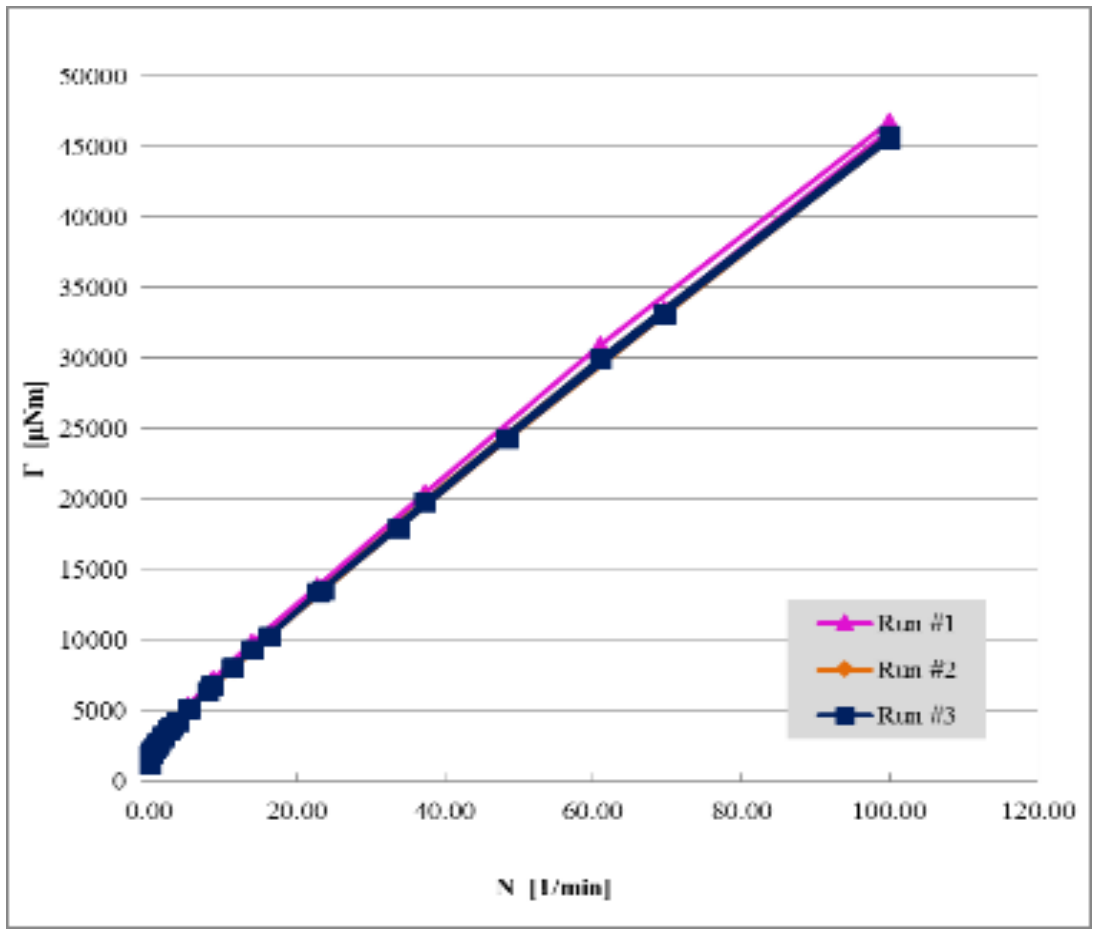

Figure A - 24: Torque vs. Angular Speed using Double spiral spindle on Mix\#7, with $20 \%$ beads by volume. Portrays data from Table A-32. 
Table A - 33: Measured data for Mix\#7 with 0 \% beads using Six-blade Vane.

\begin{tabular}{|c|c|c|c|c|c|c|c|c|}
\hline \multicolumn{6}{|c|}{6 Blade Vane } & \multirow{2}{*}{\multicolumn{3}{|c|}{$\begin{array}{c}\text { Bead } \% \\
0 \%\end{array}$}} \\
\hline Geom.: & \multicolumn{3}{|l|}{$R H N-83 C$} & \multicolumn{2}{|c|}{ Z43S } & & & \\
\hline $\mathrm{Ru}$ & $\mathrm{n} \# 1$ & Run & n \#2 & Rur & \#3 & \multirow{2}{*}{\multicolumn{3}{|c|}{$\begin{array}{c}\text { Average } \\
\text { values }\end{array}$}} \\
\hline NIST Code: & SMC-101A & NIST Code: & $S M C-101 B$ & NIST Code: & SMC-101C & & & \\
\hline $\mathrm{N}$ & $\Gamma$ & $\mathrm{N}$ & $\Gamma$ & $\mathrm{N}$ & $\Gamma$ & $\mathbf{N}$ & $\Gamma$ & $\Gamma / \boldsymbol{N}$ (Angular \\
\hline $1 / \mathrm{min}$ & $\mu \mathrm{Nm}$ & $1 / \mathrm{min}$ & $\mu \mathrm{Nm}$ & $1 / \mathrm{min}$ & $\mu \mathrm{Nm}$ & $\mathrm{rad} / \mathrm{s}$ & $\mathrm{Nm}$ & momentum) \\
\hline 0.098 & 416 & 0.098 & 393 & 0.0981 & 387 & 0.010 & $3.99 E-04$ & 3.88E-02 \\
\hline 0.163 & 546 & 0.163 & 520 & 0.163 & 516 & 0.017 & $5.27 E-04$ & 3.09E-02 \\
\hline 0.268 & 587 & 0.268 & 557 & 0.268 & 561 & 0.028 & $5.68 E-04$ & $2.03 E-02$ \\
\hline 0.439 & 620 & 0.44 & 594 & 0.441 & 596 & 0.046 & $6.03 E-04$ & $1.31 E-02$ \\
\hline 0.72 & 668 & 0.719 & 653 & 0.721 & 633 & 0.075 & $6.51 E-04$ & 8.64E-03 \\
\hline 1.18 & 735 & 1.18 & 714 & 1.18 & 703 & 0.124 & 7.17E-04 & $5.81 E-03$ \\
\hline 1.93 & 827 & 1.93 & 813 & 1.93 & 794 & 0.202 & $8.11 E-04$ & $4.01 E-03$ \\
\hline 3.16 & 958 & 3.16 & 946 & 3.16 & 934 & 0.331 & $9.46 E-04$ & $2.86 E-03$ \\
\hline 5.18 & 1150 & 5.18 & 1130 & 5.18 & 1120 & 0.542 & $1.13 E-03$ & $2.09 E-03$ \\
\hline 8.48 & 1420 & 8.48 & 1410 & 8.48 & 1400 & 0.888 & $1.41 E-03$ & $1.59 E-03$ \\
\hline 13.9 & 1820 & 13.9 & 1800 & 13.9 & 1800 & 1.456 & $1.81 E-03$ & $1.24 E-03$ \\
\hline 22.8 & 2390 & 22.8 & 2370 & 22.8 & 2380 & 2.388 & $2.38 E-03$ & $9.97 E-04$ \\
\hline 37.3 & 3280 & 37.3 & 3260 & 37.3 & 3270 & 3.906 & $3.27 E-03$ & 8.37E-04 \\
\hline 61 & 4640 & 61 & 4620 & 61 & 4650 & 6.388 & $4.64 E-03$ & $7.26 E-04$ \\
\hline 100 & 6760 & 100 & 6750 & 100 & 6790 & 10.472 & $6.77 E-03$ & $6.46 E-04$ \\
\hline 100 & 6730 & 100 & 6740 & 100 & 6780 & 10.472 & $6.75 E-03$ & $6.45 E-04$ \\
\hline 69.5 & 5050 & 69.5 & 5060 & 69.5 & 5090 & 7.278 & $5.07 E-03$ & $6.96 E-04$ \\
\hline 48.3 & 3860 & 48.3 & 3860 & 48.3 & 3880 & 5.058 & $3.87 E-03$ & 7.64E-04 \\
\hline 33.6 & 3000 & 33.6 & 3000 & 33.6 & 3010 & 3.519 & $3.00 E-03$ & 8.54E-04 \\
\hline 23.4 & 2370 & 23.4 & 2370 & 23.4 & 2390 & 2.450 & $2.38 E-03$ & $9.70 E-04$ \\
\hline 16.2 & 1920 & 16.2 & 1910 & 16.2 & 1920 & 1.696 & $1.92 E-03$ & $1.13 E-03$ \\
\hline 11.3 & 1580 & 11.3 & 1580 & 11.3 & 1590 & 1.183 & $1.58 E-03$ & $1.34 E-03$ \\
\hline 7.85 & 1320 & 7.85 & 1320 & 7.85 & 1330 & 0.822 & $1.32 E-03$ & $1.61 E-03$ \\
\hline 5.45 & 1140 & 5.45 & 1130 & 5.45 & 1130 & 0.571 & $1.13 E-03$ & 1.99E-03 \\
\hline 3.79 & 985 & 3.79 & 980 & 3.79 & 985 & 0.397 & $9.83 E-04$ & $2.48 E-03$ \\
\hline 2.64 & 880 & 2.64 & 865 & 2.64 & 869 & 0.276 & $8.71 E-04$ & $3.15 E-03$ \\
\hline 1.83 & 779 & 1.83 & 776 & 1.83 & 778 & 0.192 & $7.78 E-04$ & $4.06 E-03$ \\
\hline 1.27 & 713 & 1.27 & 705 & 1.27 & 716 & 0.133 & $7.11 E-04$ & $5.35 E-03$ \\
\hline 0.885 & 660 & 0.884 & 657 & 0.886 & 658 & 0.093 & $6.58 E-04$ & 7.10E-03 \\
\hline 0.615 & 614 & 0.616 & 622 & 0.616 & 613 & 0.064 & $6.16 E-04$ & $9.56 E-03$ \\
\hline 0.428 & 579 & 0.429 & 580 & 0.429 & 585 & 0.045 & $5.81 E-04$ & $1.30 E-02$ \\
\hline 0.294 & 573 & 0.299 & 568 & 0.297 & 570 & 0.031 & $5.70 E-04$ & $1.84 E-02$ \\
\hline 0.207 & 526 & 0.205 & 553 & 0.208 & 524 & 0.022 & $5.34 E-04$ & $2.47 E-02$ \\
\hline 0.142 & 532 & 0.144 & 521 & 0.144 & 527 & 0.015 & $5.27 E-04$ & $3.51 E-02$ \\
\hline 0.0994 & 516 & 0.0982 & 521 & 0.0994 & 518 & 0.010 & $5.18 E-04$ & $5.00 E-02$ \\
\hline
\end{tabular}


Table A - 34: Measured data for Mix\#7 with 20 \% beads using Six-blade Vane.

\begin{tabular}{|c|c|c|c|c|c|c|c|c|}
\hline \multicolumn{6}{|c|}{6 Blade Vane } & \multicolumn{3}{|c|}{$\begin{array}{c}\text { Bead } \% \\
20 \% \\
\end{array}$} \\
\hline Run & $\# 1$ & Run & $\# 2$ & Run & \#3 & \multirow{2}{*}{\multicolumn{3}{|c|}{$\begin{array}{c}\text { Average } \\
\text { values }\end{array}$}} \\
\hline NIST Code: & $S M C-101 \mathrm{~J}$ & NIST Code: & $S M C-101 K$ & NIST Code: & SMC-101L & & & \\
\hline $\mathrm{N}$ & $\Gamma$ & $\mathrm{N}$ & $\Gamma$ & $\mathrm{N}$ & $\Gamma$ & $\mathbf{N}$ & $\Gamma$ & $\boldsymbol{\Gamma} / \boldsymbol{N}$ (Angular \\
\hline $1 / \mathrm{min}$ & $\mu \mathrm{Nm}$ & $1 / \mathrm{min}$ & $\mu \mathrm{Nm}$ & $1 / \mathrm{min}$ & $\mu \mathrm{Nm}$ & $\mathrm{rad} / \mathrm{s}$ & $\mathrm{Nm}$ & momentum) \\
\hline 0.0976 & 576 & 0.0977 & 554 & 0.0976 & 577 & 0.010 & $5.69 E-04$ & $5.57 E-02$ \\
\hline 0.163 & 728 & 0.163 & 710 & 0.164 & 726 & 0.017 & 7.21E-04 & $4.22 E-02$ \\
\hline 0.268 & 793 & 0.268 & 777 & 0.268 & 785 & 0.028 & $7.85 E-04$ & $2.80 E-02$ \\
\hline 0.44 & 847 & 0.44 & 831 & 0.44 & 847 & 0.046 & $8.42 E-04$ & $1.83 E-02$ \\
\hline 0.719 & 920 & 0.719 & 907 & 0.72 & 921 & 0.075 & $9.16 E-04$ & $1.22 E-02$ \\
\hline 1.18 & 1020 & 1.18 & 1020 & 1.18 & 1040 & 0.124 & $1.03 E-03$ & 8.31E-03 \\
\hline 1.93 & 1190 & 1.93 & 1180 & 1.93 & 1200 & 0.202 & $1.19 E-03$ & $5.89 E-03$ \\
\hline 3.16 & 1410 & 3.16 & 1410 & 3.16 & 1440 & 0.331 & $1.42 E-03$ & $4.29 E-03$ \\
\hline 5.18 & 1730 & 5.18 & 1760 & 5.18 & 1800 & 0.542 & $1.76 E-03$ & $3.25 E-03$ \\
\hline 8.48 & 2220 & 8.48 & 2250 & 8.48 & 2300 & 0.888 & $2.26 E-03$ & $2.54 E-03$ \\
\hline 13.9 & 2940 & 13.9 & 3000 & 13.9 & 3080 & 1.456 & $3.01 E-03$ & 2.07E-03 \\
\hline 22.8 & 4060 & 22.8 & 4130 & 22.7 & 4240 & 2.384 & $4.14 E-03$ & $1.74 E-03$ \\
\hline 37.3 & 5680 & 37.3 & 5820 & 37.3 & 6000 & 3.906 & $5.83 E-03$ & $1.49 E-03$ \\
\hline 61 & 8050 & 61 & 8410 & 61.1 & 8690 & 6.391 & $8.38 E-03$ & $1.31 E-03$ \\
\hline 100 & 11600 & 100 & 12300 & 100 & 12800 & 10.472 & $1.22 E-02$ & $1.17 E-03$ \\
\hline 100 & 11500 & 100 & 12200 & 100 & 12800 & 10.472 & $1.22 E-02$ & $1.16 E-03$ \\
\hline 69.5 & 8600 & 69.5 & 9080 & 69.5 & 9500 & 7.278 & $9.06 E-03$ & $1.24 E-03$ \\
\hline 48.3 & 6510 & 48.3 & 6820 & 48.3 & 7110 & 5.058 & $6.81 E-03$ & $1.35 E-03$ \\
\hline 33.6 & 4970 & 33.6 & 5200 & 33.6 & 5400 & 3.519 & $5.19 E-03$ & $1.48 E-03$ \\
\hline 23.4 & 3890 & 23.3 & 4040 & 23.4 & 4160 & 2.447 & 4.03E-03 & $1.65 E-03$ \\
\hline 16.2 & 3080 & 16.2 & 3190 & 16.2 & 3280 & 1.696 & $3.18 E-03$ & $1.88 E-03$ \\
\hline 11.3 & 2480 & 11.3 & 2550 & 11.3 & 2620 & 1.183 & $2.55 E-03$ & $2.15 E-03$ \\
\hline 7.85 & 2020 & 7.85 & 2080 & 7.84 & 2120 & 0.822 & $2.07 E-03$ & $2.52 E-03$ \\
\hline 5.45 & 1690 & 5.45 & 1720 & 5.45 & 1770 & 0.571 & $1.73 E-03$ & $3.03 E-03$ \\
\hline 3.79 & 1430 & 3.79 & 1460 & 3.8 & 1500 & 0.397 & $1.46 E-03$ & $3.68 E-03$ \\
\hline 2.64 & 1230 & 2.64 & 1260 & 2.64 & 1290 & 0.276 & $1.26 E-03$ & $4.56 E-03$ \\
\hline 1.83 & 1080 & 1.83 & 1110 & 1.83 & 1130 & 0.192 & $1.11 E-03$ & 5.77E-03 \\
\hline 1.28 & 969 & 1.27 & 990 & 1.28 & 1000 & 0.134 & $9.86 E-04$ & $7.38 E-03$ \\
\hline 0.886 & 883 & 0.885 & 907 & 0.885 & 905 & 0.093 & $8.98 E-04$ & $9.69 E-03$ \\
\hline 0.616 & 810 & 0.616 & 840 & 0.615 & 844 & 0.064 & $8.31 E-04$ & $1.29 E-02$ \\
\hline 0.428 & 758 & 0.43 & 775 & 0.429 & 792 & 0.045 & $7.75 E-04$ & $1.73 E-02$ \\
\hline 0.297 & 727 & 0.297 & 736 & 0.297 & 759 & 0.031 & $7.41 E-04$ & $2.38 E-02$ \\
\hline 0.206 & 693 & 0.207 & 700 & 0.207 & 697 & 0.022 & $6.97 E-04$ & $3.22 E-02$ \\
\hline 0.144 & 659 & 0.144 & 667 & 0.143 & 713 & 0.015 & $6.80 E-04$ & $4.52 E-02$ \\
\hline 0.0995 & 639 & 0.0993 & 684 & 0.101 & 682 & 0.010 & $6.68 E-04$ & $6.39 E-02$ \\
\hline
\end{tabular}




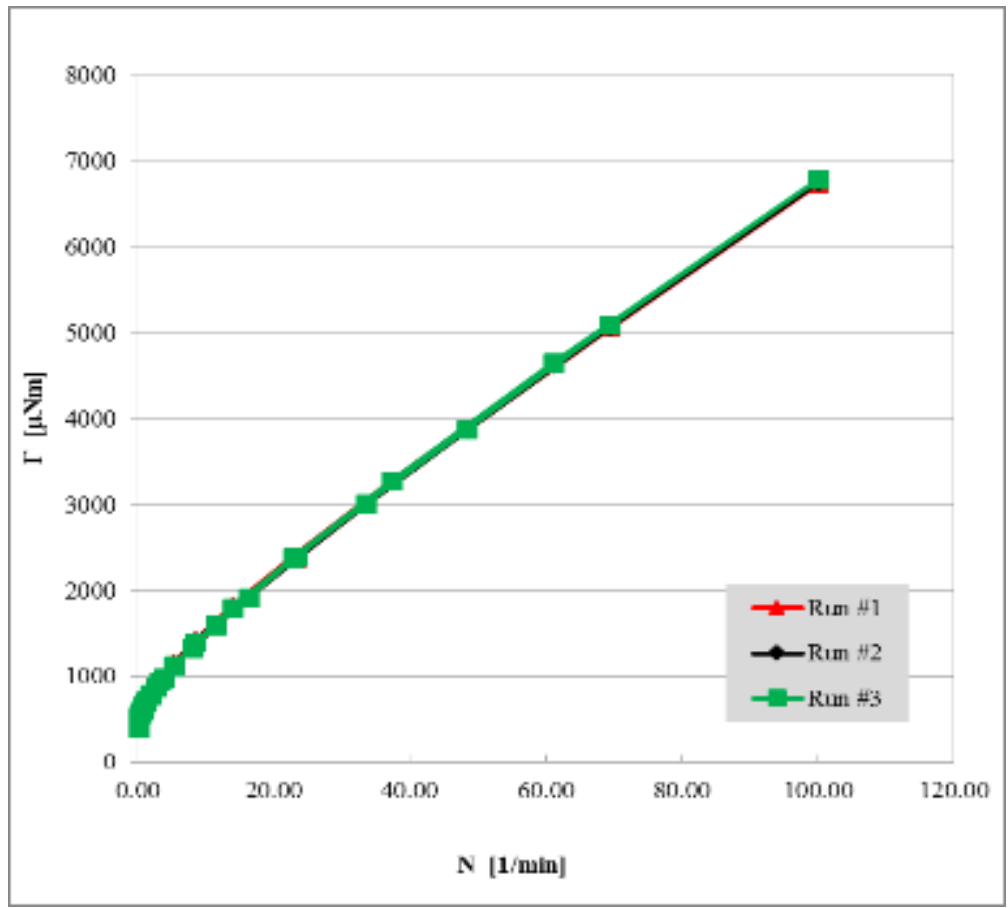

Figure A - 33: Torque vs. Angular Speed using six-blade vane on Mix\#7, with $0 \%$ beads by volume. Portrays data from Table A-33.

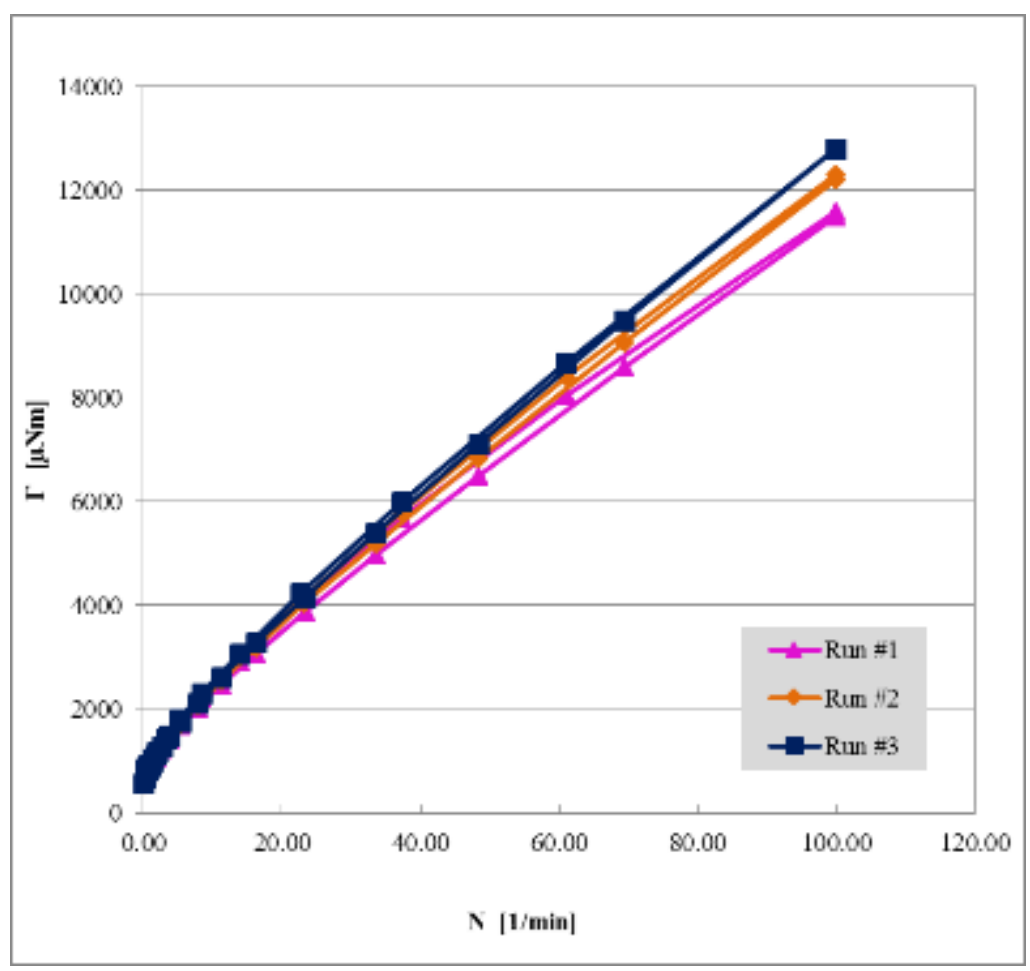

Figure A - 34: Torque vs. Angular Speed using six-blade vane on Mix\#7, with $20 \%$ beads by volume. Portrays data from Table A-34. 


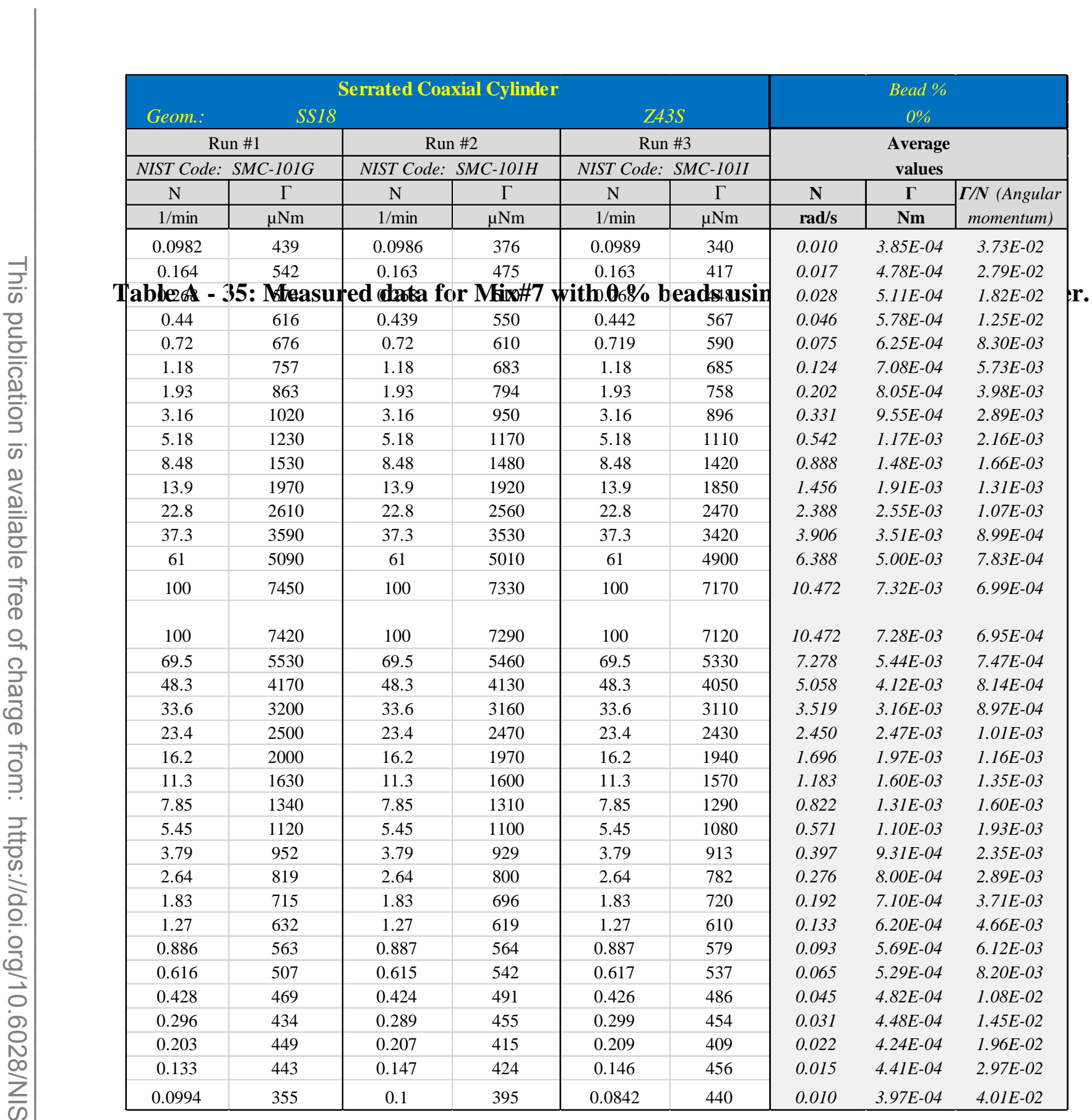


Table A - 36: Measured data for Mix\#7 with 20 \% beads using Serrated Coaxial Cylinder.

\begin{tabular}{|c|c|c|c|c|c|c|c|c|}
\hline \multicolumn{6}{|c|}{ Serrated Coaxial Cylinder } & \multirow{2}{*}{\multicolumn{3}{|c|}{$\begin{array}{c}\text { Bead } \% \\
20 \%\end{array}$}} \\
\hline Geom.: & SS18 & & & \multicolumn{2}{|c|}{ Z43S } & & & \\
\hline \multicolumn{2}{|c|}{ Run \#1 } & \multicolumn{2}{|c|}{ Run \#2 } & \multicolumn{2}{|c|}{ Run \#3 } & \multirow{2}{*}{\multicolumn{3}{|c|}{$\begin{array}{c}\text { Average } \\
\text { values }\end{array}$}} \\
\hline \multicolumn{2}{|c|}{ NIST Code: SMC-101P } & \multicolumn{2}{|c|}{ NIST Code: SMC-101Q } & \multicolumn{2}{|c|}{ NIST Code: SMC-101R } & & & \\
\hline $\mathrm{N}$ & $\Gamma$ & $\mathrm{N}$ & $\Gamma$ & $\mathrm{N}$ & $\Gamma$ & $\mathbf{N}$ & $\Gamma$ & $\Gamma / N$ (Angular \\
\hline $1 / \mathrm{min}$ & $\mu \mathrm{Nm}$ & $1 / \mathrm{min}$ & $\mu \mathrm{Nm}$ & $1 / \mathrm{min}$ & $\mu \mathrm{Nm}$ & $\mathrm{rad} / \mathrm{s}$ & $\mathrm{Nm}$ & momentum) \\
\hline 0.0979 & 638 & 0.0981 & 629 & 0.0978 & 618 & 0.010 & $6.28 E-04$ & $6.13 E-02$ \\
\hline 0.164 & 761 & 0.164 & 761 & 0.164 & 776 & 0.017 & $7.66 E-04$ & $4.46 E-02$ \\
\hline 0.268 & 788 & 0.268 & 813 & 0.269 & 831 & 0.028 & $8.11 E-04$ & $2.88 E-02$ \\
\hline 0.44 & 832 & 0.44 & 889 & 0.439 & 909 & 0.046 & 8.77E-04 & $1.90 E-02$ \\
\hline 0.719 & 913 & 0.725 & 991 & 0.719 & 992 & 0.076 & $9.65 E-04$ & $1.28 E-02$ \\
\hline 1.18 & 1030 & 1.18 & 1110 & 1.18 & 1140 & 0.124 & $1.09 E-03$ & 8.85E-03 \\
\hline 1.93 & 1210 & 1.93 & 1320 & 1.93 & 1330 & 0.202 & $1.29 E-03$ & $6.37 E-03$ \\
\hline 3.16 & 1470 & 3.16 & 1570 & 3.16 & 1630 & 0.331 & $1.56 E-03$ & 4.70E-03 \\
\hline 5.18 & 1830 & 5.18 & 1940 & 5.17 & 2010 & 0.542 & $1.93 E-03$ & $3.55 E-03$ \\
\hline 8.48 & 2330 & 8.48 & 2480 & 8.48 & 2560 & 0.888 & $2.46 E-03$ & $2.77 E-03$ \\
\hline 13.9 & 3080 & 13.9 & 3290 & 13.9 & 3390 & 1.456 & $3.25 E-03$ & $2.24 E-03$ \\
\hline 22.8 & 4190 & 22.8 & 4490 & 22.8 & 4650 & 2.388 & $4.44 E-03$ & $1.86 E-03$ \\
\hline 37.3 & 5890 & 37.3 & 6350 & 37.3 & 6560 & 3.906 & $6.27 E-03$ & $1.60 E-03$ \\
\hline 61 & 8550 & 61 & 9140 & 61 & 9460 & 6.388 & $9.05 E-03$ & $1.42 E-03$ \\
\hline 100 & 12700 & 100 & 13500 & 100 & 13900 & 10.472 & $1.34 E-02$ & $1.28 E-03$ \\
\hline 100 & 12700 & 100 & 13400 & 100 & 13700 & 10.472 & $1.33 E-02$ & $1.27 E-03$ \\
\hline 69.5 & 9410 & 69.5 & 9940 & 69.5 & 10200 & 7.278 & $9.85 E-03$ & $1.35 E-03$ \\
\hline 48.3 & 7060 & 48.3 & 7460 & 48.3 & 7670 & 5.058 & $7.40 E-03$ & $1.46 E-03$ \\
\hline 33.6 & 5360 & 33.6 & 5650 & 33.6 & 5820 & 3.519 & $5.61 E-03$ & $1.59 E-03$ \\
\hline 23.4 & 4140 & 23.4 & 4350 & 23.4 & 4480 & 2.450 & 4.32E-03 & $1.76 E-03$ \\
\hline 16.2 & 3240 & 16.2 & 3410 & 16.2 & 3510 & 1.696 & $3.39 E-03$ & $2.00 E-03$ \\
\hline 11.3 & 2590 & 11.3 & 2730 & 11.3 & 2800 & 1.183 & $2.71 E-03$ & $2.29 E-03$ \\
\hline 7.85 & 2110 & 7.85 & 2220 & 7.85 & 2280 & 0.822 & $2.20 E-03$ & $2.68 E-03$ \\
\hline 5.45 & 1740 & 5.46 & 1840 & 5.46 & 1880 & 0.571 & $1.82 E-03$ & $3.19 E-03$ \\
\hline 3.79 & 1480 & 3.79 & 1560 & 3.79 & 1590 & 0.397 & $1.54 E-03$ & 3.89E-03 \\
\hline 2.64 & 1260 & 2.64 & 1340 & 2.64 & 1360 & 0.276 & $1.32 E-03$ & $4.77 E-03$ \\
\hline 1.83 & 1090 & 1.83 & 1160 & 1.83 & 1190 & 0.192 & $1.15 E-03$ & $5.98 E-03$ \\
\hline 1.27 & 968 & 1.27 & 1020 & 1.27 & 1040 & 0.133 & $1.01 E-03$ & 7.59E-03 \\
\hline 0.886 & 862 & 0.886 & 919 & 0.888 & 944 & 0.093 & $9.08 E-04$ & $9.78 E-03$ \\
\hline 0.616 & 788 & 0.619 & 875 & 0.612 & 862 & 0.064 & $8.42 E-04$ & $1.31 E-02$ \\
\hline 0.432 & 757 & 0.428 & 762 & 0.43 & 782 & 0.045 & 7.67E-04 & $1.70 E-02$ \\
\hline 0.304 & 728 & 0.295 & 750 & 0.299 & 735 & 0.031 & 7.38E-04 & $2.35 E-02$ \\
\hline 0.205 & 678 & 0.207 & 734 & 0.196 & 768 & 0.021 & $7.27 E-04$ & $3.42 E-02$ \\
\hline 0.146 & 673 & 0.14 & 664 & 0.141 & 692 & 0.015 & $6.76 E-04$ & $4.54 E-02$ \\
\hline 0.103 & 580 & 0.0997 & 684 & 0.106 & 687 & 0.011 & $6.50 E-04$ & $6.04 E-02$ \\
\hline
\end{tabular}




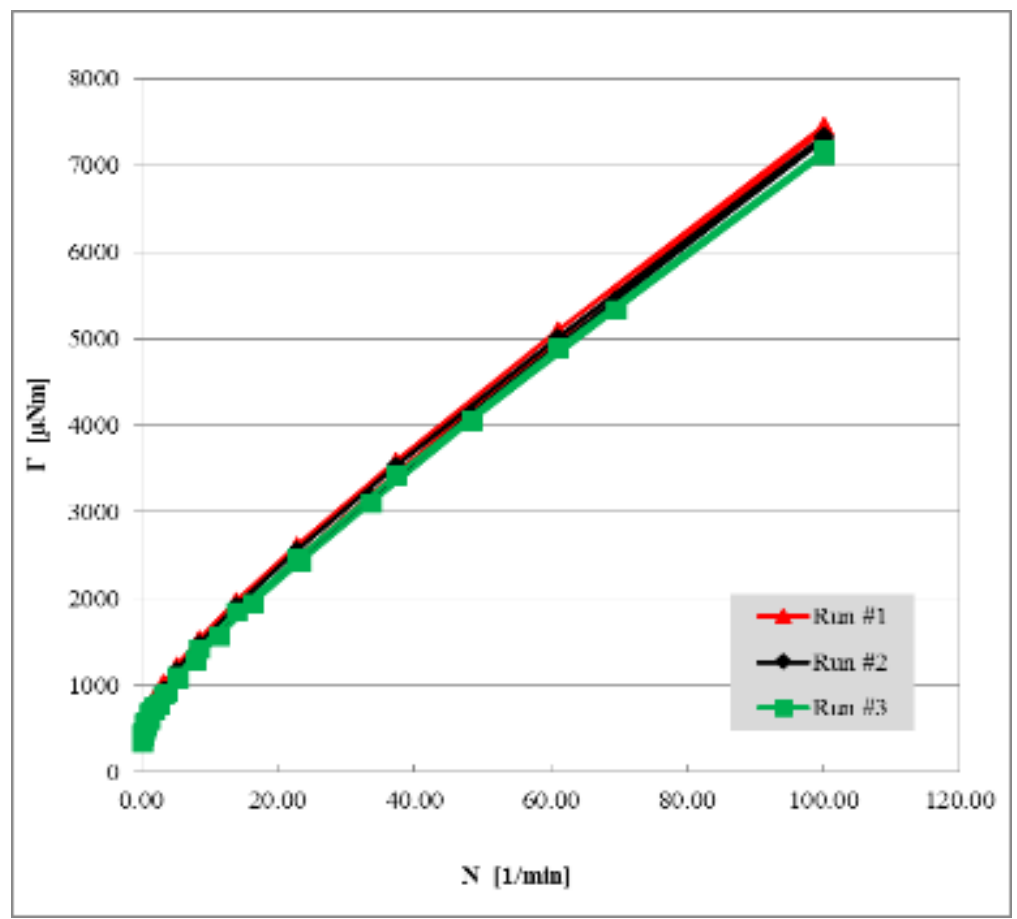

Figure A - 25: Torque vs. Angular Speed using Serrated Coaxial Cylinder on Mix\#7, with 0 \% beads by volume. Portrays data from Table A-35.

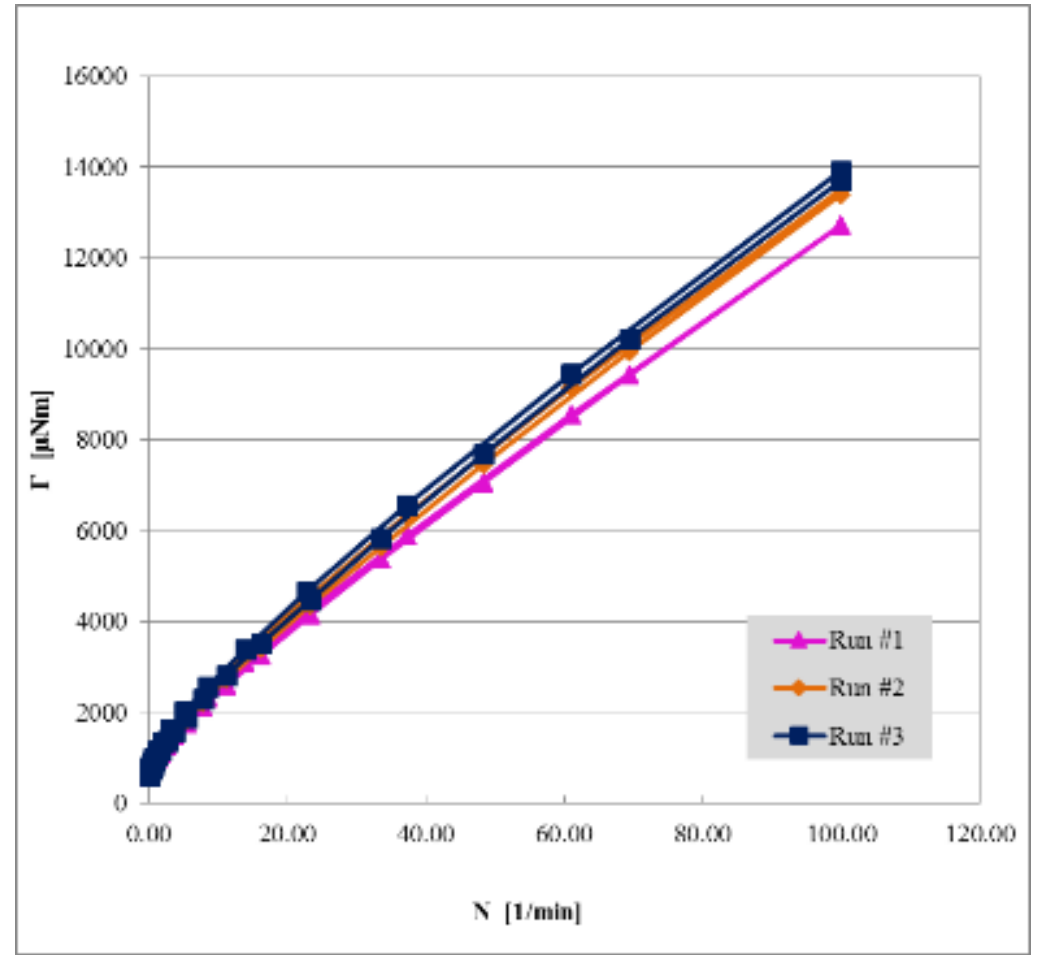

Figure A - 26: Torque vs. Angular Speed using Serrated Coaxial Cylinder on Mix\#7, with 20 \% beads by volume. Portrays data from Table A-36.

A - 55 
Table A - 37: Measured data for Mix\#8 with 0 \% beads using Double spiral spindle.

\begin{tabular}{|c|c|c|c|c|c|c|c|c|}
\hline \multicolumn{6}{|c|}{ Double Spiral } & \multicolumn{3}{|c|}{$\begin{array}{c}\text { Bead } \% \\
0 \%\end{array}$} \\
\hline \multicolumn{2}{|c|}{ Run \#1 } & \multicolumn{2}{|c|}{ Run \#2 } & \multicolumn{2}{|c|}{ Run \#3 } & \multirow{2}{*}{\multicolumn{3}{|c|}{$\begin{array}{c}\text { Average } \\
\text { values }\end{array}$}} \\
\hline NIST Code: & SMC-103G & NIST Code: & SMC-103H & NIST Code: & SMC-103I & & & \\
\hline \begin{tabular}{l|l}
$\mathrm{N}$ \\
\end{tabular} & $\Gamma$ & $\mathrm{N}$ & $\Gamma$ & $\mathrm{N}$ & $\Gamma$ & $\mathbf{N}$ & $\Gamma$ & $\Gamma / \mathbf{N}$ (Angular \\
\hline $1 / \mathrm{min}$ & $\mu \mathrm{Nm}$ & $1 / \mathrm{min}$ & $\mu \mathrm{Nm}$ & $1 / \mathrm{min}$ & $\mu \mathrm{Nm}$ & $\mathrm{rad} / \mathrm{s}$ & $\mathrm{Nm}$ & momentum) \\
\hline 0.0942 & 1030 & 0.0945 & 979 & 0.0947 & 969 & 0.010 & $9.93 E-04$ & $1.00 E-01$ \\
\hline 0.161 & 1520 & 0.161 & 1470 & 0.161 & 1430 & 0.017 & $1.47 E-03$ & $8.74 E-02$ \\
\hline 0.268 & 1720 & 0.268 & 1680 & 0.267 & 1640 & 0.028 & $1.68 E-03$ & $5.99 E-02$ \\
\hline 0.44 & 1830 & 0.439 & 1790 & 0.439 & 1750 & 0.046 & $1.79 E-03$ & $3.89 E-02$ \\
\hline 0.72 & 1950 & 0.72 & 1920 & 0.72 & 1880 & 0.075 & $1.92 E-03$ & $2.54 E-02$ \\
\hline 1.18 & 2120 & 1.18 & 2090 & 1.18 & 2070 & 0.124 & $2.09 E-03$ & $1.69 E-02$ \\
\hline 1.93 & 2370 & 1.93 & 2350 & 1.93 & 2340 & 0.202 & $2.35 E-03$ & $1.16 E-02$ \\
\hline 3.16 & 2770 & 3.16 & 2770 & 3.16 & 2760 & 0.331 & $2.77 E-03$ & 8.36E-03 \\
\hline 5.18 & 3400 & 5.18 & 3410 & 5.18 & 3410 & 0.542 & $3.41 E-03$ & $6.28 E-03$ \\
\hline 8.48 & 4360 & 8.48 & 4400 & 8.48 & 4430 & 0.888 & $4.40 E-03$ & $4.95 E-03$ \\
\hline 13.9 & 5920 & 13.9 & 5990 & 13.9 & 6080 & 1.456 & $6.00 E-03$ & $4.12 E-03$ \\
\hline 22.8 & 8360 & 22.8 & 8450 & 22.8 & 8510 & 2.388 & $8.44 E-03$ & $3.53 E-03$ \\
\hline 37.3 & 12200 & 37.3 & 12300 & 37.3 & 12500 & 3.906 & $1.23 E-02$ & $3.16 E-03$ \\
\hline 61.1 & 18200 & 61.1 & 18500 & 61.1 & 18800 & 6.398 & $1.85 E-02$ & $2.89 E-03$ \\
\hline 100 & 27600 & 100 & 28000 & 100 & 28400 & 10.472 & $2.80 E-02$ & $2.67 E-03$ \\
\hline 100 & 27400 & 100 & 27800 & 100 & 28300 & 10.472 & $2.78 E-02$ & $2.66 E-03$ \\
\hline 69.5 & 20000 & 69.5 & 20300 & 69.5 & 20700 & 7.278 & $2.03 E-02$ & $2.79 E-03$ \\
\hline 48.3 & 14700 & 48.3 & 15000 & 48.3 & 15200 & 5.058 & $1.50 E-02$ & $2.96 E-03$ \\
\hline 33.6 & 11000 & 33.6 & 11200 & 33.6 & 11400 & 3.519 & $1.12 E-02$ & $3.18 E-03$ \\
\hline 23.4 & 8380 & 23.4 & 8510 & 23.4 & 8650 & 2.450 & $8.51 E-03$ & $3.47 E-03$ \\
\hline 16.2 & 6480 & 16.2 & 6570 & 16.2 & 6680 & 1.696 & $6.58 E-03$ & $3.88 E-03$ \\
\hline 11.3 & 5110 & 11.3 & 5190 & 11.3 & 5270 & 1.183 & 5.19E-03 & $4.39 E-03$ \\
\hline 7.85 & 4130 & 7.85 & 4190 & 7.85 & 4250 & 0.822 & 4.19E-03 & $5.10 E-03$ \\
\hline 5.46 & 3420 & 5.46 & 3470 & 5.46 & 3520 & 0.572 & $3.47 E-03$ & $6.07 E-03$ \\
\hline 3.79 & 2910 & 3.79 & 2950 & 3.79 & 2980 & 0.397 & $2.95 E-03$ & $7.42 E-03$ \\
\hline 2.64 & 2530 & 2.64 & 2560 & 2.64 & 2590 & 0.276 & $2.56 E-03$ & $9.26 E-03$ \\
\hline 1.83 & 2250 & 1.83 & 2280 & 1.83 & 2300 & 0.192 & $2.28 E-03$ & 1.19E-02 \\
\hline 1.27 & 2040 & 1.27 & 2060 & 1.27 & 2090 & 0.133 & $2.06 E-03$ & $1.55 E-02$ \\
\hline 0.887 & 1890 & 0.886 & 1900 & 0.887 & 1930 & 0.093 & $1.91 E-03$ & $2.05 E-02$ \\
\hline 0.616 & 1760 & 0.615 & 1790 & 0.616 & 1810 & 0.064 & $1.79 E-03$ & 2.77E-02 \\
\hline 0.43 & 1680 & 0.428 & 1690 & 0.43 & 1750 & 0.045 & $1.71 E-03$ & 3.80E-02 \\
\hline 0.296 & 1610 & 0.296 & 1620 & 0.298 & 1660 & 0.031 & $1.63 E-03$ & $5.25 E-02$ \\
\hline 0.206 & 1540 & 0.207 & 1580 & 0.208 & 1600 & 0.022 & $1.57 E-03$ & 7.26E-02 \\
\hline 0.144 & 1500 & 0.143 & 1520 & 0.143 & 1560 & 0.015 & $1.53 E-03$ & $1.02 E-01$ \\
\hline 0.0991 & 1470 & 0.0996 & 1480 & 0.1 & 1510 & 0.010 & $1.49 E-03$ & $1.43 E-01$ \\
\hline
\end{tabular}


Table A - 38: Measured data for Mix\#8 with 20 \% beads using Double spiral spindle.

\begin{tabular}{|c|c|c|c|c|c|c|c|c|}
\hline \multicolumn{6}{|c|}{ Double Spiral } & \multirow{2}{*}{\multicolumn{3}{|c|}{$\begin{array}{c}\text { Bead } \% \\
20 \%\end{array}$}} \\
\hline \multicolumn{2}{|c|}{ Geom.: $\quad$ RHN-83A } & & & \multicolumn{2}{|l|}{ Cup: } & & & \\
\hline \multicolumn{2}{|c|}{ Run \#1 } & \multicolumn{2}{|c|}{ Run \#2 } & \multicolumn{2}{|c|}{ Run \#3 } & \multirow{2}{*}{\multicolumn{3}{|c|}{$\begin{array}{c}\text { Average } \\
\text { values }\end{array}$}} \\
\hline NIST Code: & SMC-103P & NIST Code: & $S M C-103 Q$ & NIST Code: & MC-103R & & & \\
\hline $\mathrm{N}$ & $\Gamma$ & $\mathrm{N}$ & $\Gamma$ & $\mathrm{N}$ & $\Gamma$ & $\mathbf{N}$ & $\Gamma$ & $\boldsymbol{\Gamma} / \mathbf{N}$ (Angular \\
\hline $1 / \mathrm{min}$ & $\mu \mathrm{Nm}$ & $1 / \mathrm{min}$ & $\mu \mathrm{Nm}$ & $1 / \mathrm{min}$ & $\mu \mathrm{Nm}$ & $\mathrm{rad} / \mathrm{s}$ & $\mathrm{Nm}$ & momentum) \\
\hline 0.0931 & 1340 & 0.0924 & 1350 & 0.0927 & 1390 & 0.010 & $1.36 E-03$ & $1.40 E-01$ \\
\hline 0.161 & 1930 & 0.161 & 1960 & 0.161 & 2000 & 0.017 & $1.96 E-03$ & $1.16 E-01$ \\
\hline 0.268 & 2190 & 0.269 & 2220 & 0.269 & 2290 & 0.028 & $2.23 E-03$ & $7.94 E-02$ \\
\hline 0.439 & 2360 & 0.439 & 2400 & 0.439 & 2460 & 0.046 & $2.41 E-03$ & $5.24 E-02$ \\
\hline 0.72 & 2590 & 0.721 & 2650 & 0.719 & 2710 & 0.075 & $2.65 E-03$ & $3.51 E-02$ \\
\hline 1.18 & 2920 & 1.18 & 2970 & 1.18 & 3060 & 0.124 & $2.98 E-03$ & $2.41 E-02$ \\
\hline 1.93 & 3460 & 1.93 & 3490 & 1.93 & 3600 & 0.202 & $3.52 E-03$ & $1.74 E-02$ \\
\hline 3.16 & 4320 & 3.16 & 4280 & 3.16 & 4420 & 0.331 & 4.34E-03 & $1.31 E-02$ \\
\hline 5.18 & 5650 & 5.18 & 5560 & 5.18 & 5720 & 0.542 & $5.64 E-03$ & $1.04 E-02$ \\
\hline 8.48 & 7710 & 8.48 & 7540 & 8.49 & 7800 & 0.888 & $7.68 E-03$ & $8.65 E-03$ \\
\hline 13.9 & 10700 & 13.9 & 10600 & 13.9 & 10900 & 1.456 & $1.07 E-02$ & 7.37E-03 \\
\hline 22.8 & 14900 & 22.7 & 15100 & 22.8 & 15600 & 2.384 & $1.52 E-02$ & $6.38 E-03$ \\
\hline 37.3 & 22200 & 37.3 & 22500 & 37.3 & 23400 & 3.906 & $2.27 E-02$ & $5.81 E-03$ \\
\hline 61 & 33600 & 61.1 & 34100 & 61.1 & 35400 & 6.395 & $3.44 E-02$ & $5.37 E-03$ \\
\hline 100 & 50800 & 100 & 51900 & 100 & 53900 & 10.472 & $5.22 E-02$ & $4.98 E-03$ \\
\hline 100 & 50200 & 100 & 51500 & 100 & 53500 & 10.472 & $5.17 E-02$ & $4.94 E-03$ \\
\hline 69.5 & 36400 & 69.5 & 37300 & 69.5 & 38800 & 7.278 & $3.75 E-02$ & $5.15 E-03$ \\
\hline 48.3 & 26600 & 48.3 & 27400 & 48.3 & 28400 & 5.058 & $2.75 E-02$ & $5.43 E-03$ \\
\hline 33.6 & 19700 & 33.6 & 20300 & 33.6 & 21100 & 3.519 & $2.04 E-02$ & $5.79 E-03$ \\
\hline 23.4 & 14800 & 23.3 & 15200 & 23.4 & 15800 & 2.447 & $1.53 E-02$ & $6.24 E-03$ \\
\hline 16.2 & 11200 & 16.2 & 11600 & 16.2 & 12000 & 1.696 & $1.16 E-02$ & $6.84 E-03$ \\
\hline 11.3 & 8670 & 11.3 & 8900 & 11.3 & 9240 & 1.183 & 8.94E-03 & $7.55 E-03$ \\
\hline 7.85 & 6820 & 7.85 & 7060 & 7.85 & 7290 & 0.822 & $7.06 E-03$ & 8.58E-03 \\
\hline 5.46 & 5500 & 5.46 & 5700 & 5.46 & 5870 & 0.572 & $5.69 E-03$ & $9.95 E-03$ \\
\hline 3.79 & 4560 & 3.79 & 4690 & 3.79 & 4840 & 0.397 & $4.70 E-03$ & $1.18 E-02$ \\
\hline 2.63 & 3830 & 2.64 & 3950 & 2.64 & 4080 & 0.276 & $3.95 E-03$ & $1.43 E-02$ \\
\hline 1.83 & 3320 & 1.83 & 3420 & 1.83 & 3510 & 0.192 & $3.42 E-03$ & $1.78 E-02$ \\
\hline 1.27 & 2940 & 1.28 & 3020 & 1.28 & 3110 & 0.134 & $3.02 E-03$ & $2.26 E-02$ \\
\hline 0.887 & 2640 & 0.886 & 2730 & 0.885 & 2790 & 0.093 & $2.72 E-03$ & 2.93E-02 \\
\hline 0.617 & 2420 & 0.614 & 2500 & 0.615 & 2560 & 0.064 & $2.49 E-03$ & $3.87 E-02$ \\
\hline 0.428 & 2250 & 0.429 & 2320 & 0.427 & 2370 & 0.045 & $2.31 E-03$ & $5.16 E-02$ \\
\hline 0.298 & 2120 & 0.296 & 2190 & 0.297 & 2240 & 0.031 & $2.18 E-03$ & $7.02 E-02$ \\
\hline 0.207 & 2030 & 0.205 & 2080 & 0.207 & 2130 & 0.022 & $2.08 E-03$ & $9.63 E-02$ \\
\hline 0.145 & 1950 & 0.142 & 2030 & 0.144 & 2050 & 0.015 & $2.01 E-03$ & $1.34 E-01$ \\
\hline 0.0998 & 1900 & 0.102 & 2000 & 0.101 & 1990 & 0.011 & $1.96 E-03$ & $1.86 E-01$ \\
\hline
\end{tabular}




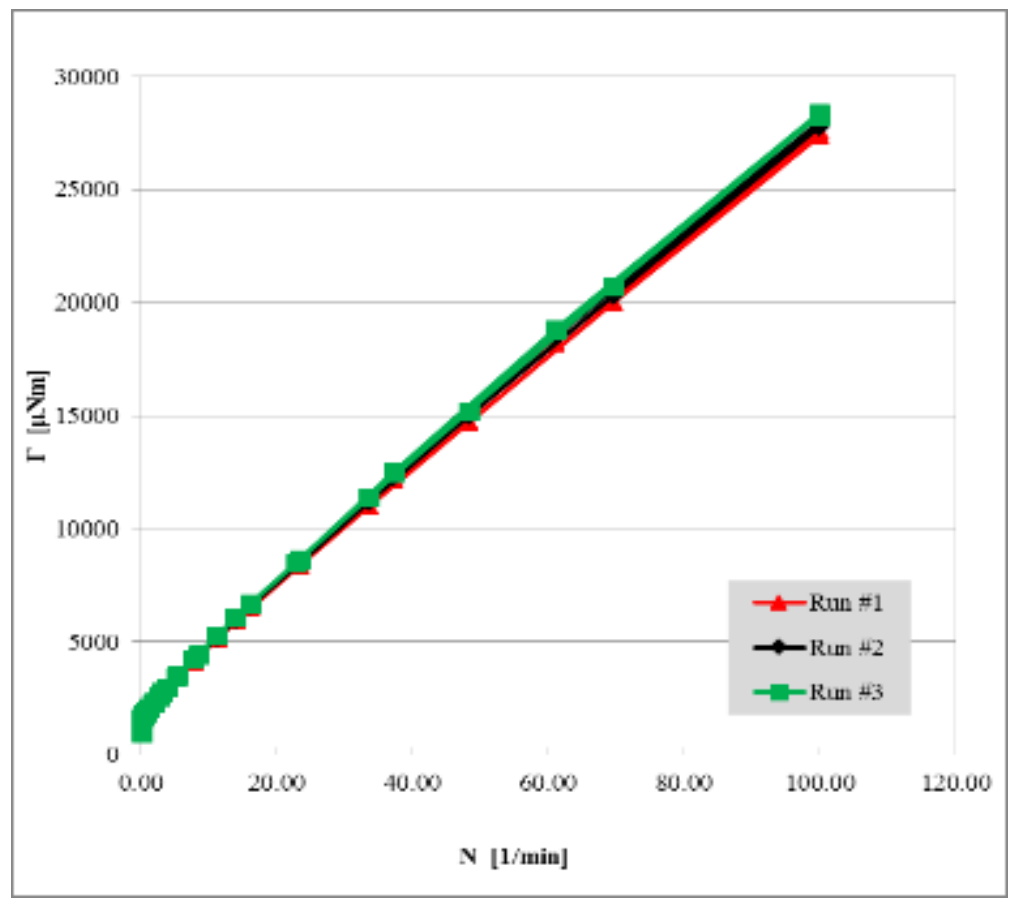

Figure A - 37: Torque vs. Angular Speed using Double spiral spindle on Mix\#8, with $0 \%$ beads by volume. Portrays data from Table A-37.

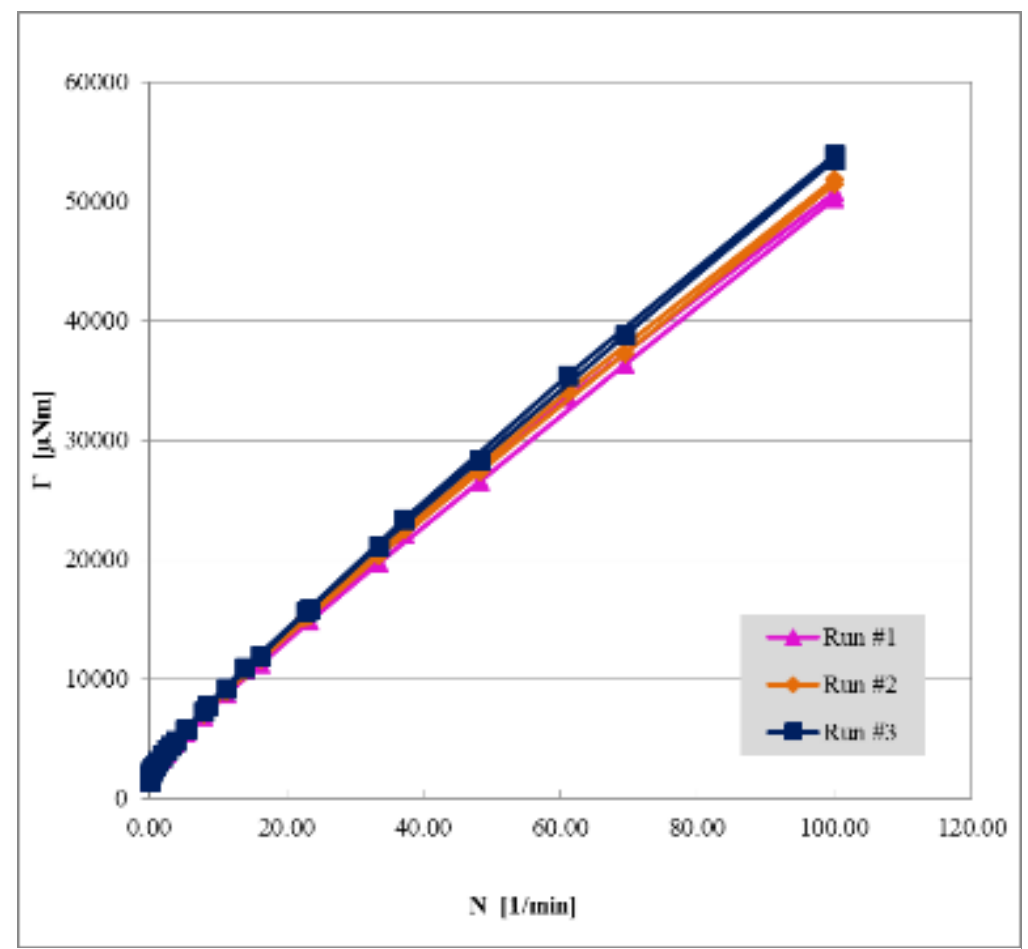

Figure A - 38: Torque vs. Angular Speed using Double spiral spindle on Mix\#8, with $20 \%$ beads by volume. Portrays data from Table A-38. 
Table A - 39: Measured data for Mix\#8 with 0 \% beads using Six-blade Vane.

\begin{tabular}{|c|c|c|c|c|c|c|c|c|}
\hline \multicolumn{6}{|c|}{6 Blade Vane } & \multicolumn{3}{|c|}{$\begin{array}{c}\text { Bead } \% \\
0 \%\end{array}$} \\
\hline Run & \#1 & Run & \#2 & & \#3 & & Average & \\
\hline NIST Code: & $S M C-103 D$ & NIST Code: & SMC-103E & NIST Code: & SMC-103F & & values & \\
\hline \begin{tabular}{l|l}
$\mathrm{N}$ \\
\end{tabular} & $\Gamma$ & $\mathrm{N}$ & $\Gamma$ & $\mathrm{N}$ & $\Gamma$ & $\mathbf{N}$ & $\Gamma$ & (Angular \\
\hline $1 / \mathrm{min}$ & $\mu \mathrm{Nm}$ & $1 / \mathrm{min}$ & $\mu \mathrm{Nm}$ & $1 / \mathrm{min}$ & $\mu \mathrm{Nm}$ & $\mathrm{rad} / \mathrm{s}$ & $\mathrm{Nm}$ & momentum \\
\hline 0.0979 & 425 & 0.0979 & 401 & 0.0977 & 421 & 0.010 & 4.16E-04 & $4.06 E-02$ \\
\hline 0.163 & 557 & 0.163 & 537 & 0.163 & 570 & 0.017 & $5.55 E-04$ & $3.25 E-02$ \\
\hline 0.268 & 595 & 0.268 & 580 & 0.268 & 611 & 0.028 & $5.95 E-04$ & $2.12 E-02$ \\
\hline 0.439 & 629 & 0.439 & 614 & 0.439 & 645 & 0.046 & $6.29 E-04$ & 1.37E-02 \\
\hline 0.72 & 679 & 0.72 & 664 & 0.72 & 695 & 0.075 & $6.79 E-04$ & $9.01 E-03$ \\
\hline 1.18 & 749 & 1.18 & 735 & 1.18 & 767 & 0.124 & $7.50 E-04$ & $6.07 E-03$ \\
\hline 1.93 & 849 & 1.93 & 834 & 1.93 & 867 & 0.202 & $8.50 E-04$ & $4.21 E-03$ \\
\hline 3.16 & 984 & 3.16 & 975 & 3.16 & 1010 & 0.331 & $9.90 E-04$ & $2.99 E-03$ \\
\hline 5.18 & 1180 & 5.18 & 1180 & 5.18 & 1210 & 0.542 & $1.19 E-03$ & $2.19 E-03$ \\
\hline 8.48 & 1470 & 8.48 & 1470 & 8.48 & 1510 & 0.888 & $1.48 E-03$ & 1.67E-03 \\
\hline 13.9 & 1890 & 13.9 & 1900 & 13.9 & 1940 & 1.456 & $1.91 E-03$ & $1.31 E-03$ \\
\hline 22.8 & 2500 & 22.8 & 2530 & 22.8 & 2570 & 2.388 & $2.53 E-03$ & $1.06 E-03$ \\
\hline 37.3 & 3450 & 37.3 & 3480 & 37.3 & 3550 & 3.906 & $3.49 E-03$ & $8.94 E-04$ \\
\hline 61 & 4880 & 61 & 4960 & 61 & 5040 & 6.388 & $4.96 E-03$ & $7.76 E-04$ \\
\hline 100 & 7130 & 100 & 7270 & 100 & 7390 & 10.472 & $7.26 E-03$ & $6.94 E-04$ \\
\hline 100 & 7100 & 100 & 7250 & 100 & 7360 & 10.472 & $7.24 E-03$ & $6.91 E-04$ \\
\hline 69.5 & 5330 & 69.5 & 5420 & 69.5 & 5500 & 7.278 & $5.42 E-03$ & $7.44 E-04$ \\
\hline 48.3 & 4050 & 48.3 & 4120 & 48.3 & 4170 & 5.058 & 4.11E-03 & $8.13 E-04$ \\
\hline 33.6 & 3140 & 33.6 & 3190 & 33.6 & 3220 & 3.519 & $3.18 E-03$ & $9.05 E-04$ \\
\hline 23.4 & 2480 & 23.4 & 2510 & 23.4 & 2540 & 2.450 & $2.51 E-03$ & $1.02 E-03$ \\
\hline 16.2 & 1990 & 16.2 & 2020 & 16.2 & 2040 & 1.696 & $2.02 E-03$ & $1.19 E-03$ \\
\hline 11.3 & 1640 & 11.3 & 1660 & 11.3 & 1670 & 1.183 & $1.66 E-03$ & $1.40 E-03$ \\
\hline 7.85 & 1370 & 7.85 & 1390 & 7.85 & 1390 & 0.822 & $1.38 E-03$ & $1.68 E-03$ \\
\hline 5.45 & 1160 & 5.46 & 1180 & 5.45 & 1180 & 0.571 & 1.17E-03 & $2.05 E-03$ \\
\hline 3.79 & 1010 & 3.79 & 1020 & 3.79 & 1030 & 0.397 & $1.02 E-03$ & $2.57 E-03$ \\
\hline 2.64 & 886 & 2.63 & 900 & 2.64 & 904 & 0.276 & 8.97E-04 & $3.25 E-03$ \\
\hline 1.83 & 797 & 1.83 & 811 & 1.83 & 811 & 0.192 & $8.06 E-04$ & $4.21 E-03$ \\
\hline 1.27 & 723 & 1.27 & 735 & 1.28 & 739 & 0.133 & 7.32E-04 & $5.49 E-03$ \\
\hline 0.887 & 669 & 0.887 & 675 & 0.885 & 681 & 0.093 & $6.75 E-04$ & 7.27E-03 \\
\hline 0.616 & 633 & 0.618 & 637 & 0.618 & 640 & 0.065 & $6.37 E-04$ & $9.85 E-03$ \\
\hline 0.43 & 601 & 0.426 & 602 & 0.428 & 595 & 0.045 & $5.99 E-04$ & $1.34 E-02$ \\
\hline 0.298 & 566 & 0.295 & 586 & 0.296 & 568 & 0.031 & $5.73 E-04$ & $1.85 E-02$ \\
\hline 0.205 & 562 & 0.208 & 550 & 0.206 & 569 & 0.022 & $5.60 E-04$ & $2.59 E-02$ \\
\hline 0.143 & 522 & 0.143 & 525 & 0.144 & 555 & 0.015 & $5.34 E-04$ & $3.56 E-02$ \\
\hline 0.0986 & 518 & 0.102 & 523 & 0.1 & 538 & 0.010 & $5.26 E-04$ & $5.02 E-02$ \\
\hline
\end{tabular}


Table A - 40: Measured data for Mix\#8 with 20 \% beads using Six-blade Vane.

\begin{tabular}{|c|c|c|c|c|c|c|c|c|}
\hline \multicolumn{6}{|c|}{6 Blade Vane } & \multirow{2}{*}{\multicolumn{3}{|c|}{$\begin{array}{c}\text { Bead } \% \\
20 \%\end{array}$}} \\
\hline Geom.: & $R H N-83 C$ & & & \multicolumn{2}{|c|}{ Z43S } & & & \\
\hline \multicolumn{2}{|c|}{ Run \#1 } & \multicolumn{2}{|c|}{ Run \#2 } & \multicolumn{2}{|c|}{ Run \#3 } & \multirow{2}{*}{\multicolumn{3}{|c|}{$\begin{array}{c}\text { Average } \\
\text { values }\end{array}$}} \\
\hline NIST Code: & SMC-103M & NIST Code: & $S M C-103 N$ & NIST Code & $C-1030$ & & & \\
\hline $\mathrm{N}$ & $\Gamma$ & $\mathrm{N}$ & $\Gamma$ & $\mathrm{N}$ & $\Gamma$ & $\mathbf{N}$ & $\Gamma$ & (Angular \\
\hline $1 / \mathrm{min}$ & $\mu \mathrm{Nm}$ & $1 / \mathrm{min}$ & $\mu \mathrm{Nm}$ & $1 / \mathrm{min}$ & $\mu \mathrm{Nm}$ & $\mathrm{rad} / \mathrm{s}$ & $\mathrm{Nm}$ & momentum) \\
\hline 0.0974 & 581 & 0.0973 & 582 & 0.0972 & 604 & 0.010 & $5.89 E-04$ & $5.78 E-02$ \\
\hline 0.163 & 737 & 0.163 & 749 & 0.164 & 782 & 0.017 & $7.56 E-04$ & $4.42 E-02$ \\
\hline 0.268 & 799 & 0.269 & 817 & 0.268 & 849 & 0.028 & $8.22 E-04$ & $2.92 E-02$ \\
\hline 0.439 & 871 & 0.439 & 879 & 0.44 & 924 & 0.046 & $8.91 E-04$ & $1.94 E-02$ \\
\hline 0.72 & 945 & 0.719 & 958 & 0.718 & 1000 & 0.075 & $9.68 E-04$ & $1.29 E-02$ \\
\hline 1.18 & 1080 & 1.18 & 1080 & 1.18 & 1130 & 0.124 & $1.10 E-03$ & 8.87E-03 \\
\hline 1.93 & 1240 & 1.93 & 1260 & 1.93 & 1320 & 0.202 & $1.27 E-03$ & $6.30 E-03$ \\
\hline 3.16 & 1500 & 3.16 & 1520 & 3.16 & 1580 & 0.331 & $1.53 E-03$ & 4.63E-03 \\
\hline 5.18 & 1860 & 5.18 & 1900 & 5.18 & 1980 & 0.542 & $1.91 E-03$ & $3.53 E-03$ \\
\hline 8.48 & 2400 & 8.48 & 2450 & 8.48 & 2530 & 0.888 & $2.46 E-03$ & 2.77E-03 \\
\hline 13.9 & 3230 & 13.9 & 3270 & 13.9 & 3370 & 1.456 & $3.29 E-03$ & $2.26 E-03$ \\
\hline 22.8 & 4440 & 22.8 & 4510 & 22.8 & 4670 & 2.388 & $4.54 E-03$ & $1.90 E-03$ \\
\hline 37.3 & 6220 & 37.3 & 6370 & 37.3 & 6630 & 3.906 & $6.41 E-03$ & $1.64 E-03$ \\
\hline 61.1 & 8820 & 61.1 & 9180 & 61 & 9590 & 6.395 & $9.20 E-03$ & $1.44 E-03$ \\
\hline 100 & 12900 & 100 & 13500 & 100 & 14200 & 10.472 & $1.35 E-02$ & $1.29 E-03$ \\
\hline 100 & 12800 & 100 & 13400 & 100 & 14000 & 10.472 & $1.34 E-02$ & $1.28 E-03$ \\
\hline 69.5 & 9530 & 69.5 & 9950 & 69.5 & 10300 & 7.278 & 9.93E-03 & $1.36 E-03$ \\
\hline 48.3 & 7160 & 48.3 & 7470 & 48.3 & 7720 & 5.058 & $7.45 E-03$ & $1.47 E-03$ \\
\hline 33.6 & 5460 & 33.6 & 5670 & 33.6 & 5890 & 3.519 & $5.67 E-03$ & $1.61 E-03$ \\
\hline 23.3 & 4210 & 23.4 & 4360 & 23.3 & 4500 & 2.443 & $4.36 E-03$ & $1.78 E-03$ \\
\hline 16.2 & 3320 & 16.2 & 3410 & 16.2 & 3520 & 1.696 & $3.42 E-03$ & $2.01 E-03$ \\
\hline 11.3 & 2650 & 11.3 & 2720 & 11.3 & 2800 & 1.183 & $2.72 E-03$ & $2.30 E-03$ \\
\hline 7.85 & 2160 & 7.85 & 2220 & 7.85 & 2270 & 0.822 & $2.22 E-03$ & $2.70 E-03$ \\
\hline 5.46 & 1780 & 5.45 & 1840 & 5.45 & 1870 & 0.571 & $1.83 E-03$ & $3.20 E-03$ \\
\hline 3.79 & 1510 & 3.79 & 1550 & 3.79 & 1590 & 0.397 & $1.55 E-03$ & $3.91 E-03$ \\
\hline 2.64 & 1300 & 2.64 & 1330 & 2.64 & 1360 & 0.276 & $1.33 E-03$ & $4.81 E-03$ \\
\hline 1.83 & 1140 & 1.83 & 1170 & 1.83 & 1200 & 0.192 & $1.17 E-03$ & $6.11 E-03$ \\
\hline 1.27 & 1020 & 1.27 & 1040 & 1.27 & 1070 & 0.133 & $1.04 E-03$ & $7.84 E-03$ \\
\hline 0.887 & 922 & 0.882 & 941 & 0.889 & 953 & 0.093 & $9.39 E-04$ & $1.01 E-02$ \\
\hline 0.616 & 844 & 0.615 & 867 & 0.617 & 880 & 0.065 & 8.64E-04 & $1.34 E-02$ \\
\hline 0.428 & 793 & 0.427 & 812 & 0.426 & 837 & 0.045 & 8.14E-04 & $1.82 E-02$ \\
\hline 0.297 & 745 & 0.302 & 783 & 0.299 & 776 & 0.031 & $7.68 E-04$ & $2.45 E-02$ \\
\hline 0.207 & 709 & 0.206 & 719 & 0.208 & 735 & 0.022 & $7.21 E-04$ & 3.33E-02 \\
\hline 0.144 & 683 & 0.141 & 708 & 0.145 & 709 & 0.015 & $7.00 E-04$ & 4.66E-02 \\
\hline 0.0996 & 657 & 0.102 & 691 & 0.0974 & 703 & 0.010 & $6.84 E-04$ & $6.55 E-02$ \\
\hline
\end{tabular}




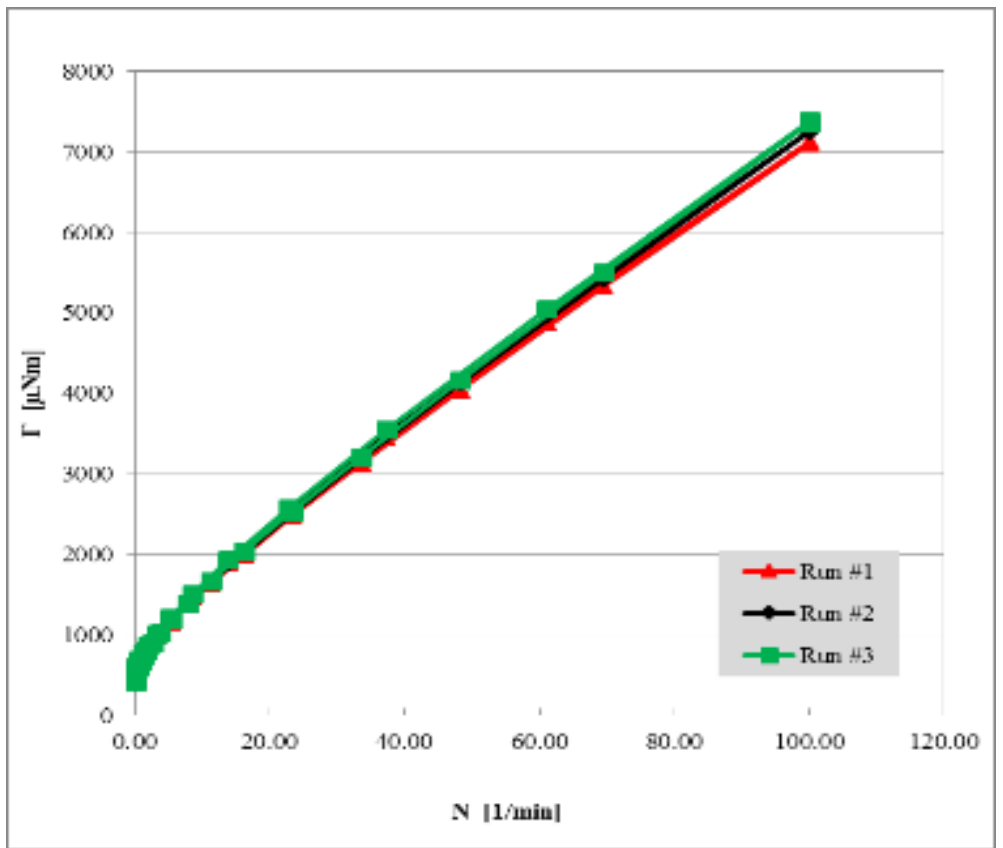

Figure A - 27: Torque vs. Angular Speed using six-blade vane on Mix\#8, with $0 \%$ beads by volume. Portrays data from Table A-39.

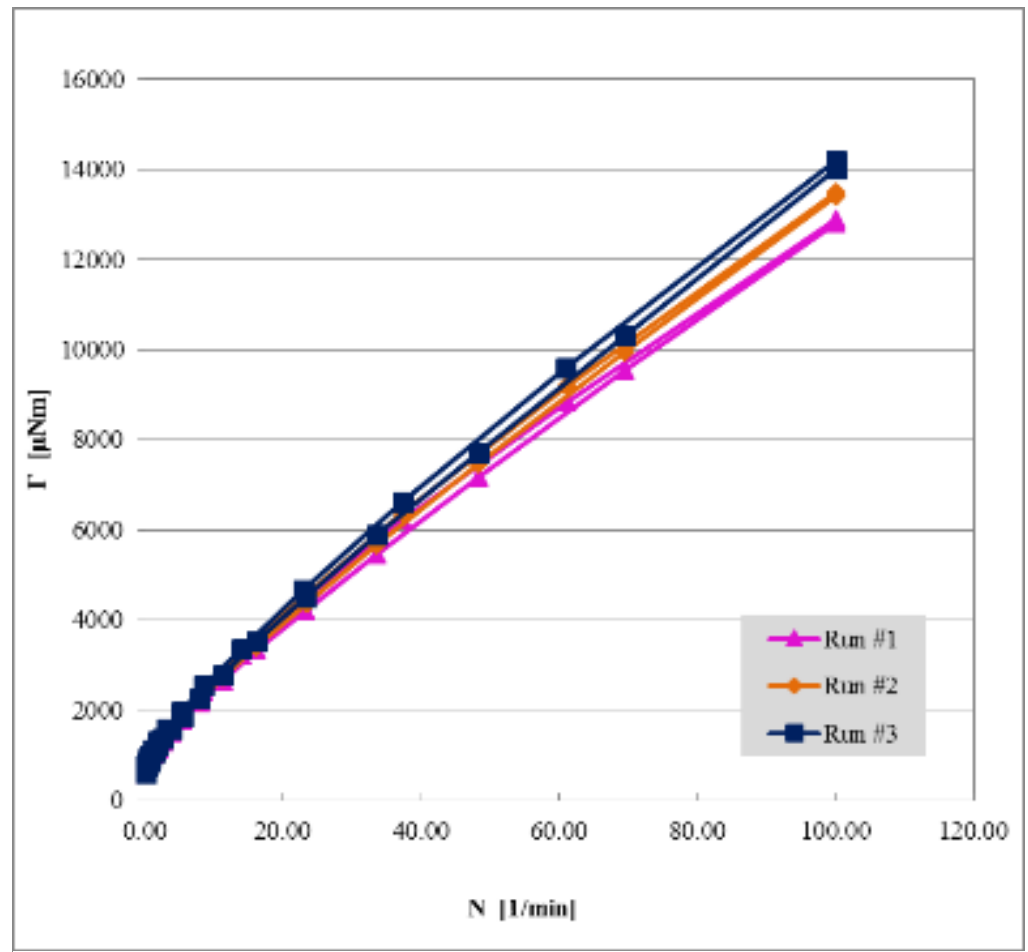

Figure A - 28: Torque vs. Angular Speed using six-blade vane on Mix\#8, with $20 \%$ beads by volume. Portrays data from Table A-40. 
Table A - 41: Measured data for Mix\#8 with 0 \% beads using Serrated Coaxial Cylinder.

\begin{tabular}{|c|c|c|c|c|c|c|c|c|}
\hline \multicolumn{6}{|c|}{ Serrated Coaxial Cylinder } & \multirow{2}{*}{\multicolumn{3}{|c|}{$\begin{array}{c}\text { Bead } \% \\
0 \%\end{array}$}} \\
\hline Geom.: & SS18 & & & \multicolumn{2}{|c|}{ Z43S } & & & \\
\hline \multicolumn{2}{|c|}{ Run \#1 } & \multicolumn{2}{|c|}{ Run \#2 } & \multicolumn{2}{|c|}{ Run \#3 } & \multirow{2}{*}{\multicolumn{3}{|c|}{$\begin{array}{c}\text { Average } \\
\text { values }\end{array}$}} \\
\hline NIST Code: & $S M C-103 A$ & NIST Code: & MC-103B & NIST Cod & $C-103 C$ & & & \\
\hline $\mathrm{N}$ & $\Gamma$ & $\mathrm{N}$ & $\Gamma$ & $\mathrm{N}$ & $\Gamma$ & $\mathbf{N}$ & $\Gamma$ & $\boldsymbol{\Gamma} / \mathbf{N}$ (Angular \\
\hline $1 / \mathrm{min}$ & $\mu \mathrm{Nm}$ & $1 / \mathrm{min}$ & $\mu \mathrm{Nm}$ & $1 / \mathrm{min}$ & $\mu \mathrm{Nm}$ & $\mathrm{rad} / \mathrm{s}$ & $\mathrm{Nm}$ & momentum) \\
\hline 0.0983 & 460 & 0.0984 & 411 & 0.0979 & 413 & 0.010 & $4.28 E-04$ & $4.16 E-02$ \\
\hline 0.164 & 563 & 0.164 & 521 & 0.164 & 551 & 0.017 & $5.45 E-04$ & $3.17 E-02$ \\
\hline 0.268 & 591 & 0.268 & 561 & 0.269 & 596 & 0.028 & $5.83 E-04$ & 2.07E-02 \\
\hline 0.439 & 630 & 0.44 & 610 & 0.439 & 636 & 0.046 & $6.25 E-04$ & $1.36 E-02$ \\
\hline 0.72 & 687 & 0.72 & 674 & 0.719 & 700 & 0.075 & $6.87 E-04$ & $9.12 E-03$ \\
\hline 1.18 & 766 & 1.18 & 758 & 1.18 & 778 & 0.124 & 7.67E-04 & $6.21 E-03$ \\
\hline 1.93 & 873 & 1.93 & 871 & 1.93 & 893 & 0.202 & 8.79E-04 & $4.35 E-03$ \\
\hline 3.16 & 1020 & 3.16 & 1030 & 3.16 & 1060 & 0.331 & $1.04 E-03$ & 3.13E-03 \\
\hline 5.18 & 1240 & 5.18 & 1240 & 5.18 & 1270 & 0.542 & $1.25 E-03$ & $2.30 E-03$ \\
\hline 8.48 & 1540 & 8.48 & 1550 & 8.48 & 1590 & 0.888 & $1.56 E-03$ & $1.76 E-03$ \\
\hline 13.9 & 1980 & 13.9 & 2000 & 13.9 & 2050 & 1.456 & $2.01 E-03$ & $1.38 E-03$ \\
\hline 22.8 & 2600 & 22.8 & 2650 & 22.8 & 2700 & 2.388 & $2.65 E-03$ & $1.11 E-03$ \\
\hline 37.3 & 3560 & 37.3 & 3640 & 37.3 & 3710 & 3.906 & $3.64 E-03$ & $9.31 E-04$ \\
\hline 61 & 5040 & 61 & 5180 & 61 & 5280 & 6.388 & $5.17 E-03$ & 8.09E-04 \\
\hline 100 & 7440 & 100 & 7550 & 100 & 7650 & 10.472 & $7.55 E-03$ & $7.21 E-04$ \\
\hline 100 & 7290 & 100 & 7460 & 100 & 7570 & 10.472 & $7.44 E-03$ & $7.10 E-04$ \\
\hline 69.5 & 5450 & 69.5 & 5570 & 69.5 & 5660 & 7.278 & $5.56 E-03$ & 7.64E-04 \\
\hline 48.3 & 4130 & 48.3 & 4210 & 48.3 & 4270 & 5.058 & $4.20 E-03$ & $8.31 E-04$ \\
\hline 33.6 & 3170 & 33.6 & 3220 & 33.6 & 3270 & 3.519 & $3.22 E-03$ & $9.15 E-04$ \\
\hline 23.4 & 2480 & 23.4 & 2520 & 23.4 & 2560 & 2.450 & $2.52 E-03$ & $1.03 E-03$ \\
\hline 16.2 & 1980 & 16.2 & 2010 & 16.2 & 2040 & 1.696 & $2.01 E-03$ & $1.18 E-03$ \\
\hline 11.3 & 1620 & 11.3 & 1640 & 11.3 & 1660 & 1.183 & $1.64 E-03$ & $1.39 E-03$ \\
\hline 7.85 & 1330 & 7.85 & 1350 & 7.85 & 1370 & 0.822 & $1.35 E-03$ & $1.64 E-03$ \\
\hline 5.45 & 1120 & 5.45 & 1130 & 5.46 & 1150 & 0.571 & $1.13 E-03$ & $1.98 E-03$ \\
\hline 3.79 & 952 & 3.79 & 963 & 3.79 & 982 & 0.397 & $9.66 E-04$ & $2.43 E-03$ \\
\hline 2.64 & 820 & 2.64 & 832 & 2.64 & 851 & 0.276 & $8.34 E-04$ & $3.02 E-03$ \\
\hline 1.83 & 717 & 1.83 & 730 & 1.83 & 751 & 0.192 & 7.33E-04 & $3.82 E-03$ \\
\hline 1.27 & 645 & 1.27 & 643 & 1.27 & 670 & 0.133 & $6.53 E-04$ & $4.91 E-03$ \\
\hline 0.886 & 571 & 0.884 & 616 & 0.887 & 605 & 0.093 & $5.97 E-04$ & $6.44 E-03$ \\
\hline 0.612 & 538 & 0.623 & 561 & 0.617 & 566 & 0.065 & $5.55 E-04$ & 8.59E-03 \\
\hline 0.421 & 497 & 0.438 & 625 & 0.422 & 579 & 0.045 & $5.67 E-04$ & $1.27 E-02$ \\
\hline 0.301 & 445 & 0.301 & 512 & 0.303 & 486 & 0.032 & $4.81 E-04$ & $1.52 E-02$ \\
\hline 0.207 & 458 & 0.211 & 499 & 0.216 & 592 & 0.022 & $5.16 E-04$ & $2.33 E-02$ \\
\hline 0.148 & 469 & 0.152 & 497 & 0.148 & 464 & 0.016 & 4.77E-04 & $3.05 E-02$ \\
\hline 0.103 & 431 & 0.11 & 528 & 0.0895 & 522 & 0.011 & $4.94 E-04$ & $4.68 E-02$ \\
\hline
\end{tabular}


Table A - 42: Measured data for Mix\#8 with 20 \% beads using Serrated Coaxial Cylinder.

\begin{tabular}{|c|c|c|c|c|c|c|c|c|}
\hline \multicolumn{6}{|c|}{ Serrated Coaxial Cylinder } & \multirow{2}{*}{\multicolumn{3}{|c|}{$\begin{array}{c}\text { Bead } \% \\
20 \%\end{array}$}} \\
\hline Geom.: & SS18 & & & \multicolumn{2}{|c|}{ Z43S } & & & \\
\hline \multicolumn{2}{|c|}{ Run \#1 } & \multicolumn{2}{|c|}{ Run \#2 } & \multicolumn{2}{|c|}{ Run \#3 } & \multirow{2}{*}{\multicolumn{3}{|c|}{$\begin{array}{c}\text { Average } \\
\text { values }\end{array}$}} \\
\hline NIST Code: & SMC-103J & NIST Cod & $I C-103 K$ & NIST Co & $I C-103 L$ & & & \\
\hline $\mathrm{N}$ & $\Gamma$ & $\mathrm{N}$ & $\Gamma$ & $\mathrm{N}$ & $\Gamma$ & $\mathbf{N}$ & $\Gamma$ & $\boldsymbol{\Gamma} / \mathbf{N}$ (Angular \\
\hline $1 / \mathrm{min}$ & $\mu \mathrm{Nm}$ & $1 / \mathrm{min}$ & $\mu \mathrm{Nm}$ & $1 / \mathrm{min}$ & $\mu \mathrm{Nm}$ & $\mathrm{rad} / \mathrm{s}$ & $\mathrm{Nm}$ & momentum) \\
\hline 0.0972 & 632 & 0.0982 & 584 & 0.098 & 590 & $1.02 E-02$ & $6.02 E-04$ & $5.88 E-02$ \\
\hline 0.164 & 784 & 0.164 & 714 & 0.164 & 729 & $1.72 E-02$ & $7.42 E-04$ & $4.32 E-02$ \\
\hline 0.269 & 801 & 0.269 & 762 & 0.268 & 784 & $2.81 E-02$ & $7.82 E-04$ & $2.78 E-02$ \\
\hline 0.439 & 818 & 0.44 & 814 & 0.44 & 838 & $4.60 E-02$ & $8.23 E-04$ & $1.79 E-02$ \\
\hline 0.719 & 900 & 0.719 & 897 & 0.719 & 931 & 7.53E-02 & $9.09 E-04$ & $1.21 E-02$ \\
\hline 1.18 & 1010 & 1.18 & 1030 & 1.18 & 1050 & $1.24 E-01$ & $1.03 E-03$ & 8.34E-03 \\
\hline 1.93 & 1180 & 1.93 & 1200 & 1.93 & 1240 & $2.02 E-01$ & $1.21 E-03$ & 5.97E-03 \\
\hline 3.16 & 1430 & 3.16 & 1450 & 3.16 & 1500 & 3.31E-01 & $1.46 E-03$ & $4.41 E-03$ \\
\hline 5.18 & 1760 & 5.18 & 1800 & 5.18 & 1870 & $5.42 E-01$ & $1.81 E-03$ & $3.34 E-03$ \\
\hline 8.48 & 2250 & 8.48 & 2310 & 8.48 & 2380 & 8.88E-01 & $2.31 E-03$ & $2.61 E-03$ \\
\hline 13.9 & 2950 & 13.9 & 3060 & 13.9 & 3140 & $1.46 E+00$ & $3.05 E-03$ & $2.10 E-03$ \\
\hline 22.8 & 3990 & 22.8 & 4150 & 22.8 & 4290 & $2.39 E+00$ & 4.14E-03 & $1.74 E-03$ \\
\hline 37.3 & 5580 & 37.3 & 5850 & 37.3 & 6030 & $3.91 E+00$ & $5.82 E-03$ & $1.49 E-03$ \\
\hline 61 & 8040 & 61 & 8410 & 61.1 & 8650 & $6.39 E+00$ & 8.37E-03 & $1.31 E-03$ \\
\hline 100 & 11900 & 100 & 12400 & 100 & 12600 & $1.05 E+01$ & $1.23 E-02$ & $1.17 E-03$ \\
\hline 100 & 11800 & 100 & 12200 & 100 & 12500 & $1.05 E+01$ & $1.22 E-02$ & $1.16 E-03$ \\
\hline 69.5 & 8810 & 69.5 & 9110 & 69.5 & 9280 & $7.28 E+00$ & $9.07 E-03$ & $1.25 E-03$ \\
\hline 48.3 & 6610 & 48.3 & 6820 & 48.3 & 6940 & $5.06 E+00$ & 6.79E-03 & $1.34 E-03$ \\
\hline 33.6 & 5020 & 33.6 & 5170 & 33.6 & 5270 & $3.52 E+00$ & $5.15 E-03$ & $1.46 E-03$ \\
\hline 23.4 & 3870 & 23.4 & 3980 & 23.4 & 4060 & $2.45 E+00$ & $3.97 E-03$ & $1.62 E-03$ \\
\hline 16.2 & 3040 & 16.2 & 3130 & 16.2 & 3180 & $1.70 E+00$ & $3.12 E-03$ & $1.84 E-03$ \\
\hline 11.3 & 2440 & 11.3 & 2510 & 11.3 & 2540 & $1.18 E+00$ & $2.50 E-03$ & $2.11 E-03$ \\
\hline 7.85 & 1990 & 7.85 & 2040 & 7.85 & 2070 & $8.22 E-01$ & $2.03 E-03$ & $2.47 E-03$ \\
\hline 5.45 & 1650 & 5.45 & 1700 & 5.45 & 1720 & $5.71 E-01$ & $1.69 E-03$ & $2.96 E-03$ \\
\hline 3.79 & 1390 & 3.79 & 1430 & 3.79 & 1450 & 3.97E-01 & $1.42 E-03$ & $3.59 E-03$ \\
\hline 2.64 & 1200 & 2.64 & 1230 & 2.64 & 1250 & $2.76 E-01$ & $1.23 E-03$ & $4.44 E-03$ \\
\hline 1.83 & 1040 & 1.83 & 1070 & 1.83 & 1080 & $1.92 E-01$ & $1.06 E-03$ & $5.55 E-03$ \\
\hline 1.27 & 918 & 1.27 & 947 & 1.28 & 964 & $1.33 E-01$ & $9.43 E-04$ & 7.07E-03 \\
\hline 0.886 & 855 & 0.886 & 847 & 0.887 & 880 & $9.28 E-02$ & 8.61E-04 & $9.27 E-03$ \\
\hline 0.615 & 757 & 0.617 & 771 & 0.61 & 822 & $6.43 E-02$ & $7.83 E-04$ & $1.22 E-02$ \\
\hline 0.418 & 752 & 0.427 & 777 & 0.422 & 753 & $4.42 E-02$ & $7.61 E-04$ & $1.72 E-02$ \\
\hline 0.29 & 675 & 0.297 & 777 & 0.294 & 734 & $3.08 E-02$ & 7.29E-04 & $2.37 E-02$ \\
\hline 0.214 & 616 & 0.205 & 633 & 0.211 & 710 & $2.20 E-02$ & $6.53 E-04$ & 2.97E-02 \\
\hline 0.149 & 667 & 0.133 & 669 & 0.143 & 620 & $1.48 E-02$ & $6.52 E-04$ & 4.39E-02 \\
\hline 0.104 & 592 & 0.107 & 607 & 0.104 & 627 & $1.10 E-02$ & $6.09 E-04$ & $5.54 E-02$ \\
\hline
\end{tabular}




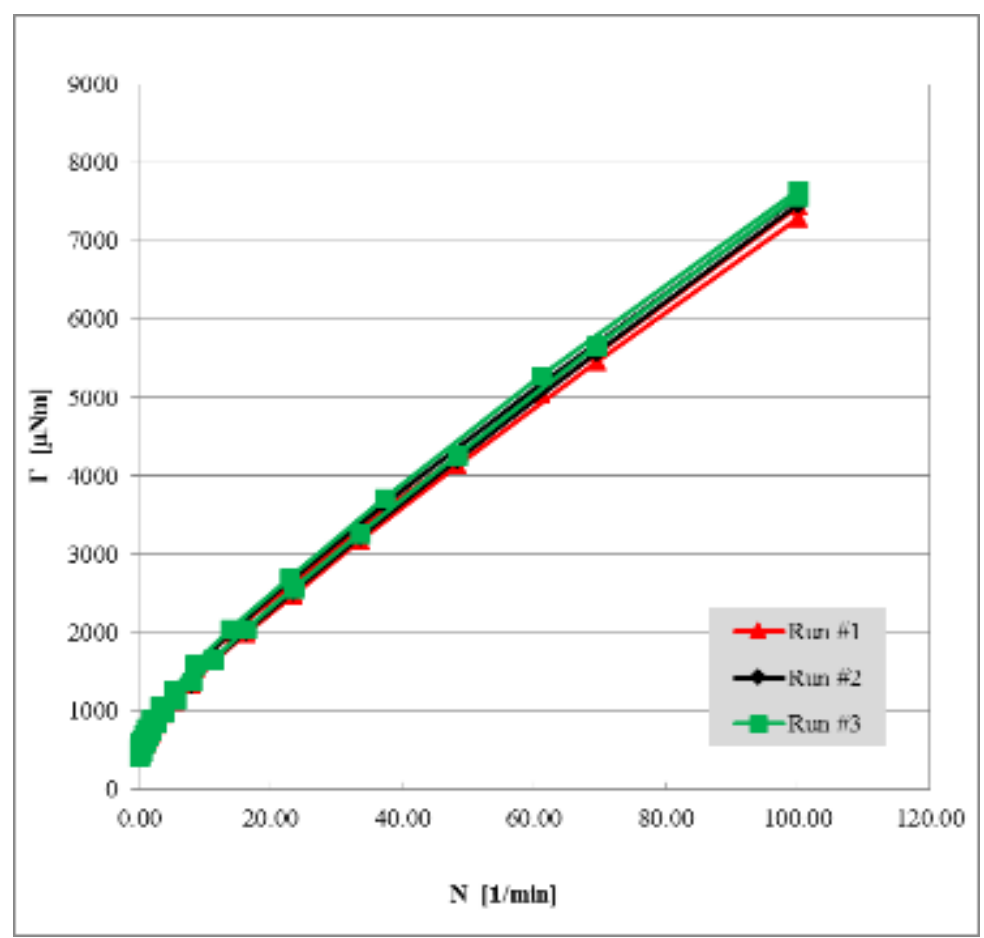

Figure A - 29: Torque vs. Angular Speed using Serrated Coaxial Cylinder on Mix\#8, with 0 \% beads by volume. Portrays data from Table A-41.

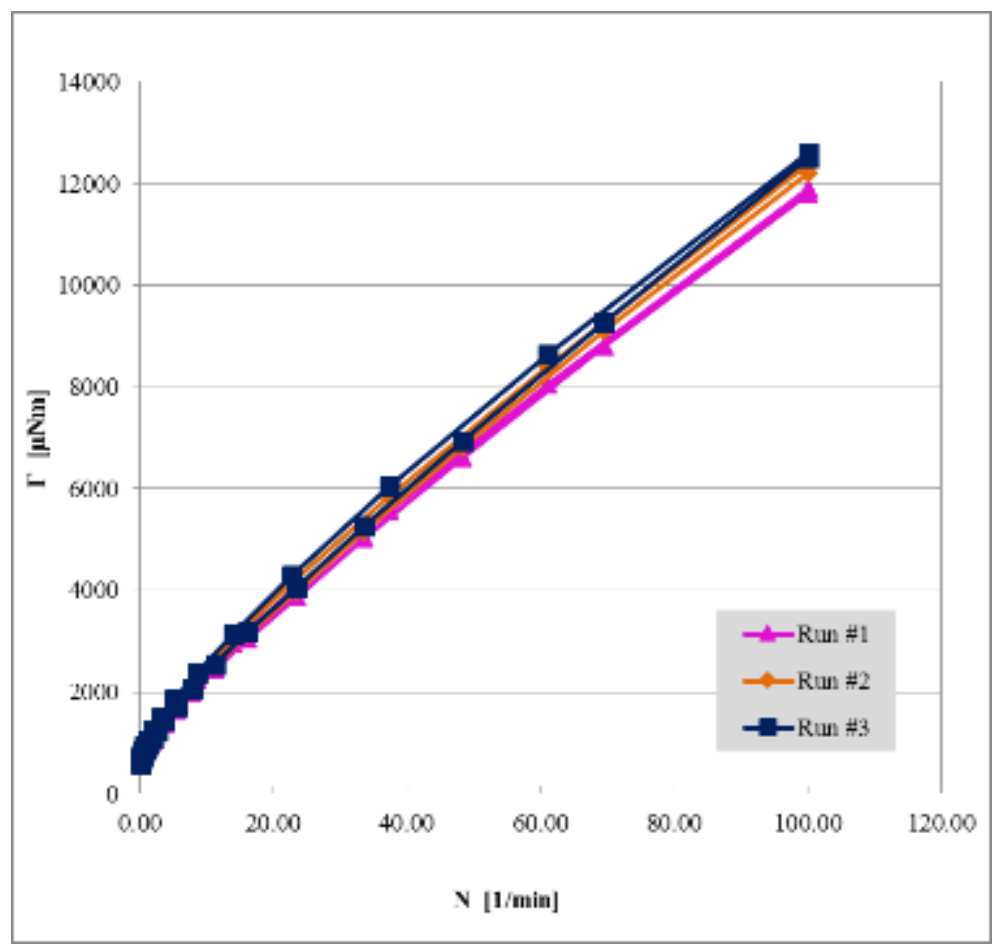

Figure A - 30: Torque vs. Angular Speed using Serrated Coaxial Cylinder on Mix\#8, with $20 \%$ beads by volume. Portrays data from Table A-42. 
Table A - 43: Measured data for Mix\#9 with 0 \% beads using Double spiral spindle.

\begin{tabular}{|c|c|c|c|c|c|c|c|c|}
\hline \multicolumn{6}{|c|}{ Double Spiral } & \multirow{2}{*}{\multicolumn{3}{|c|}{$\begin{array}{c}\text { Bead } \% \\
0 \%\end{array}$}} \\
\hline Geom.: & \multicolumn{3}{|l|}{$R H N-83 A$} & \multicolumn{2}{|l|}{ Cup: } & & & \\
\hline \multicolumn{2}{|c|}{ Run \#1 } & \multicolumn{2}{|c|}{ Run \#2 } & \multicolumn{2}{|c|}{ Run \#3 } & \multirow{2}{*}{\multicolumn{3}{|c|}{$\begin{array}{c}\text { Average } \\
\text { values }\end{array}$}} \\
\hline NIST Code: & SMC-109D & NIST Code: & $S M C-109 E$ & NIST Code: & SMC-109F & & & \\
\hline $\mathrm{N}$ & $\Gamma$ & $\mathrm{N}$ & $\Gamma$ & $\mathrm{N}$ & $\Gamma$ & $\mathbf{N}$ & $\Gamma$ & $\Gamma / \boldsymbol{N}$ (Angular \\
\hline $1 / \mathrm{min}$ & $\mu \mathrm{Nm}$ & $1 / \mathrm{min}$ & $\mu \mathrm{Nm}$ & $1 / \mathrm{min}$ & $\mu \mathrm{Nm}$ & $\mathrm{rad} / \mathrm{s}$ & $\mathrm{Nm}$ & momentum) \\
\hline 0.0941 & 1060 & 0.0946 & 983 & 0.0944 & 998 & 0.010 & $1.01 E-03$ & $1.03 E-01$ \\
\hline 0.161 & 1560 & 0.161 & 1460 & 0.161 & 1480 & 0.017 & $1.50 E-03$ & 8.90E-02 \\
\hline 0.268 & 1770 & 0.268 & 1660 & 0.268 & 1690 & 0.028 & $1.71 E-03$ & $6.08 E-02$ \\
\hline 0.439 & 1880 & 0.44 & 1790 & 0.439 & 1810 & 0.046 & $1.83 E-03$ & $3.97 E-02$ \\
\hline 0.72 & 2000 & 0.72 & 1920 & 0.72 & 1950 & 0.075 & $1.96 E-03$ & $2.60 E-02$ \\
\hline 1.18 & 2170 & 1.18 & 2110 & 1.18 & 2140 & 0.124 & $2.14 E-03$ & $1.73 E-02$ \\
\hline 1.93 & 2430 & 1.93 & 2380 & 1.93 & 2420 & 0.202 & $2.41 E-03$ & $1.19 E-02$ \\
\hline 3.16 & 2840 & 3.16 & 2810 & 3.16 & 2850 & 0.331 & $2.83 E-03$ & 8.56E-03 \\
\hline 5.18 & 3470 & 5.18 & 3460 & 5.18 & 3510 & 0.542 & $3.48 E-03$ & $6.42 E-03$ \\
\hline 8.48 & 4460 & 8.48 & 4470 & 8.48 & 4540 & 0.888 & $4.49 E-03$ & $5.06 E-03$ \\
\hline 13.9 & 6050 & 13.9 & 6090 & 13.9 & 6210 & 1.456 & $6.12 E-03$ & $4.20 E-03$ \\
\hline 22.8 & 8540 & 22.8 & 8580 & 22.8 & 8730 & 2.388 & $8.62 E-03$ & $3.61 E-03$ \\
\hline 37.3 & 12500 & 37.3 & 12500 & 37.3 & 12800 & 3.906 & $1.26 E-02$ & $3.23 E-03$ \\
\hline 61.1 & 18600 & 61.1 & 18700 & 61.1 & 19100 & 6.398 & $1.88 E-02$ & $2.94 E-03$ \\
\hline 100 & 28100 & 100 & 28400 & 100 & 28900 & 10.472 & $2.85 E-02$ & $2.72 E-03$ \\
\hline 100 & 27800 & 100 & 28200 & 100 & 28800 & 10.472 & $2.83 E-02$ & $2.70 E-03$ \\
\hline 69.5 & 20300 & 69.5 & 20600 & 69.5 & 21100 & 7.278 & $2.07 E-02$ & $2.84 E-03$ \\
\hline 48.3 & 15100 & 48.3 & 15300 & 48.3 & 15600 & 5.058 & $1.53 E-02$ & $3.03 E-03$ \\
\hline 33.6 & 11300 & 33.6 & 11400 & 33.6 & 11700 & 3.519 & $1.15 E-02$ & $3.26 E-03$ \\
\hline 23.4 & 8570 & 23.4 & 8690 & 23.4 & 8860 & 2.450 & 8.71E-03 & $3.55 E-03$ \\
\hline 16.2 & 6640 & 16.2 & 6730 & 16.2 & 6860 & 1.696 & $6.74 E-03$ & $3.97 E-03$ \\
\hline 11.3 & 5250 & 11.3 & 5320 & 11.3 & 5410 & 1.183 & $5.33 E-03$ & $4.50 E-03$ \\
\hline 7.85 & 4240 & 7.85 & 4300 & 7.85 & 4380 & 0.822 & $4.31 E-03$ & $5.24 E-03$ \\
\hline 5.46 & 3520 & 5.46 & 3560 & 5.46 & 3620 & 0.572 & $3.57 E-03$ & $6.24 E-03$ \\
\hline 3.79 & 2990 & 3.79 & 3030 & 3.79 & 3070 & 0.397 & $3.03 E-03$ & $7.63 E-03$ \\
\hline 2.64 & 2600 & 2.64 & 2630 & 2.64 & 2670 & 0.276 & $2.63 E-03$ & $9.53 E-03$ \\
\hline 1.83 & 2320 & 1.83 & 2340 & 1.83 & 2370 & 0.192 & $2.34 E-03$ & $1.22 E-02$ \\
\hline 1.27 & 2100 & 1.27 & 2130 & 1.27 & 2150 & 0.133 & $2.13 E-03$ & $1.60 E-02$ \\
\hline 0.886 & 1940 & 0.886 & 1960 & 0.886 & 1980 & 0.093 & $1.96 E-03$ & $2.11 E-02$ \\
\hline 0.616 & 1820 & 0.616 & 1830 & 0.616 & 1860 & 0.065 & $1.84 E-03$ & $2.85 E-02$ \\
\hline 0.428 & 1720 & 0.427 & 1740 & 0.429 & 1780 & 0.045 & $1.75 E-03$ & $3.90 E-02$ \\
\hline 0.298 & 1660 & 0.297 & 1670 & 0.297 & 1690 & 0.031 & $1.67 E-03$ & $5.37 E-02$ \\
\hline 0.207 & 1600 & 0.21 & 1620 & 0.206 & 1630 & 0.022 & $1.62 E-03$ & $7.43 E-02$ \\
\hline 0.144 & 1560 & 0.146 & 1580 & 0.145 & 1590 & 0.015 & $1.58 E-03$ & $1.04 E-01$ \\
\hline 0.0993 & 1520 & 0.1 & 1540 & 0.099 & 1570 & 0.010 & $1.54 E-03$ & $1.48 E-01$ \\
\hline
\end{tabular}


Table A - 44: Measured data for Mix\#9 with $20 \%$ beads using Double spiral spindle.

\begin{tabular}{|c|c|c|c|c|c|c|c|c|}
\hline \multicolumn{6}{|c|}{ Double Spiral } & \multirow{2}{*}{\multicolumn{3}{|c|}{$\begin{array}{c}\text { Bead } \% \\
20 \%\end{array}$}} \\
\hline Geom.: & \multicolumn{3}{|l|}{$R H N-83 A$} & \multicolumn{2}{|c|}{ Cup: } & & & \\
\hline \multicolumn{2}{|c|}{ Run \#1 } & \multicolumn{2}{|c|}{ Run \#2 } & \multicolumn{2}{|c|}{ Run \#3 } & \multirow{2}{*}{\multicolumn{3}{|c|}{$\begin{array}{c}\text { Average } \\
\text { values }\end{array}$}} \\
\hline NIST Code: & SMC-109M & NIST Code: & SMC-109N & NIST Code & AC-109O & & & \\
\hline $\mathrm{N}$ & $\Gamma$ & $\mathrm{N}$ & $\Gamma$ & $\mathrm{N}$ & $\Gamma$ & $\mathbf{N}$ & $\Gamma$ & $\Gamma / N$ (Angular \\
\hline $1 / \mathrm{min}$ & $\mu \mathrm{Nm}$ & $1 / \mathrm{min}$ & $\mu \mathrm{Nm}$ & $1 / \mathrm{min}$ & $\mu \mathrm{Nm}$ & $\mathrm{rad} / \mathrm{s}$ & $\mathrm{Nm}$ & momentum) \\
\hline 0.0925 & 1420 & 0.0937 & 1300 & 0.0935 & 1320 & 0.010 & $1.35 E-03$ & $1.38 E-01$ \\
\hline 0.161 & 2010 & 0.162 & 1860 & 0.162 & 1870 & 0.017 & $1.91 E-03$ & $1.13 E-01$ \\
\hline 0.269 & 2260 & 0.269 & 2100 & 0.268 & 2120 & 0.028 & $2.16 E-03$ & $7.68 E-02$ \\
\hline 0.438 & 2440 & 0.44 & 2280 & 0.438 & 2300 & 0.046 & $2.34 E-03$ & 5.09E-02 \\
\hline 0.72 & 2670 & 0.718 & 2520 & 0.721 & 2560 & 0.075 & $2.58 E-03$ & $3.43 E-02$ \\
\hline 1.18 & 2990 & 1.18 & 2880 & 1.18 & 2910 & 0.124 & $2.93 E-03$ & $2.37 E-02$ \\
\hline 1.93 & 3530 & 1.93 & 3400 & 1.93 & 3430 & 0.202 & $3.45 E-03$ & $1.71 E-02$ \\
\hline 3.16 & 4360 & 3.16 & 4200 & 3.16 & 4220 & 0.331 & $4.26 E-03$ & $1.29 E-02$ \\
\hline 5.18 & 5660 & 5.19 & 5410 & 5.18 & 5430 & 0.543 & $5.50 E-03$ & $1.01 E-02$ \\
\hline 8.49 & 7650 & 8.49 & 7250 & 8.49 & 7270 & 0.889 & 7.39E-03 & 8.31E-03 \\
\hline 13.9 & 10500 & 13.9 & 10100 & 13.9 & 10200 & 1.456 & $1.03 E-02$ & $7.05 E-03$ \\
\hline 22.7 & 14700 & 22.8 & 14400 & 22.8 & 14500 & 2.384 & $1.45 E-02$ & $6.10 E-03$ \\
\hline 37.3 & 21900 & 37.3 & 21400 & 37.3 & 21600 & 3.906 & $2.16 E-02$ & $5.54 E-03$ \\
\hline 61.1 & 33000 & 61.1 & 32400 & 61 & 32700 & 6.395 & 3.27E-02 & $5.11 E-03$ \\
\hline 100 & 49900 & 100 & 49300 & 100 & 49800 & 10.472 & $4.97 E-02$ & $4.74 E-03$ \\
\hline 100 & 49200 & 100 & 49000 & 100 & 49500 & 10.472 & $4.92 E-02$ & $4.70 E-03$ \\
\hline 69.5 & 35600 & 69.5 & 35500 & 69.5 & 36000 & 7.278 & $3.57 E-02$ & $4.91 E-03$ \\
\hline 48.3 & 26000 & 48.3 & 26100 & 48.3 & 26400 & 5.058 & $2.62 E-02$ & $5.17 E-03$ \\
\hline 33.6 & 19300 & 33.6 & 19300 & 33.6 & 19600 & 3.519 & $1.94 E-02$ & $5.51 E-03$ \\
\hline 23.4 & 14500 & 23.4 & 14500 & 23.4 & 14700 & 2.450 & $1.46 E-02$ & $5.94 E-03$ \\
\hline 16.2 & 11000 & 16.2 & 11000 & 16.2 & 11200 & 1.696 & $1.11 E-02$ & $6.52 E-03$ \\
\hline 11.3 & 8520 & 11.3 & 8550 & 11.3 & 8680 & 1.183 & $8.58 E-03$ & $7.25 E-03$ \\
\hline 7.84 & 6730 & 7.85 & 6750 & 7.85 & 6870 & 0.822 & $6.78 E-03$ & $8.26 E-03$ \\
\hline 5.45 & 5440 & 5.46 & 5470 & 5.46 & 5560 & 0.571 & $5.49 E-03$ & $9.61 E-03$ \\
\hline 3.79 & 4500 & 3.79 & 4540 & 3.79 & 4600 & 0.397 & $4.55 E-03$ & $1.15 E-02$ \\
\hline 2.63 & 3810 & 2.63 & 3840 & 2.63 & 3920 & 0.275 & $3.86 E-03$ & $1.40 E-02$ \\
\hline 1.83 & 3310 & 1.83 & 3340 & 1.83 & 3390 & 0.192 & $3.35 E-03$ & $1.75 E-02$ \\
\hline 1.27 & 2930 & 1.27 & 2960 & 1.27 & 3010 & 0.133 & $2.97 E-03$ & $2.23 E-02$ \\
\hline 0.887 & 2640 & 0.885 & 2670 & 0.882 & 2740 & 0.093 & $2.68 E-03$ & $2.90 E-02$ \\
\hline 0.617 & 2440 & 0.614 & 2480 & 0.617 & 2490 & 0.065 & $2.47 E-03$ & $3.83 E-02$ \\
\hline 0.429 & 2280 & 0.429 & 2300 & 0.429 & 2320 & 0.045 & $2.30 E-03$ & $5.12 E-02$ \\
\hline 0.298 & 2150 & 0.298 & 2170 & 0.294 & 2220 & 0.031 & $2.18 E-03$ & $7.02 E-02$ \\
\hline 0.207 & 2040 & 0.208 & 2060 & 0.204 & 2150 & 0.022 & $2.08 E-03$ & $9.64 E-02$ \\
\hline 0.144 & 1970 & 0.144 & 2010 & 0.144 & 2020 & 0.015 & $2.00 E-03$ & $1.33 E-01$ \\
\hline 0.0997 & 1920 & 0.1 & 1950 & 0.0966 & 1980 & 0.010 & $1.95 E-03$ & $1.89 E-01$ \\
\hline
\end{tabular}




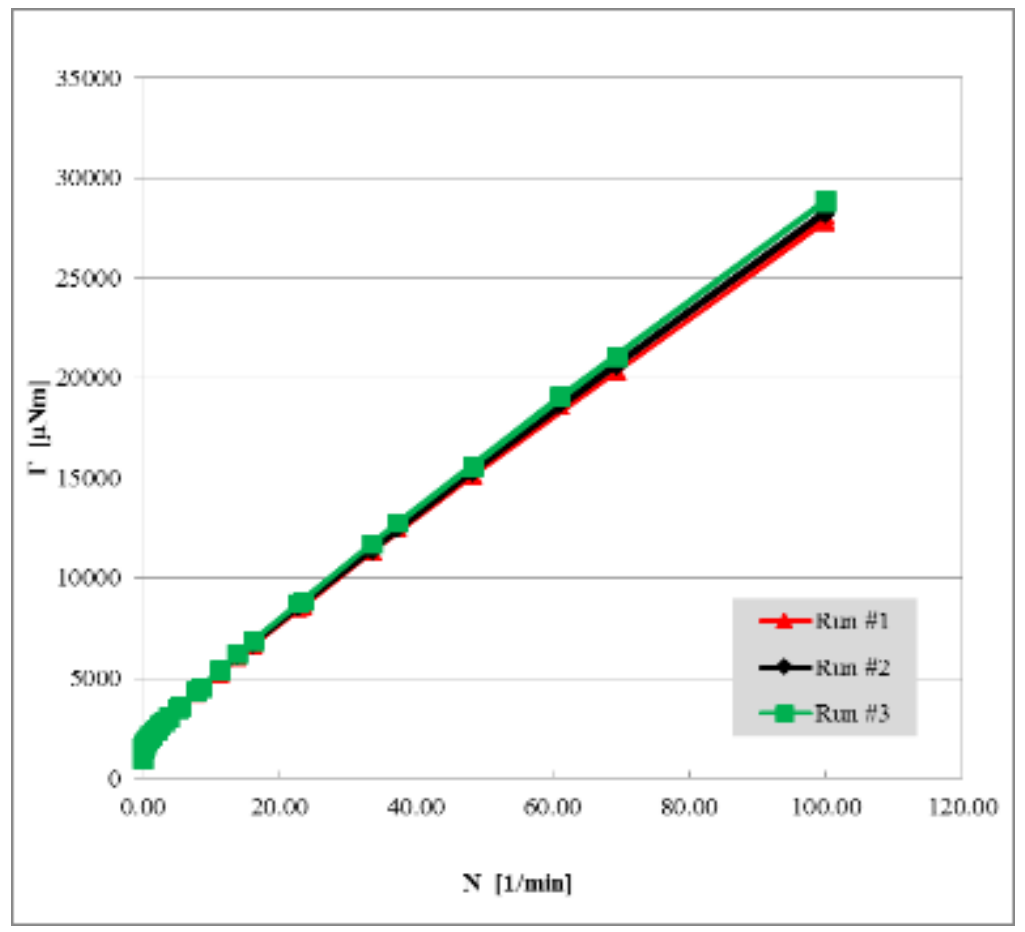

Figure A - 31: Torque vs. Angular Speed using Double spiral spindle on Mix\#9, with $0 \%$ beads by volume. Portrays data from Table A-43.

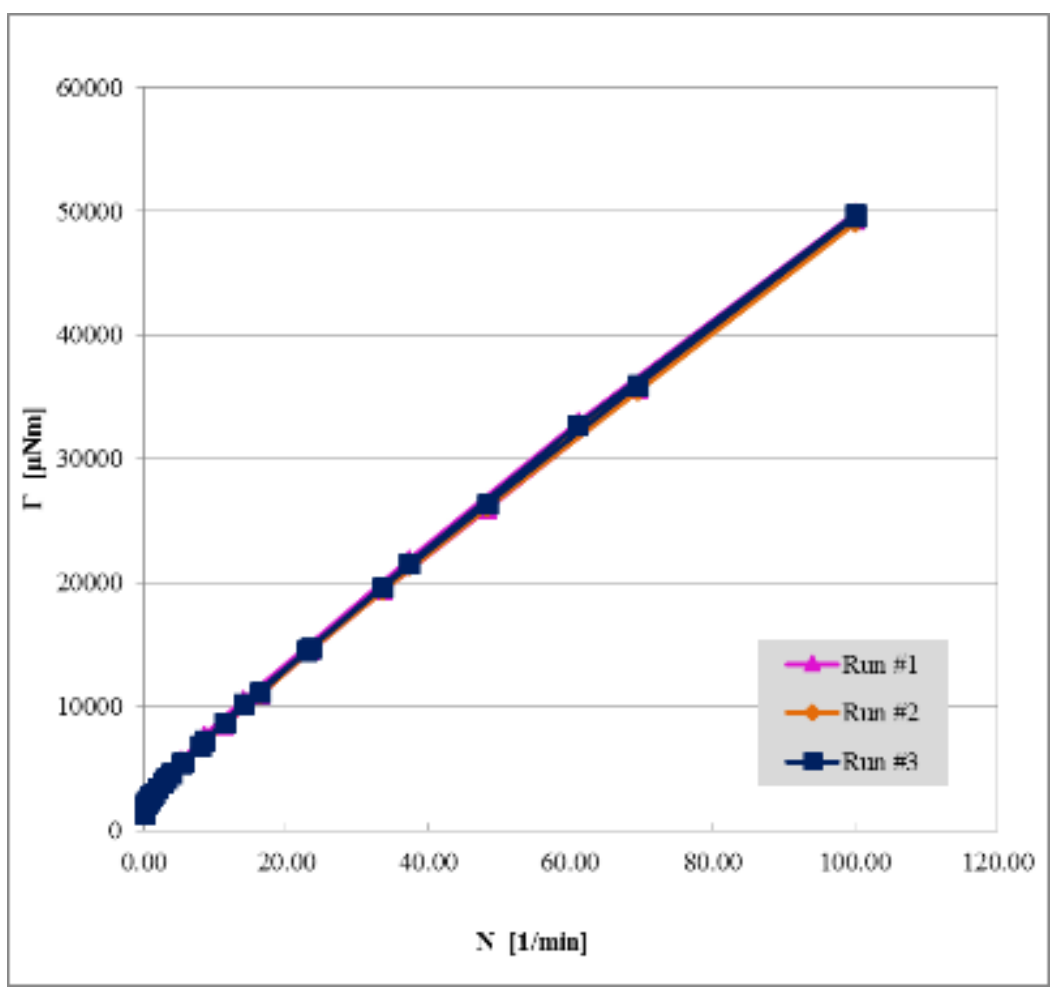

Figure A - 32: Torque vs. Angular Speed using Double spiral spindle on Mix\#9, with $20 \%$ beads by volume. Portrays data from Table A-44. 
Table A - 45: Measured data for Mix\#9 with 0 \% beads using Six-blade Vane.

\begin{tabular}{|c|c|c|c|c|c|c|c|c|}
\hline \multicolumn{6}{|c|}{6 Blade Vane } & \multirow{2}{*}{\multicolumn{3}{|c|}{$\begin{array}{c}\text { Bead } \% \\
0 \%\end{array}$}} \\
\hline Geom.: & $R H N-83$ & & & \multicolumn{2}{|c|}{ Z43S } & & & \\
\hline \multicolumn{2}{|c|}{ Run \#1 } & \multicolumn{2}{|c|}{ Run \#2 } & \multicolumn{2}{|c|}{ Run \#3 } & \multirow{2}{*}{\multicolumn{3}{|c|}{$\begin{array}{c}\text { Average } \\
\text { values }\end{array}$}} \\
\hline \multicolumn{2}{|c|}{ NIST Code: SMC-109A } & \multicolumn{2}{|c|}{ NIST Code: SMC-109B } & \multicolumn{2}{|c|}{ NIST Code: SMC-109C } & & & \\
\hline $\mathrm{N}$ & $\Gamma$ & $\mathrm{N}$ & $\Gamma$ & $\mathrm{N}$ & $\Gamma$ & $\mathbf{N}$ & $\Gamma$ & $\boldsymbol{\Gamma} / \mathbf{N}$ (Angular \\
\hline $1 / \mathrm{min}$ & $\mu \mathrm{Nm}$ & $1 / \mathrm{min}$ & $\mu \mathrm{Nm}$ & $1 / \mathrm{min}$ & $\mu \mathrm{Nm}$ & $\mathrm{rad} / \mathrm{s}$ & $\mathrm{Nm}$ & momentum) \\
\hline 0.0978 & 440 & 0.0976 & 409 & 0.0977 & 419 & 0.010 & 4.23E-04 & $4.13 E-02$ \\
\hline 0.163 & 584 & 0.163 & 563 & 0.163 & 575 & 0.017 & $5.74 E-04$ & 3.36E-02 \\
\hline 0.268 & 629 & 0.268 & 609 & 0.268 & 620 & 0.028 & $6.19 E-04$ & $2.21 E-02$ \\
\hline 0.439 & 665 & 0.439 & 643 & 0.439 & 655 & 0.046 & $6.54 E-04$ & $1.42 E-02$ \\
\hline 0.72 & 717 & 0.72 & 695 & 0.72 & 707 & 0.075 & $7.06 E-04$ & $9.37 E-03$ \\
\hline 1.18 & 791 & 1.18 & 767 & 1.18 & 781 & 0.124 & $7.80 E-04$ & $6.31 E-03$ \\
\hline 1.93 & 891 & 1.93 & 868 & 1.93 & 886 & 0.202 & 8.82E-04 & $4.36 E-03$ \\
\hline 3.16 & 1030 & 3.16 & 1020 & 3.16 & 1030 & 0.331 & $1.03 E-03$ & $3.10 E-03$ \\
\hline 5.18 & 1240 & 5.18 & 1220 & 5.18 & 1240 & 0.542 & $1.23 E-03$ & $2.27 E-03$ \\
\hline 8.48 & 1540 & 8.48 & 1510 & 8.48 & 1540 & 0.888 & $1.53 E-03$ & $1.72 E-03$ \\
\hline 13.9 & 1980 & 13.9 & 1950 & 13.9 & 1980 & 1.456 & $1.97 E-03$ & $1.35 E-03$ \\
\hline 22.8 & 2610 & 22.8 & 2580 & 22.8 & 2620 & 2.388 & $2.60 E-03$ & $1.09 E-03$ \\
\hline 37.3 & 3580 & 37.3 & 3560 & 37.3 & 3610 & 3.906 & $3.58 E-03$ & $9.17 E-04$ \\
\hline 61 & 5060 & 61 & 5070 & 61 & 5140 & 6.388 & $5.09 E-03$ & $7.97 E-04$ \\
\hline 100 & 7370 & 100 & 7410 & 100 & 7520 & 10.472 & 7.43E-03 & 7.10E-04 \\
\hline 100 & 7320 & 100 & 7390 & 100 & 7510 & 10.472 & $7.41 E-03$ & 7.07E-04 \\
\hline 69.5 & 5490 & 69.5 & 5540 & 69.5 & 5620 & 7.278 & $5.55 E-03$ & 7.63E-04 \\
\hline 48.3 & 4180 & 48.3 & 4220 & 48.3 & 4270 & 5.058 & $4.22 E-03$ & 8.35E-04 \\
\hline 33.6 & 3240 & 33.6 & 3260 & 33.6 & 3300 & 3.519 & $3.27 E-03$ & $9.28 E-04$ \\
\hline 23.4 & 2560 & 23.4 & 2570 & 23.4 & 2600 & 2.450 & $2.58 E-03$ & $1.05 E-03$ \\
\hline 16.2 & 2060 & 16.2 & 2070 & 16.2 & 2090 & 1.696 & $2.07 E-03$ & $1.22 E-03$ \\
\hline 11.3 & 1690 & 11.3 & 1700 & 11.3 & 1710 & 1.183 & $1.70 E-03$ & $1.44 E-03$ \\
\hline 7.85 & 1410 & 7.85 & 1420 & 7.85 & 1440 & 0.822 & $1.42 E-03$ & $1.73 E-03$ \\
\hline 5.46 & 1200 & 5.45 & 1210 & 5.45 & 1220 & 0.571 & $1.21 E-03$ & $2.12 E-03$ \\
\hline 3.79 & 1040 & 3.79 & 1050 & 3.79 & 1060 & 0.397 & $1.05 E-03$ & $2.65 E-03$ \\
\hline 2.64 & 920 & 2.64 & 924 & 2.64 & 930 & 0.276 & $9.25 E-04$ & $3.34 E-03$ \\
\hline 1.83 & 824 & 1.83 & 832 & 1.83 & 833 & 0.192 & 8.30E-04 & 4.33E-03 \\
\hline 1.27 & 767 & 1.27 & 752 & 1.27 & 760 & 0.133 & $7.60 E-04$ & $5.71 E-03$ \\
\hline 0.887 & 706 & 0.886 & 700 & 0.886 & 709 & 0.093 & $7.05 E-04$ & $7.60 E-03$ \\
\hline 0.615 & 645 & 0.616 & 650 & 0.614 & 667 & 0.064 & $6.54 E-04$ & $1.02 E-02$ \\
\hline 0.429 & 626 & 0.428 & 616 & 0.429 & 615 & 0.045 & $6.19 E-04$ & $1.38 E-02$ \\
\hline 0.297 & 578 & 0.296 & 596 & 0.299 & 597 & 0.031 & $5.90 E-04$ & $1.90 E-02$ \\
\hline 0.208 & 563 & 0.203 & 587 & 0.205 & 582 & 0.022 & 5.77E-04 & $2.68 E-02$ \\
\hline 0.144 & 551 & 0.145 & 551 & 0.145 & 545 & 0.015 & $5.49 E-04$ & $3.62 E-02$ \\
\hline 0.0991 & 537 & 0.0985 & 550 & 0.101 & 559 & 0.010 & $5.49 E-04$ & $5.26 E-02$ \\
\hline
\end{tabular}


Table A - 46: Measured data for Mix\#9 with 20 \% beads using Six-blade Vane.

\begin{tabular}{|c|c|c|c|c|c|c|c|c|}
\hline \multicolumn{6}{|c|}{6 Blade Vane } & \multicolumn{3}{|c|}{$\begin{array}{c}\text { Bead } \% \\
20 \%\end{array}$} \\
\hline Run & & RuI & & & & \multirow{2}{*}{\multicolumn{3}{|c|}{$\begin{array}{c}\text { Average } \\
\text { values }\end{array}$}} \\
\hline NIST Code: & $S M C-109 \mathrm{~J}$ & NIST Code: & SMC-109K & NIST Code & AC-109L & & & \\
\hline $\mathrm{N}$ & $\Gamma$ & $\mathrm{N}$ & $\Gamma$ & $\mathrm{N}$ & $\Gamma$ & $\mathbf{N}$ & $\Gamma$ & $\boldsymbol{\Gamma} / \boldsymbol{N}$ (Angular \\
\hline $1 / \mathrm{min}$ & $\mu \mathrm{Nm}$ & $1 / \mathrm{min}$ & $\mu \mathrm{Nm}$ & $1 / \mathrm{min}$ & $\mu \mathrm{Nm}$ & $\mathrm{rad} / \mathrm{s}$ & $\mathrm{Nm}$ & momentum) \\
\hline 0.0973 & 615 & 0.0973 & 614 & 0.0973 & 596 & 0.010 & $6.08 E-04$ & 5.97E-02 \\
\hline 0.163 & 776 & 0.163 & 793 & 0.163 & 758 & 0.017 & $7.76 E-04$ & $4.54 E-02$ \\
\hline 0.268 & 825 & 0.268 & 842 & 0.269 & 818 & 0.028 & $8.28 E-04$ & $2.95 E-02$ \\
\hline 0.44 & 897 & 0.439 & 914 & 0.44 & 886 & 0.046 & 8.99E-04 & $1.95 E-02$ \\
\hline 0.72 & 966 & 0.721 & 987 & 0.72 & 968 & 0.075 & $9.74 E-04$ & $1.29 E-02$ \\
\hline 1.18 & 1080 & 1.18 & 1110 & 1.18 & 1090 & 0.124 & $1.09 E-03$ & $8.85 E-03$ \\
\hline 1.93 & 1240 & 1.93 & 1280 & 1.93 & 1260 & 0.202 & $1.26 E-03$ & $6.23 E-03$ \\
\hline 3.16 & 1470 & 3.16 & 1540 & 3.16 & 1530 & 0.331 & $1.51 E-03$ & 4.57E-03 \\
\hline 5.18 & 1820 & 5.18 & 1910 & 5.18 & 1890 & 0.542 & $1.87 E-03$ & $3.45 E-03$ \\
\hline 8.48 & 2300 & 8.49 & 2430 & 8.48 & 2440 & 0.888 & 2.39E-03 & $2.69 E-03$ \\
\hline 13.9 & 3070 & 13.9 & 3240 & 13.9 & 3250 & 1.456 & $3.19 E-03$ & $2.19 E-03$ \\
\hline 22.8 & 4230 & 22.8 & 4430 & 22.8 & 4490 & 2.388 & $4.38 E-03$ & $1.84 E-03$ \\
\hline 37.3 & 5910 & 37.3 & 6200 & 37.3 & 6360 & 3.906 & $6.16 E-03$ & $1.58 E-03$ \\
\hline 61 & 8350 & 61 & 8900 & 61.1 & 9210 & 6.391 & $8.82 E-03$ & $1.38 E-03$ \\
\hline 100 & 11900 & 100 & 12900 & 100 & 13500 & 10.472 & $1.28 E-02$ & $1.22 E-03$ \\
\hline 100 & 11600 & 100 & 12800 & 100 & 13400 & 10.472 & $1.26 E-02$ & $1.20 E-03$ \\
\hline 69.5 & 8700 & 69.5 & 9490 & 69.5 & 9890 & 7.278 & $9.36 E-03$ & $1.29 E-03$ \\
\hline 48.3 & 6600 & 48.3 & 7110 & 48.3 & 7420 & 5.058 & $7.04 E-03$ & $1.39 E-03$ \\
\hline 33.6 & 5100 & 33.6 & 5430 & 33.6 & 5650 & 3.519 & $5.39 E-03$ & $1.53 E-03$ \\
\hline 23.4 & 4020 & 23.4 & 4220 & 23.4 & 4360 & 2.450 & $4.20 E-03$ & $1.71 E-03$ \\
\hline 16.2 & 3170 & 16.2 & 3340 & 16.2 & 3450 & 1.696 & $3.32 E-03$ & $1.96 E-03$ \\
\hline 11.3 & 2540 & 11.3 & 2660 & 11.3 & 2750 & 1.183 & $2.65 E-03$ & $2.24 E-03$ \\
\hline 7.85 & 2080 & 7.84 & 2180 & 7.85 & 2230 & 0.822 & $2.16 E-03$ & $2.63 E-03$ \\
\hline 5.45 & 1730 & 5.46 & 1810 & 5.46 & 1850 & 0.571 & $1.80 E-03$ & $3.14 E-03$ \\
\hline 3.79 & 1480 & 3.79 & 1540 & 3.79 & 1560 & 0.397 & $1.53 E-03$ & $3.85 E-03$ \\
\hline 2.64 & 1280 & 2.64 & 1330 & 2.64 & 1350 & 0.276 & $1.32 E-03$ & $4.77 E-03$ \\
\hline 1.83 & 1120 & 1.83 & 1170 & 1.83 & 1180 & 0.192 & $1.16 E-03$ & $6.04 E-03$ \\
\hline 1.28 & 1010 & 1.27 & 1040 & 1.27 & 1070 & 0.133 & $1.04 E-03$ & $7.80 E-03$ \\
\hline 0.886 & 912 & 0.887 & 943 & 0.888 & 960 & 0.093 & $9.38 E-04$ & $1.01 E-02$ \\
\hline 0.614 & 867 & 0.615 & 870 & 0.615 & 901 & 0.064 & $8.79 E-04$ & $1.37 E-02$ \\
\hline 0.428 & 784 & 0.43 & 817 & 0.43 & 838 & 0.045 & 8.13E-04 & $1.81 E-02$ \\
\hline 0.296 & 755 & 0.296 & 782 & 0.296 & 792 & 0.031 & $7.76 E-04$ & $2.50 E-02$ \\
\hline 0.208 & 738 & 0.209 & 747 & 0.205 & 735 & 0.022 & $7.40 E-04$ & $3.41 E-02$ \\
\hline 0.143 & 689 & 0.144 & 710 & 0.142 & 721 & 0.015 & 7.07E-04 & $4.72 E-02$ \\
\hline 0.099 & 682 & 0.103 & 701 & 0.101 & 695 & 0.011 & 6.93E-04 & $6.55 E-02$ \\
\hline
\end{tabular}




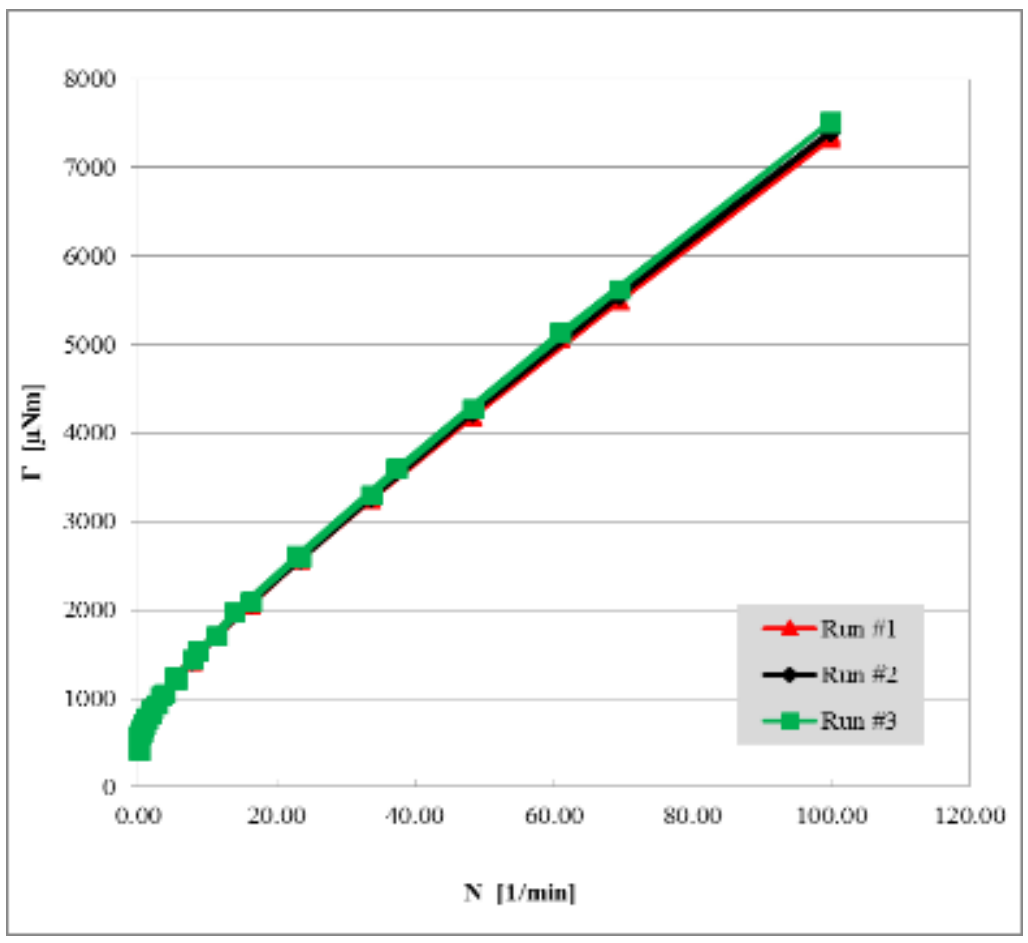

Figure A - 45: Torque vs. Angular Speed using six-blade vane on Mix\#9, with $0 \%$ beads by volume. Portrays data from Table A-45.

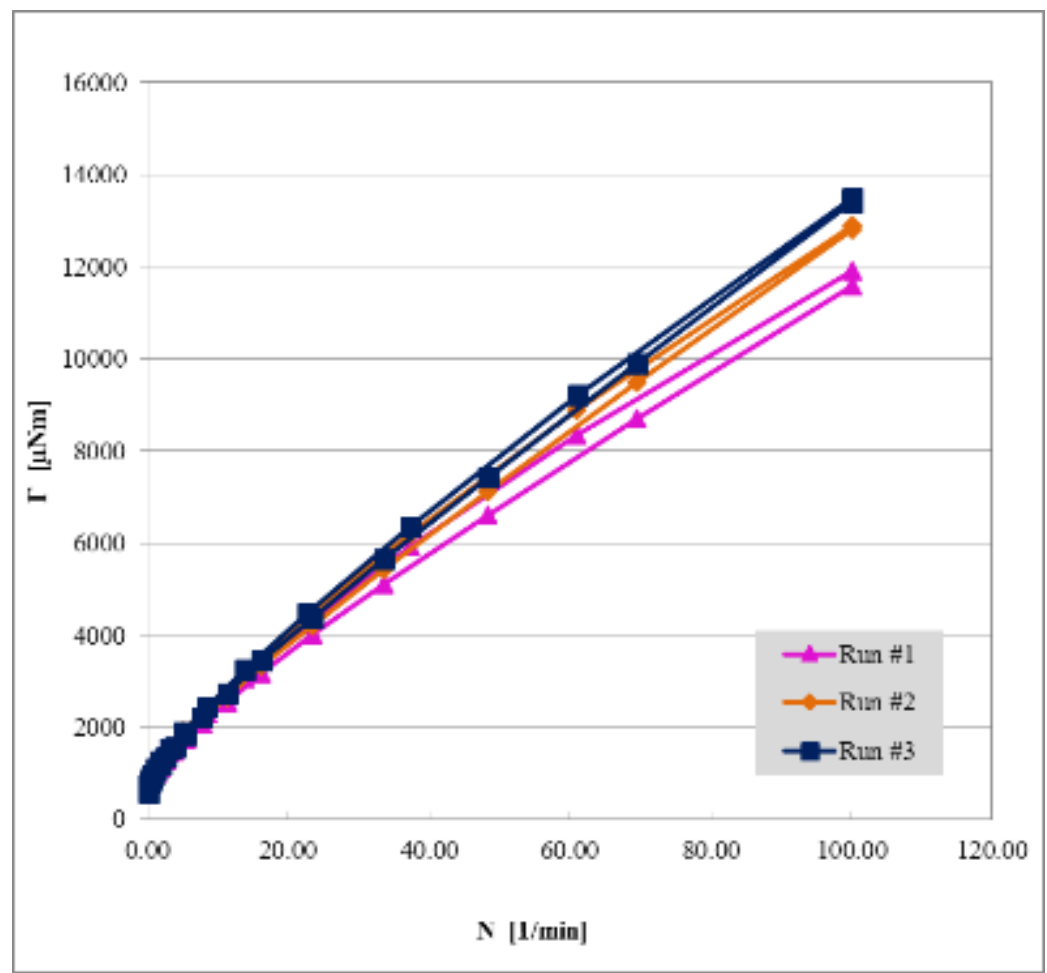

Figure A - 46: Torque vs. Angular Speed using six-blade vane on Mix\#9, with $20 \%$ beads by volume. Portrays data from Table A-46. 
Table A - 47: Measured data for Mix\#9 with 0 \% beads using Serrated Coaxial Cylinder.

\begin{tabular}{|c|c|c|c|c|c|c|c|c|}
\hline \multicolumn{6}{|c|}{ Serrated Coaxial Cylinder } & \multirow{2}{*}{\multicolumn{3}{|c|}{$\begin{array}{c}\text { Bead } \% \\
0 \%\end{array}$}} \\
\hline \multicolumn{2}{|c|}{ Geom.: } & & & \multicolumn{2}{|c|}{ Z43S } & & & \\
\hline Rur & \#1 & & \#2 & & & \multirow{2}{*}{\multicolumn{3}{|c|}{$\begin{array}{c}\text { Average } \\
\text { values }\end{array}$}} \\
\hline NIST Code: & $S M C-109 G$ & NIST Code & $S M C-109 H$ & NIST Cod & $S M C-109 I$ & & & \\
\hline $\mathrm{N}$ & $\Gamma$ & $\mathrm{N}$ & $\Gamma$ & $\mathrm{N}$ & $\Gamma$ & $\mathbf{N}$ & $\Gamma$ & $\Gamma / N$ (Angular \\
\hline $1 / \mathrm{min}$ & $\mu \mathrm{Nm}$ & $1 / \mathrm{min}$ & $\mu \mathrm{Nm}$ & $1 / \mathrm{min}$ & $\mu \mathrm{Nm}$ & $\mathrm{rad} / \mathrm{s}$ & Nm & momentum) \\
\hline 0.0979 & 480 & 0.0977 & 436 & 0.098 & 461 & 0.010 & $4.59 E-04$ & $4.48 E-02$ \\
\hline 0.164 & 629 & 0.164 & 591 & 0.164 & 612 & 0.017 & $6.11 E-04$ & $3.56 E-02$ \\
\hline 0.268 & 671 & 0.268 & 634 & 0.268 & 657 & 0.028 & $6.54 E-04$ & $2.33 E-02$ \\
\hline 0.44 & 723 & 0.44 & 685 & 0.44 & 713 & 0.046 & $7.07 E-04$ & $1.53 E-02$ \\
\hline 0.72 & 789 & 0.72 & 759 & 0.72 & 792 & 0.075 & $7.80 E-04$ & $1.03 E-02$ \\
\hline 1.18 & 885 & 1.18 & 856 & 1.17 & 925 & 0.123 & 8.89E-04 & 7.21E-03 \\
\hline 1.93 & 1010 & 1.93 & 1040 & 1.93 & 1030 & 0.202 & $1.03 E-03$ & $5.08 E-03$ \\
\hline 3.16 & 1190 & 3.16 & 1170 & 3.16 & 1210 & 0.331 & 1.19E-03 & $3.60 E-03$ \\
\hline 5.18 & 1430 & 5.18 & 1420 & 5.18 & 1470 & 0.542 & $1.44 E-03$ & $2.65 E-03$ \\
\hline 8.48 & 1790 & 8.48 & 1790 & 8.48 & 1850 & 0.888 & $1.81 E-03$ & $2.04 E-03$ \\
\hline 13.9 & 2300 & 13.9 & 2300 & 13.9 & 2390 & 1.456 & 2.33E-03 & $1.60 E-03$ \\
\hline 22.8 & 3050 & 22.8 & 3080 & 22.8 & 3190 & 2.388 & $3.11 E-03$ & $1.30 E-03$ \\
\hline 37.3 & 4200 & 37.3 & 4260 & 37.3 & 4410 & 3.906 & $4.29 E-03$ & $1.10 E-03$ \\
\hline 61 & 5950 & 61 & 6060 & 61 & 6270 & 6.388 & $6.09 E-03$ & $9.54 E-04$ \\
\hline 100 & 8750 & 100 & 8800 & 100 & 9030 & 10.472 & $8.86 E-03$ & $8.46 E-04$ \\
\hline 100 & 8580 & 100 & 8700 & 100 & 8910 & 10.472 & $8.73 E-03$ & 8.34E-04 \\
\hline 69.5 & 6420 & 69.5 & 6520 & 69.5 & 6670 & 7.278 & $6.54 E-03$ & 8.98E-04 \\
\hline 48.3 & 4840 & 48.3 & 4910 & 48.3 & 5020 & 5.058 & $4.92 E-03$ & $9.73 E-04$ \\
\hline 33.6 & 3700 & 33.6 & 3750 & 33.6 & 3830 & 3.519 & $3.76 E-03$ & $1.07 E-03$ \\
\hline 23.4 & 2880 & 23.4 & 2910 & 23.4 & 2980 & 2.450 & $2.92 E-03$ & $1.19 E-03$ \\
\hline 16.2 & 2290 & 16.2 & 2310 & 16.2 & 2360 & 1.696 & $2.32 E-03$ & $1.37 E-03$ \\
\hline 11.3 & 1850 & 11.3 & 1870 & 11.3 & 1910 & 1.183 & $1.88 E-03$ & $1.59 E-03$ \\
\hline 7.85 & 1520 & 7.85 & 1540 & 7.85 & 1570 & 0.822 & $1.54 E-03$ & $1.88 E-03$ \\
\hline 5.45 & 1270 & 5.45 & 1290 & 5.45 & 1320 & 0.571 & $1.29 E-03$ & $2.27 E-03$ \\
\hline 3.79 & 1080 & 3.79 & 1100 & 3.79 & 1130 & 0.397 & $1.10 E-03$ & $2.78 E-03$ \\
\hline 2.64 & 924 & 2.64 & 968 & 2.64 & 977 & 0.276 & $9.56 E-04$ & $3.46 E-03$ \\
\hline 1.83 & 804 & 1.83 & 872 & 1.83 & 874 & 0.192 & $8.50 E-04$ & $4.44 E-03$ \\
\hline 1.28 & 719 & 1.27 & 755 & 1.27 & 750 & 0.133 & $7.41 E-04$ & $5.56 E-03$ \\
\hline 0.885 & 634 & 0.889 & 659 & 0.885 & 690 & 0.093 & $6.61 E-04$ & $7.12 E-03$ \\
\hline 0.616 & 590 & 0.614 & 611 & 0.619 & 641 & 0.065 & $6.14 E-04$ & $9.51 E-03$ \\
\hline 0.422 & 579 & 0.422 & 575 & 0.422 & 604 & 0.044 & $5.86 E-04$ & $1.33 E-02$ \\
\hline 0.299 & 497 & 0.298 & 548 & 0.304 & 569 & 0.031 & $5.38 E-04$ & $1.71 E-02$ \\
\hline 0.211 & 525 & 0.209 & 545 & 0.207 & 629 & 0.022 & $5.66 E-04$ & $2.59 E-02$ \\
\hline 0.149 & 523 & 0.143 & 574 & 0.142 & 605 & 0.015 & 5.67E-04 & $3.74 E-02$ \\
\hline 0.0952 & 493 & 0.11 & 537 & 0.0896 & 654 & 0.010 & 0.000561 & 0.054548899 \\
\hline
\end{tabular}


Table A - 48: Measured data for Mix\#9 with 20 \% beads using Serrated Coaxial Cylinder.

\begin{tabular}{|c|c|c|c|c|c|c|c|c|}
\hline \multicolumn{6}{|c|}{ Serrated Coaxial Cylinder } & \multirow{2}{*}{\multicolumn{3}{|c|}{$\begin{array}{c}\text { Bead } \% \\
20 \%\end{array}$}} \\
\hline Geom.: & SS18 & & & \multicolumn{2}{|c|}{ Z43S } & & & \\
\hline \multicolumn{2}{|c|}{ Run \#1 } & \multicolumn{2}{|c|}{ Run \#2 } & \multicolumn{2}{|c|}{ Run \#3 } & \multirow{2}{*}{\multicolumn{3}{|c|}{$\begin{array}{c}\text { Average } \\
\text { values }\end{array}$}} \\
\hline NIST Code: & SMC-109P & NIST Code & IC-109Q & NIST Cod & $C-109 R$ & & & \\
\hline $\mathrm{N}$ & $\Gamma$ & $\mathrm{N}$ & $\Gamma$ & $\mathrm{N}$ & $\Gamma$ & $\mathbf{N}$ & $\Gamma$ & $\Gamma / N$ (Angular \\
\hline $1 / \mathrm{min}$ & $\mu \mathrm{Nm}$ & $1 / \mathrm{min}$ & $\mu \mathrm{Nm}$ & $1 / \mathrm{min}$ & $\mu \mathrm{Nm}$ & $\mathrm{rad} / \mathrm{s}$ & $\mathrm{Nm}$ & momentum) \\
\hline 0.0975 & 719 & 0.0978 & 625 & 0.0972 & 681 & 0.010 & $6.75 E-04$ & $6.61 E-02$ \\
\hline 0.164 & 880 & 0.164 & 790 & 0.167 & 846 & 0.017 & 8.39E-04 & $4.85 E-02$ \\
\hline 0.269 & 885 & 0.268 & 851 & 0.266 & 930 & 0.028 & 8.89E-04 & $3.17 E-02$ \\
\hline 0.439 & 930 & 0.441 & 929 & 0.441 & 1000 & 0.046 & $9.53 E-04$ & $2.07 E-02$ \\
\hline 0.719 & 1020 & 0.721 & 1050 & 0.723 & 1090 & 0.076 & $1.05 E-03$ & $1.40 E-02$ \\
\hline 1.18 & 1150 & 1.18 & 1180 & 1.18 & 1260 & 0.124 & $1.20 E-03$ & $9.68 E-03$ \\
\hline 1.93 & 1340 & 1.93 & 1370 & 1.93 & 1480 & 0.202 & $1.40 E-03$ & $6.91 E-03$ \\
\hline 3.16 & 1610 & 3.16 & 1650 & 3.16 & 1760 & 0.331 & $1.67 E-03$ & $5.06 E-03$ \\
\hline 5.18 & 2000 & 5.18 & 2060 & 5.18 & 2200 & 0.542 & $2.09 E-03$ & $3.85 E-03$ \\
\hline 8.48 & 2540 & 8.48 & 2630 & 8.48 & 2830 & 0.888 & 2.67E-03 & $3.00 E-03$ \\
\hline 13.9 & 3340 & 13.9 & 3500 & 13.9 & 3750 & 1.456 & $3.53 E-03$ & $2.43 E-03$ \\
\hline 22.8 & 4540 & 22.8 & 4790 & 22.8 & 5150 & 2.388 & $4.83 E-03$ & $2.02 E-03$ \\
\hline 37.3 & 6370 & 37.3 & 6790 & 37.3 & 7270 & 3.906 & $6.81 E-03$ & $1.74 E-03$ \\
\hline 61 & 9140 & 61 & 9710 & 61.1 & 10500 & 6.391 & $9.78 E-03$ & $1.53 E-03$ \\
\hline 100 & 13500 & 100 & 14300 & 100 & 15300 & 10.472 & $1.44 E-02$ & $1.37 E-03$ \\
\hline 100 & 13500 & 100 & 14100 & 100 & 15200 & 10.472 & $1.43 E-02$ & $1.36 E-03$ \\
\hline 69.5 & 10100 & 69.5 & 10500 & 69.5 & 11300 & 7.278 & $1.06 E-02$ & $1.46 E-03$ \\
\hline 48.3 & 7580 & 48.3 & 7910 & 48.3 & 8460 & 5.058 & $7.98 E-03$ & $1.58 E-03$ \\
\hline 33.6 & 5750 & 33.6 & 6000 & 33.6 & 6420 & 3.519 & $6.06 E-03$ & $1.72 E-03$ \\
\hline 23.4 & 4430 & 23.4 & 4610 & 23.4 & 4930 & 2.450 & $4.66 E-03$ & $1.90 E-03$ \\
\hline 16.2 & 3480 & 16.2 & 3620 & 16.2 & 3860 & 1.696 & $3.65 E-03$ & $2.15 E-03$ \\
\hline 11.3 & 2780 & 11.3 & 2890 & 11.3 & 3070 & 1.183 & $2.91 E-03$ & $2.46 E-03$ \\
\hline 7.85 & 2260 & 7.85 & 2350 & 7.85 & 2500 & 0.822 & $2.37 E-03$ & $2.88 E-03$ \\
\hline 5.45 & 1870 & 5.46 & 1950 & 5.46 & 2060 & 0.571 & $1.96 E-03$ & $3.43 E-03$ \\
\hline 3.79 & 1580 & 3.79 & 1650 & 3.79 & 1730 & 0.397 & $1.65 E-03$ & $4.17 E-03$ \\
\hline 2.64 & 1350 & 2.63 & 1410 & 2.63 & 1480 & 0.276 & $1.41 E-03$ & $5.13 E-03$ \\
\hline 1.83 & 1180 & 1.83 & 1240 & 1.83 & 1280 & 0.192 & $1.23 E-03$ & $6.44 E-03$ \\
\hline 1.27 & 1070 & 1.27 & 1080 & 1.27 & 1140 & 0.133 & $1.10 E-03$ & $8.25 E-03$ \\
\hline 0.887 & 979 & 0.886 & 979 & 0.885 & 1040 & 0.093 & $9.99 E-04$ & $1.08 E-02$ \\
\hline 0.611 & 864 & 0.617 & 888 & 0.607 & 980 & 0.064 & $9.11 E-04$ & $1.42 E-02$ \\
\hline 0.429 & 841 & 0.422 & 897 & 0.432 & 864 & 0.045 & 8.67E-04 & $1.94 E-02$ \\
\hline 0.298 & 794 & 0.301 & 769 & 0.299 & 875 & 0.031 & $8.13 E-04$ & $2.59 E-02$ \\
\hline 0.207 & 679 & 0.198 & 806 & 0.201 & 886 & 0.021 & 7.90E-04 & $3.74 E-02$ \\
\hline 0.149 & 737 & 0.138 & 720 & 0.148 & 822 & 0.015 & $7.60 E-04$ & $5.00 E-02$ \\
\hline 0.101 & 668 & 0.087 & 780 & 0.101 & 701 & 0.010 & 7.16E-04 & 7.10E-02 \\
\hline
\end{tabular}




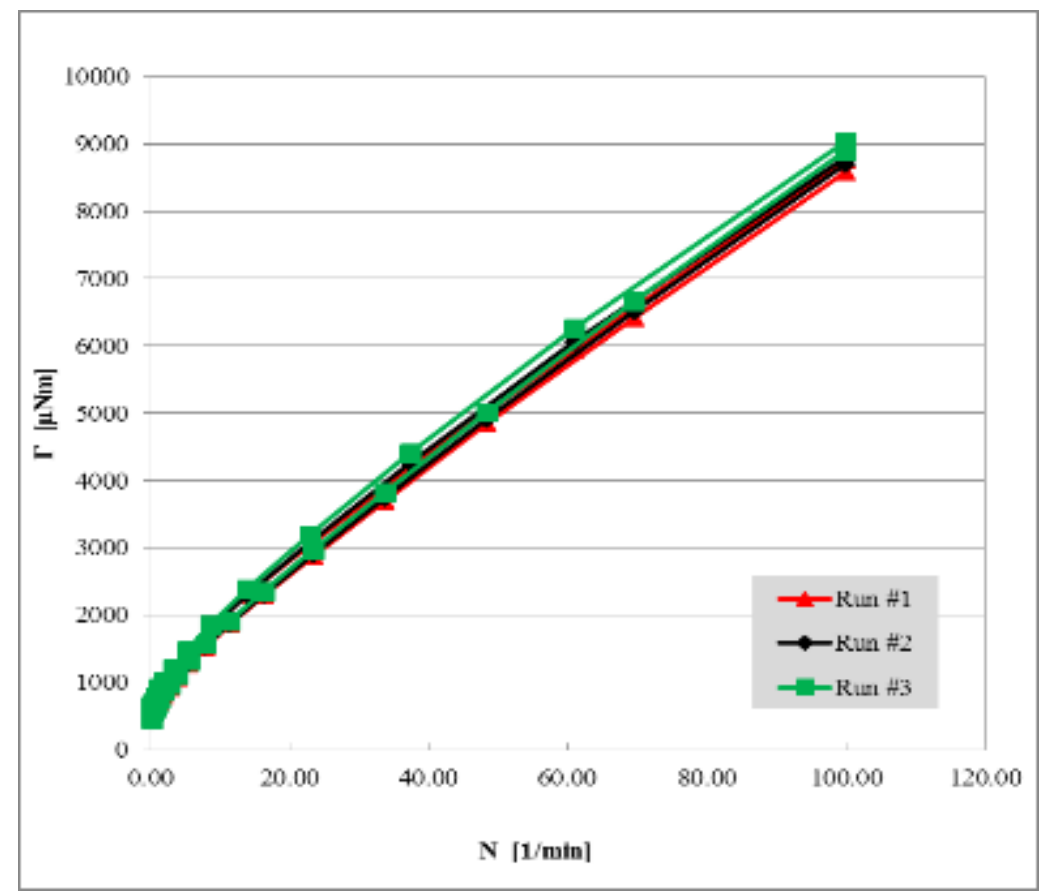

Figure A - 33: Torque vs. Angular Speed using Serrated Coaxial Cylinder on Mix\#9, with $0 \%$ beads by volume. Portrays data from Table A-47.

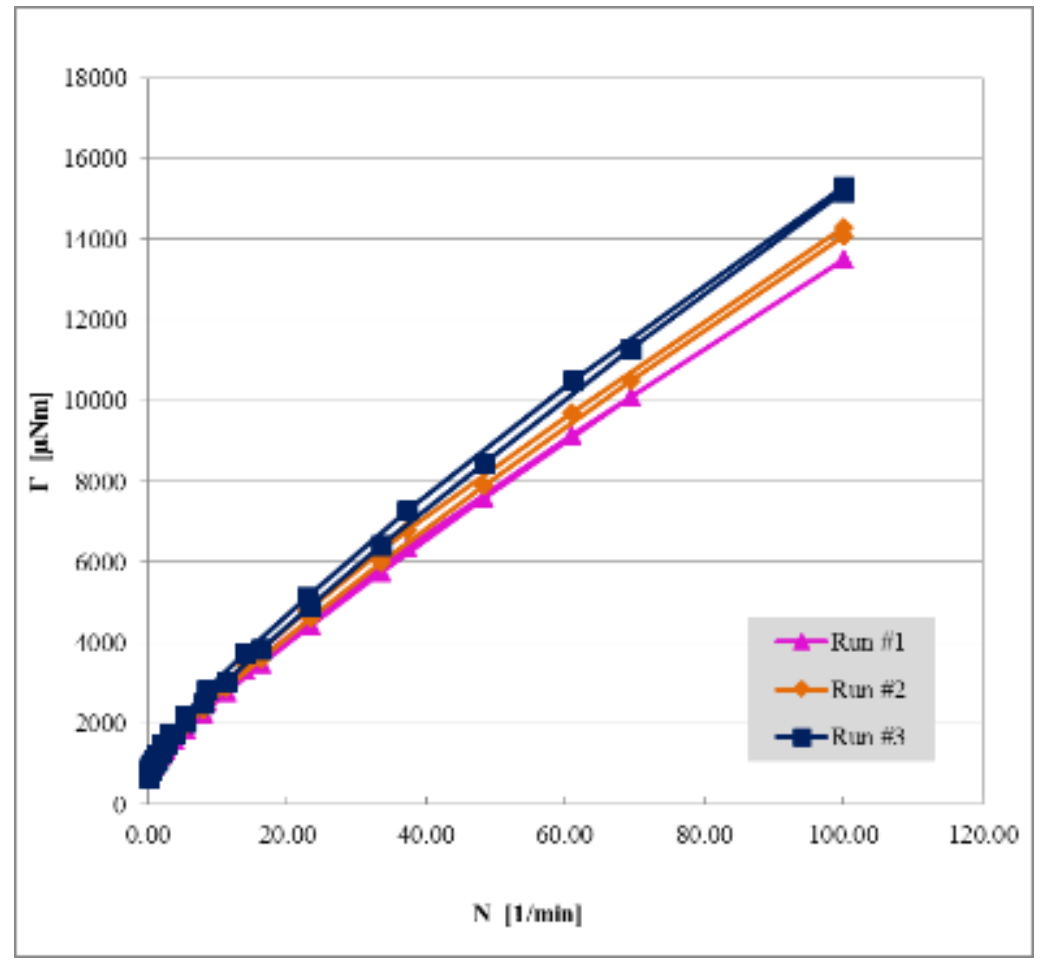

Figure A - 34: Torque vs. Angular Speed using Serrated Coaxial Cylinder on Mix\#9, with $20 \%$ beads by volume. Portrays data from Table A-48. 
Table A - 49: Measured data for Mix\#10 with 0 \% beads using Double spiral spindle.

\begin{tabular}{|c|c|c|c|c|c|c|c|c|}
\hline \multicolumn{6}{|c|}{ Double Spiral } & \multirow{2}{*}{\multicolumn{3}{|c|}{$\begin{array}{c}\text { Bead } \% \\
0 \%\end{array}$}} \\
\hline Geom.: & \multicolumn{3}{|l|}{$R H N-83 A$} & \multicolumn{2}{|l|}{ Cup: } & & & \\
\hline \multicolumn{2}{|c|}{ Run \#1 } & \multicolumn{2}{|c|}{ Run \#2 } & \multicolumn{2}{|c|}{ Run \#3 } & \multirow{2}{*}{\multicolumn{3}{|c|}{$\begin{array}{c}\text { Average } \\
\text { values }\end{array}$}} \\
\hline \multicolumn{2}{|c|}{ NIST Code: SMC-111G } & \multicolumn{2}{|c|}{ NIST Code: SMC-111H } & \multicolumn{2}{|c|}{ NIST Code: SMC-111I } & & & \\
\hline $\mathrm{N}$ & $\Gamma$ & $\mathrm{N}$ & $\Gamma$ & $\mathrm{N}$ & $\Gamma$ & $\mathbf{N}$ & $\Gamma$ & $\Gamma / N$ (Angular \\
\hline $1 / \mathrm{min}$ & $\mu \mathrm{Nm}$ & $1 / \mathrm{min}$ & $\mu \mathrm{Nm}$ & $1 / \mathrm{min}$ & $\mu \mathrm{Nm}$ & $\mathrm{rad} / \mathrm{s}$ & $\mathrm{Nm}$ & momentum) \\
\hline 0.094 & 1050 & 0.0941 & 1020 & 0.0939 & 1020 & 0.010 & $1.03 E-03$ & $1.05 E-01$ \\
\hline 0.161 & 1580 & 0.161 & 1540 & 0.161 & 1550 & 0.017 & $1.56 E-03$ & $9.23 E-02$ \\
\hline 0.268 & 1790 & 0.268 & 1780 & 0.268 & 1790 & 0.028 & $1.79 E-03$ & 6.37E-02 \\
\hline 0.439 & 1910 & 0.439 & 1900 & 0.44 & 1910 & 0.046 & $1.91 E-03$ & 4.14E-02 \\
\hline 0.72 & 2050 & 0.72 & 2040 & 0.72 & 2060 & 0.075 & $2.05 E-03$ & $2.72 E-02$ \\
\hline 1.18 & 2240 & 1.18 & 2230 & 1.18 & 2250 & 0.124 & $2.24 E-03$ & $1.81 E-02$ \\
\hline 1.93 & 2520 & 1.93 & 2520 & 1.93 & 2560 & 0.202 & $2.53 E-03$ & $1.25 E-02$ \\
\hline 3.16 & 2970 & 3.16 & 2970 & 3.16 & 3020 & 0.331 & $2.99 E-03$ & $9.03 E-03$ \\
\hline 5.18 & 3660 & 5.18 & 3670 & 5.18 & 3730 & 0.542 & $3.69 E-03$ & $6.80 E-03$ \\
\hline 8.48 & 4740 & 8.48 & 4750 & 8.48 & 4830 & 0.888 & 4.77E-03 & $5.38 E-03$ \\
\hline 13.9 & 6420 & 13.9 & 6470 & 13.9 & 6630 & 1.456 & $6.51 E-03$ & $4.47 E-03$ \\
\hline 22.8 & 9040 & 22.8 & 9130 & 22.8 & 9340 & 2.388 & $9.17 E-03$ & $3.84 E-03$ \\
\hline 37.3 & 13200 & 37.3 & 13300 & 37.3 & 13700 & 3.906 & $1.34 E-02$ & $3.43 E-03$ \\
\hline 61.1 & 19700 & 61.1 & 20000 & 61.1 & 20500 & 6.398 & $2.01 E-02$ & $3.14 E-03$ \\
\hline 100 & 29700 & 100 & 30300 & 100 & 31100 & 10.472 & $3.04 E-02$ & $2.90 E-03$ \\
\hline 100 & 29500 & 100 & 30100 & 100 & 31000 & 10.472 & $3.02 E-02$ & $2.88 E-03$ \\
\hline 69.5 & 21600 & 69.5 & 22000 & 69.5 & 22700 & 7.278 & $2.21 E-02$ & $3.04 E-03$ \\
\hline 48.3 & 16000 & 48.3 & 16300 & 48.3 & 16700 & 5.058 & $1.63 E-02$ & $3.23 E-03$ \\
\hline 33.6 & 12000 & 33.6 & 12200 & 33.6 & 12500 & 3.519 & $1.22 E-02$ & $3.48 E-03$ \\
\hline 23.4 & 9100 & 23.4 & 9260 & 23.4 & 9510 & 2.450 & $9.29 E-03$ & 3.79E-03 \\
\hline 16.2 & 7040 & 16.2 & 7160 & 16.2 & 7340 & 1.696 & 7.18E-03 & 4.23E-03 \\
\hline 11.3 & 5560 & 11.3 & 5650 & 11.3 & 5790 & 1.183 & $5.67 E-03$ & $4.79 E-03$ \\
\hline 7.85 & 4490 & 7.85 & 4560 & 7.85 & 4660 & 0.822 & $4.57 E-03$ & $5.56 E-03$ \\
\hline 5.46 & 3710 & 5.46 & 3770 & 5.46 & 3850 & 0.572 & $3.78 E-03$ & $6.61 E-03$ \\
\hline 3.79 & 3150 & 3.79 & 3190 & 3.79 & 3260 & 0.397 & $3.20 E-03$ & 8.06E-03 \\
\hline 2.64 & 2740 & 2.64 & 2770 & 2.64 & 2830 & 0.276 & $2.78 E-03$ & $1.01 E-02$ \\
\hline 1.83 & 2430 & 1.83 & 2460 & 1.83 & 2500 & 0.192 & $2.46 E-03$ & $1.29 E-02$ \\
\hline 1.27 & 2200 & 1.27 & 2230 & 1.27 & 2270 & 0.133 & $2.23 E-03$ & $1.68 E-02$ \\
\hline 0.885 & 2040 & 0.886 & 2060 & 0.883 & 2100 & 0.093 & $2.07 E-03$ & $2.23 E-02$ \\
\hline 0.615 & 1900 & 0.617 & 1920 & 0.617 & 1950 & 0.065 & $1.92 E-03$ & $2.98 E-02$ \\
\hline 0.428 & 1800 & 0.428 & 1810 & 0.428 & 1840 & 0.045 & $1.82 E-03$ & $4.05 E-02$ \\
\hline 0.295 & 1720 & 0.296 & 1750 & 0.296 & 1770 & 0.031 & $1.75 E-03$ & $5.64 E-02$ \\
\hline 0.207 & 1670 & 0.207 & 1680 & 0.206 & 1700 & 0.022 & $1.68 E-03$ & $7.78 E-02$ \\
\hline 0.143 & 1640 & 0.143 & 1630 & 0.142 & 1660 & 0.015 & $1.64 E-03$ & $1.10 E-01$ \\
\hline 0.1 & 1590 & 0.1 & 1590 & 0.1 & 1630 & 0.010 & $1.60 E-03$ & $1.53 E-01$ \\
\hline
\end{tabular}


Table A - 50: Measured data for Mix\#10 with $20 \%$ beads using Double spiral spindle.

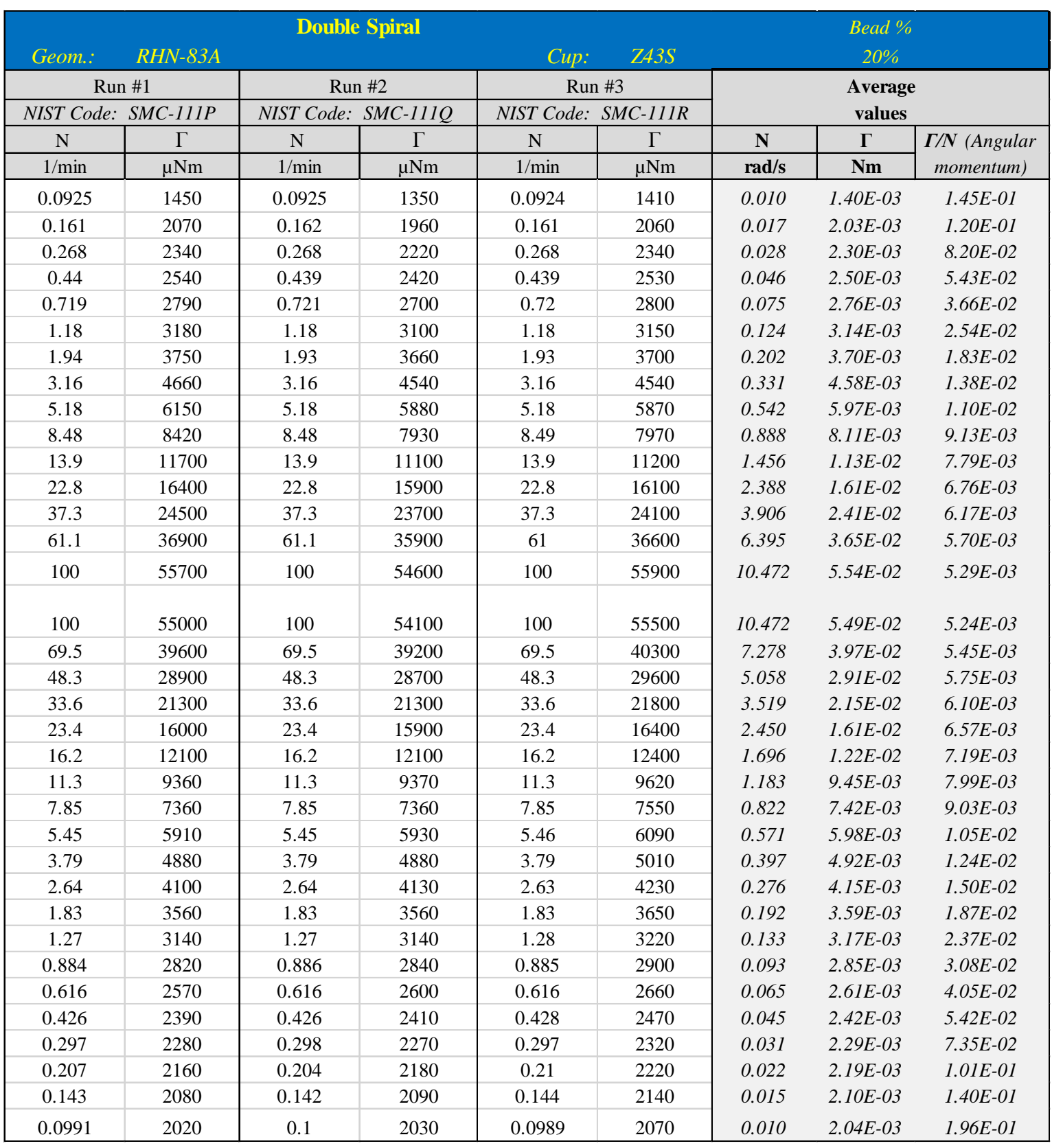




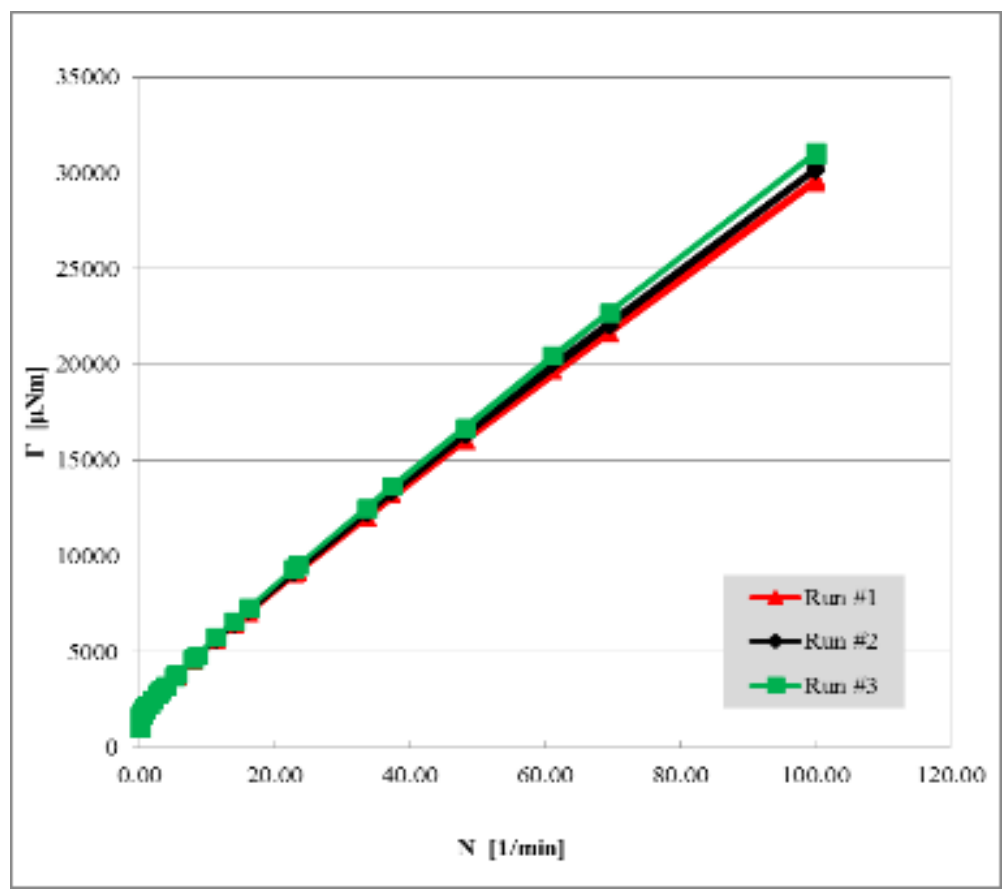

Figure A - 35: Torque vs. Angular Speed using Double spiral spindle on Mix\#10, with $0 \%$ beads by volume. Portrays data from Table A-49.

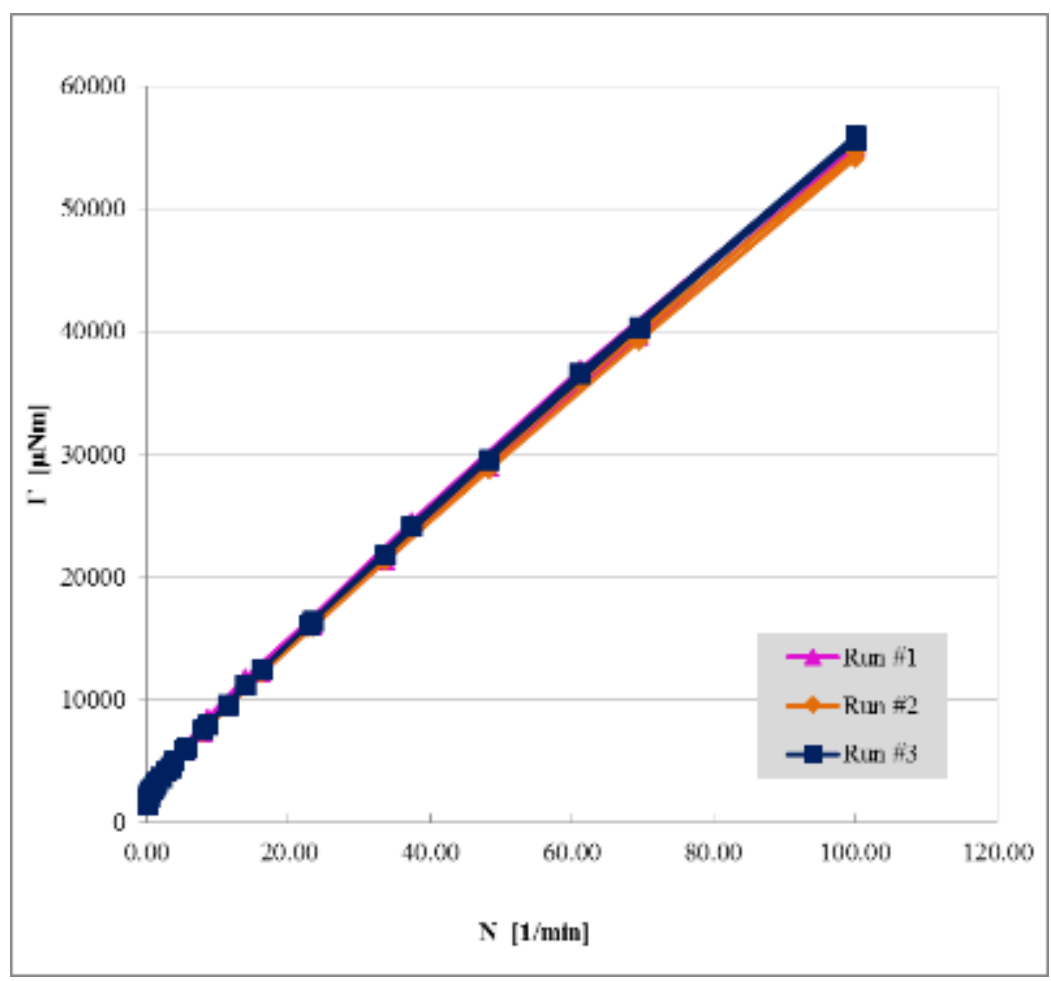

Figure A - 36: Torque vs. Angular Speed using Double spiral spindle on Mix\#10, with $20 \%$ beads by volume. Portrays data from Table A-50. 
Table A - 51: Measured data for Mix\#10 with 0 \% beads using Six-blade Vane.

\begin{tabular}{|c|c|c|c|c|c|c|c|c|}
\hline \multicolumn{6}{|c|}{6 Blade Vane } & \multirow{2}{*}{\multicolumn{3}{|c|}{$\begin{array}{c}\text { Bead } \% \\
0 \%\end{array}$}} \\
\hline Geom.: & RHN-83C & & & \multicolumn{2}{|c|}{ Z43S } & & & \\
\hline \multicolumn{2}{|c|}{ Run \#1 } & \multicolumn{2}{|c|}{ Run \#2 } & \multicolumn{2}{|c|}{ Run \#3 } & \multirow{2}{*}{\multicolumn{3}{|c|}{$\begin{array}{c}\text { Average } \\
\text { values }\end{array}$}} \\
\hline NIST Code: & SMC-111A & NIST Code: & $S M C-111 B$ & NIST Code: & $S M C-111 C$ & & & \\
\hline $\mathrm{N}$ & $\Gamma$ & $\mathrm{N}$ & $\Gamma$ & $\mathrm{N}$ & $\Gamma$ & $\mathbf{N}$ & $\Gamma$ & $\Gamma / \mathbf{N}$ (Angular \\
\hline $1 / \mathrm{min}$ & $\mu \mathrm{Nm}$ & $1 / \mathrm{min}$ & $\mu \mathrm{Nm}$ & $1 / \mathrm{min}$ & $\mu \mathrm{Nm}$ & $\mathrm{rad} / \mathrm{s}$ & $\mathrm{Nm}$ & momentum) \\
\hline 0.0977 & 454 & 0.0976 & 444 & 0.0975 & 437 & 0.0102 & $4.45 E-04$ & 4.35E-02 \\
\hline 0.163 & 602 & 0.163 & 595 & 0.163 & 597 & 0.0171 & $5.98 E-04$ & $3.50 E-02$ \\
\hline 0.268 & 644 & 0.268 & 636 & 0.268 & 640 & 0.0281 & $6.40 E-04$ & $2.28 E-02$ \\
\hline 0.439 & 681 & 0.439 & 672 & 0.439 & 675 & 0.0460 & $6.76 E-04$ & $1.47 E-02$ \\
\hline 0.72 & 734 & 0.72 & 724 & 0.72 & 728 & 0.0754 & 7.29E-04 & $9.66 E-03$ \\
\hline 1.18 & 808 & 1.18 & 799 & 1.18 & 803 & 0.1236 & 8.03E-04 & $6.50 E-03$ \\
\hline 1.93 & 910 & 1.93 & 901 & 1.93 & 906 & 0.2021 & $9.06 E-04$ & $4.48 E-03$ \\
\hline 3.16 & 1060 & 3.16 & 1050 & 3.16 & 1050 & 0.3309 & $1.05 E-03$ & $3.18 E-03$ \\
\hline 5.18 & 1260 & 5.18 & 1260 & 5.18 & 1270 & 0.5424 & $1.26 E-03$ & $2.33 E-03$ \\
\hline 8.48 & 1570 & 8.48 & 1560 & 8.48 & 1570 & 0.8880 & $1.57 E-03$ & $1.76 E-03$ \\
\hline 13.9 & 2010 & 13.9 & 2000 & 13.9 & 2020 & 1.4556 & $2.01 E-03$ & $1.38 E-03$ \\
\hline 22.8 & 2650 & 22.8 & 2650 & 22.8 & 2670 & 2.3876 & $2.66 E-03$ & $1.11 E-03$ \\
\hline 37.3 & 3630 & 37.3 & 3650 & 37.3 & 3680 & 3.9060 & $3.65 E-03$ & $9.35 E-04$ \\
\hline 61 & 5130 & 61 & 5170 & 61 & 5220 & 6.3879 & 5.17E-03 & 8.10E-04 \\
\hline 100 & 7460 & 100 & 7530 & 100 & 7640 & 10.4720 & $7.54 E-03$ & $7.20 E-04$ \\
\hline 100 & 7430 & 100 & 7500 & 100 & 7620 & 10.4720 & $7.52 E-03$ & $7.18 E-04$ \\
\hline 69.5 & 5580 & 69.5 & 5630 & 69.5 & 5710 & 7.2780 & $5.64 E-03$ & $7.75 E-04$ \\
\hline 48.3 & 4250 & 48.3 & 4280 & 48.3 & 4350 & 5.0580 & 4.29E-03 & 8.49E-04 \\
\hline 33.6 & 3290 & 33.6 & 3320 & 33.6 & 3360 & 3.5186 & $3.32 E-03$ & $9.45 E-04$ \\
\hline 23.4 & 2600 & 23.4 & 2620 & 23.4 & 2650 & 2.4504 & $2.62 E-03$ & $1.07 E-03$ \\
\hline 16.2 & 2100 & 16.2 & 2110 & 16.2 & 2140 & 1.6965 & $2.12 E-03$ & $1.25 E-03$ \\
\hline 11.3 & 1720 & 11.3 & 1730 & 11.3 & 1750 & 1.1833 & $1.73 E-03$ & $1.46 E-03$ \\
\hline 7.85 & 1450 & 7.85 & 1460 & 7.85 & 1470 & 0.8221 & $1.46 E-03$ & $1.78 E-03$ \\
\hline 5.45 & 1230 & 5.46 & 1240 & 5.46 & 1250 & 0.5714 & $1.24 E-03$ & $2.17 E-03$ \\
\hline 3.79 & 1070 & 3.79 & 1080 & 3.79 & 1080 & 0.3969 & $1.08 E-03$ & $2.71 E-03$ \\
\hline 2.64 & 943 & 2.63 & 950 & 2.64 & 954 & 0.2761 & $9.49 E-04$ & $3.44 E-03$ \\
\hline 1.83 & 841 & 1.83 & 847 & 1.83 & 854 & 0.1916 & $8.47 E-04$ & $4.42 E-03$ \\
\hline 1.28 & 773 & 1.27 & 780 & 1.27 & 797 & 0.1333 & $7.83 E-04$ & $5.87 E-03$ \\
\hline 0.884 & 707 & 0.889 & 721 & 0.889 & 723 & 0.0929 & 7.17E-04 & $7.72 E-03$ \\
\hline 0.616 & 661 & 0.615 & 666 & 0.616 & 669 & 0.0645 & $6.65 E-04$ & $1.03 E-02$ \\
\hline 0.427 & 629 & 0.426 & 641 & 0.428 & 638 & 0.0447 & $6.36 E-04$ & $1.42 E-02$ \\
\hline 0.296 & 601 & 0.298 & 612 & 0.298 & 616 & 0.0311 & $6.10 E-04$ & $1.96 E-02$ \\
\hline 0.208 & 569 & 0.206 & 578 & 0.208 & 589 & 0.0217 & $5.79 E-04$ & 2.67E-02 \\
\hline 0.142 & 564 & 0.145 & 561 & 0.145 & 557 & 0.0151 & $5.61 E-04$ & $3.72 E-02$ \\
\hline 0.0988 & 552 & 0.103 & 566 & 0.1 & 532 & 0.0105 & $5.50 E-04$ & $5.22 E-02$ \\
\hline
\end{tabular}


Table A - 52: Measured data for Mix\#10 with 20 \% beads using Six-blade Vane.

\begin{tabular}{|c|c|c|c|c|c|c|c|c|}
\hline \multicolumn{6}{|c|}{6 Blade Vane } & \multirow{2}{*}{\multicolumn{3}{|c|}{$\begin{array}{c}\text { Bead } \% \\
20 \%\end{array}$}} \\
\hline Geom.: & RHN-83C & & & \multicolumn{2}{|c|}{ Z43S } & & & \\
\hline \multicolumn{2}{|c|}{ Run \#1 } & \multicolumn{2}{|c|}{ Run \#2 } & \multicolumn{2}{|c|}{ Run \#3 } & \multirow{2}{*}{\multicolumn{3}{|c|}{$\begin{array}{c}\text { Average } \\
\text { values }\end{array}$}} \\
\hline NIST Code: & $S M C-111 \mathrm{~J}$ & NIST Code: & $S M C-111 K$ & NIST Cod & MC-111L & & & \\
\hline $\mathrm{N}$ & $\Gamma$ & $\mathrm{N}$ & $\Gamma$ & $\mathrm{N}$ & $\Gamma$ & $\mathbf{N}$ & $\Gamma$ & $\Gamma / N$ (Angular \\
\hline $1 / \mathrm{min}$ & $\mu \mathrm{Nm}$ & $1 / \mathrm{min}$ & $\mu \mathrm{Nm}$ & $1 / \mathrm{min}$ & $\mu \mathrm{Nm}$ & $\mathrm{rad} / \mathrm{s}$ & $\mathrm{Nm}$ & momentum) \\
\hline 0.0974 & 618 & 0.0974 & 602 & 0.0972 & 597 & 0.010 & $6.06 E-04$ & 5.94E-02 \\
\hline 0.163 & 779 & 0.163 & 775 & 0.163 & 755 & 0.017 & $7.70 E-04$ & $4.51 E-02$ \\
\hline 0.268 & 849 & 0.268 & 835 & 0.269 & 826 & 0.028 & $8.37 E-04$ & $2.98 E-02$ \\
\hline 0.439 & 907 & 0.439 & 893 & 0.439 & 892 & 0.046 & 8.97E-04 & $1.95 E-02$ \\
\hline 0.721 & 978 & 0.719 & 965 & 0.721 & 977 & 0.075 & $9.73 E-04$ & $1.29 E-02$ \\
\hline 1.18 & 1080 & 1.18 & 1090 & 1.18 & 1100 & 0.124 & $1.09 E-03$ & 8.82E-03 \\
\hline 1.93 & 1250 & 1.93 & 1260 & 1.93 & 1280 & 0.202 & $1.26 E-03$ & $6.25 E-03$ \\
\hline 3.16 & 1490 & 3.16 & 1510 & 3.16 & 1540 & 0.331 & $1.51 E-03$ & $4.57 E-03$ \\
\hline 5.18 & 1830 & 5.18 & 1870 & 5.18 & 1920 & 0.542 & $1.87 E-03$ & $3.45 E-03$ \\
\hline 8.48 & 2330 & 8.48 & 2390 & 8.48 & 2450 & 0.888 & $2.39 E-03$ & 2.69E-03 \\
\hline 13.9 & 3110 & 13.9 & 3180 & 13.9 & 3270 & 1.456 & $3.19 E-03$ & 2.19E-03 \\
\hline 22.8 & 4280 & 22.8 & 4380 & 22.8 & 4500 & 2.388 & $4.39 E-03$ & $1.84 E-03$ \\
\hline 37.3 & 5980 & 37.3 & 6140 & 37.3 & 6370 & 3.906 & $6.16 E-03$ & $1.58 E-03$ \\
\hline 61.1 & 8440 & 61 & 8880 & 61.1 & 9210 & 6.395 & $8.84 E-03$ & $1.38 E-03$ \\
\hline 100 & 12200 & 100 & 12900 & 100 & 13500 & 10.472 & $1.29 E-02$ & $1.23 E-03$ \\
\hline 100 & 12000 & 100 & 12800 & 100 & 13400 & 10.472 & $1.27 E-02$ & $1.22 E-03$ \\
\hline 69.5 & 8990 & 69.5 & 9510 & 69.5 & 9930 & 7.278 & $9.48 E-03$ & $1.30 E-03$ \\
\hline 48.3 & 6780 & 48.3 & 7160 & 48.3 & 7450 & 5.058 & $7.13 E-03$ & $1.41 E-03$ \\
\hline 33.6 & 5200 & 33.6 & 5450 & 33.6 & 5650 & 3.519 & $5.43 E-03$ & $1.54 E-03$ \\
\hline 23.3 & 4080 & 23.4 & 4220 & 23.4 & 4370 & 2.447 & $4.22 E-03$ & $1.73 E-03$ \\
\hline 16.2 & 3220 & 16.2 & 3340 & 16.2 & 3440 & 1.696 & 3.33E-03 & $1.96 E-03$ \\
\hline 11.3 & 2600 & 11.3 & 2680 & 11.3 & 2740 & 1.183 & $2.67 E-03$ & $2.26 E-03$ \\
\hline 7.85 & 2120 & 7.84 & 2190 & 7.84 & 2230 & 0.821 & $2.18 E-03$ & $2.65 E-03$ \\
\hline 5.46 & 1760 & 5.45 & 1820 & 5.45 & 1840 & 0.571 & $1.81 E-03$ & $3.16 E-03$ \\
\hline 3.79 & 1500 & 3.79 & 1540 & 3.79 & 1560 & 0.397 & $1.53 E-03$ & $3.86 E-03$ \\
\hline 2.64 & 1300 & 2.64 & 1330 & 2.63 & 1350 & 0.276 & $1.33 E-03$ & $4.80 E-03$ \\
\hline 1.83 & 1140 & 1.83 & 1170 & 1.84 & 1180 & 0.192 & $1.16 E-03$ & $6.06 E-03$ \\
\hline 1.27 & 1020 & 1.27 & 1050 & 1.27 & 1060 & 0.133 & $1.04 E-03$ & $7.84 E-03$ \\
\hline 0.889 & 944 & 0.887 & 984 & 0.884 & 948 & 0.093 & $9.59 E-04$ & $1.03 E-02$ \\
\hline 0.615 & 872 & 0.61 & 899 & 0.615 & 880 & 0.064 & 8.84E-04 & $1.38 E-02$ \\
\hline 0.43 & 812 & 0.427 & 805 & 0.43 & 844 & 0.045 & $8.20 E-04$ & $1.83 E-02$ \\
\hline 0.297 & 794 & 0.297 & 765 & 0.301 & 778 & 0.031 & 7.79E-04 & $2.49 E-02$ \\
\hline 0.206 & 726 & 0.207 & 732 & 0.208 & 739 & 0.022 & 7.32E-04 & $3.38 E-02$ \\
\hline 0.142 & 709 & 0.143 & 694 & 0.143 & 723 & 0.015 & 7.09E-04 & $4.74 E-02$ \\
\hline 0.0988 & 683 & 0.101 & 682 & 0.0997 & 710 & 0.010 & $6.92 E-04$ & $6.62 E-02$ \\
\hline
\end{tabular}




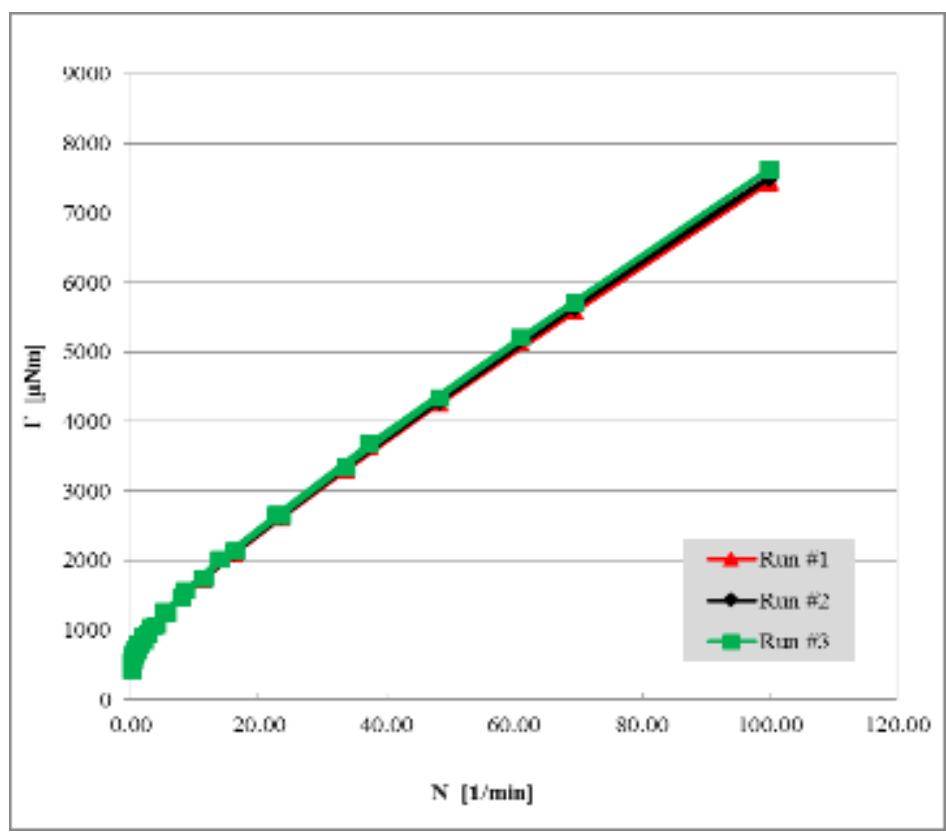

Figure A - 37: Torque vs. Angular Speed using sixblade vane on Mix\#10, with $0 \%$ beads by volume. Portrays data from Table A-51.

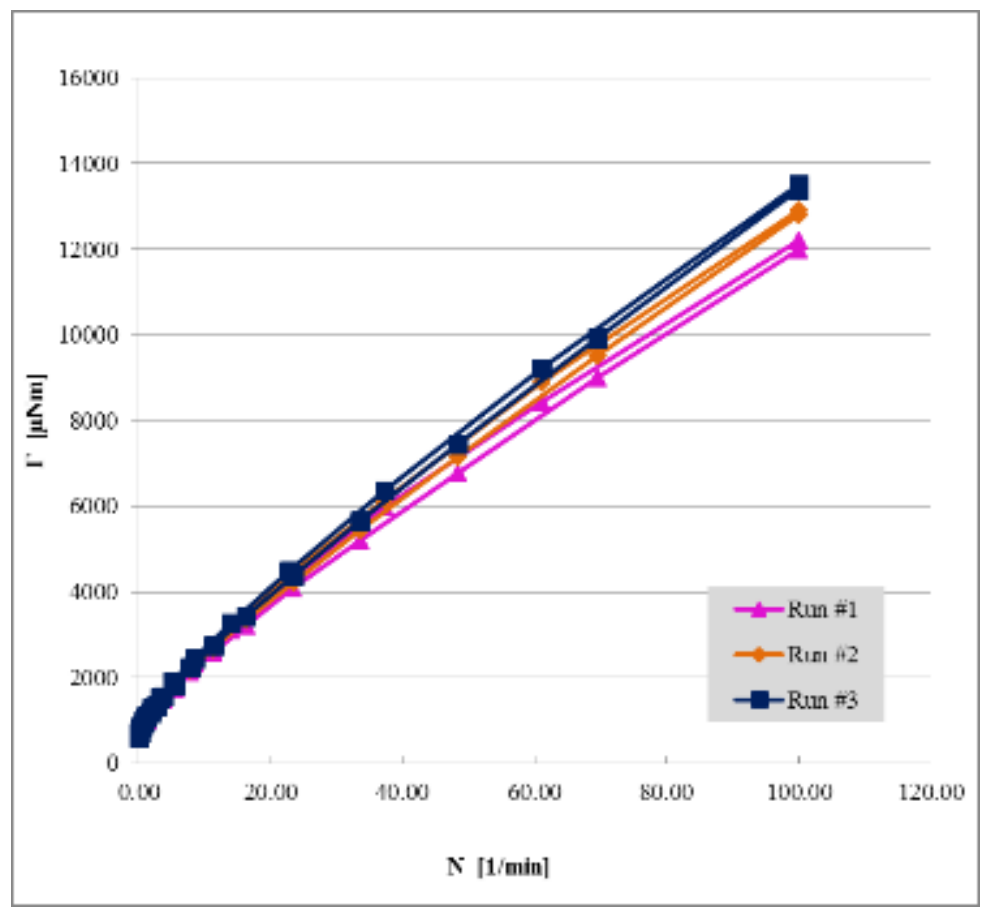

Figure A - 38: Torque vs. Angular Speed using sixblade vane on Mix\#10, with $20 \%$ beads by volume. Portrays data from Table A-52. 
Table A - 53: Measured data for Mix\#10 with 0 \% beads using Serrated Coaxial Cylinder.

\begin{tabular}{|c|c|c|c|c|c|c|c|c|}
\hline \multicolumn{6}{|c|}{ Serrated Coaxial Cylinder } & \multirow{2}{*}{\multicolumn{3}{|c|}{$\begin{array}{c}\text { Bead } \% \\
0 \%\end{array}$}} \\
\hline Geom.: & SS1E & & & \multicolumn{2}{|c|}{ Z43S } & & & \\
\hline \multicolumn{2}{|c|}{ Run \#1 } & \multicolumn{2}{|c|}{ Run \#2 } & \multicolumn{2}{|c|}{ Run \#3 } & \multirow{2}{*}{\multicolumn{3}{|c|}{$\begin{array}{c}\text { Average } \\
\text { values }\end{array}$}} \\
\hline \multicolumn{2}{|c|}{ NIST Code: SMC-111D } & \multicolumn{2}{|c|}{ NIST Code: SMC-111E } & \multicolumn{2}{|c|}{ NIST Code: SMC-111F } & & & \\
\hline $\mathrm{N}$ & $\Gamma$ & $\mathrm{N}$ & $\Gamma$ & $\mathrm{N}$ & $\Gamma$ & $\mathbf{N}$ & $\Gamma$ & $\boldsymbol{\Gamma} / \mathbf{N}$ (Angular \\
\hline $1 / \mathrm{min}$ & $\mu \mathrm{Nm}$ & $1 / \mathrm{min}$ & $\mu \mathrm{Nm}$ & $1 / \mathrm{min}$ & $\mu \mathrm{Nm}$ & $\mathrm{rad} / \mathrm{s}$ & $\mathrm{Nm}$ & momentum) \\
\hline 0.0981 & 469 & 0.0981 & 447 & 0.0984 & 409 & 0.010 & $4.42 E-04$ & $4.29 E-02$ \\
\hline 0.164 & 600 & 0.164 & 586 & 0.164 & 522 & 0.017 & $5.69 E-04$ & 3.32E-02 \\
\hline 0.268 & 638 & 0.268 & 634 & 0.268 & 579 & 0.028 & $6.17 E-04$ & $2.20 E-02$ \\
\hline 0.44 & 685 & 0.439 & 688 & 0.439 & 649 & 0.046 & $6.74 E-04$ & $1.46 E-02$ \\
\hline 0.72 & 751 & 0.72 & 760 & 0.72 & 734 & 0.075 & $7.48 E-04$ & $9.93 E-03$ \\
\hline 1.18 & 841 & 1.18 & 885 & 1.18 & 847 & 0.124 & $8.58 E-04$ & $6.94 E-03$ \\
\hline 1.93 & 965 & 1.93 & 991 & 1.93 & 974 & 0.202 & $9.77 E-04$ & $4.83 E-03$ \\
\hline 3.16 & 1140 & 3.16 & 1170 & 3.16 & 1190 & 0.331 & $1.17 E-03$ & $3.53 E-03$ \\
\hline 5.18 & 1390 & 5.18 & 1420 & 5.18 & 1430 & 0.542 & $1.41 E-03$ & $2.61 E-03$ \\
\hline 8.48 & 1730 & 8.48 & 1780 & 8.48 & 1790 & 0.888 & $1.77 E-03$ & $1.99 E-03$ \\
\hline 13.9 & 2220 & 13.9 & 2290 & 13.9 & 2310 & 1.456 & $2.27 E-03$ & $1.56 E-03$ \\
\hline 22.8 & 2950 & 22.8 & 3050 & 22.8 & 3090 & 2.388 & $3.03 E-03$ & $1.27 E-03$ \\
\hline 37.3 & 4060 & 37.3 & 4210 & 37.3 & 4260 & 3.906 & $4.18 E-03$ & $1.07 E-03$ \\
\hline 61 & 5750 & 61 & 5970 & 61 & 6050 & 6.388 & $5.92 E-03$ & $9.27 E-04$ \\
\hline 100 & 8440 & 100 & 8660 & 100 & 8690 & 10.472 & 8.60E-03 & $8.21 E-04$ \\
\hline 100 & 8360 & 100 & 8550 & 100 & 8580 & 10.472 & $8.50 E-03$ & $8.11 E-04$ \\
\hline 69.5 & 6250 & 69.5 & 6400 & 69.5 & 6440 & 7.278 & $6.36 E-03$ & $8.74 E-04$ \\
\hline 48.3 & 4720 & 48.3 & 4830 & 48.3 & 4850 & 5.058 & $4.80 E-03$ & $9.49 E-04$ \\
\hline 33.6 & 3610 & 33.6 & 3700 & 33.6 & 3710 & 3.519 & 3.67E-03 & $1.04 E-03$ \\
\hline 23.4 & 2810 & 23.4 & 2880 & 23.4 & 2890 & 2.450 & $2.86 E-03$ & $1.17 E-03$ \\
\hline 16.2 & 2240 & 16.2 & 2290 & 16.2 & 2290 & 1.696 & $2.27 E-03$ & $1.34 E-03$ \\
\hline 11.3 & 1810 & 11.3 & 1850 & 11.3 & 1850 & 1.183 & $1.84 E-03$ & $1.55 E-03$ \\
\hline 7.85 & 1490 & 7.85 & 1530 & 7.85 & 1530 & 0.822 & $1.52 E-03$ & $1.84 E-03$ \\
\hline 5.45 & 1250 & 5.45 & 1280 & 5.45 & 1270 & 0.571 & $1.27 E-03$ & $2.22 E-03$ \\
\hline 3.79 & 1060 & 3.79 & 1080 & 3.79 & 1080 & 0.397 & $1.07 E-03$ & $2.70 E-03$ \\
\hline 2.64 & 906 & 2.64 & 928 & 2.63 & 932 & 0.276 & $9.22 E-04$ & $3.34 E-03$ \\
\hline 1.83 & 788 & 1.83 & 810 & 1.83 & 830 & 0.192 & 8.09E-04 & $4.22 E-03$ \\
\hline 1.27 & 694 & 1.27 & 741 & 1.27 & 724 & 0.133 & $7.20 E-04$ & $5.41 E-03$ \\
\hline 0.886 & 619 & 0.883 & 659 & 0.885 & 683 & 0.093 & $6.54 E-04$ & $7.06 E-03$ \\
\hline 0.611 & 597 & 0.615 & 659 & 0.628 & 633 & 0.065 & $6.30 E-04$ & $9.73 E-03$ \\
\hline 0.429 & 512 & 0.433 & 594 & 0.428 & 639 & 0.045 & $5.82 E-04$ & $1.29 E-02$ \\
\hline 0.298 & 468 & 0.299 & 514 & 0.293 & 531 & 0.031 & $5.04 E-04$ & $1.62 E-02$ \\
\hline 0.204 & 479 & 0.202 & 555 & 0.202 & 552 & 0.021 & $5.29 E-04$ & $2.49 E-02$ \\
\hline 0.146 & 550 & 0.128 & 492 & 0.14 & 571 & 0.014 & $5.38 E-04$ & $3.72 E-02$ \\
\hline 0.101 & 405 & 0.107 & 538 & 0.104 & 515 & 0.011 & $4.86 E-04$ & $4.46 E-02$ \\
\hline
\end{tabular}


Table A - 54: Measured data for Mix\#10 with 20 \% beads using Serrated Coaxial Cylinder.

\begin{tabular}{|c|c|c|c|c|c|c|c|c|}
\hline \multicolumn{6}{|c|}{ Serrated Coaxial Cylinder } & \multirow{2}{*}{\multicolumn{3}{|c|}{$\begin{array}{c}\text { Bead } \% \\
20 \%\end{array}$}} \\
\hline Geom.: & SS1E & & & \multicolumn{2}{|c|}{ Z43S } & & & \\
\hline \multicolumn{2}{|c|}{ Run \#1 } & \multicolumn{2}{|c|}{ Run \#2 } & \multicolumn{2}{|c|}{ Run \#3 } & \multirow{2}{*}{\multicolumn{3}{|c|}{$\begin{array}{c}\text { Average } \\
\text { values }\end{array}$}} \\
\hline \multicolumn{2}{|c|}{ NIST Code: SMC-111M } & \multicolumn{2}{|c|}{ NIST Code: SMC-111N } & \multicolumn{2}{|c|}{ NIST Code: SMC-111O } & & & \\
\hline $\mathrm{N}$ & $\Gamma$ & $\mathrm{N}$ & $\Gamma$ & $\mathrm{N}$ & $\Gamma$ & $\mathbf{N}$ & $\Gamma$ & $\boldsymbol{\Gamma} / \mathbf{N}$ (Angular \\
\hline $1 / \mathrm{min}$ & $\mu \mathrm{Nm}$ & $1 / \mathrm{min}$ & $\mu \mathrm{Nm}$ & $1 / \min$ & $\mu \mathrm{Nm}$ & $\mathrm{rad} / \mathrm{s}$ & $\mathrm{Nm}$ & momentum) \\
\hline 0.0978 & 611 & 0.0976 & 599 & 0.0977 & 607 & 0.010 & $6.06 E-04$ & $5.92 E-02$ \\
\hline 0.164 & 736 & 0.164 & 758 & 0.164 & 775 & 0.017 & $7.56 E-04$ & 4.40E-02 \\
\hline 0.268 & 771 & 0.268 & 810 & 0.268 & 831 & 0.028 & 8.04E-04 & $2.86 E-02$ \\
\hline 0.439 & 824 & 0.44 & 876 & 0.439 & 895 & 0.046 & 8.65E-04 & $1.88 E-02$ \\
\hline 0.72 & 905 & 0.72 & 964 & 0.719 & 992 & 0.075 & $9.54 E-04$ & $1.27 E-02$ \\
\hline 1.18 & 1030 & 1.18 & 1100 & 1.18 & 1120 & 0.124 & $1.08 E-03$ & $8.77 E-03$ \\
\hline 1.93 & 1190 & 1.93 & 1280 & 1.93 & 1310 & 0.202 & $1.26 E-03$ & $6.23 E-03$ \\
\hline 3.16 & 1430 & 3.16 & 1540 & 3.16 & 1590 & 0.331 & $1.52 E-03$ & $4.59 E-03$ \\
\hline 5.18 & 1780 & 5.18 & 1910 & 5.18 & 1970 & 0.542 & $1.89 E-03$ & $3.48 E-03$ \\
\hline 8.48 & 2270 & 8.48 & 2440 & 8.48 & 2530 & 0.888 & $2.41 E-03$ & $2.72 E-03$ \\
\hline 13.9 & 3000 & 13.9 & 3240 & 13.9 & 3350 & 1.456 & $3.20 E-03$ & $2.20 E-03$ \\
\hline 22.8 & 4090 & 22.8 & 4440 & 22.8 & 4600 & 2.388 & $4.38 E-03$ & $1.83 E-03$ \\
\hline 37.3 & 5780 & 37.3 & 6300 & 37.3 & 6540 & 3.906 & $6.21 E-03$ & $1.59 E-03$ \\
\hline 61 & 8330 & 61 & 9090 & 61 & 9430 & 6.388 & $8.95 E-03$ & $1.40 E-03$ \\
\hline 100 & 12400 & 100 & 13300 & 100 & 13800 & 10.472 & $1.32 E-02$ & $1.26 E-03$ \\
\hline 100 & 12300 & 100 & 13200 & 100 & 13600 & 10.472 & $1.30 E-02$ & $1.24 E-03$ \\
\hline 69.5 & 9180 & 69.5 & 9830 & 69.5 & 10100 & 7.278 & $9.70 E-03$ & $1.33 E-03$ \\
\hline 48.3 & 6910 & 48.3 & 7380 & 48.3 & 7610 & 5.058 & 7.30E-03 & $1.44 E-03$ \\
\hline 33.6 & 5270 & 33.6 & 5610 & 33.6 & 5780 & 3.519 & $5.55 E-03$ & $1.58 E-03$ \\
\hline 23.4 & 4080 & 23.4 & 4320 & 23.4 & 4450 & 2.450 & $4.28 E-03$ & $1.75 E-03$ \\
\hline 16.2 & 3210 & 16.2 & 3390 & 16.2 & 3490 & 1.696 & 3.36E-03 & $1.98 E-03$ \\
\hline 11.3 & 2580 & 11.3 & 2710 & 11.3 & 2790 & 1.183 & $2.69 E-03$ & $2.28 E-03$ \\
\hline 7.85 & 2100 & 7.85 & 2210 & 7.85 & 2270 & 0.822 & $2.19 E-03$ & $2.67 E-03$ \\
\hline 5.46 & 1750 & 5.45 & 1840 & 5.46 & 1880 & 0.571 & $1.82 E-03$ & $3.19 E-03$ \\
\hline 3.79 & 1470 & 3.79 & 1560 & 3.79 & 1590 & 0.397 & $1.54 E-03$ & $3.88 E-03$ \\
\hline 2.64 & 1260 & 2.64 & 1330 & 2.64 & 1360 & 0.276 & $1.32 E-03$ & 4.76E-03 \\
\hline 1.83 & 1120 & 1.83 & 1160 & 1.83 & 1190 & 0.192 & $1.16 E-03$ & $6.04 E-03$ \\
\hline 1.27 & 975 & 1.27 & 1030 & 1.27 & 1050 & 0.133 & $1.02 E-03$ & $7.66 E-03$ \\
\hline 0.881 & 940 & 0.888 & 930 & 0.887 & 939 & 0.093 & $9.36 E-04$ & $1.01 E-02$ \\
\hline 0.617 & 793 & 0.611 & 898 & 0.617 & 862 & 0.064 & $8.51 E-04$ & $1.32 E-02$ \\
\hline 0.438 & 770 & 0.43 & 783 & 0.424 & 808 & 0.045 & $7.87 E-04$ & $1.75 E-02$ \\
\hline 0.283 & 738 & 0.297 & 777 & 0.3 & 809 & 0.031 & $7.75 E-04$ & $2.52 E-02$ \\
\hline 0.205 & 677 & 0.207 & 690 & 0.212 & 725 & 0.022 & $6.97 E-04$ & $3.20 E-02$ \\
\hline 0.147 & 627 & 0.145 & 753 & 0.148 & 825 & 0.015 & 7.35E-04 & $4.79 E-02$ \\
\hline 0.101 & 686 & 0.0814 & 698 & 0.0916 & 715 & 0.010 & $7.00 E-04$ & 7.32E-02 \\
\hline
\end{tabular}




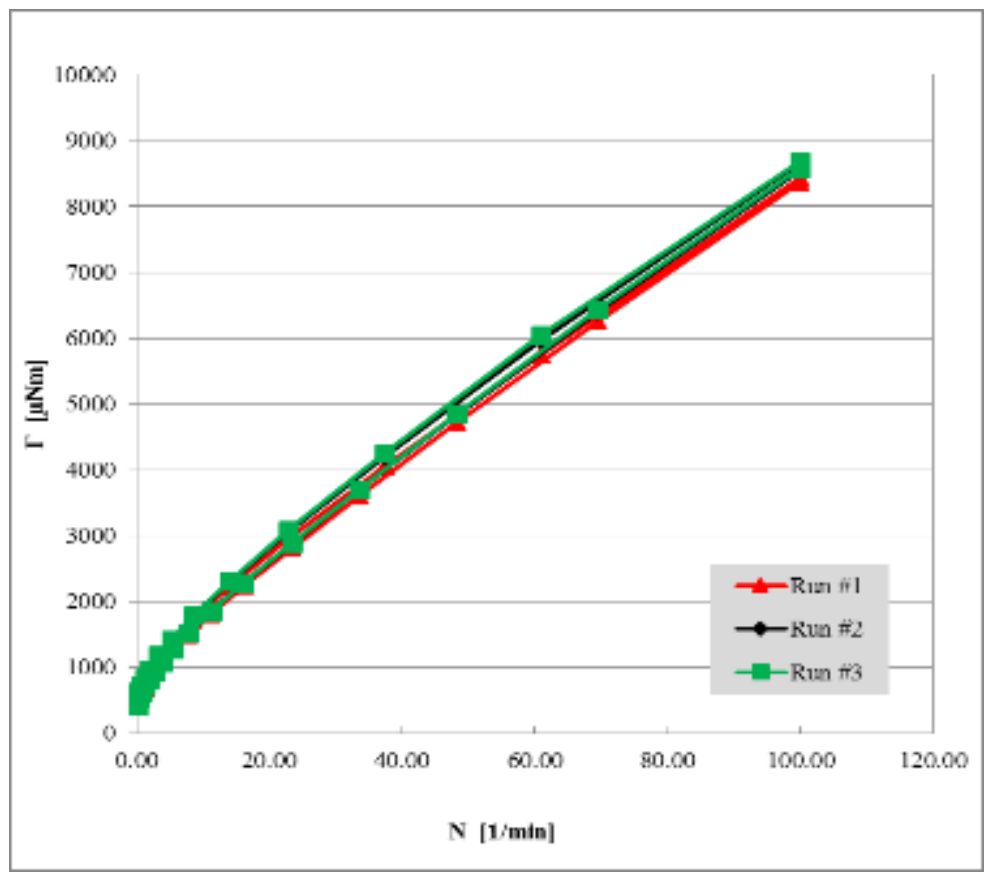

Figure A - 39: Torque vs. Angular Speed using Serrated Coaxial Cylinder on Mix\#10, with 0 \% beads by volume. Portrays data from Table A-53.

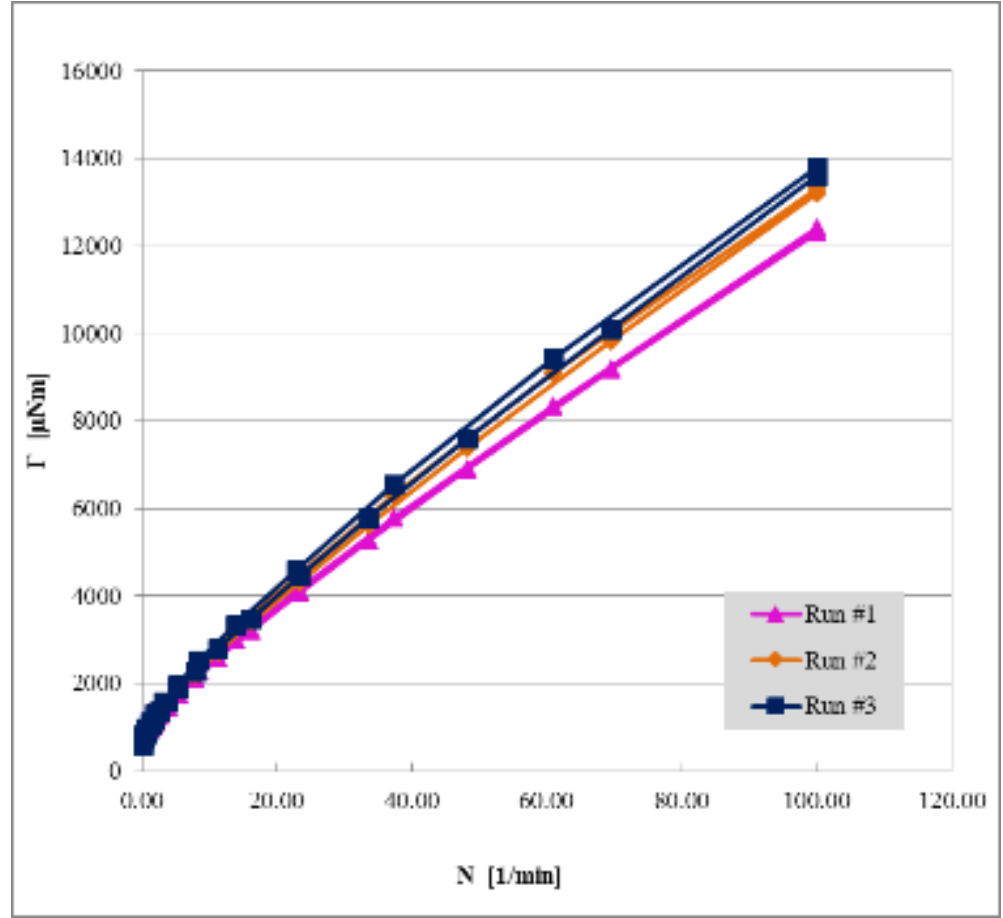

Figure A - 40: Torque vs. Angular Speed using Serrated Coaxial Cylinder on Mix\#10, with $20 \%$ beads by volume. Portrays data from Table A-54. 
Table A - 55: Measured data for Mix\#11 with 0 \% beads using Double spiral spindle.

\begin{tabular}{|c|c|c|c|c|c|c|c|c|}
\hline \multicolumn{6}{|c|}{ Double Spiral } & \multirow{2}{*}{\multicolumn{3}{|c|}{$\begin{array}{c}\text { Bead } \% \\
0 \%\end{array}$}} \\
\hline Geom.: & \multicolumn{3}{|l|}{$R H N-83 A$} & \multicolumn{2}{|r|}{ Z43S } & & & \\
\hline \multicolumn{2}{|c|}{ Run $\# 1$} & \multicolumn{2}{|c|}{ Run \#2 } & \multicolumn{2}{|c|}{ Run \#3 } & \multirow{2}{*}{\multicolumn{3}{|c|}{$\begin{array}{c}\text { Average } \\
\text { values }\end{array}$}} \\
\hline \multicolumn{2}{|c|}{ NIST Code: SMC-113D } & \multicolumn{2}{|c|}{ NIST Code: SMC-113E } & \multicolumn{2}{|c|}{ NIST Code: SMC-113F } & & & \\
\hline $\mathrm{N}$ & $\Gamma$ & $\mathrm{N}$ & $\Gamma$ & $\mathrm{N}$ & $\Gamma$ & $\mathbf{N}$ & $\Gamma$ & $\boldsymbol{\Gamma} / \mathbf{N}$ (Angular \\
\hline $1 / \mathrm{min}$ & $\mu \mathrm{Nm}$ & $1 / \mathrm{min}$ & $\mu \mathrm{Nm}$ & $1 / \mathrm{min}$ & $\mu \mathrm{Nm}$ & $\mathrm{rad} / \mathrm{s}$ & $\mathrm{Nm}$ & momentum) \\
\hline 0.0943 & 1020 & 0.0945 & 959 & 0.0944 & 998 & 0.010 & $9.92 E-04$ & $1.00 E-01$ \\
\hline 0.161 & 1500 & 0.161 & 1430 & 0.161 & 1480 & 0.017 & $1.47 E-03$ & $8.72 E-02$ \\
\hline 0.268 & 1700 & 0.268 & 1640 & 0.268 & 1690 & 0.028 & $1.68 E-03$ & $5.97 E-02$ \\
\hline 0.44 & 1800 & 0.44 & 1760 & 0.44 & 1810 & 0.046 & $1.79 E-03$ & $3.88 E-02$ \\
\hline 0.72 & 1910 & 0.72 & 1880 & 0.72 & 1930 & 0.075 & $1.91 E-03$ & $2.53 E-02$ \\
\hline 1.18 & 2080 & 1.18 & 2050 & 1.18 & 2110 & 0.124 & $2.08 E-03$ & $1.68 E-02$ \\
\hline 1.93 & 2320 & 1.93 & 2300 & 1.93 & 2370 & 0.202 & $2.33 E-03$ & $1.15 E-02$ \\
\hline 3.16 & 2710 & 3.16 & 2710 & 3.16 & 2780 & 0.331 & $2.73 E-03$ & $8.26 E-03$ \\
\hline 5.18 & 3320 & 5.18 & 3330 & 5.18 & 3420 & 0.542 & $3.36 E-03$ & $6.19 E-03$ \\
\hline 8.48 & 4260 & 8.48 & 4290 & 8.48 & 4410 & 0.888 & 4.32E-03 & $4.86 E-03$ \\
\hline 13.9 & 5780 & 13.9 & 5860 & 13.9 & 6000 & 1.456 & $5.88 E-03$ & $4.04 E-03$ \\
\hline 22.8 & 8160 & 22.8 & 8220 & 22.8 & 8470 & 2.388 & $8.28 E-03$ & $3.47 E-03$ \\
\hline 37.3 & 11900 & 37.3 & 12000 & 37.3 & 12400 & 3.906 & $1.21 E-02$ & $3.10 E-03$ \\
\hline 61.1 & 17700 & 61.1 & 18000 & 61.1 & 18500 & 6.398 & $1.81 E-02$ & $2.82 E-03$ \\
\hline 100 & 26800 & 100 & 27200 & 100 & 28100 & 10.472 & $2.74 E-02$ & 2.61E-03 \\
\hline 100 & 26600 & 100 & 27100 & 100 & 27900 & 10.472 & $2.72 E-02$ & $2.60 E-03$ \\
\hline 69.5 & 19400 & 69.5 & 19800 & 69.5 & 20400 & 7.278 & $1.99 E-02$ & $2.73 E-03$ \\
\hline 48.3 & 14400 & 48.3 & 14700 & 48.3 & 15100 & 5.058 & $1.47 E-02$ & $2.91 E-03$ \\
\hline 33.6 & 10800 & 33.6 & 11000 & 33.6 & 11300 & 3.519 & $1.10 E-02$ & $3.14 E-03$ \\
\hline 23.4 & 8190 & 23.4 & 8350 & 23.4 & 8590 & 2.450 & 8.38E-03 & $3.42 E-03$ \\
\hline 16.2 & 6350 & 16.2 & 6470 & 16.2 & 6650 & 1.696 & $6.49 E-03$ & $3.83 E-03$ \\
\hline 11.3 & 5020 & 11.3 & 5110 & 11.3 & 5250 & 1.183 & $5.13 E-03$ & $4.33 E-03$ \\
\hline 7.85 & 4070 & 7.85 & 4140 & 7.85 & 4240 & 0.822 & $4.15 E-03$ & $5.05 E-03$ \\
\hline 5.46 & 3380 & 5.46 & 3430 & 5.46 & 3510 & 0.572 & $3.44 E-03$ & $6.02 E-03$ \\
\hline 3.79 & 2870 & 3.79 & 2920 & 3.79 & 2980 & 0.397 & $2.92 E-03$ & 7.37E-03 \\
\hline 2.64 & 2500 & 2.64 & 2540 & 2.64 & 2590 & 0.276 & $2.54 E-03$ & $9.20 E-03$ \\
\hline 1.83 & 2230 & 1.83 & 2260 & 1.83 & 2300 & 0.192 & $2.26 E-03$ & $1.18 E-02$ \\
\hline 1.27 & 2020 & 1.27 & 2050 & 1.27 & 2090 & 0.133 & $2.05 E-03$ & $1.54 E-02$ \\
\hline 0.886 & 1870 & 0.886 & 1890 & 0.886 & 1930 & 0.093 & $1.90 E-03$ & $2.04 E-02$ \\
\hline 0.615 & 1750 & 0.616 & 1770 & 0.616 & 1800 & 0.064 & $1.77 E-03$ & $2.75 E-02$ \\
\hline 0.428 & 1670 & 0.428 & 1680 & 0.427 & 1720 & 0.045 & $1.69 E-03$ & $3.77 E-02$ \\
\hline 0.3 & 1600 & 0.297 & 1620 & 0.298 & 1650 & 0.031 & $1.62 E-03$ & $5.20 E-02$ \\
\hline 0.206 & 1550 & 0.207 & 1580 & 0.207 & 1580 & 0.022 & $1.57 E-03$ & $7.25 E-02$ \\
\hline 0.144 & 1500 & 0.144 & 1510 & 0.145 & 1540 & 0.015 & $1.52 E-03$ & $1.00 E-01$ \\
\hline 0.0994 & 1470 & 0.0997 & 1480 & 0.101 & 1510 & 0.010 & $1.49 E-03$ & $1.42 E-01$ \\
\hline
\end{tabular}


Table A - 56: Measured data for Mix\#11 with 20 \% beads using Double spiral spindle.

\begin{tabular}{|c|c|c|c|c|c|c|c|c|}
\hline \multicolumn{6}{|c|}{ Double Spiral } & \multirow{2}{*}{\multicolumn{3}{|c|}{$\begin{array}{c}\text { Bead } \% \\
20 \%\end{array}$}} \\
\hline Geom.: & \multicolumn{3}{|l|}{ RHN-83A } & \multicolumn{2}{|r|}{ Z43S } & & & \\
\hline \multicolumn{2}{|c|}{ Run \#1 } & \multicolumn{2}{|c|}{ Run \#2 } & \multicolumn{2}{|c|}{ Run \#3 } & \multirow{2}{*}{\multicolumn{3}{|c|}{$\begin{array}{l}\text { Average } \\
\text { values }\end{array}$}} \\
\hline \multicolumn{2}{|c|}{ NIST Code: SMC-113M } & \multicolumn{2}{|c|}{ NIST Code: SMC-113N } & \multicolumn{2}{|c|}{ NIST Code: SMC-113O } & & & \\
\hline $\mathrm{N}$ & $\Gamma$ & $\mathrm{N}$ & $\Gamma$ & $\mathrm{N}$ & $\Gamma$ & $\mathbf{N}$ & $\Gamma$ & \multirow{2}{*}{$\begin{array}{c}\Gamma / \boldsymbol{N} \text { (Angular } \\
\text { momentum) }\end{array}$} \\
\hline $1 / \mathrm{min}$ & $\mu \mathrm{Nm}$ & $1 / \mathrm{min}$ & $\mu \mathrm{Nm}$ & $1 / \mathrm{min}$ & $\mu \mathrm{Nm}$ & $\mathrm{rad} / \mathrm{s}$ & $\mathrm{Nm}$ & \\
\hline 0.0929 & 1350 & 0.0938 & 1290 & 0.0925 & 1330 & 0.010 & $1.32 E-03$ & $1.36 E-01$ \\
\hline 0.161 & 1900 & 0.16 & 1850 & 0.161 & 1900 & 0.017 & $1.88 E-03$ & $1.12 E-01$ \\
\hline 0.268 & 2120 & 0.268 & 2110 & 0.267 & 2160 & 0.028 & $2.13 E-03$ & $7.60 E-02$ \\
\hline 0.44 & 2300 & 0.439 & 2270 & 0.439 & 2340 & 0.046 & $2.30 E-03$ & $5.01 E-02$ \\
\hline 0.72 & 2500 & 0.72 & 2500 & 0.72 & 2570 & 0.075 & $2.52 E-03$ & $3.35 E-02$ \\
\hline 1.18 & 2840 & 1.18 & 2800 & 1.18 & 2880 & 0.124 & $2.84 E-03$ & $2.30 E-02$ \\
\hline 1.93 & 3320 & 1.93 & 3270 & 1.93 & 3370 & 0.202 & $3.32 E-03$ & $1.64 E-02$ \\
\hline 3.17 & 4090 & 3.16 & 4000 & 3.16 & 4110 & 0.331 & $4.07 E-03$ & $1.23 E-02$ \\
\hline 5.18 & 5320 & 5.18 & 5130 & 5.18 & 5290 & 0.542 & $5.25 E-03$ & $9.67 E-03$ \\
\hline 8.49 & 7210 & 8.48 & 6930 & 8.49 & 7130 & 0.889 & $7.09 E-03$ & $7.98 E-03$ \\
\hline 13.9 & 10000 & 13.9 & 9720 & 13.9 & 10000 & 1.456 & $9.91 E-03$ & $6.81 E-03$ \\
\hline 22.8 & 14000 & 22.8 & 13900 & 22.8 & 14300 & 2.388 & $1.41 E-02$ & $5.89 E-03$ \\
\hline 37.3 & 20800 & 37.3 & 20700 & 37.3 & 21300 & 3.906 & $2.09 E-02$ & $5.36 E-03$ \\
\hline 61 & 31300 & 61.1 & 31300 & 61 & 32300 & 6.391 & $3.16 E-02$ & 4.95E-03 \\
\hline 100 & 47300 & 100 & 47500 & 100 & 49200 & 10.472 & $4.80 E-02$ & $4.58 E-03$ \\
\hline 100 & 46800 & 100 & 47200 & 100 & 48900 & 10.472 & $4.76 E-02$ & $4.55 E-03$ \\
\hline 69.5 & 33900 & 69.5 & 34300 & 69.5 & 35600 & 7.278 & $3.46 E-02$ & $4.75 E-03$ \\
\hline 48.3 & 24800 & 48.3 & 25200 & 48.3 & 26100 & 5.058 & $2.54 E-02$ & $5.02 E-03$ \\
\hline 33.6 & 18400 & 33.6 & 18700 & 33.6 & 19400 & 3.519 & $1.88 E-02$ & $5.35 E-03$ \\
\hline 23.4 & 13800 & 23.4 & 14000 & 23.4 & 14500 & 2.450 & $1.41 E-02$ & $5.75 E-03$ \\
\hline 16.2 & 10500 & 16.2 & 10700 & 16.2 & 11100 & 1.696 & $1.08 E-02$ & $6.35 E-03$ \\
\hline 11.3 & 8120 & 11.3 & 8290 & 11.3 & 8570 & 1.183 & 8.33E-03 & 7.04E-03 \\
\hline 7.84 & 6430 & 7.85 & 6540 & 7.85 & 6760 & 0.822 & $6.58 E-03$ & 8.00E-03 \\
\hline 5.46 & 5210 & 5.45 & 5320 & 5.46 & 5470 & 0.571 & $5.33 E-03$ & $9.33 E-03$ \\
\hline 3.79 & 4300 & 3.8 & 4400 & 3.79 & 4530 & 0.397 & $4.41 E-03$ & $1.11 E-02$ \\
\hline 2.64 & 3650 & 2.64 & 3730 & 2.64 & 3840 & 0.276 & $3.74 E-03$ & $1.35 E-02$ \\
\hline 1.83 & 3180 & 1.83 & 3240 & 1.83 & 3330 & 0.192 & $3.25 E-03$ & $1.70 E-02$ \\
\hline 1.27 & 2810 & 1.27 & 2860 & 1.27 & 2950 & 0.133 & $2.87 E-03$ & $2.16 E-02$ \\
\hline 0.887 & 2540 & 0.885 & 2590 & 0.887 & 2650 & 0.093 & $2.59 E-03$ & $2.79 E-02$ \\
\hline 0.618 & 2330 & 0.615 & 2380 & 0.615 & 2440 & 0.065 & $2.38 E-03$ & 3.69E-02 \\
\hline 0.428 & 2160 & 0.43 & 2220 & 0.428 & 2280 & 0.045 & $2.22 E-03$ & $4.95 E-02$ \\
\hline 0.298 & 2040 & 0.297 & 2090 & 0.297 & 2150 & 0.031 & $2.09 E-03$ & $6.72 E-02$ \\
\hline 0.206 & 1970 & 0.208 & 2000 & 0.207 & 2040 & 0.022 & $2.00 E-03$ & $9.24 E-02$ \\
\hline 0.144 & 1900 & 0.144 & 1930 & 0.143 & 1960 & 0.015 & $1.93 E-03$ & $1.28 E-01$ \\
\hline 0.102 & 1840 & 0.0996 & 1880 & 0.1 & 1920 & 0.011 & $1.88 E-03$ & $1.79 E-01$ \\
\hline
\end{tabular}




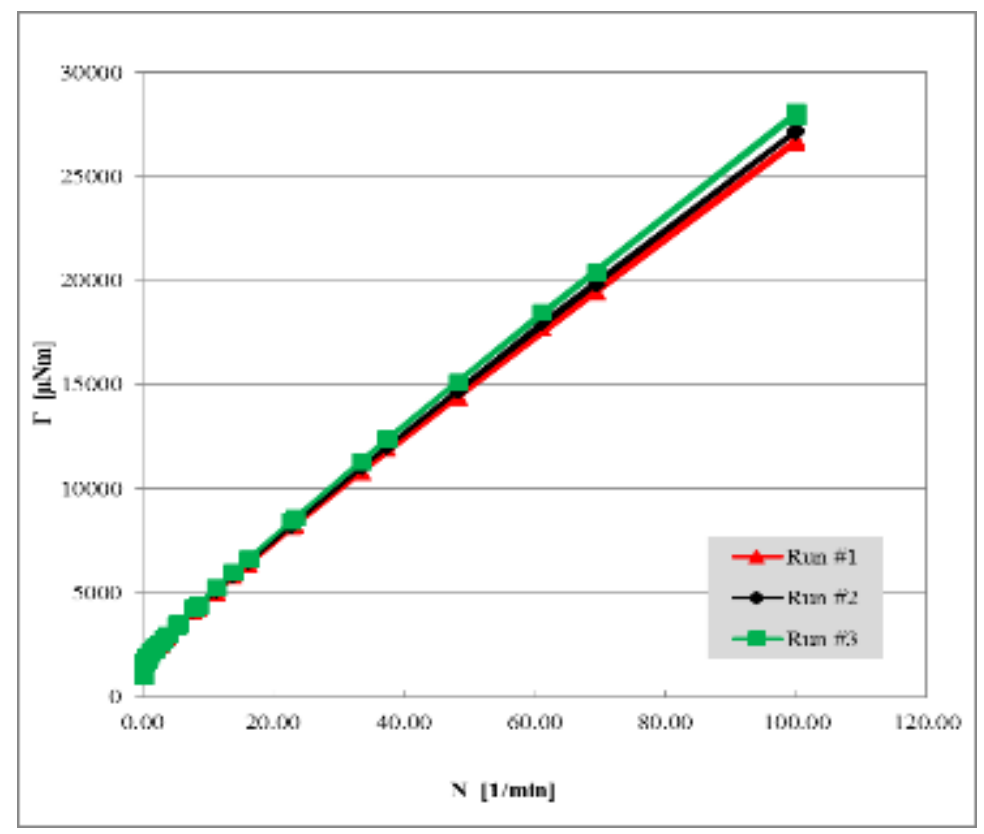

Figure A - 41: Torque vs. Angular Speed using Double spiral spindle on Mix\#11, with $0 \%$ beads by volume. Portrays data from Table A-55.

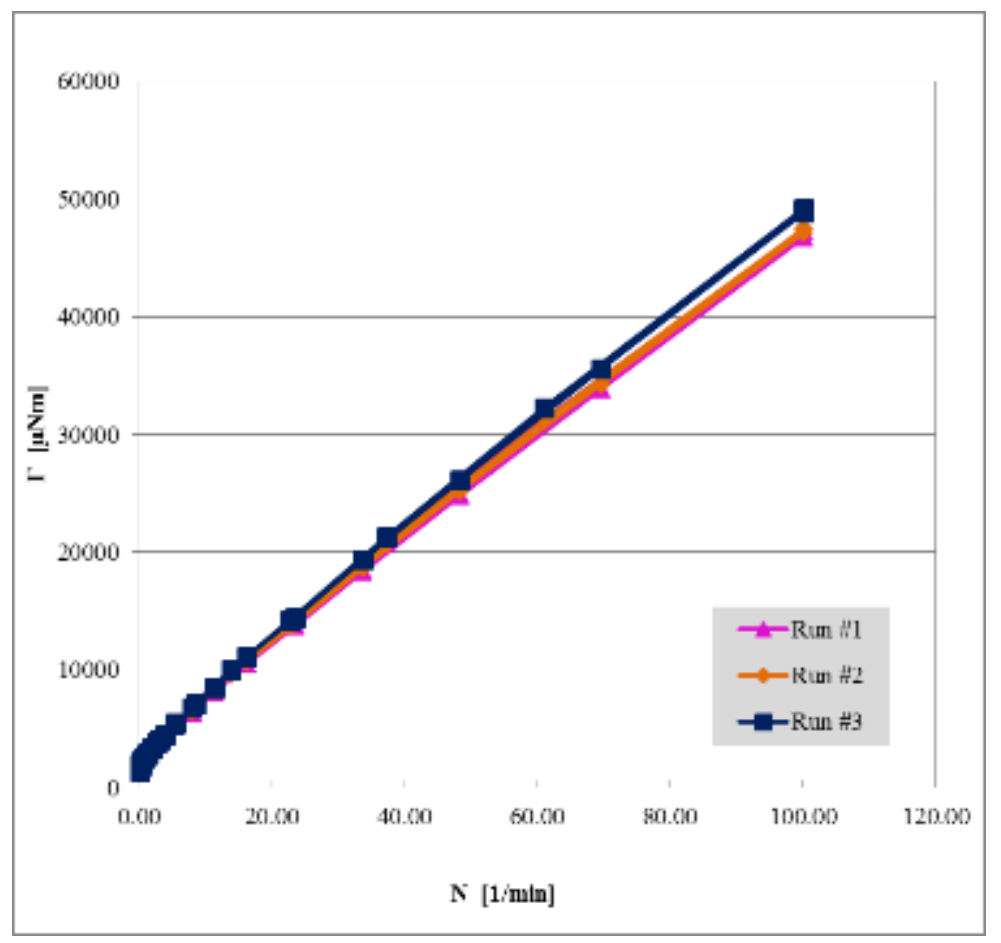

Figure A - 42: Torque vs. Angular Speed using Double spiral spindle on Mix\#11, with 20 \% beads by volume. Portrays data from Table A-56. 
Table A - 57: Measured data for Mix\#11 with 0 \% beads using Six-blade Vane.

\begin{tabular}{|c|c|c|c|c|c|c|c|c|}
\hline \multicolumn{6}{|c|}{6 Blade Vane } & \multicolumn{3}{|c|}{$\begin{array}{c}\text { Bead } \% \\
0 \%\end{array}$} \\
\hline \multicolumn{2}{|c|}{ Run \#1 } & \multicolumn{2}{|c|}{ Run \#2 } & \multicolumn{2}{|c|}{ Run \#3 } & \multirow{2}{*}{\multicolumn{3}{|c|}{$\begin{array}{c}\text { Average } \\
\text { values }\end{array}$}} \\
\hline NIST Code: & $S M C-113 A$ & NIST Code: & $S M C-113 B$ & NIST Cod & $C-113 C$ & & & \\
\hline $\mathrm{N}$ & $\Gamma$ & $\mathrm{N}$ & $\Gamma$ & $\mathrm{N}$ & $\Gamma$ & $\mathbf{N}$ & $\Gamma$ & $\boldsymbol{\Gamma} / \mathbf{N}$ (Angular \\
\hline $1 / \mathrm{min}$ & $\mu \mathrm{Nm}$ & $1 / \mathrm{min}$ & $\mu \mathrm{Nm}$ & $1 / \mathrm{min}$ & $\mu \mathrm{Nm}$ & $\mathrm{rad} / \mathrm{s}$ & $\mathrm{Nm}$ & momentum) \\
\hline 0.0987 & 441 & 0.0977 & 415 & 0.0978 & 412 & 0.010 & $4.23 E-04$ & 4.12E-02 \\
\hline 0.164 & 512 & 0.163 & 563 & 0.163 & 559 & 0.017 & $5.45 E-04$ & $3.18 E-02$ \\
\hline 0.268 & 545 & 0.268 & 602 & 0.268 & 602 & 0.028 & $5.83 E-04$ & $2.08 E-02$ \\
\hline 0.439 & 583 & 0.439 & 633 & 0.439 & 634 & 0.046 & $6.17 E-04$ & $1.34 E-02$ \\
\hline 0.72 & 650 & 0.72 & 682 & 0.72 & 683 & 0.075 & $6.72 E-04$ & 8.91E-03 \\
\hline 1.18 & 713 & 1.18 & 751 & 1.18 & 753 & 0.124 & 7.39E-04 & $5.98 E-03$ \\
\hline 1.93 & 805 & 1.93 & 847 & 1.93 & 849 & 0.202 & 8.34E-04 & $4.12 E-03$ \\
\hline 3.16 & 933 & 3.16 & 982 & 3.16 & 990 & 0.331 & $9.68 E-04$ & $2.93 E-03$ \\
\hline 5.18 & 1120 & 5.18 & 1180 & 5.18 & 1180 & 0.542 & $1.16 E-03$ & $2.14 E-03$ \\
\hline 8.48 & 1390 & 8.48 & 1460 & 8.48 & 1470 & 0.888 & $1.44 E-03$ & $1.62 E-03$ \\
\hline 13.9 & 1790 & 13.9 & 1870 & 13.9 & 1890 & 1.456 & $1.85 E-03$ & $1.27 E-03$ \\
\hline 22.8 & 2380 & 22.8 & 2470 & 22.8 & 2510 & 2.388 & $2.45 E-03$ & $1.03 E-03$ \\
\hline 37.3 & 3290 & 37.3 & 3400 & 37.3 & 3450 & 3.906 & $3.38 E-03$ & 8.65E-04 \\
\hline 61 & 4670 & 61 & 4820 & 61 & 4900 & 6.388 & $4.80 E-03$ & $7.51 E-04$ \\
\hline 100 & 6850 & 100 & 7050 & 100 & 7160 & 10.472 & $7.02 E-03$ & $6.70 E-04$ \\
\hline 100 & 6860 & 100 & 7030 & 100 & 7170 & 10.472 & $7.02 E-03$ & $6.70 E-04$ \\
\hline 69.5 & 5150 & 69.5 & 5270 & 69.5 & 5370 & 7.278 & $5.26 E-03$ & 7.23E-04 \\
\hline 48.3 & 3930 & 48.3 & 4010 & 48.3 & 4090 & 5.058 & $4.01 E-03$ & $7.93 E-04$ \\
\hline 33.6 & 3050 & 33.6 & 3110 & 33.6 & 3160 & 3.519 & $3.11 E-03$ & $8.83 E-04$ \\
\hline 23.4 & 2410 & 23.4 & 2450 & 23.4 & 2490 & 2.450 & $2.45 E-03$ & $1.00 E-03$ \\
\hline 16.2 & 1950 & 16.2 & 1980 & 16.2 & 2010 & 1.696 & $1.98 E-03$ & $1.17 E-03$ \\
\hline 11.3 & 1600 & 11.3 & 1630 & 11.3 & 1650 & 1.183 & $1.63 E-03$ & $1.37 E-03$ \\
\hline 7.85 & 1340 & 7.85 & 1360 & 7.85 & 1380 & 0.822 & $1.36 E-03$ & $1.65 E-03$ \\
\hline 5.45 & 1140 & 5.46 & 1160 & 5.46 & 1170 & 0.571 & $1.16 E-03$ & $2.02 E-03$ \\
\hline 3.79 & 989 & 3.79 & 1010 & 3.79 & 1020 & 0.397 & $1.01 E-03$ & $2.54 E-03$ \\
\hline 2.64 & 872 & 2.64 & 885 & 2.64 & 894 & 0.276 & $8.84 E-04$ & $3.20 E-03$ \\
\hline 1.83 & 788 & 1.83 & 793 & 1.83 & 809 & 0.192 & 7.97E-04 & $4.16 E-03$ \\
\hline 1.28 & 717 & 1.27 & 730 & 1.27 & 733 & 0.133 & $7.27 E-04$ & $5.45 E-03$ \\
\hline 0.887 & 669 & 0.887 & 669 & 0.882 & 680 & 0.093 & $6.73 E-04$ & $7.26 E-03$ \\
\hline 0.618 & 621 & 0.616 & 626 & 0.618 & 630 & 0.065 & $6.26 E-04$ & $9.68 E-03$ \\
\hline 0.424 & 582 & 0.428 & 590 & 0.426 & 604 & 0.045 & $5.92 E-04$ & $1.33 E-02$ \\
\hline 0.298 & 567 & 0.3 & 564 & 0.298 & 585 & 0.031 & $5.72 E-04$ & $1.83 E-02$ \\
\hline 0.207 & 535 & 0.207 & 544 & 0.205 & 553 & 0.022 & $5.44 E-04$ & $2.52 E-02$ \\
\hline 0.142 & 543 & 0.141 & 534 & 0.138 & 548 & 0.015 & $5.42 E-04$ & $3.69 E-02$ \\
\hline 0.0994 & 496 & 0.101 & 503 & 0.101 & 530 & 0.011 & $5.10 E-04$ & $4.84 E-02$ \\
\hline
\end{tabular}


Table A - 58: Measured data for Mix\#11 with 20 \% beads using Six-blade Vane.

\begin{tabular}{|c|c|c|c|c|c|c|c|c|}
\hline \multicolumn{6}{|c|}{6 Blade Vane } & \multicolumn{3}{|c|}{$\begin{array}{c}\text { Bead \% } \\
20 \%\end{array}$} \\
\hline \multirow{2}{*}{\multicolumn{2}{|c|}{ Run \#1 }} & \multirow{2}{*}{\multicolumn{2}{|c|}{ Run \#2 }} & \multicolumn{2}{|c|}{ Z43S } & \multirow{3}{*}{\multicolumn{3}{|c|}{$\begin{array}{c}\text { Average } \\
\text { values }\end{array}$}} \\
\hline & & & & \multicolumn{2}{|c|}{ Run \#3 } & & & \\
\hline NIST Code: & $S M C-113 \mathrm{~J}$ & NIST Code & MC-113K & NIST Coc & $1 \mathrm{C}-113 \mathrm{~L}$ & & & \\
\hline $\mathrm{N}$ & $\Gamma$ & $\mathrm{N}$ & $\Gamma$ & $\mathrm{N}$ & $\Gamma$ & $\mathbf{N}$ & $\Gamma$ & $\boldsymbol{\Gamma} / \mathbf{N}$ (Angular \\
\hline $1 / \mathrm{min}$ & $\mu \mathrm{Nm}$ & $1 / \mathrm{min}$ & $\mu \mathrm{Nm}$ & $1 / \mathrm{min}$ & $\mu \mathrm{Nm}$ & $\mathrm{rad} / \mathrm{s}$ & $\mathrm{Nm}$ & momentum) \\
\hline 0.0976 & 585 & 0.0975 & 570 & 0.0973 & 590 & 0.010 & $5.82 E-04$ & $5.70 E-02$ \\
\hline 0.163 & 732 & 0.163 & 719 & 0.163 & 752 & 0.017 & $7.34 E-04$ & $4.30 E-02$ \\
\hline 0.268 & 791 & 0.268 & 775 & 0.268 & 807 & 0.028 & 7.91E-04 & $2.82 E-02$ \\
\hline 0.44 & 844 & 0.44 & 839 & 0.44 & 868 & 0.046 & $8.50 E-04$ & $1.85 E-02$ \\
\hline 0.72 & 927 & 0.719 & 921 & 0.719 & 955 & 0.075 & $9.34 E-04$ & $1.24 E-02$ \\
\hline 1.18 & 1040 & 1.18 & 1040 & 1.18 & 1070 & 0.124 & $1.05 E-03$ & $8.50 E-03$ \\
\hline 1.93 & 1190 & 1.93 & 1210 & 1.93 & 1250 & 0.202 & $1.22 E-03$ & $6.02 E-03$ \\
\hline 3.16 & 1410 & 3.16 & 1440 & 3.16 & 1480 & 0.331 & $1.44 E-03$ & 4.36E-03 \\
\hline 5.18 & 1730 & 5.18 & 1800 & 5.18 & 1850 & 0.542 & $1.79 E-03$ & $3.31 E-03$ \\
\hline 8.48 & 2210 & 8.48 & 2300 & 8.48 & 2360 & 0.888 & $2.29 E-03$ & $2.58 E-03$ \\
\hline 13.9 & 2940 & 13.9 & 3060 & 13.9 & 3150 & 1.456 & $3.05 E-03$ & $2.10 E-03$ \\
\hline 22.8 & 4050 & 22.8 & 4220 & 22.8 & 4310 & 2.388 & 4.19E-03 & $1.76 E-03$ \\
\hline 37.3 & 5660 & 37.3 & 5950 & 37.3 & 6030 & 3.906 & $5.88 E-03$ & $1.51 E-03$ \\
\hline 61.1 & 8060 & 61 & 8550 & 61 & 8650 & 6.391 & $8.42 E-03$ & $1.32 E-03$ \\
\hline 100 & 11600 & 100 & 12400 & 100 & 12700 & 10.472 & $1.22 E-02$ & $1.17 E-03$ \\
\hline 100 & 11500 & 100 & 12300 & 100 & 12600 & 10.472 & $1.21 E-02$ & $1.16 E-03$ \\
\hline 69.5 & 8590 & 69.5 & 9180 & 69.5 & 9370 & 7.278 & $9.05 E-03$ & $1.24 E-03$ \\
\hline 48.3 & 6490 & 48.3 & 6880 & 48.3 & 7020 & 5.058 & $6.80 E-03$ & $1.34 E-03$ \\
\hline 33.6 & 4980 & 33.6 & 5240 & 33.6 & 5330 & 3.519 & $5.18 E-03$ & $1.47 E-03$ \\
\hline 23.4 & 3900 & 23.4 & 4040 & 23.3 & 4130 & 2.447 & $4.02 E-03$ & $1.64 E-03$ \\
\hline 16.2 & 3090 & 16.2 & 3200 & 16.2 & 3240 & 1.696 & $3.18 E-03$ & $1.87 E-03$ \\
\hline 11.3 & 2480 & 11.3 & 2580 & 11.3 & 2600 & 1.183 & $2.55 E-03$ & $2.16 E-03$ \\
\hline 7.85 & 2030 & 7.85 & 2080 & 7.85 & 2110 & 0.822 & $2.07 E-03$ & $2.52 E-03$ \\
\hline 5.46 & 1690 & 5.45 & 1740 & 5.46 & 1750 & 0.571 & $1.73 E-03$ & $3.02 E-03$ \\
\hline 3.79 & 1430 & 3.79 & 1470 & 3.79 & 1480 & 0.397 & $1.46 E-03$ & $3.68 E-03$ \\
\hline 2.64 & 1240 & 2.64 & 1260 & 2.64 & 1270 & 0.276 & $1.26 E-03$ & $4.55 E-03$ \\
\hline 1.83 & 1090 & 1.83 & 1120 & 1.83 & 1130 & 0.192 & $1.11 E-03$ & $5.81 E-03$ \\
\hline 1.27 & 986 & 1.28 & 1010 & 1.27 & 996 & 0.133 & 9.97E-04 & $7.48 E-03$ \\
\hline 0.885 & 889 & 0.887 & 898 & 0.886 & 908 & 0.093 & $8.98 E-04$ & $9.68 E-03$ \\
\hline 0.615 & 837 & 0.613 & 840 & 0.612 & 847 & 0.064 & $8.41 E-04$ & $1.31 E-02$ \\
\hline 0.425 & 768 & 0.431 & 788 & 0.43 & 789 & 0.045 & 7.82E-04 & $1.74 E-02$ \\
\hline 0.297 & 747 & 0.296 & 741 & 0.297 & 747 & 0.031 & $7.45 E-04$ & $2.40 E-02$ \\
\hline 0.205 & 705 & 0.209 & 709 & 0.206 & 724 & 0.022 & $7.13 E-04$ & $3.29 E-02$ \\
\hline 0.143 & 676 & 0.145 & 689 & 0.144 & 694 & 0.015 & $6.86 E-04$ & $4.55 E-02$ \\
\hline 0.0996 & 639 & 0.0993 & 656 & 0.0999 & 668 & 0.010 & $6.54 E-04$ & $6.27 E-02$ \\
\hline
\end{tabular}




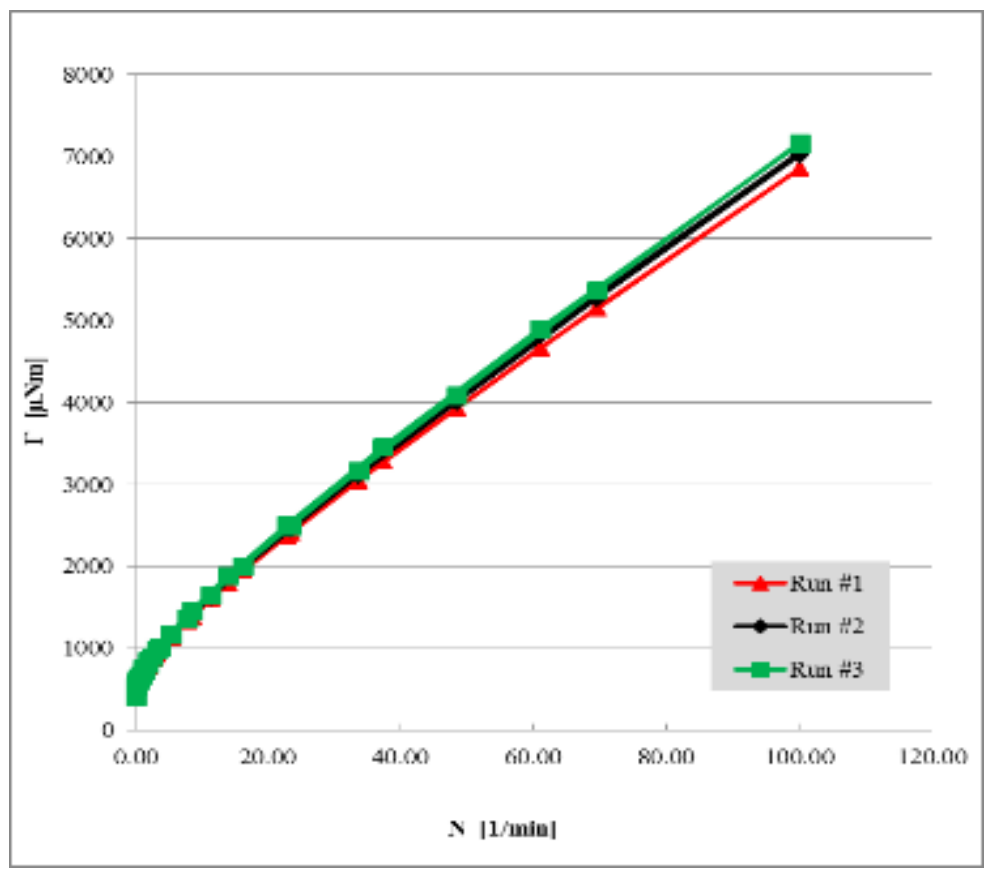

Figure A - 57: Torque vs. Angular Speed using sixblade vane on Mix\#11, with $0 \%$ beads by volume. Portrays data from Table A-57.

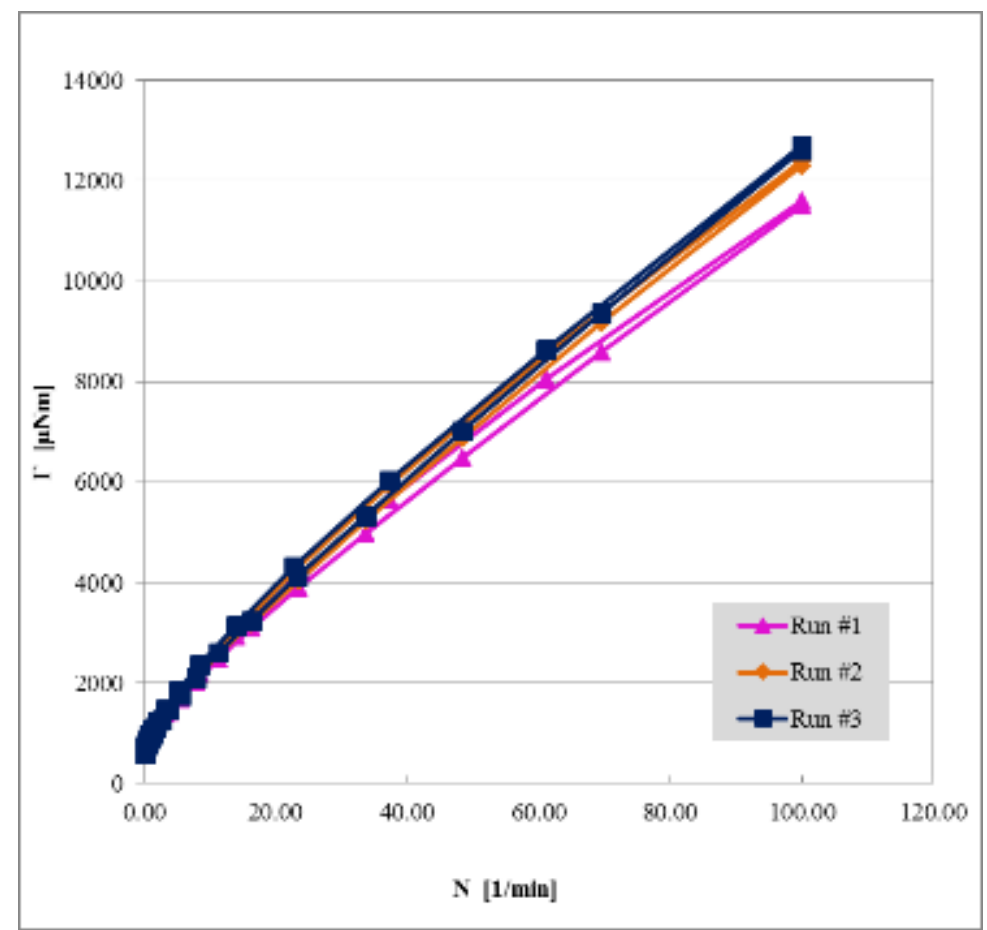

Figure A - 58: Torque vs. Angular Speed using sixblade vane on Mix\#11, with $20 \%$ beads by volume. Portrays data from Table A-58. 
Table A - 59: Measured data for Mix\#11 with 0 \% beads using Serrated Coaxial Cylinder.

\begin{tabular}{|c|c|c|c|c|c|c|c|c|}
\hline \multicolumn{6}{|c|}{ Serrated Coaxial Cylinder } & \multirow{2}{*}{\multicolumn{3}{|c|}{$\begin{array}{c}\text { Bead } \% \\
0 \%\end{array}$}} \\
\hline Geom.: & SS18 & & & \multicolumn{2}{|c|}{ Z43S } & & & \\
\hline \multicolumn{2}{|c|}{ Run \#1 } & \multicolumn{2}{|c|}{ Run \#2 } & \multicolumn{2}{|c|}{ Run \#3 } & \multirow{2}{*}{\multicolumn{3}{|c|}{$\begin{array}{c}\text { Average } \\
\text { values }\end{array}$}} \\
\hline \multicolumn{2}{|c|}{ NIST Code: SMC-113G } & \multicolumn{2}{|c|}{ NIST Code: SMC-113H } & \multicolumn{2}{|c|}{ NIST Code: SMC-113I } & & & \\
\hline $\mathrm{N}$ & $\Gamma$ & $\mathrm{N}$ & $\Gamma$ & $\mathrm{N}$ & $\Gamma$ & $\mathbf{N}$ & $\Gamma$ & $\Gamma / N$ (Angular \\
\hline $1 / \mathrm{min}$ & $\mu \mathrm{Nm}$ & $1 / \mathrm{min}$ & $\mu \mathrm{Nm}$ & $1 / \mathrm{min}$ & $\mu \mathrm{Nm}$ & $\mathrm{rad} / \mathrm{s}$ & $\mathrm{Nm}$ & momentum) \\
\hline 0.0978 & 444 & 0.0984 & 436 & 0.0983 & 426 & 0.010 & $4.35 E-04$ & $4.23 E-02$ \\
\hline 0.164 & 598 & 0.164 & 561 & 0.164 & 549 & 0.017 & $5.69 E-04$ & $3.32 E-02$ \\
\hline 0.268 & 638 & 0.269 & 615 & 0.268 & 618 & 0.028 & $6.24 E-04$ & $2.22 E-02$ \\
\hline 0.439 & 682 & 0.44 & 669 & 0.439 & 676 & 0.046 & $6.76 E-04$ & $1.47 E-02$ \\
\hline 0.72 & 746 & 0.721 & 749 & 0.72 & 745 & 0.075 & 7.47E-04 & $9.90 E-03$ \\
\hline 1.18 & 839 & 1.18 & 857 & 1.18 & 853 & 0.124 & 8.50E-04 & $6.88 E-03$ \\
\hline 1.93 & 959 & 1.93 & 983 & 1.93 & 985 & 0.202 & $9.76 E-04$ & 4.83E-03 \\
\hline 3.16 & 1140 & 3.16 & 1160 & 3.16 & 1170 & 0.331 & $1.16 E-03$ & $3.50 E-03$ \\
\hline 5.18 & 1370 & 5.18 & 1420 & 5.18 & 1430 & 0.542 & $1.41 E-03$ & $2.59 E-03$ \\
\hline 8.48 & 1710 & 8.48 & 1770 & 8.48 & 1800 & 0.888 & $1.76 E-03$ & $1.98 E-03$ \\
\hline 13.9 & 2200 & 13.9 & 2270 & 13.9 & 2320 & 1.456 & $2.26 E-03$ & $1.55 E-03$ \\
\hline 22.8 & 2910 & 22.8 & 3020 & 22.8 & 3100 & 2.388 & $3.01 E-03$ & $1.26 E-03$ \\
\hline 37.3 & 4020 & 37.3 & 4160 & 37.3 & 4280 & 3.906 & $4.15 E-03$ & $1.06 E-03$ \\
\hline 61 & 5700 & 61 & 5930 & 61 & 6070 & 6.388 & $5.90 E-03$ & $9.24 E-04$ \\
\hline 100 & 8290 & 100 & 8580 & 100 & 8740 & 10.472 & $8.54 E-03$ & $8.15 E-04$ \\
\hline 100 & 8180 & 100 & 8460 & 100 & 8620 & 10.472 & $8.42 E-03$ & $8.04 E-04$ \\
\hline 69.5 & 6120 & 69.5 & 6340 & 69.5 & 6460 & 7.278 & $6.31 E-03$ & $8.67 E-04$ \\
\hline 48.3 & 4620 & 48.3 & 4770 & 48.3 & 4860 & 5.058 & $4.75 E-03$ & $9.39 E-04$ \\
\hline 33.6 & 3530 & 33.6 & 3640 & 33.6 & 3710 & 3.519 & $3.63 E-03$ & $1.03 E-03$ \\
\hline 23.4 & 2750 & 23.4 & 2830 & 23.4 & 2880 & 2.450 & $2.82 E-03$ & $1.15 E-03$ \\
\hline 16.2 & 2180 & 16.2 & 2250 & 16.2 & 2280 & 1.696 & $2.24 E-03$ & $1.32 E-03$ \\
\hline 11.3 & 1760 & 11.3 & 1820 & 11.3 & 1840 & 1.183 & $1.81 E-03$ & $1.53 E-03$ \\
\hline 7.85 & 1460 & 7.85 & 1500 & 7.85 & 1520 & 0.822 & $1.49 E-03$ & $1.82 E-03$ \\
\hline 5.45 & 1210 & 5.46 & 1260 & 5.45 & 1280 & 0.571 & $1.25 E-03$ & $2.19 E-03$ \\
\hline 3.79 & 1030 & 3.79 & 1090 & 3.79 & 1090 & 0.397 & $1.07 E-03$ & $2.70 E-03$ \\
\hline 2.64 & 882 & 2.63 & 930 & 2.64 & 943 & 0.276 & $9.18 E-04$ & 3.33E-03 \\
\hline 1.82 & 792 & 1.83 & 810 & 1.83 & 831 & 0.191 & $8.11 E-04$ & $4.24 E-03$ \\
\hline 1.27 & 716 & 1.27 & 727 & 1.28 & 741 & 0.133 & $7.28 E-04$ & $5.46 E-03$ \\
\hline 0.887 & 609 & 0.885 & 670 & 0.887 & 663 & 0.093 & $6.47 E-04$ & $6.97 E-03$ \\
\hline 0.614 & 567 & 0.623 & 619 & 0.616 & 605 & 0.065 & $5.97 E-04$ & $9.23 E-03$ \\
\hline 0.425 & 595 & 0.428 & 537 & 0.432 & 571 & 0.045 & $5.68 E-04$ & $1.27 E-02$ \\
\hline 0.297 & 511 & 0.297 & 514 & 0.306 & 598 & 0.031 & $5.41 E-04$ & $1.72 E-02$ \\
\hline 0.217 & 547 & 0.208 & 587 & 0.193 & 587 & 0.022 & $5.74 E-04$ & $2.66 E-02$ \\
\hline 0.143 & 576 & 0.141 & 497 & 0.113 & 594 & 0.014 & $5.56 E-04$ & $4.01 E-02$ \\
\hline 0.1 & 522 & 0.0819 & 517 & 0.102 & 452 & 0.010 & $4.97 E-04$ & $5.02 E-02$ \\
\hline
\end{tabular}


Table A - 60: Measured data for Mix\#11 with 20 \% beads using Serrated Coaxial Cylinder.

\begin{tabular}{|c|c|c|c|c|c|c|c|c|}
\hline \multicolumn{6}{|c|}{ Serrated Coaxial Cylinder } & \multirow{2}{*}{\multicolumn{3}{|c|}{$\begin{array}{c}\text { Bead } \% \\
20 \%\end{array}$}} \\
\hline Geom.: & SS18 & & & \multicolumn{2}{|c|}{ Z43S } & & & \\
\hline \multicolumn{2}{|c|}{ Run \#1 } & \multicolumn{2}{|c|}{ Run \#2 } & \multicolumn{2}{|c|}{ Run \#3 } & \multirow{2}{*}{\multicolumn{3}{|c|}{$\begin{array}{c}\text { Average } \\
\text { values }\end{array}$}} \\
\hline NIST Code: & $S M C-113 P$ & NIST Cod & $C-113 Q$ & NIST Co & $S M C-113 R$ & & & \\
\hline $\mathrm{N}$ & $\Gamma$ & $\mathrm{N}$ & $\Gamma$ & $\mathrm{N}$ & $\Gamma$ & $\mathbf{N}$ & $\Gamma$ & $\Gamma / N$ (Angular \\
\hline $1 / \mathrm{min}$ & $\mu \mathrm{Nm}$ & $1 / \mathrm{min}$ & $\mu \mathrm{Nm}$ & $1 / \mathrm{min}$ & $\mu \mathrm{Nm}$ & $\mathrm{rad} / \mathrm{s}$ & $\mathrm{Nm}$ & momentum) \\
\hline 0.0975 & 667 & 0.0982 & 609 & 0.0982 & 617 & 0.010 & $6.31 E-04$ & $6.15 E-02$ \\
\hline 0.164 & 814 & 0.164 & 716 & 0.164 & 748 & 0.017 & $7.59 E-04$ & $4.42 E-02$ \\
\hline 0.269 & 818 & 0.268 & 781 & 0.268 & 818 & 0.028 & $8.06 E-04$ & 2.87E-02 \\
\hline 0.44 & 862 & 0.439 & 855 & 0.439 & 904 & 0.046 & $8.74 E-04$ & $1.90 E-02$ \\
\hline 0.72 & 948 & 0.719 & 968 & 0.72 & 1010 & 0.075 & $9.75 E-04$ & $1.29 E-02$ \\
\hline 1.18 & 1070 & 1.18 & 1110 & 1.18 & 1160 & 0.124 & $1.11 E-03$ & $9.01 E-03$ \\
\hline 1.93 & 1260 & 1.93 & 1320 & 1.93 & 1370 & 0.202 & $1.32 E-03$ & $6.51 E-03$ \\
\hline 3.16 & 1520 & 3.16 & 1600 & 3.16 & 1660 & 0.331 & $1.59 E-03$ & $4.81 E-03$ \\
\hline 5.18 & 1890 & 5.18 & 2010 & 5.18 & 2080 & 0.542 & $1.99 E-03$ & $3.67 E-03$ \\
\hline 8.48 & 2420 & 8.48 & 2590 & 8.48 & 2690 & 0.888 & $2.57 E-03$ & $2.89 E-03$ \\
\hline 13.9 & 3200 & 13.9 & 3430 & 13.9 & 3580 & 1.456 & $3.40 E-03$ & $2.34 E-03$ \\
\hline 22.8 & 4370 & 22.8 & 4710 & 22.8 & 4910 & 2.388 & $4.66 E-03$ & $1.95 E-03$ \\
\hline 37.3 & 6170 & 37.3 & 6620 & 37.3 & 6950 & 3.906 & $6.58 E-03$ & $1.68 E-03$ \\
\hline 61 & 8860 & 61 & 9470 & 61 & 9930 & 6.388 & $9.42 E-03$ & $1.47 E-03$ \\
\hline 100 & 13100 & 100 & 13900 & 100 & 14600 & 10.472 & $1.39 E-02$ & $1.32 E-03$ \\
\hline 100 & 13000 & 100 & 13800 & 100 & 14500 & 10.472 & $1.38 E-02$ & $1.31 E-03$ \\
\hline 69.5 & 9770 & 69.5 & 10300 & 69.5 & 10800 & 7.278 & $1.03 E-02$ & $1.41 E-03$ \\
\hline 48.3 & 7350 & 48.3 & 7720 & 48.3 & 8080 & 5.058 & 7.72E-03 & $1.53 E-03$ \\
\hline 33.6 & 5580 & 33.6 & 5860 & 33.6 & 6130 & 3.519 & $5.86 E-03$ & $1.66 E-03$ \\
\hline 23.4 & 4300 & 23.4 & 4500 & 23.4 & 4720 & 2.450 & $4.51 E-03$ & $1.84 E-03$ \\
\hline 16.2 & 3360 & 16.2 & 3520 & 16.2 & 3680 & 1.696 & $3.52 E-03$ & $2.07 E-03$ \\
\hline 11.3 & 2680 & 11.3 & 2810 & 11.3 & 2930 & 1.183 & $2.81 E-03$ & $2.37 E-03$ \\
\hline 7.85 & 2180 & 7.85 & 2280 & 7.85 & 2370 & 0.822 & $2.28 E-03$ & $2.77 E-03$ \\
\hline 5.46 & 1790 & 5.45 & 1880 & 5.46 & 1960 & 0.571 & $1.88 E-03$ & $3.28 E-03$ \\
\hline 3.79 & 1510 & 3.79 & 1590 & 3.79 & 1650 & 0.397 & $1.58 E-03$ & $3.99 E-03$ \\
\hline 2.64 & 1300 & 2.64 & 1370 & 2.64 & 1410 & 0.276 & $1.36 E-03$ & $4.92 E-03$ \\
\hline 1.84 & 1140 & 1.83 & 1170 & 1.83 & 1220 & 0.192 & $1.18 E-03$ & $6.13 E-03$ \\
\hline 1.27 & 986 & 1.27 & 1030 & 1.28 & 1140 & 0.133 & $1.05 E-03$ & $7.89 E-03$ \\
\hline 0.885 & 931 & 0.888 & 951 & 0.878 & 980 & 0.093 & $9.54 E-04$ & $1.03 E-02$ \\
\hline 0.624 & 817 & 0.613 & 869 & 0.613 & 943 & 0.065 & $8.76 E-04$ & $1.36 E-02$ \\
\hline 0.43 & 756 & 0.431 & 800 & 0.419 & 857 & 0.045 & $8.04 E-04$ & $1.80 E-02$ \\
\hline 0.296 & 717 & 0.281 & 813 & 0.291 & 783 & 0.030 & $7.71 E-04$ & $2.54 E-02$ \\
\hline 0.212 & 793 & 0.187 & 928 & 0.198 & 908 & 0.021 & $8.76 E-04$ & $4.21 E-02$ \\
\hline 0.138 & 685 & 0.146 & 705 & 0.148 & 708 & 0.015 & $6.99 E-04$ & $4.64 E-02$ \\
\hline 0.106 & 704 & 0.111 & 699 & 0.092 & 668 & 0.011 & $6.90 E-04$ & $6.40 E-02$ \\
\hline
\end{tabular}




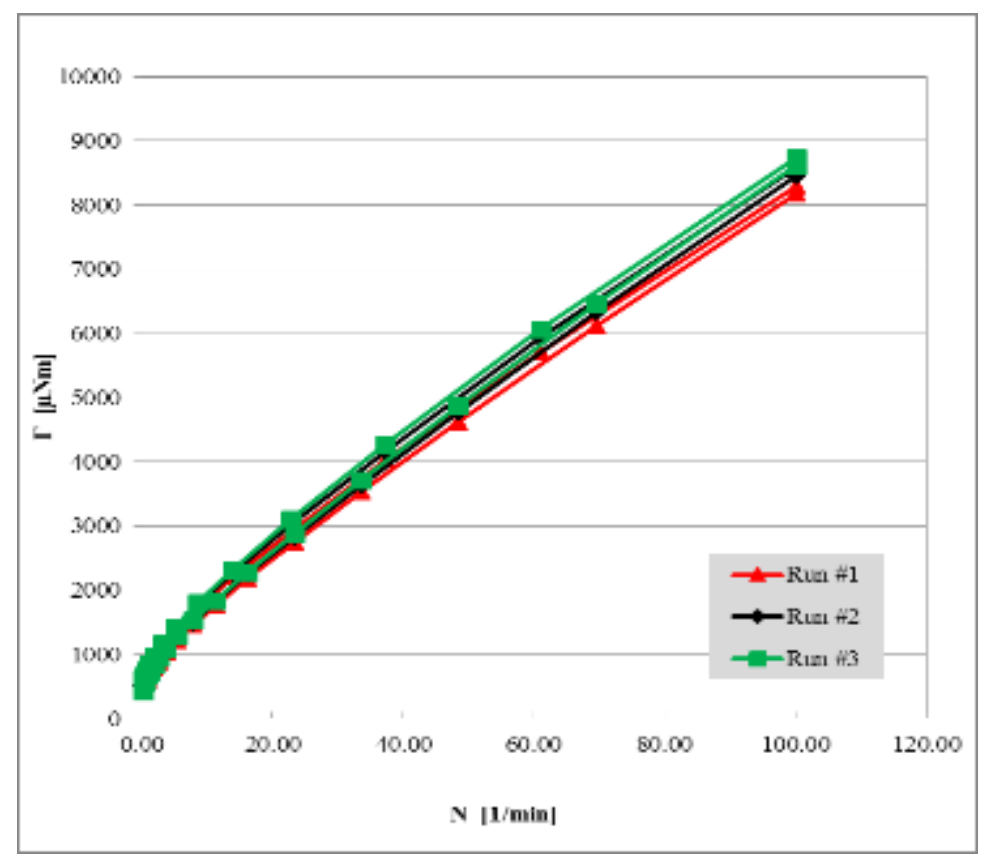

Figure A - 43: Torque vs. Angular Speed using Serrated Coaxial Cylinder on Mix\#11, with 0 \% beads by volume. Portrays data from Table A-59.

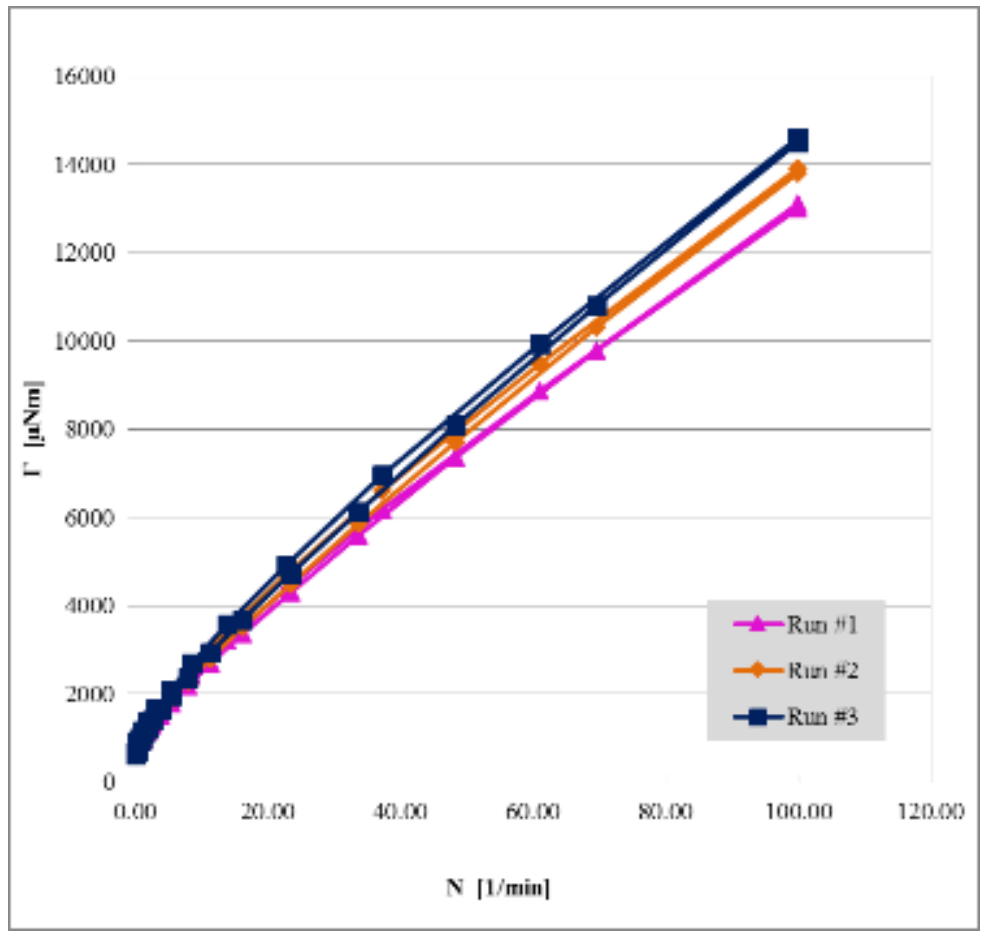

Figure A - 44: Torque vs. Angular Speed using Serrated Coaxial Cylinder on Mix\#11, with $20 \%$ beads by volume. Portrays data from Table A-60. 


\section{Appendix B: Data for Calibration}

This appendix summarizes the average values calculated from the original data shown in Appendix A. These average data sets were combined into a set of average values (green colored columns) for each spindle and mortar composition (bead \%). The combined average data sets shown in this appendix were used during the calibration process described in Section 4.3 to convert the original raw data into fundamental units. 
Table B - 1: Average values for SRM paste with $0 \%$ beads (by volume) performed prior to the $20 \%$ mortar tests using a double spiral spindle geometry. [NIST Codes]

\begin{tabular}{|c|c|c|c|c|c|c|c|c|c|}
\hline \multicolumn{10}{|c|}{ Double Spiral [RHN-83A] } \\
\hline \multicolumn{10}{|c|}{$0 \%$ Beads (Pre-20\%) } \\
\hline \multicolumn{2}{|c|}{ Mix \#7 [SMC-101] } & \multicolumn{2}{|c|}{ Mix \#8 [SMC-103] } & \multicolumn{2}{|c|}{ Mix \#9 [SMC-109] } & \multicolumn{2}{|c|}{ Mix \#10 [SMC-111] } & \multicolumn{2}{|c|}{ Mix \#11 [SMC-113 } \\
\hline $\begin{array}{c}\mathrm{N} \\
{[1 / \mathrm{min}]}\end{array}$ & $\begin{array}{c}\Gamma \\
{[\mu \mathrm{Nm}]}\end{array}$ & $\begin{array}{c}\mathrm{N} \\
{[1 / \mathrm{min}]}\end{array}$ & $\begin{array}{c}\Gamma \\
{[\mu \mathrm{Nm}]}\end{array}$ & $\begin{array}{c}\mathrm{N} \\
{[1 / \mathrm{min}]}\end{array}$ & $\begin{array}{c}\Gamma \\
{[\mu \mathrm{Nm}]}\end{array}$ & $\begin{array}{c}\mathrm{N} \\
{[1 / \mathrm{min}]}\end{array}$ & $\begin{array}{c}\Gamma \\
{[\mu \mathrm{Nm}]}\end{array}$ & $\begin{array}{c}\mathrm{N} \\
{[1 / \mathrm{min}]}\end{array}$ & $\left.\begin{array}{c}\Gamma \\
{[\mu \mathrm{Nm}]}\end{array}\right]$ \\
\hline 0.09 & 953 & 0.09 & 993 & 0.09 & 1014 & 0.09 & 1030 & 0.09 & 992 \\
\hline 0.16 & 1410 & 0.16 & 1473 & 0.16 & 1500 & 0.16 & 1557 & 0.16 & 1470 \\
\hline 0.27 & 1593 & 0.27 & 1680 & 0.27 & 1707 & 0.27 & 1787 & 0.27 & 1677 \\
\hline 0.44 & 1703 & 0.44 & 1790 & 0.44 & 1827 & 0.44 & 1907 & 0.44 & 1790 \\
\hline 0.72 & 1820 & 0.72 & 1917 & 0.72 & 1957 & 0.72 & 2050 & 0.72 & 1907 \\
\hline 1.18 & 1993 & 1.18 & 2093 & 1.18 & 2140 & 1.18 & 2240 & 1.18 & 2080 \\
\hline 1.93 & 2237 & 1.93 & 2353 & 1.93 & 2410 & 1.93 & 2533 & 1.93 & 2330 \\
\hline 3.16 & 2617 & 3.16 & 2767 & 3.16 & 2833 & 3.16 & 2987 & 3.16 & 2733 \\
\hline 5.18 & 3200 & 5.18 & 3407 & 5.18 & 3480 & 5.18 & 3687 & 5.18 & 3357 \\
\hline 8.48 & 4107 & 8.48 & 4397 & 8.48 & 4490 & 8.48 & 4773 & 8.48 & 4320 \\
\hline 13.90 & 5527 & 13.90 & 5997 & 13.90 & 6117 & 13.90 & 6507 & 13.90 & 5880 \\
\hline 22.80 & 7760 & 22.80 & 8440 & 22.80 & 8617 & 22.80 & 9170 & 22.80 & 8283 \\
\hline 37.30 & 11267 & 37.30 & 12333 & 37.30 & 12600 & 37.30 & 13400 & 37.30 & 12100 \\
\hline 61.10 & 16800 & 61.10 & 18500 & 61.10 & 18800 & 61.10 & 20067 & 61.10 & 18067 \\
\hline 100.00 & 25467 & 100.00 & 28000 & 100.00 & 28467 & 100.00 & 30367 & 100.00 & 27367 \\
\hline 100.00 & 25333 & 100.00 & 27833 & 100.00 & 28267 & 100.00 & 30200 & 100.00 & 27200 \\
\hline 69.50 & 18533 & 69.50 & 20333 & 69.50 & 20667 & 69.50 & 22100 & 69.50 & 19867 \\
\hline 48.30 & 13733 & 48.30 & 14967 & 48.30 & 15333 & 48.30 & 16333 & 48.30 & 14733 \\
\hline 33.60 & 10300 & 33.60 & 11200 & 33.60 & 11467 & 33.60 & 12233 & 33.60 & 11033 \\
\hline 23.40 & 7843 & 23.40 & 8513 & 23.40 & 8707 & 23.40 & 9290 & 23.40 & 8377 \\
\hline 16.20 & 6093 & 16.20 & 6577 & 16.20 & 6743 & 16.20 & 7180 & 16.20 & 6490 \\
\hline 11.30 & 4837 & 11.30 & 5190 & 11.30 & 5327 & 11.30 & 5667 & 11.30 & 5127 \\
\hline 7.85 & 3927 & 7.85 & 4190 & 7.85 & 4307 & 7.85 & 4570 & 7.85 & 4150 \\
\hline 5.46 & 3270 & 5.46 & 3470 & 5.46 & 3567 & 5.46 & 3777 & 5.46 & 3440 \\
\hline 3.79 & 2790 & 3.79 & 2947 & 3.79 & 3030 & 3.79 & 3200 & 3.79 & 2923 \\
\hline 2.64 & 2433 & 2.64 & 2560 & 2.64 & 2633 & 2.64 & 2780 & 2.64 & 2543 \\
\hline 1.83 & 2173 & 1.83 & 2277 & 1.83 & 2343 & 1.83 & 2463 & 1.83 & 2263 \\
\hline 1.27 & 1980 & 1.27 & 2063 & 1.27 & 2127 & 1.27 & 2233 & 1.27 & 2053 \\
\hline 0.89 & 1830 & 0.89 & 1907 & 0.89 & 1960 & 0.88 & 2067 & 0.89 & 1897 \\
\hline 0.62 & 1720 & 0.62 & 1787 & 0.62 & 1837 & 0.62 & 1923 & 0.62 & 1773 \\
\hline 0.43 & 1633 & 0.43 & 1707 & 0.43 & 1747 & 0.43 & 1817 & 0.43 & 1690 \\
\hline 0.30 & 1567 & 0.30 & 1630 & 0.30 & 1673 & 0.30 & 1747 & 0.30 & 1623 \\
\hline 0.21 & 1517 & 0.21 & 1573 & 0.21 & 1617 & 0.21 & 1683 & 0.21 & 1570 \\
\hline 0.14 & 1473 & 0.14 & 1527 & 0.15 & 1577 & 0.14 & 1643 & 0.14 & 1517 \\
\hline 0.10 & 1440 & 0.10 & 1487 & 0.10 & 1543 & 0.10 & 1603 & 0.10 & 1487 \\
\hline
\end{tabular}


Table B - 2: Average values for SRM paste with 0 \% beads (by volume) performed prior to the $40 \%$ mortar tests using a double spiral spindle geometry. [NIST Codes]

\begin{tabular}{|c|c|c|c|c|c|c|c|c|c|}
\hline \multicolumn{10}{|c|}{ Double Spiral [RHN-83A] } \\
\hline \multicolumn{10}{|c|}{$0 \%$ Beads (Pre-40\%) } \\
\hline \multicolumn{2}{|c|}{ Mix \#2 [SMC-79] } & \multicolumn{2}{|c|}{ Mix \#3 [SMC-81] } & \multicolumn{2}{|c|}{ Mix \#4 [SMC-85] } & \multicolumn{2}{|c|}{ Mix \#5 [SMC-95] } & \multicolumn{2}{|c|}{ Mix \#6 [SMC-97] } \\
\hline $\begin{array}{c}\mathrm{N} \\
{[1 / \mathrm{min}]}\end{array}$ & $\begin{array}{c}\Gamma \\
{[\mu \mathrm{Nm}]}\end{array}$ & $\begin{array}{c}\mathrm{N} \\
{[1 / \mathrm{min}]}\end{array}$ & $\begin{array}{c}\Gamma \\
{[\mu \mathrm{Nm}]}\end{array}$ & $\begin{array}{c}\mathrm{N} \\
{[1 / \mathrm{min}]}\end{array}$ & $\begin{array}{c}\Gamma \\
{[\mu \mathrm{Nm}]}\end{array}$ & $\begin{array}{c}\mathrm{N} \\
{[1 / \mathrm{min}]}\end{array}$ & $\begin{array}{c}\Gamma \\
{[\mu \mathrm{Nm}]}\end{array}$ & $\begin{array}{c}\mathrm{N} \\
{[1 / \mathrm{min}]}\end{array}$ & $\begin{array}{c}\Gamma \\
{[\mu \mathrm{Nm}]}\end{array}$ \\
\hline 0.10 & 1181 & 0.10 & 1370 & 0.09 & 999 & 0.10 & 1098 & 0.09 & 1023 \\
\hline 0.16 & 1507 & 0.16 & 1567 & 0.16 & 1460 & 0.16 & 1407 & 0.16 & 1510 \\
\hline 0.27 & 1660 & 0.27 & 1670 & 0.27 & 1650 & 0.27 & 1547 & 0.27 & 1717 \\
\hline 0.44 & 1770 & 0.44 & 1767 & 0.44 & 1760 & 0.44 & 1643 & 0.44 & 1840 \\
\hline 0.72 & 1893 & 0.72 & 1893 & 0.72 & 1883 & 0.72 & 1760 & 0.72 & 1977 \\
\hline 1.18 & 2083 & 1.18 & 2073 & 1.18 & 2060 & 1.18 & 1940 & 1.18 & 2160 \\
\hline 1.93 & 2347 & 1.93 & 2340 & 1.93 & 2310 & 1.93 & 2193 & 1.93 & 2443 \\
\hline 3.16 & 2760 & 3.16 & 2760 & 3.16 & 2707 & 3.16 & 2587 & 3.16 & 2873 \\
\hline 5.18 & 3393 & 5.18 & 3407 & 5.18 & 3317 & 5.18 & 3187 & 5.18 & 3530 \\
\hline 8.48 & 4373 & 8.48 & 4393 & 8.48 & 4250 & 8.48 & 4103 & 8.48 & 4543 \\
\hline 13.90 & 5900 & 13.90 & 5913 & 13.90 & 5730 & 13.90 & 5527 & 13.90 & 6163 \\
\hline 22.80 & 8307 & 22.80 & 8377 & 22.80 & 8047 & 22.80 & 7813 & 22.80 & 8670 \\
\hline 37.30 & 12100 & 37.30 & 12233 & 37.30 & 11733 & 37.30 & 11367 & 37.30 & 12633 \\
\hline 61.00 & 18033 & 61.10 & 18267 & 61.10 & 17467 & 61.10 & 16867 & 61.10 & 18833 \\
\hline 100.00 & 27233 & 100.00 & 27733 & 100.00 & 26433 & 100.00 & 25467 & 100.00 & 28433 \\
\hline 100.00 & 27133 & 100.00 & 27567 & 100.00 & 26267 & 100.00 & 25333 & 100.00 & 28300 \\
\hline 69.50 & 19833 & 69.50 & 20167 & 69.50 & 19167 & 69.50 & 18567 & 69.50 & 20733 \\
\hline 48.30 & 14700 & 48.30 & 14900 & 48.30 & 14167 & 48.30 & 13767 & 48.30 & 15300 \\
\hline 33.60 & 10967 & 33.60 & 11100 & 33.60 & 10600 & 33.60 & 10333 & 33.60 & 11500 \\
\hline 23.40 & 8363 & 23.40 & 8440 & 23.40 & 8093 & 23.40 & 7857 & 23.40 & 8740 \\
\hline 16.20 & 6477 & 16.20 & 6527 & 16.20 & 6280 & 16.20 & 6090 & 16.20 & 6777 \\
\hline 11.30 & 5120 & 11.30 & 5150 & 11.30 & 4980 & 11.30 & 4823 & 11.30 & 5363 \\
\hline 7.85 & 4143 & 7.85 & 4167 & 7.85 & 4043 & 7.85 & 3907 & 7.85 & 4343 \\
\hline 5.45 & 3437 & 5.46 & 3450 & 5.46 & 3360 & 5.46 & 3240 & 5.46 & 3603 \\
\hline 3.79 & 2920 & 3.79 & 2930 & 3.79 & 2867 & 3.79 & 2757 & 3.79 & 3070 \\
\hline 2.64 & 2540 & 2.64 & 2547 & 2.64 & 2503 & 2.64 & 2403 & 2.64 & 2673 \\
\hline 1.83 & 2263 & 1.83 & 2270 & 1.83 & 2237 & 1.83 & 2137 & 1.83 & 2377 \\
\hline 1.27 & 2053 & 1.27 & 2060 & 1.27 & 2030 & 1.27 & 1940 & 1.27 & 2160 \\
\hline 0.89 & 1893 & 0.89 & 1900 & 0.89 & 1880 & 0.89 & 1790 & 0.89 & 1997 \\
\hline 0.62 & 1773 & 0.62 & 1777 & 0.62 & 1767 & 0.62 & 1680 & 0.62 & 1863 \\
\hline 0.43 & 1693 & 0.43 & 1690 & 0.43 & 1683 & 0.43 & 1593 & 0.43 & 1773 \\
\hline 0.30 & 1613 & 0.30 & 1617 & 0.30 & 1613 & 0.30 & 1527 & 0.30 & 1703 \\
\hline 0.21 & 1563 & 0.21 & 1560 & 0.21 & 1557 & 0.21 & 1470 & 0.21 & 1643 \\
\hline 0.14 & 1520 & 0.14 & 1517 & 0.14 & 1517 & 0.14 & 1430 & 0.14 & 1597 \\
\hline 0.10 & 1483 & 0.10 & 1480 & 0.10 & 1487 & 0.10 & 1400 & 0.10 & 1560 \\
\hline
\end{tabular}


Table B - 3: Average data set for the combined measured values of SRM paste ( 0 \% beads) using Double spiral spindle geometry.

\begin{tabular}{|cc|cc|}
\hline \multicolumn{4}{c|}{ Double Spiral } \\
\hline \multicolumn{4}{c|}{$\mathbf{0} \%$ Average Values } \\
\hline $\mathbf{N}$ & & $\boldsymbol{\Gamma}$ \\
{$[1 / \mathrm{min}]$} & $S D$ & {$[\mu \mathrm{Nm}]$} & $S D$ \\
\hline $\mathbf{0 . 1 0}$ & $1.3 E-03$ & $\mathbf{1 0 6 5}$ & 119 \\
$\mathbf{0 . 1 6}$ & $7.1 E-04$ & $\mathbf{1 4 8 6}$ & 51 \\
$\mathbf{0 . 2 7}$ & $1.8 E-04$ & $\mathbf{1 6 6 9}$ & 62 \\
$\mathbf{0 . 4 4}$ & $4.0 E-04$ & $\mathbf{1 7 8 0}$ & 69 \\
$\mathbf{0 . 7 2}$ & $3.0 E-04$ & $\mathbf{1 9 0 6}$ & 76 \\
$\mathbf{1 . 1 8}$ & $0.0 E+00$ & $\mathbf{2 0 8 6}$ & 79 \\
$\mathbf{1 . 9 3}$ & $2.2 E-16$ & $\mathbf{2 3 5 0}$ & 92 \\
$\mathbf{3 . 1 6}$ & $0.0 E+00$ & $\mathbf{2 7 6 2}$ & 111 \\
$\mathbf{5 . 1 8}$ & $0.0 E+00$ & $\mathbf{3 3 9 6}$ & 141 \\
$\mathbf{8 . 4 8}$ & $1.8 E-15$ & $\mathbf{4 3 7 5}$ & 191 \\
$\mathbf{1 3 . 9 0}$ & $1.8 E-15$ & $\mathbf{5 9 2 6}$ & 282 \\
$\mathbf{2 2 . 8 0}$ & $3.6 E-15$ & $\mathbf{8 3 4 8}$ & 399 \\
$\mathbf{3 7 . 3 0}$ & $7.1 E-15$ & $\mathbf{1 2 1 7 7}$ & 600 \\
$\mathbf{6 1 . 0 9}$ & $3.0 E-02$ & $\mathbf{1 8 1 7 0}$ & 930 \\
$\mathbf{1 0 0 . 0 0}$ & $0.0 E+00$ & $\mathbf{2 7 4 9 7}$ & 1408 \\
$\mathbf{1 0 0 . 0 0}$ & $0.0 E+00$ & $\mathbf{2 7 3 4 3}$ & 1397 \\
$\mathbf{6 9 . 5 0}$ & $0.0 E+00$ & $\mathbf{1 9 9 9 7}$ & 1024 \\
$\mathbf{4 8 . 3 0}$ & $7.1 E-15$ & $\mathbf{1 4 7 9 3}$ & 745 \\
$\mathbf{3 3 . 6 0}$ & $7.1 E-15$ & $\mathbf{1 1 0 7 3}$ & 555 \\
$\mathbf{2 3 . 4 0}$ & $3.6 E-15$ & $\mathbf{8 4 2 2}$ & 414 \\
$\mathbf{1 6 . 2 0}$ & $3.6 E-15$ & $\mathbf{6 5 2 3}$ & 313 \\
$\mathbf{1 1 . 3 0}$ & $1.8 E-15$ & $\mathbf{5 1 5 8}$ & 241 \\
$\mathbf{7 . 8 5}$ & $0.0 E+00$ & $\mathbf{4 1 7 5}$ & 188 \\
$\mathbf{5 . 4 6}$ & $3.0 E-03$ & $\mathbf{3 4 6 1}$ & 151 \\
$\mathbf{3 . 7 9}$ & $4.4 E-16$ & $\mathbf{2 9 4 3}$ & 124 \\
$\mathbf{2 . 6 4}$ & $0.0 E+00$ & $\mathbf{2 5 6 2}$ & 105 \\
$\mathbf{1 . 8 3}$ & $4.4 E-16$ & $\mathbf{2 2 8 0}$ & 90 \\
$\mathbf{1 . 2 7}$ & $2.2 E-16$ & $\mathbf{2 0 7 0}$ & 81 \\
$\mathbf{0 . 8 9}$ & $5.0 E-04$ & $\mathbf{1 9 1 2}$ & 75 \\
$\mathbf{0 . 6 2}$ & $2.6 E-04$ & $\mathbf{1 7 9 0}$ & 66 \\
$\mathbf{0 . 4 3}$ & $4.5 E-04$ & $\mathbf{1 7 0 3}$ & 61 \\
$\mathbf{0 . 3 0}$ & $6.7 E-04$ & $\mathbf{1 6 3 1}$ & 60 \\
$\mathbf{0 . 2 1}$ & $3.6 E-04$ & $\mathbf{1 5 7 5}$ & 58 \\
$\mathbf{0 . 1 4}$ & $5.8 E-04$ & $\mathbf{1 5 3 2}$ & 58 \\
$\mathbf{0 . 1 0}$ & $2.1 E-04$ & $\mathbf{1 4 9 7}$ & 56 \\
\hline
\end{tabular}


Table B - 4: Average values for 20 \% mortar mixes (by volume) using a Double spiral spindle geometry. [NIST Codes]

\begin{tabular}{|c|c|c|c|c|c|c|c|c|c|c|c|c|c|}
\hline \multicolumn{10}{|c|}{ Double Spiral [RHN-83A] } & & & & \\
\hline \multicolumn{10}{|c|}{$20 \%$ Beads } & \multicolumn{4}{|c|}{ Double Spiral } \\
\hline \multicolumn{2}{|c|}{ Mix \#7 [SMC-101] } & \multicolumn{2}{|c|}{ Mix \#8 [SMC-103] } & \multicolumn{2}{|c|}{ Mix \#9 [SMC-109] } & \multicolumn{2}{|c|}{ Mix \#10 [SMC-111] } & \multicolumn{2}{|c|}{ Mix \#11 [SMC-113] } & \multicolumn{4}{|c|}{$20 \%$ Average Values } \\
\hline $\begin{array}{c}\mathrm{N} \\
{[1 / \mathrm{min}]}\end{array}$ & $\begin{array}{c}\Gamma \\
{[\mu \mathrm{Nm}]}\end{array}$ & $\begin{array}{c}\mathrm{N} \\
{[1 / \mathrm{min}]}\end{array}$ & $\begin{array}{c}\Gamma \\
{[\mu \mathrm{Nm}]}\end{array}$ & $\begin{array}{c}\mathrm{N} \\
{[1 / \mathrm{min}]}\end{array}$ & $\begin{array}{c}\Gamma \\
{[\mu \mathrm{Nm}]}\end{array}$ & \begin{tabular}{|c|}
$\mathbf{N}$ \\
{$[1 / \mathrm{min}]$}
\end{tabular} & $\begin{array}{c}\Gamma \\
{[\mu \mathrm{Nm}]}\end{array}$ & \begin{tabular}{|c}
$\mathrm{N}$ \\
{$[1 / \mathrm{min}]$}
\end{tabular} & $\begin{array}{c}\Gamma \\
{[\mu \mathrm{Nm}]}\end{array}$ & $\begin{array}{c}\mathbf{N} \\
{[1 / \mathrm{min}]}\end{array}$ & $S D$ & $\begin{array}{c}\boldsymbol{\Gamma} \\
{[\mu \mathrm{Nm}]}\end{array}$ & $S D$ \\
\hline 0.09 & 1407 & 0.09 & 1360 & 0.09 & 1347 & 0.09 & 1403 & 0.09 & 1323 & 0.09 & $7.6 E-04$ & 1368 & 32 \\
\hline 0.16 & 1850 & 0.16 & 1963 & 0.16 & 1913 & 0.16 & 2030 & 0.16 & 1883 & 0.16 & $3.9 E-04$ & 1928 & 63 \\
\hline 0.27 & 2057 & 0.27 & 2233 & 0.27 & 2160 & 0.27 & 2300 & 0.27 & 2130 & 0.27 & $3.9 E-04$ & 2176 & 84 \\
\hline 0.44 & 2220 & 0.44 & 2407 & 0.44 & 2340 & 0.44 & 2497 & 0.44 & 2303 & 0.44 & $2.7 E-04$ & 2353 & 94 \\
\hline 0.72 & 2433 & 0.72 & 2650 & 0.72 & 2583 & 0.72 & 2763 & 0.72 & 2523 & 0.72 & 1.6E-04 & 2591 & 112 \\
\hline 1.18 & 2757 & 1.18 & 2983 & 1.18 & 2927 & 1.18 & 3143 & 1.18 & 2840 & 1.18 & $0.0 E+00$ & 2930 & 132 \\
\hline 1.93 & 3253 & 1.93 & 3517 & 1.93 & 3453 & 1.93 & 3703 & 1.93 & 3320 & 1.93 & $1.3 E-03$ & 3449 & 158 \\
\hline 3.16 & 4010 & 3.16 & 4340 & 3.16 & 4260 & 3.16 & 4580 & 3.16 & 4067 & 3.16 & $1.3 E-03$ & 4251 & 204 \\
\hline 5.18 & 5167 & 5.18 & 5643 & 5.18 & 5500 & 5.18 & 5967 & 5.18 & 5247 & 5.18 & $1.3 E-03$ & 5505 & 288 \\
\hline 8.48 & 6923 & 8.48 & 7683 & 8.49 & 7390 & 8.48 & 8107 & 8.49 & 7090 & 8.49 & $2.7 E-03$ & 7439 & 423 \\
\hline 13.90 & 9493 & 13.90 & 10733 & 13.90 & 10267 & 13.90 & 11333 & 13.90 & 9907 & 13.90 & $0.0 E+00$ & 10347 & 640 \\
\hline 22.77 & 13533 & 22.77 & 15200 & 22.77 & 14533 & 22.80 & 16133 & 22.80 & 14067 & 22.78 & 1.6E-02 & 14693 & 905 \\
\hline 37.30 & 20033 & 37.30 & 22700 & 37.30 & 21633 & 37.30 & 24100 & 37.30 & 20933 & 37.30 & $0.0 E+00$ & 21880 & 1412 \\
\hline 61.03 & 30333 & 61.07 & 34367 & 61.07 & 32700 & 61.07 & 36467 & 61.03 & 31633 & 61.05 & $1.6 E-02$ & 33100 & 2140 \\
\hline 100.00 & 46133 & 100.00 & 52200 & 100.00 & 49667 & 100.00 & 55400 & 100.00 & 48000 & 100.00 & $0.0 E+00$ & 50280 & 3246 \\
\hline 100.00 & 45733 & 100.00 & 51733 & 100.00 & 49233 & 100.00 & 54867 & 100.00 & 47633 & 100.00 & $0.0 E+00$ & 49840 & 3192 \\
\hline 69.50 & 33200 & 69.50 & 37500 & 69.50 & 35700 & 69.50 & 39700 & 69.50 & 34600 & 69.50 & $0.0 E+00$ & 36140 & 2269 \\
\hline 48.30 & 24333 & 48.30 & 27467 & 48.30 & 26167 & 48.30 & 29067 & 48.30 & 25367 & 48.30 & $0.0 E+00$ & 26480 & 1650 \\
\hline 33.60 & 18033 & 33.60 & 20367 & 33.60 & 19400 & 33.60 & 21467 & 33.60 & 18833 & 33.60 & $0.0 E+00$ & 19620 & 1196 \\
\hline 23.37 & 13567 & 23.37 & 15267 & 23.40 & 14567 & 23.40 & 16100 & 23.40 & 14100 & 23.39 & $1.6 E-02$ & 14720 & 888 \\
\hline 16.20 & 10333 & 16.20 & 11600 & 16.20 & 11067 & 16.20 & 12200 & 16.20 & 10767 & 16.20 & $0.0 E+00$ & 11193 & 651 \\
\hline 11.30 & 8017 & 11.30 & 8937 & 11.30 & 8583 & 11.30 & 9450 & 11.30 & 8327 & 11.30 & $0.0 E+00$ & 8663 & 496 \\
\hline 7.85 & 6363 & 7.85 & 7057 & 7.85 & 6783 & 7.85 & 7423 & 7.85 & 6577 & 7.85 & $1.6 E-03$ & 6841 & 371 \\
\hline 5.46 & 5150 & 5.46 & 5690 & 5.46 & 5490 & 5.45 & 5977 & 5.46 & 5333 & 5.46 & $2.1 E-03$ & 5528 & 286 \\
\hline 3.79 & 4277 & 3.79 & 4697 & 3.79 & 4547 & 3.79 & 4923 & 3.79 & 4410 & 3.79 & $1.6 E-03$ & 4571 & 225 \\
\hline 2.64 & 3627 & 2.64 & 3953 & 2.63 & 3857 & 2.64 & 4153 & 2.64 & 3740 & 2.64 & $3.3 E-03$ & 3866 & 181 \\
\hline 1.83 & 3153 & 1.83 & 3417 & 1.83 & 3347 & 1.83 & 3590 & 1.83 & 3250 & 1.83 & $0.0 E+00$ & 3351 & 149 \\
\hline 1.27 & 2793 & 1.28 & 3023 & 1.27 & 2967 & 1.27 & 3167 & 1.27 & 2873 & 1.27 & $2.5 E-03$ & 2965 & 128 \\
\hline 0.89 & 2523 & 0.89 & 2720 & 0.88 & 2683 & 0.89 & 2853 & 0.89 & 2593 & 0.89 & $6.5 E-04$ & 2675 & 113 \\
\hline 0.62 & 2323 & 0.62 & 2493 & 0.62 & 2470 & 0.62 & 2610 & 0.62 & 2383 & 0.62 & $3.3 E-04$ & 2456 & 98 \\
\hline 0.43 & 2173 & 0.43 & 2313 & 0.43 & 2300 & 0.43 & 2423 & 0.43 & 2220 & 0.43 & $9.4 E-04$ & 2286 & 86 \\
\hline 0.30 & 2060 & 0.30 & 2183 & 0.30 & 2180 & 0.30 & 2290 & 0.30 & 2093 & 0.30 & $5.0 E-04$ & 2161 & 80 \\
\hline 0.21 & 1970 & 0.21 & 2080 & 0.21 & 2083 & 0.21 & 2187 & 0.21 & 2003 & 0.21 & 4.0E-04 & 2065 & 75 \\
\hline 0.14 & 1893 & 0.14 & 2010 & 0.14 & 2000 & 0.14 & 2103 & 0.14 & 1930 & 0.14 & $6.0 E-04$ & 1987 & 72 \\
\hline 0.10 & 1823 & 0.10 & 1963 & 0.10 & 1950 & 0.10 & 2040 & 0.10 & 1880 & 0.10 & 8.3E-04 & 1931 & 74 \\
\hline
\end{tabular}


Table B - 5: Average values for 40 \% mortar mixes (by volume) using a Double spiral spindle geometry. [NIST Codes]

\begin{tabular}{|c|c|c|c|c|c|c|c|c|c|c|c|c|c|}
\hline \multicolumn{10}{|c|}{ Double Spiral [RHN-83A] } & & & & \\
\hline \multicolumn{10}{|c|}{$40 \%$ Beads } & \\
\hline \multicolumn{2}{|c|}{ Mix \#2 [SMC-79] } & \multicolumn{2}{|c|}{ Mix \#3 [SMC-81] } & \multicolumn{2}{|c|}{ Mix \#4 [SMC-85] } & \multicolumn{2}{|c|}{ Mix \#5 [SMC-95] } & \multicolumn{2}{|c|}{ Mix \#6 [SMC-97] } & \multicolumn{4}{|c|}{$40 \%$ Average Values } \\
\hline $\begin{array}{c}N \\
{[1 / \mathrm{min}]}\end{array}$ & $\begin{array}{c}\Gamma \\
{[\mu \mathrm{Nm}]}\end{array}$ & $\begin{array}{c}\mathrm{N} \\
{[1 / \mathrm{min}]}\end{array}$ & $\begin{array}{c}\Gamma \\
{[\mu \mathrm{Nm}]}\end{array}$ & $\begin{array}{c}\mathrm{N} \\
{[1 / \mathrm{min}]}\end{array}$ & $\begin{array}{c}\Gamma \\
{[\mu \mathrm{Nm}]}\end{array}$ & $\begin{array}{c}\mathrm{N} \\
{[1 / \mathrm{min}]}\end{array}$ & $\begin{array}{c}\Gamma \\
{[\mu \mathrm{Nm}]}\end{array}$ & $\begin{array}{c}\mathrm{N} \\
{[1 / \mathrm{min}]}\end{array}$ & $\begin{array}{c}\Gamma \\
{[\mu \mathrm{Nm}]}\end{array}$ & $\underset{[1 / \mathrm{min}]}{\mathbf{N}}$ & $S D$ & $\begin{array}{c}\boldsymbol{\Gamma} \\
{[\mu \mathrm{Nm}]}\end{array}$ & $S D$ \\
\hline 0.09 & 2203 & 0.09 & 2693 & 0.21 & 3193 & 0.09 & 2243 & 0.08 & 2793 & 0.11 & 0.047 & 2625 & 369 \\
\hline 0.16 & 3113 & 0.16 & 3557 & 0.25 & 3850 & 0.16 & 3177 & 0.16 & 4070 & 0.18 & 0.037 & 3553 & 372 \\
\hline 0.27 & 3630 & 0.27 & 4140 & 0.32 & 4197 & 0.27 & 3660 & 0.26 & 4857 & 0.28 & 0.023 & 4097 & 447 \\
\hline 0.44 & 4127 & 0.44 & 4683 & 0.44 & 4567 & 0.44 & 4233 & 0.44 & 5597 & 0.44 & 0.003 & 4641 & 520 \\
\hline 0.72 & 4763 & 0.72 & 5437 & 0.72 & 5177 & 0.72 & 4883 & 0.73 & 6427 & 0.72 & 0.003 & 5337 & 593 \\
\hline 1.18 & 5613 & 1.18 & 6450 & 1.18 & 6113 & 1.18 & 5743 & 1.17 & 7557 & 1.18 & 0.005 & 6295 & 695 \\
\hline 1.93 & 6873 & 1.93 & 8030 & 1.93 & 7430 & 1.93 & 7063 & 1.94 & 9333 & 1.93 & 0.003 & 7746 & 886 \\
\hline 3.16 & 8910 & 3.15 & 10533 & 3.16 & 9490 & 3.17 & 9140 & 3.17 & 12167 & 3.16 & 0.006 & 10048 & 1196 \\
\hline 5.18 & 12000 & 5.17 & 14333 & 5.18 & 12800 & 5.18 & 12433 & 5.19 & 16500 & 5.18 & 0.005 & 13613 & 1644 \\
\hline 8.48 & 17067 & 8.48 & 19900 & 8.48 & 18067 & 8.50 & 17300 & 8.48 & 23267 & 8.48 & 0.008 & 19120 & 2300 \\
\hline 13.90 & 24200 & 13.90 & 27867 & 13.90 & 25467 & 13.90 & 24433 & 13.90 & 32867 & 13.90 & 0.000 & 26967 & 3223 \\
\hline 22.77 & 34400 & 22.77 & 39333 & 22.80 & 35967 & 22.80 & 35067 & 22.80 & 46400 & 22.79 & 0.016 & 38233 & 4422 \\
\hline 37.30 & 50667 & 37.30 & 58067 & 37.30 & 52633 & 37.27 & 51567 & 37.30 & 67433 & 37.29 & 0.013 & 56073 & 6235 \\
\hline 61.03 & 74900 & 61.10 & 84000 & 61.07 & 77000 & 61.07 & 76100 & 61.10 & 95833 & 61.07 & 0.025 & 81567 & 7806 \\
\hline 100.00 & 105000 & 100.00 & 116333 & 100.00 & 91733 & 100.00 & 111667 & 100.00 & 134333 & 100.00 & 0.000 & 111813 & 13980 \\
\hline 100.00 & 100267 & 100.00 & 111333 & 100.00 & 86000 & 100.00 & 109000 & 99.97 & 127333 & 99.99 & 0.013 & 106787 & 13582 \\
\hline 69.57 & 71700 & 69.57 & 79133 & 69.53 & 61600 & 69.53 & 78367 & 69.57 & 90100 & 69.55 & 0.016 & 76180 & 9380 \\
\hline 48.30 & 52400 & 48.33 & 57400 & 48.37 & 45100 & 48.37 & 56967 & 48.37 & 65500 & 48.35 & 0.027 & 55473 & 6686 \\
\hline 33.60 & 38900 & 33.60 & 42233 & 33.60 & 34167 & 33.60 & 42167 & 33.60 & 48100 & 33.60 & 0.000 & 41113 & 4568 \\
\hline 23.37 & 29733 & 23.40 & 31633 & 23.37 & 27267 & 23.40 & 31733 & 23.40 & 35900 & 23.39 & 0.016 & 31253 & 2834 \\
\hline 16.20 & 23533 & 16.23 & 25200 & 16.20 & 22700 & 16.23 & 23967 & 16.20 & 27633 & 16.21 & 0.016 & 24607 & 1715 \\
\hline 11.30 & 18100 & 11.30 & 19867 & 11.30 & 18500 & 11.30 & 18300 & 11.30 & 22367 & 11.30 & 0.000 & 19427 & 1595 \\
\hline 7.85 & 14033 & 7.86 & 15600 & 7.85 & 14333 & 7.85 & 14167 & 7.84 & 17667 & 7.85 & 0.007 & 15160 & 1372 \\
\hline 5.45 & 11100 & 5.46 & 12267 & 5.45 & 11267 & 5.46 & 11200 & 5.46 & 14033 & 5.46 & 0.005 & 11973 & 1113 \\
\hline 3.78 & 8987 & 3.79 & 9903 & 3.80 & 9077 & 3.79 & 8947 & 3.80 & 11200 & 3.79 & 0.006 & 9623 & 863 \\
\hline 2.64 & 7377 & 2.64 & 8103 & 2.63 & 7470 & 2.64 & 7410 & 2.64 & 9210 & 2.64 & 0.003 & 7914 & 701 \\
\hline 1.82 & 6183 & 1.83 & 6750 & 1.83 & 6287 & 1.83 & 6217 & 1.84 & 7730 & 1.83 & 0.004 & 6633 & 585 \\
\hline 1.27 & 5297 & 1.28 & 5757 & 1.27 & 5410 & 1.27 & 5320 & 1.28 & 6560 & 1.27 & 0.002 & 5669 & 475 \\
\hline 0.88 & 4613 & 0.89 & 5020 & 0.88 & 4777 & 0.89 & 4663 & 0.89 & 5700 & 0.89 & 0.002 & 4955 & 398 \\
\hline 0.61 & 4120 & 0.62 & 4510 & 0.62 & 4253 & 0.62 & 4137 & 0.61 & 5067 & 0.62 & 0.002 & 4417 & 353 \\
\hline 0.43 & 3733 & 0.42 & 4063 & 0.43 & 3863 & 0.43 & 3770 & 0.43 & 4560 & 0.43 & 0.002 & 3998 & 303 \\
\hline 0.30 & 3433 & 0.30 & 3770 & 0.30 & 3583 & 0.30 & 3487 & 0.30 & 4200 & 0.30 & 0.001 & 3695 & 277 \\
\hline 0.21 & 3220 & 0.21 & 3477 & 0.21 & 3370 & 0.21 & 3253 & 0.21 & 3920 & 0.21 & 0.001 & 3448 & 253 \\
\hline 0.15 & 3047 & 0.14 & 3277 & 0.14 & 3187 & 0.14 & 3093 & 0.14 & 3717 & 0.14 & 0.001 & 3264 & 240 \\
\hline 0.10 & 2933 & 0.10 & 3157 & 0.10 & 3067 & 0.10 & 2983 & 0.10 & 3570 & 0.10 & 0.000 & 3142 & 227 \\
\hline
\end{tabular}


Table B - 6: Average values for SRM paste with $0 \%$ beads (by volume) performed prior to the $20 \%$ mortar tests using six-blade vane geometry. [NIST Codes]

\begin{tabular}{|c|c|c|c|c|c|c|c|c|c|}
\hline \multicolumn{10}{|c|}{6 Blade Vane [RHN-83C] } \\
\hline \multicolumn{10}{|c|}{$0 \%$ Beads (Pre-20\%) } \\
\hline \multicolumn{2}{|c|}{ Mix \#7 [SMC-101] } & \multicolumn{2}{|c|}{ Mix \#8 [SMC-103] } & \multicolumn{2}{|c|}{ Mix \#9 [SMC-109] } & \multicolumn{2}{|c|}{ Mix \#10 [SMC-111] } & \multicolumn{2}{|c|}{ Mix \#11 [SMC-113 } \\
\hline $\begin{array}{c}\mathrm{N} \\
{[1 / \mathrm{min}]}\end{array}$ & $\begin{array}{c}\mathrm{M} \\
{[\mu \mathrm{Nm}]}\end{array}$ & $\begin{array}{c}\mathrm{N} \\
{[1 / \mathrm{min}]}\end{array}$ & $\begin{array}{c}\mathrm{M} \\
{[\mu \mathrm{Nm}]}\end{array}$ & $\begin{array}{c}\mathrm{N} \\
{[1 / \mathrm{min}]}\end{array}$ & $\begin{array}{c}\mathrm{M} \\
{[\mu \mathrm{Nm}]}\end{array}$ & $\begin{array}{c}\mathrm{N} \\
{[1 / \mathrm{min}]}\end{array}$ & $\begin{array}{c}\mathrm{M} \\
{[\mu \mathrm{Nm}]}\end{array}$ & $\begin{array}{c}\mathrm{N} \\
{[1 / \mathrm{min}]}\end{array}$ & $\begin{array}{c}\mathrm{M} \\
{[\mu \mathrm{Nm}]}\end{array}$ \\
\hline 0.10 & 399 & 0.10 & 416 & 0.10 & 423 & 0.10 & 445 & 0.10 & 423 \\
\hline 0.16 & 527 & 0.16 & 555 & 0.16 & 574 & 0.16 & 598 & 0.16 & 545 \\
\hline 0.27 & 568 & 0.27 & 595 & 0.27 & 619 & 0.27 & 640 & 0.27 & 583 \\
\hline 0.44 & 603 & 0.44 & 629 & 0.44 & 654 & 0.44 & 676 & 0.44 & 617 \\
\hline 0.72 & 651 & 0.72 & 679 & 0.72 & 706 & 0.72 & 729 & 0.72 & 672 \\
\hline 1.18 & 717 & 1.18 & 750 & 1.18 & 780 & 1.18 & 803 & 1.18 & 739 \\
\hline 1.93 & 811 & 1.93 & 850 & 1.93 & 882 & 1.93 & 906 & 1.93 & 834 \\
\hline 3.16 & 946 & 3.16 & 990 & 3.16 & 1027 & 3.16 & 1053 & 3.16 & 968 \\
\hline 5.18 & 1133 & 5.18 & 1190 & 5.18 & 1233 & 5.18 & 1263 & 5.18 & 1160 \\
\hline 8.48 & 1410 & 8.48 & 1483 & 8.48 & 1530 & 8.48 & 1567 & 8.48 & 1440 \\
\hline 13.90 & 1807 & 13.90 & 1910 & 13.90 & 1970 & 13.90 & 2010 & 13.90 & 1850 \\
\hline 22.80 & 2380 & 22.80 & 2533 & 22.80 & 2603 & 22.80 & 2657 & 22.80 & 2453 \\
\hline 37.30 & 3270 & 37.30 & 3493 & 37.30 & 3583 & 37.30 & 3653 & 37.30 & 3380 \\
\hline 61.00 & 4637 & 61.00 & 4960 & 61.00 & 5090 & 61.00 & 5173 & 61.00 & 4797 \\
\hline 100.00 & 6767 & 100.00 & 7263 & 100.00 & 7433 & 100.00 & 7543 & 100.00 & 7020 \\
\hline 100.00 & 6750 & 100.00 & 7237 & 100.00 & 7407 & 100.00 & 7517 & 100.00 & 7020 \\
\hline 69.50 & 5067 & 69.50 & 5417 & 69.50 & 5550 & 69.50 & 5640 & 69.50 & 5263 \\
\hline 48.30 & 3867 & 48.30 & 4113 & 48.30 & 4223 & 48.30 & 4293 & 48.30 & 4010 \\
\hline 33.60 & 3003 & 33.60 & 3183 & 33.60 & 3267 & 33.60 & 3323 & 33.60 & 3107 \\
\hline 23.40 & 2377 & 23.40 & 2510 & 23.40 & 2577 & 23.40 & 2623 & 23.40 & 2450 \\
\hline 16.20 & 1917 & 16.20 & 2017 & 16.20 & 2073 & 16.20 & 2117 & 16.20 & 1980 \\
\hline 11.30 & 1583 & 11.30 & 1657 & 11.30 & 1700 & 11.30 & 1733 & 11.30 & 1627 \\
\hline 7.85 & 1323 & 7.85 & 1383 & 7.85 & 1423 & 7.85 & 1460 & 7.85 & 1360 \\
\hline 5.45 & 1133 & 5.45 & 1173 & 5.45 & 1210 & 5.46 & 1240 & 5.46 & 1157 \\
\hline 3.79 & 983 & 3.79 & 1020 & 3.79 & 1050 & 3.79 & 1077 & 3.79 & 1006 \\
\hline 2.64 & 871 & 2.64 & 897 & 2.64 & 925 & 2.64 & 949 & 2.64 & 884 \\
\hline 1.83 & 778 & 1.83 & 806 & 1.83 & 830 & 1.83 & 847 & 1.83 & 797 \\
\hline 1.27 & 711 & 1.27 & 732 & 1.27 & 760 & 1.27 & 783 & 1.27 & 727 \\
\hline 0.89 & 658 & 0.89 & 675 & 0.89 & 705 & 0.89 & 717 & 0.89 & 673 \\
\hline 0.62 & 616 & 0.62 & 637 & 0.62 & 654 & 0.62 & 665 & 0.62 & 626 \\
\hline 0.43 & 581 & 0.43 & 599 & 0.43 & 619 & 0.43 & 636 & 0.43 & 592 \\
\hline 0.30 & 570 & 0.30 & 573 & 0.30 & 590 & 0.30 & 610 & 0.30 & 572 \\
\hline 0.21 & 534 & 0.21 & 560 & 0.21 & 577 & 0.21 & 579 & 0.21 & 544 \\
\hline 0.14 & 527 & 0.14 & 534 & 0.14 & 549 & 0.14 & 561 & 0.14 & 542 \\
\hline 0.10 & 518 & 0.10 & 526 & 0.10 & 549 & 0.10 & 550 & 0.10 & 510 \\
\hline
\end{tabular}


Table B - 7: Average values for SRM paste with $0 \%$ beads (by volume) performed prior to the $40 \%$ mortar tests using six-blade vane geometry. [NIST Codes]

\begin{tabular}{|c|c|c|c|c|c|c|c|c|c|}
\hline \multicolumn{10}{|c|}{6 Blade Vane [RHN-83C] } \\
\hline \multicolumn{10}{|c|}{$0 \%$ Beads (Pre-40\%) } \\
\hline \multicolumn{2}{|c|}{ Mix \#2 [SMC-79] } & \multicolumn{2}{|c|}{ Mix \#3 [SMC-81] } & \multicolumn{2}{|c|}{ Mix \#4 [SMC-85] } & \multicolumn{2}{|c|}{ Mix \#5 [SMC-95] } & \multicolumn{2}{|c|}{ Mix \#6 [SMC-97] } \\
\hline $\begin{array}{c}\mathrm{N} \\
{[1 / \mathrm{min}]}\end{array}$ & $\begin{array}{c}\mathrm{M} \\
{[\mu \mathrm{Nm}]}\end{array}$ & $\begin{array}{c}\mathrm{N} \\
{[1 / \mathrm{min}]}\end{array}$ & $\begin{array}{c}\mathrm{M} \\
{[\mu \mathrm{Nm}]}\end{array}$ & $\begin{array}{c}\mathrm{N} \\
{[1 / \mathrm{min}]}\end{array}$ & $\begin{array}{c}\mathrm{M} \\
{[\mu \mathrm{Nm}]}\end{array}$ & $\begin{array}{c}\mathrm{N} \\
{[1 / \mathrm{min}]}\end{array}$ & $\begin{array}{c}\mathrm{M} \\
{[\mu \mathrm{Nm}]}\end{array}$ & $\begin{array}{c}\mathrm{N} \\
{[1 / \mathrm{min}]}\end{array}$ & $\begin{array}{c}\mathrm{M} \\
{[\mu \mathrm{Nm}]}\end{array}$ \\
\hline 0.10 & 451 & 0.10 & 463 & 0.10 & 486 & 0.10 & 433 & 0.10 & 429 \\
\hline 0.16 & 550 & 0.16 & 562 & 0.16 & 549 & 0.16 & 526 & 0.16 & 566 \\
\hline 0.27 & 589 & 0.27 & 600 & 0.27 & 584 & 0.27 & 566 & 0.27 & 606 \\
\hline 0.44 & 627 & 0.44 & 638 & 0.44 & 624 & 0.44 & 600 & 0.44 & 641 \\
\hline 0.72 & 680 & 0.72 & 689 & 0.72 & 677 & 0.72 & 654 & 0.72 & 693 \\
\hline 1.18 & 755 & 1.18 & 762 & 1.18 & 751 & 1.18 & 721 & 1.18 & 766 \\
\hline 1.93 & 858 & 1.93 & 864 & 1.93 & 855 & 1.93 & 823 & 1.93 & 866 \\
\hline 3.16 & 1008 & 3.16 & 1008 & 3.16 & 1001 & 3.16 & 956 & 3.16 & 1006 \\
\hline 5.18 & 1217 & 5.18 & 1217 & 5.18 & 1203 & 5.18 & 1150 & 5.18 & 1210 \\
\hline 8.48 & 1517 & 8.48 & 1507 & 8.48 & 1503 & 8.48 & 1427 & 8.48 & 1503 \\
\hline 13.90 & 1960 & 13.90 & 1943 & 13.90 & 1943 & 13.90 & 1833 & 13.90 & 1933 \\
\hline 22.80 & 2607 & 22.80 & 2577 & 22.80 & 2583 & 22.80 & 2427 & 22.80 & 2557 \\
\hline 37.30 & 3610 & 37.30 & 3557 & 37.30 & 3580 & 37.30 & 3337 & 37.30 & 3510 \\
\hline 61.00 & 5153 & 61.00 & 5053 & 61.00 & 5117 & 61.00 & 4727 & 61.00 & 4953 \\
\hline 100.00 & 7567 & 100.00 & 7407 & 100.00 & 7533 & 100.00 & 6890 & 100.00 & 7213 \\
\hline 100.00 & 7550 & 100.00 & 7390 & 100.00 & 7547 & 100.00 & 6867 & 100.00 & 7193 \\
\hline 69.50 & 5650 & 69.50 & 5550 & 69.50 & 5657 & 69.50 & 5157 & 69.50 & 5403 \\
\hline 48.30 & 4293 & 48.30 & 4223 & 48.30 & 4297 & 48.30 & 3933 & 48.30 & 4127 \\
\hline 33.60 & 3317 & 33.60 & 3263 & 33.60 & 3320 & 33.60 & 3047 & 33.60 & 3203 \\
\hline 23.40 & 2610 & 23.40 & 2570 & 23.40 & 2617 & 23.40 & 2410 & 23.40 & 2537 \\
\hline 16.20 & 2093 & 16.20 & 2067 & 16.20 & 2100 & 16.20 & 1940 & 16.20 & 2047 \\
\hline 11.30 & 1713 & 11.30 & 1697 & 11.30 & 1720 & 11.30 & 1600 & 11.30 & 1690 \\
\hline 7.85 & 1437 & 7.85 & 1417 & 7.85 & 1437 & 7.85 & 1340 & 7.85 & 1417 \\
\hline 5.45 & 1217 & 5.45 & 1203 & 5.45 & 1220 & 5.45 & 1140 & 5.45 & 1207 \\
\hline 3.79 & 1050 & 3.79 & 1037 & 3.79 & 1050 & 3.79 & 987 & 3.79 & 1050 \\
\hline 2.64 & 922 & 2.64 & 913 & 2.64 & 924 & 2.64 & 870 & 2.64 & 924 \\
\hline 1.83 & 824 & 1.83 & 816 & 1.83 & 825 & 1.83 & 778 & 1.83 & 828 \\
\hline 1.27 & 746 & 1.27 & 740 & 1.27 & 748 & 1.27 & 707 & 1.27 & 755 \\
\hline 0.89 & 687 & 0.89 & 680 & 0.89 & 688 & 0.89 & 653 & 0.89 & 698 \\
\hline 0.62 & 642 & 0.62 & 633 & 0.62 & 639 & 0.62 & 607 & 0.62 & 648 \\
\hline 0.43 & 603 & 0.43 & 597 & 0.43 & 600 & 0.43 & 571 & 0.43 & 617 \\
\hline 0.30 & 570 & 0.30 & 565 & 0.30 & 569 & 0.30 & 543 & 0.30 & 585 \\
\hline 0.21 & 545 & 0.21 & 541 & 0.21 & 545 & 0.21 & 520 & 0.21 & 567 \\
\hline 0.14 & 523 & 0.14 & 521 & 0.14 & 525 & 0.14 & 501 & 0.14 & 554 \\
\hline 0.10 & 509 & 0.10 & 506 & 0.10 & 511 & 0.10 & 487 & 0.10 & 530 \\
\hline
\end{tabular}


Table B - 8: Average data set for the combined measured values of SRM paste ( $0 \%$ beads) using six blade vane geometry.

\begin{tabular}{|cccc|}
\hline \multicolumn{4}{|c|}{ 6 Blade Vane } \\
\hline \multicolumn{4}{c}{ 0\% Average Values } \\
\hline $\mathbf{N}$ & & $\mathbf{M}$ \\
{$[1 / \mathrm{min}]$} & $S D$ & {$[\mu N \mathrm{Nm}$} & $S D$ \\
\hline $\mathbf{0 . 1 0}$ & $4.8 E-04$ & $\mathbf{4 3 7}$ & 24 \\
$\mathbf{0 . 1 6}$ & $2.1 E-04$ & $\mathbf{5 5 5}$ & 20 \\
$\mathbf{0 . 2 7}$ & $1.0 E-04$ & $\mathbf{5 9 5}$ & 21 \\
$\mathbf{0 . 4 4}$ & $4.3 E-04$ & $\mathbf{6 3 1}$ & 22 \\
$\mathbf{0 . 7 2}$ & $4.0 E-04$ & $\mathbf{6 8 3}$ & 22 \\
$\mathbf{1 . 1 8}$ & $0.0 E+00$ & $\mathbf{7 5 4}$ & 25 \\
$\mathbf{1 . 9 3}$ & $2.2 E-16$ & $\mathbf{8 5 5}$ & 26 \\
$\mathbf{3 . 1 6}$ & $0.0 E+00$ & $\mathbf{9 9 6}$ & 31 \\
$\mathbf{5 . 1 8}$ & $0.0 E+00$ & $\mathbf{1 1 9 8}$ & 38 \\
$\mathbf{8 . 4 8}$ & $1.8 E-15$ & $\mathbf{1 4 8 9}$ & 47 \\
$\mathbf{1 3 . 9 0}$ & $1.8 E-15$ & $\mathbf{1 9 1 6}$ & 62 \\
$\mathbf{2 2 . 8 0}$ & $3.6 E-15$ & $\mathbf{2 5 3 8}$ & 85 \\
$\mathbf{3 7 . 3 0}$ & $7.1 E-15$ & $\mathbf{3 4 9 7}$ & 121 \\
$\mathbf{6 1 . 0 0}$ & $0.0 E+00$ & $\mathbf{4 9 6 6}$ & 178 \\
$\mathbf{1 0 0 . 0 0}$ & $0.0 E+00$ & $\mathbf{7 2 6 4}$ & 272 \\
$\mathbf{1 0 0 . 0 0}$ & $0.0 E+00$ & $\mathbf{7 2 4 8}$ & 273 \\
$\mathbf{6 9 . 5 0}$ & $0.0 E+00$ & $\mathbf{5 4 3 5}$ & 202 \\
$\mathbf{4 8 . 3 0}$ & $7.1 E-15$ & $\mathbf{4 1 3 8}$ & 149 \\
$\mathbf{3 3 . 6 0}$ & $7.1 E-15$ & $\mathbf{3 2 0 3}$ & 111 \\
$\mathbf{2 3 . 4 0}$ & $3.6 E-15$ & $\mathbf{2 5 2 8}$ & 84 \\
$\mathbf{1 6 . 2 0}$ & $3.6 E-15$ & $\mathbf{2 0 3 5}$ & 66 \\
$\mathbf{1 1 . 3 0}$ & $1.8 E-15$ & $\mathbf{1 6 7 2}$ & 50 \\
$\mathbf{7 . 8 5}$ & $0.0 E+00$ & $\mathbf{1 4 0 0}$ & 43 \\
$\mathbf{5 . 4 5}$ & $2.7 E-03$ & $\mathbf{1 1 9 0}$ & 35 \\
$\mathbf{3 . 7 9}$ & $4.4 E-16$ & $\mathbf{1 0 3 1}$ & 29 \\
$\mathbf{2 . 6 4}$ & $1.3 E-03$ & $\mathbf{9 0 8}$ & 25 \\
$\mathbf{1 . 8 3}$ & $4.4 E-16$ & $\mathbf{8 1 3}$ & 22 \\
$\mathbf{1 . 2 7}$ & $1.5 E-03$ & $\mathbf{7 4 1}$ & 22 \\
$\mathbf{0 . 8 9}$ & $6.4 E-04$ & $\mathbf{6 8 4}$ & 19 \\
$\mathbf{0 . 6 2}$ & $7.0 E-04$ & $\mathbf{6 3 7}$ & 17 \\
$\mathbf{0 . 4 3}$ & $7.8 E-04$ & $\mathbf{6 0 2}$ & 18 \\
$\mathbf{0 . 3 0}$ & $6.5 E-04$ & $\mathbf{5 7 5}$ & 17 \\
$\mathbf{0 . 2 1}$ & $7.0 E-04$ & $\mathbf{5 5 1}$ & 18 \\
$\mathbf{0 . 1 4}$ & $1.2 E-03$ & $\mathbf{5 3 4}$ & 17 \\
$\mathbf{0 . 1 0}$ & $4.5 E-04$ & $\mathbf{5 2 0}$ & 19 \\
\hline & & & \\
\hline
\end{tabular}


Table B - 9: Average values for $20 \%$ mortar mixes (by volume) using six-blade vane geometry. [NIST Codes]

\begin{tabular}{|c|c|c|c|c|c|c|c|c|c|c|c|c|c|}
\hline \multicolumn{10}{|c|}{6 Blade Vane [RHN-83C] } & \multirow{2}{*}{\multicolumn{4}{|c|}{6 Blade Vane [RHN-83C] }} \\
\hline \multicolumn{10}{|c|}{$20 \%$ Beads } & & & & \\
\hline \multicolumn{2}{|c|}{ Mix \#7 [SMC-101] } & \multicolumn{2}{|c|}{ Mix \#8 [SMC-103] } & \multicolumn{2}{|c|}{ Mix \#9 [SMC-109] } & \multicolumn{2}{|c|}{ Mix \#10 [SMC-111] } & \multicolumn{2}{|c|}{ Mix \#11 [SMC-113] } & \multicolumn{4}{|c|}{$20 \%$ Average Values } \\
\hline $\begin{array}{c}\mathrm{N} \\
{[1 / \mathrm{min}]}\end{array}$ & $\begin{array}{c}\mathrm{M} \\
{[\mu \mathrm{Nm}]}\end{array}$ & $\begin{array}{c}\mathrm{N} \\
{[1 / \mathrm{min}]} \\
\end{array}$ & $\begin{array}{c}\mathrm{M} \\
{[\mu \mathrm{Nm}]}\end{array}$ & $\begin{array}{c}\mathrm{N} \\
{[1 / \mathrm{min}]} \\
\end{array}$ & $\begin{array}{c}\mathrm{M} \\
{[\mu \mathrm{Nm}]}\end{array}$ & $\begin{array}{c}\mathrm{N} \\
{[1 / \mathrm{min}]}\end{array}$ & $\begin{array}{c}\mathrm{M} \\
{[\mu \mathrm{Nm}]}\end{array}$ & \begin{tabular}{|c|}
$\mathrm{N}$ \\
{$[1 / \mathrm{min}]$} \\
\end{tabular} & $\begin{array}{c}\mathrm{M} \\
{[\mu \mathrm{Nm}]}\end{array}$ & $\begin{array}{c}\mathbf{N} \\
{[1 / \mathrm{min}]}\end{array}$ & $S D$ & $\begin{array}{c}\mathbf{M} \\
{[\mu \mathrm{Nm}]}\end{array}$ & $S D$ \\
\hline 0.10 & 569 & 0.10 & 589 & 0.10 & 608 & 0.10 & 606 & 0.10 & 582 & 0.10 & 0.000 & 591 & 591 \\
\hline 0.16 & 721 & 0.16 & 756 & 0.16 & 776 & 0.16 & 770 & 0.16 & 734 & 0.16 & 0.000 & 751 & 751 \\
\hline 0.27 & 785 & 0.27 & 822 & 0.27 & 828 & 0.27 & 837 & 0.27 & 791 & 0.27 & 0.000 & 813 & 813 \\
\hline 0.44 & 842 & 0.44 & 891 & 0.44 & 899 & 0.44 & 897 & 0.44 & 850 & 0.44 & 0.000 & 876 & 876 \\
\hline 0.72 & 916 & 0.72 & 968 & 0.72 & 974 & 0.72 & 973 & 0.72 & 934 & 0.72 & 0.001 & 953 & 953 \\
\hline 1.18 & 1027 & 1.18 & 1097 & 1.18 & 1093 & 1.18 & 1090 & 1.18 & 1050 & 1.18 & 0.000 & 1071 & 1071 \\
\hline 1.93 & 1190 & 1.93 & 1273 & 1.93 & 1260 & 1.93 & 1263 & 1.93 & 1217 & 1.93 & 0.000 & 1241 & 1241 \\
\hline 3.16 & 1420 & 3.16 & 1533 & 3.16 & 1513 & 3.16 & 1513 & 3.16 & 1443 & 3.16 & 0.000 & 1485 & 1485 \\
\hline 5.18 & 1763 & 5.18 & 1913 & 5.18 & 1873 & 5.18 & 1873 & 5.18 & 1793 & 5.18 & 0.000 & 1843 & 1843 \\
\hline 8.48 & 2257 & 8.48 & 2460 & 8.48 & 2390 & 8.48 & 2390 & 8.48 & 2290 & 8.48 & 0.001 & 2357 & 2357 \\
\hline 13.90 & 3007 & 13.90 & 3290 & 13.90 & 3187 & 13.90 & 3187 & 13.90 & 3050 & 13.90 & 0.000 & 3144 & 3144 \\
\hline 22.77 & 4143 & 22.80 & 4540 & 22.80 & 4383 & 22.80 & 4387 & 22.80 & 4193 & 22.79 & 0.013 & 4329 & 4329 \\
\hline 37.30 & 5833 & 37.30 & 6407 & 37.30 & 6157 & 37.30 & 6163 & 37.30 & 5880 & 37.30 & 0.000 & 6088 & 6088 \\
\hline 61.03 & 8383 & 61.07 & 9197 & 61.03 & 8820 & 61.07 & 8843 & 61.03 & 8420 & 61.05 & 0.016 & 8733 & 8733 \\
\hline 100.00 & 12233 & 100.00 & 13533 & 100.00 & 12767 & 100.00 & 12867 & 100.00 & 12233 & 100.00 & 0.000 & 12727 & 12727 \\
\hline 100.00 & 12167 & 100.00 & 13400 & 100.00 & 12600 & 100.00 & 12733 & 100.00 & 12133 & 100.00 & 0.000 & 12607 & 12607 \\
\hline 69.50 & 9060 & 69.50 & 9927 & 69.50 & 9360 & 69.50 & 9477 & 69.50 & 9047 & 69.50 & 0.000 & 9374 & 9374 \\
\hline 48.30 & 6813 & 48.30 & 7450 & 48.30 & 7043 & 48.30 & 7130 & 48.30 & 6797 & 48.30 & 0.000 & 7047 & 7047 \\
\hline 33.60 & 5190 & 33.60 & 5673 & 33.60 & 5393 & 33.60 & 5433 & 33.60 & 5183 & 33.60 & 0.000 & 5375 & 5375 \\
\hline 23.37 & 4030 & 23.33 & 4357 & 23.40 & 4200 & 23.37 & 4223 & 23.37 & 4023 & 23.37 & 0.021 & 4167 & 4167 \\
\hline 16.20 & 3183 & 16.20 & 3417 & 16.20 & 3320 & 16.20 & 3333 & 16.20 & 3177 & 16.20 & 0.000 & 3286 & 3286 \\
\hline 11.30 & 2550 & 11.30 & 2723 & 11.30 & 2650 & 11.30 & 2673 & 11.30 & 2553 & 11.30 & 0.000 & 2630 & 2630 \\
\hline 7.85 & 2073 & 7.85 & 2217 & 7.85 & 2163 & 7.84 & 2180 & 7.85 & 2073 & 7.85 & 0.002 & 2141 & 2141 \\
\hline 5.45 & 1727 & 5.45 & 1830 & 5.46 & 1797 & 5.45 & 1807 & 5.46 & 1727 & 5.45 & 0.002 & 1777 & 1777 \\
\hline 3.79 & 1463 & 3.79 & 1550 & 3.79 & 1527 & 3.79 & 1533 & 3.79 & 1460 & 3.79 & 0.001 & 1507 & 1507 \\
\hline 2.64 & 1260 & 2.64 & 1330 & 2.64 & 1320 & 2.64 & 1327 & 2.64 & 1257 & 2.64 & 0.001 & 1299 & 1299 \\
\hline 1.83 & 1107 & 1.83 & 1170 & 1.83 & 1157 & 1.83 & 1163 & 1.83 & 1113 & 1.83 & 0.001 & 1142 & 1142 \\
\hline 1.28 & 986 & 1.27 & 1043 & 1.27 & 1040 & 1.27 & 1043 & 1.27 & 997 & 1.27 & 0.002 & 1022 & 1022 \\
\hline 0.89 & 898 & 0.89 & 939 & 0.89 & 938 & 0.89 & 959 & 0.89 & 898 & 0.89 & 0.001 & 926 & 926 \\
\hline 0.62 & 831 & 0.62 & 864 & 0.61 & 879 & 0.61 & 884 & 0.61 & 841 & 0.61 & 0.001 & 860 & 860 \\
\hline 0.43 & 775 & 0.43 & 814 & 0.43 & 813 & 0.43 & 820 & 0.43 & 782 & 0.43 & 0.001 & 801 & 801 \\
\hline 0.30 & 741 & 0.30 & 768 & 0.30 & 776 & 0.30 & 779 & 0.30 & 745 & 0.30 & 0.001 & 762 & 762 \\
\hline 0.21 & 697 & 0.21 & 721 & 0.21 & 740 & 0.21 & 732 & 0.21 & 713 & 0.21 & 0.000 & 721 & 721 \\
\hline 0.14 & 680 & 0.14 & 700 & 0.14 & 707 & 0.14 & 709 & 0.14 & 686 & 0.14 & 0.000 & 696 & 696 \\
\hline 0.10 & 668 & 0.10 & 684 & 0.10 & 693 & 0.10 & 692 & 0.10 & 654 & 0.10 & 0.001 & 678 & 678 \\
\hline
\end{tabular}


Table B - 10: Average values for $40 \%$ mortar mixes (by volume) using six-blade vane geometry. [NIST Codes]

\begin{tabular}{|c|c|c|c|c|c|c|c|c|c|c|c|c|c|}
\hline \multicolumn{10}{|c|}{6 Blade Vane [RHN-83C] } & \multirow{2}{*}{\multicolumn{4}{|c|}{6 Blade Vane [RHN-83C] }} \\
\hline \multicolumn{10}{|c|}{$40 \%$ Beads } & & & & \\
\hline \multicolumn{2}{|c|}{ Mix \#2 [SMC-79] } & \multicolumn{2}{|c|}{ Mix \#3 [SMC-81] } & \multicolumn{2}{|c|}{ Mix \#4 [SMC-85] } & \multicolumn{2}{|c|}{ Mix \#5 [SMC-95] } & \multicolumn{2}{|c|}{ Mix \#6 [SMC-97] } & \multicolumn{4}{|c|}{$40 \%$ Average Values } \\
\hline $\begin{array}{c}\mathrm{N} \\
{[1 / \mathrm{min}]}\end{array}$ & $\begin{array}{c}\mathrm{M} \\
{[\mu \mathrm{Nm}]}\end{array}$ & $\begin{array}{c}\mathrm{N} \\
{[1 / \mathrm{min}]}\end{array}$ & $\begin{array}{c}\mathrm{M} \\
{[\mu \mathrm{Nm}]}\end{array}$ & $\begin{array}{c}\mathbf{N} \\
{[1 / \mathrm{min}]}\end{array}$ & $\begin{array}{c}\mathrm{M} \\
{[\mu \mathrm{Nm}]}\end{array}$ & $\begin{array}{c}\mathrm{N} \\
{[1 / \mathrm{min}]}\end{array}$ & $\begin{array}{c}\mathrm{M} \\
{[\mu \mathrm{Nm}]}\end{array}$ & $\begin{array}{c}\mathrm{N} \\
{[1 / \mathrm{min}]}\end{array}$ & $\begin{array}{c}\mathrm{M} \\
{[\mu \mathrm{Nm}]}\end{array}$ & $\begin{array}{c}N \\
{[1 / \mathrm{min}]}\end{array}$ & $S D$ & $\underset{[\mu \mathrm{Nm}]}{\mathbf{M}}$ & $S D$ \\
\hline 0.10 & 1077 & 0.10 & 1067 & 0.09 & 1423 & 0.10 & 1009 & 0.10 & 1223 & 0.10 & 0.001 & 1160 & 150 \\
\hline 0.16 & 1347 & 0.16 & 1340 & 0.16 & 1813 & 0.16 & 1273 & 0.16 & 1503 & 0.16 & 0.000 & 1455 & 194 \\
\hline 0.27 & 1470 & 0.27 & 1457 & 0.27 & 1960 & 0.27 & 1377 & 0.27 & 1640 & 0.27 & 0.002 & 1581 & 208 \\
\hline 0.44 & 1567 & 0.44 & 1610 & 0.44 & 2120 & 0.44 & 1493 & 0.44 & 1770 & 0.44 & 0.001 & 1712 & 223 \\
\hline 0.72 & 1747 & 0.72 & 1767 & 0.72 & 2347 & 0.72 & 1657 & 0.73 & 1950 & 0.72 & 0.003 & 1893 & 246 \\
\hline 1.18 & 2030 & 1.18 & 2043 & 1.18 & 2680 & 1.18 & 1903 & 1.18 & 2193 & 1.18 & 0.002 & 2170 & 271 \\
\hline 1.93 & 2403 & 1.94 & 2420 & 1.93 & 3170 & 1.93 & 2223 & 1.94 & 2587 & 1.93 & 0.004 & 2561 & 326 \\
\hline 3.17 & 2957 & 3.17 & 2977 & 3.16 & 3923 & 3.17 & 2713 & 3.16 & 3143 & 3.16 & 0.004 & 3143 & 414 \\
\hline 5.18 & 3770 & 5.18 & 3773 & 5.17 & 4933 & 5.18 & 3477 & 5.19 & 3937 & 5.18 & 0.005 & 3978 & 500 \\
\hline 8.48 & 4897 & 8.48 & 4943 & 8.48 & 6410 & 8.48 & 4510 & 8.48 & 5090 & 8.48 & 0.001 & 5170 & 649 \\
\hline 13.90 & 6573 & 13.90 & 6543 & 13.90 & 8410 & 13.90 & 6033 & 13.90 & 6747 & 13.90 & 0.000 & 6861 & 810 \\
\hline 22.77 & 8957 & 22.80 & 8903 & 22.77 & 11267 & 22.77 & 8307 & 22.77 & 9143 & 22.77 & 0.013 & 9315 & 1015 \\
\hline 37.30 & 12600 & 37.30 & 12300 & 37.30 & 15367 & 37.30 & 11600 & 37.30 & 12700 & 37.30 & 0.000 & 12913 & 1286 \\
\hline 61.03 & 17500 & 61.10 & 17100 & 61.07 & 21367 & 61.07 & 16400 & 61.07 & 17700 & 61.07 & 0.021 & 18013 & 1735 \\
\hline 100.00 & 25300 & 100.00 & 24700 & 100.00 & 29667 & 100.00 & 23267 & 100.00 & 25033 & 100.00 & 0.000 & 25593 & 2154 \\
\hline 100.00 & 24433 & 99.93 & 23867 & 100.00 & 28033 & 100.00 & 22500 & 100.00 & 23733 & 99.99 & 0.027 & 24513 & 1870 \\
\hline 69.50 & 18367 & 69.50 & 17500 & 69.50 & 21567 & 69.50 & 16500 & 69.50 & 17400 & 69.50 & 0.000 & 18267 & 1753 \\
\hline 48.30 & 13900 & 48.30 & 13033 & 48.30 & 16000 & 48.30 & 12333 & 48.30 & 13033 & 48.30 & 0.000 & 13660 & 1271 \\
\hline 33.60 & 10667 & 33.60 & 9997 & 33.60 & 12233 & 33.60 & 9317 & 33.60 & 10000 & 33.60 & 0.000 & 10443 & 992 \\
\hline 23.37 & 8227 & 23.40 & 7673 & 23.40 & 9427 & 23.33 & 7123 & 23.37 & 7620 & 23.37 & 0.025 & 8014 & 788 \\
\hline 16.20 & 6380 & 16.20 & 6000 & 16.20 & 7373 & 16.20 & 5537 & 16.20 & 5970 & 16.20 & 0.000 & 6252 & 621 \\
\hline 11.30 & 5057 & 11.30 & 4743 & 11.30 & 5840 & 11.30 & 4347 & 11.30 & 4713 & 11.30 & 0.000 & 4940 & 503 \\
\hline 7.85 & 4043 & 7.85 & 3807 & 7.84 & 4617 & 7.85 & 3497 & 7.85 & 3790 & 7.85 & 0.005 & 3951 & 375 \\
\hline 5.45 & 3270 & 5.46 & 3080 & 5.45 & 3760 & 5.46 & 2837 & 5.46 & 3073 & 5.46 & 0.002 & 3204 & 310 \\
\hline 3.80 & 2703 & 3.79 & 2547 & 3.79 & 3093 & 3.79 & 2353 & 3.79 & 2553 & 3.79 & 0.002 & 2650 & 248 \\
\hline 2.64 & 2260 & 2.64 & 2127 & 2.63 & 2617 & 2.64 & 1973 & 2.63 & 2133 & 2.64 & 0.003 & 2222 & 217 \\
\hline 1.83 & 1930 & 1.83 & 1823 & 1.84 & 2207 & 1.83 & 1710 & 1.83 & 1853 & 1.83 & 0.003 & 1905 & 167 \\
\hline 1.27 & 1690 & 1.27 & 1593 & 1.28 & 1927 & 1.27 & 1493 & 1.27 & 1617 & 1.27 & 0.002 & 1664 & 146 \\
\hline 0.89 & 1497 & 0.89 & 1413 & 0.88 & 1703 & 0.89 & 1333 & 0.89 & 1433 & 0.89 & 0.001 & 1476 & 125 \\
\hline 0.62 & 1347 & 0.61 & 1277 & 0.62 & 1537 & 0.62 & 1213 & 0.62 & 1297 & 0.62 & 0.001 & 1334 & 110 \\
\hline 0.43 & 1233 & 0.43 & 1170 & 0.43 & 1410 & 0.43 & 1107 & 0.43 & 1210 & 0.43 & 0.001 & 1226 & 102 \\
\hline 0.30 & 1147 & 0.30 & 1097 & 0.30 & 1300 & 0.30 & 1033 & 0.30 & 1120 & 0.30 & 0.002 & 1139 & 89 \\
\hline 0.21 & 1083 & 0.21 & 1037 & 0.21 & 1207 & 0.21 & 974 & 0.21 & 1040 & 0.21 & 0.001 & 1068 & 77 \\
\hline 0.14 & 1013 & 0.14 & 980 & 0.14 & 1167 & 0.14 & 934 & 0.14 & 1015 & 0.14 & 0.000 & 1022 & 78 \\
\hline 0.10 & 974 & 0.10 & 944 & 0.10 & 1103 & 0.10 & 896 & 0.10 & 982 & 0.10 & 0.000 & 980 & 69 \\
\hline
\end{tabular}


Table B - 11: Average values for SRM paste with $0 \%$ beads (by volume) performed prior to the $20 \%$ mortar tests using serrated coaxial cylinder geometry. [NIST Codes]

\begin{tabular}{|c|c|c|c|c|c|c|c|c|c|}
\hline \multicolumn{10}{|c|}{ Serrated Coaxial Cylinder [SS18] } \\
\hline \multicolumn{10}{|c|}{$0 \%$ Beads (Pre-20\%) } \\
\hline \multicolumn{2}{|c|}{ Mix \#7 [SMC-101] } & \multicolumn{2}{|c|}{ Mix \#8 [SMC-103] } & \multicolumn{2}{|c|}{ Mix \#9 [SMC-109] } & \multicolumn{2}{|c|}{ Mix \#10 [SMC-111] } & \multicolumn{2}{|c|}{ Mix \#11 [SMC-113 } \\
\hline $\begin{array}{c}\mathrm{N} \\
{[1 / \mathrm{min}]}\end{array}$ & $\begin{array}{c}\mathrm{M} \\
{[\mu \mathrm{Nm}]}\end{array}$ & $\begin{array}{c}\mathrm{N} \\
{[1 / \mathrm{min}]}\end{array}$ & $\begin{array}{c}\mathrm{M} \\
{[\mu \mathrm{Nm}]}\end{array}$ & $\begin{array}{c}\mathrm{N} \\
{[1 / \mathrm{min}]}\end{array}$ & $\begin{array}{c}\mathrm{M} \\
{[\mu \mathrm{Nm}]}\end{array}$ & $\begin{array}{c}\mathrm{N} \\
{[1 / \mathrm{min}]}\end{array}$ & $\begin{array}{c}\mathrm{M} \\
{[\mu \mathrm{Nm}]}\end{array}$ & $\begin{array}{c}\mathrm{N} \\
{[1 / \mathrm{min}]}\end{array}$ & $\begin{array}{c}\mathrm{M} \\
{[\mu \mathrm{Nm}]}\end{array}$ \\
\hline 0.10 & 385 & 0.10 & 428 & 0.10 & 459 & 0.10 & 442 & 0.10 & 435 \\
\hline 0.16 & 478 & 0.16 & 545 & 0.16 & 611 & 0.16 & 569 & 0.16 & 569 \\
\hline 0.27 & 511 & 0.27 & 583 & 0.27 & 654 & 0.27 & 617 & 0.27 & 624 \\
\hline 0.44 & 578 & 0.44 & 625 & 0.44 & 707 & 0.44 & 674 & 0.44 & 676 \\
\hline 0.72 & 625 & 0.72 & 687 & 0.72 & 780 & 0.72 & 748 & 0.72 & 747 \\
\hline 1.18 & 708 & 1.18 & 767 & 1.18 & 889 & 1.18 & 858 & 1.18 & 850 \\
\hline 1.93 & 805 & 1.93 & 879 & 1.93 & 1027 & 1.93 & 977 & 1.93 & 976 \\
\hline 3.16 & 955 & 3.16 & 1037 & 3.16 & 1190 & 3.16 & 1167 & 3.16 & 1157 \\
\hline 5.18 & 1170 & 5.18 & 1250 & 5.18 & 1440 & 5.18 & 1413 & 5.18 & 1407 \\
\hline 8.48 & 1477 & 8.48 & 1560 & 8.48 & 1810 & 8.48 & 1767 & 8.48 & 1760 \\
\hline 13.90 & 1913 & 13.90 & 2010 & 13.90 & 2330 & 13.90 & 2273 & 13.90 & 2263 \\
\hline 22.80 & 2547 & 22.80 & 2650 & 22.80 & 3107 & 22.80 & 3030 & 22.80 & 3010 \\
\hline 37.30 & 3513 & 37.30 & 3637 & 37.30 & 4290 & 37.30 & 4177 & 37.30 & 4153 \\
\hline 61.00 & 5000 & 61.00 & 5167 & 61.00 & 6093 & 61.00 & 5923 & 61.00 & 5900 \\
\hline 100.00 & 7317 & 100.00 & 7547 & 100.00 & 8860 & 100.00 & 8597 & 100.00 & 8537 \\
\hline 100.00 & 7277 & 100.00 & 7440 & 100.00 & 8730 & 100.00 & 8497 & 100.00 & 8420 \\
\hline 69.50 & 5440 & 69.50 & 5560 & 69.50 & 6537 & 69.50 & 6363 & 69.50 & 6307 \\
\hline 48.30 & 4117 & 48.30 & 4203 & 48.30 & 4923 & 48.30 & 4800 & 48.30 & 4750 \\
\hline 33.60 & 3157 & 33.60 & 3220 & 33.60 & 3760 & 33.60 & 3673 & 33.60 & 3627 \\
\hline 23.40 & 2467 & 23.40 & 2520 & 23.40 & 2923 & 23.40 & 2860 & 23.40 & 2820 \\
\hline 16.20 & 1970 & 16.20 & 2010 & 16.20 & 2320 & 16.20 & 2273 & 16.20 & 2237 \\
\hline 11.30 & 1600 & 11.30 & 1640 & 11.30 & 1877 & 11.30 & 1837 & 11.30 & 1807 \\
\hline 7.85 & 1313 & 7.85 & 1350 & 7.85 & 1543 & 7.85 & 1517 & 7.85 & 1493 \\
\hline 5.45 & 1100 & 5.45 & 1133 & 5.45 & 1293 & 5.45 & 1267 & 5.45 & 1250 \\
\hline 3.79 & 931 & 3.79 & 966 & 3.79 & 1103 & 3.79 & 1073 & 3.79 & 1070 \\
\hline 2.64 & 800 & 2.64 & 834 & 2.64 & 956 & 2.64 & 922 & 2.64 & 918 \\
\hline 1.83 & 710 & 1.83 & 733 & 1.83 & 850 & 1.83 & 809 & 1.83 & 811 \\
\hline 1.27 & 620 & 1.27 & 653 & 1.27 & 741 & 1.27 & 720 & 1.27 & 728 \\
\hline 0.89 & 569 & 0.89 & 597 & 0.89 & 661 & 0.88 & 654 & 0.89 & 647 \\
\hline 0.62 & 529 & 0.62 & 555 & 0.62 & 614 & 0.62 & 630 & 0.62 & 597 \\
\hline 0.43 & 482 & 0.43 & 567 & 0.42 & 586 & 0.43 & 582 & 0.43 & 568 \\
\hline 0.29 & 448 & 0.30 & 481 & 0.30 & 538 & 0.30 & 504 & 0.30 & 541 \\
\hline 0.21 & 424 & 0.21 & 516 & 0.21 & 566 & 0.20 & 529 & 0.21 & 574 \\
\hline 0.14 & 441 & 0.15 & 477 & 0.14 & 567 & 0.14 & 538 & 0.13 & 556 \\
\hline 0.09 & 397 & 0.10 & 494 & 0.10 & 561 & 0.10 & 486 & 0.09 & 497 \\
\hline
\end{tabular}


Table B - 12: Average values for SRM paste with $0 \%$ beads (by volume) performed prior to the $\mathbf{4 0} \%$ mortar tests using serrated coaxial cylinder geometry. [NIST Codes]

\begin{tabular}{|c|c|c|c|c|c|c|c|c|c|}
\hline \multicolumn{10}{|c|}{ Serrated Coaxial Cylinder [SS18] } \\
\hline \multicolumn{10}{|c|}{$0 \%$ Beads (Pre-40\%) } \\
\hline \multicolumn{2}{|c|}{ Mix \#2 [SMC-79] } & \multicolumn{2}{|c|}{ Mix \#3 [SMC-81] } & \multicolumn{2}{|c|}{ Mix \#4 [SMC-85] } & \multicolumn{2}{|c|}{ Mix \#5 [SMC-95] } & \multicolumn{2}{|c|}{ Mix \#6 [SMC-97] } \\
\hline $\begin{array}{c}\mathrm{N} \\
{[1 / \mathrm{min}]}\end{array}$ & $\begin{array}{c}\mathrm{M} \\
{[\mu \mathrm{Nm}]}\end{array}$ & $\begin{array}{c}\mathrm{N} \\
{[1 / \mathrm{min}]}\end{array}$ & $\begin{array}{c}\mathrm{M} \\
{[\mu \mathrm{Nm}]}\end{array}$ & $\begin{array}{c}\mathrm{N} \\
{[1 / \mathrm{min}]}\end{array}$ & $\begin{array}{c}\mathrm{M} \\
{[\mu \mathrm{Nm}]}\end{array}$ & $\begin{array}{c}\mathrm{N} \\
{[1 / \mathrm{min}]}\end{array}$ & $\begin{array}{c}\mathrm{M} \\
{[\mu \mathrm{Nm}]}\end{array}$ & $\begin{array}{c}\mathrm{N} \\
{[1 / \mathrm{min}]}\end{array}$ & $\begin{array}{c}\mathbf{M} \\
{[\mu \mathrm{Nm}]}\end{array}$ \\
\hline 0.10 & 395 & 0.10 & 372 & 0.10 & 379 & 0.10 & 387 & 0.10 & 454 \\
\hline 0.16 & 478 & 0.16 & 454 & 0.16 & 450 & 0.16 & 465 & 0.16 & 577 \\
\hline 0.27 & 518 & 0.27 & 494 & 0.27 & 487 & 0.27 & 499 & 0.27 & 615 \\
\hline 0.44 & 570 & 0.44 & 545 & 0.44 & 533 & 0.44 & 541 & 0.44 & 662 \\
\hline 0.72 & 641 & 0.72 & 616 & 0.72 & 595 & 0.72 & 600 & 0.72 & 727 \\
\hline 1.18 & 733 & 1.18 & 708 & 1.18 & 680 & 1.18 & 681 & 1.18 & 816 \\
\hline 1.93 & 860 & 1.93 & 824 & 1.93 & 794 & 1.93 & 792 & 1.93 & 936 \\
\hline 3.16 & 1034 & 3.16 & 986 & 3.16 & 951 & 3.16 & 946 & 3.16 & 1103 \\
\hline 5.18 & 1280 & 5.18 & 1213 & 5.18 & 1170 & 5.18 & 1160 & 5.18 & 1337 \\
\hline 8.48 & 1630 & 8.48 & 1533 & 8.48 & 1480 & 8.48 & 1467 & 8.48 & 1667 \\
\hline 13.90 & 2130 & 13.90 & 1993 & 13.90 & 1923 & 13.90 & 1910 & 13.90 & 2143 \\
\hline 22.80 & 2857 & 22.80 & 2667 & 22.80 & 2567 & 22.80 & 2540 & 22.80 & 2833 \\
\hline 37.30 & 3973 & 37.30 & 3707 & 37.30 & 3553 & 37.30 & 3510 & 37.30 & 3897 \\
\hline 61.10 & 5680 & 61.00 & 5317 & 61.00 & 5073 & 61.00 & 4997 & 61.00 & 5517 \\
\hline 100.00 & 8273 & 100.00 & 7753 & 100.00 & 7457 & 100.00 & 7330 & 100.00 & 7990 \\
\hline 100.00 & 8207 & 100.00 & 7670 & 100.00 & 7393 & 100.00 & 7250 & 100.00 & 7913 \\
\hline 69.50 & 6167 & 69.50 & 5743 & 69.50 & 5543 & 69.50 & 5433 & 69.50 & 5940 \\
\hline 48.30 & 4650 & 48.30 & 4337 & 48.30 & 4190 & 48.30 & 4107 & 48.30 & 4487 \\
\hline 33.60 & 3553 & 33.60 & 3320 & 33.60 & 3217 & 33.60 & 3150 & 33.60 & 3443 \\
\hline 23.40 & 2760 & 23.40 & 2587 & 23.40 & 2517 & 23.40 & 2463 & 23.40 & 2693 \\
\hline 16.20 & 2190 & 16.20 & 2053 & 16.20 & 1997 & 16.20 & 1957 & 16.20 & 2147 \\
\hline 11.30 & 1763 & 11.30 & 1663 & 11.30 & 1623 & 11.30 & 1590 & 11.30 & 1743 \\
\hline 7.85 & 1443 & 7.85 & 1363 & 7.85 & 1337 & 7.85 & 1307 & 7.85 & 1443 \\
\hline 5.46 & 1200 & 5.45 & 1130 & 5.45 & 1113 & 5.45 & 1090 & 5.45 & 1207 \\
\hline 3.79 & 1011 & 3.79 & 957 & 3.79 & 943 & 3.79 & 923 & 3.79 & 1027 \\
\hline 2.64 & 864 & 2.64 & 820 & 2.64 & 809 & 2.64 & 792 & 2.64 & 888 \\
\hline 1.83 & 748 & 1.83 & 711 & 1.83 & 703 & 1.83 & 689 & 1.83 & 775 \\
\hline 1.27 & 656 & 1.27 & 625 & 1.27 & 618 & 1.27 & 607 & 1.27 & 687 \\
\hline 0.89 & 582 & 0.89 & 555 & 0.89 & 551 & 0.89 & 541 & 0.89 & 643 \\
\hline 0.62 & 523 & 0.62 & 498 & 0.62 & 497 & 0.62 & 487 & 0.62 & 573 \\
\hline 0.43 & 475 & 0.43 & 452 & 0.43 & 454 & 0.43 & 446 & 0.43 & 522 \\
\hline 0.30 & 436 & 0.30 & 416 & 0.30 & 418 & 0.30 & 410 & 0.30 & 486 \\
\hline 0.21 & 405 & 0.21 & 385 & 0.21 & 389 & 0.21 & 383 & 0.20 & 478 \\
\hline 0.14 & 380 & 0.14 & 362 & 0.14 & 366 & 0.14 & 360 & 0.14 & 437 \\
\hline 0.10 & 360 & 0.10 & 342 & 0.10 & 348 & 0.10 & 341 & 0.10 & 434 \\
\hline
\end{tabular}


Table B - 13: Average data set for the combined measured values of SRM paste ( $0 \%$ beads) using serrated coaxial cylinder geometry.

\begin{tabular}{|cc|cc|}
\hline \multicolumn{4}{c|}{ Coaxial Cylinder } \\
\hline \multicolumn{4}{c}{ 0\% Average Values } \\
\hline $\mathbf{N}$ & & $\mathbf{M}$ \\
{$[1 / \mathrm{min}]$} & $S D$ & {$[\mu \mathrm{Nm}]$} & $S D$ \\
\hline $\mathbf{0 . 1 0}$ & $4.2 E-04$ & $\mathbf{4 1 4}$ & 32 \\
$\mathbf{0 . 1 6}$ & $2.0 E-04$ & $\mathbf{5 2 0}$ & 57 \\
$\mathbf{0 . 2 7}$ & $1.3 E-04$ & $\mathbf{5 6 0}$ & 61 \\
$\mathbf{0 . 4 4}$ & $4.0 E-04$ & $\mathbf{6 1 1}$ & 62 \\
$\mathbf{0 . 7 2}$ & $2.1 E-04$ & $\mathbf{6 7 7}$ & 66 \\
$\mathbf{1 . 1 8}$ & $1.0 E-03$ & $\mathbf{7 6 9}$ & 74 \\
$\mathbf{1 . 9 3}$ & $2.2 E-16$ & $\mathbf{8 8 7}$ & 82 \\
$\mathbf{3 . 1 6}$ & $0.0 E+00$ & $\mathbf{1 0 5 3}$ & 90 \\
$\mathbf{5 . 1 8}$ & $0.0 E+00$ & $\mathbf{1 2 8 4}$ & 103 \\
$\mathbf{8 . 4 8}$ & $1.8 E-15$ & $\mathbf{1 6 1 5}$ & 124 \\
$\mathbf{1 3 . 9 0}$ & $1.8 E-15$ & $\mathbf{2 0 8 9}$ & 153 \\
$\mathbf{2 2 . 8 0}$ & $3.6 E-15$ & $\mathbf{2 7 8 1}$ & 204 \\
$\mathbf{3 7 . 3 0}$ & $7.1 E-15$ & $\mathbf{3 8 4 1}$ & 281 \\
$\mathbf{6 1 . 0 1}$ & $3.0 E-02$ & $\mathbf{5 4 6 7}$ & 393 \\
$\mathbf{1 0 0 . 0 0}$ & $0.0 E+00$ & $\mathbf{7 9 6 6}$ & 541 \\
$\mathbf{1 0 0 . 0 0}$ & $0.0 E+00$ & $\mathbf{7 8 8 0}$ & 523 \\
$\mathbf{6 9 . 5 0}$ & $0.0 E+00$ & $\mathbf{5 9 0 3}$ & 394 \\
$\mathbf{4 8 . 3 0}$ & $7.1 E-15$ & $\mathbf{4 4 5 6}$ & 291 \\
$\mathbf{3 3 . 6 0}$ & $7.1 E-15$ & $\mathbf{3 4 1 2}$ & 218 \\
$\mathbf{2 3 . 4 0}$ & $3.6 E-15$ & $\mathbf{2 6 6 1}$ & 164 \\
$\mathbf{1 6 . 2 0}$ & $3.6 E-15$ & $\mathbf{2 1 1 5}$ & 128 \\
$\mathbf{1 1 . 3 0}$ & $1.8 E-15$ & $\mathbf{1 7 1 4}$ & 99 \\
$\mathbf{7 . 8 5}$ & $0.0 E+00$ & $\mathbf{1 4 1 1}$ & 83 \\
$\mathbf{5 . 4 5}$ & $3.0 E-03$ & $\mathbf{1 1 7 8}$ & 71 \\
$\mathbf{3 . 7 9}$ & $4.4 E-16$ & $\mathbf{1 0 0 0}$ & 62 \\
$\mathbf{2 . 6 4}$ & $1.3 E-03$ & $\mathbf{8 6 0}$ & 55 \\
$\mathbf{1 . 8 3}$ & $1.0 E-03$ & $\mathbf{7 5 4}$ & 52 \\
$\mathbf{1 . 2 7}$ & $1.5 E-03$ & $\mathbf{6 6 6}$ & 48 \\
$\mathbf{0 . 8 9}$ & $7.9 E-04$ & $\mathbf{6 0 0}$ & 45 \\
$\mathbf{0 . 6 2}$ & $8.4 E-04$ & $\mathbf{5 5 0}$ & 49 \\
$\mathbf{0 . 4 3}$ & $2.1 E-03$ & $\mathbf{5 1 3}$ & 55 \\
$\mathbf{0 . 3 0}$ & $1.9 E-03$ & $\mathbf{4 6 8}$ & 47 \\
$\mathbf{0 . 2 1}$ & $2.5 E-03$ & $\mathbf{4 6 5}$ & 73 \\
$\mathbf{0 . 1 4}$ & $4.3 E-03$ & $\mathbf{4 4 8}$ & 78 \\
$\mathbf{0 . 1 0}$ & $2.7 E-03$ & $\mathbf{4 2 6}$ & 76 \\
\hline & & & \\
& & \\
\hline
\end{tabular}


Table B - 14: Average values for 20 \% mortar mixes (by volume) using serrated coaxial cylinder geometry. [NIST Codes]

\begin{tabular}{|c|c|c|c|c|c|c|c|c|c|c|c|c|c|}
\hline \multicolumn{10}{|c|}{ Serrated Coaxial Cylinder [SS18] } & \multirow{3}{*}{\multicolumn{4}{|c|}{$\begin{array}{c}\text { Serrated Coaxial Cylinder [SS18 } \\
20 \% \text { Average Values }\end{array}$}} \\
\hline \multicolumn{10}{|c|}{$20 \%$ Beads } & & & & \\
\hline \multicolumn{2}{|c|}{ Mix \#7 [SMC-101] } & \multicolumn{2}{|c|}{ Mix \#8 [SMC-103] } & \multicolumn{2}{|c|}{ Mix \#9 [SMC-109] } & \multicolumn{2}{|c|}{ Mix \#10 [SMC-111] } & \multicolumn{2}{|c|}{ Mix \#11 [SMC-113] } & & & & \\
\hline $\begin{array}{c}\mathrm{N} \\
{[1 / \mathrm{min}]}\end{array}$ & $\begin{array}{c}\mathrm{M} \\
{[\mu \mathrm{Nm}]}\end{array}$ & $\begin{array}{c}\mathrm{N} \\
{[1 / \mathrm{min}]}\end{array}$ & $\begin{array}{c}\mathrm{M} \\
{[\mu \mathrm{Nm}]}\end{array}$ & $\begin{array}{c}\mathrm{N} \\
{[1 / \mathrm{min}]}\end{array}$ & $\begin{array}{c}\mathrm{M} \\
{[\mu \mathrm{Nm}]}\end{array}$ & $\begin{array}{c}\mathrm{N} \\
{[1 / \mathrm{min}]}\end{array}$ & $\begin{array}{c}\mathrm{M} \\
{[\mu \mathrm{Nm}]}\end{array}$ & $\begin{array}{c}\mathrm{N} \\
{[1 / \mathrm{min}]}\end{array}$ & $\begin{array}{c}\mathrm{M} \\
{[\mu \mathrm{Nm}]}\end{array}$ & $\begin{array}{c}N \\
{[1 / \min ]}\end{array}$ & $S D$ & $\begin{array}{c}\mathrm{M} \\
{[\mu \mathrm{Nm}]}\end{array}$ & $S D$ \\
\hline 0.10 & 628 & 0.10 & 602 & 0.10 & 675 & 0.10 & 606 & 0.10 & 631 & 0.1 & 0.000 & 0.1 & 26 \\
\hline 0.16 & 766 & 0.16 & 742 & 0.17 & 839 & 0.16 & 756 & 0.16 & 759 & 0.2 & 0.000 & 0.2 & 34 \\
\hline 0.27 & 811 & 0.27 & 782 & 0.27 & 889 & 0.27 & 804 & 0.27 & 806 & 0.3 & 0.000 & 0.3 & 37 \\
\hline 0.44 & 877 & 0.44 & 823 & 0.44 & 953 & 0.44 & 865 & 0.44 & 874 & 0.4 & 0.000 & 0.4 & 42 \\
\hline 0.72 & 965 & 0.72 & 909 & 0.72 & 1053 & 0.72 & 954 & 0.72 & 975 & 0.7 & 0.001 & 0.7 & 47 \\
\hline 1.18 & 1093 & 1.18 & 1030 & 1.18 & 1197 & 1.18 & 1083 & 1.18 & 1113 & 1.2 & 0.000 & 1.2 & 54 \\
\hline 1.93 & 1287 & 1.93 & 1207 & 1.93 & 1397 & 1.93 & 1260 & 1.93 & 1317 & 1.9 & 0.000 & 1.9 & 63 \\
\hline 3.16 & 1557 & 3.16 & 1460 & 3.16 & 1673 & 3.16 & 1520 & 3.16 & 1593 & 3.2 & 0.000 & 3.2 & 72 \\
\hline 5.18 & 1927 & 5.18 & 1810 & 5.18 & 2087 & 5.18 & 1887 & 5.18 & 1993 & 5.2 & 0.001 & 5.2 & 94 \\
\hline 8.48 & 2457 & 8.48 & 2313 & 8.48 & 2667 & 8.48 & 2413 & 8.48 & 2567 & 8.5 & 0.000 & 8.5 & 123 \\
\hline 13.90 & 3253 & 13.90 & 3050 & 13.90 & 3530 & 13.90 & 3197 & 13.90 & 3403 & 13.9 & 0.000 & 13.9 & 166 \\
\hline 22.80 & 4443 & 22.80 & 4143 & 22.80 & 4827 & 22.80 & 4377 & 22.80 & 4663 & 22.8 & 0.000 & 22.8 & 236 \\
\hline 37.30 & 6267 & 37.30 & 5820 & 37.30 & 6810 & 37.30 & 6207 & 37.30 & 6580 & 37.3 & 0.000 & 37.3 & 338 \\
\hline 61.00 & 9050 & 61.03 & 8367 & 61.03 & 9783 & 61.00 & 8950 & 61.00 & 9420 & 61.0 & 0.016 & 61.0 & 476 \\
\hline 100.00 & 13367 & 100.00 & 12300 & 100.00 & 14367 & 100.00 & 13167 & 100.00 & 13867 & 100.0 & 0.000 & 100.0 & 695 \\
\hline 100.00 & 13267 & 100.00 & 12167 & 100.00 & 14267 & 100.00 & 13033 & 100.00 & 13767 & 100.0 & 0.000 & 100.0 & 708 \\
\hline 69.50 & 9850 & 69.50 & 9067 & 69.50 & 10633 & 69.50 & 9703 & 69.50 & 10290 & 69.5 & 0.000 & 69.5 & 534 \\
\hline 48.30 & 7397 & 48.30 & 6790 & 48.30 & 7983 & 48.30 & 7300 & 48.30 & 7717 & 48.3 & 0.000 & 48.3 & 404 \\
\hline 33.60 & 5610 & 33.60 & 5153 & 33.60 & 6057 & 33.60 & 5553 & 33.60 & 5857 & 33.6 & 0.000 & 33.6 & 305 \\
\hline 23.40 & 4323 & 23.40 & 3970 & 23.40 & 4657 & 23.40 & 4283 & 23.40 & 4507 & 23.4 & 0.000 & 23.4 & 232 \\
\hline 16.20 & 3387 & 16.20 & 3117 & 16.20 & 3653 & 16.20 & 3363 & 16.20 & 3520 & 16.2 & 0.000 & 16.2 & 179 \\
\hline 11.30 & 2707 & 11.30 & 2497 & 11.30 & 2913 & 11.30 & 2693 & 11.30 & 2807 & 11.3 & 0.000 & 11.3 & 138 \\
\hline 7.85 & 2203 & 7.85 & 2033 & 7.85 & 2370 & 7.85 & 2193 & 7.85 & 2277 & 7.9 & 0.000 & 7.9 & 111 \\
\hline 5.46 & 1820 & 5.45 & 1690 & 5.46 & 1960 & 5.46 & 1823 & 5.46 & 1877 & 5.5 & 0.003 & 5.5 & 88 \\
\hline 3.79 & 1543 & 3.79 & 1423 & 3.79 & 1653 & 3.79 & 1540 & 3.79 & 1583 & 3.8 & 0.000 & 3.8 & 75 \\
\hline 2.64 & 1320 & 2.64 & 1227 & 2.63 & 1413 & 2.64 & 1317 & 2.64 & 1360 & 2.6 & 0.003 & 2.6 & 61 \\
\hline 1.83 & 1147 & 1.83 & 1063 & 1.83 & 1233 & 1.83 & 1157 & 1.83 & 1177 & 1.8 & 0.001 & 1.8 & 55 \\
\hline 1.27 & 1009 & 1.27 & 943 & 1.27 & 1097 & 1.27 & 1018 & 1.27 & 1052 & 1.3 & 0.002 & 1.3 & 51 \\
\hline 0.89 & 908 & 0.89 & 861 & 0.89 & 999 & 0.89 & 936 & 0.88 & 954 & 0.9 & 0.001 & 0.9 & 46 \\
\hline 0.62 & 842 & 0.61 & 783 & 0.61 & 911 & 0.62 & 851 & 0.62 & 876 & 0.6 & 0.002 & 0.6 & 42 \\
\hline 0.43 & 767 & 0.42 & 761 & 0.43 & 867 & 0.43 & 787 & 0.43 & 804 & 0.4 & 0.003 & 0.4 & 38 \\
\hline 0.30 & 738 & 0.29 & 729 & 0.30 & 813 & 0.29 & 775 & 0.29 & 771 & 0.3 & 0.004 & 0.3 & 30 \\
\hline 0.20 & 727 & 0.21 & 653 & 0.20 & 790 & 0.21 & 697 & 0.20 & 876 & 0.2 & 0.004 & 0.2 & 78 \\
\hline 0.14 & 676 & 0.14 & 652 & 0.15 & 760 & 0.15 & 735 & 0.14 & 699 & 0.1 & 0.002 & 0.1 & 39 \\
\hline 0.10 & 650 & 0.11 & 609 & 0.10 & 716 & 0.09 & 700 & 0.10 & 690 & 0.1 & 0.005 & 0.1 & 39 \\
\hline
\end{tabular}


Table B - 15: Average values for $40 \%$ mortar mixes (by volume) using serrated coaxial cylinder geometry. [NIST Codes]

\begin{tabular}{|c|c|c|c|c|c|c|c|c|c|c|c|c|c|}
\hline \multicolumn{10}{|c|}{ Serrated Coaxial Cylinder [SS18] } & \multirow{3}{*}{\multicolumn{4}{|c|}{$\begin{array}{c}\text { Serrated Coaxial Cylinder [SS18] } \\
40 \% \text { Average Values }\end{array}$}} \\
\hline \multicolumn{10}{|c|}{$40 \%$ Beads } & & & & \\
\hline \multicolumn{2}{|c|}{ Mix \#2 [SMC-79] } & \multicolumn{2}{|c|}{ Mix \#3 [SMC-81] } & \multicolumn{2}{|c|}{ Mix \#4 [SMC-85] } & \multicolumn{2}{|c|}{ Mix \#5 [SMC-95] } & \multicolumn{2}{|c|}{ Mix \#6 [SMC-97] } & & & & \\
\hline $\begin{array}{c}N \\
{[1 / \mathrm{min}]}\end{array}$ & $\begin{array}{c}\mathrm{M} \\
{[\mu \mathrm{Nm}]}\end{array}$ & $\begin{array}{c}\mathrm{N} \\
{[1 / \mathrm{min}]}\end{array}$ & $\begin{array}{c}\mathrm{M} \\
{[\mu \mathrm{Nm}]}\end{array}$ & $\begin{array}{c}\mathrm{N} \\
{[1 / \mathrm{min}]}\end{array}$ & $\begin{array}{c}\mathrm{M} \\
{[\mu \mathrm{Nm}]}\end{array}$ & $\begin{array}{c}\mathrm{N} \\
{[1 / \mathrm{min}]}\end{array}$ & $\begin{array}{c}\mathrm{M} \\
{[\mu \mathrm{Nm}]}\end{array}$ & $\begin{array}{c}\mathrm{N} \\
{[1 / \mathrm{min}]}\end{array}$ & $\begin{array}{c}\mathrm{M} \\
{[\mu \mathrm{Nm}]}\end{array}$ & $\begin{array}{c}\mathbf{N} \\
{[1 / \mathrm{min}]}\end{array}$ & $S D$ & $\begin{array}{c}\mathbf{M} \\
{[\mu \mathrm{Nm}]}\end{array}$ & $S D$ \\
\hline 0.10 & 952 & 0.10 & 1307 & 0.10 & 1343 & 0.10 & 1147 & 0.10 & 1197 & 0.10 & 0.001 & 1189 & 138 \\
\hline 0.16 & 1090 & 0.16 & 1580 & 0.16 & 1540 & 0.16 & 1317 & 0.16 & 1387 & 0.16 & 0.000 & 1383 & 175 \\
\hline 0.27 & 1119 & 0.27 & 1630 & 0.27 & 1557 & 0.27 & 1350 & 0.27 & 1440 & 0.27 & 0.000 & 1419 & 178 \\
\hline 0.44 & 1187 & 0.44 & 1697 & 0.44 & 1647 & 0.44 & 1420 & 0.44 & 1510 & 0.44 & 0.001 & 1492 & 181 \\
\hline 0.72 & 1333 & 0.72 & 1853 & 0.72 & 1793 & 0.72 & 1557 & 0.72 & 1667 & 0.72 & 0.001 & 1641 & 185 \\
\hline 1.18 & 1567 & 1.18 & 2087 & 1.18 & 2063 & 1.18 & 1753 & 1.18 & 1927 & 1.18 & 0.000 & 1879 & 196 \\
\hline 1.93 & 1890 & 1.93 & 2503 & 1.93 & 2433 & 1.93 & 2103 & 1.93 & 2303 & 1.93 & 0.000 & 2247 & 224 \\
\hline 3.17 & 2383 & 3.16 & 3063 & 3.16 & 2990 & 3.16 & 2593 & 3.16 & 2860 & 3.16 & 0.002 & 2778 & 254 \\
\hline 5.18 & 3087 & 5.18 & 3920 & 5.18 & 3787 & 5.18 & 3317 & 5.18 & 3683 & 5.18 & 0.001 & 3559 & 310 \\
\hline 8.48 & 4143 & 8.48 & 5187 & 8.48 & 4927 & 8.49 & 4360 & 8.49 & 4833 & 8.48 & 0.004 & 4690 & 382 \\
\hline 13.90 & 5680 & 13.90 & 7020 & 13.90 & 6583 & 13.90 & 5873 & 13.90 & 6487 & 13.90 & 0.000 & 6329 & 489 \\
\hline 22.80 & 7953 & 22.80 & 9730 & 22.80 & 8947 & 22.77 & 8050 & 22.80 & 8930 & 22.79 & 0.013 & 8722 & 656 \\
\hline 37.30 & 11333 & 37.30 & 13733 & 37.30 & 12367 & 37.30 & 11133 & 37.30 & 12467 & 37.30 & 0.000 & 12207 & 932 \\
\hline 61.07 & 16067 & 61.03 & 18600 & 61.07 & 16467 & 61.10 & 15533 & 61.03 & 16567 & 61.06 & 0.025 & 16647 & 1042 \\
\hline 100.00 & 22067 & 99.97 & 24600 & 100.00 & 22333 & 100.00 & 21467 & 100.00 & 23233 & 99.99 & 0.013 & 22740 & 1090 \\
\hline 100.00 & 21667 & 100.00 & 24800 & 99.97 & 22467 & 99.97 & 20100 & 99.97 & 22667 & 99.98 & 0.016 & 22340 & 1526 \\
\hline 69.50 & 16933 & 69.50 & 19733 & 69.50 & 17833 & 69.50 & 15400 & 69.50 & 17667 & 69.50 & 0.000 & 17513 & 1404 \\
\hline 48.30 & 12900 & 48.30 & 14967 & 48.30 & 13533 & 48.30 & 11867 & 48.30 & 13500 & 48.30 & 0.000 & 13353 & 1007 \\
\hline 33.60 & 10033 & 33.60 & 11433 & 33.60 & 10347 & 33.60 & 9450 & 33.60 & 10350 & 33.60 & 0.000 & 10323 & 645 \\
\hline 23.40 & 7690 & 23.40 & 8690 & 23.33 & 7890 & 23.37 & 7327 & 23.33 & 7877 & 23.37 & 0.030 & 7895 & 447 \\
\hline 16.20 & 5900 & 16.20 & 6700 & 16.20 & 6117 & 16.20 & 5690 & 16.20 & 6073 & 16.20 & 0.000 & 6096 & 337 \\
\hline 11.30 & 4597 & 11.30 & 5210 & 11.30 & 4797 & 11.30 & 4447 & 11.30 & 4737 & 11.30 & 0.000 & 4757 & 257 \\
\hline 7.85 & 3660 & 7.85 & 4123 & 7.85 & 3800 & 7.85 & 3530 & 7.85 & 3750 & 7.85 & 0.001 & 3773 & 198 \\
\hline 5.46 & 2930 & 5.45 & 3293 & 5.46 & 3077 & 5.45 & 2853 & 5.45 & 3027 & 5.46 & 0.003 & 3036 & 150 \\
\hline 3.79 & 2403 & 3.79 & 2700 & 3.79 & 2523 & 3.79 & 2350 & 3.79 & 2477 & 3.79 & 0.000 & 2491 & 120 \\
\hline 2.64 & 1997 & 2.64 & 2230 & 2.64 & 2097 & 2.63 & 1963 & 2.64 & 2073 & 2.64 & 0.003 & 2072 & 93 \\
\hline 1.83 & 1697 & 1.83 & 1880 & 1.83 & 1787 & 1.83 & 1667 & 1.83 & 1750 & 1.83 & 0.001 & 1756 & 75 \\
\hline 1.27 & 1467 & 1.27 & 1623 & 1.28 & 1543 & 1.27 & 1450 & 1.28 & 1520 & 1.27 & 0.003 & 1521 & 62 \\
\hline 0.89 & 1283 & 0.89 & 1410 & 0.89 & 1350 & 0.89 & 1273 & 0.89 & 1333 & 0.89 & 0.000 & 1330 & 49 \\
\hline 0.62 & 1140 & 0.62 & 1257 & 0.62 & 1207 & 0.62 & 1137 & 0.62 & 1207 & 0.62 & 0.000 & 1189 & 45 \\
\hline 0.43 & 1030 & 0.43 & 1123 & 0.43 & 1083 & 0.43 & 1022 & 0.43 & 1097 & 0.43 & 0.001 & 1071 & 39 \\
\hline 0.30 & 941 & 0.30 & 1031 & 0.30 & 992 & 0.30 & 940 & 0.29 & 991 & 0.30 & 0.001 & 979 & 35 \\
\hline 0.21 & 872 & 0.21 & 949 & 0.21 & 921 & 0.21 & 867 & 0.21 & 930 & 0.21 & 0.001 & 908 & 33 \\
\hline 0.14 & 806 & 0.14 & 877 & 0.14 & 856 & 0.14 & 808 & 0.14 & 952 & 0.14 & 0.000 & 860 & 54 \\
\hline 0.10 & 764 & 0.10 & 828 & 0.10 & 798 & 0.10 & 758 & 0.10 & 887 & 0.10 & 0.000 & 807 & 47 \\
\hline
\end{tabular}

